Masarykova univerzita

Pedagogická fakulta

\title{
Sprachkorpora
}

\section{in Unterricht und Forschung DaF/DaZ}

Tomáš Kán̆a

Brno 2014 
Redaktion: Mag. phil. Sandra Reitbrecht

Rezension: $\quad$ prof. PhDr. Peter Durčo, CSc. (Bratislava/ Trnava)

Mag. Dr. Brigitte Sorger (Wien)

\author{
(C) 2014 Tomáš Káňa \\ (C) 2014 Masarykova univerzita
}

ISBN 978-80-210-6994-7 


\section{Inhaltsverzeichnis}

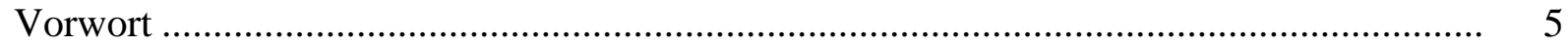

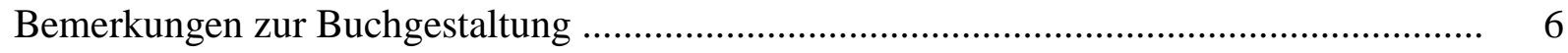

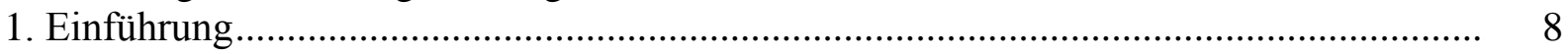

1.1 Zugang zur Sprache über Suchmaschinen ................................................... 9

1.2 Korpustools in gängigen Programmen ........................................................ 11

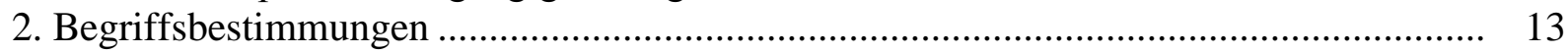

2.1. Korpus und Sprachkorpus .......................................................................... 13

2.2 Wissenschaften rund um moderne Korpora .............................................. 21

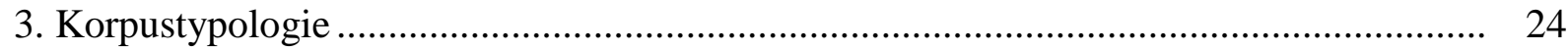

3.1 Stadium der gespeicherten Sprache ............................................................ 24

3.1.1 Synchrone Korpora .................................................................. 24

3.1.2 Diachrone Korpora …....................................................................... 25

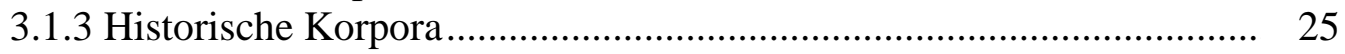

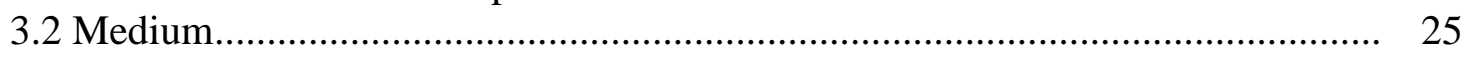

3.2.1 Korpora der geschriebenen Sprache …........................................ 25

3.2.2 Korpora der gesprochenen Sprache ............................................... 26

3.3 Repräsentativität ........................................................................... 26

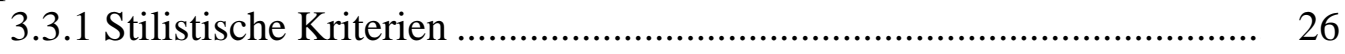

3.3.2 Kriterium der Ausgewogenheit.......................................................... 27

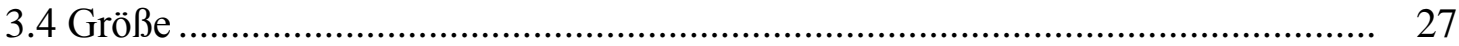

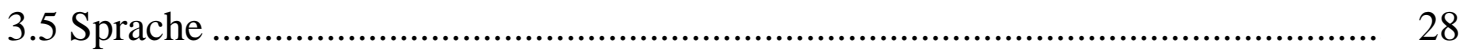

3.5.1 Monolinguale Korpora..................................................................... 28

3.5.2 Bilinguale und Multilinguale Korpora........................................... 28

3.6 Technische Eigenschaften .................................................................. 29

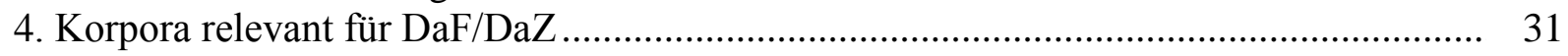

4.1 Nationale Korpora (außer mit Deutsch) .................................................... 33

4.1.1 Englisch .............................................................................. 33

4.1.2 Französisch ...................................................................... 35

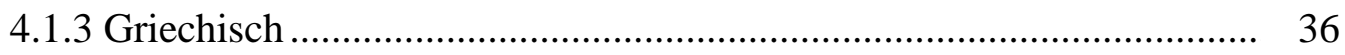

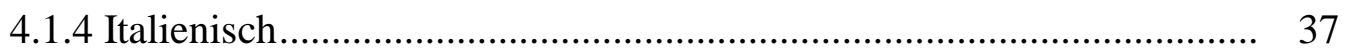

4.1.5 Kroatisch ............................................................................ 38

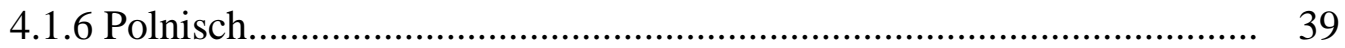

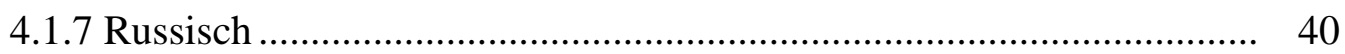

4.1.8 Slowenisch .......................................................................... 41

4.1.9 Slowakisch ............................................................................ 41

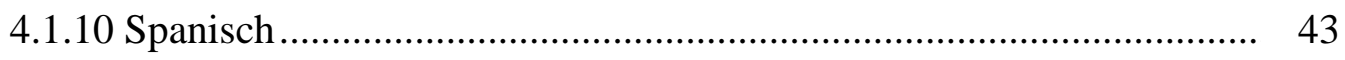

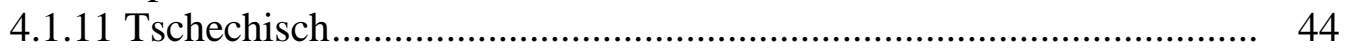

4.1.12 Türkisch ..................................................................... 45

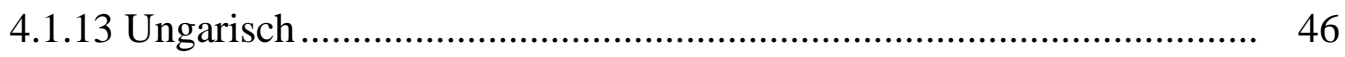

4.2 Korpora mit Deutsch ......................................................................... 48

4.2.1 Korpora mit (mehrheitlich) geschriebenem Deutsch ........................ 49

4.2.1.1 Wortschatz Leipzig ........................................................ 49

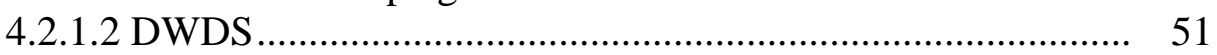

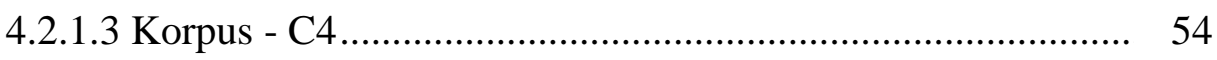

4.2.1.4 DeReKo......................................................................... 56

4.2.1.4.1 Recherche in allen Archiven ....................................... 57

4.2.1.4.2 Recherche im morphosyntaktisch annotierten Teil.......... 63

4.2.2 Korpora mit gesprochenem Deutsch ........................................... 70 


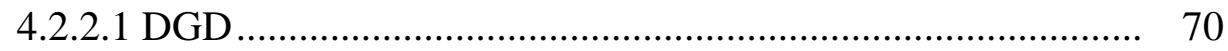

4.2.2.2 DWDS - gesprochene Sprache............................................. 73

4.2.3 Korpora mit historischen deutschen Texten ........................................ 74

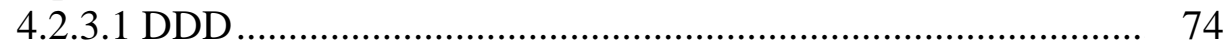

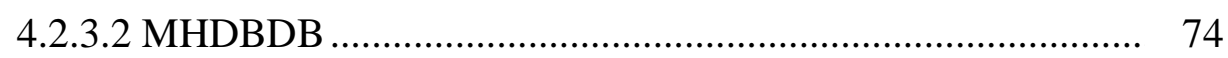

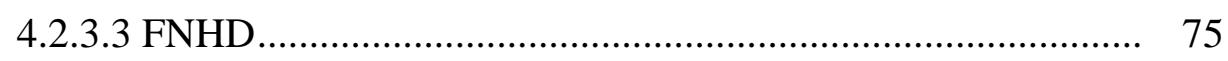

4.2.3.4 DeReKo - historische Texte) …………………………....... 75

4.2.4 Parallelkorpora mit Deutsch .............................................................. 76

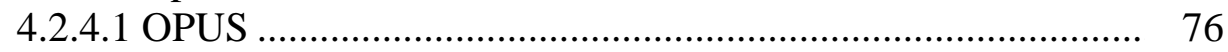

4.2.4.2 InterCorp …………………………………………..... 77

4.2.4.2.1 Texte und Sprachen des InterCorp................................... 78

4.2.4.2.2 Arbeit mit dem InterCorp................................................ 81

5. Konkordanzprogramme und korpusähnliche Instrumente …………………………….... 105

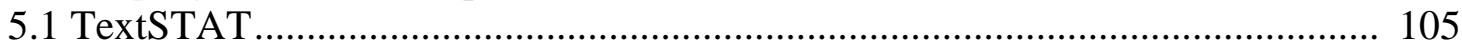

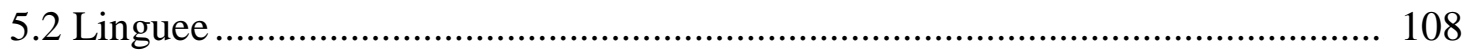

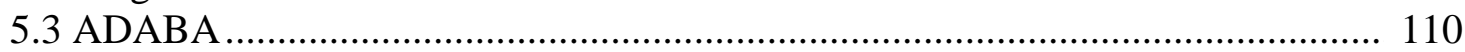

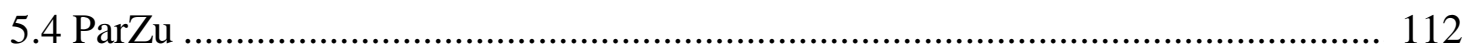

6. Korpusarbeit - Studien ……………………………………………………………. 115

Studie 1: Suche nach der ,richtigen“ Aussprache .................................................... 117

Studie 2: Suche nach dem ,richtigen“ Schriftbild..................................................... 125

Studie 3: Grammatik auf einen Klick ................................................................. 128

Studie 4: Entdeckung der Flexionsformen ......................................................... 135

Studie 5: Ermittlung einer Wortfamilie .................................................................... 140

Studie 6: Wortbildung .................................................................................... 144

Studie 7: Verben mit Zusatz ........................................................................... 146

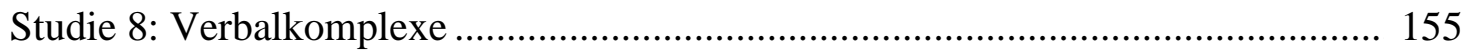

Studie 9: Präposition pro ………………………………………………......... 162

Studie 10: Kollokationen, syntagmatische Muster, Chunks...................................... 166

Studie 11: Quasi-Anglizismen.............................................................................. 177

Studie 12: Ein Blick in die Geschichte vom Hit.................................................. 180

Studie 13: Illokutionsverben............................................................................. 183

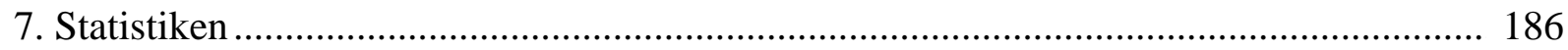

7.1 Morphologische Kategorien ....................................................................... 186

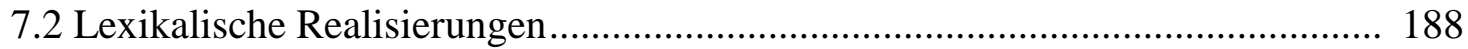

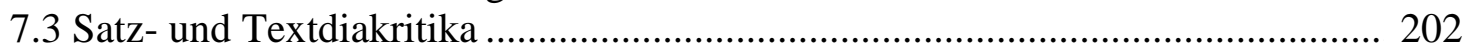

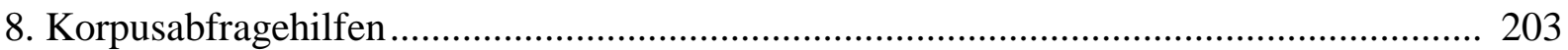

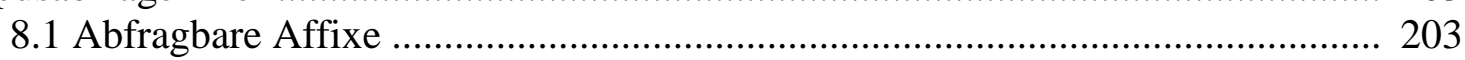

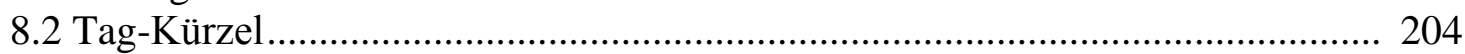

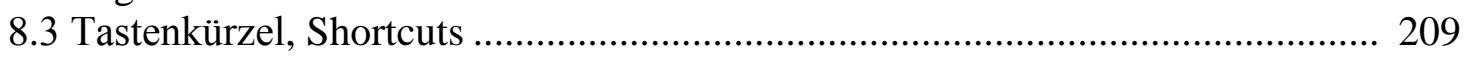

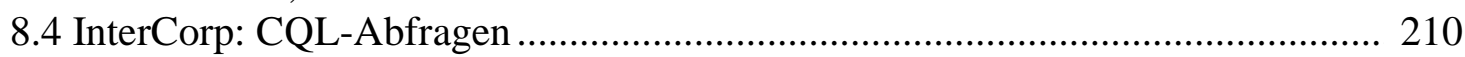

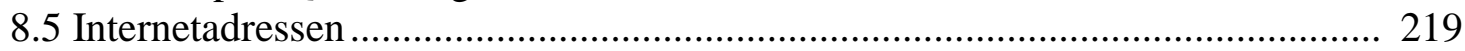

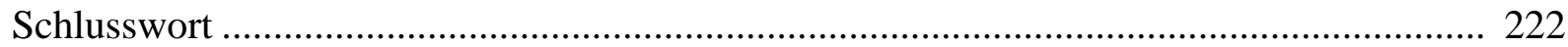

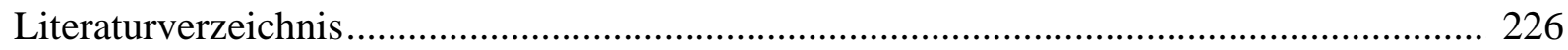

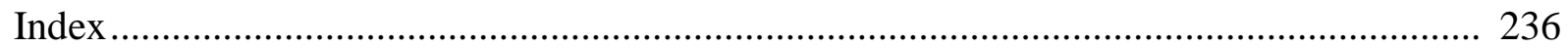




\section{Vorwort}

Ein schneller Zugang zu Informationen ist heutzutage ebenso selbstverständlich wie die allgemeine Verbreitung der Computertechnik. Beides beeinflusst auch die heutige Sprachwissenschaft und Sprach(en)vermittlung. Tatsache bleibt jedoch, dass viele Studierende, (auch junge) Wissenschaftler/-innen und (lt. Erfahrungen aus Schulen) fast alle Lehrenden die Möglichkeiten der heutigen Instrumente für Forschung und Lehre nicht kennen oder kaum verwenden. Diese Tatsache kann unterschiedliche Gründe haben, die häufigsten aber sind das fehlende Wissen von der Existenz dieser Instrumente oder die Angst vor ihrer Komplexität, ihre (vermeintliche oder auch tatsächliche) Unübersichtlichkeit und die Aufwendigkeit ihrer Nutzung. Außer Kraft sollen beide Argumente mit diesem Buch gesetzt werden, welches jedoch mehrere Ziele anstrebt:

1) einen Überblick über die wichtigsten Begriffe der Korpuslinguistik, sowie der Typologie und Eigenschaften der größten Korpora zu geben. Das Auswahlverfahren beschränke ich auf diejenigen Instrumente, die

a) dem Bereich DaF/DaZ nützlich sein könnten;

b) kontrastiven Einblick unterstützen und ermöglichen;

c) Schüler/-innen, Studierende und Lehrkräfte ansprechen können.

2) den Umgang mit Korpora einfach und transparent darzustellen und so die potentielle Angst vor den technisch oft aufwendigen Instrumenten abzubauen.

3) die Verbindung zwischen der Korpuslinguistik und DaF/DaZ herzustellen.

4) mit Studien zu einigen Phänomenen neue Erkenntnisse über die deutsche Sprache zu bringen. So leistet diese Monographie auch der (zum Teil) kontrastiven quantitativen Forschung der deutschen Sprache einen Beitrag.

In allen Punkten soll die Verbindung zu DaF/DaZ transparent und nachvollziehbar sein, auf Lerner- oder Fehlerkorpora wird jedoch nicht eingegangen. Alle Beispiele und Studien sollen auch als Anregungen für weitere und tiefere Forschungsfragen verstanden werden. Naturgemäß werden viele Aspekte ausgeblendet, einzelne Beispiele nicht bis ins letzte Detail interpretiert. Auch viele elektronische Instrumente werden nicht erwähnt, um die Übersichtlichkeit des Buches in Grenzen zu halten. In erster Linie sollen hier die vielfältigen Arbeitsmöglichkeiten mit elektronischen Instrumenten vorgestellt und die ersten Impulse für ihre Anwendung im Fremdsprachenunterricht initiiert werden.

Die technischen Anforderungen und das computertechnische Wissen der p.t. Leserschaft können minimalst sein: es reichen Basiskenntnisse im Umgang mit Computer und den gängigsten Programmen (Word, Excel, übliche Internetbrowser). Es ist keine Liebe zum PC erforderlich, sehr wohl aber die Liebe zur Sprache und eine gewisse Entdeckungslust. 


\section{Bemerkungen zur Buchgestaltung}

Diese Publikation ist auch als ein „Kochbuch“ für die Korpusarbeit gedacht und gestaltet. Es muss zwar nicht chronologisch gelesen werden, für diejenigen, die mit der Korpusarbeit beginnen, ist es jedoch ratsam.

Nach der Erklärung der wichtigsten korpuslinguistischen Begriffe (Kap. 2) wird die Korpustypologie skizziert (Kap. 3). Im Kap. 4 (Korpora relevant für DaF/DaZ) werden konkrete Korpora vorgestellt: zuerst einige nationale Korpora von Sprachen, die im mitteleuropäischen Raum erfahrungsgemäß mehr Interessent/-innen haben als andere Sprachen (4.1), dann Korpora mit der deutschen Sprache (4.2) und zum Schluss das Projekt InterCorp, in dem teilweise alle Sprachen aus den Kap. 4.1 und 4.2 integriert werden. Während die nationalen Korpora anderer Sprachen im Kap. 4.1 nur kurz vorgestellt werden, wird in den beiden folgenden Kapiteln näher auf die Funktionen der Korpora eingegangen, um die Möglichkeiten ihrer Nutzung darzustellen. $\mathrm{Zu}$ den meisten Korpora, die an verschiedenen Institutionen auf dem deutschsprachigen Gebiet entstanden sind, gibt es gute Beschreibungen in Form der Manuale oder Hilfeportale (Hinweise sind im Text). Die Beschreibungen in diesem Buch sind ,vorentlastend“ und bemühen sich, über einen einfachen Weg zu vernünftigen Rechercheergebnissen zu gelangen. Der Korpusmanager KonText (InterCorp) wird detailliert (im Kap. 4.2.4.2) beschrieben, weil zu diesem ein Manual auf Deutsch weder existiert noch geplant ist. Im Kap. 5 werden einige elektronische Instrumente beschrieben, die zwar keine Korpora sind, ihre Verwendung und Einsatz im Fremdsprachenunterricht kann jedoch ähnliche Hilfe leisten wie Korpora.

Das darauf folgende Kap. 6. (Studien) präsentiert konkrete Forschungsfragen und detaillierte Beschreibungen, wie man zu Ergebnissen kommt, und wie diese interpretiert werden können. Es ist ratsam, zuerst diese Grundinformationen über die Korpora im Kap. 4 zu lesen, im Internet nachzuschauen und dann den einzelnen Schritten im Kap. 6 zu folgen und womöglich gleichzeitig auch direkt im Internet durchzuführen.

Das vorletzte Kap. 7. (Statistiken) beinhaltet Listen mit statistischen Angaben über die deutsche Sprache, darauf (im Kap. 8) folgen Tabellen mit Kürzeln, Zeichen und Abfragemöglichkeiten, die für die Korpusarbeit erfahrungsgemäß nützlich sind.

Da sich die Schnittstellen einzelner Korpusmanager von einander gravierend unterscheiden, wurden im ganzen Buch folgende graphische Vereinheitlichungen durchgeführt, um die Bildhaftigkeit der einzelnen Schritte zu gewährleisten:

- Die Ikonen und/ oder Beschriftungen, wie sie auf dem Bildschirm erscheinen, werden in Calibri geschrieben.

- Ikonen zum Anklicken sind unterstrichen.

- Der nächste Schritt, Übergang zur nächsten Ikone oder zum nächsten Level wird durch einen Pfeil $(\rightarrow)$ markiert.

- Die Bestätigungstasten und Suchbuttons, die man anklicken muss, um zu den Ergebnissen (oder zum nächsten Schritt) zu gelangen, sind durch inverse Schrift (etwa Search) gekennzeichnet.

- Das konkrete Bild der Abfragen mit Sonderzeichen, Abfolge, Leerzeichen etc. wird in Calibri (10 Punkte) geschrieben.

- Sonderzeichen, die in den Abfragen vorkommen können, werden bei der Beschreibung der einzelnen Korpora erklärt und im Kap. 8 noch einmal zusammengefasst.

- Über Schrägstrich (/) werden im laufenden Text Synonyme oder Alternativen geschrieben. 
Konkordanzen, syntagmatische Muster und andere Belege entsprechen dem Originalschriftbild aus dem Korpus - sie wurden nicht der jetzt geltenden Rechtschreibung angepasst, in einigen Belegen ist die Tokenisierung sichtbar (z.B. Leerzeichen vor einem Punkt oder Komma).

Bei Abfragen, die anspruchsvoller sind als einfache Abfragen nach einer Wortform, werden oft sog. reguläre Ausdrücke verwendet. Diese Ausdrücke (engl. regular expressions) sowie die Syntax ihrer Eingabe ins Suchfeld sind nur teilweise standardisiert. Aus diesem Grund sind die regulären Ausdrücke nicht zusammengefasst und werden bei den Beschreibungen der Abfragen beim jeweiligen Korpus angeführt. Falls sinnvoll wird auch ihre Bedeutung erklärt. 


\section{Einführung}

Die Sprache ist für jede/-n Nutzer/-in ein selbstverständliches Kommunikationsmittel. Wir reden, schreiben, hören und lesen täglich unzählige Aussagen (Texte), über deren Form wir kaum nachdenken. Es gibt aber immer wieder Situationen, in denen wir uns die Frage stellen, ob diese oder jene Formulierung wohl richtig, üblich, normal oder akzeptabel, ja verständlich ist. Solche Fragen könnten (bzw. sollten) sich vielleicht öfter Schüler/-innen, Student/-innen und Lehrer/-innen stellen, die letzteren auch mit dem Gedanken im Hintergrund, wie sie das Wissen über die Sprache vermitteln und das Können ihrer Schüler/-innen weiterentwickeln.

Die Impulsfragen könnten auch wie folgt lauten: Wie finde ich schnell kurze Texte zu einem bestimmten Thema? Wie kann ich schnell zusätzliche Grammatikübungen angepasst an die konkrete Varietät des Deutschen zusammenstellen? Darüber hinaus stellen die Lernenden oft Fragen über die Sprache, auf die die Lehrer/-in vielleicht keine eindeutige Antwort (nicht einmal aus den Nachschlagewerken) parat hat. Solche Fragen sind in der Ganzheit der Sprache oft marginal, stellen jedoch einen Baustein im ganzen Mosaik des Systems der Sprache dar. Jede/-r Muttersprachler/-in kann zwar aus dem Stegreif Position beziehen, ob

(1) Vergleich mit oder Vergleich zu für ihn/sie ,richtig klingt““. Offen bleibt dabei allerdings, ob er/sie sich nicht womöglich vom eigenen Gefühl (Idiolekt) täuschen lässt?

Weitere aus dem ,pädagogischen Leben“ willkürlich gegriffene Fragen sind:

(2) Was ist im Deutschen gängiger: sprechen von oder sprechen über?

(3) Was entspricht den Verbindungen unter (1) und (2) im Englischen (Polnischen, Ungarischen, Türkischen)?

(4) Sagt man eher der Pool oder das Pool?

(5) Soll man jetzt eher Peking oder Beijing/ Bombay oder Mumbai sagen und schreiben?

(6) Was sagt die Norm zu (1) bis (5)?

(7) Was ist die Norm?

Die Fragen (1), (2), (4) sind typisch linguistische Fragen, die in der kommunikativen Sprach(en)vermittlung auf wenig Resonanz stoßen. Betrachtet man jedoch die Entwicklung in der Methodik der letzten Jahre, stellt man ein „Anzeichen für eine Weiterentwicklung von der kommunikativen zur kognitiven Wende [fest], wonach nicht mehr nur Sprachkönnen, als Ziel angestrebt wird, sondern das Wissen über die Sprache (wieder) in den Vordergrund rückt" (Rösch 2011:89-91). Dies bedeutet jedoch keine Rückkehr zur GrammatikÜbersetzungsmethode, sondern, wie Rösch (2011: 90) schreibt, eher „die Erweiterung des Begriffes Grammatik zur kommunikativen Grammatik“. Diesem Gedanken liegt der lexikalische Ansatz im Sinne des „lexical approach“ (Lewis 1993) nahe, in dem vorgefertigte Sprachelemente, sog. „chunks“, die Basisbausteine von Texten bilden. Somit bleibt der Text im Mittelpunkt der Sprachenvermittlung (das ist ja wichtig, denn die Kommunikation erfolgt in Texten), der Weg zur Textrezeption und -produktion wird jedoch mit solchen Bausteinen gepflastert, die über die Grenze eines Wortes hinausreichen, also mit Wortverbindungen und Phrasen. Diese Bausteine lassen sich mit den modernen (Sprach)Analyseinstrumenten relativ einfach erkunden, präsentieren und auch in Übungen umwandeln.

Viele linguistische, sprachenpolitische oder philosophische Fragen (wie die Beispiele unter (1) bis (7)) lassen sich erfolgreich mithilfe von Fachpublikationen beantworten, oft findet man die Antwort auch im Internet. Aber die einfache Frage danach, was gängiger ist, kann niemand ohne repräsentative Belege beantworten. In diesem Wort ,gängig“ steckt der Schlüssel zu Reflexionen über die Sprache an sich, aber auch über die zielführende Vermittlung von Sprache(n). Jede/-r Sprecher/-in hat zwar das Recht, die Sprache kreativ zu 
verwenden, neue, ungewöhnliche Konstruktionen zu bilden, keine/r hat jedoch das Recht zu sagen „, $X$ sagt man nicht!“ oder sogar „, $X$ ist falsch!“, wenn es gleich mehrere Belege für $X$ gibt. (Über diese streng präskriptive Phase ist hoffentlich jede demokratische Sprachenkommunität hinweg.)

Die Sprache als ein lebendes und lebendiges System ändert sich unabhängig von einem Individuum. Es ist ein System, das sich durch das Wiederholen der Elemente kennzeichnet. In anderen Worten: je öfter etwas $(X)$ gesagt wird, desto größer ist die Wahrscheinlichkeit, dass dieses $X$ von der Kommunität als richtig empfunden wird und (irgendwann auch) in normativen Werken standardisiert werden kann.

Die Betrachtungen einer Fremdsprache sollen sich auf diejenigen Aspekte konzentrieren, die allgemein gültig, verständlich und üblich sind. Diesem Ansatz folgend werden in diesem Buch Beispiele präsentiert, wie man sich die Arbeit mit der Erkundung der Sprache und der Erschließung einzelner Elemente erleichtern kann. Dazu kommen auch sprachkontrastive Einblicke auf einzelne Phänomene. So können auch Interferenzen oder Transfererscheinungen aufgedeckt werden.

\subsection{Zugang zur Sprache über Suchmaschinen}

Vieles lässt sich mit Suchmaschinen (z.B. Google, Altavista, Yahoo!) auch über die Sprache(n) finden: interessante Artikel, Wörterbücher, Diskussionsforen über sprachliche Elemente, Unterrichtsmaterialien u.a., wie es auch die folgenden Beispiele verdeutlichen.

Einmal wollte ich wissen, ob die Wortzusammensetzung KorruptionsUntersuchungsausschuss auch außerhalb der österreichischen Realität existiert: am 16.4.2012 fanden sich unter Google.com 27.000 (!) Nachweise . Aus den Homepage-Adressen der ersten Seiten ließ sich erahnen, dass es sich hauptsächlich um österreichische Medien und Portale handelte (Domäne: .at).

Dieselbe Abfrage stellte ich am 9.1.2014 an Google.com. Die Suche ergab „nur“ über 22.400 „Treffer“, bei yahoo.at (erweitert auf die ganze Welt) lediglich 2.620 Ergebnisse. Leider ist aus diesen Angaben nicht ersichtlich, ob das Wort (noch) im Umlauf ist, wie sich die Frequenz veränderte, in welchen Texten und wie oft es vorkommt.

Aus der schrumpfenden Anzahl der Belege lässt sich nur erahnen, dass der Begriff nicht mehr so gebräuchlich ist. Viele Artikel sind offensichtlich aus dem Internet verschwunden. Es lässt sich auch nicht überprüfen, ob die Anzahl von 27.000 im Jahr 2012 tatsächlich gestimmt hat, ob ich mich vielleicht um eine Null nicht vertippt habe.

In einem Sprachkorpus (DeReKo) war am 16.4.2012 kein einziger Treffer. Zwei Jahre später, (9.1.2014) fand ich zwar nur 37 Treffer, aber auch aus diesen Daten lassen sich bereits Informationen ableiten, die (anhand der geringen Belegmenge) mit etwas Vorsicht Folgendes aussagen:

a) Die Wortkopplung Korruptions-Untersuchungsausschuss kommt fast ausschließlich in österreichischen Texten vor.

b) Weniger überraschend ist, dass es sich um Zeitungstexte über Politik handelt.

c) Am häufigsten wurde der Wortkomplex im März 2012 verwendet.

Es sind zwar keine überraschende Ergebnisse, es sind aber Beweise über das gesuchte Wortgeschöpf. 
Als ein Problempunkt tauchte in einem DaF-Seminar die Frage nach der korrekten Präposition auf: Ist die Verbindung im Vergleich mit (etwas) oder im Vergleich zu (etwas) richtig? Die übliche Praxis sagt: man sucht auf gut Glück, was es gibt: ins Google-Suchfeld schreibt man „Vergleich mit oder zu“ und bekommt Links zu mehr oder weniger seriösen Artikeln oder Foren.

Im Forum Deutsch als Fremdsprache (1996-2012) löste man die Problematik folgend: Frage:

Im Vergleich mit/oder zu?

geschrieben von: Hussein ()

Datum: 18. Mai 2009 19:25

Hallo zusammen,

man hat mich korrigiert, in dem man den Satz "Im Vergleich mit..." durch den Satz "Im Vergleich zu..." ersetzt hat. Könntet ihr mir mal sagen, was der Unterschied dazwischen ist. Im Wörterbuch stehen aber beide Möglichkeiten.

Antwort:

Re: Im Vergleich mit/oder zu?

geschrieben von: oberhaenslir ()

Datum: 18. Mai 2009 23:02

Standarddeutsch: etwas mit etwas vergleichen; jemanden mit jemandem vergleichen

Korrekt ist also 'im Vergleich mit':

"Schweizer Hochschulen halten im Vergleich mit Deutschland und Österreich gut mit."

Falsch ist 'im Vergleich zu':

"Wie schneidet ein heutiges Gerät im Vergleich zu einem Geschirrspüler vor zwanzig Jahren beim Wasserverbrauch ab?"

Andere Forumsteilnehmer versuchen die Antwort zu relativieren:

Re: Im Vergleich mit/oder zu?

geschrieben von: Franziska ()

Datum: 19. Mai 2009 16:51

Ich glaube, heutzutage wird im Alltag mehr "Im Vergleich zu" verwendet - das könnte ein Grund für die Korrektur sein, Hussein. Aber sicher sind beide richtig. Dieses "zu" drückt wohl mehr die Richtung aus, in die der Vergleich abzielt. Es klingt ein bisschen energischer. "Mit" klingt gleichwertiger, freundlicher.

"Im Vergleich zu mir hat er keine Ahnung, ha!"

"Im Vergleich mit dieser Teesorte schmeckt die andere etwas lieblicher."

Aber das ist nur so ein Gefühl - bevor wieder Jeros obligatorisches "Hm" kommt.

Es herrscht also Unsicherheit. Ob Hussein jetzt weiß, was er das nächste Mal schreiben soll, ist zu bezweifeln. Die Frage bleibt unbefriedigend beantwortet.

Die Google-Suche nach den genauen Verbindungen ,im Vergleich mit“ und ,im Vergleich zu“ zeigt ziemlich deutlich, dass die Verbindung mit der Präposition $z u$ überwiegt (187 Mio. Suchergebnisse zu 51 Mio. Suchergebnissen mit der Verbindung im Vergleich mit). Einige von den Ergebnissen repräsentieren Artikel, Foren und Polemiken zu dieser Problematik (wie hier oben angeführt), für das wahre Bild der Verbindung sind sie also irrelevant. Texte, in denen diese Verbindungen in einem natürlichen Kontext vorkommen, sind schwer herauszufiltern. Dabei lässt sich das Problem anhand von einigen realen Textpassagen induktiv ziemlich schnell und zufriedenstellend beantworten:

Sie wiegen ja viele Tonnen, und im Vergleich

Das Wasser war kalt im Vergleich

Es war sogar ganz außerordentlich klein im Vergleich wird festgelegt, und dadurch im Vergleich

- doch im Vergleich

Die glauben doch, daß im Vergleich zu ihrer Größe ist das Gehirn relativ klein.

zu der warmen Luft

zu anderen Ländern, wie zum Beispiel Deutschland

zu allen anderen,

zu der Macht, die

zu ihrer Arbeit alles andere völlig bedeutungslos ist 
Es waren freie und glückliche Jahre im Vergleich

Ein Übel, das nichts ist im Vergleich

Aber dieser Tick war gar nichts im Vergleich

manche der Aussagen des Thomas Morus wirken im Vergleich zu den Zeiten, die mich im Kloster erwarteten,

mit dem, was dich in der Hölle erwartet,

zum Verhalten seines Vaters, der sich...

mit diesem 20. Jahrhundert frappant

Aus diesen zehn (zufällig aus 1.533 ausgewählten) Beispielen aus dem Korpus InterCorp ist auf den ersten Blick ersichtlich, dass

1) kein Beleg aus einer linguistischen Polemik über die Konkurrenzformen stammt;

2) beide Präpositionen gebraucht werden;

3) mehrheitlich $z u$ verwendet wird. Das Verhältnis ist nicht 2:8, wie es hier den Anschein erweckend dargestellt ist, sondern etwa 1:100. Die Verbindung im Vergleich mit ist also eher selten.

Es ist unumstritten, dass die Suchmaschinen wertvolle Daten liefern, aus denen man sich einen ersten Überblick verschaffen kann: sie sind blitzschnell, liefern eine große Menge von Ergebnissen, darüber hinaus sind die Daten höchstaktuell - entsprechen dem momentanen Zustand im Internet. Aus den Ergebnissen der Internetsuchen kann man bestimmt sehr gut die erste Orientierung auch über sprachliche Phänomene gewinnen ${ }^{1}$. Zumindest die simple Verifizierung, ob das Abgefragte existiert oder nicht, kann wertvoll sein. Ob man aus Internetbelegen Beispiele zum Sprachgebrauch präsentieren, Übungen effektiv zusammenstellen und überhaupt die Sprache mit bloßen Internetrecherchen systematisch erkunden kann, ist jedoch ernsthaft anzuzweifeln.

\subsection{Korpustools in gängigen Programmen}

Ein „Korpus“ kann jeder durchschnittliche PC User erstellen und so die Arbeit mit den Daten üben.

Die Vorstellung von den elementaren Korpusfunktionen verschafft man sich über einige Tools heute gängiger Computer-Programme:

MS-Word hat beispielsweise diese sprachlichen Tools:

- auf der Registerkarte Überprüfen in der Gruppe Dokumentprüfung $\rightarrow$ Wörterzählen gibt es statistische Angaben zum Text: Seiten, Absätze, Zeilen, Zeichen (mit oder ohne Leerzeichen) Rechtschreib- und Grammatikkorrektor

- Suchen von Wort(teilen) über die Tastenkombination Strg+F

- (Übersetzungsdienste nur online)

Google-Suche verfügt unter Erweiterte Suche über diese Suchmöglichkeiten:

Suche nach

- all diesen Wörtern: Diese Funktion ermöglicht teilweise lemmatisierte Suche, d.h. die Abfrage kalt UND kochen ergibt auch kalten (Tee), kalte (Küche) etc. und weiter im Text kochen.

- der exakten Phrase

- einem/ keinem dieser Wörter

1 Die Validität der Ergebnisse lässt sich dann etwa mit dem Informationswert der Wikipedia-Artikel vergleichen: Vieles entspricht der Realität, ist aber manchmal einseitig, nicht genügend belegt oder zu oberflächlich. 
Diese Tools sind hilfreich für die Suche nach dem gewünschten Thema. Was die Ermittlung der Sprache anlagt, haben die Programme allerdings ziemlich eingeschränkte Möglichkeiten. Dem ist es so aus dem einfachen Grund: sie sind nicht primär für Fragen über die Sprache per se bestimmt.

Die wichtigsten Unterschiede zwischen einer Suchmaschine und einem Korpus bezüglich der Spracherkundung sind in der folgenden Tabelle zu sehen.

\begin{tabular}{|c|c|c|}
\hline & Suchmaschine & Korpus \\
\hline Geschwindigkeit & schnell & oft langsam \\
\hline Benutzerfreundlichkeit & einfach, intuitiv & $\begin{array}{l}\text { oft kompliziert, ohne Einleitung } \\
\text { kaum verständlich }\end{array}$ \\
\hline Daten(Text)menge & riesig & oft (sehr) eingeschränkt \\
\hline Wesen der Sprache & nur geschrieben & $\begin{array}{l}\text { oft nur geschrieben, aber auch } \\
\text { gesprochen }\end{array}$ \\
\hline Sprache der Texte & eingeschränkt eindeutig & eindeutig \\
\hline Originalsprache d. Texte & kaum feststellbar & eindeutig feststellbar \\
\hline Zeit der Verfassung & kaum feststellbar & eindeutig feststellbar \\
\hline Textfunktion & keine Angaben & (eindeutig) zuordenbar \\
\hline Verfasser/-in(nen) & schwer feststellbar & relativ eindeutig \\
\hline $\begin{array}{l}\text { Strukturiertes Suchen im } \\
\text { Text (in Texten) }\end{array}$ & nicht möglich & möglich \\
\hline Suche nach Wortteilen & eingeschränkt möglich & möglich \\
\hline Statistische Angaben & $\begin{array}{l}\text { fast keine (nur Anzahl der } \\
\text { Ergebnisse) }\end{array}$ & vielfältig \\
\hline
\end{tabular}

Tab. 1: Vergleich einiger Eigenschaften: Suchmaschine - Korpus

Die Unterschiede werden auch aus den Bestimmungen der Instrumente klar: eine Suche im Internet ist der Suche in einem Bibliothekskatalog oder einer Enzyklopädie ähnlich. Dort findet man jedoch (fast) keine sprachlichen, grammatikalischen Informationen über die gesuchten Begriffe. Dafür gibt es andere Quellen: Wörterbücher und Grammatiken. Korpora hingegen sind keine Suchmaschinen nach Themen, sondern nach sprachlichen Erscheinungen, die dann interpretiert werden müssen.

Beide Instrumente können sich in der Sprachforschung und -vermittlung gut ergänzen: die Internetsuchmaschinen liefern „frische“ Daten über den Stand der Internetsprache, Korpora können wiederum gut auch über (vergangene) gesellschaftliche Themen informieren: Schaut man im Korpus ${ }^{3}$ nach dem Wort Impeachment nach, stellt man sofort fest, womit sich die Welt (unter anderen wichtigen Ereignissen) in den Jahren 1998 und 1999 befasst hat. Google bietet heutzutage (nur) Hinweise zu Seiten mit Erklärungen dieses Wortes.

2 Z.B. Suche nach zwei Wörtern in einem Satz, oder Wortanfang/-ende, nach grammatikalischen Angaben (z.B. nur Adjektive, die auf - $e l$ enden).

3 DeReKo: W - Archiv der geschriebenen Sprache. 


\section{Begriffsbestimmungen}

Für ein reibungsloses Verständnis des Inhalts dieses Buches werden hier die wichtigsten Begriffe definiert und erklärt, wie sie in der Korpuslinguistik üblicherweise verstanden werden. In diesem Kapitel werden sie thematisch geordnet: von Korpus und Sprachkorpus ausgehend bis zu einigen korpustechnischen Begriffen wie Parser oder Alignment, die für die Recherche zwar entbehrlich sind, in Korpusmanuals oder in der Fachliteratur jedoch oft vorkommen. Alphabetisch werden die einzelnen Begriffe noch einmal (mit einer kurzen Definition) im Register angeführt.

\subsection{Korpus und Sprachkorpus}

Ein Korpus ist lateinisch „Körper“. Selbst das deutsche Wort Körper ist eine sprachliche Entlehnung aus dem Lateinischen und fungiert laut Kluge (2002: 530) in der deutschen Sprache seit dem 13. Jh.:

„....mhd. korper, körper, fnhd. auch körpel mit Dissimilierung des zweiten $r$ Entlehnung. Entlehnt aus 1. corpus (-poris) n. "Leib" (der spätere Umlaut ist nicht ausreichend erklärt). Ersetzt die älteren Wörter Leib und Leiche.“

In der heutigen deutschen Sprache gehen mehrere Lexeme auf diese gemeinsame Wurzel (Etymon) zurück: Körper, Korps, Korpuskel, korpulent, und natürlich auch Korpus oder Corpus. Laut DUDEN (1996: 885-886 und 2006 [CD-ROM]) ist der Korpus die Bezeichnung des menschlichen Körpers) und das Korpus eine Sammlung bzw. der Körper eines Instruments:

${ }^{1}$ Korpus, der; -, -se [lat. corpus, $\uparrow$ Körper]: 1. (ugs. schrezh.) menschlicher Körper ... 2. (bild. Kunst) Christusfigur am Kruzifix. 3. <o. Pl.> (Fachspr.) (bei Möbeln) das massive, die eigentliche Gestalt ausmachende Teil ohne die Einsatzteile ... 4. (schweiz.) Ladentisch; [Büro]möbel mit Fächern ...;

${ }^{2}$ Korpus, Corpus, das; -, Korpora bzw. Corpora [lat. corpus, = Gesamtwerk, Sammlung...]: 1. (Sprachw.) Sammlung einer begrenzten Anzahl von Texten, Äußerungen o. Ä. als Grundlage für sprachwissenschaftliche Untersuchungen. 2. <heute meist: der; o. Pl.> Klangkörper bes. eines Saiteninstruments;

${ }^{3}$ Korpus, die; (Druckw.) Schriftgrad von 10 Punkt; Garmond.

Im Kontrast $\mathrm{zu}$ den angeführten Angaben bringt eine Recherche im größten deutschen Korpus/ Corpus Ergebnisse, die auf den folgenden Gebrauchsusus hindeuten:

- Das Schriftbild mit „C“ kommt in modernen deutschen Texten der letzten 20 Jahre nur in einer lateinischen Verbindung vor: z.B. Corpus delicti, Corpus Christi, Ave verum corpus, Corpus iuris Civilis.

- Bis auf Corpus Christi ist das Lexem immer nur sächlich. In der Verbindung Corpus Christi, bzw. nur elliptisch Corpus ist es ausschließlich männlich ${ }^{4}$.

- Korpus mit $K$ geschrieben ist fast immer maskulin, egal ob es sich um einen menschlichen Körper, das Geschöpf eines Instrumentes oder eine Sammlung handelt (alle folgenden Belege sind aus dem DeReKo, W - Archiv der geschriebenen Sprache):

Die rhythmisch pointierte Sprache der eigenwilligen Geige kommt am eindrucksvollsten bei ihren perkussiven Elementen zur Entfaltung, wenn er die Saiten zupft und den Korpus als Trommel zusammenwirken läßt.

Eine repräsentative und hinreichend große Menge an verfügbaren schriftlichen Quellen, genannt der Korpus, aus einer Sprache wird als Grundlage für die Auswertung verwendet.

4 Ein Beleg aus einem Dutzend im DeReKo: Das neue, fast drei Meter hohe Kreuz ist aus Holz und trägt keinen Corpus. 
Deswegen wird auch in manchen Kinos von Wolfram Weber weiter ein analoger Zelluloid-Projektor stehen. Sogar wenn der gesamte Korpus der Filmgeschichte einst digitalisiert sein sollte.

- Ein seltener Fall ist das neutrale Genus von Korpus im Sinne einer Sammlung, wie das folgende Textbeispiel zeigt:

Kurt Ostbahn. [...] Nach drei überfüllten Abenden en suite hängt der echteste aller Wiener noch zwei Auftritte im "Zelt" an. Der Doppler nennt sich das Ereignis, bei dem das gesamte historische Korpus des Kurti-Schaffens - auf zwei Konzerte verteilt - ausgebreitet werden wird, natürlich ohne auch nur eine Nummer doppelt zu spielen.

Der nächste interessante Punkt im oben genannten Lexikoneintrag (Duden Universalwörterbuch 1996, und in allen späteren Versionen) stellt die Bedeutungserklärung dieses Lexems dar. An der ersten Stelle (dies bedeutet wohl die häufigste Verwendung) steht:

1. (ugs. schrezh.) menschlicher Körper ...

Sieht man das typische Umfeld ${ }^{5}$ von Korpus in der geschriebenen deutschen Sprache an, findet man keinerlei Hinweise auf umgangssprachliche oder scherzhafte Elemente:

ein gusseiserner Korpus

Wegekreuz ... mit|bezeichnet ... Korpus bezeichnet ...

der|die Saiten und|auf den Korpus

spätbarockes Friedhofskreuz mit Korpus ... 1755

Friedhofskreuz mit Korpus

einen ... hohlen [...] Korpus der

mit hohlem Korpus

ein Kruzifix [mit ...] Korpus

des|Hohler Korpus ... die|der ... Zargen mit Tonabnehmer

mit massivem [...] Korpus

Trotz der Diskrepanz zwischen der Norm und dem realen Gebrauch wird in dieser Monographie die pragmatische Lösung verfolgt, nämlich konsequent der Terminus das Korpus verwendet, weil in der korpuslinguistischen Fachliteratur das neutrale Geschlecht und die Schreibweise mit $K$ immer noch überwiegen. Mit Korpus ist hier nur das Sprachkorpus gemeint.

Der heutige Begriff Sprachkorpus bezeichnet eine elektronische Sammlung/ Datenbank natürlicher Texte, die (meist) in voller Länge gespeichert wurden ${ }^{6}$. Diese Datenbank ist strukturiert und mit einem Korpusmanager versehen. Diese zwei Eigenschaften heben ein Korpus von einer einfachen Textdatenbank oder von einer elektronischen Bibliothek ab. (Dazu auch Káňa/ Peloušková 2005 und McEnery/ Wilson 2001: 30). Als ein Sprachkorpus kann nicht jede (wenn auch wertvolle) elektronische Datenbank bezeichnet werden. Die Mindestanforderungen an die Suchmöglichkeiten in einem Sprachkorpus sind: Abfragen nach Wörtern und Wortteilen, statistische Angaben über die Ergebnisse, Angaben über die Größe und Zusammenstellung des Korpus. Ein Sprachkorpus ist auch kein Wörterbuch, reflektierte User können es jedoch als solches verwenden.

Ein Sprachkorpus ist also eine elektronische Textdatenbank, in der man effektiv nach sprachlichen Phänomenen suchen kann.

Sog. ,syntagmatische Muster“ (Belica 1995).

Die Vorreiter elektronischer Korpora waren Kartotheken, in denen nur Textsequenzen eingetragen wurden. 
Sprachkorpora hat es schon lange im vorelektronischen Zeitalter gegeben. Unentbehrlich waren sie für die Erstellungen seriöser Wörterbücher, wie im Vorwort zu Band 1 des Grimmschen DWB (1854-1961: XXXVII) angeführt wird:

„Wörter verlangen beispiele, die beispiele gewähr, ohne welche ihre beste kraft verloren gienge. wie könnten stellen (loci) heiszen, deren stelle ungenannt bliebe? der name ihres urhebers reicht nicht aus, sie müssen aufgeschlagen werden können; aus der leichtigkeit dieses nachschlagens entspringt ein groszer reiz, denn wie genau auch die belege ausgehoben seien, der leser hat nicht selten das bedürfnis sie in ihrem vollständigeren zusammenhang einzusehen: indem er weiter vordringt, findet er dicht neben den beigebrachten ausdrücken noch etwas anderes, unmitgetheilt gebliebenes, wodurch ihm das verständnis vollends erschlossen wird. auch in der classischen philologie ist es hergebracht die quelle anzuführen, aus der entnommen wurde. unbelegte citate sind unordentlich zusammengerafte, unbeglaubigte, unbeeidete zeugen.“

Prinzipiell wurde jede Sammlung von Texten, die für Zwecke sprachlicher Untersuchungen zusammengestellt wurde, als ein Sprachkorpus bezeichnet. In der heutigen üblichen Verwendung beschränkt sich der Begriff auf elektronische Sprachkorpora.

Ein Korpusmanager („Korpussuchmaschine“) ermöglicht eine effektive Suche nach sprachlichen Elementen. Korpusmanager sind aufwendige Text-Analyse-Systeme, die gezielte, auch kompliziertere linguistische Recherchen ermöglichen.

Die Suche erfolgt in Form einer Abfrage. Allerdings ist die Sprache des Korpusmanagers anders als die „normale“ Sprache. Deswegen muss die Abfrage in die Korpussprache „,̈̈bersetzt“" werden. Die Sprache heißt corpus query language (CQL).

CQL (Corpus Query Language) ist eine spezielle „Sprache“, in der man mit dem KorpusManager (z.B. CQP - Corpus Query Processor) kommuniziert, d.h. die Abfrage erstellt. Für die Abfragestellung gibt es (je nach Korpusmanager) entweder die sog. „graphische“ Eingabe oder die ,zeilenorientierte ${ }^{7 *}$ Eingabe der Abfrage. Die graphische Eingabe wird durch Klicken auf Ikonen, die zur Auswahl stehen, zusammengestellt. Die zeilenorientierte Eingabe der Abfrage wird direkt ins Suchfeld getippt.

Die Formulierung der Abfragen ist fast in jedem Korpusmanager anders. Ohne Basiswissen über das Korpus, den Korpusmanager und dessen Eigenschaften ist eine kompliziertere Abfragestellung oft schwierig, manchmal sogar unmöglich. Aus diesen Gründen muss jeder Nutzer die Manuale (falls sie vorhanden sind) der Korpusmanager studieren, die Eigenschaften kennen oder einfach probieren, was geht. (Diesen langwierigen Prozess soll dieses Buch erleichtern und gleichzeitig dafür plädieren, dass das Bassiswissen über diese Instrumente in jedem Sprach(en)unterrricht verbreitet wird.)

Der Begriff Abfrage beinhaltet 1) die Einstellung der Suche, 2) die Einstellung der Ergebnispräsentation und 3) die Formulierung der Frage an den Korpusmanager im Suchfeld (Suchfeldeingabe).

Die Suchfeldeingabe (oder nur Eingabe) ist eine Kombination von Graphemen, Zeichen und anderen Elementen, die ins Suchfeld des Korpusmanagers eingegeben werden muss, um die gewünschte sprachliche Erscheinung aus den Korpustexten abrufen zu können.

7 Begriff, der in COSMAS II verwendet wird. 


\section{Übersicht der Abfragemöglichkeiten}

Die Möglichkeiten der Abfrage sind grundsätzlich diese:

Suche nach

1) einzelnen Wörtern bzw. Zeichenketten, die ein Bestandteil eines Wortes sind

2) Grundformen

3) Wortkombinationen

4) Kombinationen (von Wörtern, Zeichenketten etc.) mit Abstand voneinander

5) morphosyntaktischen Kategorien (in Kombination mit Abstand)

6) Satzelementen

Die Möglichkeiten der Suchabfragen variieren nach Korpustyp. Als Ergebnis einer Korpusabfrage erscheinen auf dem Bildschirm Konkordanzen.

Die Konkordanzen in der Korpuslinguistik ${ }^{8}$ sind Ergebnisse einer Suchanfrage. Es sind Belege aus den Korpustexten. Sie erscheinen in Form einer Konkordanzzeile (Abb. 1), in der Mitte steht das gesuchte Element vom umliegenden Text graphisch hervorgehoben (in der Abb. 1 Schaf), genannt KWIC (steht für Key Word In Context), oft wird es auch Node oder Treffer genannt. Rechts und links auf derselben Zeile sind Wörter aus der Umgebung im Text:

\begin{tabular}{|rll|}
\hline Wenn sie oben am Hügel standen, sahen sie & Schafe & und Ziegen auf einem grünen Rasen springen . \\
Der Pater begann zu glauben, das gute & Schaf & aus des Herren Herde sei nicht richtig im Kopf \\
, ich bin das schwarze & Schaf & der Familie, ein Windhund. \\
(...) erfuhr Esteban Trueba von dem schwarzen & Schaf & in seiner Familie nur durch den (...) Briefwechsel \\
Gib mir Kraft, die Sünderin zu bessern, das verirrte & Schaf & in deine Herde zurückzubringen!
\end{tabular}
Abb. 1: Konkordanzen zur Abfrage Schaf (InterCorp_de)

$\mathrm{Zu}$ diesen Konkordanzen gibt es auch Angaben über die Texte, aus denen sie stammen. Sie werden jedoch (je nach Korpusmanager) unterschiedlich ausführlich angegeben.

$\mathrm{Zu}$ den ersten Texten, die als „Sprachkorpora“ verwendet wurden, zählt die Bibel. Um in der Heiligen Schrift besser suchen zu können, wurden „Konkordanzen“ erstellt: ein Verzeichnis der Wörter mit Kennzeichnung der Stelle, wo sie vorkommen. Die erste Konkordanz entstand zur Vulgata Anfang des 13. Jahrhunderts, später folgten Konkordanzen zu den BibelÜbersetzungen. Heute ist die Suche in mehreren deutschen Übersetzungen auch online möglich - beispielsweise über Bibel Online (2014), wie die Abb. 2 zeigt:

1.) Und ich will meiner Herde helfen, daß sie nicht mehr sollen zum Raub werden, und will richten zwischen Schaf und Schaf. (Hesekiel 34.22)

2.) Aber zu euch, meine Herde, spricht der Herr, HERR also: Siehe, ich will richten zwischen Schaf und Schaf und zwischen Widdern und Böcken. (Hesekiel 34.17)

3.) Wenn jemand einen Ochsen oder ein Schaf stiehlt und schlachtet's oder verkauft's, der soll fünf Ochsen für einen Ochsen wiedergeben und vier Schafe für ein Schaf. (2. Mose 21.37)

4.) Es sei ein Ochs oder Schaf, so soll man's nicht mit seinem Jungen auf einen Tag schlachten. (3. Mose 22.28) Abb. 2: Konkordanzen der Abfrage Schaf, Bibel Online (Übersetzung: M. Luther, Ausgabe 1912).

Diese modernen Instrumente verwenden für die Suche spezielle Konkordanzprogramme. Es sind elektronische Instrumente für die Suche nach Wortformen in beliebigen elektronischen

8 Das Wort Konkordanz hat mehrere Bedeutungen. In der Schweiz wird mit Konkordanz u.a. die Koalitionsregierung bezeichnet. 
Texten. Auch die Basis jedes Korpusmanagers bildet ein Konkordanzprogramm. Das Ergebnis der Suche mit einem Konkordanzprogramm sind Konkordanzen in Konkordanzzeilen. (Im Internet findet man unter den Begriffen Konkordanzprogramm oder concordancer viele Konkordanzprogramme mit unterschiedlich komplexer Bedienung und diversen Funktionen.)

Bei der Auswertung der Konkordanzen/ Treffer sind weitere Funktionen der Korpusmanager wichtig, die die Interpretation der gewonnenen Daten erleichtern oder gar erst ermöglichen. Die üblichsten Funktionen, die in den meisten Korpora vorkommen, sind: Filter, Frequenzund Kollokationsanalysen.

Der Filter ermöglicht die Belege zu sortieren. Prinzipiell kann man positive und negative Filter einsetzen.

In jedem Korpus gibt es mehrere Angaben zu den Frequenzen (Häufigkeitsmaße) von KWIC. Grundsätzlich existieren in jedem Korpus zwei Frequenzgruppen:

1) Frequenzangaben zum KWIC:

absolute Frequenz: exakte Anzahl des Vorkommen (der Treffer) im Korpus;

relative Frequenz: Angabe über das Vorkommen bezogen auf eine Menge (z.B. die Gesamtgröße des Korpus oder das absolute Vorkommen des gesuchten Elements). In vielen Korpora wird der (hypothetische) Richtwert pro eine Million Worte (p.m.W.)/ instances per million (i.p.m.) berechnet.

Häufigkeitsklassen: Werte von 0 bis n. Die Häufigkeitsklassen werden oft auf das Vorkommen des am meisten frequentierten Wortes in der Sprache bezogen: je kleiner die Ziffer, desto wahrscheinlicher ist das Vorkommen des Wortes im (beliebigen) Text. Das Zentrum des Wortschatzes (allgemein verständliche, hochfrequentierte Wörter) fällt in die Häufigkeitsklassen 0 bis 13 (insgesamt 6.700 Grundformen). Wörter mit der Häufigkeitsklasse höher als 18 kann man als äußerst selten bezeichnen.

Die Häufigkeitsklasse 0 haben im Deutschen die Wörter der, die und und (vgl. DeReWo 2009).

2) Frequenzangaben des KWIC zu anderen Wörtern:

Kookkurrenzen, bzw. Kollokationen.

Obwohl die Kookkurrenz als ein gemeinsames Erscheinen (ohne Betonung der semantischen Bindung) verstanden wird und die Kollokation als eine semantische Verbindung mehrerer Elemente (vgl. auch Durčo 1994: 16) definiert werden, erscheinen in der korpuslinguistischen Literatur beide Begriffe mehr oder weniger als Synonyme.

Die Berechnung der Kookkurrenzen/Kollokationen zeigt das typische Umfeld des KWIC. Ein übersichtliches Tutorial zur Kookkurrenzanalyse ist auch auf der Homepage des IDSMannheim (siehe Belica 1995) zu finden.

Die Kollokationen werden nach unterschiedlichen (statistischen) Formeln berechnet, die den Kollokationswert/ das Signifikanzmaß ergeben. Dieser Wert (jeweils eine Nummer) kann man sich als ein Signal für die Wahrscheinlichkeit des gemeinsamen Vorkommens vom KIWC und seinem Kollokationspartner (Kollokator) vorstellen. Je nach den Variablen, die in diese Berechnung einbezogen werden (z.B. Größe des Korpus, Häufigkeit des KWICs und/ oder 
des Kollokationspartners, Anzahl der Dokumente im Korpus etc.), und nach dem Algorithmus, der die Signifikanz berechnet, werden auch die Signifikanzmaße benannt: mutual information score (MI-score), test score (T-score), log likelihood ratio (LLR) um nur die üblichsten zu nennen. Die Werte werden bei den Kookkurrenz-/Kollokationsanalysen in jedem Korpus automatisch mitgeliefert (siehe auch die Beschreibung von DWDS, DeReKo und InterCorp).

Mi-Score (mutual information) ist ein Wert, der die Wahrscheinlichkeit der Kookkurrenz von KWIC und dem Kookkurrenzpartner (Kollokator) anzeigt. Ist der Wert höher als 10.000, handelt es sich höchstwahrscheinlich um feste Verbindungen (bzw. Namen, die sich im Korpus wiederholen).

T-Score (test score) ,relativiert“ den Mi-score, zeigt Partner, die weniger häufig, jedoch immer noch signifikant vorkommen. Die Reihung nach dem T-Score zeigt (je nach Einstellung) deutlich auch das „grammatikalische Umfeld“ vom KWIC: Funktionswörter, Präpositionen, Satzzeichen.

LLR (log likelyhood ratio) bedeutet „Interner Wert für die ermittelte Stärke der lexikalischen Kohäsion“ (Belica 1995). Es ist immer eine positive Nummer, sie kann beliebig hoch sein (je frequentierter beide Kollokationspartner sind, desto höher ist sie). Die Werte der Kollokatoren sinken bis 0. Dieses Signifikanzmaß ist ein guter Kompromiss zwischen dem Mi-Score und dem T-Score und wird in den meisten Korpora berechnet.

logDice ist eine Berechnung, die für Lexikographen empfohlen wird (Rychlý 2008: 6), da sie typische (aber nicht unbedingt häufige) Partner aufdeckt, untypische jedoch ausblendet. Aus diesem Grund könnte sie auch für den DaF/DaZ-Unterricht verwendet werden. Leider werden diese Werte nicht von jedem Korpusmanager ${ }^{9}$ berechnet. Die Vorteile dieses Maßes listet Rychlý (2008: 9) folgend auf:

- Der maximale Wert kann 14 sein, üblicherweise ist er unter 10.

- Der Wert 0 (oder negativer Wert) heißt (absolut) keine Signifikanz.

- Das Maß ist von der Größe des Korpus unabhängig.

Im beliebig großen (Sub)Korpus kann man sich also sicher sein, dass die Kollokatoren mit dem Wert über 10 sehr häufig mit dem KWIC vorkommen (d.h. diese sollten die Lerner/ Lernerinnen unbedingt beherrschen), dafür können die nahe 0 ausgeblendet werden. Als Beispiel kann man sich die typischen Kollokatoren zur Verbform fragst anschauen:

\begin{tabular}{|c|c|c|c|c|c|c|}
\hline & Filter & & Freq & T-score & MI & logDice \\
\hline 1. & p $/ \underline{n}$ & Wieso & 5 & 2.235 & 10.670 & 7.002 \\
\hline 2. & - $\mathrm{p} / \underline{\mathrm{n}}$ & Warum & 23 & 4.792 & 10.212 & 6.742 \\
\hline 3. & $\mathrm{p} / \underline{\mathrm{n}}$ & du & 155 & 12.439 & 10.116 & 6.681 \\
\hline 4. & $\mathrm{p} / \underline{\mathrm{n}}$ & Ernst & 4 & 1.998 & 10.331 & 6.666 \\
\hline 5. & $\mathrm{p} / \underline{\mathrm{n}}$ & $\mathrm{Du}$ & 37 & 6.076 & 9.923 & 6.473 \\
\hline 6. & $\mathrm{p} / \underline{\mathrm{n}}$ & zuviel & 3 & 1.730 & 10.125 & 6.426 \\
\hline 7. & $\mathrm{p} / \underline{\mathrm{n}}$ & warum & 12 & 3.457 & 9.027 & 5.563 \\
\hline 8. & $\mathrm{p} / \underline{\mathrm{n}}$ & dich & 20 & 4.463 & 8.965 & 5.516 \\
\hline
\end{tabular}

Abb. 3: Kollokationspartner zur Abfrage fragst (InterCorp_de)

9 DWDS und InterCorp berechnen diesen Wert. 
Für die übliche Nutzung der Korpora (wenn man nicht in die Tiefe der mathematischen Wahrscheinlichkeitsberechnung eintauchen will) zeigt sich bezüglich der Signifikanzmaße des Vorkommens sprachlicher Elemente der folgende Weg als leicht begehbar und hilfreich:

1) Für den Vergleich der Signifikanz unter verschiedenen Kollokationspartnern immer dieselbe Berechnung (z.B. LLR) verwenden. (Dies gilt als grundsätzliche Faustregel in der Forschung.)

2) Beim Untersuchen nur eines Kollokationspaars immer mehrere Berechnungen vergleichen.

3) Die Belege (Konkordanzzeilen mit den Kollokationspartnern) sollten immer stichprobenartig angeschaut werden, um zu überprüfen, ob die Ergebnisse der Berechnung nachvollziehbar sind.

$\mathrm{Zu}$ den weiteren wichtigen Eigenschaften der Korpora gehören Annotationen. Es sind zusätzliche Informationen zu den Korpustexten (äußere Annotation) und zu den einzelnen Wörtern im Korpus (innere Annotation).

Äußere Annotationen, genannt Metainformationen, beinhalten bibliographische Angaben zum Text und zu seinem Ursprung (Autor/in, Erscheinungsjahr, Quelle), aber auch andere, für die Untersuchung der Sprache wichtige Informationen wie Textsorte oder grobe stilistische Zuordnung. Für Gender-Studien beispielsweise sind bestimmt die Angaben über das Geschlecht des/der Verfassers/ in, Übersetzers/ in interessant. Die letztgenannten Angaben werden jedoch in den Korpora selten angegeben.

Innere Annotationen beziehen sich auf den Text: sie liefern Informationen zu jedem Wort im Text, über seine morphosyntaktischen Eigenschaften (Tagging) und syntaktisch-semantischen Rollen (Parsing).

Ein Tagger ist ein Programm, das durch Tagging jedem Wort im Text seine morphosyntaktische Eigenschaft zuweist: Der Tagger bestimmt die Grundform des Wortes, die Wortart, ggfs. auch andere morphosyntaktische Kategorien (Genus, Kasus, Numerus, Steigerungsform, Kategorie des Verbs, Tempus, Modus, in einigen Sprachen auch Aspekt). Das Ergebnis vom automatischen Tagging (ein Prozess, in dem der Text mit einem Tagger analysiert wird) ist in Abb. 4 und 5 zu sehen.

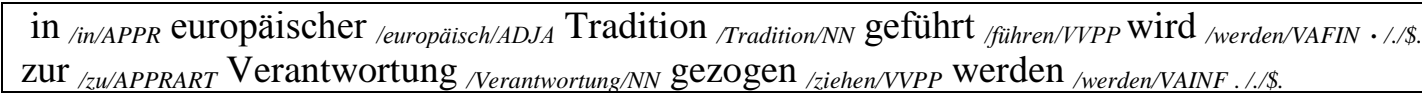

Abb. 4: Morphosyntaktisch annotierter und lemmatisierter Text - ein Auschnitt (InterCorp_de)

Parser ist ein Programm, das Sätze automatisch analysiert und einzelne Wörter im Satz syntaktisch annotiert, d.h. jedem Wort werden Informationen über seine syntaktischsemantische Funktion im Satz zugewiesen (Abb. 5). Obwohl das Wort Parsing (Part-ofSpeech-Analyse) treffender das Prinzip von Tagging charakterisiert, versteht man ,unter Parsing [...] heute eher solche Analyseprozesse, die substantiell über das bloße Annotieren eines Textes mit Wortarten hinausgehen und die grammatische Struktur einer Äußerung aufdecken“"(Carstensen et al. 2010: 303). 


\section{Parsing mit ParZu}

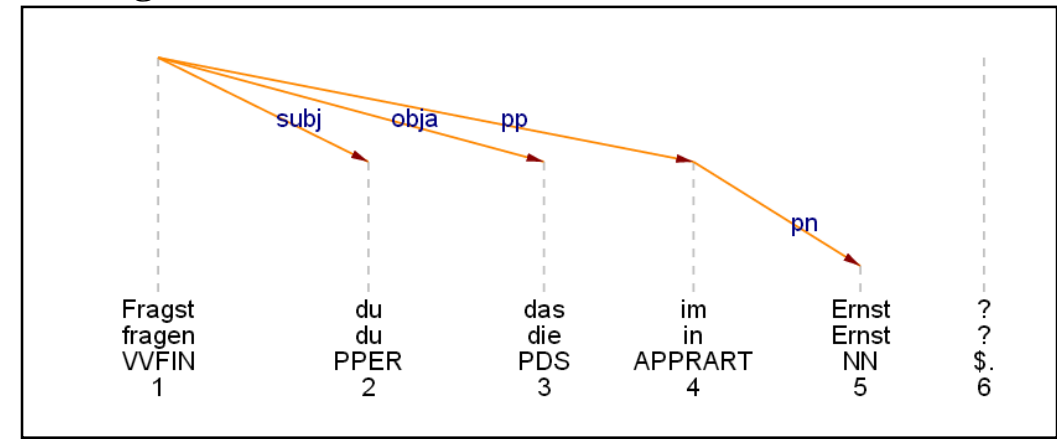

Abb. 5: Geparster Satz Fragst du das im Ernst? (ParZu)

Geparste Texte werden in Treebanks gespeichert. Treebanks (deutsch auch Baumbanken) sind Korpora, die das Suchen nach sprachlichen (semantischen und syntaktischen) Tiefenstrukturen ermöglichen. Die Texte müssen dafür vorher über einen Parser aufbereitet werden.

Neben den oben genannten Begriffen, die sich auf alle Korpora beziehen können, gibt es auch einige spezielle Fachbezeichnungen, die (ausschließlich) in Verbindung mit Parallelkorpora zu finden sind.

In Parallelkorpora werden übersetzte Texte in mehreren Sprachen eingespeist. Die Textsammlung für eine Sprache, zu der es in demselben Parallelkorpus Pendants noch in einer anderen Sprache gibt, heißt Parallele. Die sprachlichen Pendants/ Parallelen werden durch einen Zuordnungsprozess, sog. Alignment, einander zugewiesen.

Allgemein bedeutet Alignment die Anpassung und „Zuweisung“ zu einer anderen Parallele oder medialen Form des Textes. Im Parallelkorpus werden am häufigsten Textsegmente auf der Höhe eines Satzes einander angepasst (Abb. 6). In multimedialen Korpora wird als Alignment die Synchronisierung des Textes mit dem Ton bezeichnet.

\begin{tabular}{|c|c|c|}
\hline de & CS & en \\
\hline $\begin{array}{l}\text { Sie flogen kreuz und quer, um } \\
\text { einander irrezuführen }\end{array}$ & $\begin{array}{l}\text { Poletovali sem a tam, aby se } \\
\text { navzájem svedli s cesty. }\end{array}$ & $\begin{array}{l}\text { They flew hither and thither, to } \\
\text { entice one another astray. }\end{array}$ \\
\hline $\begin{array}{l}\text { Herr Präsident ! In dieser Debatte } \\
\text { geht es kreuz und quer }\end{array}$ & $\begin{array}{l}\text { Pane předsedající , rozprava se } \\
\text { pohybuje napříč různými } \\
\text { směrnicemi . }\end{array}$ & $\begin{array}{l}\text { Mr President, this debate criss- } \\
\text { crosses the Directives. }\end{array}$ \\
\hline
\end{tabular}

Abb. 6: Segmente in drei Sprachparallelen (InterCorp_de)

Segmente sind Teile der Korpustexte, die nach unterschiedlichen Kriterien bestimmt werden - oft sind es Absätze oder Sätze. In einem Parallelkorpus handelt es sich um minimale Einheiten, die in zwei oder mehreren Sprachen aligniert (einander zugewiesen) werden, wie in der Abb. 6. 


\subsection{Wissenschaften rund um moderne Korpora}

Die heutigen elektronischen Sprachkorpora könnte es nicht ohne Computer geben. Erst die Entwicklung der EDV hat es ermöglicht, große Datenmengen von sprachlichen Erscheinungen zu verwalten. Dazu entstand auch ein neues Fach an der Schnittstelle der Linguistik und Computertechnik: die Computerlinguistik.

Die Computerlinguistik „beschäftigt sich mit der maschinellen Verarbeitung natürlicher Sprache“" (Carstensen 2010: 1), sie versucht sprachliche Strukturen und Prozesse mit Hilfe der EDV-Technik zu beschreiben und zu simulieren (vgl. Metzler 2000: 129), sie unterstützt die Korpuslinguistik. Die Simulierung der menschlichen Sprache ist jedoch bisher mehr oder weniger gescheitert (z.B. Versuche der maschinellen Übersetzung). Die Beschreibung der Sprache erfolgt wiederum mit der Technik viel effektiver als früher. Man kann es mit einer Recherche in einem Bibliothekskatalog vergleichen: wie früher in Karteikatalogen der Bibliotheken gesucht wurde und wie heute mit elektronischen Katalogen recherchiert wird.

Die Anfänge der Computerlinguistik reichen bis in die ersten Jahre nach dem 2. Weltkrieg, in die USA, wo eben die ersten Versuche mit der maschinellen Übersetzung gemacht wurden. Der Begriff „,computational linguistics“ wurde in den USA seit Anfang der 1960-er Jahre verwendet (Leech 1991: 8-10). Bis heute hat sich die Computerlinguistik als eine interdisziplinäre Wissenschaft (zwischen der angewandten Informatik und Linguistik) etabliert. Ihr Bereich reicht vom elektronischen Erfassen der Sprache über das Generieren von maschinellen Äußerungen, bis zur automatischen Transformation der geschriebenen zur gesprochenen (relativ einfach) Sprache und umgekehrt (bis heute nicht lückenlos gelöst).

Der wohl wichtigste Schwerpunkt der Computerlinguistik liegt in der Korpuslinguistik.

Die Korpuslinguistik ist jener Teil der Linguistik, der die Sprache systematisch anhand großer Mengen von realisierten sprachlichen Äußerungen (Parole) untersucht. Das Ziel des „korpuslinguistischen“ Ansatzes in der modernen Linguistik ist es, die Strukturen und Funktionen der natürlichen Sprache besser und realitätsnäher zu beschreiben (vgl. dazu Biber 1998).

Gerhard Budin (2011: 17) sagt zur Stellung der Korpuslinguistik in den Wissenschaften:

„Die Corpuslinguistik ist eine interdisziplinäre, methodologische Grundlage für die computergestützte Forschung. Sie vernetzt im Kontext der Computerlinguistik die Sprachwissenschaft mit der Informatik und ermöglicht das Arbeiten auf einer breiten empirischen Basis.“

Dass diese „breite empirische Basis“ wichtig ist, lässt sich leicht am Beispiel aus einem renommierten Buch, das die Problematik der deutschen Wortbildung ausführlich behandelt, demonstrieren. In seiner Publikation Wortbildung der deutschen Gegenwartssprache" schreibt Wolfgang Fleischer (1969: 117):

„In Österreich sind z.B. geläufig die Formen (...) Ausnahmszustand, Fabriksmarke, Gepäcksaufgabe, Zugsführer, Aufnahmsprüfung, Ausnahmszustand; in der Schweiz z.B. (...) Sportsmeldung, Zugsverbindung u.ä.“

Eine einfache Recherche im Korpus bezeugt, wie frequentiert diese Formen im heutigen Deutsch sind: Im IDS-Korpus gibt es null Treffer/ Belege zum Wort Ausnahmszustand, dagegen zeigt die Streuung (das Vorkommen umgerechnet auf 1 Mio. Wörter) der Form Ausnahmezustand ganz deutlich, dass diese Form in allen drei Varietäten der deutschen Sprache gleich häufig verwendet wird. Genauso unüblich scheint das in der Schweiz „geläufige Fugen-S“, wie Fleischer (1969: 117) schreibt, im Wort Sportśsmeldung: keine Treffer für Sportsmeldung, gegen 23 Treffer für Sportmeldung. 
Die Korpuslinguistik kann auch die Theorien und Hypothesen der generativen Grammatik verifizieren oder widerlegen (vgl. Metzler 2000: 384). Dies wird aber von den meisten Generativisten abgelehnt (vgl. Lewandowski 1994: 612).

Die Korpuslinguistik ist keine neue „Wissenschaft“, sondern nur ein Zugang, eine Sichtweise auf die Sprache. Zu Recht erntet sie Kritik seitens der Verfechter/-innen der mentalistischen, generativistischen Auffassung der Sprache (z.B. Chomsky 1986: 19), da kein Korpus alle Äußerungen einer Sprache erfassen kann. Auf der anderen Seite sieht man auch anhand von einigen wenigen Belegen, was in der Sprache überwiegt und typisch ist. Die Abstraktion des Typischen soll dann die Grundlage für die Grammatik der Sprache darstellen. Bisher wurden normative Grammatiken von nur wenigen Sprachen anhand von Korpusdaten erstellt. Selbst die letzte neubearbeitete Duden-Grammatik (2005) führt zwar Beispiele zu vielen grammatikalischen Erscheinungen aus dem Korpus DeReKo an, ignoriert jedoch sehr oft die Abweichungen in einzelnen Varietäten des Deutschen - so z.B. die Verbalkomplexe (2005: 481), wo die „österreichische“ Reihenfolge trotz vieler Belege im Korpus (DeReKo) unerwähnt, dadurch implizit als falsch bezeichnet wird (dazu auch hier Studie 8). Viele offizielle Referenzwerke basieren auch heute noch auf ungenügenden Belegsammlungen bzw. immer noch auf dem „Gefühl“ ihrer Autor/-innen (nicht anders ist es im Fall Österreichisches Wörterbuch, teilweise auch Duden Deutsches Universalwörterbuch u.a.) Es muss betont werden, dass es im korpuslinguistischen Ansatz auf keinen Fall darum geht, was in der Sprache korrekt oder inkorrekt ist, sondern darum, welches Phänomen in der Verwendung der Sprache typisch ist, welches untypisch, selten oder rar ist. In diesem Zusammenhang darf der Korpuseinsatz in $\mathrm{DaF} / \mathrm{DaZ}$-Unterricht und -forschung nicht unerwähnt bleiben. $\mathrm{DaF} / \mathrm{DaZ}$ passiert eben auch nicht in einer ,idealen Sprachwelt" - es ist jeweils in einem konkreten Kontext eingebettet, der bei der Vermittlung des Deutschen berücksichtigt werden muss. Erst in diesem Kontext sucht man das Typische, bzw. das für die Lerner/-innen Hilfreiche. Dem soll auch die Wahl des passenden Instruments entsprechen. So gesehen grenzt die Korpuslinguistik auch an die Methodologie der Fremdsprachenvermittlung - diese Schnittstelle wurde allerdings noch wenig beschrieben und erforscht.

Die Vorreiter des korpusunterstützten Fremdsprachenunterrichts waren Artikel, Bücher, sogar kleine Wörterbücher über Fehler, die die Lerner/-innen wiederholt begehen, z.B. Swans' (1982 u. weitere Auflagen) Practical English usage; Wörterbücher der Falschen Freunde bei deutschen Englischlernenden (z.B. von Barnickel (1992)); Sparlings' (1989): English or Czenglish über die Interferenzfehler im Englischen bei tschechischen Englischlernenden u.a.m. Mit den EDV-Möglichkeiten kann sich eigentlich jede/-r ein Korpus erstellen, in dem effektiv gesucht werden kann. Ein aufwendigeres Instrument für die Fehlerforschung (Falko) ist bereits an der Humboldt-Universität zu Berlin entstanden (siehe Reznicek et al. 2012). Wie man sich selbstständig ein Korpus einfach erstellen kann, wird im Kap. 5.1 (TextSTAT) beschrieben.

An und für sich ist die Erstellung eines Korpus und die Arbeit mit ihm nichts Neues (Francis 1992: 17). Die ersten Korpora entstanden als Basis für die Zusammenstellung von Wörterbüchern und Grammatiken - in Form von unzähligen Karteizetteln. Zum Beispiel die traditionelle Sprachkartei der Dudenredaktion verfügte über drei Millionen authentischer Sprachbelege (Drosdowski 1985: 87). Die Anfänge der Korpuslinguistik reichen also tief in die Geschichte der Sprachforschung.

Die heutige Korpuslinguistik arbeitet ausschließlich mit elektronischen Sprachkorpora und zeigt so den Trend in der modernen Philologie an. Ihre Bedeutung ist vor allem im 
Zusammenhang mit dem Aufkommen der EDV- Technik angestiegen. Mit der Verbreitung des Internets ist diese moderne Methode der Sprachwissenschaft und der Sprachvermittlung für beinahe jedermann zugänglich. 


\section{Korpustypologie}

Die Vielfalt der Korpora lässt sich etwa mit der Vielfalt der Wörterbücher und Enzyklopädien vergleichen: für jeden Zweck gibt es das passende Werk, man muss nur wissen, was man braucht. Wer die Bedeutung eines Wortes sucht, greift zum Bedeutungswörterbuch, äquivalente Bezeichnungen in einer anderen Sprache findet man in einem Übersetzungswörterbuch. Wer etwa mehr über eine Sache wissen will, nimmt eine Enzyklopädie zur Hand. Heutzutage wird die Suche noch erleichtert und beschleunigt, denn die meisten modernen Lexika gibt es auch in elektronischer Form.

Ähnlich ist es mit elektronischen Korpora: Wer im Internet sucht, der wird oft fündig. Und wenn kein passendes Korpus existiert, lässt es sich irgendwie erstellen. Eine selbstständige Erstellung von einsprachigen Korpora geht relativ leicht z.B. mit dem Programm TextSTAT (siehe Kap. 5.1). Das Erstellen eines Parallelkorpus ist schon aufwendiger, aber auch nicht unmöglich (z.B. mit dem kostenpflichtigen Programm ParaConc von M. Barlow (2009)). Auf jeden Fall müsste man bei diesen in Selbsthilfe erstellten Korpora auf viele hilfreiche und angenehme Eigenschaften und Tools verzichten. Deshalb sehe ich die Nutzung der bereits bestehenden, oft professionell erstellten Korpora als den sinnvollsten Weg.

Eine übersichtliche Graphik mit Kriterien, nach denen Korpora (allgemein) aufgeteilt werden können, präsentieren Lemnitzer/ Zinsmeister (2010: 103). Eine ähnliches Schema (ergänzt um einige Eigenschaften, auf die man bei der Korpuswahl Rücksicht nehmen soll) präsentiert auf seiner Homepage auch Stephen Berman (2013).

Die folgende Typologie reißt die Grundaufteilung der Korpuslandschaft auf, dabei werden auch zu jedem Typ die Namen der entsprechenden Korpora aufgelistet. Die Vertreter der einzelnen Korpustypen werden hier in Abkürzung angeführt. Eine Übersicht mit vollen Namen und Internetadressen befindet sich ab der Seite 219.

Alle hier erwähnten Korpora sind einfach zugänglich. Sie können m.E. den DaF/DaZ-Bereich in der Lehre und Forschung unterstützen.

Die einzelnen Korpustypen schließen einander nicht aus. Viele Korpora können verschiedenen Zwecken dienen (diese werden bei einigen Korpora im Kap. 4 näher charakterisiert) - je nachdem, wie das Korpus aufgebaut ist, welche Eigenschaften der Korpusmanager hat und welche Abfrage möglich ist.

\subsection{Stadium der gespeicherten Sprache}

\subsubsection{Synchrone Korpora}

Korpora der Gegenwartssprache (synchrone Korpora) beinhalten aktuelle Texte der letzten Dekaden. Meist sind es Texte, die nach dem 2. Weltkrieg entstanden sind. Als Schwelle zur „heutigen“ Sprache gilt das Jahr 1989 (Kocek et al 2000: 13).

Einen nicht geringen Teil der nationalen Korpora bilden aus pragmatischen Gründen auch Texte vor 1945, v.a. belletristische Texte, wegen ihrer Sprache, die (immer noch) als modern gilt (Kafka, Böll, sogar Hašek und sein Švejk). Im Unterschied zu Gebrauchstexten veraltet 
die Belletristik wesentlich langsamer - u.a. auch dank der überarbeiteten neuen Auflagen der alten Werke.

Synchrone Korpora mit Deutsch (nach Textvolumen absteigend): geschriebene Sprache: DeReKo, deTenTen, Wortschatz Leipzig, DWDS, deWac, InterCorp_de, OPUS;

gesprochene Sprache: DGD, DWDS - gesprochene Sprache, GeWiss, BAS

\subsubsection{Diachrone Korpora}

Mit Diachronie bezeichnete de Saussure einzelne aufeinander folgende Entwicklungsstadien der Sprache. Diachrone Korpora sollen helfen, die Sprache(n) auf der Zeitachse zu betrachten, Änderungen und Tendenzen in der Entwicklung der Sprache/den Sprachen besser zu verstehen. Jedes Korpus, das Texte aus mehreren Jahrzehnten beinhaltet, und dessen Tools es ermöglichen, die Texte nach der Zeit ihres Entstehens/ Herausgebens zu filtern, kann als ein diachrones Korpus bezeichnet werden.

Diachrone Korpora des Deutschen (letzte Jahrhunderte):

DWDS (20. Jh.), DeReKo (18. - 21. Jh.)

\subsubsection{Historische Korpora}

In die historischen Korpora werden Texte aus einem älteren, bereits überwundenen Stadium der Sprache eingespeist. Sie dienen in erster Linie als Forschungsquellen für das Studium der jeweiligen Entwicklungsphase der Sprache. Darüber hinaus fungieren sie als eine Datenbank von historischen Texten, die sonst nur schwer zugänglich wären. Für DaF (jedoch eher im universitären Bereich, speziell der Auslandsgermanistik) haben sie insofern Bedeutung, da zum vollkommenen Studium der Sprache auch ein Einblick in ihre historische Entwicklung gehört. Für DaZ spielen sie keine Rolle.

Historische Korpora des Deutschen (nach Entwicklungsstadium der Sprache):

Althochdeutsch: DDD (Deutsch Diachron Digital: Referenzkorpus Altdeutsch)

Mittelhochdeutsch: MHDBDB (Mittelhochdeutsche Begriffsdatenbank)

Frühneuhochdeutsch: FnhdC (Das Bonner Frühneuhochdeutschkorpus)

alle historische Stadien: DDD (Deutsch Diachron Digital)

Die Begriffe „diachrone“ und „historische“ Korpora werden oft willkürlich und synonym verwendet. Die richtige Bezeichnung für historische Korpora ist „Korpora mit historischen Texten“. Alle Korpora, in denen Texte aus mehreren Jahren oder Dekaden gespeichert sind, können eigentlich als „diachrone Korpora“ bezeichnet werden.

\subsection{Medium}

\subsubsection{Korpora der geschriebenen Sprache}

Die geschriebene Sprache lässt sich viel einfacher digitalisieren und in eine (von einem Korpusmanager) lesbare Form umwandeln als die gesprochene Sprache. Deswegen haben Korpora der geschriebenen Sprache viel mehr Vertreter in der Korpuswelt. Korpora der geschriebenen Sprache beinhalten digitalisierte authentische Texte, die in irgendeiner geschriebenen Form (gedruckt oder elektronisch) öffentlich erschienen sind. 
Elektronisch verfasste Texte bilden das Gros aller großen Korpora, viele Texte werden jedoch immer noch eingescannt.

Korpora der geschriebenen deutschen Sprache:

DeReKo, deTenTen (nur Internettexte), Wortschatz Leipzig (nur Internettexte), DWDS, deWac (nur Internettexte), InterCorp_de, OPUS (nur Internettexte)

\subsubsection{Korpora der gesprochenen Sprache}

Obwohl (immer noch) mehr gesprochen als geschrieben wird, bilden Korpora der gesprochenen Sprache nur einen Bruchteil in der Korpuslandschaft aller Sprachen. Für viele Sprachen gibt es sie noch gar nicht. Der Grund liegt in ihrer aufwendigen technischen Aufbereitung: Korpora der gesprochenen Sprache sind transkribierte und digitalisierte gesprochene Texte. Das weitere Manko dieser Instrumente ist ihre Authentizität (ebenso wie häufig auch bei Hörtexten in Lehrbüchern): die Sprechereignisse sind oft gesteuert, künstlich aufgebaut (teilweise DGD), oder repräsentieren gar das Vorlesen eines vorgefertigten, geschriebenen Textes (adaba). Für die Erforschung der Aussprache sind aber auch diese Vorgangsweisen legitim.

Das Erstellen eines repräsentativen Korpus der gesprochenen Sprache scheitert grundsätzlich an zwei Punkten: 1) die heutige Technik ist noch nicht so weit, alle Nuancen in der Aussprache unterschiedlicher Sprecher zu erfassen (v.a. wenn es sich auch um dialektale Färbungen handelt); 2) das Aufnehmen von Privatgesprächen betrachtet die europäische Gesetzeslage als widerrechtlich, falls es ohne ausdrückliches Erlaubnis der beteiligten Personen erfolgt ${ }^{10}$.

Korpora der deutschen gesprochenen Sprache:

DGD, BAS, DWDS - gesprochene Sprache

Lemnitzer/ Zinsmeister (2010: 103) listen noch „multimodale Korpora“ auf - darunter verstehen sie Korpora mit Audio- und/oder Videoaufnahmen. Nach dieser Definition sind alle hier angeführten Korpora der gesprochenen Sprache „multimodal“, da jeweils auch Audiofiles abrufbar sind.

\subsection{Repräsentativität}

\subsubsection{Stilistische Kriterien}

Allgemeine Korpora

Allgemeine Korpora versuchen Texte aller Textsorten aus (möglichst) vielen Kommunikationsbereichen und stilistischen Ebenen abzudecken.

Allgemeine Korpora mit Deutsch:

DeReKo, DWDS, deTenTen

\section{Spezifische Korpora}

Spezifische Korpora werden für die Erforschung nur eines kleineren Segments der Sprache aufgebaut. Sie beinhalten Texte, die ein gemeinsames Spezifikum verbindet - z.B. Fachbereich (medizinische oder naturwissenschaftliche Texte ...), Stil (journalistische oder

10 Meine Redakteurin meint, ich soll es begrüßen, was ich grundsätzlich auch tue. Dennoch schadet dieses Gesetz der Erstellung der Korpora der gesprochenen Sprache. 
dramatische Texte, Texte im Dialekt...), Autor (Goethe, Orwell...), andere besondere Zwecke (Fehlerkorpus, Lernerkorpus, Varietätenkorpus...)

\section{Spezifische Korpora mit Deutsch:}

Viele größere Korpora ermöglichen auch ein spezifisches Korpus als Subkorpus zu erstellen und nur in diesem zu recherchieren. Beispielsweise im DeReKo gibt es das Korpus GOE mit Goethes Werken oder ein (allerdings nicht öffentliches) Korpus IKO, wo ausschließlich Zeitungsinterviews gespeichert sind u.v.a.m. (siehe Cosmas II $\rightarrow$ Hilfeportal $\rightarrow$ Organisation des Textmaterials).

Deutsch ist vertreten auch in verschiedenen spezifischen Parallelkorpora - z.B. im MULTEXT-East corpus (George Orwells Novelle 1984 in 11 Sprachen), im OPUSCorpus (Filmuntertitel, EU-Verfassung).

\section{Weitere spezifische Korpora:}

Varietätenkorpus: Korpus C4 (im Aufbau)

Fehlerkorpus (Lernerkorpus): Falko

\subsubsection{Kriterium der Ausgewogenheit}

\section{Ausgewogene Korpora}

Über genaue Kriterien für die Erstellung eines ausgewogenen Korpus herrscht kein Konsens. Beispielsweise folgen zwei repräsentative „Nationalkorpora“ unterschiedlichen Ausgewogenheitskriterien: Im DeReKo überwiegen Zeitungstexte, im ČNK (Referenzkorpus SYN 2005) jedoch Belletristik.

In einem ausgewogenen, repräsentativen und allgemeinen Korpus soll das Verhältnis der Textsorten und Textstile dem Verhältnis in der realen sprachlichen Welt entsprechen. Das heißt, dass den größten Teil der Korpora unbedingt die gesprochene Sprache bilden müsste. Da dies (noch) nicht möglich ist (siehe oben), werden als ausgewogene Korpora solche bezeichnet, die ein stabiles Verhältnis der einzelnen geschriebenen Textsorten beinhalten. Große nationale Korpora werden laufend um Textsorten ergänzt um ihre Ausgewogenheit zu gewährleisten.

Deutsche Korpora mit repräsentativ ausgewogenen Daten: DeReKo, DWDS

\section{Opportunistisch gebildete Korpora}

Es sind Korpora, die keinen Anspruch auf Abdeckung der Kriterien der Ausgewogenheit haben, bzw. sie erfüllen diese Kriterien nicht.

\section{Nicht ausgewogene Korpora mit Deutsch}

ausschließlich Internettexte: Wortschatz-Portal, deTenTen, deWac

Protokolle, Festschriften: OPUS-Corpus

Belletristik und Publizistik: InterCorp

\subsection{Größe}

Das Kriterium der Korpusgröße hängt unmittelbar mit der Repräsentativität der gespeicherten Daten und dadurch auch mit der Validität der Ergebnisse, die man aus Abfragen gewinnt, zusammen. Grundsätzlich gilt, dass kleinere oder kleine Korpora ${ }^{11}$ zwar übersichtlicher, daher auch (oft) fehlerfrei sind, sie aber für die Erforschung oder Darstellung allgemeiner

11 Ein kleines Korpus ist z.B. DeuCze (siehe Kotulková et al. 2005-2010). 
sprachlicher Phänomene keine repräsentativen Daten liefern können. Gut können sie nur ein kleines, spezifisches Segment oder ein hoch frequentiertes Element der Sprache aufdecken.

Ein kleines Korpus lässt sich grundsätzlich aus jedem größeren Korpus (als Sub-Korpus) erstellen. Im korpuslinguistischen Ansatz der Sprach(en)forschung und -vermittlung gilt: je größer das Korpus, desto repräsentativer, sicherer, überzeugender sind die Rechercheergebnisse. Die Grundstrukturen und die hochfrequentierten Elemente der Sprache lassen sich zwar tatsächlich schon anhand von kürzeren Texten erfassen, wie aus dem Kap. 5.1 ersichtlich ist, viele Fragen (z.B. über die Lexik, Peripherie der Sprache, Dialekte) können aber nicht einmal die jetzigen Korpora mit Milliarden Wörtern beantworten.

Sehr große Korpora mit Deutsch:

DeReKo (8,9 Mrd. Wörter), deTenTen (1 Mrd. Wörter), deWac (1,4 Mrd. Wörter)

\subsection{Sprache}

\subsubsection{Monolinguale Korpora}

In einsprachige (monolinguale) Korpora werden Texte einer Sprache eingespeist, ungeachtet dessen, ob es sich um Originaltexte oder um Übersetzungen aus anderen Sprachen handelt. Korpora mit plurizentrischen Sprachen (Deutsch, Englisch, Spanisch) haben entweder überhaupt selbstständige Instrumente für die jeweilige Varietät (DWDS, Schweizer Textkorpus) oder - falls sie „unter einem Dach“ verwaltet werden - manchmal Tools, die das Filtern von einzelnen Varietäten ermöglichen (DeReKo). Viele Korpora ermöglichen dies jedoch nicht (Wortschatz-Portal, deWac).

Monolinguale Korpora mit Deutsch:

DeReKo (D-A-CH), AAC (nur Österreich), DWDS (nur Deutschland), Schweizer Textkorpus (nur Schweiz); DGD, deWac, deTenTen, Wortschatz-Leipzig.

Nationalkorpora anderer Sprachen siehe Kap. 4.1.

\subsubsection{Bilinguale und Multilinguale Korpora}

Zwei- und mehrsprachige Korpora können theoretisch noch in zwei Kategorien unterteilt werden: Vergleichskorpora und Parallelkorpora.

In Vergleichskorpora findet man Texte zum selben Thema, wobei diese Texte unabhängig voneinander als Originale entstanden sind. Das heißt, zwischen zwei Texten des Vergleichskorpus wurde keine übersetzerische Arbeit geleistet. Als Vergleichskorpus könnten beispielsweise Nachrichten zum selben Thema in unterschiedlichen Medien und Sprachen dienen. Als große Quelle von Texten für ein Vergleichskorpus können auch diejenigen Artikel in Wikipedia dienen, die nicht als Übersetzungen gekennzeichnet sind (vgl. Wikipedia: Übersetzungen (2014)).

Parallelkorpora sind Datenbanken mit „offiziellen“ Übersetzungen, also mit denselben Texten, die in mehreren Sprachen existieren und ihre kommunikative Funktion erfüllen.

Beim Vergleich dieser beiden Subkategorien eröffnet sich automatisch die Frage nach der (1) Vergleichbarkeit der Texte: können wir zwei unabhängig voneinander verfasste Texte als zwei gleiche sprachliche Handlungen betrachten? Können wir auch Übersetzungen aus „dritten Sprachen“ vergleichen (z.B. deutsche und englische Übersetzungen des ungarischen 
Schriftstellers István Örkény „One Minute Stories/ Minutengeschichten“); (2) Authentizität der Texte: eine Übersetzung ist kein frei formulierter Text. Darf er also die „reale“ Sprache repräsentieren?; (3) Kompetenz der Übersetzer/-innen und die damit verbundene Gefahr der Übersetzungsfehler.

Diese Fragen muss man bei der Arbeit mit Parallelkorpora immer im Auge behalten. Es sind eben Faktoren, die das Bild (d.h. die Ergebnisse der Recherche) verzerren können. Auf der anderen Seite gibt es wohl keine andere Möglichkeit, Sprachen untereinander zu vergleichen. Wir können uns nur darauf verlassen, dass die Menge und Vielfalt der Texte im Korpus die genannten Fehlerquellen wieder ausgleichen.

Bilinguale und multilinguale Korpora mit Deutsch: InterCorp, OPUS-Corpus

\subsection{Technische Eigenschaften}

Eigentlich sind es die technischen Eigenschaften, die aus einer simplen Textsammlung ein Korpus machen. In diesem Punkt unterscheidet sich auch jede Korpusarbeit vom Googeln.

Viele Korpustools und Zusatzinformationen sind auf den ersten Blick irrelevant. Sie sind aber für die Suche im Korpus sehr wichtig. Darüber hinaus helfen sie besser zu verstehen, wie die Korpora funktionieren und aufgebaut sind. Nach Berman (2013, ergänzt) lassen sich folgende korpustechnische Eigenschaften nennen:

- Metadaten: Informationen über den Aufbau und Inhalt des Korpus. Nach einzelnen Posten der Metadaten lassen sich Subkorpora erstellen: z.B. Texte aus einer Zeitperiode (DeReKo, DWDS), eines Autors (InterCorp), Texte, die nur Frauen übersetzt haben (InterCorp) u.a.m.

- Linguistische Aufbereitung: hinzugefügte linguistisch relevante Markierungen im Korpus

- keine: rohe sprachliche Daten (Texte)

- Tokenisierung: Segmentierung der Texte in kleinere Einheiten: Absätze, Sätze, Wörter und Satzzeichen (alle gängigen Korpora); Phrasen, Konstituenten (geparste Korpora)

- Lemmatisierung: Zuweisung der flektierten Formen zu einer Grundform (lemmatisierte Korpora)

- Annotation: Markierung von (morpho)syntaktischen, semantischen u.a. Eigenschaften:

- Tagging (Morphosyntax, z.T. auch Semantik): Wortart, Flexionsmerkmale, Eigennamen (getaggte Korpora)

- Parsing (Syntax): Konstituenten, syntaktische Funktionen, topologische Felder (geparste Korpora).

- Phonetik/Prosodie/Intonation (DGD)

- Fehler: evtl. auf allen Ebenen, z.B. Rechtschreibung, Kongruenz, Wortstellung, Tempus usw., dienlich für Sprachunterrichts- sowie Spracherwerbsforschung (Falko)

- Word Sketches: automatische Berechnung und Darstellung der Eigenschaften und des Verhaltens eines Wortes im Korpus. (deTenTen, teilweise auch DWDS) 
$\mathrm{Zu}$ bemerken ist, dass all diese technischen Korpusfinessen mehrheitlich automatisch durchgeführt werden, dadurch stößt man immer wieder auf fehlerhafte Angaben. Dies ist ein Preis für die große Menge der Daten, die mit manueller Aufbereitung gar nicht oder nur mit einem enormen Aufwand freigegeben werden könnten. 


\section{Korpora relevant für DaF/DaZ}

Der folgenden Auswahl mit einer kurzen Beschreibung der einzelnen Korpora liegen diese Überlegungen zu Grunde:

1) Brauchen DaF/DaZ-Lehrer/-innen und Schüler/-innen überhaupt Korpora? Wenn ja, wozu?

2) Von welchen Korpora könnten sie am meisten Gebrauch machen?

3) Welche Korpustools und Korpuseigenschaften sind am ehesten einsetzbar und hilfreich im DaF/DaZ-Unterricht?

Gleich die erste Frage ist schwierig zu beantworten: Für die Sprachenvermittlung ist an sich natürlich kein Korpus notwendig. Der Sprach(en)unterricht läuft in den meisten Fällen auch heute ohne Korpora und mit Erfolg. Es wäre auch nicht sinnvoll Korpora im Unterricht zwanghaft einzusetzen. Die Arbeit mit fast allen Korpusmanagern (also die Bedienung dieser Instrumente) erfordert einige technische und linguistische Vorkenntnisse. Die Interpretation der gewonnenen Daten benötigt auch gewisse Erfahrungen mit dem Sprachgebrauch. Dies alles darf man von vielen Kursteilnehmer/-innen (vor allem im DaZ-Bereich) nicht erwarten. Darüber hinaus ist auch das sichere Erlernen der Korpusbedienung zeitaufwändig, noch dazu werden die meisten Korpusmanager verbessert, das Bild der Schnittstelle verändert sich und so könnte man leicht verzweifeln und skeptisch werden, weil man etwas gelernt hat, plötzlich aber alles anders ist und man wieder wie am Anfang sucht.

Auf alle Fälle ist es aber dennoch wichtig, zumindest die Information über die Existenz von Korpora zu vermitteln und diese als eine zuverlässige Informationsquelle über die Sprache darzustellen.

Korpora können und sollen den (Fremd-)Sprachenunterricht nicht steuern, sie können und müssen ihn unterstützen, denn die kommunikativ-pragmatische Methode des modernen Sprachunterrichts erfordert die Arbeit mit authentischen Texten, die Vermittlung realer Sprachmittel und häufiger Strukturen. Das alles ist in einem natürlichen Kontext im Korpus explizit oder implizit beinhaltet.

Die meisten DaF-Schüler/-innen sind im Umgang mit Computern gewandt. Bei den DaZLernenden (v.a. bei den Erwachsenen) ist dies nicht automatisch zu erwarten. Für sie könnte die Arbeit mit einem elektronischen Instrument zwar eine (zusätzliche) Belastung sein. Auf der anderen Seite könnte es für sie auch eine positive Herausforderung darstellen, sich mit ihrer eigenen Sprache zu befassen und diese vielleicht mit dem Deutschen zu vergleichen vorausgesetzt, dass es dazu entsprechende Korpora gibt. Beim jetzigen Stand der DaZKlientel klingt dieses Statement etwas naiv, man soll jedoch bedenken, dass sich das Korpusangebot stets erweitert.

Selbst die Grundkenntnisse über Korpora und die Fähigkeit, die einfachsten Recherchen durchführen zu können, unterstützen das autonome Lernen sowohl im induktiven als auch im deduktiven Weg: Lernende können selbst Regelmäßigkeiten in der Sprache aufdecken, sie können auch bereits gelernte Strukturen überprüfen. Dabei sollen sie sich aber bewusst sein, dass sie sich in der realen Sprache bewegen, in der die Theorie immer wieder an ihre Grenzen stößt. Diese Tatsache soll auch zur kritischen Stellungnahme zu theoretischen Grundlagen und Darstellungen in Grammatiken führen. 
Bei der Wahl aus dem Korpusangebot müssen wir uns damit begnügen, was es gibt. Es gibt schon viele nutzbare Korpora, allerdings klaffen in der Landschaft auch ziemlich viele Löcher, die noch gefüllt werden müssen.

Falls wir Korpora im DaF/DaZ-Unterricht einsetzen wollen, wäre es sicher sinnvoll auch die nationalen (einsprachigen) Korpora der jeweiligen Ausgangssprache zu erwähnen. Auch im DaZ-Unterricht wäre es sinnvoll, sich einen Überblick zu verschaffen, ob es vielleicht nicht ein Korpus für die Erstsprache der Lernenden gibt.

In europäischen Dimensionen sollen wir zumindest eine grobe Vorstellung davon haben, welche Korpora unsere Nachbarsprachen zur Verfügung haben.

Die eingesetzten Korpora und Korpusinstrumente sollen zugänglich, möglichst repräsentativ und relativ einfach und übersichtlich sein. Die Bedienung vieler Korpora sieht auf den ersten Blick zwar ziemlich komplex aus und kann von der Arbeit fast abschrecken (DeReKo, InterCorp), gut gewählte Beispiele von einfachen Abfragen können jedoch die Arbeit vorentlasten und so bildhaft darstellen, wie schnell man eigentlich Vieles über den Sprachgebrauch erfahren kann - wie das folgende Beispiel verdeutlicht. Die Abfrage (Korpus InterCorp_de) lautete: ich rede. Aus mehreren Dutzend Konkordanzen werden hier nur einige präsentiert, um die Übersichtlichkeit zu bewahren:

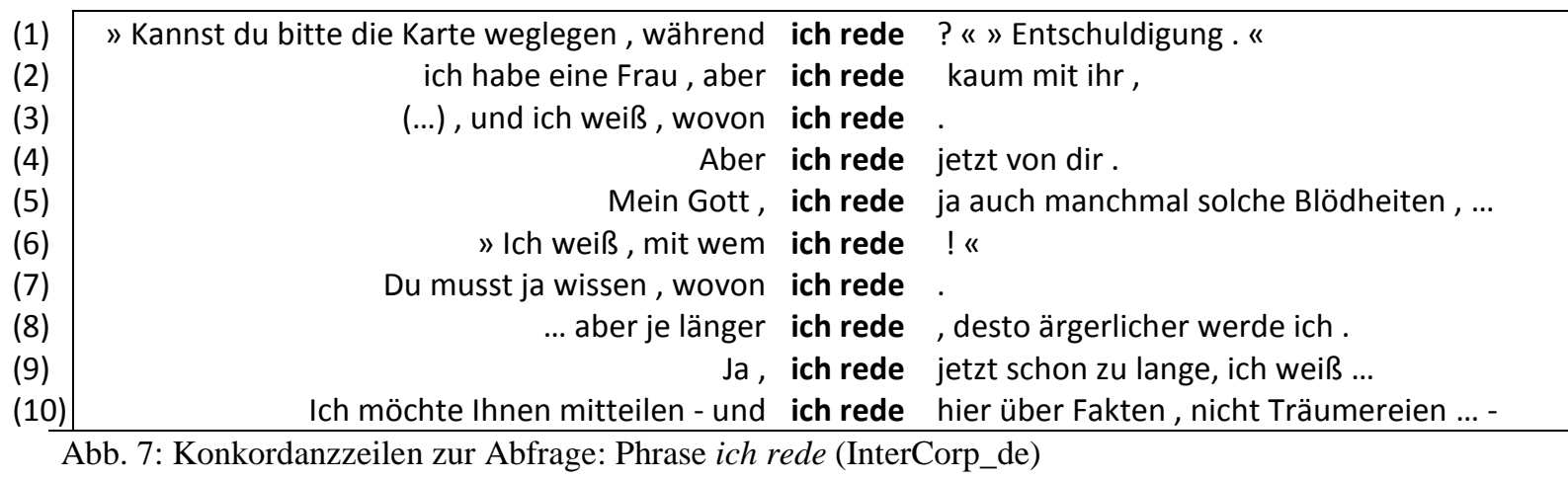

Auch ein/e Anfänger/-in kann sich ein Bild machen, wie die Verbindung ich rede in unterschiedliche Sätze eingebettet werden kann: ich rede (1); ich rede (etwas) (5); ich rede (wie) (8), (9); ich rede mit (jemandem) (2), (6); ich rede von (3), (4), (7); ich rede über (10).

Diese Überlegungen zur Sinnhaftigkeit des Korpuseinsatzes münden in die folgende Auflistung einiger (nationaler) Korpora von Sprachen, die in Mitteleuropa am häufigsten anzutreffen sind. Gemeint sind Korpora der offiziellen Landessprachen einzelner mitteleuropäischer Länder (Französisch, Polnisch, Slowakisch, Tschechisch, Ungarisch), aber auch Sprachen, die viele Migranten/-innen sprechen (Russisch, Griechisch, Türkisch). Selbstverständlich werden auch einige Korpora des Englischen erwähnt. Darauf folgt eine detailliertere Übersicht über Korpora, in denen die deutsche Sprache gespeichert ist. 


\subsection{NATIONALE KORPORA (außer mit Deutsch)}

Die (nationalen) Korpora der einzelnen Sprachen sind hier alphabetisch aufgelistet. In die Auswahl kamen Korpora der Nachbarsprachen der deutschsprachigen Länder und anderer wichtiger Sprachen. Ihre Namen sind zuerst linear (wortwörtlich) ins Deutsche übersetzt, dann folgen ihre offiziellen Namen in der Landesprache und - falls vorhanden - auch die offizielle englische Bezeichnung. Vertreten sind „große“, aber auch „kleinere“ Sprachen. Die Auswahl der Korpora unterliegt folgenden Kriterien: Zugänglichkeit, Aufwendigkeit. Hier werden nur leicht zugängliche Korpora erwähnt, in denen einfach und direkt (über eine Internetschnittstelle) recherchiert werden kann. Nach dem Bild der Startseite ${ }^{12}$ wird als Beispiel für die Arbeit mit dem jeweiligen Korpus ein Bild mit einigen Konkordanzzeilen angeführt. Die Konkordanzen werden durch die entsprechende Abfrage eingeleitet: in Klammer stehen Buttons oder Suchfeldnamen (in der Landessprache oder auf Englisch), in die die Abfrage eingegeben wird, bzw. die Beschreibung des Weges zu ihnen. Nach dem Doppelpunkt steht die Abfrage in der Abfragesyntax, die der Korpusmanager verlangt.

\subsubsection{Englisch}

\section{Britisches Nationalkorpus (BNC)/ British National Corpus}

http://www.natcorp.ox.ac.uk/

Das Britische Nationalkorpus beinhaltet 100 Mio. Wörter: $90 \%$ geschriebenes und 10\% gesprochenes Britisches Englisch ,aus den späten Jahren des 20. Jahrhunderts“ (BNC $2009 \rightarrow$ About BNC).

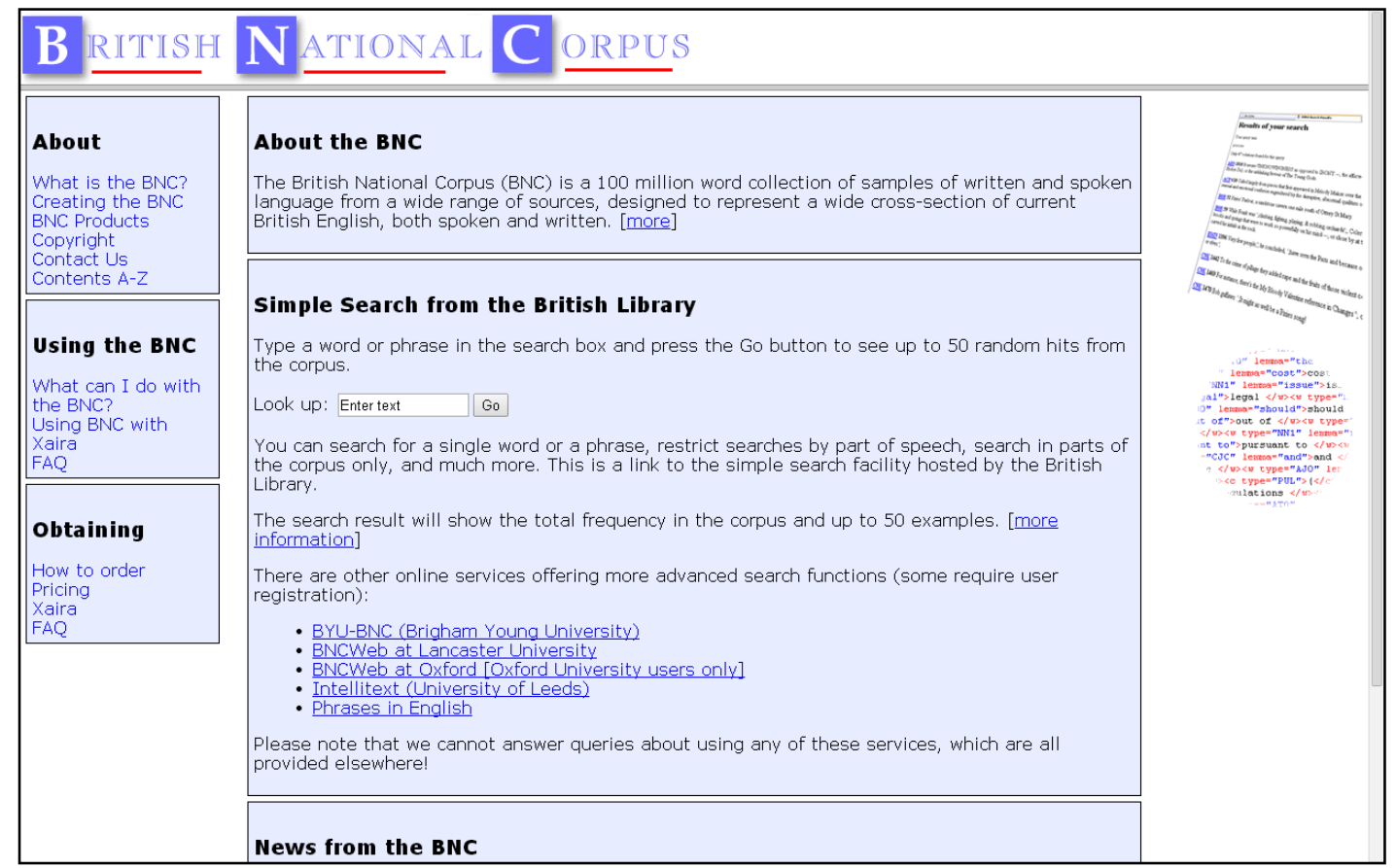

Abb. 8: BNC-Startseite (2014)

12 Stand Frühjahr 2014 - dieses wird sich mit großer Wahrscheinlichkeit mit der Zeit ändern, da auch die Korpusseiten einem regelmäßigen Lifting der graphischen Gestaltung unterzogen werden. 


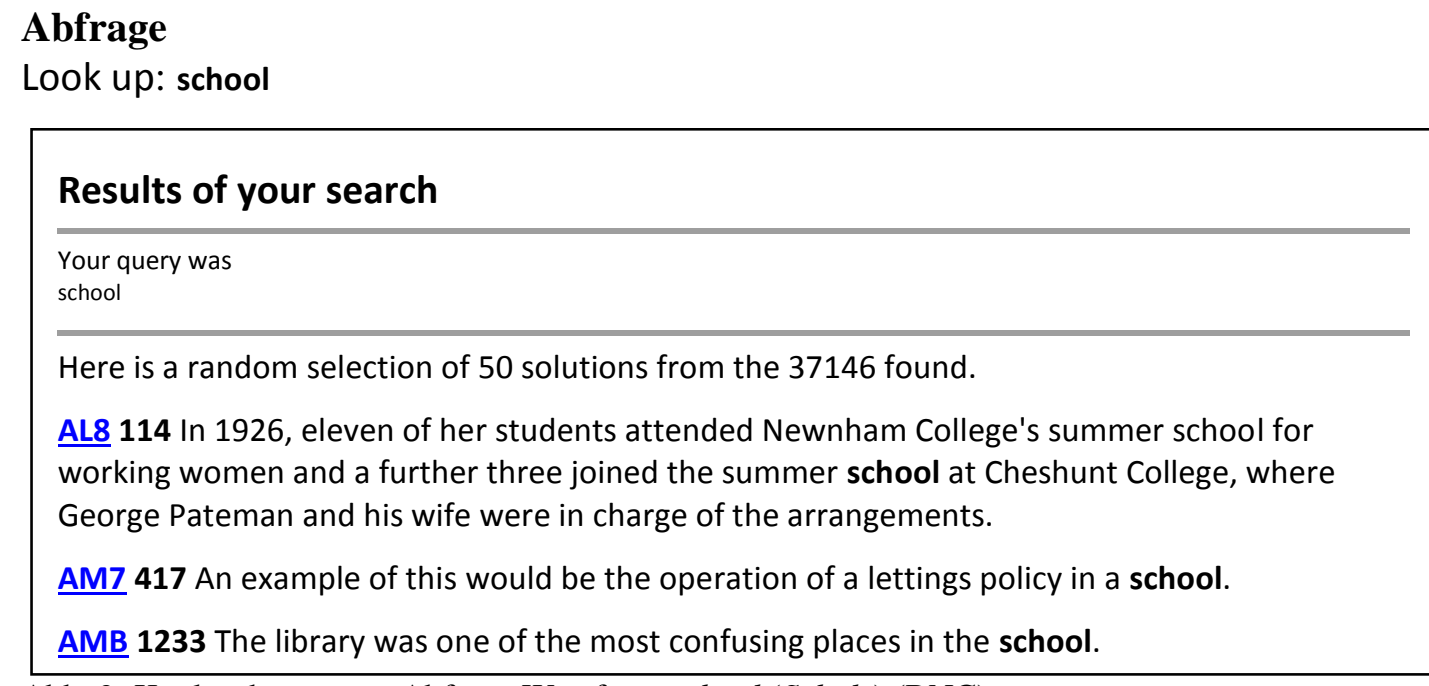

Abb. 9: Konkordanzen zur Abfrage Wortform school (Schule) (BNC)

Volles Korpusservice (Suchmöglichkeiten nach unterschiedlichen Attributen, statistische Funktionen etc.) ist mittels Xaira-Programm möglich. Download und Manual dazu sind auf der Homepage des BNC zu finden. Die Daten des BNC sind auch (teilweise) unter InterCorp recherchierbar.

Das Korpus ist lemmatisiert und morphosyntaktisch annotiert.

\section{Andere Korpora mit Englisch}

Andere Korpora mit Englisch sind auf mehreren Web-Seiten aufgelistet. Siehe z.B. unter Braun (2005) oder Davies (2014).

Ein interessantes Korpus ist bestimmt das GloWbE (Corpus of Global Web-based English: http://corpus2.byu.edu/glowbe/ ) mit fast 2 Mrd. laufenden Wörtern aus 20 Ländern, in denen Englisch als eine übliche Kommunikationssprache verwendet wird. Der Korpusmanager erlaubt Abfragen, bzw. eine Darstellung der Ergebnisse nach einzelnen Ländern, daher ist es auch ein wertvolles Instrument für die Untersuchung der einzelnen Varietäten des Englischen. Im Korpus sind ausschließlich Texte aus Internetseiten gespeichert.

Englisch ist auch in vielen Parallelkorpora vertreten. 


\subsubsection{Französisch}

\section{Textkorpus FRANTEXT/ Base textuelle FRANTEXT}

http://www.frantext.fr/ctlf/

Die „Textdatabase“ (wörtliche Übersetzung) beinhaltet Texte geschriebener französischer Sprache vom 12. bis zum 21. Jahrhundert mit dem Umfang von über 271 Mio. Wörtern. Die Schnittstelle sowie alle Informationen (bis auf eine kurze Projektdarstellung in Englisch) sind ausschließlich auf Französisch.
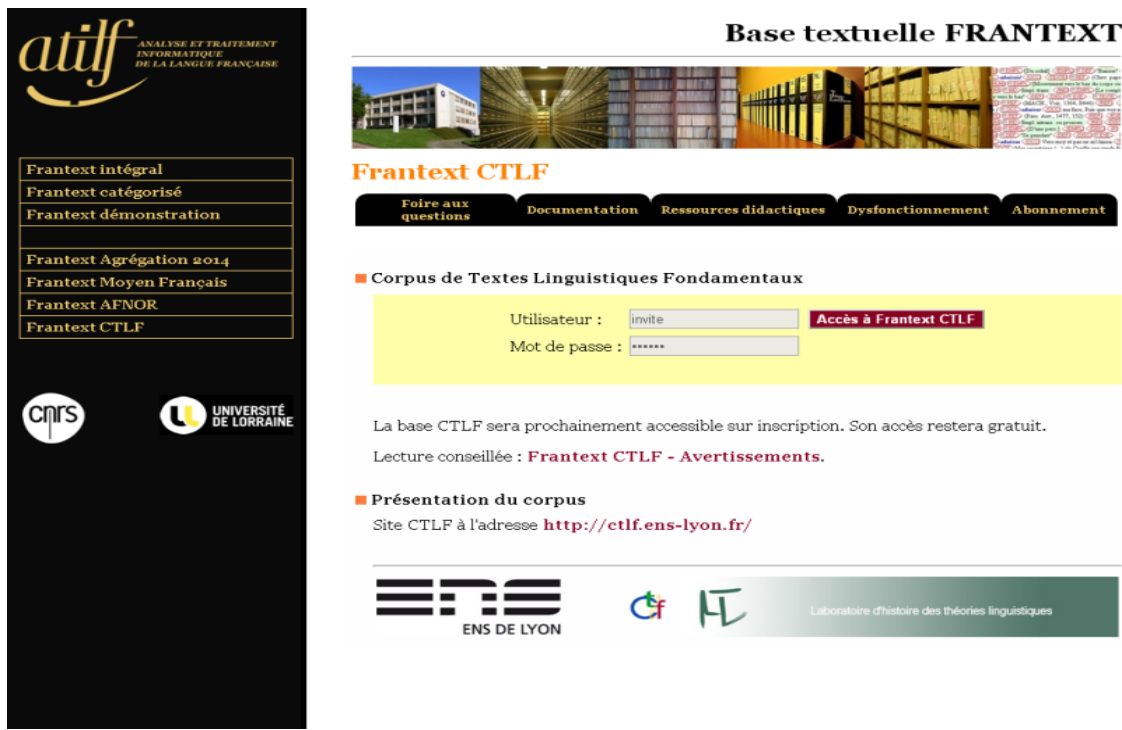

Présentation du corpus

Site CTLF à l'adresse http://ctlf.ens-lyon.fr/

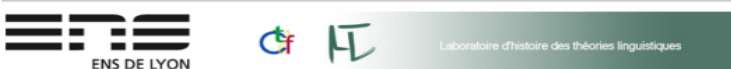

Abb. 10: Frantext-Startseit (2014)

\section{Abfrage}

Mot à rechercher: école expression régulière

\begin{tabular}{|c|c|c|c|c|}
\hline \multicolumn{3}{|c|}{ Résultats 1 à 20 / 557} & \multirow[b]{2}{*}{ école } & \multirow[b]{2}{*}{ de. l'artiste. L'observation montrant de } \\
\hline [1] & $\mathrm{CO02}$ & maniere, laquelle fait reconnoître la main ou l' & & \\
\hline [2] & $\mathrm{C002}$ & compositions : raison pourquoi ils avoient des & écoles & où les anciens enseignoient aux enfans ces \\
\hline [3] & $\mathrm{C} 002$ & , qui, au rapport de Suidas, tint une & école & de grammaire à Alexandrie, puis à Constantinople \\
\hline [4] & $\mathrm{C} 004$ & de différentes manieres dans les diverses & Écoles & de Peinture, sans cesser d'être le même ; le fond \\
\hline [5] & $\mathrm{CO04}$ & , dont on s'est servi jusqu'à présent dans les & Écoles & , pour nommer les parties de la frase ? Non, on est \\
\hline [6] & $\mathrm{CO04}$ & singularite que j'ai, abandonné ces termes de l' & École & ; mais uniquement, parce qu'ils m'ont paru ne pas \\
\hline [7] & $\mathrm{C} 055$ & Non, sans doute. II n'était plus ce temps où des & écoles & publiques, ouvertes et entretenues à grands \\
\hline [8] & C061 & disparut l'esprit exclusivement patricien de l' & école & classique, que commencèrent à s'introduire \\
\hline [9] & C061 & ), globe, mode, proche (prŏpius), rose, & école & sole (sŏlea), viole, v.-fr. voche (vŏco \\
\hline [10] & C061 & de o devant $m$ et $n$ : pomme, don, raison, bon, & école & - 2) De u bref ou de y : trop (lat.moy. truppus \\
\hline
\end{tabular}

Abb. 11: Konkordanzen zur Abfrage: Lemma école (Lemma Schule) (Frantext)

Frantext ist lemmatisiert und morphosyntaktisch annotiert.

\section{Andere Korpora mit Französisch}

Ein großes Korpus mit französischen Internettexten ist frWaC mit 1,6 Mrd. Positionen. Dieses Korpus ist ebenfalls lemmatisiert und morphosyntaktisch annotiert. Es ist zugänglich und recherchierbar auch über KontText auf der Homepage des ČNK (2014).

Es gibt auch ein Korpus des gesprochenen Französischen (etwa 400.000 Wörter) aus unterschiedlichen frankofonen Regionen - CRFP. 


\subsubsection{Griechisch}

\section{Nationales Korpus Griechischer Sprache (EThEG)}

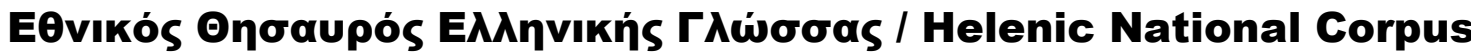

http://hnc.ilsp.gr/en/

Es ist ein repräsentatives Korpus des gegenwärtigen Griechisch: 47 Millionen laufende Wörter, ausschließlich modernes (nach 1990) geschriebenes Griechisch, stilistisch ausgewogen. Das Korpus wird laufend erweitert.

Interface und Manual sind auf Griechisch und Englisch.

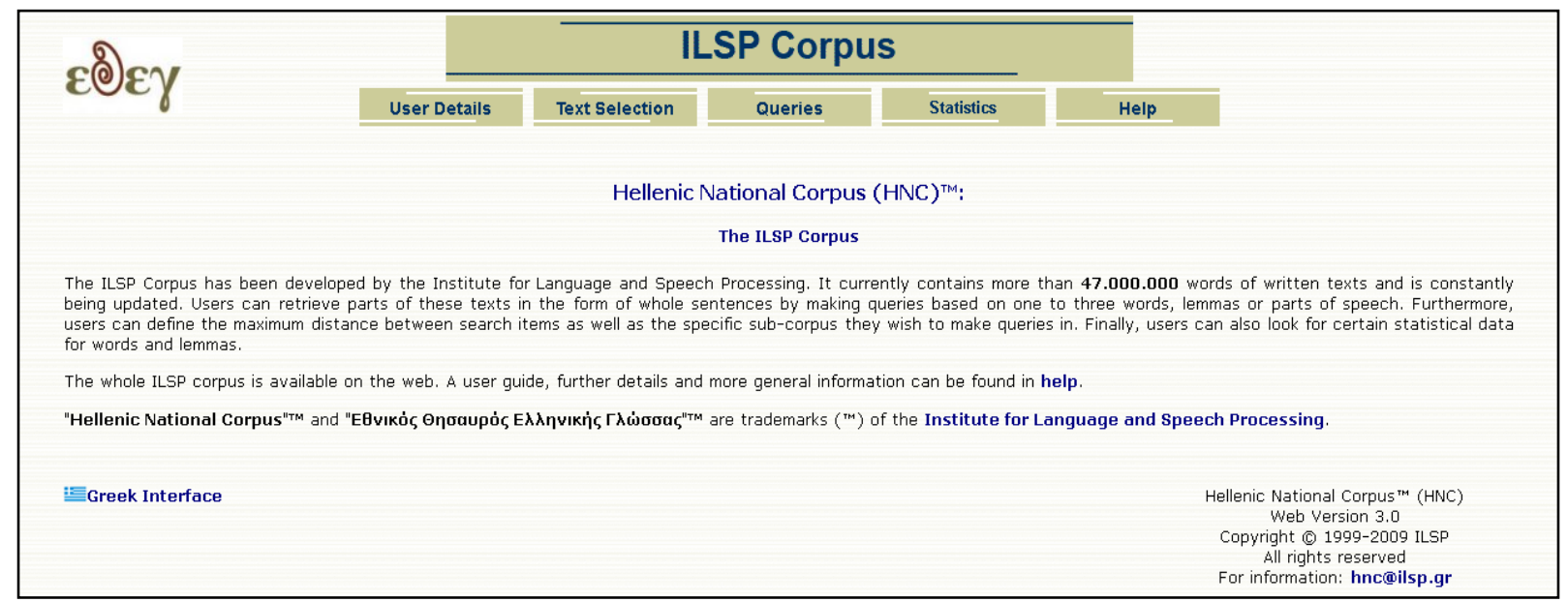

Abb. 12: EThEG/ HNC-Startseite (2014)

\section{Abfrage}

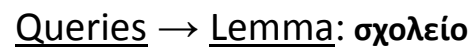

\begin{tabular}{|c|c|}
\hline$\underline{1}$ & 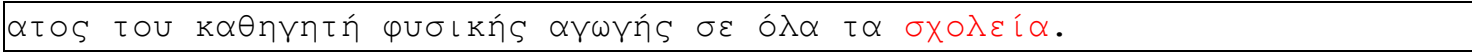 \\
\hline$\underline{2}$ & 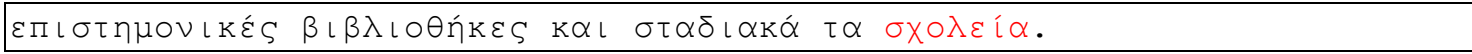 \\
\hline$\underline{\underline{3}}$ & 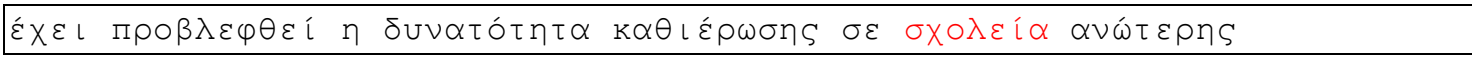 \\
\hline$\underline{4}$ & 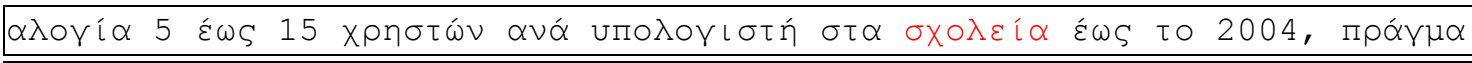 \\
\hline
\end{tabular}

Abb. 13: Konkordanzen zur Abfrage: Lemma $\sigma \chi 0 \lambda \varepsilon i o$ (Lemma Schule) (EThEG/ HNC)

EThEG ist lemmatisiert und morphosyntaktisch annotiert. 


\subsubsection{Italienisch}

\section{Korpus des geschriebenen Italienisch(CORIS) \\ Corpus di italiano scritto / Corpus of written Italian}

http://corpora.dslo.unibo.it

Das Korpus hat über 130 Mio. Wörter der geschriebenen Sprache, ist repräsentativ für das moderne Italienisch und stilistisch ausgewogen. Es wird sukzessive erweitert.

Die Homepage ist auf Italienisch und Englisch, das Abfrageformular auf Englisch.

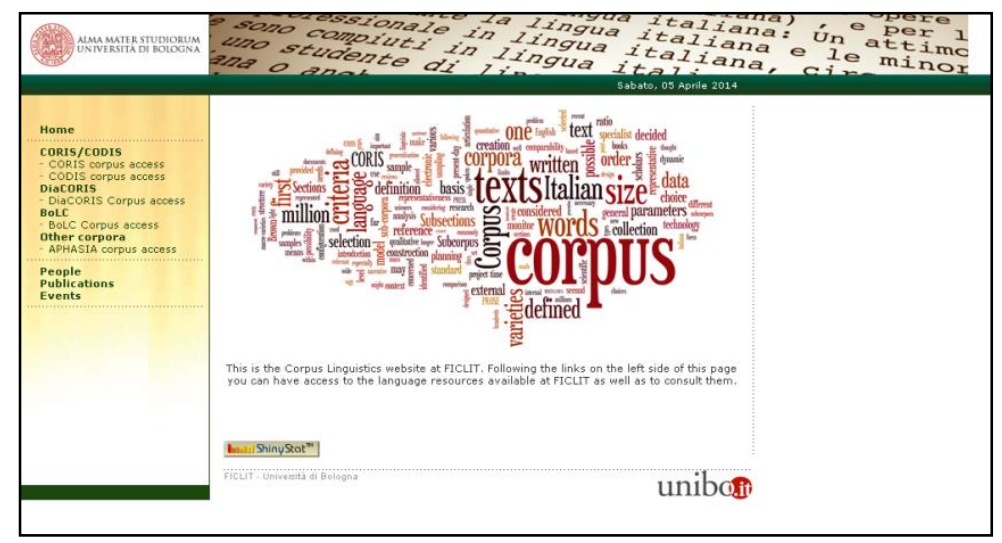

Abb. 14: CORIS-Startseite (2014)

\section{Abfrage \\ Query: [lemma="scuola"]}

\begin{tabular}{|c|c|c|}
\hline \multicolumn{3}{|l|}{ Corpus query result } \\
\hline \multicolumn{3}{|l|}{ Query: [lemma="scuola"] } \\
\hline Sorted: 0 & & \\
\hline \multicolumn{3}{|c|}{ Number of concordances: $30 / 30801$} \\
\hline , qualcuno all ' interno della & scuola & avesse saputo o intuito quant \\
\hline ure indicate. Iscrizione alla & scuola & materna Per_quanto riguarda I \\
\hline re_a innumerevoli parrocchie, & scuole & , gruppi locali . Nata nel di \\
\hline t La gastronomia italiana, la & scuola & di cucina, un elenco di rist \\
\hline Papini con il suo Chiudiamo le & scuole & Lo scrittore denunciava « I \\
\hline alla fine del primo giorno di & scuola & è parso raggiante. Ha subi \\
\hline muro del convento adiacente la & scuola & e si mosse per prenderla. Po \\
\hline né è mai stato il ruolo delle & scuole & di retorica nei secoli passat \\
\hline non aveva mai avito problemi a & scuola & ; invece pensò come poteva fa \\
\hline a ? Anche voi uomini avete una & scuola & ? - soffia il Piccolo Drago s \\
\hline
\end{tabular}

Abb. 15: Konkordanzen zur Abfrage: Lemma scuola (Lemma Schule) (CORIS)

CORIS ist lemmatisiert und morphosyntaktisch annotiert. Die Abfragesyntax entspricht derjenigen, die im CQL-Mode im InterCorp verwendet wird. (Es gilt natürlich nicht für die morphosyntaktischen Zeichen (Tags).)

\section{Andere Korpora mit Italienisch}

Ein großes Korpus für Italienisch ist auch das "LA REPUBBLICA" CORPUS mit 380 Mio. Wörtern aus der gleichnamigen Tageszeitung oder das Korpus aus italienischen Internettexten (itWaC). Das Korpus itWaC ist gemeinsam mit dem Web-Korpus des Deutschen (deWaC), Französischen (frWaC) und des britischen Englisch ( $\mathrm{ukWaC}$ ) auch über die Homepage des ČNK (2014) zugänglich und recherchierbar. 


\subsubsection{Kroatisch}

\section{Kroatisches Nationalkorpus (HNK)}

\section{Hrvatski nacionalni korpus / Croatian National Corpus}

http://www.hnk.ffzg.hr/

Direktlink zu NoSketch: http://filip.ffzg.hr/bonito2/run.cgi/first_form

Das Korpus hat d.Z. rund 115 Mio. laufende Wörter, wird regelmäßig ergänzt und ist stilistisch ausgewogen.

Zugang über Bonito oder NoSketch Engine. Das Interface und Manual sind auf Kroatisch und Englisch.

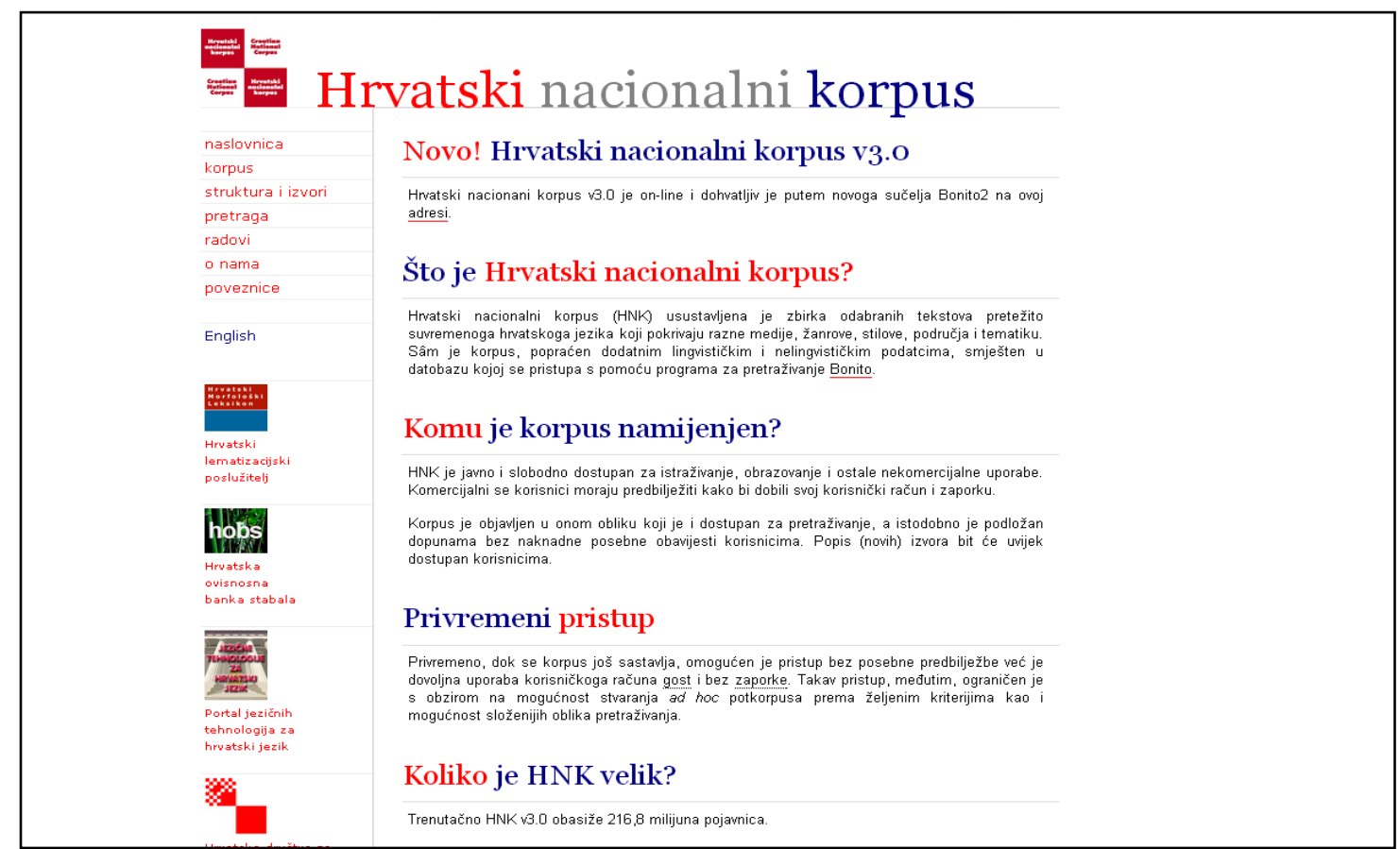

Abb. 16: HNK-Startseite (2014)

Abfrage
Query Type: Lemma $\rightarrow$ Lemma: škola

\begin{tabular}{|l||c|l||l||}
\hline doc\#11 & s radovima djece iz osnovnih & škola & , uz tvrdnju da će ' svima onima koji su \\
\hline \hline doc\#26 & više od 700 svjedodžbi za srednje & škole & i fakultete , \\
\hline \hline doc\#30 & u crkvi i u Hrvatskoj osnovnoj & školi & i gimnaziji u Budimpešti . \\
\hline \hline doc\#67 & na stranim sveučilištima ili srednjim & školama & , a određenom broju smo \\
\hline \hline doc\#68 & planova je i osnivanje posebne & škole & u Opatiji za obrazovanje managemanta . \\
\hline \hline
\end{tabular}

Abb. 17: Konkordanzen zur Abfrage: Lemma škola (Lemma Schule) (HNK)

Das HNK ist lemmatisiert und morphosyntaktisch annotiert. 


\subsubsection{Polnisch}

\section{Nationalkorpus der Polnischen Sprache (NKJP)}

\section{Narodowy Korpus Języka Polskiego / National Corpus of Polish}

\section{http://nkjp.pl}

Es ist ein stilistisch ausgewogenes Korpus mit 1.800 Millionen laufenden Wörtern der polnischen, ausschließlich geschriebenen Sprache (aufgeteilt in Presse-Texte und Bücher). Das Interface und eine Kurzbeschreibung sind auf Polnisch und Englisch.

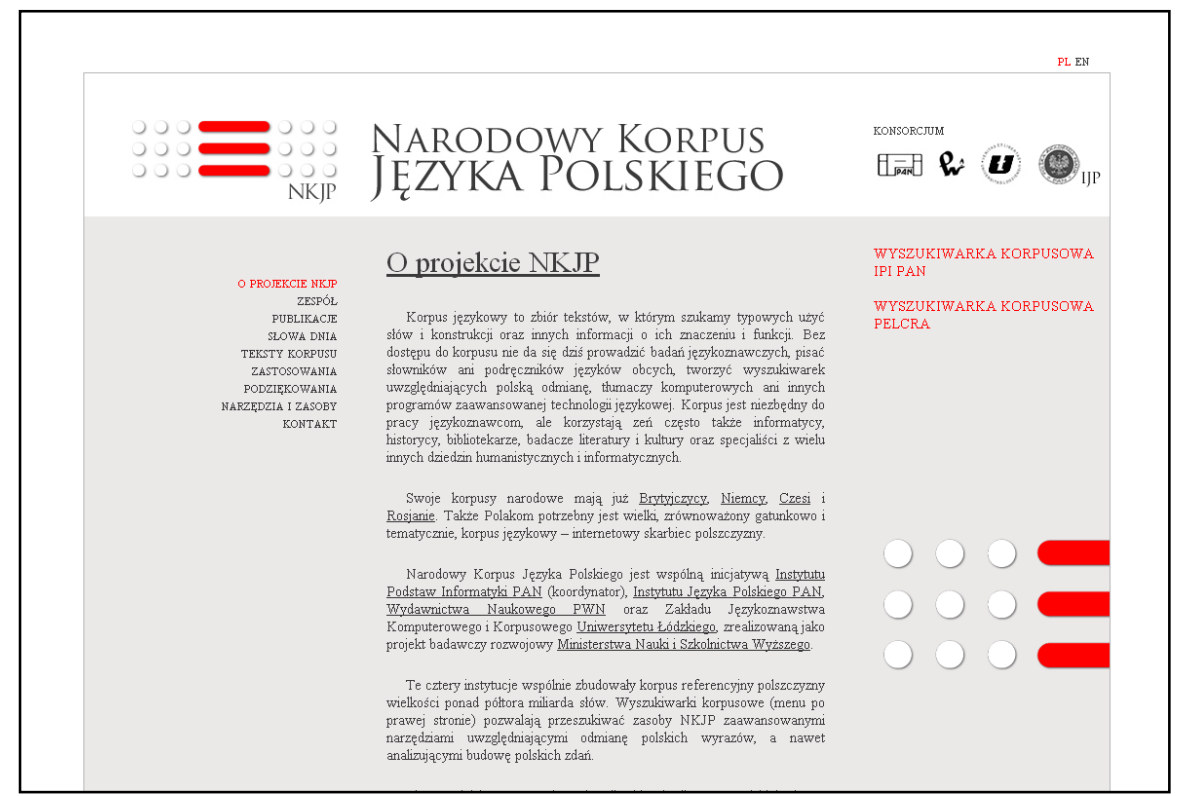

Abb. 18: NPKJP - Startseite (2014)

\section{Abfrage \\ Query/Zapytanie: [base="szkoła"]}

\begin{tabular}{|r|l|l|}
\hline -W dzień jest w & szkole [szkoła:subst:sg:loc:f] & , ale nie mogę jej \\
\hline - Gdzie ona chodzi do & szkoły [szkoła:subst:sg:gen:f] & ? - Tutaj, niedaleko \\
\hline - A ja zaraz po & szkole [szkoła:subst:sg:loc:f] & . Uśmiechał się kiedy ruszyli \\
budynku z cyklu "1000 & szkół [szkoła:subst:pl:gen:f] & na 1000-lecie". Ze \\
\hline było go więcej w wiejskich & szkołach [szkoła:subst:pl:Ioc:f] & . W województwie łódzkim skrzyżowano \\
\hline słyszała już takie nazwisko w & szkole [szkoła:subst:sg:loc:f] & , przyznała tę kwaterę właśnie \\
\hline bitew podręczniki historii obowiązujące w & szkołach [szkoła:subst:pl:loc:f] & Obozu Pokoju przyznawały na skąpo \\
\hline dzieci przepytywanych na lekcjach w & szkole [szkoła:subst:sg:loc:f] & , co też w domach \\
\hline zebrał informacje o nastrojach w w & szkole [szkoła:subst:sg:loc:f] & podstawowej im. "Walczącej \\
\hline ulic, budynków, & szkół [szkoła:subst:pl:gen:f] & , mostów, placów, \\
\hline
\end{tabular}

Abb. 19: Konkordanzen zur Abfrage: Lemma szkoła (Lemma Schule) (NPKJP)

Das Korpus ist lemmatisiert und morphosyntaktisch annotiert. Die Abfrage nach einem Grundwort (Lemma) liefert automatisch bei jedem KWIC auch morphologische Angaben (hier Wortart, Numerus, Kasus, Genus). 


\subsubsection{Russisch}

\section{Nationalkorpus der russischen Sprache (NKRJa) \\ Национальный корпус русского языка / Russian National Corpus}

http://www.ruscorpora.ru/

Das Russische Nationalkorpus hat über 149 Mio. Tokens (entspricht etwa 100 Mio. Wörtern). Mehr als die Hälfte bilden Sachtexte, etwa 40\% Belletristik und weniger als 4\% gesprochene Sprache.

Die geschriebenen Texte stammen aus der Mitte des 18. Jahrhunderts bis heute, gesprochene Texte wurden zwischen 1950 und heute aufgenommen und für das Korpus aufbereitet.

Die Interface sowie eine gute Beschreibung des Korpus inkl. einiger statistischer Angaben zu den Texten und Tokens gibt es sowohl auf Russisch als auch auf Englisch.

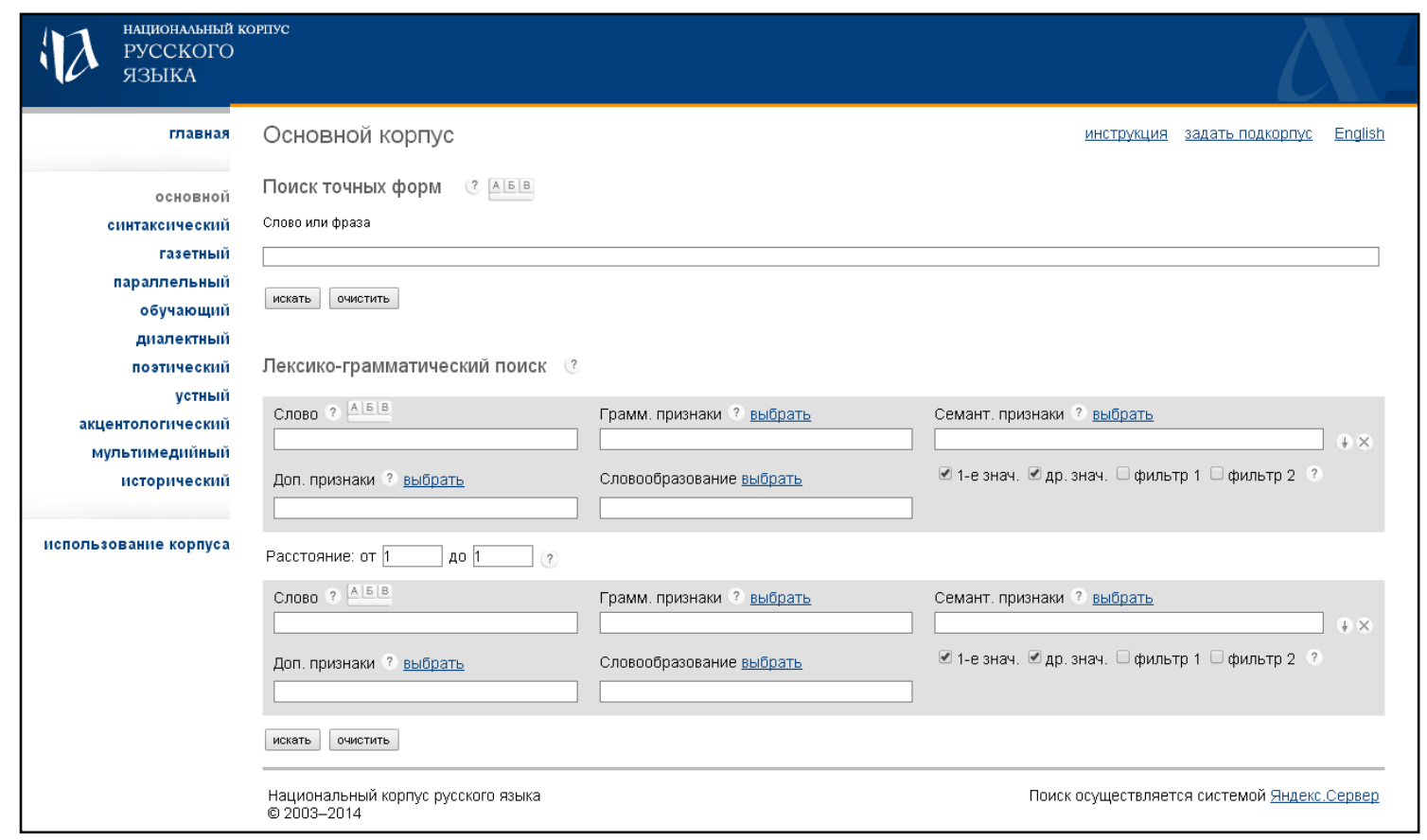

Abb. 20: NKRJa/ RNC - Abfrageseite (2014)

\section{Abfrage \\ Слово/ Word ${ }^{13}$ : школа}

\begin{tabular}{|c|c|c|}
\hline тебе понятие о той великой & школе & жизни, которая образует истинных человеков $\leftarrow \ldots \rightarrow$ \\
\hline 03-06-00139а, и Програ́ммой подде́ржки нау́чных & школ & , грант N 005-97893. $\leftarrow \ldots \rightarrow$ \\
\hline Я же по́мню, как в & шко́ле & уви́жу таку́ю схе́му в кабине́те $\leftarrow \ldots \rightarrow$ \\
\hline вре́мя Ершо́в, впосле́дствии возгла́вивший программистскую & шко́лу & в Новосибирске, ещё рабо́тал в $\leftarrow \ldots \rightarrow$ \\
\hline в ча́стных ли́бо в воскре́сных & шко́лах & , "общеобразова́тельная же шко́ла не должна́ $\leftarrow \ldots \rightarrow$ \\
\hline при́нципами; напро́тив, оно́ изоби́лует разли́чными & шко́лами & , мно́гие из кото́рых тракту́ют одни́ $\leftarrow \ldots \rightarrow$ \\
\hline нау́к, профе́ссора Госуда́рственного университе́та - Вь́сшей & шко́лы & эконо́мики \\
\hline пройду́т торже́ственные собра́ния, в подше́фных & шко́лах & - встре́чи с молодёжью, а в $\leftarrow \ldots \rightarrow$ \\
\hline Говори́шь, в & шко́ле & опя́ть ничего́ не за́дали? $\leftarrow \ldots \rightarrow$ \\
\hline день, когда́ Па́влик верну́лся из & шко́лы & , на́спех поел, кое-ка́к что́-то накалякал $\leftarrow \ldots \rightarrow$ \\
\hline
\end{tabular}

Abb. 21: Konkordanzen zur Abfrage Lemma школа (Lemma Schule) (NKRJa/ RNC)

Das Korpus ist lemmatisiert und morphosyntaktisch annotiert.

13 Word = Lemma bei Eingabe der Grundform 


\subsubsection{Slowenisch}

\section{Korpus der slowenischen Sprache (FIDAPLUS) \\ FIDAPLUS Korpus slovenskega jezika}

http://www.fidaplus.net/

Korpus slovenskega jezika FIDAPLUS ist ein stilistisch ausgewogenes Korpus mit über 600 Millionen Textwörtern. Interface und Manual sind auf Slowenisch, Grundinformationen auch auf Englisch.

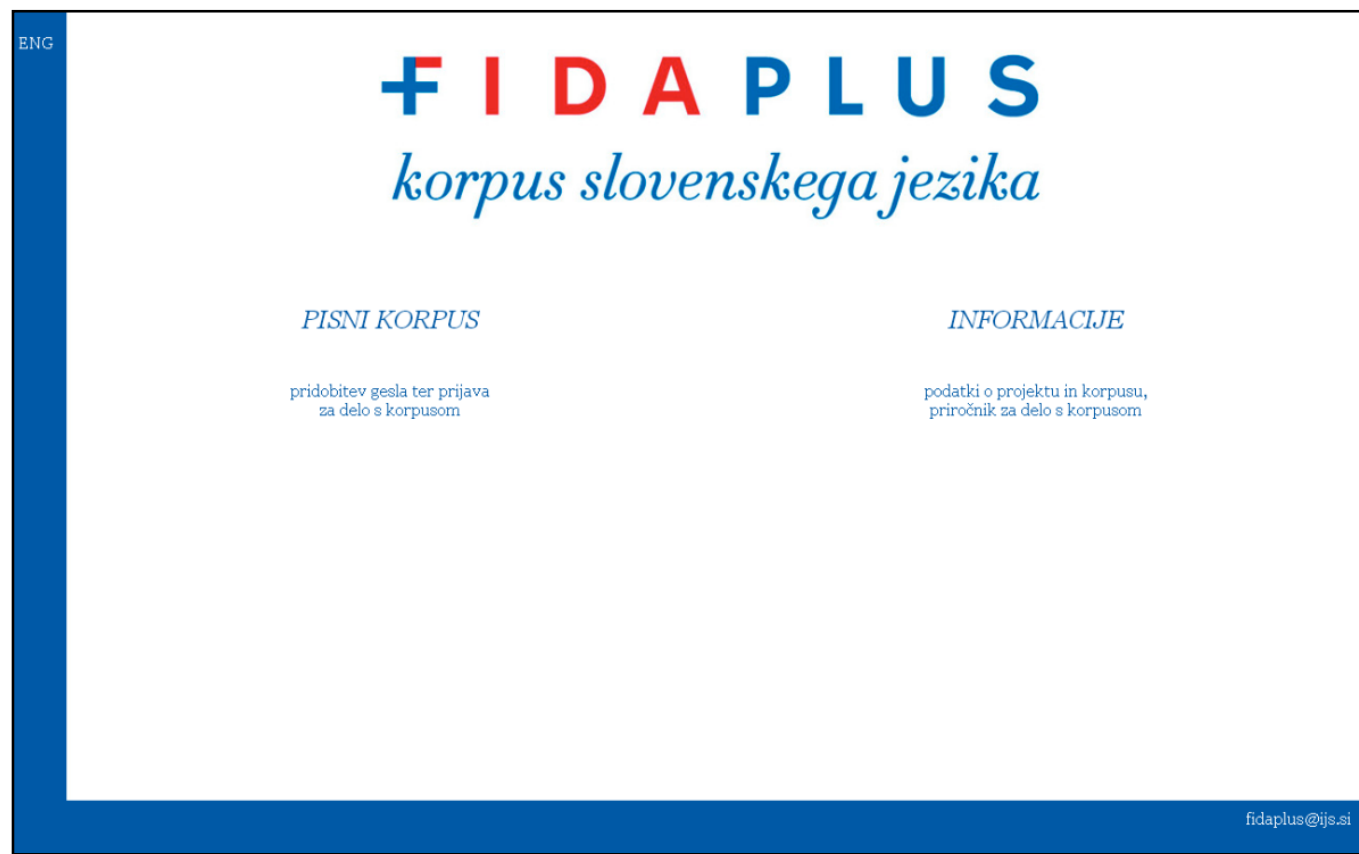

Abb. 22: FIDAPLUS-Startseite (2014)

\section{Abfrage}

Osnovno iskanje: \#1šola

\begin{tabular}{|c|c|}
\hline DELO........ 0000007 & Škoda vnovi gostinski šoli - Povodenj je v celjski občini hudo prizadejala vsto šolskih \\
\hline DELO........ 0000007 & škode. Precej je poškodovana tudi nova stavba srednje gostinske šole in ob njej nova telovadnica. Na obeh objektih je \\
\hline DELO ........ 0000007 & bo potrebno v celoti zamenjati, vkletni etaži nove šole pa so uničeni tlaki in stene. Na srečo ne \\
\hline DELO ........ 0000013 & otrok v oddaljene kraje zaradi prostorske stiske v obeh čmomaljskih šolah in na pritiske občine in najemnika kotlarne na občane naselja \\
\hline NOVI. TEDNIK. 0000005 & mestni občini so se v projekt vključili dijaki Srednje ekonomske šole ter poslomna enota Telekoma Celje, katere ekipa je poskrbela \\
\hline MENS. HEALT . . 0001116 & izkaznico, prosim? Kje si ti, v srednji šoli ? Leto dni je že od tega, sinko moj \\
\hline MENS. HEALT . . 0001118 & Kdo bo pospravil kopalnico, kdo bo peljal otroke v šolo, kdo se v družbi vede bolj prostaško, kdo \\
\hline DNEVNIK ..... 0000208 & . Nagrade je prejelo tudi 15 dijakov z dolenjskih srednjih šol . Med nagrajenci so štirje mladi raziskovalci iz Ruske federacije \\
\hline DNEVNIK..... 0000232 & Učenci ribenske osnowne šole čakajo na šolski avtobus še vedno kar na cesti \\
\hline DNEVNIK . . . . 0000234 & Zaradi predvidene popotresne obnove šole učenci obiskujejo pouk na Bledu \\
\hline
\end{tabular}

Abb. 23: Konkordanzen zur Abfrage: Lemma šola (Lemma Schule) (FIDAPLUS)

Das Korpus ist lemmatisiert und morphosyntaktisch annotiert. 


\subsubsection{Slowakisch}

\section{Slowakisches Nationalkorpus (SNK)}

\section{Slovenský národný korpus / Slovak National Corpus}

Die Version 6.1 public (aus Herbst 2013) beinhaltet geschriebene slowakische Texte aus den letzten Jahren. Deutlich überwiegen publizistische Texte (fast 70\%), belletristische betragen etwa $14 \%$, Fachtexte 15\%. Das Korpus 6.1 hat 655 Mio. Wörter.

Für registrierte Nutzer sind auch andere Korpora recherchierbar: Korpus der gesprochenen slowakischen Sprache, einige Web-Korpora, sowie (noch relativ kleine) Parallelkorpora Slowakisch - Bulgarisch, Englisch, Latein, Russisch, Tschechisch, Ungarisch.

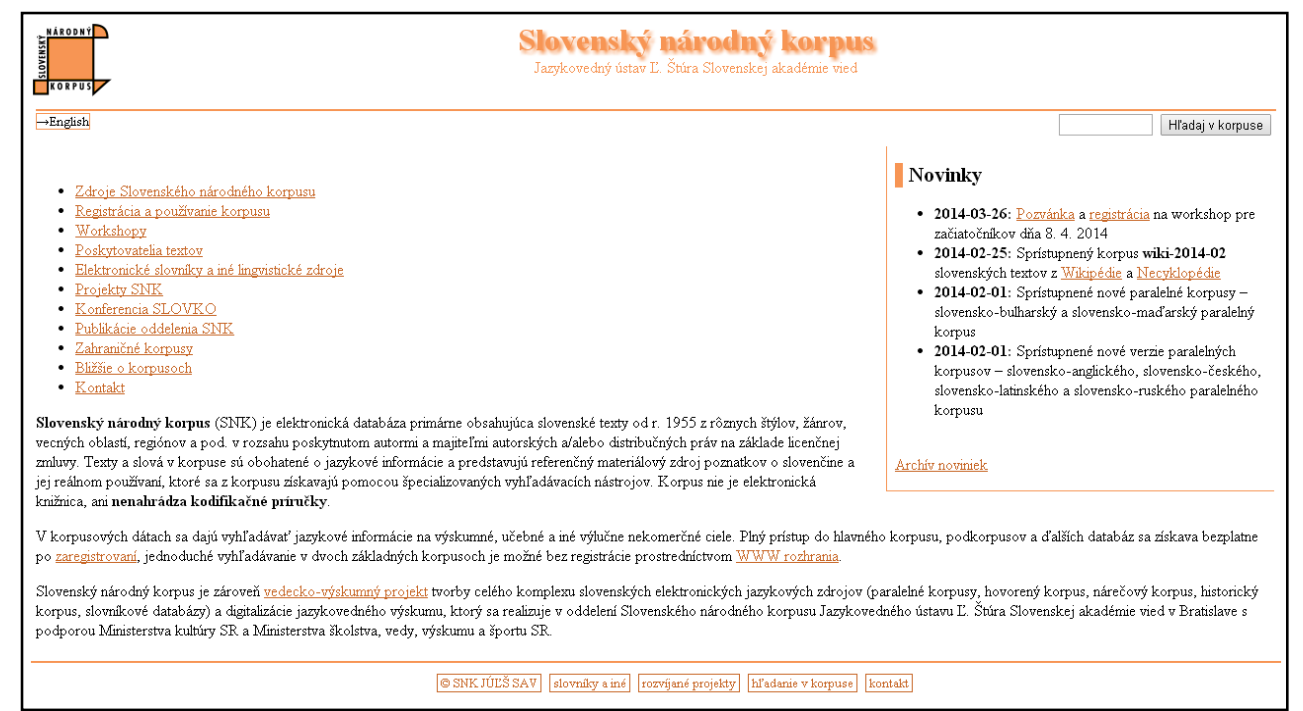

Abb. 24: SNK-Starseite (2014)

\section{Abfrage \\ Query Type: Lemma $\rightarrow$ Lemma: škola}

\begin{tabular}{|c|c|}
\hline ? Kňazi potrebujú poznat' umenie . Po skončení školy & prichádzajú do kostolov, o ktorých vedia len \\
\hline Tvorivá dielňa Krása z hliny bude v základnej škole & určená pre všetkých, ktorí si budú chciet' \\
\hline \multicolumn{2}{|c|}{ potrebám chlapcov. Na systéme wučovania na základných školách sa podpísala najmä ženská ruka, a preto } \\
\hline iniciátor projektu. Podla neho sa deti v škole & učia rozličné veci, ale mali by sa \\
\hline ceslovenskej wýtvarnej sútaži , ale výtvarná wýchova v škole & vaj nepatrí k jeho najoblúbenejším predmetom . ", \\
\hline 19. júla v areáli amfiteátra a základnej školy & v Malatinej na Orave . Úvod sobotného programu \\
\hline , a tak poskytli argumenty pre vznik d'alších škôl & Kvantitatíny rozvoj univerzít má však aj iný \\
\hline sa narodil v Starom Smokovci . Do l'udovej školy & chodil v Poprade, do gymnázia v Kežmarku \\
\hline ôsmom, ani M . Horkheimer a frankfurtská škola & . V Nemecku siaha tradícia myslenia o politike \\
\hline že nedokáže pokračovat' v štúdiu ani na inej škole & . Určitá čast' šikanovaných vidí v samovražde jediné \\
\hline
\end{tabular}

Das Korpus ist lemmatisiert und morphosyntaktisch annotiert. 


\subsubsection{Spanisch:}

\section{Korpus des Spanischen/ CORPUS DEL ESPAÑOL}

http://www.corpusdelespanol.org/

Das Korpus beinhaltet Texte ab dem Jahr 1200 bis heute, inkludiert auch Texte gesprochener Sprache aus dem 20. und 21. Jahrhundert.

Manual und Interface sind auf Englisch.

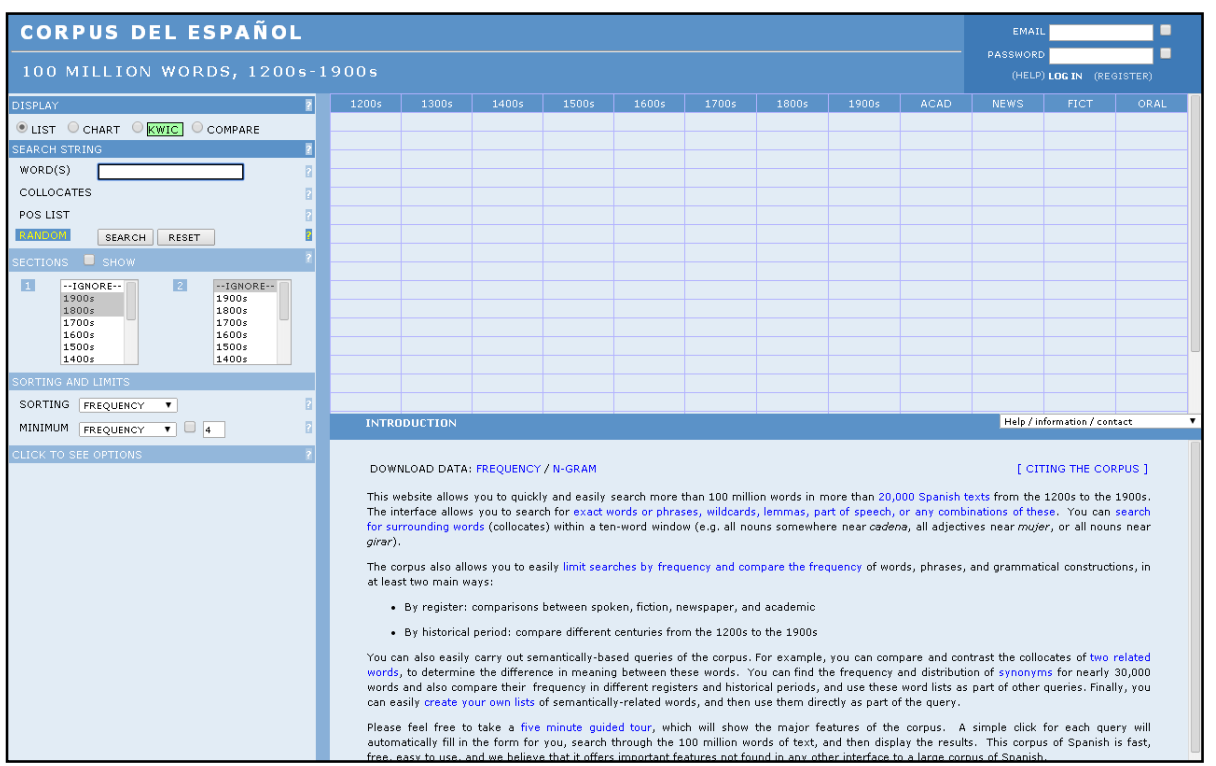

Abb. 26: Corpus del Español - Start- und Abfrageseite (2014)

\section{Abfrage \\ WORD(S): [escuela]}

\begin{tabular}{|c|c|c|}
\hline por la que agora tengo. Juan.$-i$ Tienen & escuelas & - Infinitas. Los señores, y primer \\
\hline ". Se considera que la etapa culminante de la " & escuela & bolera " discurre entre 1835 y 1880 , en coincid \\
\hline Massachusetts (MIT)- - o del norte de California donde hay & escuelas & como Stanford, que producen una corriente con \\
\hline en lo que tienen de fundamental y común a & escuelas & conservadoras $? i$ No es esto lo que he hecho \\
\hline conocen á Cristo ; los que nunca & escuela & de Cristo no se puede decir que niegan á Cristo \\
\hline en primaria . Pero después teníamos en & escuela & de educación, que preparaba a los pedagogos \\
\hline en esta parte más novedad & escuelas & de toda pensión o retribución particular. Cabalm \\
\hline frecuentes en los porteros de las mismas & escuela & debe ser para el niño un lugar sano y fortifica \\
\hline gran neoconfuciano Zhu XI & escuela & En el siglo XIV estas doctrinas fuer \\
\hline haréis más llevadera & escuela & donde habréis aprendido que el hombre para se \\
\hline la subvención anual (grant ) votada & escuelas & elementales, el mismo Departamento creó cier \\
\hline a escuelas prácticas para agricultura, pero verdader & escuelas & eminentemente prácticas , no impartir la enser̂́ \\
\hline y seudo - filosófico, comunes a las di & escuelas & en que se divide y $\mathrm{s}$ \\
\hline
\end{tabular}

Abb. 27: Konkordanzen zur Abfrage escuela (Lemma Schule) (Corpus del Español)

Das Korpus ist lemmatisiert und morphosyntaktisch annotiert.

Von demselben Autor (Mark Davies), mit der gleichen Schnittstelle und mit gleichen Recherchemöglichkeiten gibt es auf der Homepage http://corpus.byu.edu/corpora.asp Links $\mathrm{zu}$ anderen hilfreichen Korpora: die meisten sind $\mathrm{zu}$ (amerikanischem, kanadischem und britischem) Englisch, aber auch ein relativ großes Korpus (45 Mio. Wörter) zum Portugiesischen. 


\subsubsection{Tschechisch}

\section{Tschechisches Nationalkorpus (ČNK) \\ Český národní korpus/ Czech National Corpus}

https://www.korpus.cz/

Das Instrument besteht aus mehreren Korpora. Im synchronen Teil der geschriebenen Sprache befinden sich mehr als $2 \mathrm{Mrd}$. Wörter. Die gesprochene tschechische Sprache wird mit Transkripten von fast 3 Mio. Wörtern repräsentiert, zu ihnen ist auch immer die Tonspur abrufbar.

Historische tschechische Texte sind im Diakorp zu finden (fast 5 Mio. Wörter).

Über die Schnittstelle KonText des Tschechischen Nationalkorpus gelangen registrierte Nutzer auch zu großen Korpora anderer Sprachen und auch zum InterCorp.

Das Interface und die Beschreibungen sind auf Tschechisch und Englisch.

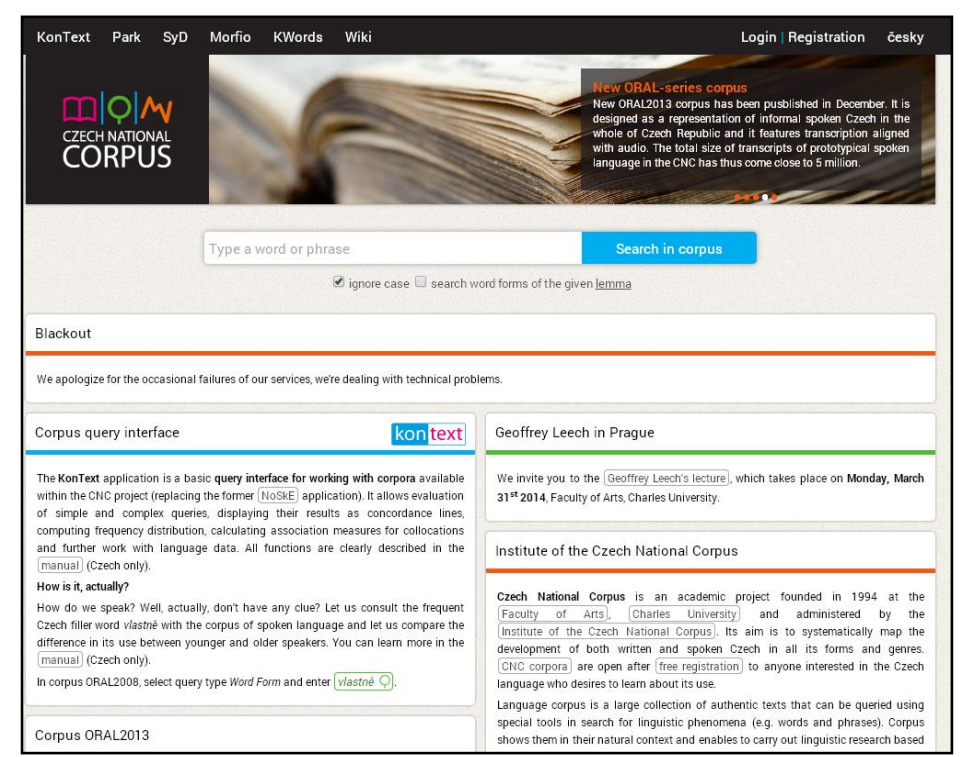

Abb. 28: С̆NK-Startseite (2014)

Abfrage

Query Type: Lemma $\rightarrow$ Lemma: škola

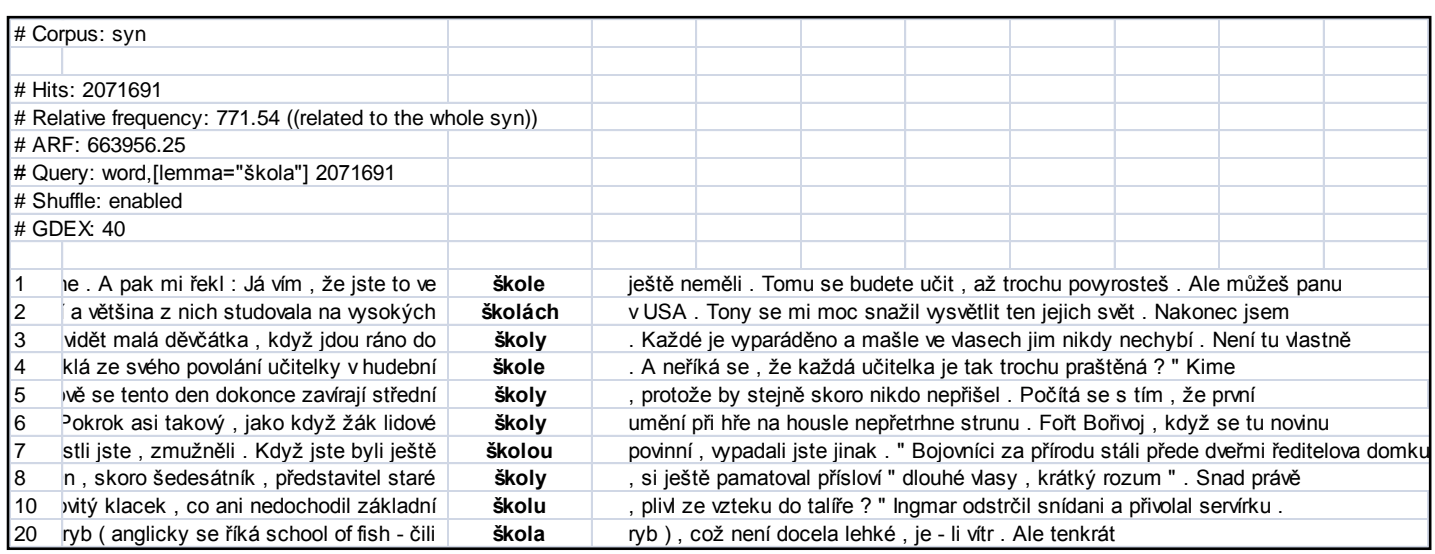

Abb. 29: Konkordanzen zur Abfrage: Lemma škola (Lemma Schule) (ČNK)

Das Korpus ist lemmatisiert und morphosyntaktisch annotiert. 


\subsubsection{Türkisch}

\section{Türkisches Nationalkorpus (TUD)}

\section{Türkçe Ulusal Derlemi / Turkish National Corpus}

\section{http://www.tnc.org.tr/index.php/en/}

Das Türkische Nationalkorpus beinhaltet etwa 50 Millionen Wörter in Texten aus den Jahren 1990 - 2009, etwa 2\% bilden Transkripte der gesprochenen Sprache. Es ist stilistisch ausgewogen. Das Interface und die Beschreibung sind auf Türkisch und Englisch.

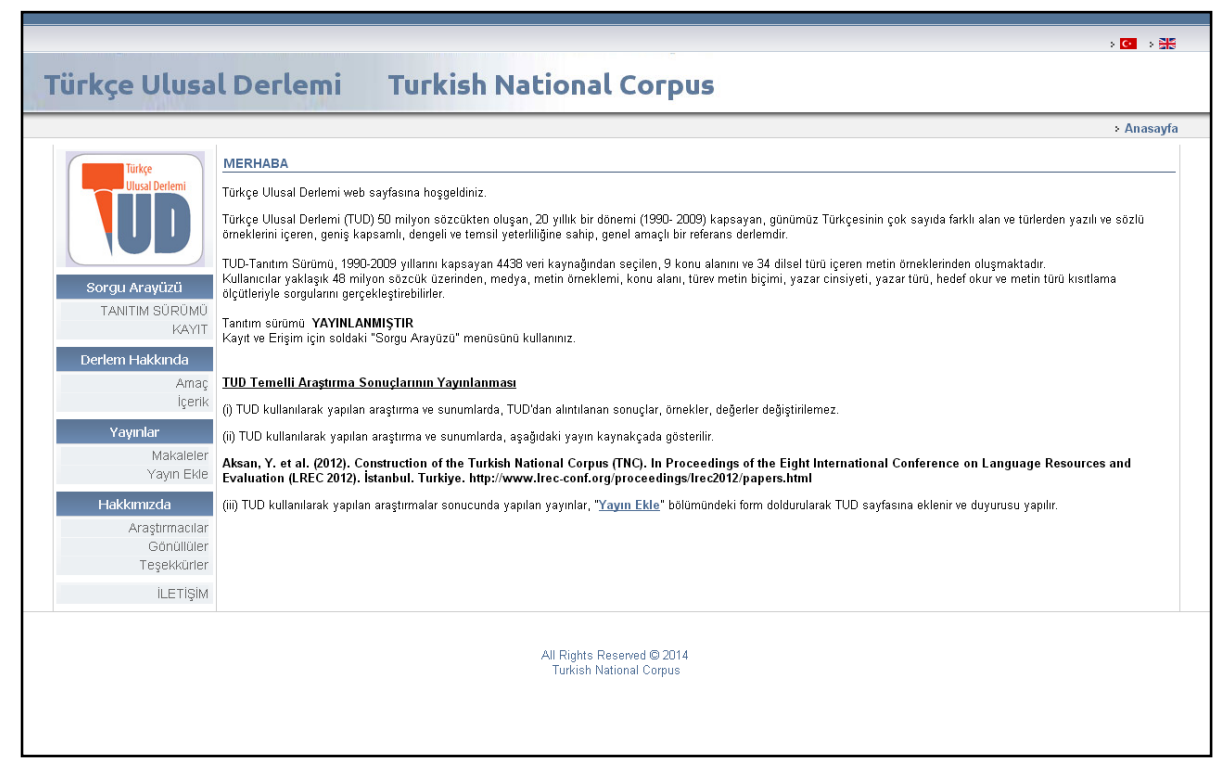

Abb. 30: TNC-Startseite (2014)

\section{Abfrage \\ Query Term: okul*}

\begin{tabular}{|c|c|c|c|}
\hline 1 & konuştu; - "Hocam, biz hedefteyiz. & Okuldan & ayrılmak istiyoruz. Biliyorsunuz dün \\
\hline 2 & göreceğimiz çok açıktır. Bunun için & okullarda, & Prof. Kalter'in dediği gibi mesela \\
\hline 3 & rahibeler yılar. Romana konu olan & okul & 150 yıl önce açılan Özel \\
\hline 4 & içindi senin özbenliğinin. Bak baba, & okula & iki yıl erken başlatılmış her \\
\hline 5 & Devlet Üretme Çiftlikleri, tarım meslek & okulları, & tohum ve arazi ıslah istasyonları \\
\hline 6 & muhit, gittiği yazlık yer, okuduğu & okul, & babasının işi, kıyafetlerinin markası, taktığı \\
\hline 7 & zekâlı bedevi, kuş beyniyle bizim & okula & nasıl birincilikle girdi, anlamıyorum. Oğlu \\
\hline 8 & Enstitüsü mezunu, daha sonra o & okulun & adı Öğretmen Okulu olarak değiştirilmiş, \\
\hline 9 & tılarımızın ortasında Ali'yle gelmiştik okula? & Okulun & o büyük konferans salonunda kura \\
\hline 10 & miyorlardı. İşleriyle öylesine doluydular ki! & Okulda & yoruldukları yetmiyor gibi çalışmalarını, e \\
\hline
\end{tabular}

Abb. 31: Konkordanzen zur Abfrage: Wortform okul- (Wortform Schule mit offenem Ende) (TUD)

Das TUD ist (noch) nicht morphosyntaktisch annotiert und lemmatisiert. 


\subsubsection{Ungarisch}

\section{Das ungarische Nationalkorpus (MNSz)}

\section{Magyar Nemzeti Szövegtár / Hungarian National Corpus}

http://corpus.nytud.hu/mnsz/

Das Ungarische Nationalkorpus umfasst fast 188 Mio. laufender Wörter in Texten aus den letzten Jahrzehnten. Fast die Hälfte der Texte stammt aus Presse und Publizistik. Neben der üblichen Recherche (nach unterschiedlichen Attributen) ist auch die Eingrenzung der Region möglich, dadurch können Varianten des Ungarischen (aus Ungarn, der Slowakei, Transkarpatien, Transsylvanien und Vojvodina) verglichen werden.

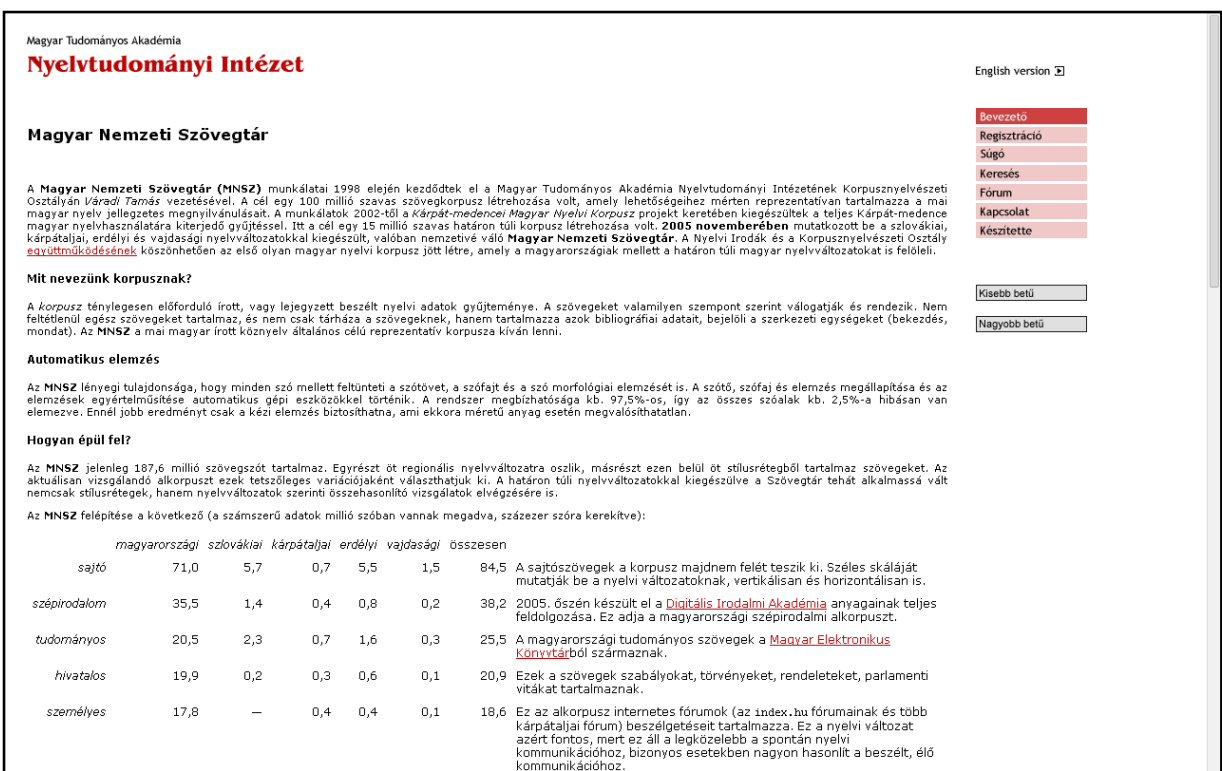

Abb. 32: MNSz-Startseite (2014)

\section{Abfrage}

Query $\rightarrow$ Stem: iskola

\begin{tabular}{|c|c|c|c|c|}
\hline \multirow{2}{*}{\multicolumn{4}{|c|}{$\begin{array}{l}\text { Region: Hungary, Slovakia, Subcarpathia, Transylvania, Vojvodina } \\
\text { Subcorpus: media, literature, scientific, official, informal }\end{array}$}} & \\
\hline & & & & \\
\hline & \\
\hline \multicolumn{5}{|c|}{ Size of corpus queried: 187644886 words } \\
\hline \multicolumn{5}{|c|}{ Number of matches: $64094 \quad 341,57$ / million words } \\
\hline 1. & & Az erõs akaratú Márton István & iskolája N.PSe3.NOM & 1804-tõl, a református konvent \\
\hline$\overline{2}$. & & : 1. óvodákra 2. általános & iskolákra N.PL.SUB & 3. gimnáziumokra, szakközépiskolákra, \\
\hline$\overline{4 .}$ & & a cél, hogy az & iskoláknak N.PL.DAT & elvegyék a kedvét a mérlegképes \\
\hline$\underline{5}$ & & , hogy az egyház és & iskolája N.PSe3.NOM & felfelé ívelõ pályára kerüljön. \\
\hline 6. & & hogy a hibát nemcsak az & iskolában N.INE & kellene keresnie? Azt állítja \\
\hline$\underline{7}$. & & betakarítási munkák, egy hét & iskola N.NOM & közben / helyett; káposzta \\
\hline $1 \overline{4}$. & & . Akik Zsonnát ismerték az & iskolából $N . E L A$ & vagy Bísztõ Gyalla és Karmenják \\
\hline 15. & hogy világn & lézeti beállítottság nélkül nincs & iskola N.NOM & , ahogy nincs világnézet nélkül \\
\hline 16. & & , a minõség iránt fogékony & iskolákról N.PL.DEL & , és a fasornak talán \\
\hline 18. & óvodá & ások napi szállítása a központi & iskolába N.ILL & , mert annyira lecsökkentették a \\
\hline$\underline{20 .}$ & & jövõje van egy ilyen kicsi & iskolának N.DAT & ? Nem kerülnek -e hátrányba \\
\hline
\end{tabular}

Abb. 33: Konkordanzen zur Abfrage: Lemma iskola (Lemma Schule) (MNSz)

Das MNSz ist lemmatisiert und morphosyntaktisch annotiert. 


\section{Schlussbemerkung zu nationalen Korpora}

Neben den vorhin erwähnten Korpora gibt es im Internet eine Menge computerlesbarer Textsammlungen, die auch (zu Recht) als Korpora bezeichnet und zur Verfügung gestellt werden. Für ihre Verwendung als vollwertige Sprachkorpora benötigt man jedoch einen Korpusmanager, was die Arbeit wegen der aufwendigen Aufbereitung erschwert und für viele potenzielle Nutzer uninteressant macht.

Es fehlen aber noch jegliche Korpora mit Sprachen vieler Minderheiten: Romani, Vietnamesisch, Kurdisch, um einige zu nennen. Für das Türkische gibt es zwar ein Nationalkorpus, ein deutsch-türkisches Parallelkorpus wird erst im Rahmen des Projektes InterCorp geplant. 


\subsection{KORPORA mit DEUTSCH}

Die deutsche Sprache ist in verschiedenen Korpusinstrumenten gut erfasst. Einzelne Institute, die sich mit der Korpuserstellung befassen, finden sich im ganzen deutschsprachigen Raum. Da aber unter den Instituten kaum eine Zusammenarbeit besteht, sind die Schnittstellen, Korpustools, Eigenschaften und Abfragemodi bei jedem Korpus anders, was für ihre Nutzer gewisse Schwierigkeiten mit sich bringt. Bei der Arbeit mit Korpora aus verschiedenen Institutionen ist also eine große Flexibilität gefragt.

Der Umgang mit jedem Korpusmanager braucht Routine. Viele Eigenschaften bzw. Abfragemöglichkeiten sind oft in einer eigenen Korpussprache verschlüsselt, Beschreibungen irgendwo auf der Homepage versteckt oder gar nicht zu finden. Die folgende Übersicht soll die Arbeit mit den Korpora erleichtern, die Eigenschaften kurz zusammenfassen und die wichtigsten Abfragen (inkl. einiger Rechercheergebnisse) darstellen.

Die Reihenfolge, in der die einzelnen Korpora vorgestellt werden, wurde aufgrund der Komplexität der Instrumente vorgenommen (dies ist natürlich auch subjektiv). Erfahrungsgemäß ist es ratsam, mit einem einfachen Instrument zu beginnen, deswegen wird mit dem Wortschatz-Portal der Universität Leipzig angefangen, danach folgt das DWDS (Berlin-Brandenburgische Akademie der Wissenschaften) und das im Aufbau befindliche plurizentrische Projekt Korpus-C4.

Die größten und wohl auch komplexesten Korpora für Deutsch sind in Mannheim zu Hause: Das Deutsche Referenz Korpus für die geschriebene Sprache und die Datenbank für gesprochenes Deutsch. Die Übersicht wird um Korpora mit historischen Texten ergänzt.

Es gibt natürlich auch noch andere hilfreiche Korpora für Deutsch, die hier aber nicht näher erwähnt werden: Die meisten von ihnen (deWaC, AAC, Korpus Südtirol, Schweizer Textkorpus) sind in andere Projekte oder Großkorpora integriert, weitere sind erschwert zugänglich (TenTen corpora $^{14}$ ), bzw. für DaF/DaZ nur wenig relevant.

14 Webkorpora mit mindestens einer Billion $\left(10^{10}\right)$ Wörtern. Ein großer Vorteil dieses Korpus ist das Tagging mit RFTAgger. Dieser ermöglicht auch das Suchen nach Kasus, Genus und anderen morphosyntaktischen Kategorien, die über andere Tagger nicht abrufbar sind (vgl. Schmid/ Laws 2008). 


\subsubsection{Korpora mit (mehrheitlich) geschriebenem Deutsch}

\subsubsection{Wortschatz Leipzig}

http://wortschatz.uni-leipzig.de/

Dieses Instrument verbindet ein elektronisches Wörterbuch mit einem großen Textkorpus. $\mathrm{Zu}$ den abgefragten Elementen werden morphologische, syntaktische und lexikalische Angaben angezeigt.

Das Korpus ist lemmatisiert - den einzelnen Wortformen werden Grundformen zugeordnet, im Ergebnis werden alle Formen aufgelistet. In den Konkordanzen erscheint jedoch als KWIC nur die abgefragte Form.

Es ist ein automatisch und „opportunistisch erstelltes Korpus“ (wie seine Autoren schreiben), daher soll man die Recherchergebnisse mit Vorsicht betrachten.

\section{Abfragen:}

Die Erstellung einer Abfrage ist sehr einfach. Ins Suchfeld schreibt man bloß das gewünschte Wort oder die gewünschte Verbindung. Andere Einstellungen sind nicht notwendig.

Da es sich primär um ein Instrument für die Erschließung des Wortschatzes handelt, sind die Abfragemöglichkeiten dementsprechend beschränkt. Grundsätzlich können nur Wortformen (mit der Möglichkeit Groß-/Kleinschreibung $\mathrm{zu}$ beachten) abgefragt werden. Wortkombinationen sind nur in der Abfolge abfragbar, in der sie ins Suchfeld eingegeben werden.

Abfragemöglichkeiten im Wortschatz - Leipzig

Der Abstand (ein Leerzeichen) ist mit einem Mittelpunkt (·) markiert.

\begin{tabular}{|l|l|}
\hline Eingabe & Ergebnis (Beschreibung) \\
\hline Arzt & Arzt (nur diese Wortform) \\
\hline Arzt* & $\begin{array}{l}\text { Arzt, Arztabrechnung, Arztakten, Arztangebot ... (automatische Ordnung nach Formen, } \\
\text { alphabetisch absteigend) }\end{array}$ \\
\hline *arzt & $\begin{array}{l}\text { Notarzt, Chefarzt, Hausarzt, Zahnarzt ... (automatische Ordnung nach Frequenz, } \\
\text { absteigend) }\end{array}$ \\
\hline böhmisch*·Dorf* & böhmisches Dorf, böhmische Dörfer \\
\hline
\end{tabular}

Tab. 2: Abfragen Wortschatz-Portal

Eingabe der Abfrage

Beispiel: Wortform Hörer

- Einstellungen: keine erforderlich

- Abfrage (Suchfeldeingabe): Hörer

Ergebnis: Angaben und Belege in Form einer Tabelle (Abb. 34) mit morphologischen, grammatischen und semantischen Angaben, sowie Belegen (Kontext von mindestens einem Satz) und Listen mit (automatisch errechneten) Kollokationspartnern. 


\section{Ergebnis der Abfrage Hörer:}

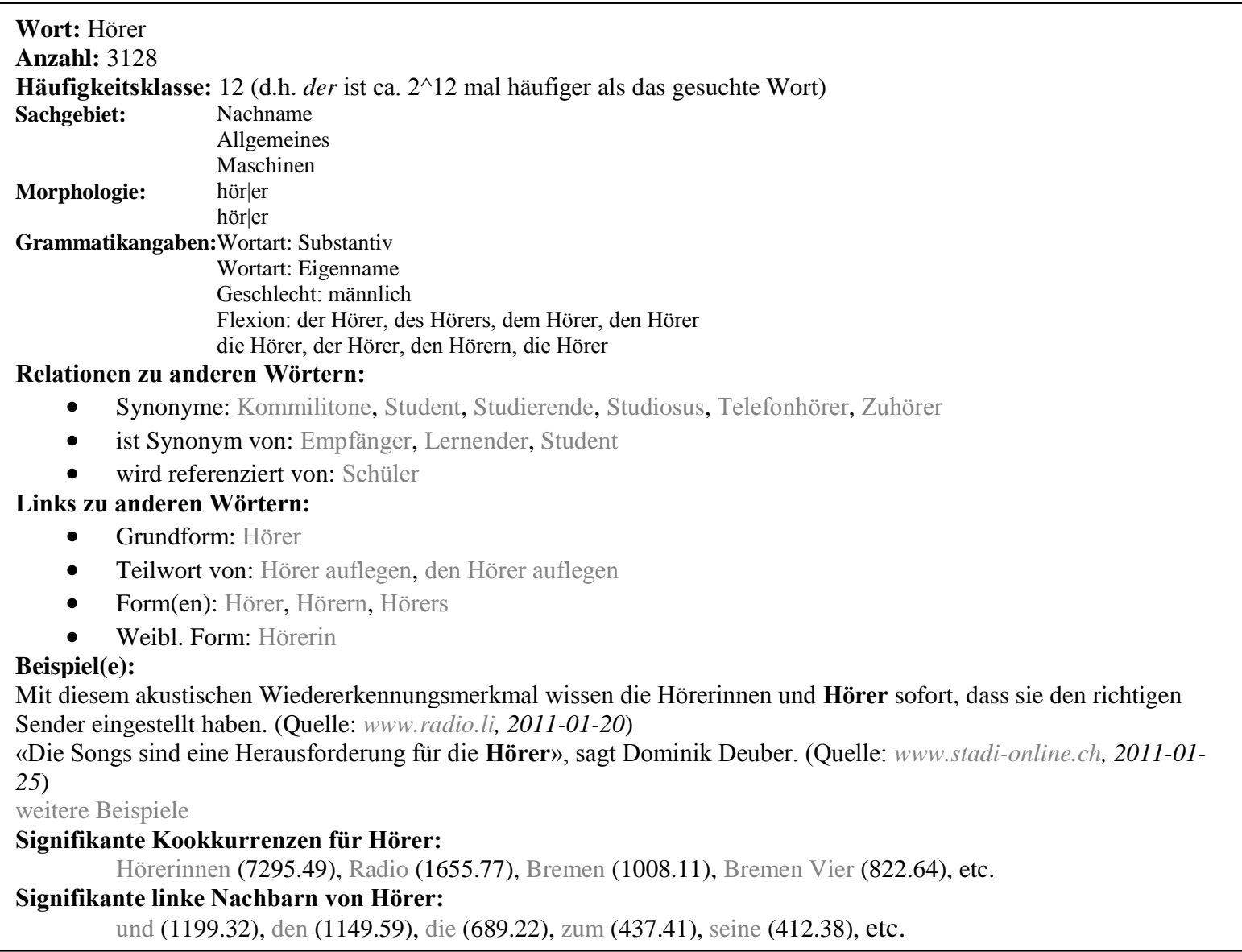

Abb. 34: Abfrage Hörer (Wortschatz-Portal)

Die Graphische Darstellung kann sehr schnell feste Verbindungen aufdecken - diese sind bei anderen Korpusinstrumenten eher schwierig zu identifizieren. Die häufigsten Kollokatoren/ Nachbarn/ Kookkurrenten werden auch in Form einer Graphik (,Spinnennetz“) angezeigt:

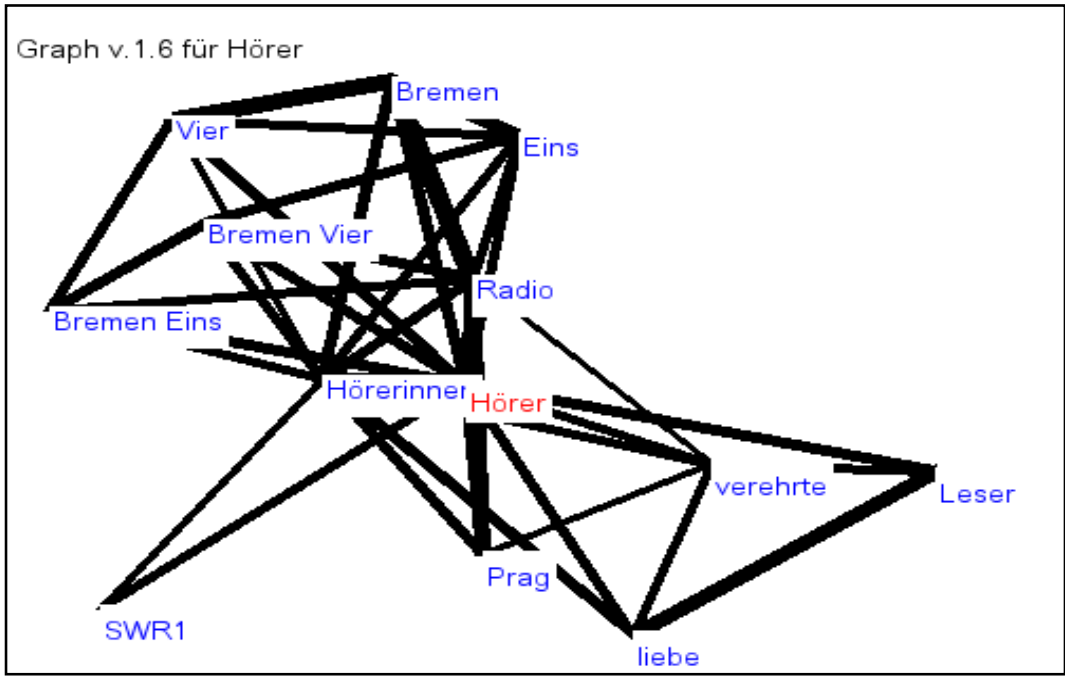

Abb. 35: Graphische Darstellung der Kollokatoren zur Abfrage Hörer (Wortschatz-Portal) 
Aus der Graphik ist ersichtlich, dass das Wort Hörer selten ohne die movierte Form Hörerin vorkommt. Darüber hinaus sieht man deutlich, dass das Wort Hörer im heutigen Deutsch vorrangig in der Bedeutung Radio-Zuhörer verwendet wird und viel seltener als Synonym zu Student oder als Kopfhörer (eines Telefons).

Auf dem Wortschatz-Portal sind auch Wörterbücher von anderen 230 Sprachen. Alle wurden automatisch erstellt, das Korpus besteht aus Internetseiten der jeweiligen sprachlichen Domänen. Dieses Instrument eignet sich gut zur Erklärung einiger Basisfunktionen von Korpora: Darstellung der Belege, Errechnung von Frequenzen, Ermittlung der Kollokationspartner, Erschließung des Kontextes (Umfelds) u.a. Der besondere Wert des Wortschatz-Portals liegt in der übersichtlichen und graphisch gelungenen Präsentation der Ergebnisse.

\subsubsection{Das Digitale Wörterbuch der deutschen Sprache (DWDS)}

www.dwds.de

Das Das Digitale Wörterbuch der deutschen Sprache (DWDS) sind eigentlich mehrere (Sub)Korpora. Die Texte stammen aus Zeitungen, Belletristik und Gebrauchsliteratur. Das Gros bilden Texte aus dem 20. Jahrhundert, welche stilistisch ausgewogen sind.

Das DWDS erfordert eine Anmeldung, mit der man Zugang zum größten Teil der Texte erhält.

Die Eigenschaften sind unter Ressourcen (rechts oben) sehr gut beschrieben.

Ins Suchfeld (nach der ersten Abfrage erscheint es links oben) wird die Abfrage eingegeben (das gewünschte Wort/ Wortteil/ Verbindung etc.), rechts davon wählt man die Ansicht (Standard-, Wörterbuchansicht etc.).

In der Standard- und in der Wörterbuchansicht kann man auch die Aussprache (allerdings nur bei Abfrage einzelner Wörter) anhören. Die Tonspur für einzelne Wörter haben professionelle Sprecher/-innen besprochen. Es handelt sich also um kein Korpus der gesprochenen Sprache! Korpora mit Vertonung können beispielsweise als phonetische Hilfe im Unterricht eingesetzt werden. Da es sich um ein Korpus mit ausschließlich bundesdeutschen Texten handelt, befolgt die Aussprache auch die bundesdeutsche Aussprachennorm (siehe dazu auch Kap. 5).

Die Syntax der häufigsten Abfragen befindet sich unter dem Button Hilfe zur Suche (auf der Homepage oben rechts) und wird auch hier zusammengefasst und ergänzt:

\section{Abfragemöglichkeiten im DWDS}

Der Abstand (ein Leerzeichen) ist mit einem Mittelpunkt (·) markiert.

\begin{tabular}{|l|l|}
\hline Eingabe & Ergebnis (Beschreibung) \\
\hline Arzt & Arzt, Arztes, Ärzte ... (alle flektierten Formen von Arzt) \\
\hline @Arzt & Arzt (nur diese Wortform) \\
\hline Arzt* & Arzt, Arztbesuch, Arztberuf ... (mit Arzt- beginnende Wörter) \\
\hline *arzt & Sportarzt, Hausarzt ... (auf -arzt endende Wörter) \\
\hline "gut·Arzt" & $\begin{array}{l}\text { guter Arzt, bessere Arzt, bester Arzt, besten Arztes, gute Ärzte .... } \\
\text { (beide Wörter lemmatisiert) }\end{array}$ \\
\hline
\end{tabular}




\begin{tabular}{|c|c|}
\hline /arzt/ & $\begin{array}{l}\text { Zahnarzt, Oberarzt, knarzt, verwarzt, verharzt } \ldots \text { (alle } \\
\text { Formen mit der Buchstabenkombination arzt) }\end{array}$ \\
\hline$\$ p=V^{*} \cdot$ with$\cdot / a r z t /$ & $\begin{array}{l}\text { geharzt, knarzt, knarzten, verharzt, vewarzt (alle Verben mit } \\
\text { der Buchstabenkombination arzt) }\end{array}$ \\
\hline "das·gute·Beispiel" & $\begin{array}{l}\text { das gute Beispiel, das beste Beispiel, die besseren Beispiele } \\
\ldots \text { (alle Formen aller Wörter) }\end{array}$ \\
\hline "Kanzler·\#1·Schröder" & $\begin{array}{l}\text { Kanzler Schröder, Kanzler Gerhard Schröder ... (Kanzler } \\
\text { und Schröder im Abstand von höchstens einem Wort) }\end{array}$ \\
\hline "Kanzler·\#3·Schröder" & $\begin{array}{l}\text { Kanzler aller Moleküle Gerhard Schröder will Innovationen } \\
\text { fördern; Kanzler und begnadeten Wahlmatador Schröder ... } \\
\text { (Kanzler und Schröder im Abstand von höchstens drei } \\
\text { Wörtern) }\end{array}$ \\
\hline 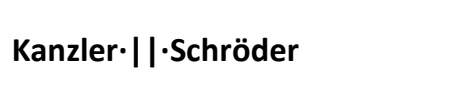 & $\begin{array}{l}\text { Alle Sätze, in denen entweder das Wort Kanzler oder } \\
\text { Schröder oder beide Wörter vorkommen. }\end{array}$ \\
\hline 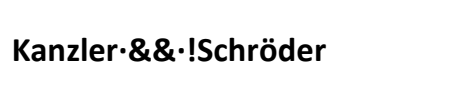 & $\begin{array}{l}\text { Alle Sätze, in denen Kanzler, aber nicht Schröder } \\
\text { vorkommen. }\end{array}$ \\
\hline$\$ p=N E \cdot$ with $\cdot$ Herzog & Roman Herzog, Peter Herzog ... (Eigennamen ${ }^{15}$ Herzog) \\
\hline "Einfluss·\#2·\$p=NE" & $\begin{array}{l}\text { Einfluß Smetanas, Einfluss in der EU ...(Einfluss }(\beta) \text { im } \\
\left.\text { Abstand von max. } 2 \text { Positionen von einem Eigennamen }{ }^{15}\right)\end{array}$ \\
\hline$\$ p=N N \cdot$ with $\cdot * l e i n$ & $\begin{array}{l}\text { Häuflein, Kräutlein, Büchlein ... (Substantive }{ }^{15} \text {, die auf -lein } \\
\text { enden. Ausgeschlossen.) }\end{array}$ \\
\hline$\$ p=A D J A \cdot$ with $\cdot *$ sam & $\begin{array}{l}\text { schweigsam, ehrsam, wundersam, achtsam } \\
\text { (Adjektive }{ }^{15} \text { auf -sam) }\end{array}$ \\
\hline$\$ p=A D J A \cdot w i t h \cdot m i n i *$ & $\begin{array}{l}\text { minimal, minimalistisch, miniaturhaft ... (Adjektive }{ }^{15} \text { auf } \\
\text { mini-) }\end{array}$ \\
\hline "gehen·\#5·aus·with·\$p=PTKVZ" & $\begin{array}{l}\text { Das Licht ging aus (...), Mir ging die Luft aus. Wir gehen } \\
\text { also nicht von der Sprechhandlung aus (...) ... (Verb }{ }^{15} \\
\text { ausgehen mit abgetrenntem aus, maximaler Abstand } 5 \\
\text { Positionen) }\end{array}$ \\
\hline \multicolumn{2}{|c|}{ "sein·with·\$p=VVFIN·\#20·\$p=VVPP·\#0·@worden" } \\
\hline \multicolumn{2}{|c|}{$\begin{array}{l}\text {... im September } 1980 \text { waren Teile des Streckennetzes stillgelegt worden; das } \\
\text { anzuerkennen sei auf Grund der längst überholten These von der Einheit eines } \\
\text { Volkes unmöglich gemacht worden; jedoch waren sie gleichzeitig darauf } \\
\text { aufmerksam gemacht worden (Verb sein als finites Verb }{ }^{15} \text {, max. } 20 \text { Positionen } \\
\text { davon Partizipium eines Vollverbs gefolgt von worden) }\end{array}$} \\
\hline 1 & $\begin{array}{l}\text { (Suche nach Komma. Satzzeichen wie ",.?!; werden mit } \\
\text { Backslash maskiert!) }\end{array}$ \\
\hline
\end{tabular}

Tab. 3: Abfragen DWDS

Weitere Abfragemöglichkeiten sind unter DWDS $\rightarrow$ Hilfe zur Suche zu finden.

Das Korpus ist mit dem Stuttgarter Tagset (STTS) morphologisch getagget und lemmatisiert. Dasselbe Tagset findet man in den meisten Korpora der deutschen Sprache (DeReKo, deutsche Parallele des InterCorp, deWaC).

15 Andere abfragbare morphosyntaktische Kategorien entsprechen dem Stuttgarter Tagset (STTS). Sie sind im Kap. 8.2 angeführt. 


\section{Eingabe der Abfrage}

Vor der Abfrage sind keine Einstellungen notwendig. Die Ansicht (Default: DWDSStandardansicht) kann auch nach Abrufen der Ergebnisse geändert werden.

\section{Beispiel: Wortform Hit}

- Einstellungen: keine erforderlich

- Abfrage (Suchfeldeingabe): Hit

Ergebnis: Je nach Ansichteinstellung erscheinen Fenster mit verschiedenen Angaben.

\section{DWDS-Wörterbuch:}

\section{Hit}

mask., $-\mathrm{s},-\mathrm{s}$

Herkunft Englisch

Erfolgsschlager, Spitzenschlager

eine Schallplatte mit den neuesten Hits

kaufen

Dazu: Hitparade

Abb. 36: Fenster: DWDS-Wörterbuch

\section{DWDS-Kernkorpus 20}

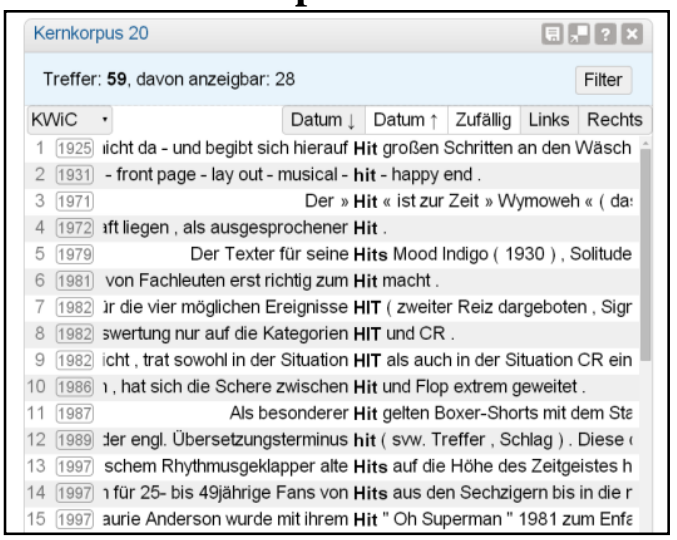

Abb. 38: Fenster: DWDS-Konkordanzen

\begin{tabular}{|c|c|c|c|c|}
\hline "Rank & Stammform & Wortart & logDice & Frequenz \\
\hline "1 & Medley & Substantiv & & 11 18" \\
\hline "2 & Coverversioner & r Substantiv & & 11 17" \\
\hline "3 & Oldies & Substantiv & & $1144 "$ \\
\hline "4 & Song & Substantiv & & 11 39" \\
\hline "5 & wie Lady & PP & & $116^{\prime \prime}$ \\
\hline "6 & Evergreens & Substantiv & & $1018 "$ \\
\hline "7 & Flops & Substantiv & & $1025 "$ \\
\hline "8 & für Kids & PP & & $1050 "$ \\
\hline "9 & Lied & Substantiv & & $1033^{\prime \prime}$ \\
\hline "10 & Tantiemen & Substantiv & & $96 "$ \\
\hline "11 & landete & Verb & & $9237^{\prime \prime}$ \\
\hline
\end{tabular}

Abb. 37: Fenster: DWDS-Wortprofil - Präpositionalgruppe

\section{Wortverlauf (Basis DWDS-Kernkorpus)}

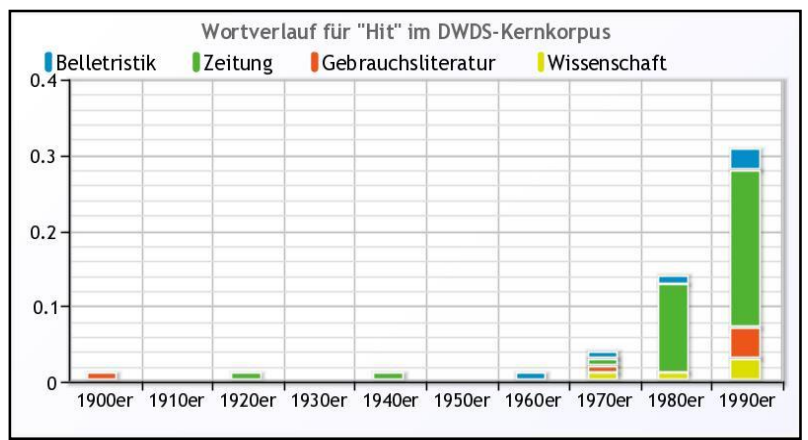

Abb. 39: Fenster: DWDS-Kernkorpus - Wortverlauf

In weiteren Fenstern der Standardansicht gibt es Angaben zu Etymologie, Open Thesaurus, Konkordanzen aus einzelnen Korpora (Zeit-Korpus, Kern-Korpus etc.) und das aus den Konkordanzen errechnete Wortprofil mit Angaben, die das Wort - je nach seiner Wortart - in seinen typischen Funktionen und Positionen im Satz zeigen: Überblick (Kookkurrenzen), Attribute zum Wort (falls vorhanden), Präpositionalgruppen, in denen das Wort vorkommt, Subjekte und Objekte, die das Wort (falls ein Verb) regiert, und andere mehr. Im Prinzip sind diese Informationen ident mit sog. Word Sketches - automatisch berechneten Eigenschaften der Wörter (siehe Sketch Engine 2014 und Kilgariff et al. 2014).

Es ist auch ratsam, andere Ansichten zu aktivieren, um sich ein Bild zu verschaffen, welche Informationen der Korpusmanager errechnen kann.

Das DWDS ist ein relativ einfaches Korpusinstrument mit umfangreichen Möglichkeiten. Nach einer kurzen Einschulung kann es jeder Lernende auch als Nachschlagewerk nutzen. 
Die einzelnen Korpora des DWDS beinhalten nur Texte aus Deutschland. Ein Teil von ihnen wird daher zum Bestandteil eines geplanten plurizentrischen Korpus mit einzelnen Varietäten des Deutschen. Dazu: DWDS (2014) unter Ressourcen $\rightarrow \underline{\text { Korpora }} \rightarrow \underline{1 \text {. Referenzkorpora, }}$ Absatz C4-Korpus.

\subsubsection{Korpus-C4}

http://www.korpus-c4.org/

Dieses Korpus - eigentlich ein System von 4 Korpora - soll die deutsche Sprache des 20. Jahrhunderts plurizentrisch abdecken. Auf der Homepage des Projekts erfährt man Näheres unter den einzelnen Ikonen INFORMATIONEN TEILPROJEKTE STRUKTUR:

INFORMATIONEN Zusammensetzung des Korpus (s. auch hier unter Struktur) und das Abfragesystem:

Über die Zusammensetzung und das Abfragesystem wird wörtlich geschrieben (2014):

„Zusammensetzung

Am Korpus C4 beteiligt sind das $>>$ Digitale Wörterbuch der deutschen Sprache des 20. Jahrhunderts (DWDS) aus Berlin, das >> Austrian Academy Corpus (AAC) aus Wien, das $\gg$ Korpus Südtirol aus Bozen und das $\gg$ SCHWEIZER TEXT KORPUS (CHTK) aus Basel.

Das Projekt verfolgt das Ziel, die deutsche Standardsprache des 20. Jahrhunderts möglichst ausgewogen zu erfassen und online zugänglich zu machen. Momentan setzt sich das Korpus aus 20 Mio. Textwörtern des DWDS, 4.1 Mio. Textwörtern des AAC, 1.7 Mio. Textwörtern des Korpus Südtirol und 20 Mio. Textwörtern des CHTK zusammen.

Abfragesystem

Eine technische Besonderheit des Korpus $\mathrm{C} 4$ ist die verteilte Abfrage. Jedes Teilprojekt stellt seine Daten auf einem eigenen Server zur Verfügung, und erst zum Zeitpunkt der Abfrage werden die Resultate von den einzelnen Servern abgeholt und zusammengesetzt. Die Zusammenführung der Teilkorpora zu einem gemeinsamen ganzen Korpus C4 ist also nur virtuell. Technisch nutzt das Korpus dazu vor allem Funktionen der linguistischen Suchmaschine DDC, die vom Berliner Teilprojekt DWDS entwickelt wurde.“

$\underline{\text { TEILPROJEKTE }}$

STRUKTUR

Beschreibung der einzelnen „Landeskorpora“"

Aktuelle detaillierte Struktur der einzelnen Korpora in den einzelnen Quartalen des 20. Jahrhunderts

Ausgewogen ist im Moment nur der Teil des DWDS. Die anderen Korpora werden erst aufgebaut.

Die Ikone BEISPIELE zeigt die Abfragemöglichkeiten, die sich zwar im Wesentlichen mit den Abfragen an das DWDS decken (vgl. CHTK 2008-2014 $\rightarrow$ Beispiele), die Syntax der Abfragen weicht jedoch teilweise ab.

\section{Abfragemöglichkeiten im Korpus-C4}

Bei der Suche wird automatisch die Groß-/Kleinschreibung beachtet. Der Abstand (ein Leerzeichen) ist mit einem Mittelpunkt (·) markiert. 


\begin{tabular}{|c|c|}
\hline Eingabe & Ergebnis (Beschreibung) \\
\hline Gärten & Gärten (nur diese Wortform) \\
\hline \$Lemma=Garten & Garten, Gartens, Gärten ... (alle flektierten Formen von Garten) \\
\hline Garten* & Gartenkonzert, Gartengesellschaft, Gartenarbeit ... \\
\hline *garten & Obstgarten, Versuchsgarten, Kindergarten ... \\
\hline "schöne·Gärten" & schöne Gärten (Verbindung in exakt dieser Form) \\
\hline "\$Lemma=schön·\$Lemma=Garten" & $\begin{array}{l}\text { schöner Garten, schöne Gärten (Verbindung jeglicher Form von } \\
\text { schön mit jeglicher Form von Garten) }\end{array}$ \\
\hline "\$Lemma=schön·\#3·\$Lemma=Garten" & $\begin{array}{l}\text { um den schönen Weg zwischen den Gärten (...) zu genießen } \\
\text { (Lemma schön und Lemma Garten, max. Abstand } 3 \text { Wörter) }\end{array}$ \\
\hline wild·\&\&·Garten & $\begin{array}{l}\text {...der ungepflegte Garten wucherte wild und bunt ... (wild und } \\
\text { Garten in einem Satz) }\end{array}$ \\
\hline Haus.||-Garten & $\begin{array}{l}\text { freie Bewegung in Garten, Haus und um's Haus gestattet (Sätze mit } \\
\text { Haus oder Garten oder mit beiden Wörtern) }\end{array}$ \\
\hline Garten $\& \& \cdot ! \$$ Lemma=schön & Garten (Sätze mit Garten, jedoch ohne jegliche Form von schön) \\
\hline
\end{tabular}

Tab. 4: Abfragen Korpus C4

Eingabe der Abfrage

Zum Abfragefenster gelangt man über

$\underline{\text { Korpus }} \rightarrow \geq$ C4-Suche beim SCHWEIZER TEXT KORPUS, Basel

Vor der Abfrage sind keine Einstellungen notwendig.

Beispiel: Suche nach der Wortform Hit

- Einstellungen: keine erforderlich

- Abfrage (Suchfeldeingabe): Hit

\section{Ergebnis:}

\begin{tabular}{|c|c|c|}
\hline 1975 & te Hit & \\
\hline 197 & Ihr Bruder Markus stellt den Kassettenrecorder an und lässt einen Hit & laufen \\
\hline 1976 & ... auf sie, es gibt Streit. 1 Kann jemand von euch die Fremdwörter Hit & $\mathrm{m} \ldots$ \\
\hline 1976 & & \\
\hline 197 & .. Der Kasperli sang bei seinem er & «Trul \\
\hline 1986 & ... eindringen, anprangern, all & , Job, Quiz, \\
\hline 1989 & ach einem Monat buchstäblic & der \\
\hline 1991 & ... Zeitungspapier am Laufmeter, auch das ist spannend; der absolute Hit & waren aber Holzrugel, e \\
\hline 1993 & die Vereinsmänner immer wichtigere Preise auspackten: Der grosse Hit & war ein Schwarzweiss-Fernseher. Und eine ... \\
\hline
\end{tabular}

Abb. 40: Konkordanzen zur Abfrage: Wortform Hit (CHTK)

Die abgerufenen Konkordanzen lassen sich filtern und sortieren: über die Ikonen Filter Sortieren Export Optionen Beispiele werden einfache und übersichtliche Formulare abgerufen, die man anwenden kann.

Auch das Export-Format (TXT) ist sehr gut und einfach lesbar (auch wenn es auf den ersten Blick nicht so aussieht): Die Daten werden aus einem Notizblock (TXT) einfach in eine Excel-Tabelle kopiert (TXT: Strg+A $\rightarrow$ Strg+C $\rightarrow$ EXCEL: Strg+V). In Excel werden die folgenden Angaben ausgewiesen: die gewählten Metainformationen (Spalte A), der Kontext links vom KWIC (Spalte B), der Kontext rechts vom KWIC (Spalte C), wie in der Abb. 40 dargestellt wird. So lassen sich die Konkordanzen sehr einfach sortieren. 
Das Korpus C4 würde sich sehr gut für die plurizentrische Arbeit eignen (zugänglich und recherchierbar ist im Moment das Schweizer Textkorpus, aus dem die Konkordanzen in der Abb. 40 stammen). Leider sind die einzelnen Teile noch nicht ausgewogen und wegen der Größe der Teile ist man auch weit von der Repräsentativität des sprachlichen Materials entfernt. Für die plurizentrische Arbeit wird jedoch das DeReKo empfohlen.

Mangelhaft bzw. gar nicht vorhanden sind im C4 die statistischen Funktionen. Auf der anderen Seite ist das Korpus sehr einfach und intuitiv zu bedienen; es eignet sich dadurch als ein guter Einstieg in die Korpuslinguistik.

\subsubsection{Das Deutsche Referenzkorpus (DeReKo)}

https://cosmas2.ids-mannheim.de/cosmas2-web/

Das Deutsche Referenzkorpus ist auch unter dem Namen IDS-Korpus oder Cosmas II bekannt. Die Bezeichnungen sind jedoch nicht ganz richtig: das Institut für Deutsche Sprache in Mannheim entwickelt und verwaltet mehrere Korpora (z.B. auch die DGD), Cosmas II hingegen ist ein vom IDS entwickelter Korpusmanager (Corpus Search, Management and Analysis System, Version II). Unter diesem Manager laufen alle IDS-Korpora der geschriebenen Sprache.

Das DeReKo ist momentan das größte Korpus für Deutsch weltweit, es enthält über 6 Milliarden Textwörter (Stand 2013) und wird sukzessive und regelmäßig erweitert. Die Bedienung aller IDS-Korpora ist ziemlich komplex. Für jede/-n, der/die sich mit der deutschen Sprache befasst, ist es dennoch, ratsam zumindest die Basisfunktionen von Cosmas II (Korpusmanager) sicher zu beherrschen. Es gibt leicht zugängliche Quellen, die die Bedienung sehr gut beschreiben: als Einstieg empfiehlt sich die Anleitung von Stephen Berman (2013). Weiterhin verfügt Cosmas II über ein sehr gutes Hilfe-Portal (Cosmas II 2012). Das Wesentliche (kombiniert aus beiden Quellen und ergänzt) wird hier beschrieben.

Der Zugang zum Korpus der geschriebenen Sprache erfolgt entweder direkt über die InternetSchnittstelle (http://www.ids-mannheim.de/cosmas2/web-app/) oder über eine WindowsApplikation, die auf die Festplatte heruntergeladen werden muss.

Installieren der Cosmas $\mathbf{I I}_{\text {win }}$ Suchmaschine

\begin{tabular}{|lr|}
\hline Ins Google- oder Adressen-Suchfeld http://www1.ids-mannheim.de/start/ & $\begin{array}{r}\text { eingeben } \\
\text { Forschung } \rightarrow \text { laufende Projekte } \rightarrow \underline{\text { Cosmas } I I} \rightarrow \text { Cosmas II }_{\text {win }}\end{array}$ \\
$\begin{array}{c}\text { Detaillierte Informationen } \\
\text { Applikation herunterladen und installieren }(. . .) .\end{array}$ & anklicken. \\
\hline
\end{tabular}

Beim Herunterladen und Installieren der Anleitung folgen. Es ist sehr einfach!

Zugang zur Cosmas $\mathrm{II}_{\text {web-Schnittstelle }}$

\begin{tabular}{|lr|}
\hline Ins Google- oder Adressen-Suchfeld http://www.ids-mannheim.de/cosmas2/ & $\begin{array}{r}\text { eingeben } \\
\text { anklicken. }\end{array}$ \\
Cosmas $\|_{\text {web }}$ & \\
\hline $\begin{array}{l}\text { Aktuelle Version: } \\
\text { Applikation starten. }\end{array}$ & anklicken \\
\hline
\end{tabular}

Für die Recherche sowohl über die Suchmaschine als auch über das Web benötigt man eine Registrierung. 
Die Funktionen von CosmasII ${ }_{\text {win }}$ und CosmasII ${ }_{\text {web }}$ sind fast ident. CosmasII ${ }_{\text {win }}$ ermöglicht die sog. ,graphische Suche“: das gewünschte Kästchen (links) auf die graue Fläche ziehen, durch einen Doppelklick öffnen und ins Suchfeld den Suchbegriff eingeben. Des Weiteren hat es einige zusätzliche Funktionen, die CosmasII ${ }_{\text {web }}$ nicht hat. Für den ersten Einstieg wird CosmasII $_{\text {web }}$ empfohlen, die folgende Beschreibung bezieht sich terminologisch auch auf die Web-Applikation.

\section{Abfragen}

Die sprachlichen Elemente, die sich mit Cosmas II abfragen lassen, sind übersichtlich unter: Online Hilfe $\rightarrow$ Suchanfrage $\rightarrow$ Zeilenangabe $\rightarrow$ Beispiele aufgeführt. Die Syntax der häufigsten Abfragen ist hier mit Erklärungen ab Seite $60 \mathrm{zu}$ finden.

Im Folgenden werden einige Recherchen Schritt für Schritt beschrieben, somit wird die (oft mühsame) Suche im umfangreichen Hilfe-Portal hinfällig.

\subsection{Recherche in allen Archiven}

Einstieg: Anmeldung $\rightarrow$ Login $\rightarrow$ Recherche

Der Weg zur Abfrage geht über die folgenden Schritte $(\mathrm{A} . \rightarrow \mathrm{B} . \rightarrow$ C. $\rightarrow$ D. $)$ :
A. Auswahl des Archivs
B. Auswahl des Korpus
C. Einstellung der Optionen
D. Suchanfrage

\section{A. Auswahl des Archivs}

DeReKo Archive:

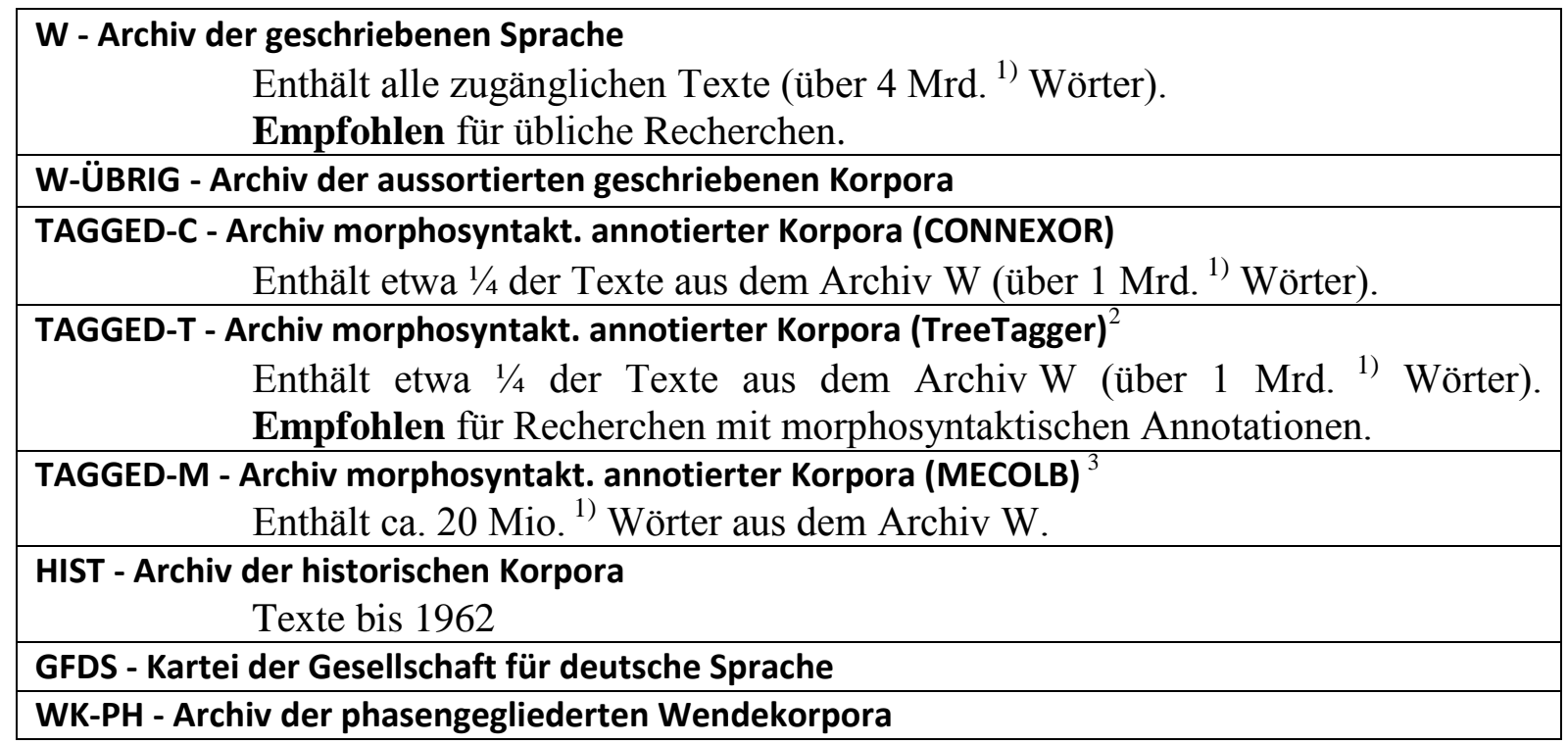

\section{Anmerkungen:}

1) Die Angaben über die Größe der Archive beziehen sich nur auf öffentlich zugängliche Texte.

2) Das Archiv TAGGED-T wird deswegen empfohlen, da es mit demselben Tagset annotiert wird als andere hier erwähnte Korpora (DWDS, InterCorp).

3) Das Archiv TAGGED-M ermöglicht auch Abfragen nach morphologischen Kategorien, die sonst nicht abgefragt werden können (Kasus, Genus). Es beinhaltet nur bundesdeutsche Texte und ist sehr klein. 


\section{B. Auswahl des Korpus}

Empfehlenswert ist alle vordefinierten/ alle öffentlichen Korpora auszuwählen. Beliebige Subkorpora kann man über die Ikone Korpusverwaltung erstellen.

\section{Einstellung der Optionen}

Vor der Sucheingabe soll zuerst auch der Suchmodus eingestellt werden (unter Optionen). Bitte nicht vergessen, nach der jeweiligen Einstellung die Ikone Übernehmen anzuklicken.

Empfehlung: um die Einstellung für die nächste „Sitzung“ zu speichern, muss nach Abschluss der Recherchen die Webseite über Logout verlassen werden. Nach dem einfachen Schließen der Web-Seite werden die Einstellungen auf die Standardwerte zurückgesetzt.

Die meisten Optionen sind selbsterklärend. Kommentiert werden hier nur die erfahrungsgemäß problematischen Punkte.

\begin{tabular}{|c|c|}
\hline \multicolumn{2}{|l|}{ Suchmodalitäten } \\
\hline Groß- / Kleinschreibung beachten für 1. Zeichen & aus-/einschalten \\
\hline Groß- / Kleinschreibung beachten für andere Zeichen & aus-/einschalten \\
\hline Diakritische Zeichen beachten & aus-/einschalten \\
\hline \multicolumn{2}{|l|}{ Expansionslisten (Liste der Formen) } \\
\hline Expansionslisten anzeigen & aus-/einschalten \\
\hline mit Häufigkeiten (langsam) & alternativ aus-/abwählen \\
\hline ohne Häufigkeiten (mittel) & alternativ aus-/abwählen \\
\hline ohne Korpusvalidierung (schnell) & alternativ aus-/abwählen \\
\hline Sortierung ${ }^{1)}$ & Auswahl \\
\hline Zusammenfassung ab & Zahl eingeben \\
\hline \multicolumn{2}{|l|}{ Begrenzung der Ergebnismenge (Falls zu viele zu erwarten sind.) } \\
\hline alle Treffer & alternativ aus-/abwählen \\
\hline Zufallsauswahl & alternativ aus-/abwählen \\
\hline durch Zufallsauswahl eingrenzen auf: & Zahl eingeben \\
\hline Zufallsgenerierung: fest $^{2)}$ variabel & alternativ aus-/abwählen \\
\hline & Übernehmen anklicken \\
\hline
\end{tabular}

Anmerkungen:

1) Nach jeder Präsentation der Ergebnisse setzt sich die Sortierung auf alphabetisch aufsteigend zurück.

2) Auswahlmöglichkeit fest bedeutet: bei der nächsten Abfrage desselben Phänomens bekommt man dieselben Konkordanzen

\section{Lemmatisierung}

Lemmatisierung

Flexionsformen

immer eingeschaltet

Komposita

aus-/einschalten

Sonstige Wortbildungsformen ${ }^{1)}$

aus-/einschalten

Spezialfälle ${ }^{2)}$

\section{Anmerkungen:}

1) Einschalten, wenn z.B. nach Affixen gesucht wird.

2) Wörter mit Bindestrich, Nummern etc. 


\begin{tabular}{|lr|}
\hline Korpuspräsentation & \\
\hline $\begin{array}{l}\text { Korpuspräsentation } \\
\text { Korpusansicht }\end{array}$ & Auswahl \\
\hline Sekundäre Sortierung: & Auswahl \\
Prozentuelle Verteilung & aus-/einschalten \\
Darstellung & aus-/einschalten \\
\hline & Übernehmen anklicken \\
\hline
\end{tabular}

\begin{tabular}{|c|c|}
\hline $\begin{array}{l}\text { Ergebnispräsentation } \\
\text { Ergebnisansicht: }\end{array}$ & Auswahl \\
\hline Sekundäre Sortierung der Ergebnisse: & Auswahl \\
\hline \multicolumn{2}{|c|}{ Berechnung des Häufigkeitsmaßes (Frequenzen und Distribution) } \\
\hline Maß für die Häufigkeit berechnen und anzeigen & aus-/einschalten \\
\hline Relative Häufigkeiten & alternativ aus-/abwählen \\
\hline $\begin{array}{l}\text { in Prozent } \\
\text { pro Million Worte }\end{array}$ & alternativ aus-/abwählen \\
\hline Differenzenkoeffizient & alternativ aus-/abwählen \\
\hline Häufigkeitsklassen mit folgender Referenz: & alternativ aus-/abwählen \\
\hline Einzelwort, Groß/Klein/Diakr. beachten & \\
\hline $\begin{array}{l}\text { Einzelwort, Groß/Klein/Diakr. ignorieren } \\
\text { Lemma } \\
\text { automatisch }\end{array}$ & alternativ aus-/abwählen \\
\hline \multirow[t]{2}{*}{ Darstellung: Häufigkeitsmaß grau darstellen } & aus-/einschalten \\
\hline & Übernehmen anklicken \\
\hline
\end{tabular}

KWIC/Volltext
\begin{tabular}{|lr|}
\hline Treffermarkierung: Markierungsfarbe & Auswahl \\
\hline KWIC-Anzeige: Volltext-Anzeige & Eingaben und Auswahl \\
Angaben über die Präsentation der Konkordanzen und Textabschnitte & Zahl eingeben \\
\hline Seitengröße & \\
\hline Alphabetische Sortierung ${ }^{1)}$ & immer eingeschaltet/ Auswahl \\
\hline 1.Kriterium: & aus-/einschalten/ Auswahl \\
\hline 2.Kriterium: & aus-/einschalten/ Auswahl \\
\hline 3.Kriterium: & aus-/einschalten \\
\hline Sonderzeichen ignorieren & alternativ aus-/abwählen \\
\hline Zufällige Sortierung: fest variabel ${ }^{2)}$ & Übernehmen anklicken \\
\hline
\end{tabular}

\section{Anmerkungen:}

1) Sortierung nach der unmittelbaren Umgebung des KWICs (bis zu 3 Positionen links oder rechts).

2) Auswahlmöglichkeit fest bedeutet: bei der nächsten Abfrage desselben Phänomens bekommt man dieselben Ergebnisse.

\section{Suchanfrage}

Die Eingabe der Abfrage erfolgt über den Button Suchanfrage. Beim Schreiben der Sucheingabe ins Suchfeld muss man immer den Abstand zwischen den einzelnen Wörtern/ Zeichenketten und Sonderzeichen beachten! Leerzeichen werden hier als ein Mittelpunkt $(\cdot)$ gekennzeichnet.

Beim Suchen nach Elementen mit Abstand (Wortkombinationen) immer die folgende Funktion überprüfen: 
Die Abfrage wird ins Suchfeld Eingabe geschrieben und durch Klicken auf Suchen aktiviert. Eingabe: irgendwas

\begin{tabular}{|r|}
\hline Suchen anklicken \\
(Ergebnisse anklicken)
\end{tabular}

Die Ergebnisse sind Konkordanzzeilen, die dann sortiert und gespeichert werden können (Abb. 41).

Konkordanzen (10 Beispiele, Zufallsauswahl):

\begin{tabular}{|lrl}
\hline A13 3 Enzelhaft gesetzt zu werden, weil irgendwas passiert war. Einmal sprangen alle \\
A13 & einer Band, die niemandem mehr irgendwas beweisen und keinen zeitgeistigen \\
A13 & sein.» Wo steht hier irgendwo irgendwas von Koran, Hadith oder Teilauszüge \\
A13 & auch nur, um sich vor irgendwas zu drücken, oder? Das Weltgeschehen \\
A13 & hochgeladen, in dem ein Model irgendwas erzählt. Frei nach dem Motto: \\
A13 & des Weges noch im Tal irgendwas. Dafür sahen sie sich tief \\
A13 & wollen die Jungen häufig in irgendwas belehren. Die Jugendlichen wissen \\
A13 & Liner hat zu keiner Zeit irgendwas des Geldes willen gemalt. Mehrmals \\
A13 Rechtschreibung jedenfalls. Heureusement, irgendwas mit Stunden, schlägt Bazyli vor. \\
A13
\end{tabular}

Abb. 41 Konkordanzen zur Abfrage irgendwas (DeReKo, W-Archiv)

\section{Abfragemöglichkeiten im Cosmas II}

Die Möglichkeiten der Abfragen weichen von den üblichen Korpusabfragetypen nicht ab. Die Syntax der Abfragen mag jedoch am Anfang etwas ungewohnt wirken. Für eine bessere Orientierung werden die Abfragen hier in vier Gruppen mit steigender Komplexität aufgeteilt. In der rechten Spalte werden typische Ergebnisse und ein Kommentar angeführt.

\section{Suche nach:}

1) einzelnen Wörtern bzw. Zeichenketten, die Bestandteil eines Wortes sind;

2) Wortkombinationen;

3) Kombinationen mit Abstand;

4) morphosyntaktischen Kategorien (in Kombination mit Abstand).

\section{Abfragen: Wörter/ ununterbrochene Zeichenketten}

\begin{tabular}{|l|l|}
\hline Eingabe & Ergebnis, Kommentar \\
\hline Arzt & $\begin{array}{l}\text { Nur die Wortform Arzt, jedoch nach Einstellung der Suchmodalitäten (Optionen - } \\
\text { Suchmodalitäten). Falls alles abgewählt, dann auch Ärzt, ARZT oder ARzt } \text { etc. }\end{array}$ \\
\hline \&Arzt & Arzt lemmatisiert (alle flektierten Formen von Arzt): Arzt, Arztes, Arzte ... \\
\hline Arzt* & $\begin{array}{l}\text { Alle Wörter, die mit Arzt- beginnen: Arzt, Arztpraxen, Arztpraxis, Arztbesuch, Arzthelferin sind } \\
\text { die häufigsten im DeReKo, W-Archiv. }\end{array}$ \\
\hline *arzt & $\begin{array}{l}\text { Alle Wörter, die auf -arzt enden: Notarzt, Chefarzt, Hausarzt, Zahnarzt, Tierarzt, Facharzt, } \\
\text { Oberarzt, Kinderarzt, Augenarzt sind die häufigsten im DeReKo, W-Archiv. }\end{array}$ \\
\hline$++a r t ~$ & $\begin{array}{l}\text { Alle Wörter, die auf -art enden und maximal aus 5 Buchstaben bestehen: Art, hart, Wart, } \\
\text { Chart, smart sind die häufigsten im DeReKo, W-Archiv. }\end{array}$ \\
\hline ?art & $\begin{array}{l}\text { Alle Wörter, die auf -art enden und genau aus } 4 \text { Buchstaben bestehen: hart, Part, Bart, zart... } \\
\text { sind die häufigsten im DeReKo, W-Archiv. }\end{array}$ \\
\hline \&-schaft & $\begin{array}{l}\text { Alle Wörter mit dem Suffix -schaft: Mannschaft, Gesellschaft, Wirtschaft, Staatsanwaltschaft, } \\
\text { Meisterschaft... sind die häufigsten im DeReKo, W-Archiv. }\end{array}$ \\
\hline \&wider- & $\begin{array}{l}\text { Alle Wörter mit dem Präfix wider-: Widerstand, widersprachen, widerlegt, Widerlegung, } \\
\text { Widerhaken sind die häufigsten im DeReKo, W-Archiv. }\end{array}$ \\
\hline
\end{tabular}

Tab. 5: Abfragen COSMAS II (Wörter) 
Erklärungen und Bemerkungen:

\begin{tabular}{|l|l|}
\hline Symbol & Bedeutung \\
\hline$\&$ & $\begin{array}{l}\text { „Lemmatisator“: Sucht alle Formen des Grundwortes bzw. alle Wörter mit dem eingegebenen } \\
\text { Affix }{ }^{6} \text {. Kein Leerzeichen zw. \& und der Grundform, bzw. dem Affix. }\end{array}$ \\
\hline$*$ & „Platzhalter“: öffnet die Grenze der Zeichenkette um eine beliebige Anzahl an Zeichen \\
\hline+ & „Platzhalter“: öffnet die Grenze der Zeichenkette um ein oder kein Zeichen. \\
\hline$?$ & „Platzhalter“: öffnet die Grenze der Zeichenkette um genau ein beliebiges Zeichen. \\
\hline
\end{tabular}

Abfragen: Wortkombinationen (ununterbrochene Zeichenketten)

Der Abstand (ein Leerzeichen) ist mit einem Mittelpunkt (·) markiert.

\begin{tabular}{|c|c|}
\hline Eingabe & Ergebnis, Kommentar \\
\hline bester·Arzt & Genaue Verbindung von den Wörtern: bester Arzt \\
\hline \&gut $\cdot \& A r z t$ & $\begin{array}{l}\text { Verbindung guter Arzt in unterschiedlichen Formen: guter Arzt, gute Ärzte, } \\
\text { besten Ärzte sind die häufigsten Formen im DeReKo, W-Archiv }\end{array}$ \\
\hline$d++\cdot$ \&gut $\cdot$ \&Arzt & $\begin{array}{l}\text { Verbindung guter Arzt in unterschiedlichen Formen mit bestimmtem Artikel } \\
\text { (oder einem anderen Wort, das auf } d \text { beginnt und max. } 3 \text { Buchstaben hat): die } \\
\text { besten Ärzte, der beste Arzt, den besten Ärzten sind die häufigsten Formen im } \\
\text { DeReKo, W-Archiv. }\end{array}$ \\
\hline Kanzlerin·oder·Merkel & Alle Texte, in denen die Wörter Kanzlerin oder Merkel vorkommen. \\
\hline Merkel·„,oder“ & $\begin{array}{l}\text { Genaue Verbindung von Merkel und oder: ... Merkel oder Außenminister ...; } \\
\text { Merkel oder Bundespräsident; ... Merkel oder Edmund Stoiber ... }\end{array}$ \\
\hline Kanzlerin·nicht·Merkel+ & Alle Texte, in denen Kanzlerin, aber nicht Merkel/ Merkels vorkommen. \\
\hline Merkel· und $\cdot$ Obama & en die Wörter Merkel und Obama vorkommen. \\
\hline
\end{tabular}

Tab. 6: Abfragen COSMAS II (Wortkombinationen)

Erklärungen und Bemerkungen:

\begin{tabular}{|c|c|}
\hline Symbol & Bedeutung \\
\hline oder, nicht, und & $\begin{array}{l}\text { Diese Wörter (und nur diese) sind sogenannte „logische Operatoren“, deswegen } \\
\text { müssen sie in Anführungszeichen gesetzt werden, wenn wir sie abrufen wollen } \\
\text { (mehr dazu siehe Cosmas II } \rightarrow \underline{\text { Hilfe } \rightarrow \text { Logische Operatoren) }}\end{array}$ \\
\hline
\end{tabular}

\section{Abfragen: Kombinationen mit Abstand - allgemein}

Der Abstand (ein Leerzeichen) ist mit einem Mittelpunkt (·) markiert.

\begin{tabular}{|l|l|}
\hline Eingabe & Ergebnis, Kommentar \\
\hline Kanzlerin·/w1·Merkel & $\begin{array}{l}\text { Zwei nebeneinanderstehende Wörter: Kanzlerin Merkel, Merkel Kanzlerin } \\
\text { (Kanzlerin und Merkel im Abstand von einem Wort, beliebige Reihenfolge) }\end{array}$ \\
\hline Kanzlerin·/+w1·Merkel & Zwei nebeneinanderstehende Wörter: Kanzlerin Merkel in dieser Reihenfolge \\
\hline Kanzlerin·/-w1·Merkel & $\begin{array}{l}\text { Zwei nebeneinanderstehende Wörter: Kanzlerin Merkel in umgekehrter } \\
\text { Reihenfolge, also nur Merkel Kanzlerin }\end{array}$ \\
\hline Merkel·/s0·Obama & $\begin{array}{l}\text { Sätze, in denen die Wörter Obama und Merkel (in beliebiger Reihenfolge) } \\
\text { vorkommen. }\end{array}$ \\
\hline Kanzlerin·\%Merkel & Sätze, in denen das Wort Kanzlerin vorkommt, jedoch nicht das Wort Merkel. \\
\hline
\end{tabular}

Tab. 7: Abfragen COSMAS II (Kombinationen mit Abstand)

16 Bei der Affixsuche muss die Option ,sonstige Wortformen“ eingeschaltet werden (Optionen $\rightarrow$ Lemmatisierung $\rightarrow$ Sonstige Wortbildungsformen). Die Liste mit suchbaren Affixen ist auch hier im Kap. 8.1. 
Erklärungen und Bemerkungen

\begin{tabular}{|c|c|}
\hline Symbol & Bedeutung \\
\hline /w2 & $\begin{array}{l}\text { Dieses Symbol bedeutet Wortabstand (w für „Wort“) zwischen zwei Elementen. Die Ziffer (hier } \\
\text { 2) bedeutet den Abstand von zwei Wörtern, d.h. zwischen den abgefragten Wörtern liegt noch } \\
\text { ein anderes. Die Reihenfolge ist beliebig. Die Reihenfolge wird durch die Plus- und } \\
\text { Minuszeichen bestimmt. Das Pluszeichen (+) bedeutet „diese Reihenfolge“, das Minuszeichen (- } \\
\text { ) bedeutet ,umgekehrte Reihenfolge“ (siehe Ergebnis Kanzlerin } /+ \text { w1 } \cdot \text { Merkel und Kanzlerin } /- \\
\text { w1·Merkel) }\end{array}$ \\
\hline$/ \mathrm{s} 0$ & $\begin{array}{l}\text { Das Zeichen s bedeutet „Satz“, die Ziffer } 0 \text { denselben Satz. Analog zu Wort (w) können } \\
\text { Elemente in nacheinander folgenden Sätzen gesucht werden (/s1 bedeutet in } 2 \text { nacheinander } \\
\text { folgenden Sätzen, /s } 2 \text { im Abstand von maximal } 2 \text { Sätzen etc.) }\end{array}$ \\
\hline$/ \mathrm{p} 0$ & $\begin{array}{l}\text { Das Zeichen p bedeutet „Absatz“. Analog zu den Abstandsoperatoren w und s können Elemente } \\
\text { auch im Abstand von Absätzen gesucht werden. }\end{array}$ \\
\hline
\end{tabular}

\section{Abfragen: Kombinationen mit Abstand - strukturelle Fragen innerhalb eines Satzes}

Der Abstand (ein Leerzeichen) ist mit einem Mittelpunkt (·) markiert.

\begin{tabular}{|c|c|c|c|}
\hline Eingabe & \multicolumn{3}{|c|}{ Ergebnis, Kommentar } \\
\hline \&erwidern·/w0; & \multicolumn{3}{|c|}{$\begin{array}{l}\text { Suche nach dem Wort erwidern (in unterschiedlichen Konjugationsformen), nach } \\
\text { dem ein Beistrich folgt: erwidert, aber ...; erwiderte, dass ... }\end{array}$} \\
\hline \&fordern $/ \mathrm{s0} \cdot$ auf & \multicolumn{3}{|c|}{$\begin{array}{l}\text { Suche nach Verben mit (trennbarem) Verbzusatz (traditionell „trennbares Präfix““ } \\
\text { genannt). Diese Abfrage findet jedoch auch Sätze, in denen beide Elemente } \\
\text { vorkommen, bilden gemeinsam jedoch nicht das Lexem auffordern. (Sie fordert } \\
\text { zwar einen Rechtsanspruch zur Rückkehr auf eine Vollzeitstelle,...). Deswegen ist } \\
\text { es sinnvoller die folgende Abfrage zu wählen: }\end{array}$} \\
\hline \multicolumn{2}{|c|}{ \&fordern $/ \mathrm{s} 0$ auf \#IN(R)·<s $>$} & \multicolumn{2}{|c|}{$\begin{array}{l}\text { Suche nach Verben mit (trennbarem) Verbzusatz (traditionell „trennbares } \\
\text { Präfix““ genannt), wobei der Verbzusatz am Ende des Satzes steht. }\end{array}$} \\
\hline Wo+++ $\cdot / \mathrm{s} 0 \cdot 1 ?$ & \multicolumn{3}{|c|}{ Fragesätze, die mit Wo, Wozu, Wofür ... beginnen. } \\
\hline Wie+++·/s0·! & \multicolumn{3}{|c|}{ Exklamative Sätze beginnend mit Wie, Wieso ... } \\
\hline Wer $/ \mathrm{s} 0 \cdot$ & \multicolumn{3}{|c|}{ Aussagesätze, die mit Wer beginnen. } \\
\hline \multicolumn{2}{|c|}{ Wer $\# I I N(L) \cdot<s>\cdot / s 0 \cdot$ gedreht } & $\begin{array}{l}\text { Wer } \\
\text { drel } \\
\text { ged } \\
\text { ged }\end{array}$ & $\begin{array}{l}\text { Anfang des Satzes, in demselben Satz auch das Partizip Perfekt von } \\
\text { Wer hat an der Uhr gedreht? Wer hat hier denn seine Runden } \\
\text { Wer wissen und vor allem sehen möchte, wo berühmte Filme } \\
\text { urden, }\end{array}$ \\
\hline \multicolumn{3}{|c|}{$\begin{array}{l}\text { Wer } \# I N(L) \cdot<s>\cdot / s 0 \cdot \text { gedreht } \cdot \# I N(R) \\
<S>\end{array}$} & $\begin{array}{l}\text { Suche nach Wer am Anfang und gedreht am Ende desselben } \\
\text { Satzes: Wer hat daran gedreht? Wer hat an der Uhr gedreht ... }\end{array}$ \\
\hline \&Einbahn·\#IN <ü> & \multicolumn{3}{|c|}{ Suche nach allen Formen des Wortes Einbahn in allen Überschriften. } \\
\hline
\end{tabular}

Tab. 8: Abfragen COSMAS II (Kombinationen im Satz)

Erklärungen und Bemerkungen

\begin{tabular}{|l|l|}
\hline Symbol & Bedeutung \\
\hline$\# I N(L)<s>$ & $\begin{array}{l}\text { Dieses Symbol hinter einem Abfrageelement (hier Wer) bedeutet Anfang eines Satzes } \\
(\mathrm{L}=\text { "links" }) .\end{array}$ \\
\hline$\# I N(R)<s>$ & $\begin{array}{l}\text { Dieses Symbol hinter einem Abfrageelement (hier gedreht) bedeutet Ende eines Satzes } \\
(\mathrm{R}=\text { "rechts"). }\end{array}$ \\
\hline$<s>$ & Dieses Symbol bedeutet Satz. Es können auch andere Textteile abgerufen werden: \\
\hline$<\ddot{U}>$ & $\begin{array}{l}\text { Alle Überschriften (zu Unterkategorien siehe „Suchanfragen in Überschriften“ in Cosmas II } \\
\text { Online-Hilfe). }\end{array}$ \\
\hline
\end{tabular}


Mehr zur Abfragesyntax im COSMAS II unter: $\underline{\text { Cosmas } \text { II }_{\text {web }}} \rightarrow \underline{\text { Online-Hilfe }} \rightarrow \underline{\text { Suchanfrage }}$ $\rightarrow$ Zeileneingabe $\rightarrow$ Syntax.

\subsection{Recherche im morphosyntaktisch annotierten Teil}

Viele deutsche Wörter oder Verbindungen sind mehrdeutig. Um die Mehrdeutigkeit (Polysemie oder Homonymie) zu verringern, empfiehlt es sich, ein Korpus mit Tagging zu verwenden. Tagging ist ein Prozess, in dem jedes Wort mit einer Zusatzinformation versehen (,,annotiert") wird. Diese Information bezieht sich auf die morphosyntaktische Charakteristik des Wortes. Im Prinzip handelt es sich um morphologische, teilweise auch syntaktische Kategorien, die aus der Form und Position des Wortes im Satz abgeleitet werden können (Wortart, Numerus, Tempus etc. ).

Unter Cosmas II gibt es drei morphologisch annotierte (getaggte) Korpora: TAGGED-C, TAGGED-T und TAGGED-M.

Zur Abfrage nach Tags (morphosyntaktischen Kategorien) verwendet man in beiden Versionen von Cosmas II (Cosmas II $_{\text {web }}$ und Cosmas II $_{\text {win }}$ ) die sog. „graphische Suche“ - man wählt aus dem Angebot (Pop-up-Fenster) des morphologischen Assistenten die gewünschte Kategorie. (Im Cosmas $\mathrm{II}_{\text {win }}$ ist die graphische Suche auch im nicht-annotierten Teil möglich.)

Das IDS empfiehlt „die annotierten Korpora der neueren Archive TAGGED-C oder TAGGED-T (Stand: 2010 bzw. 2011) zu verwenden.“ (IDS online ${ }^{17}$ ). Dennoch kann auch das Archiv TAGGED-M interessant sein - es ist zwar im Vergleich sehr klein (ca. 30 Mio. laufende Wortformen), bietet aber andere Funktionen, die die größeren Archive nicht ermöglichen (siehe Thematische Auflistung der morphosyntaktischen Tags im Kap. 8.2 - in der Tabelle sind die abfragbaren Kategorien im MECOLB (Archiv TAGGED-M) und Treetagger (Archiv TAGGED-T) nebeneinander gestellt, in der letzten Spalte die Kürzel im InterCorp).

\section{Abfragen}

Zuerst muss das entsprechende Archiv und Korpus ausgewählt werden:

\section{Auswahl des Archivs und Korpus}

Archiv - TAGGED-T - Archiv morphosyntakt. annotierter Korpora (TreeTagger)

Korpus - TAGGED-T-öffentlich - alle öffentlichen Korpora des Archivs TAGGED-T

auswählen auswählen

In getaggten Archiven kann man dieselben Abfragen stellen wie im nicht-getaggtem Archiv. Beim Schreiben der Sucheingabe ins Suchfeld muss man immer den Abstand zwischen den einzelnen Wörtern/ Zeichenketten und Sonderzeichen beachten! Leerzeichen werden hier als ein Mittelpunkt (·) gekennzeichnet.

Beim Suchen nach Elementen mit Abstand (Wortkombinationen) immer die folgende Funktion überprüfen:

Weggelassener Verknüpfungsoperator bedeutet:

$\odot$ Wortabstand /+w1 O logisches "ODER"

alternativ aus-/abwählen

Für das kommende Beispiel muss Wortabstand /+w1 ausgewählt werden.

17 Bemerkungen zum Umgang mit morphosyntaktisch annotierten Korpora (Cosmas II $\rightarrow$ Textorganisation $\rightarrow$ Annotationen $\rightarrow$ Bemerkungen) 
Beispiel: Gesucht wird nach dem Wort um in der Rolle eines Verbzusatzes (umdenken, umkreisen...), der abgetrennt ist.

Zuerst wird der morphosyntaktische Assistent aktiviert: anklicken

Jetzt erscheint ein Pop-up-Fenster, in dem das gewünschte Phänomen ausgewählt werden kann.

Die Rolle „abgetrennter Verbzusatz“, nach dem jetzt gesucht wird, ist unter Partikel, abgetrennter Verbzusatz zu finden. Diese muss man ,übernehmen“.

\begin{tabular}{|l|l|}
\hline Partikel $\rightarrow$ abgetrennter Verbzusatz & Übernehmen anklicken \\
\hline
\end{tabular}

Wenn man jetzt auf Suchen klicken würde, bekäme man als Ergebnis alle abgetrennten Verbzusätze (...fest, ...ein, ...um etc.). Gesucht wird jedoch lediglich nach ...um, deswegen muss die Abfrage um ein Argument ergänzt (kombiniert) werden.

\section{Kombinationen:}

Wenn ein konkretes Wort in einer bestimmten morphosyntaktischen Rolle (als eine „morphologische Kategorie“) gesucht wird (hier: um als abgetrennter Verbzusatz), muss die Abfrage als „Null-Abstand“ formuliert werden.

Nachdem der morphologische Assistent das entsprechende morphosyntaktische Zeichen (Tag) ins Suchfeld eingegeben hat (hier: MORPH(PTK vz)), müssen noch der „Null-Abstand“ und das gewünschte Wort oder Element (hier: um) eingegeben werden:

\begin{tabular}{|lr|}
\hline /w0 & (Abstand „, $0^{“}$ ) eingeben \\
\hline um & (Wort um) eingeben \\
\hline
\end{tabular}

Die Eingabe sieht wie folgt aus (Abstand beachten!):

Eingabe:
MORPH(PTK vz)./w0.um

Suchen anklicken

Bitte bedenken Sie, dass die morphologische Annotation automatisch durchgeführt wurde und die Ergebnisse eine große Fehlerquote aufweisen. Bedenken Sie während der Aussortierung falscher Belege, wie lange eine manuelle Recherche in Büchern, Zeitungen und Zeitschriften dauern würde.

Abfragen mit Abstand: Kombinationen mit Tag (über morphologischen Assistenten) (Auswahl)

Der Abstand (ein Leerzeichen) ist mit einem Mittelpunkt (·) markiert.

\begin{tabular}{|l|l|}
\hline Eingabe & Ergebnis, Kommentar \\
\hline MORPH(N ne)·/w0·König & $\begin{array}{l}\text { König als Eigenname: Kardinal Franz König; Trainer Christian } \\
\text { König }\end{array}$ \\
\hline MORPH(N nn)·/w0·König & $\begin{array}{l}\text { König als Gattungsname (im morphosyntaktischen Assistent als } \\
\text {,normales Nominum“ bezeichnet): Simba }- \text { König der Löwen; der } \\
\text { Heilige König ... }\end{array}$ \\
\hline MORPH(AP pr)·/+w2·König & $\begin{array}{l}\text { König im Abstand von max. 2 Wörtern nach einer beliebigen } \\
\text { Präposition: ... durch König Karl I. ...; ... durch den König Herodes } \\
\ldots ; \text { Laut SP-Bürgermeister König ... }\end{array}$ \\
\hline
\end{tabular}

(Fortsetzung auf nächster Seite) 


\begin{tabular}{|c|c|}
\hline Eingabe & Ergebnis, Kommentar \\
\hline \multicolumn{2}{|c|}{ MORPH(AP·pr) /+w2·(König·/w0·MORPH(N·nn)) } \\
\hline & $\begin{array}{l}\text { König als Gattungsname im Abstand max. } 2 \text { Wörter davor eine beliebiger } \\
\text { Präposition: ... auf den König ..., ... mit einem König ... }\end{array}$ \\
\hline MORPH(PTK·vz) /w0·um & $u m$ als Verbzusatz \\
\hline \multicolumn{2}{|c|}{ MORPH(VRB·fin·a)·MORPH(VRB·inf·v) MORPH(VRB·inf·m) \#IN(R) <s> } \\
\hline & $\begin{array}{l}\text { Verbalkomplex (dreistellig) am Ende eines Satzes: da diese bereits vor vier } \\
\text { Wochen hätte erfolgen müssen. }\end{array}$ \\
\hline
\end{tabular}

Tab. 9: Abfragen COSMAS II (Kombinationen mit Tag)

\subsection{Ansichten}

Diese Funktion ist wichtig für die Entdeckung der Unterschiede im Vorkommen der KWICs. Die Ansicht kann nach unterschiedlichen Kriterien ausgewählt werden, die die Distribution des KWICs auf der stilistischen (Themen/Textsorten), regionalen (Länderansicht) oder historischen (Jahresansicht) Ebene beleuchten. Über Cosmas $\mathrm{II}_{\text {web }}$ kann man unter folgenden Ansichten wählen: Quellenansicht, Korpusansicht, Dokumentansicht, Ansicht vor/ seit (Zeitpunkt), Jahrzehntansicht, Jahresansicht, Monatsansicht, Tagesansicht, Länderansicht, Textsortenansicht, Themenansicht. Im Cosmas $\mathrm{II}_{\text {win }}$ gibt es dazu noch die Ansicht nach Wort-Types (Statistik einzelner Formen z.B. bei einer Lemmaabfrage). Unter Ergebnisse kann zwischen den einzelnen Ansichten durch Klicken auf die entsprechende Ikone gewechselt werden.

Beispiel: Gesucht wird nach den Lexemen Gehsteig (Abb. 42) und Gummibärli (Abb. 43) mit dem Ziel zu recherchieren, ob ihr Vorkommen regional bedingt ist.

\section{Länderansicht:}

Zuerst soll wieder das entsprechende Archiv und Korpus ausgewählt werden, erst dann werden die gewünschten Optionen eingestellt. Die Einstellung der Optionen ist für die Präsentation der Ergebnisse nach einzelnen Kriterien (hier Länderansicht) elementar.

Auswahl des Archivs und des Korpus

\begin{tabular}{|cc|}
\hline Archiv - W - Archiv der geschriebenen Sprache & auswählen \\
\hline Korpus - N-öffentlich - alle öffentlichen Korpora & auswählen \\
\hline
\end{tabular}

\section{Einstellung:}

\begin{tabular}{|c|c|}
\hline \multicolumn{2}{|l|}{ Korpuspräsentation } \\
\hline Korpusansicht & Länderansicht \\
\hline Sekundäre Sortierung: & Texte (absteigend) \\
\hline Prozentuelle Verteilung der Texte & einschalten \\
\hline Darstellung & einschalten \\
\hline & ernehmen anklicken \\
\hline
\end{tabular}


Optionen $\rightarrow$ Ergebnispräsentation

\begin{tabular}{|cr|}
\hline $\begin{array}{c}\text { Ergebnispräsentation } \\
\text { Ergebnisansicht: }\end{array}$ & Länderansicht \\
\hline Sekundäre Sortierung der Ergebnisse: & Texte (absteigend) \\
\hline $\begin{array}{l}\text { Berechnung des Häufigkeitsmaßes (Frequenzen und Distribution) } \\
\text { Maß für die Häufigkeit berechnen und anzeigen } \\
\text { Relative Häufigkeiten }\end{array}$ & einschalten \\
in Prozent & auswählen \\
pro Million Worte & auswählen \\
Differenzenkoeffizient & auswählen \\
Häufigkeitsklassen mit folgender Referenz: & auswählen \\
Einzelwort, Groß/Klein/Diakr. beachten & \\
Einzelwort, Groß/Klein/Diakr. ignorieren & \\
Lemma & \\
automatisch & Übernehmen anklicken \\
\hline
\end{tabular}

\section{Eingabe der Abfrage:}

Ins Suchfeld Gehsteig (lemmatisiert) eingeben:

Die Eingabe sieht wie folgt aus (Abstand beachten!):

\section{\&Gehsteig}

Nach dem eine Listen mit Formen (Gehsteige, Gehsteigs etc.) erscheint:.

Als Ergebnis bekommt man eine Tabelle mit Länderansicht, sortiert nach relativer Häufigkeit:

\begin{tabular}{|c|c|c|c|c|c|c|}
\hline & Treffer & rel. Häuf. $\nabla$ & Texte & von & bis & Land \\
\hline$\boxplus$ & 10.633 & $15.61 \mathrm{pMW}$ & 7.768 & 1991 & 2013 & $\mathrm{~A}$ \\
\hline 田 & 2.349 & 0.75 pMW & 2.079 & 1949 & 2013 & $D$ \\
\hline 田 & 171 & $0.37 \mathrm{pMW}$ & 160 & 1996 & 2013 & $\mathrm{CH}$ \\
\hline & 13.153 & $3.07 \mathrm{pMW}$ & 10.007 & 1949 & 2013 & 3 Länder \\
\hline
\end{tabular}

Abb. 42: Gehsteig (lemmatisiert), Länderansicht (DeReKo, W-Archiv)

Beim Klicken auf $⿴$ eröffnen sich die Konkordanzzeilen (Belege).

Es ist ratsam, jeweils die relativen Häufigkeiten zu beobachten. Das Beispiel in der Abb. 42 zeigt: die meisten Treffer (2. Spalte $=$ absolute Anzahl der „Treffer“/Belege im Korpus) kommen in österreichischen Texten vor und haben dort auch die höchste relative Häufigkeit (15.61 Vorkommnisse pro eine Million Worte). 
Das Beispiel in Abb. 43 (gesucht wurde nach Gummibärli) könnte jedoch bei der simplen Betrachtung der absoluten Zahlen (Treffer) den Beobachter in die Irre führen:

\begin{tabular}{|c|c|c|c|c|c|c|}
\hline & Treffer & rel. Häuf. & Texte & von & bis & Land \\
\hline 田 & 85 & 0.1834 pMW & 76 & 1996 & 2013 & $\mathrm{CH}$ \\
\hline 田 & 93 & 0.1365 pMW & 82 & 1992 & 2012 & A \\
\hline 田 & 6 & 0.0019 pMW & 5 & 2008 & 2011 & $D$ \\
\hline & 184 & $0.0430 \mathrm{pMW}$ & 163 & 1992 & 2013 & $\begin{array}{r}3 \\
\text { Länder }\end{array}$ \\
\hline
\end{tabular}

Abb. 43: Gummibärli, Länderansicht (DeReKo, W-Archiv)

Die höchste absolute Trefferanzahl ist zwar wieder in österreichischen Texten zu verzeichnen, nach relativer Häufigkeit sieht man aber, dass das Wort Gummibärli (nicht überraschend) am häufigsten in schweizerischen Texten vorkommt.

\subsection{Kookkurrenzanalyse}

Zur Entdeckung von häufigsten Verbindungen und sprachlichen Chunks ist die Berechnung der Verbindungen einzelner Lexeme wichtig. Im DeReKo heißt diese Berechnung Kookkurrenzanalyse. Diese kann zu jedem Rechercheergebnis durchgeführt werden. Diese Korpusfunktion ist im (Fremd)Sprachenunterricht für die Erschließung der Redundanzfelder beim Leseverstehen (im Sinne von Westhoff (1987: 41) wichtig.

Beispiel: Gesucht wird nach dem Verb aufsetzen (ohne abgetrenntes auf) und seinen Kookkurrenzpartnern. In anderen Worten fragen wir das Korpus: „Was setzt man am häufigsten in deutschen Texten auf?“

\section{Abfrage:}

Auswahl des Archivs und des Korpus

\begin{tabular}{|cc|}
\hline Archiv - W - Archiv der geschriebenen Sprache & auswählen \\
\hline Korpus - N-öffentlich - alle öffentlichen Korpora & auswählen \\
\hline
\end{tabular}

Einstellung der Optionen: Standardwerte

Eingabe: $\quad$ \&aufsetzen

Aus der Liste der Formen (aufgesetzt, aufsetzen, aufzusetzen, aufsetzt, aufsetzte sind die fünf häufigsten Formen) ist ersichtlich, dass jetzt nur diejenigen Formen analysiert werden, die als ein Wort (ununterbrochene Zeichenkette) vorkommen. Formen mit abgetrenntem Verbzusatz auf werden nicht berücksichtigt.

\section{Durchführung der Kookkurrenzanalyse:}


Dann eröffnet sich das Fenster, in dem die Parameter der Kookkurrenzanalyse eingestellt werden:

\begin{tabular}{|lr|}
\hline Einstellungen (Standardeinstellung) & 5 Wörter rechts \\
\hline Kontext & 5 Wörter links \\
Granularität: & grob \\
Zuverlässigkeit: & normal \\
Clusterzuordnung: & eindeutig \\
Lemmatisierung verwenden: & abwählen \\
Funktionswörter ignorieren & auswählen \\
LLR-Wert anzeigen: & auswählen \\
Nummerierung des Hauptkollokators: & auswählen \\
\hline & \\
\hline
\end{tabular}

Die Standardeinstellung (wie hier) ist empfehlenswert. Die Berechnung liefert „normale“ Kookkurrenzpartner. Erläuterungen zu den weniger verständlichen Schaltern beschreiben Perkuhn/ Belica (2004) und Belica (1995) folgend:

„Autofocus: (Typische Stellung der Kollokatoren im Kontext ermitteln?)

Ohne Autofocus wird der gesamte eingestellte Kontext betrachtet, mit Autofocus werden alle möglichen Kontexte innerhalb des vorgegebenen Kontextes ausgewertet und es wird derjenige ausgewählt, der den höchsten Signifikanzwert aufweist

Granularität: (Wie intensiv sollen Mehrwortgruppen gesucht werden?)

Die Granularität gibt an, wieviele der nach Signifikanz sortierten Kookkurrenzpartner als möglicher Kandidat eines Kookkurrenzpartners n. Stufe in Frage kommen:

fein betrachtet die meisten (alle, die unter einem internen Schwellwert liegen) [zielt auf Wortverbindungen]

mittel betrachtet weniger (alle, die unter dem Schwellwert - $10 \%$ liegen) grob betrachtet am wenigsten (nochmals - $10 \%$ ) [zielt auf Schlagwörter]

Zuverlässigkeit: (Ziehen Sie Ausbeute oder Zuverlässigkeit vor?)

Inwieweit die Abweichung "beobachtet vs. normal" als relevant eingestuft werden soll, kann in drei Abstufungen vorgegeben werden:

hoch: nur starke Abweichungen sind relevant [findet wenige Kookkurrenzpartner, aber diese zuverlässig, ignoriert aber evtl. interessante Kandidaten, z.B. zufällig aufgrund Korpusauswahl und -komposition]

normal: mittlere Abweichungen sind relevant

analytisch: schwache Abweichungen sind relevant [findet viele Kookkurrenzpartner, aber diese evtl. unzuverlässig, kann u.U. auch schlechte Kandidaten mit erfassen]

Clusterzuordnung: (Was tun, wenn ein Beleg mehreren Kollokationsclustern zugeordnet werden kann?) Bei "eindeutig" werden Ambiguitäten zugunsten des stärksten Kollokationsclusters aufgelöst. Bei "mehrfach" werden Belege in alle relevanten Kollokationscluster eingefügt."

Ist die Funktion Lemmatisierung verwenden ausgewählt, erscheinen nur die Kookkurrenzpartner/ Kollokatoren (Abb. 44). Bei nicht eingeschalteter Lemmatisierung zeigt das Programm auch

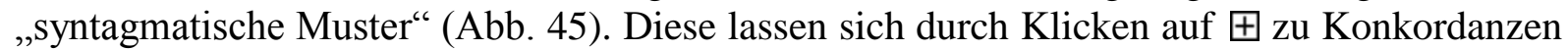
umwandeln.

\begin{tabular}{|rrrll|}
\hline \# & Total & Anzahl & LLR & Kookkurrenzen \\
1 & 5 & 5 & 824 & Krone \\
2 & 2078 & 108 & 278 & Krönchen \\
3 & 2247 & 169 & 247 & Sahne \\
4 & 2813 & 566 & 161 & Brille \\
5 & 2874 & 61 & 137 & Poker \\
\hline
\end{tabular}

Abb. 44: Kookkurrenzen zur Abfrage: Lemma aufsetzen, lemmatisiert (Belica 1995, DeReKo) 


\begin{tabular}{|c|c|c|c|c|c|c|}
\hline$\#$ & Total & Anzahl & LLR & Kookkurrenzen & & syntagmatische Muster \\
\hline 1 & 1923 & 1923 & 1166 & Krone & & $36 \%$ die Krone [...] aufgesetzt \\
\hline 2 & 2031 & 108 & 278 & Krönchen & & $40 \%$ das|ein Krönchen [...] aufsetzen \\
\hline 3 & 2505 & 474 & 238 & Brille & & $38 \%$ dieleine Brille [...] aufsetzen \\
\hline 4 & 2663 & 158 & 224 & Sahnehäubchen & $7 \%$ noch & ein|das Sahnehäubchen [...] aufgesetzt werden \\
\hline 5 & 2729 & 66 & 201 & Meisterkrone & & 63응 die Meisterkrone [...] aufsetzen \\
\hline 6 & 2875 & 146 & 174 & Kopfhörer & & $39 \%$ die Kopfhörer [...] aufgesetzt und \\
\hline 7 & 2951 & 76 & 139 & Narrenkappe & & $42 \%$ die Narrenkappe $[\ldots]$ aufgesetzt \\
\hline 8 & 3000 & 49 & 127 & WM-Krone & & $34 \%$ die WM-Krone $[\ldots]$ aufgesetzt hatte \\
\hline 9 & 3092 & 92 & 123 & i-Tüpfelchen & & $32 \%$ noch das i-Tüpfelchen aufsetzen \\
\hline 10 & 3139 & 47 & 98 & Pokerface & & $40 \%$ seinlihr Pokerface $[\ldots]$ aufgesetzt und \\
\hline
\end{tabular}

Abb. 45: Kookkurrenzen zur Abfrage: Lemma aufsetzen, Wortformen (Belica 1995, DeReKo)

Die syntagmatischen Muster sind eigentlich sprachliche Chunks. In Anbetracht der Tatsache, dass sie einen hohen Signifikanzwert aufzeichnen, sollten sie auch im Fremd- und Zweitsprachenunterrich berücksichtigt und eingesetzt werden.

\section{Bemerkungen:}

Will man die Kookkurrenzpartner oder syntagmatischen Muster exportieren, muss beim

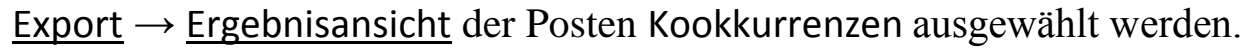

Die Kookkurrenzanalyse (in anderen Korpora auch Kollokationsanalyse genannt) ist eine Funktion, zu deren Ergebnissen ein Mensch, der nicht Jahre mit Recherchieren und Rechnen verbringen möchte, ohne ein elektronisches Korpus kaum gelangen könnte. 


\subsubsection{Korpora mit gesprochenem Deutsch}

Repräsentative und große Korpora der gesprochenen Sprache sind viel schwieriger zu erstellen als die der geschriebenen Sprache. Dennoch existieren auch auf diesem Gebiet einige Quellen: Bayerisches Archiv für Sprachsignale (BAS); Österreichisches Aussprachewörterbuch/ Österreichische Aussprachedatenbank (adaba), Korpus Gesprochene Sprache beim DWDS, die Datenbank für gesprochenes Deutsch (DGD) oder das GeWiss-Korpus der gesprochenen Wissenschaftssprache. Darüber hinaus gibt es verschiedene Kleinkorpora mit unterschiedlichen Zielsetzungen (z.B. das Bochumer Korpus der gesprochenen Sprache im Ruhrgebiet, oder diverse Projekte am Hamburger Zentrum für Sprachkorpora). Keines von diesen Instrumenten ist so groß sowie stilistisch und regional ausgewogen, dass es für die gesprochene deutsche Sprache wirklich repräsentativ wäre. Das größte von ihnen ist die DGD.

Die meisten Korpora beinhalten standardisierte Transkripte der gesprochenen Sprache, zu denen die entsprechende Tonaufnahme synchronisiert abrufbar ist.

\subsubsection{Datenbank für gesprochenes Deutsch (DGD)}

http://dgd.ids-mannheim.de/

Die DGD ist momentan die größte Datenbank der gesprochenen deutschen Sprache (über 7,5 Mio. laufender Wörter), in der ähnlich wie in einem Korpus der geschriebenen Sprache recherchiert werden kann.

Für die Recherche benötigt man eine Registrierung. (Obwohl die DGD auch vom IDSMannheim verwaltet wird, gilt für sie nicht die Registrierung zu Cosmas II!)

Die Recherche ist relativ einfach, zuvor ist es jedoch ratsam, auf der übersichtlichen „Herzlich-Willkommen-Seite“ einen kurzen Blick auf die Beschreibungen der einzelnen Teilkorpora zu werfen.

\section{Zugang zur DGD-Schnittstelle}

\begin{tabular}{|ll|}
\hline Ins Google- oder Adressen-Suchfeld http://dgd.ids-mannheim.de/ & $\begin{array}{l}\text { eingeben } \\
\text { Registrierung }\end{array}$ \\
Login & Anmelden \\
Recherche (auf der oberen Leiste) $\rightarrow$ Volltext & anklicken \\
\hline Korpuswahl (am linken Rand) & aus-/abwählen \\
\hline
\end{tabular}

\section{Eingabe der Abfrage:}

\section{Volltext-Suche}

Beim Schreiben der Sucheingabe ins Suchfeld muss man immer den Abstand zwischen den einzelnen Wörtern/ Zeichenketten und Sonderzeichen beachten! Leerzeichen werden hier als ein Mittelpunkt (·) gekennzeichnet.

Die Möglichkeiten der Abfrage sind grundsätzlich diese:

Suche nach:

1) Einzelnen Wörtern bzw. Zeichenketten, die ein Bestandteil eines Wortes sind;

2) Wortkombinationen;

3) Kombinationen mit Abstand. 
Abfragen: Wörter und Wortteile

\begin{tabular}{|l|l|}
\hline Eingabe & Ergebnis \\
\hline Arzt & Nur die Wortform Arzt \\
\hline Arzt\% & $\begin{array}{l}\text { Alle Wörter, die mit Arzt- beginnen: Arzt, Arztpraxis, Arztbesuche, Arztkoffer sind die } \\
\text { häufigsten in der DGD. }\end{array}$ \\
\hline$\% a r z t$ & $\begin{array}{l}\text { Alle Wörter, die auf -arzt enden: Notarzt, Hausarzt, Pseudoarzt, Frauenfacharzt, Dienstarzt sind } \\
\text { die häufigsten in der DGD. }\end{array}$ \\
\hline \multirow{-}{*}{ ig } & $\begin{array}{l}\text { Alle Wörter, die auf -ig enden und genau aus 6 Buchstaben bestehen: fertig, völlig, witzig, häufig } \\
\text { sind die häufigsten in der DGD. }\end{array}$ \\
\hline
\end{tabular}

Tab. 10: Abfragen DGD (Wörter und Wortteile)

Erklärungen und Bemerkungen:

\begin{tabular}{|l|l|}
\hline Symbol & Bedeutung \\
\hline$\%$ & „Platzhalter“: öffnet die Grenze der Zeichenkette um eine beliebige Anzahl an Zeichen \\
\hline - & „Platzhalter“: öffnet die Grenze der Zeichenkette um genau ein beliebiges Zeichen. \\
\hline
\end{tabular}

\section{Abfragen: Wortkombinationen}

Der Abstand (ein Leerzeichen) ist mit einem Mittelpunkt (·) markiert.

\begin{tabular}{|l|l|}
\hline Eingabe & Ergebnis \\
\hline kein·Spaß & Genaue Verbindung von Wörtern: kein Spaß \\
\hline kein\%·Spaß & Verbindung kein Spaß, kein in unterschiedlichen Formen: kein, keinen, keinerlei \\
\hline groß\&spaß & Alle Texte, in denen die Wörter groß und Spaß vorkommen. \\
\hline lach\%|läch\% & $\begin{array}{l}\text { Alle Texte, in denen eines oder beide Wörter, die auf lach- oder läch- beginnen, } \\
\text { vorkommen: lächelnd, lachen. }\end{array}$ \\
\hline lach\%\&spaß & $\begin{array}{l}\text { Alle Texte, in denen lachen, lachst, lacht etc. und Spaß vorkommen: spielen und lachen } \\
\text { miteinander...und haben alle viel spaß }\end{array}$ \\
\hline \$jung & jung lemmatisiert (alle flektierten Formen von jung): jung, jüngeren, aber auch Junge, Jungs \\
\hline
\end{tabular}

Tab. 11: Abfragen DGD (Wortkombinationen)

Erklärungen und Bemerkungen:

\begin{tabular}{|l|l|}
\hline Symbol & Bedeutung \\
\hline $\boldsymbol{\&}$ & bedeutet ,gleichzeitig“ \\
\hline I & bedeutet Alternative \\
\hline $\mathbf{\$}$ & $\begin{array}{l}\text { „Lemmatisator“: sucht alle Formen des Grundwortes. (Kein Leerzeichen zw. \$ und der } \\
\text { Grundform) }\end{array}$ \\
\hline
\end{tabular}

\section{Abfragen: Kombinationen mit Abstand}

\begin{tabular}{|l|l|}
\hline Suchanfrage & Ergebnis \\
\hline \multirow{3}{*}{ NEAR((\$geh,Schule),4,false) } & $\begin{array}{l}\text { Zwei Wörter im Abstand von max. 4 Positionen voneinander entfernt, } \\
\text { Reihenfolge beliebig. } \\
\text { (hier: ... in die Schule gegangen. Und gehst hier auch zur Schule?) }\end{array}$ \\
\hline \multirow{2}{*}{ NEAR((\$geh,Schule),2,true) } & $\begin{array}{l}\text { Zwei Wörter im Abstand von max. 2 Positionen voneinander entfernt in } \\
\text { dieser Reihenfolge. } \\
\text { (hier: Zwei gehen in die Schule ...; Denn wir gingen mit der Schule ...; Und } \\
\text { dann ging ich zur Schule.), }\end{array}$ \\
\hline
\end{tabular}

Tab. 12: Abfragen DGD (Kombinationen mit Abstand) 
Erklärungen und Bemerkungen

\begin{tabular}{|l|l|}
\hline Symbol & Bedeutung \\
\hline NEAR & bedeutet Abstand (Keine Leerzeichen nach Kommas!) \\
\hline Ziffer (hier: 4 und 2) & bedeutet Abstand (Keine Leerzeichen nach Kommas!) \\
\hline false/true & Reihenfolge: false = beliebig; true = wie angegeben \\
\hline
\end{tabular}

Als Ergebnis bekommt man Konkordanzen der Transkripte, die KWICs sind färbig gekennzeichnet.

\section{Weitere Funktionen:}

\section{Metadaten}

Unter dem Menüpunkt Metadaten (auf der oberen Leiste unter Recherche $\rightarrow$ Metadaten) ist eine struktursensitive Metadatensuche möglich. Das heißt, dass die Audiofiles (und Transkripte) sich nach unterschiedlichen Kriterien einschränken und abrufen lassen: etwa nach Region, aus der die Sprecher/-innen kommen, Geschlecht, Dauer der Aufnahme, Anzahl der Sprecher/-innen etc.

\section{Tokens}

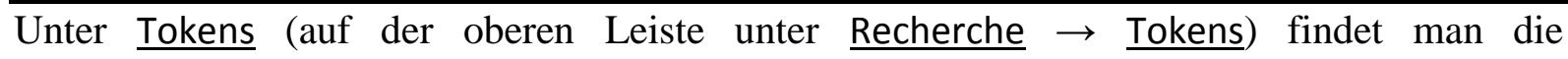
Abfragemöglichkeiten: Wort, Normalisiert, Lemma.

Wort heißt hier transkribiertes Wort, „lautgetreu“ (z.B. ['vuR $\left.\int \mathrm{t}\right]=$ wurscht).

Normalisiert bedeutet der genormten Schreibweise angepasste Form (wurst oder Wurst).

Lemmatisiert ist eine Funktion, die alle Formen, die dem gewünschten Lemma zugeordnet wurden, abruft.

Die Abfrage nach Wurst (bitte Groß-/Kleinschreibung beachten!) ergibt:

\begin{tabular}{|c|c|c|c|c|}
\hline \multicolumn{5}{|c|}{ Ergebnisse 1 bis 8 von 8 ( 0 ausgefiltert) } \\
\hline & Ereignis & & Treffer & \\
\hline 1 & FOLK002 & nein antwortet marmelad auf & wurst & hätt ich jetz mehr lust \\
\hline 2 & FOLK021 & nee wä malaria wär mir & wurscht & gewesen aber seine freundin schlägt der \\
\hline 3 & FOLK024 & mir können s ja auch mal sammlen is ja & wurscht & in welcher äh \\
\hline 4 & FOLK024 & in nem normale haushalt is is eigentlich ah & worscht & ob jetz gebortstag hosch oder net du mus \\
\hline 5 & FOLK024 & is ja irgendwie so die gschicht des is doch & wurscht & ob wir jetz enttäuscht wäre oder net entt \\
\hline 6 & FOLK066 & ah der eine der net warte konnt bis drei & würscht & roh esse also ich bitt dich \\
\hline 7 & OS-009 & dort mußten die Hochzeitsleute alle selber & Wurst & zu essen kaufen, ein Stückchen Wurst \\
\hline 8 & OS-028 & ds dann haben wir den Verwandten immer & Würste & und Fleisch hingetragen noch. Man hat's j \\
\hline
\end{tabular}

Abb. 46: Konkordanzen zur Abfrage: Lemma Wurst (DGD2)

$\mathrm{Zu}$ jedem Beispiel lassen sich die Tonaufnahmen und die Transkripte abrufen und herunterladen.

Das Korpus kann für unterschiedliche phonetische Übungen eingesetzt werden. Da zu den Transkripten Sequenzen von jeweils 15 Sek. (WMA-Format) abgerufen werden können, 
könnte man die Transkripte und Tonsequenzen mischen und zuordnen lassen. Dies geht in einem E-Learning-Portal mit ein wenig Vorwissen ziemlich einfach.

Es eignet sich natürlich auch sehr gut für sämtliche wissenschaftliche Studien in der Phonetik und in der modernen Dialektologie.

\subsubsection{DWDS - Gesprochene Sprache}

http://retro.dwds.de/

Eine weitere bemerkenswerte Quelle für das gesprochene Deutsch ist das Korpus Gesprochene Sprache beim DWDS. Mit über 2,5 Mio. Tokens deckt das Korpus das ganze 20. Jahrhundert $\mathrm{ab}$ - von Aussagen Kaiser Wilhelms bis zu Sitzungsprotokollen des Deutschen Bundestags aus dem Jahr 1999. Es handelt sich zwar nur um standardisierte Transkripte ohne Tonspur (die Aussprache wurde dem üblichen Schriftbild angepasst - siehe Abb. 47), dennoch ist es eine ausgezeichnete Quelle für Recherchen zu gesprochenem Deutsch.

Die Abfragemöglichkeiten sind ident mit anderen DWDS-Korpora (siehe in Kap. 4.2.1.2) Beispiel: In der Abb. 47 wurde die Wortform net abgerufen.

\section{Suchfeldeingabe: @net}

\begin{tabular}{|c|c|c|c|c|}
\hline \multicolumn{5}{|c|}{ Corpus: gesprochene Sprache } \\
\hline \multicolumn{5}{|c|}{ Abfrage: @net \#less_by_date[1900-01-01,2001-12-31] \#cntxt 1 :spk } \\
\hline \multicolumn{5}{|c|}{ Trefferanzahl: 10.} \\
\hline \multicolumn{5}{|c|}{ Seite: 1} \\
\hline 1 & 1938 & STRE & ... Scheißkerl ist, das ist ja ganz klar, net & wahr.... \\
\hline 2 & 1938 & STRE & ... sind wir doch ganz ehrlich, net & wahr. ... \\
\hline 3 & 1938 & STRE & ... nach München rüberfahren können, $\underline{\text { net }}$ & wahr, da Kaffee trinken können.... \\
\hline 4 & 1938 & STRE & ... bei uns wieder das Arbeiten, $\underline{\text { net }}$ & wahr. Es ist gar keine Schande, wenn wir ... \\
\hline 5 & 1938 & STRE & ..., wir sind doch beweglich, net & wahr (Zwischengelächter), aber ... \\
\hline 6 & 1989 & $J A$ & .... Ich kann (weiß i net & ) ich hab alle alle diese Sachen schriftlich, ... \\
\hline 7 & 1989 & BA & ... und da hat man ja Kraft in der Gefahr, net & wahr? Und da war ich mit, ... \\
\hline 8 & 1989 & $\mathrm{CB}$ & ..., ich hab ja das net & gewußt, einen Sauerteig gemacht, ... \\
\hline 9 & 1994 & $A D$ & ..., ich mach das net & . Ich hab das selbst ... \\
\hline
\end{tabular}

Abb. 47: Konkordanzen zur Abfrage: Wortform net (DWDS - gesprochene Sprache)

Da sich das Korpus momentan (2014) auf der alten Homepage des DWDS befindet, ist eine gesonderte Registrierung empfohlen (jedoch nicht notwendig). 


\subsubsection{Korpora mit historischen deutschen Texten}

Studierende von $\mathrm{DaF} / \mathrm{DaZ}$ sind meist Germanistikstudenten/-innen und haben oftmals eine germanistische Vorbildung. Im Studium, für die Forschung und auch als Demonstration der Vielfalt deutscher Sprache können auch historische und diachrone Korpora behilflich sein. In der Größe und Ausgewogenheit werden sie nie das Niveau von Korpora der gegenwärtigen Sprache erreichen können, aus naheliegenden Gründen wird es nie Korpora der gesprochenen Sprache aus der Zeit geben, in der Tonaufnahmen noch sehr rar waren oder gar nicht existierten. Dennoch ergänzen die Korpora mit historischen Texten das Bild über die deutsche Sprache um einen wesentlichen Aspekt.

Historische und diachrone Korpora werden oft gemeinsam mit synchronen Korpora verwaltet und bilden das „Nationale Korpus“. (z.B. Das Korpus Diakorp im Tschechischen Nationalkorpus).

Online gibt es mehrere Korpora, die nahezu alle überlieferten Stadien des Deutschen abdecken.

\subsubsection{Deutsch Diachron Digital (DDD)}

http://www.deutschdiachrondigital.de

Die Autoren schreiben über das Korpus des Althochdeutschen (DDD 2011):

„Das Referenzkorpus Altdeutsch erfasst und annotiert sämtliche althochdeutschen und altniederdeutschen Texte (ca. 750 - 1050 u.Z.). Nach Abschluss der Arbeiten wird das Korpus etwa 650.000 Wörter umfassen.“

Die Grundlage für das Korpus bilden als Referenztexte die handschriftengetreuesten gedruckten Texteditionen, soweit diese zugänglich sind. Alle Wortformen werden sowohl auf der Wortebene als auch auf der Buchstabenebene aufgenommen. Neben den Formen der Editionen werden die Schreibweise der Handschriften ebenso wie einheitlich standardisierte Wortformen in das Korpus aufgenommen. Graphische Besonderheiten wie z.B. Rubrizierung in den Manuskripten, Kursivierung, in den Editionen aufgelöste Diakritika oder Ligaturen werden im Korpus kommentiert.

Alle Wortformen sind lemmatisiert. Die linguistische Annotation umfasst die Wortarten, morphologische Informationen und Angaben zu den Sätzen, des Weiteren werden Zeilenumbrüche, Absätze und andere Mittel zur Textgliederung ebenso wie Angaben zu Versgliederung und Reimpositionen, soweit vorhanden, erfasst.

Alle in die Datenbank aufgenommenen Texte sind mit Header-Informationen versehen, die die sprachund literaturwissenschaftlich relevanten Informationen zum jeweiligen Text wie z.B. Entstehungszeit, sprachlicher Raum und Überlieferungskontext enthalten.

Momentan läuft das Korpus unter dem Korpusmanager ANNIS (Search and Visualization in Multilayer Linguistic Corpora). Beschreibungen der Abfragen sind unter https://korpling.german.hu-berlin.de/annis3/ zu finden.

\subsubsection{Mittelhochdeutsche Begriffsdatenbank (MHDBDB)}

http://mhdbdb.sbg.ac.at

Das Ziel des Projektes ist (MHDBDB 2012):

„... vollständige mittelhochdeutsche literarische Texte elektronisch bearbeiten und speichern. [Dies] ergibt damit nicht nur die größte elektronische Sammlung deutscher Texte aus dem Mittelalter, sondern auch das bislang mächtigste und umfangreichste Abrufsystem für diese Sammlung.

Fernziel ist es, die Textbasis weiter auszubauen und alle Texte voll-lemmatisiert und disambiguiert (d.h. nach Bedeutungskategorien aufgeschlüsselt) der Forschung über das Internet zur Verfügung zu stellen.“

Im Februar 2014 zählt die MHDBDB über 8 Mio. laufende Wörter in 418 Texten unterschiedlicher mittelalterlicher Quellen - von der Arthusdichtung bis zum Schwank und zu Verserzählungen (siehe MHDBDB $\rightarrow$ Textliste) 


\subsubsection{Bonner Frühneuhochdeutschkorpus (FNHD)}

http://www.korpora.org/Fnhd/

Ein Korpus des Frühneuhochdeutschen entstand in Bonn: (FnhdC 2007):

„Das Korpus besteht aus 40 Quellen, die nach Sprachlandschaften und Zeitschnitten (1350-1400, 14501500, 1550-1600 und 1650-1700) angeordnet sind. Es handelt sich um Auswahltexte mit einem Umfang von jeweils ca. 30 Normalseiten. Sämtliche Texte sind mit Wortklassenangaben und z.T. mit Formenbestimmungen annotiert.“

\subsubsection{Deutsches Referenzkorpus (DeReKo)}

https://cosmas2.ids-mannheim.de/cosmas2-web/

Ein diachrones Korpus beinhaltet auch das DeReKo. Es „enthält Texte von der zweiten Hälfte des 17. Jahrhunderts bis 1972“" (Cosmas II 2012) und befindet sich im Archiv HIST (Zugang über Cosmas II: $\underline{\text { Archiv }} \rightarrow \underline{\text { HIST-öffentlich). }}$

Die letzte Version des Archivs HIST beinhaltet laut aktueller Abfrage (3.4.2014) Texte aus den Jahren 1650 bis 1979 und umfasst knapp 66 Mio. laufende Wörter in fast 5000 Texten (Abb. 48).

(C) Institut für Deutsche Sprache, Mannheim COSMAS II-Server, C2API-Version 4.5.4 - 17. Feb. 2014

\begin{tabular}{lll}
\hline Datum & Freitag, den 4. April 2014, 9:13:51 \\
Korpus & $:$ HIST-öffentlich - alle öffentlichen Korpora des Archivs HIST \\
Archiv-Release: Deutsches Referenzkorpus (DeReKo-2010-II)
\end{tabular}

Zusammensetzung des aktiven Korpus

Korpus-Ansicht, 10 Einträge, nach »bis« aufsteigend sortiert.

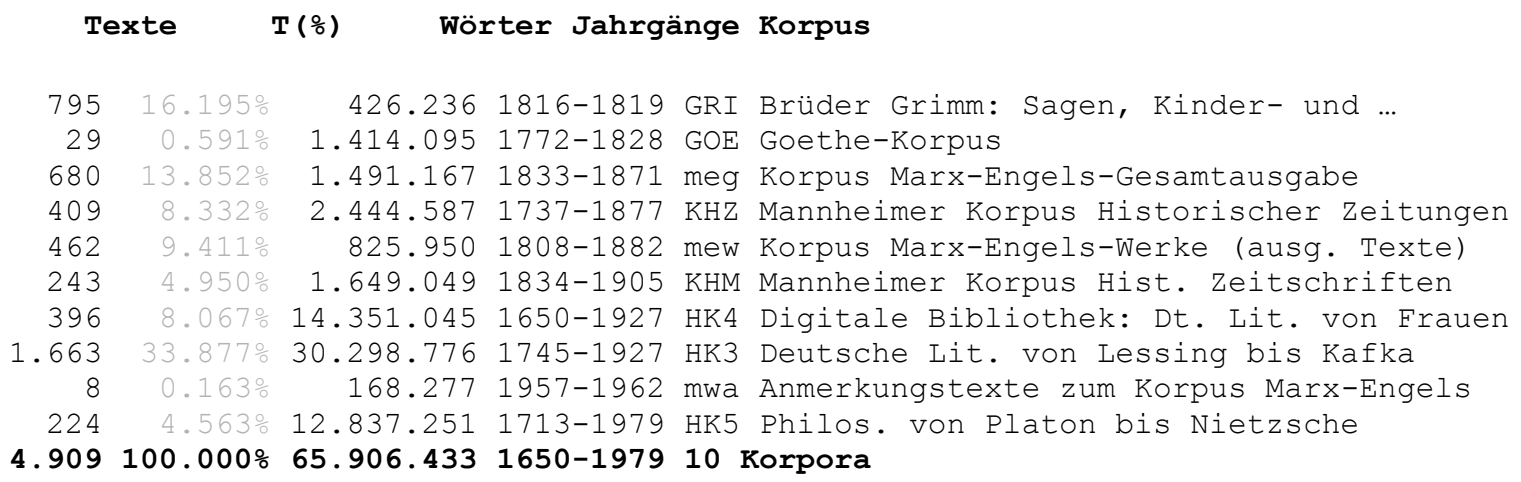

Abb. 48: Zusammensetzung des Archivs HIST (DeReKo) 


\subsubsection{Parallelkorpora mit Deutsch}

Die deutsche Sprache ist in mehreren Parallelkorpora vertreten, die unterschiedliche Größe, Eigenschaften und Recherchemöglichkeiten anbieten. Da für eine seriöse kontrastive Arbeit viele Belege aus unterschiedlichen Texten und zumindest die grundlegenden statistischen Werkzeuge benötigt werden, beschränkt sich die Vorstellung der Parallelkorpora mit Deutsch lediglich auf das OPUS-Corpus und das InterCorp. Beide bilden eine solide Basis nicht nur für verschiedene kontrastive Recherchen in zwei und mehreren Sprachen, sondern fungieren auch als einsprachige Korpora.

\subsubsection{OPUS Korpus}

http://opus.lingfil.uu.se/

Eine gute Quelle paralleler Texte bietet das „Open parallel corpus“ (kurz OPUS oder OPUSCorpus), erstellt und sukzessive erweitert von Jörg Tiedemann (d.Z. an der Universität Uppsala).

Es beinhaltet Texte, die ausschließlich öffentlich zugänglich sind, aus dem Internet heruntergeladen und automatisch annotiert wurden (vgl. Tiedemann 2012: 2214). Die meisten Texte, in denen man mit Korpustools browsen kann, sind Fachtexte (Details in Tiedemann (2009)). Das Korpus beinhaltet für die Erkundung der gesprochenen Sprache auch eine wertvolle Sammlung von Film-Untertiteln (dazu Tiedemann 2007).

Das Korpus besteht aus mehreren Sub-Korpora, in denen recherchiert werden kann (siehe Sub-corpora (downloads \& infos) auf der Hauptseite

Unter Search \& download resources auf der Hauptseite kann man sich ein Bild davon machen, in welchen Subkorpora die gewünschten Parallelen zu finden und wie groß diese sind.

Die Abfragemöglichkeiten sind eingeschränkter als beim InterCorp, dennoch im Wesentlichen ident (dies betrifft die Syntax der Abfrage, das Filtern, Frequenzen etc.). Aus diesem Grund wird auf eine detaillierte Beschreibung der einzelnen Tools im OPUS-Corpus verzichtet.

Es eignet sich auch als eine hervorragende Ergänzung zum InterCorp, weil hier Recherchen in einigen Sprachenkombinationen möglich sind, die im InterCorp noch nicht existieren: z.B. Deutsch - Türkisch, Deutsch - Chinesisch, Deutsch - Japanisch (Abb. 49).

Beispiel: Gesucht wird im Deutschen - Japanischen nach Wörtern, die im Deutschen auf Haus- beginnen:

Abfrage:

Search \& Browse $\rightarrow$ OPUS multilingual search interface $\rightarrow$ OpenSubtitles $\rightarrow \underline{\text { de }}$

\begin{tabular}{|lr|}
\hline ja (= Japanisch) & auswählen \\
\hline vertical & auswählen \\
\hline
\end{tabular}

Eingabe: [word="Haus.*"] 
Die Ergebnisse der Abfrage sind parallele Konkordanzzeilen - Sätze bzw. Repliken der Filmfiguren:

Query string: '[word="Haus.*"] :

20 hits found

\begin{tabular}{|c|c|c|}
\hline & de & ja \\
\hline $\begin{array}{l}413066 \\
413079\end{array}$ & $\begin{array}{l}\text { Ich habe ein Haus in Brentwood. } \\
\text { ' in mein Haus eingebrochen und haben meine } \\
\text { Haushälterin getötet. ' }\end{array}$ & $\begin{array}{l}\text { 私の家はブレントウッドです } \\
\text { 家に押し入って 来て家政 婦を } \\
\text { 殺したの }\end{array}$ \\
\hline 414258 & Ja , zu Hause , & ああ 家にね \\
\hline 418314 & ch nach Hause bringen & わかったよ ママ \\
\hline $\begin{array}{l}1095165 \\
1752181\end{array}$ & $\begin{array}{l}\text { Die Haustür stand letzte Nacht wieder offen . } \\
\text { Zu Hause. }\end{array}$ & $\begin{array}{l}\text { 昨日また玄関 開けっ放しだったぞ } \\
\text { またね }\end{array}$ \\
\hline 1752701 & Ich führe zurzeit zwei Haushalte & 2 世帯を世話してるので \\
\hline 1756657 & Komm einfach abends nach Hause & 夜までには帰ってきてー \\
\hline
\end{tabular}

Abb. 49: Parallele Konkordanzen zur Abfrage Haus- (OPUS-Corpus, Open Subtitles: Deutsch - Japanisch)

Die komplette Seite, Beschreibungen und die Bedienung des OPUS-Corpus sind auf Englisch.

\subsubsection{InterCorp}

http://ucnk.ff.cuni.cz/intercorp/

InterCorp besteht aus parallelen Korpora mehrerer Sprachen. Dieses multilinguale Korpus bildet einen Teil des Projektes „Das Tschechische Nationalkorpus und Korpora anderer Sprachen“. Die Basis des InterCorp wurde in den Jahren 2005 bis 2011 aufgebaut und beinhaltete damals 22 Sprachen. Mittlerweile ist die Recherche in 33 Sprachen möglich und weitere, auch außereuropäische Sprachen sollen ergänzt werden. Weitere Informationen über das Korpus in deutscher Sprache sind auf der deutschen InterCorp-Homepage zu finden (Káňa/ Vavř́ńn: 2011). Momentan liegt das Manual für das InterCorp nur auf Tschechisch, in Zukunft auch auf Englisch vor. Eine deutsche Fassung ist nicht geplant, weshalb hier die wichtigsten Arbeitsanweisungen dargestellt werden:

Das Ziel des Projektes ist die Erstellung von synchronen Parallelkorpora der tschechischen und jeweils einer anderen Sprache. So sollen akademische und nicht kommerzielle Parallelkorpora mit Tschechisch und den meisten Fremdsprachen, die an den geisteswissenschaftlichen Fakultäten in Tschechien studiert werden, entstehen.

Das Korpus InterCorp nimmt in mehreren Hinsichten eine Sonderrolle unter den Korpora des Instituts fürs Tschechische Nationalkorpus ein. Die wesentlichen Unterschiede sind folgende:

- Jede Parallele fungiert als ein vollwertiges einsprachiges Korpus mit allen zugänglichen Instrumenten (Filtern, Sortieren, Verteilung...)

- Im Unterschied zu Referenzkorpora zeichnet sich das InterCorp durch einen stetigen Zuwachs im Umfang der Paralleltexte und auch in der Anzahl der Sprachen aus. Es kann aber nicht automatisch als Referenzkorpus verwendet werden, da die Texte weder stilistisch noch regional noch originalsprachlich (nach Richtung der Übersetzung) ausgewogen sind.

Die Parallelkorpora dienen als Datenquellen für theoretische Studien, studentische Arbeiten, für die Lexikographie und vor allem auch als Unterstützung für den Fremdsprachenunterricht. 
Weiters stehen sie auch Übersetzer/-innen und der breiten Öffentlichkeit zur Verfügung. In der Anbahnungsphase des Projektes wurden die Parallelkorpora an der jeweils zuständigen Arbeitsstelle erstellt und ausprobiert. In weiteren Phasen wurden sie auf einem zentralen Server zusammengeführt und veröffentlicht.

\section{Hinweise zur Nutzung von InterCorp}

Nach der Registrierung auf der Hauptseite (inkl. einer unbürokratischen Zustimmung zu den Nutzungsbedingungen) ist der Zugang über das Web-Interface frei. Bereits registrierte Nutzer des ÚČNK/ICNC haben automatisch Zugriff auch auf das InterCorp.

\section{Registrierung:}

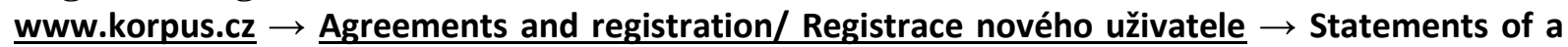
User of the ICNC Corpora (Erklärung des Benutzers der ÚČNK-Korpora) (nur Englisch)

\subsection{Texte und Sprachen des InterCorp}

Im InterCorp sind mehrheitlich manuell alignierte, d.h. in den Parallelen sich entsprechende Texte gespeichert. Laufend werden auch Texte aus Project Syndicate und Presseurop eingespeist und zugänglich gemacht. Es ist jedoch darauf hinzuweisen, dass diese weiteren Texte lediglich automatisch aligniert werden. Die Konkordanzen (Trefferzeilen) können daher in ihren Entsprechungen Fehler aufweisen.

Jeder Text im Korpus hat auch (s)ein tschechisches Pendant, mit dem er primär aligniert ist. Im Zentrum steht also jeweils ein Text auf Tschechisch, zu dem es eine oder mehrere fremdsprachige Variante(n) gibt. Der Gesamtumfang des InterCorp ändert sich mit jeder Version und ist auf der Homepage festzustellen. Die Version 6 (veröffentlicht am 8. 4. 2013) hatte 68,509.857 Positionen bzw. 56,835.000 Wörter in der deutschen Sprache (Dovalil/ Káňa/ Peloušková et al. 2013).

Deutsch kann man kontrastiv mit allen im InterCorp vertretenen Sprachen untersuchen. Das bedeutet, dass es jeweils mindestens einen Text gibt, der auf Deutsch und einer weiteren Sprache im InterCorp enthalten ist. Allerdings muss man damit rechnen, dass zu einigen Sprachen nur ganz wenige Paralleltexte vorhanden sind. Etwa die Sprachkombinationen Deutsch - Arabisch oder Deutsch - Hindi ermöglichen derzeit eine Recherche in nur einem oder zwei Texten.

Die Tabellen auf der folgenden Seite charakterisieren das InterCorp, Version 6. Sie präsentieren eine alphabetische Übersicht über die Sprachen in einzelnen Parallelen (Tab. 13) und eine Übersicht über die aktuelle Größe der jeweiligen Sprachparallele (Tab. 14). Die Angaben zur Größe werden in 1.000 Wörtern angeführt. 
Parallelen im InterCorp - Übersicht

\begin{tabular}{|c|c|c|c|c|c|c|c|}
\hline Kürzel & Link & Sprache & $\begin{array}{l}\text { Wörter } \\
10^{3}\end{array}$ & Kürzel & Link & Sprache & $\begin{array}{l}\text { Wörter } \\
10^{3}\end{array}$ \\
\hline ar & intercorp ar & Arabisch & 29 & cs & intercorp cs & Tschechisch & 99547 \\
\hline bg & intercorp bg & Bulgarisch & 26879 & es & intercorp es & Spanisch & 62865 \\
\hline da & intercorp da & Dänisch & 35785 & de & intercorp de & Deutsch & 56835 \\
\hline de & intercorp de & Deutsch & 56835 & en & intercorp en & Englisch & 54753 \\
\hline en & intercorp en & Englisch & 54753 & $\mathrm{fr}$ & intercorp $\mathrm{fr}$ & Französisch & 53936 \\
\hline et & intercorp et & Estnisch & 26862 & $\mathrm{nl}$ & intercorp nl & Niederländisch & 51817 \\
\hline $\mathrm{fi}$ & intercorp fi & Finnisch & 29040 & pt & intercorp pt & Portugiesisch & 49502 \\
\hline $\mathrm{fr}$ & intercorp fr & Französisch & 53936 & pl & intercorp pl & Polnisch & 47640 \\
\hline el & intercorp el & Griechisch & 40683 & it & intercorp it & Italienisch & 46560 \\
\hline hi & intercorp hi & Hindi & 155 & sv & intercorp sv & Schwedisch & 41694 \\
\hline it & intercorp it & Italienisch & 46560 & el & intercorp el & Griechisch & 40683 \\
\hline ca & intercorp ca & Katalanisch & 1758 & sk & intercorp sk & Slowakisch & 40108 \\
\hline $\mathrm{hr}$ & intercorp $\mathrm{hr}$ & Kroatisch & 12625 & da & intercorp da & Dänisch & 35785 \\
\hline Iv & intercorp IV & Lettisch & 31770 & hu & intercorp hu & Ungarisch & 33985 \\
\hline It & intercorp It & Litauisch & 29811 & sl & intercorp sl & Slowenisch & 33741 \\
\hline $\mathrm{mt}$ & intercorp $\mathrm{mt}$ & Maltesisch & 14133 & Iv & intercorp IV & Lettisch & 31770 \\
\hline mk & intercorp mk & Mazedonisch & 2664 & It & intercorp It & Litauisch & 29811 \\
\hline $\mathrm{nl}$ & intercorp $\mathrm{nl}$ & Niederländisch & 51817 & fi & intercorp fi & Finnisch & 29040 \\
\hline no & intercorp no & Norwegisch & 2301 & bg & intercorp bg & Bulgarisch & 26879 \\
\hline pl & intercorp pl & Polnisch & 47640 & et & intercorp et & Estnisch & 26862 \\
\hline pt & intercorp pt & Portugiesisch & 49502 & ro & intercorp ro & Rumänisch & 21995 \\
\hline ro & intercorp ro & Rumänisch & 21995 & $\mathrm{mt}$ & intercorp $\mathrm{mt}$ & Maltesisch & 14133 \\
\hline ru & intercorp ru & Russisch & 7588 & $\mathrm{hr}$ & intercorp $\mathrm{hr}$ & Kroatisch & 12625 \\
\hline$s r$ & intercorp sr & Serbisch (lat.) & 6972 & $\mathrm{ru}$ & intercorp ru & Russisch & 7588 \\
\hline sy & intercorp sy & Serbisch (kyr.) & 2443 & $s r$ & intercort sr & Serbisch (lat.) & 6972 \\
\hline sv & intercorp sv & Schwedisch & 41694 & mk & intercorp mk & Mazedonisch & 2664 \\
\hline sk & intercorp sk & Slowakisch & 40108 & sy & intercorp sy & Serbisch (kyr.) & 2443 \\
\hline sl & intercorp sl & Slowenisch & 33741 & no & intercorp no & Norwegisch & 2301 \\
\hline es & intercorp es & Spanisch & 62865 & ca & intercorp ca & Katalanisch & 1758 \\
\hline CS & intercorp cS & Tschechisch & 99547 & uk & intercorp uk & Ukrainisch & 1493 \\
\hline uk & intercorp uk & Ukrainisch & 1493 & be & intercorp be & Weißrussisch & 1308 \\
\hline hu & intercorp hu & Ungarisch & 33985 & hi & intercorp hi & Hindi & 155 \\
\hline be & intercorp be & Weißrussisch & 1308 & ar & intercorp ar & Arabisch & 29 \\
\hline
\end{tabular}

Tab. 13: Sprachen im InterCorp alphabetisch

Tab. 14: Sprachen im InterCorp nach Größe 
Morphosyntaktische Annotation im InterCorp

Im InterCorp wurden bisher Texte der folgenden Sprachen morphosyntaktisch annotiert:

\begin{tabular}{|c|c|c|c|c|}
\hline Sprache & $\begin{array}{l}\text { Taggi } \\
\text { ng }\end{array}$ & $\begin{array}{l}\text { Lemmatisi } \\
\text { erung }\end{array}$ & Sprache des Manuals & Tagger \\
\hline Bulgarisch & $\checkmark$ & & Englisch & TreeTagger \\
\hline Deutsch & $\checkmark$ & $\checkmark$ & Deutsch & TreeTagger \\
\hline Englisch & $\checkmark$ & $\checkmark$ & Englisch + Ergänzungen & TreeTagger \\
\hline Estnisch & $\checkmark$ & $\checkmark$ & Englisch, Estnisch & TreeTagger \\
\hline Französisch & $\checkmark$ & $\checkmark$ & & TreeTagger \\
\hline Italienisch & $\checkmark$ & $\checkmark$ & & TreeTagger \\
\hline Litauisch & $\checkmark$ & $\checkmark$ & & von Vidas Daudaravičius \\
\hline Niederländisch & $\checkmark$ & & & TreeTagger \\
\hline Norwegisch & $\checkmark$ & $\checkmark$ & & Oslo Bergen Tagger \\
\hline Polnisch & $\checkmark$ & $\checkmark$ & Englisch & Morfeusz, TaKIPI \\
\hline Portugiesisch & $\checkmark$ & $\checkmark$ & Spanisch & TreeTagger \\
\hline Tschechisch & $\checkmark$ & $\checkmark$ & Englisch & Morče \\
\hline Russisch & $\checkmark$ & $\checkmark$ & Englisch & TreeTagger \\
\hline Slowakisch & $\checkmark$ & $\checkmark$ & Slowakisch & Radovan Garabík, Morče \\
\hline Slowakisch & $\checkmark$ & $\checkmark$ & Slowakisch & Radovan Garabík, Morče \\
\hline Slowenisch & $\checkmark$ & $\checkmark$ & Englisch & totale \\
\hline Spanisch & $\checkmark$ & $\checkmark$ & Spanisch & TreeTagger \\
\hline Ungarisch & $\checkmark$ & & Englisch & HunPos \\
\hline $\begin{array}{l}\text { Arabisch } \\
\text { Dänisch } \\
\text { Finnisch } \\
\text { Griechisch } \\
\text { Hindi } \\
\text { Katalanisch } \\
\text { Kroatisch } \\
\text { Lettisch } \\
\text { Maltesisch } \\
\text { Mazedonisch } \\
\text { Rumänisch } \\
\text { Serbisch (kyr.) } \\
\text { Serbisch (lat.) } \\
\text { Schwedisch } \\
\text { Ukrainisch } \\
\text { Weißrussisch }\end{array}$ & & & nicht getagget & \\
\hline
\end{tabular}

Tab. 15: Übersicht der morphosyntaktischen Annotation im InterCorp (2014) 


\subsection{ARBEIT MIT DEM INTERCORP}

Die Internetseite des Tschechischen Nationalkorpus, auf der sich auch das InterCorp befindet (www.korpus.cz), ist nur in englischer und tschechischer Sprache verfasst. Grundkenntnisse des Englischen sind demnach für die Arbeit mit diesem Korpus erforderlich.

Falls die Seite auf Tschechisch erscheint, kann man auf der oberen Leiste rechts auf English umschalten. Dann erscheinen auf der Leiste diese Posten:

\section{KonText Park SyD Morfio KWords Login|New user registration česky}

Eine Registrierung über New user registration ist erforderlich, jedoch schnell und unbürokratisch.

Nach der Registrierung auf KonText klicken, dann erscheint dieses Bild:

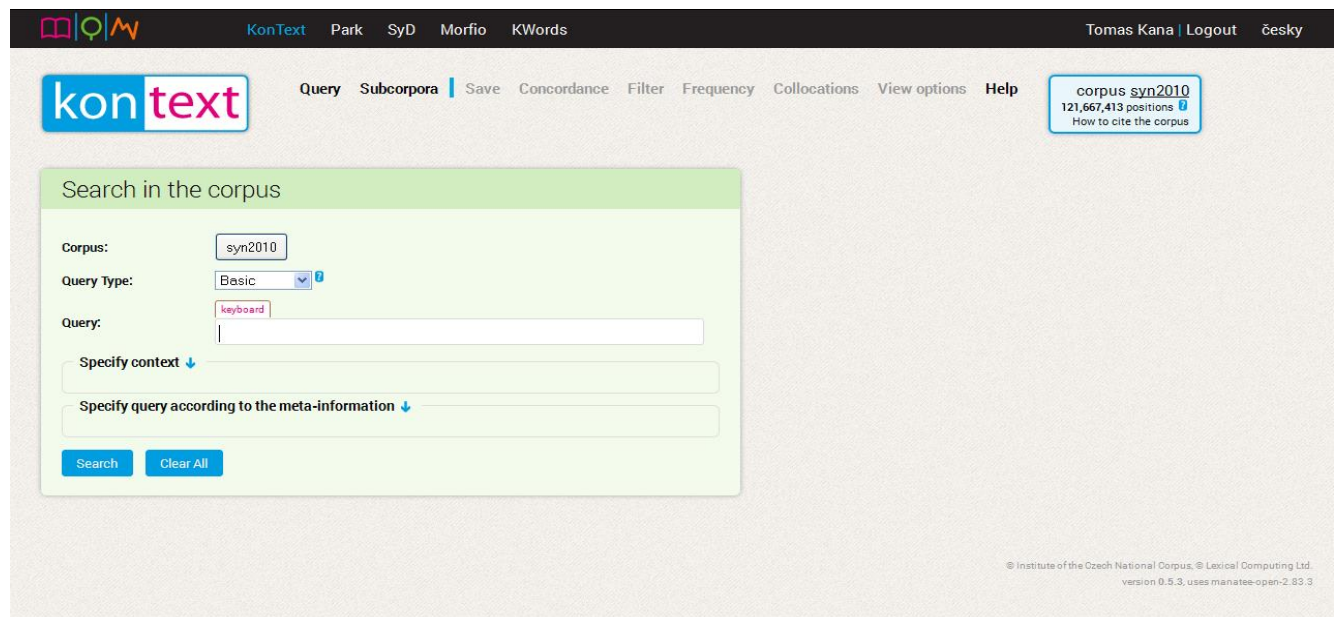

Abb. 50: KonText Startseite (Default: Korpus syn 2010)

Im grünen Viereck Search in the Corpus stellt man das gewünschte Korpus ein:

Dazu den grauen Button neben Corpus: anklicken (vorangestellt ist das Korpus syn2010 synchrones Korpus des Tschechischen, für $\mathrm{DaF} / \mathrm{DaZ}$ irrelevant), dann erscheint folgendes Angebot:

Synchronic written corpora

- Synchronic spoken corpora

- Diachronic corpora

- Foreign language corpora

- Foreign language web corpora

- Parallel corpus InterCorp

Für DaF/DaZ sind primär Korpora mit Deutsch relevant. Die findet man unter:

Foreign language web corpora

Beim Anklicken des Texts Foreign language web corpora öffnet sich das Angebot an WebKorpora. Hier ist u.a. auch das Korpus deWaC zu finden. Dieses beinhaltet Texte ausschließlich aus dem Internet, hat über 1,5 Milliarden Tokens/ Positionen (Stand: Jänner 2014) und dieselben Eigenschaften wie die deutsche Parallele im InterCorp. 


\section{Parallel corpus InterCorp}

Nach dem Klick auf Parallel corpus InterCorp öffnet sich das Angebot an sprachlichen Parallelen. Derzeit sind es Sprachen, die in den Tab. 13 und Tab. 14 angeführt sind.

Die Texte der jeweiligen Sprache haben immer ihr Pendant im Tschechischen und viele von ihnen auch in einer anderen Sprache. Es sind bereits viele Texte auf Tschechisch, Deutsch und noch in einer anderen Sprache im Korpus vorhanden. Man kann mit relativ großen Korpora (einige Mio. Wörter) mit Deutsch und noch einer anderen großen Parallelsprache arbeiten. Zu beachten ist jedoch Folgendes: je „kleiner“ oder von Mitteleuropa aus gesehen entfernter die Sprache ist, desto weniger Texte sind im Parallelkorpus zu finden.

Aus dem Angebot der Parallelen wählt man jetzt Deutsch durch einen Klick auf intercorp de aus.

Im Weiteren wird mit der deutschen Parallele (intercorp_de) gearbeitet. Die Eigenschaften (inkl. Tagging) sind für die Korpora intercorp_de und deWac ident. Sie decken sich sogar mit vielen Eigenschaften anderer deutschsprachigen Korpora (v.a. DeReKo und DWDS). Die Korpora intercorp_de und deWac unterscheiden sich vor allem in der Größe, Struktur der Texte und natürlich in der Möglichkeit, dieselben Texte in einer anderen Sprache abzurufen dies ist im deWaC nicht möglich.

\section{Beschreibung des InterCorp unter KonText}

Das Korpus InterCorp und andere Korpora des Instituts (für das Tschechische Nationalkorpus) laufen unter mehreren Schnittstellen. Die neueste heißt KonText und ist im Prinzip der Schnittstelle (No)Sketch Engine (frühere Version des InterCorp) sehr ähnlich.

Die einzelnen Funktionen und Einstellungen sind einfach und intuitiv zu bedienen. Hier werden sie in drei Kapiteln (in der logischen Abfolge zu einer Abfrage) beschrieben:

\section{A. Aktuelles Korpus \\ B. Abfragefenster \\ C. Einstellungen und Funktionen}

Diese Kapitel widerspiegeln das Bild der Arbeitsfläche (Abb. 51). In ihnen werden die Bedeutungen einzelner Buttons und Funktionen erklärt und um Beispiele ergänzt.

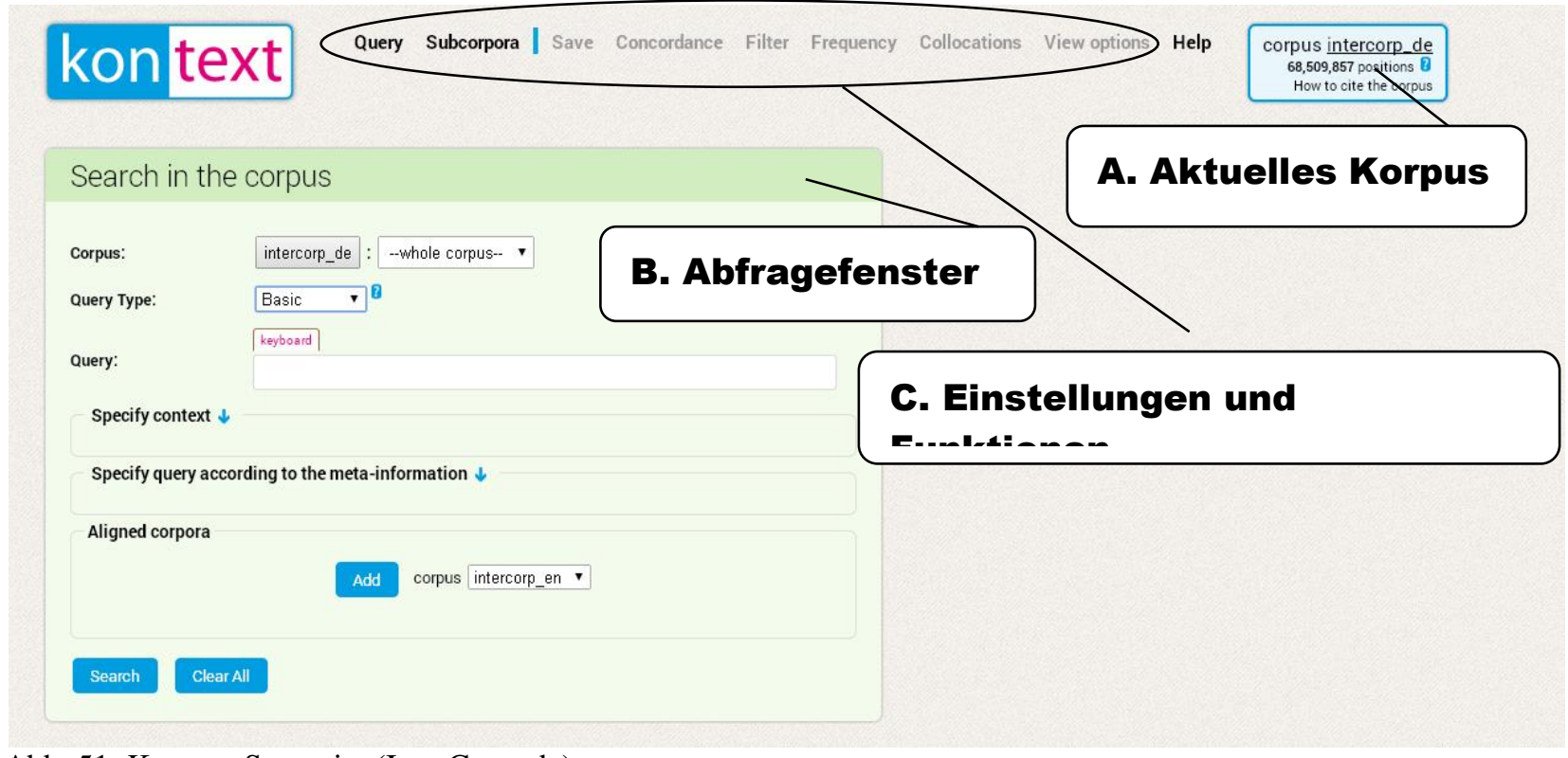

Abb. 51: Kontext-Startseite (InterCorp_de) 


\section{A. Aktuelles Korpus}

\section{corpus:}

intercorp de: Name des Korpus (hier intercorp_de = deutsche Parallele).

Durch Anklicken dieses Buttons erscheinen ausführliche Informationen über diese Parallele (die gleichzeitig auch als ein vollwertiges Korpus funktioniert).

In der Version 6 (2013) sind es für Deutsch folgende Angaben:

\begin{tabular}{|l|l|l|}
\hline \multicolumn{2}{|l|}{ Attributes } & \multicolumn{2}{l}{ word } & 885.504 & Anzahl unterschiedlicher Wörter/ Positionen \\
\hline lemma & 706.237 & Anzahl unterschiedlicher Lemmata einzelner Positionen \\
\hline tag & 54 & Anzahl der Tags (s. Tagset) \\
\hline Ic & 823.825 & Anzahl der Wörter (ohne Satzdiakritika) \\
\hline lemma_Ic & 677.282 & Anzahl der Lemmata (Satzdiakritika ausgeschlossen) \\
\hline
\end{tabular}

Tab. 16: Technische Angaben: Attribute (InterCorp_de, Version 6)

\begin{tabular}{|l|l|l|}
\hline \multicolumn{2}{|l|}{ Structures } & \multicolumn{2}{l|}{ Anzahl der Dokumente im Korpus } \\
\hline$<$ doc $>$ & 231 & Anzahl struktureller Attribute (Informationen über Texte) \\
\hline$<$ div $>$ & 89.734 & An \\
\hline$<\mathrm{p}>$ & $1,748.827$ & Anzahl der Absätze \\
\hline$<\mathrm{s}>$ & $3,714.390$ & Anzahl der Sätze \\
\hline
\end{tabular}

Tab. 17: Technische Angaben: Strukturen (InterCorp_de, Version 6)

$68,509,857$ positions $?$ How to cite the corpus

\section{Gesamtgröße des Korpus in Positionen}

Durch Anklicken des Fragezeichens erscheint eine kurze Definition des Begriffes Position.

\section{Hinweise zu den Zitierregeln}

Durch das Anklicken bekommt man bibliographische Angaben, wie jegliche Belege und Daten aus dem Korpus zu zitieren sind. Bitte bedenken Sie, dass das konsequente und richtige Zitieren die Qualität jeder Arbeit erhöht und in der wissenschaftlichen Praxis zum Standard gehört. Die Autor/-innen sind für Hinweise über die Zitate dankbar. 


\section{B. Abfragefenster/ Search in the corpus}

\begin{tabular}{|c|c|}
\hline Corpus: & Name des aktuellen Korpus \\
\hline & $\begin{array}{l}\text { Falls ein Subkorpus erstellt wurde, erscheint nach dem Korpusnamen ein } \\
\text { Fensterchen, in dem zur Auswahl steht, ob im gesamten Korpus (- whole } \\
\text { corpus }- \text { ) oder nur in einem Teil davon recherchiert wird. } \\
\text { Vorangestellt (Default) ist - whole corpus - } \\
\text { Zur Erstellung der Subkorpora: auf der oberen Leiste Subcorpora anklicken } \\
\text { (Beschreibung hier im Abschnitt C) }\end{array}$ \\
\hline Query Type: & Abfragemodus: im Auswahlfenster kann man \\
\hline & $\begin{array}{l}\text { Abfragemöglichkeiten (Attribute der Abfrage) einstellen: } \\
\text { Basic } \\
\text { Lemma } \\
\text { Phrase } \\
\text { Word Form } \\
\text { Charakter } \\
\text { CQL }\end{array}$ \\
\hline
\end{tabular}

\section{Query Type:}

Basic: $\quad$ bedeutet „Einstiegsmodus“. Gibt man eine Grundform („Wörterbuchform“) ein (z.B. lustig), sucht das Korpus nach alle Formen des Wortes (lustig, Lustig, lustigen, lustiger, lustiges, lustigste etc.). Wenn eine flektierte Form (z.B. Täler, lustiger, dich, möchte), sucht das Korpus nur diese. Im BasicModus wird die Groß-/Kleinschreibung nicht beachtet.

\section{Beispiel}

\begin{tabular}{|l|l|}
\hline Eingabe & Ergebnis (Beschreibung) \\
\hline lustig & lustig, Lustig, lustigen, lustiger, lustiges, lustigste ... (alle Formen) \\
\hline Täler & Täler, täler (nur diese Formen) \\
\hline
\end{tabular}

Konkordanzen (Zufallsauswahl):

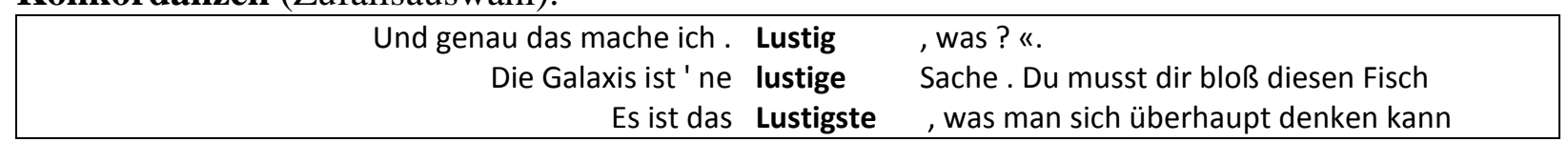

Abb. 52: Konkordanzen zur Abfrage: (Basic) lustig (InterCorp_de) 


\section{Beispiel:}

\begin{tabular}{|l|l|}
\hline Eingabe & Ergebnis (Beschreibung) \\
\hline schreiben & schreiben, schreibe, schrieb, geschrieben ... (alle Flexionsformen) \\
\hline
\end{tabular}

Konkordanzen (Beispiele, Zufallsauswahl):

\begin{tabular}{|rcl|}
\hline $\begin{array}{r}\text { der Befehl, von einem General eigenhändig } \\
\text { paar Wochen angefangen, eine Geschichte zu }\end{array}$ & $\begin{array}{c}\text { geschrieben } \\
\text { schreiben }\end{array}$ & : „ Alle sind auf der Stelle zu \\
Ich erkundigte mich, was er & schreibe & . Er beschreibe sein Leben . \\
schwierig, aber nicht schlecht & geschrieben & . Der Verfasser dieser Beiträge aus \\
die astrologische Rubrik ihrer Zeitschrift zu & schreiben & , war ich \\
Versuche gelang. Dann & schrieb & er ihr einen Brief sowohl ins Büro als \\
sie auch mich. Peter & schreibt & . Peter sagt . Alles Lüge, Lüge, Lüge \\
Mannes, von dem ich noch nie gehört hatte, & geschrieben & von einem Mann, von dem ich \\
Gleich in medias res gehen. & Schreib & !... Ich, Valerie Steinfeld, geborene \\
\hline
\end{tabular}

Abb. 54: Konkordanzen zur Abfrage: Lemma schreiben (InterCorp_de)

\section{Query Type:}

Phrase:
Abfrage nach zwei und mehr Wörtern/ Zeichenketten, die durch ein Leerzeichen (hier durch einen Punkt ," " gekennzeichnet) getrennt sind.

\begin{tabular}{|l|l|}
\hline Eingabe & Ergebnis (Beschreibung) \\
\hline rosarote·Brille & rosarote Brille (nur diese Formen) \\
\hline
\end{tabular}

Konkordanzen (Beispiele, Zufallsauswahl):

\begin{tabular}{|rll|}
\hline die Lage vor dem Eingreifen durch eine & rosarote Brille & zu sehen . Wie bereits betont \\
das Parlament bei einer derartig durch die & rosarote Brille & gesehenen Herangehensweise \\
Reis, Honig. "Aber ich versuche, eine & rosarote Brille & zu tragen. " Irgendwann werde \\
aber durch eine wesentlich weniger & rosarote Brille & . Nicht nur, dass der Begriff Kultur in \\
\hline
\end{tabular}

Abb. 55: Konkordanzen zur Abfrage Phrase: rosarote Brille (InterCorp_de)

\begin{tabular}{|l|l|}
\hline Eingabe & Ergebnis (Beschreibung) \\
\hline rosa.*.Brille & $\begin{array}{l}\text { rosa Brille, rosarote Brille, rosaroten Brille ... (alle Wörter auf } \\
\text { rosa- gefolgt von Brille) }\end{array}$ \\
\hline
\end{tabular}

Konkordanzen (Beispiele, Zufallsauswahl):

\begin{tabular}{rrl|}
\hline bei einer derartig durch die & rosarote Brille & gesehenen Herangehensweise mitmacht \\
"Aber ich versuche, eine & rosarote Brille & zu tragen ." \\
"Dann sage ich : nehmt die & rosa Brille & ab “ und spielt dabei auf \\
Wenn man dieses Bild mit einer & rosaroten Brille & betrachtet, so könnte man \\
\hline
\end{tabular}

Abb. 56: Konkordanzen zur Abfrage: Phrase rosa-Brille (InterCorp_de)

18 Diese findet man als Wörterbucheintrag: bei Substantiven, Adjektiven etc. ist es die Form im Nominativ Singular (bei Adjektiven und Adverbien Positiv), bei Verben ist es die Form im Infinitiv Präsens. 


\section{Query Type:}

Word Form: Abfrage nach einer bestimmten Form oder nach Teil eines Wortes. Die Eingabe muss eine ununterbrochene Kette von Zeichen sein (ohne Leerzeichen). Die Abfrage nach einem Teil erfolgt über Platzhalterzeichen (Tab. 18 auf der folgenden Seite).

\begin{tabular}{|l|l|}
\hline Eingabe & Ergebnis (Beschreibung) \\
\hline rosa & rosa (nur diese Form; Groß-/Kleinschreibung ausgeschaltet) \\
\hline
\end{tabular}

Konkordanzen (Beispiele, Zufallsauswahl):

\begin{tabular}{|r|}
\hline und nährte gigantische rosa und blaue Hortensiensträucher, \\
Herr Karl ihr ein rosa Netz über die Wickler legte, \\
dazu tragen und ein rosa Hemd und eine gestreifte Krawatte \\
Rosa, schreibend an Rosa und Rosa begehrend, \\
ein weißes und ein rosa Zettelchen heraus,
\end{tabular}

Abb. 57: Konkordanzen zur Abfrage rosa (Word Form)

\begin{tabular}{|l|l|}
\hline Eingabe & Ergebnis (Beschreibung) \\
\hline rosa.* & $\begin{array}{l}\text { Ein beliebiger Buchstabe links, beliebige Anzahl beliebiger Buchstaben } \\
\text { rechts: Prosa, Brosamen, Arosa, prosaisch, prosaischen, Prosawerk sind } \\
\text { die häufigsten im InterCorp_de. }\end{array}$ \\
\hline
\end{tabular}

Konkordanzen (Beispiele, Zufallsauswahl):

\begin{tabular}{|rcl|}
\hline stelle ich Ihnen die folgende & prosaische & Frage : Wie viel hat es Spanien \\
Auch in der & Prosa & gibt es kaum noch einen Autor , \\
So lautete das Eingangsgedicht des & Prosabandes & " Frühling um 1900 «, das 1936 \\
traurige Unzulänglichkeit aller & Prosadichtung & gegenüber der Aufgabe, die sie \\
ist später dann in dem erwähnten & Prosatext & festgehalten worden . \\
sein Schicksal, immer nur die & Brosamen & einsammeln zu können \\
\hline
\end{tabular}

Abb. 58: Konkordanzen zur Abfrage: Wortform -rosa- (InterCorp_de)

\begin{tabular}{|l|l|}
\hline Eingabe & Ergebnis (Beschreibung) \\
\hline \multirow{2}{*}{${ }^{\text {rosa.* }}$} & $\begin{array}{l}\text { Eine beliebige Anzahl beliebiger Buchstaben sowohl rechts als auch } \\
\text { links: Prosa, Brosamen...; blaßrosa, rosafarben(en), zartrosa sind die } \\
\text { häufigsten im InterCorp_de. }\end{array}$ \\
\hline
\end{tabular}

Konkordanzen (Beispiele, Zufallsauswahl):

\begin{tabular}{|rcl|}
\hline der Zeit das Geld ersetzte . Mit den & rosa & Zettelchen wurde alles im Kramladen \\
Der Fahrer, der Bischof & $\begin{array}{c}\text { Aringarosa } \\
\text { am Flughafen Leonardo da Vinci in Rom }\end{array}$ \\
In der Tat hätte die Bevölkerung & Ombrosas & , wäre die Sache ruchbar geworden, die \\
eingeschlossen, sind & sakrosankt & - tabu . \\
Sie hat den & prosaischen & Blick, wasserblau natürlich \\
Nasenflügel, legte einen & zartrosa & Lippenstift auf und musterte sich im \\
Edelsteins sprühten in einem & rosafarbenem & Feuer . \\
ihre langen Fingernägel waren & knallrosa & lackiert, \\
\hline
\end{tabular}

Abb. 59: Konkordanzen zur Abfrage: Worform -rosa- (InterCorp_de) 
Platzhalterzeichen und andere reguläre Ausdrücke im InterCorp:

\begin{tabular}{|c|c|c|}
\hline \multicolumn{2}{|r|}{ Symbol } & Erklärung \\
\hline • & Punkt & ein beliebiges Zeichen \\
\hline$*$ & Asterisk & beliebige Anzahl an Zeichen \\
\hline+ & Pluszeichen & $\begin{array}{l}\text { einmalige oder mehrmalige Wiederholung des vorhergehenden } \\
\text { Zeichens: } \\
\text { z.B. Buf+et+ ergibt Bufet, Buffet, Bufett, Buffett) }\end{array}$ \\
\hline [] & $\begin{array}{l}\text { Eckige } \\
\text { Klammern }\end{array}$ & $\begin{array}{l}\text { umgeben Alternativen: } \\
\text { z.B. B[ü̈]ffet ergibt Buffet und Büffet }{ }^{19} \text {. }\end{array}$ \\
\hline$\Lambda$ & Zirkumflex & $\begin{array}{l}\text { negiert die Alternative in der Klammer: } \\
\text { z.B. gr[^ü]ße ergibt größe, große, jedoch nicht grüße. }\end{array}$ \\
\hline () & $\begin{array}{l}\text { Runde } \\
\text { Klammern }\end{array}$ & $\begin{array}{l}\text { geben Prioritäten an - wie beim Rechnen: } \\
\text { z.B. Schluß|ss ergibt Schlu } \beta \text { oder } S S \\
\text { während Schlu(ß|ss) ergibt } S c h l u \beta \text { oder Schluss. }\end{array}$ \\
\hline\{\} & $\begin{array}{l}\text { Geschwungene } \\
\text { Klammern }\end{array}$ & $\begin{array}{l}\text { geben Intervall der Wiederholungen an: } \\
\text { z.B. [tag="ADJA "]\{3,5\} ergibt Adjektivhäufungen von } 3 \text { bis } 5 \\
\text { Adjektiven hintereinander: neuen globalen politischen oder } \\
\text { ausgezeichneten neuen britischen parlamentarischen sowie } \\
\text { europaweiten öffentlichen zellularen digitalen terrestrischen }\end{array}$ \\
\hline$?$ & Fragezeichen & $\begin{array}{l}\text { wiederholt das vorhergehende Zeichen oder die vorhergehende } \\
\text { Zeichenkette einmal oder null-mal: } \\
\text { z.B. (ur)?urgroßeltern ergibt Ururgroßeltern und } \\
\text { Urgroßeltern. }\end{array}$ \\
\hline$!$ & Rufzeichen & $\begin{array}{l}\text { negiert das darauffolgende (relevant für CQL-Abfrage): } \\
\text { z.B. [word=".*lein"\&!word="allein"] ergibt Fräulein, } \\
\text { Häuflein, Büchlein, klein ... }\end{array}$ \\
\hline 1 & Backslash & $\begin{array}{l}\text { muss eingesetzt werden, wenn nach einem der hier angeführten } \\
\text { Sonderzeichen gesucht wird: } \\
\text { z.B. I+ sucht nach Pluszeichen im Text und ergibt Schale mit } \\
\text { der Aufschrift MILCH + ALKOHOL oder Canal + }\end{array}$ \\
\hline
\end{tabular}

Tab. 18: Reguläre Ausdrücke im InterCorp

Shortcuts zu diesen Symbolen befinden sich im Kap. 8.3.

Query Type:

Charakter: Abfrage nach einer beliebigen Zeichenkette innerhalb eines Wortes. Dieser Modus eignet sich gut für die Erschließung von Wortfamilien.

\begin{tabular}{|l|l|}
\hline Eingabe & Ergebnis (Beschreibung) \\
\hline \multirow{3}{*}{ lust } & $\begin{array}{l}\text { ergibt alle Wörter, die die Buchstabenkombination (hier lust) } \\
\text { beinhalten: Verlust, Verlusten, Verlustrechnung, lustig, belustigt, } \\
\text { lustlos, Wollust, Cluster, illustriert, Ballustrade sind die 10 häufigsten } \\
\text { im InterCorp_de. }\end{array}$ \\
\hline
\end{tabular}

19 Nach Daten des Korpuses DeWaC ist übrigens Buffet (80\%) etwa viermal üblicher als Büffet (20\%). 
Konkordanzen (Beispiele, Zufallsauswahl):

\begin{tabular}{|rcl|}
\hline Selbstverwirklichung und dem & Bedeutungsverlust & kollektiver Interessensvertretung \\
Programm Perdita Durango Ein & lebenslustiger & Santería-Priester sucht \\
die Mini-Bar ( hier drohte schließlich & Umsatzverlust & ;-))repariert . \\
Sicherheitsgründen vor & Verlust & gelassen und konnten sich \\
Einen auch mit Bildern & illustrierten & Gang durch die Geschichte der \\
Bildung von räumlichen und sektoralen " & Clustern & " erfolgversprechend . \\
gesellten sich zu unserer & lustigen & Runde und demonstrierten ihr " \\
Auslagerung kann auch einen & Verlust & an Know-how und an Flexibilität \\
\hline
\end{tabular}

Abb. 60: Konkordanzen zur Abfrage: Wörter mit (-)lust(-) (InterCorp_de)

\section{Query Type:}

CQL
Corpus Query Language: Abfrage nach morphosyntaktischen Zeichen, die jeder Position im Korpus zugewiesen sind. Diese Abfrage ist von ihrer Aufstellung her ein wenig kompliziert, kann aber das Suchen nach vielen sprachlichen Elementen wesentlich erleichtern und Interessantes über die Sprache aufdecken.

Bei Abfragen im CQL-Modus sind immer die Sprache und das dazugehörige Tagset zu beachten! (Viele Sprachen sind im InterCorp noch nicht morphosyntaktisch annotiert - siehe Tab. 15 auf der Seite 80.) Die Verwendung eines „fremden“ Tagsets liefert verständlicherweise keine Ergebnisse - der Korpusmanager ,versteht“ die Anfrage nicht.

Die deutschen Korpora im InterCorp (intercorp_de und deWac) sind mit TreeTagger annotiert (genauso wie auch das Archiv TAGGED-T im DeReKo, das DWDS und das CHTK). Das Tagset (Verzeichnis der Zeichen für einzelne morphosyntaktische Kategorien) ist im Kap. 8.2 zu finden. Beispiele für konkrete Abfragen sind im Kap. 8.4 angeführt.

Wenn der Modus CQL eingeschaltet ist, muss die Eingabe immer in der folgenden Syntax (Eckklammern) geschrieben werden. Als Abfragevariable (Attribut) muss word (=Wortform), lemma (= Grundform/ Lemma) oder tag (=morphologisches Zeichen) eingegeben werden.

\begin{tabular}{|l|l|}
\hline Eingabe & Ergebnis (Beschreibung) \\
\hline [word=“lustig"] & $\begin{array}{l}\text { ergibt nur die Formen lustig (ident mit Query Type: Word Form - siehe } \\
\text { oben). }\end{array}$ \\
\hline
\end{tabular}

\begin{tabular}{|l|l|}
\hline Eingabe & Ergebnis (Beschreibung) \\
\hline [lemma=“lustig“] & $\begin{array}{l}\text { ergibt alle Flexionsformen des Wortes lustig: lustig, lustige, lustiges, } \\
\text { lustigste, Lustigsten etc. (ident mit Query Type: Lemma - siehe oben). }\end{array}$ \\
\hline
\end{tabular}

\begin{tabular}{|l|l|}
\hline Eingabe & Ergebnis (Beschreibung) \\
\hline [tag=“ITJ“] & $\begin{array}{l}\text { ergibt alle Interjektionen (ITJ = Interjektionen) im Korpus (bzw. alle } \\
\text { Wörter, die als Interjektionen erkannt wurden): na, ach, och, } a u, h a l l o, \\
\text { oh, } a h, a h a, n u, ~ h e \text { sind die häufigsten im InterCorp. (Au müsste } \\
\text { überprüft werden - siehe letztes Beispiel in den Konkordanzen.) }\end{array}$ \\
\hline
\end{tabular}


Die Liste der morphosyntaktischen Zeichen (Codes, die man anstatt ITJ eingeben kann,) ist im Kap. 8.2.

Konkordanzen (Beispiele, Zufallsauswahl):

\begin{tabular}{|rcl|}
\hline . Ingrid, lese ich auf dem Display. " & Hallo & ", sage ich . " Wie war die Sauftour \\
Sinn , daß er es zur Kenntnis nehme." & Ach je «, sagte sie und \\
"Jetzt sucht mich, & ha ! Jetzt bin ich es, der besser sieht ! \\
MÖBIUS Ihr besitzt Geheimsender ? EINSTEIN & Na & und ? \\
Erde saß und vergnügt vor sich hin sang: " & Hurra & , wie kann ich gut - \\
berechtigten Grimm. - Au, au, au, & au ! schrie der Kopf \\
en meine allzu mächtige Portion Mousse & au chocolat an . \\
\hline
\end{tabular}

Abb. 61: Konkordanzen zur Abfrage: Interjektionen (InterCorp_de)

\section{Kombinationen}

Die drei Attribute/ Abfragevariablen [word], [lemma] und [tag] lassen sich auch kombinieren, wenn man aus mehreren homonymen Formen nur eine abrufen will. Aus unzähligen Kombinationsmöglichkeiten werden im Folgenden drei Abfragebeispiele mit Ergebnissen angeführt.

Beispiel 1: Gesucht wird nach der Interjektion (= Code ITJ) marsch (!). Das Substantiv Marsch soll ausgeblendet werden.

\begin{tabular}{|l|l|}
\hline Eingabe (CQL) & Ergebnis (Beschreibung) \\
\hline [tag="ITJ"\&word="marsch"] & $\begin{array}{l}\text { ergibt Interjektionen (ITJ = Interjektionen), die gleichzeitig die } \\
\text { Form marsch haben. }\end{array}$ \\
\hline
\end{tabular}

Konkordanzen (Beispiele, Zufallsauswahl):

\begin{tabular}{|rrr|}
\hline , wie Leid dir DAS tun wird . Also, & marsch & in die Wanne, und jetzt (...) kein Wort \\
Magst du Kaffee ? Katrin, & marsch & ins Zimmer ! " \\
"Bißchen nassauern, was? & Marsch & , abfahren! “ Albert steht wie betäubt \\
\hline
\end{tabular}

Abb. 62: Konkordanzen zur Abfrage: Interjektion marsch (InterCorp_de)

Beispiel 2: Gesucht wird nach dem Wort(teil) $z u$, das nur als Verbzusatz vorkommt.

\begin{tabular}{|l|l|}
\hline Eingabe (CQL) & Ergebnis (Beschreibung) \\
\hline [word="zu"\&tag="PTKVZ"] & $\begin{array}{l}\text { ergibt Verbzusätze (PTKVZ = Verbzusatz), die gleichzeitig die } \\
\text { Form } z u \text { haben. }\end{array}$ \\
\hline
\end{tabular}

Konkordanzen (Beispiele, Zufallsauswahl):

\begin{tabular}{|rll|}
\hline also steht mir auch ein Drittel von allem & $\mathbf{z u}$ & . Egal \\
aber dann drückte er ein Auge & $\mathbf{z u}$ & , denn \\
Die Kommission stimmt der Empfehlung & $\mathbf{z u}$ & . \\
Sangra hörte wortlos & $\mathbf{z u}$ & . Aber als der Architekt gegangen war , \\
Der Diener ließ das aber nicht & $\mathbf{z u}$ & . " Nein «, sagte er, " Sie müssen \\
" Hört ihr da drüben eigentlich & $\mathbf{z u}$ & ? "Professor Raue-Pritsches Stimme \\
nahm die Hitze jedoch immer mehr & $\mathbf{z u}$ &, bis sie schließlich kulminierte \\
und ich stimme & $\mathbf{z u}$ & , dass die Informationen bezüglich \\
Dann nickte er mir & $\mathbf{z u}$ & und ging. \\
Harry sah eine Weile & $\mathbf{z u}$ & und stellte fest, \\
\hline
\end{tabular}

Abb. 63: Konkordanzen zur Abfrage: Verbzusatz zu (InterCorp_de) 
Beispiel 3: Gesucht wird nach einer Präposition (= Code APPR) und dem Wort König als Gattungsname (= Code NN) im Abstand von max. 2 Wörtern.

\begin{tabular}{|l|l|}
\hline Eingabe (CQL) & Ergebnis (Beschreibung) \\
\hline [tag="APPR"][]\{0,2\}[lemma="König"\&tag="NN"] & $\begin{array}{l}\text { ergibt Präposition, König als Gattungsname, } \\
\text { dazwischen 0-2 andere Wörter. }\end{array}$ \\
\hline
\end{tabular}

Konkordanzen (Beispiele, Zufallsauswahl):

\begin{tabular}{|rcl|}
\hline Ablehnung der & von den fränkischen Königen & praktizierten \\
wandte sich Judit Kinski & an den König & . " \\
, dachte der Jüngling & an den alten König & und den Marktplatz , \\
beugte sich Judit & über den König & und zog \\
und wenn es & um einen König & längstvergangener Zeiten \\
als sie auch selbst & für die Könige & der Menschen \\
und wie er & unter deren legendären Königen & damals gearbeitet \\
war nicht persönlich & mit dem König & bekannt . \\
Pluralismus & unter Königen & , die Böhmen \\
\hline
\end{tabular}

Abb. 64: Konkordanzen zur Abfrage: Präposition (2 Positionen) vor dem Appellativum König (InterCorp_de)

Vorsicht! Immer auf den Abfragemodus achten. Wird z.B. beim eingeschalteten CQL Modus ein einfaches Wort eingegeben, zeigt das Korpus keine Treffer (,Empty result" ${ }^{6)}$. 


\section{B. Abfragefenster/ Search in the corpus}

(Fortsetzung - siehe Abb. 51 auf Seite 82)

Specify context::

Beim Anklicken dieser Überschrift öffnet sich das Fenster Lemmafilter, in dem der Kontext des Suchbegriffs spezifiziert werden kann.

Diese Funktion ist wichtig, wenn die Umgebung des KWIC eingeschränkt werden soll.

Beispiel: Wo kommen im Umfeld von lustig auch Lemmata lachen und laut vor?

\begin{tabular}{|l|l|}
\hline Specify context: \\
\hline Window: & $\begin{array}{l}\text { both }=\text { suche rechts und links vom KWIC } \\
\text { right = suche nur rechts vom KWIC }\end{array}$ \\
& left = suche nur links vom KWIC \\
& $1-15$ tokens = Abstand vom KWIC \\
Lemma: & $\begin{array}{l}\text { Eingabe der gewünschten Lemmata, die im Kontext gesucht werden sollen (hier } \\
\text { lustig und lachen). Gleich daneben: Einstellung des Suchmodus: }\end{array}$ \\
\hline $\begin{array}{ll}\text { all } & \text { sucht im Umfeld sowohl (lachen) als auch (laut) } \\
\text { any of these items } & \text { sucht entweder (lachen) oder (laut) } \\
\text { none } & \text { sucht weder (lachen) noch (laut) im Umfeld (von lustig) }\end{array}$ \\
\hline
\end{tabular}

Konkordanzen (alle Belege):

Die Cousine lachte laut heraus, weil sie tatsächlich zuviel gekifft hatte und alles als lustig empfand und OCCASIONAL LOVER . " Cecilia Vanger lachte laut . "Was ist daran so lustig ?"

Abb. 65: Konkordanzen zur Abfrage: Lemma lustig im Kontext mit Lemmata lachen und laut

\section{Specify query according to the meta-information:}

Die Ergebnisse lassen sich auf bestimmte Texte einschränken. Die

Kriterien der Einschränkung sind ident mit der Funktion Erstellung eines

Subkorpus (siehe dort).

\section{Aligned corpora:}

Aus dem Angebot unter

corpus intercorp_ar

bis

intercorp_uk (siehe Tab. 13 auf S. 79) wird die gewünschte Parallele durch Klicken auf die entsprechende Sprache und Bestätigen auf den Button Add ausgewählt.

Add anklicken.

Jetzt erscheint ein Abfrageformular für die erste Parallelsprache. Wiederholt man den Vorgang, erscheint ein Formular für die zweite Parallelsprache (Abb. 66 auf der nächsten Seite). 
Es wird also in drei Sprachen recherchiert: Deutsch (als 1. Parallele) - Englisch (als 2. Parallele) - Spanisch (als 3. Parallele).

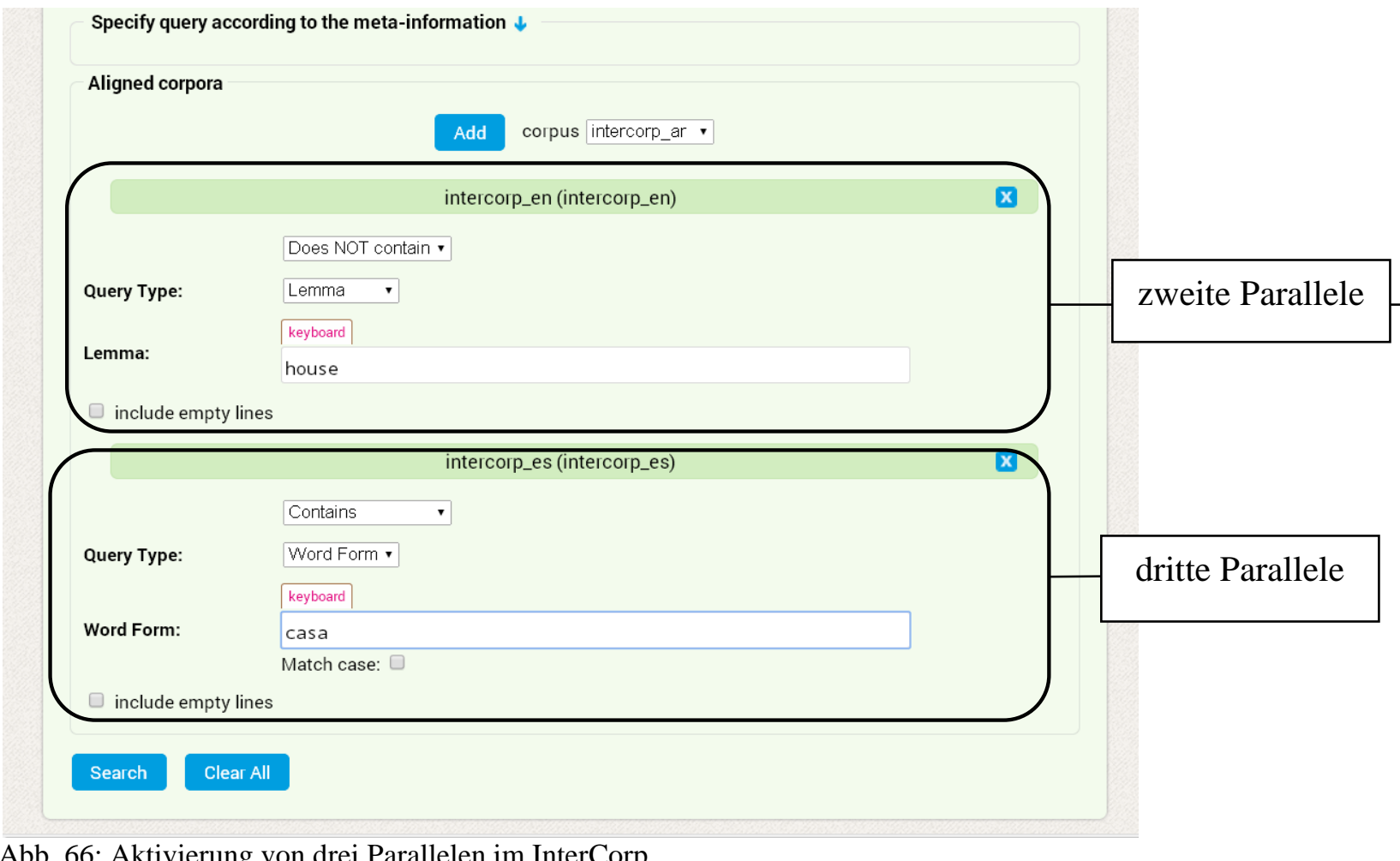

Abb. 66: Aktivierung von drei Parallelen im InterCorp

Jede Parallele kann durch Klicken auf $\mathbf{x}$ wieder gelöscht werden.

Wenn kein Formular für die Parallelsprache(n) (zweite, dritte Parallele) ausgefüllt wird, bekommt man als Suchergebnisse alle Belege angezeigt, die der Abfrage in der ersten Sprache/Parallele (hier Deutsch) entsprechen. (Im Beispiel unten sind es alle Segmente, in denen im Deutschen die Wörter Haus, Hause, Hauses, Häuser vorkommen.)

Diese Formulare dienen der sog. parallelen Suche, die die Abfrage in der ersten Sprache (hier Deutsch) einschränken: ins Suchfeld der zweiten (dritten ...) Sprache kann ein Wort, Lemma, eine Phrase etc. eingegeben werden. Wenn dies erfolgt, sucht das Korpus nur nach denjenigen Segmenten, in denen die Sucheingaben von beiden (allen drei) Sprachen vorkommen (= Auswahl: Contains) oder eben nicht vorkommen (= Auswahl: Does NOT contain).

include empty lines bedeutet, dass in den Ergebnissen auch leere Segmente erscheinen, also $\square$ (aus-/abwählen) diejenigen Segmente, die in der Parallele der zweiten (dritten ...) Sprache keine Entsprechung haben. Dies kann mehrere Ursachen haben: a) translatologische Arbeit (Übersetzungen entsprechen einander kaum); b) andere Auflage der Publikation; c) schlechtes Alignment. 


\section{Beispiel:}

Gesucht wird nach Entsprechungen des deutschen Wortes Schule im Englischen und Spanischen, wobei man im Englischen das Wort school ausblenden möchte.

\section{Search in the corpus}

\begin{tabular}{llr} 
Corpus: & intercorp_de & auswählen \\
Query Type: & Basic & auswählen \\
Query: & Schule & eingeben \\
\hline
\end{tabular}

\section{Aligned corpora}

\begin{tabular}{llr|} 
Corpus: & intercorp en & auswählen $\rightarrow$ Add \\
& Does NOT contain & auswählen \\
Query Type: & Lemma & auswählen \\
Lemma: & school & eingeben \\
\hline
\end{tabular}

dann

\begin{tabular}{|llr|}
\hline Corpus: & intercorp_es & auswählen $\rightarrow$ Add \\
& Contains & auswählen \\
Query Type: & Word form & auswählen \\
Word form: & escuela & eingeben \\
& & Search anklicken \\
\hline
\end{tabular}

Konkordanz (Beispiel, Zufallsauswahl):

\begin{tabular}{|r|l|l|r|r|l|l|}
\hline de (links) & KWIC & de (rechts) & en & es (links) & KWIC & es (r.) \\
\hline $\begin{array}{r}\text { Man hätte mich } \\
\text { von der }\end{array}$ & Schule & geworfen. & $\begin{array}{r}\text { I 'd have been kicked } \\
\text { out of the Academy. }\end{array}$ & $\begin{array}{r}\text { Me hubieran } \\
\text { echado de la }\end{array}$ & escuela &. \\
\hline
\end{tabular}

Abb. 67: Konkordanz zur Abfrage: (de) Basic: Schule - (en) NICHT Lemma school - (es) Wortform escuela (InterCorp_de-en-es)

Soll im Spanischen auch das Wort escuela ausgeblendet werden, stellt man einfach den Schalter Contains auf Does NOT contain um.

Konkordanz (Beispiel, Zufallsauswahl):

\begin{tabular}{|r|l|l|l|l|}
\hline de (links) & KWIC & de (rechts) & en & es \\
\hline $\begin{array}{r}\text { Pr war aus der } \\
\text { der und aus }\end{array}$ & Schule & hinausgeflogen . & $\begin{array}{l}\text { He 'd been kicked out of } \\
\text { both the Party and the } \\
\text { university. }\end{array}$ & $\begin{array}{l}\text { Lo habían echado del partido y } \\
\text { de la facultad. }\end{array}$ \\
\hline
\end{tabular}

Abb. 68: Konkordanz zur Abfrage: (de) Basic: Schule - (en) NICHT Lemma school - (es) NICHT Wortform escuela (InterCorp_de-en-es)

Die Konkordanzen/ Segmente erscheinen in Spalten und lassen sich einfach exportieren (siehe unter Export). 


\section{Einstellungen und Funktionen}

Auf der oberen Leiste (neben dem Logo KonText) findet man (waagrecht gereiht) folgende Posten:

Query|Subcorpora|Save $\mid$ Concordance $\mid$ Filter|Frequency|Collocations|View options|Help

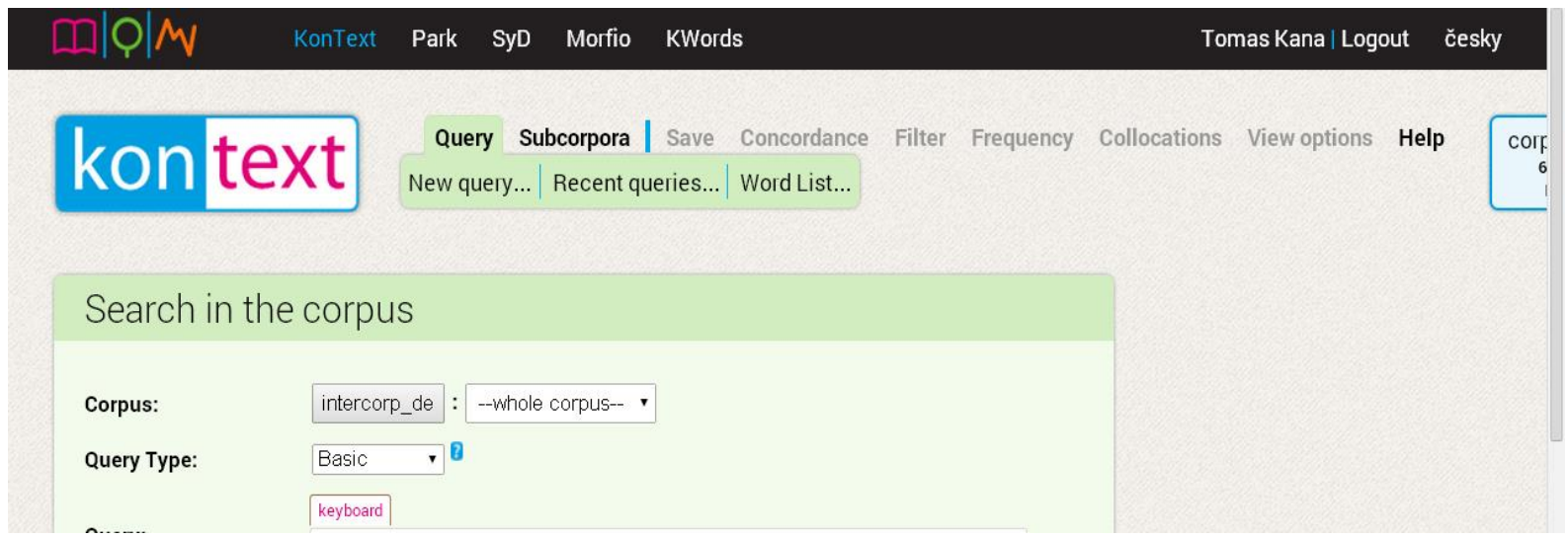

Abb. 69: Einstellungen und Funktionen: Level 1 (InterCorp)

\section{$[D \mid M$ KonText Park SyD Morfio KWords Tomas Kanal Logout česky \\ kontext \\ Query Subcorpora | Save Concordance Filter Frequency \\ New query... $\mid$ Recent queries... $\mid$ Word List...}

Abb. 70: Einstellungen und Funktionen: Level 2 (InterCorp)

Fährt man mit der Maus zu dem gewünschten Posten, öffnet sich unter ihm das weitere Angebot (hier unter Query: New query | Recent quiries | Word List).

Die Posten Save bis View Options sind erst nach dem Aufrufen der Konkordanz aktiv.

\section{Query}

New query...|Recent queries... |Word List...

Hier gelangt man zu den Abfragefenstern.

\begin{tabular}{|ll|}
\hline New query & $\begin{array}{l}\text { neue Anfrage (siehe B. Abfragefenster/Search in the corpus ab Seite } \\
84 .)\end{array}$ \\
\hline Recent queries & $\begin{array}{l}\text { zuletzt gestellte Anfragen während der letzten Sitzungen. Diese können } \\
\text { wieder abgerufen werden. }\end{array}$ \\
\hline Word List & $\begin{array}{l}\text { gibt die Frequenzliste der Wörter im aktuellen Korpus an. Die Liste } \\
\text { erscheint automatisch beginnend mit dem Wort mit der höchsten } \\
\text { Frequenz, lässt sich aber auch alphabetisch erstellen (durch Klicken auf } \\
\text { Word). }\end{array}$ \\
\hline
\end{tabular}


Diese Funktion ermöglicht die Einschränkung/ systematische Auswahl aus dem bestehenden Korpus. Wichtig ist dies für eine gezielte Suche in spezifischen Texten. Das neue Subkorpus kann man nach verschiedenen Kriterien (siehe unten) aufbauen. (Die Kriterien sind gleich wie bei der Einschränkung der Konkordanzen bzw. beim Spezifizieren der Suchkriterien - siehe Specify query according to the meta-information auf Seite 91.)

\begin{tabular}{|ll|}
\hline Create new & \\
\hline New subcorpus name: & $\begin{array}{l}\text { Benennen des neuen Subkorpus: einen kurzen, treffenden } \\
\text { Namen eingeben. }\end{array}$ \\
\hline $\begin{array}{l}\text { Specify subcorpus using: } \\
\text { Custom 'within' condition } \odot \\
\text { Attribute list O }\end{array}$ & $\begin{array}{l}\text { spezifiziert die Subkorpuscharakteristik } \\
\text { aus-/abwählen }\end{array}$ \\
\hline Custom 'within' condition & $\begin{array}{l}\text { erfordert eine gute Orientierung in den Korpustexten und in } \\
\text { ihren Bezeichnungen. Die Erstellung nach diesen Kriterien } \\
\text { ist etwas ,benutzerunfreundlich“. Einfacher ist ein }\end{array}$ \\
Attribute list $\odot$ & $\begin{array}{l}\text { Vorgehen über den Befehl Attribute list. } \\
\text { Auswahlmöglichkeiten über Aus-/Abwahlbuttons } \square\end{array}$ \\
\hline
\end{tabular}

\begin{tabular}{|c|c|}
\hline $\begin{array}{l}\text { div.group } \\
\square \text { jádro/ core } \\
\square \text { kolekce/ collections }\end{array}$ & $\begin{array}{l}\text { Das „Kernkorpus“ (jádro/ core) besteht aus manuell } \\
\text { bearbeiteten Texten. Die „Textsammlungen“ } \text { (kolekce/ }^{\circ} \text { collections) bestehen aus automatisch bearbeiteten Internettexten. }\end{array}$ \\
\hline $\begin{array}{l}\text { div.id: } \\
\square \_ \text {ACQUIS } \\
\square \_ \text {EUROPARL } . . .\end{array}$ & Technische Namen ${ }^{20}$ einzelner Texte im Korpus. \\
\hline $\begin{array}{l}\text { div.txtype: } \\
\square \text { drama } \\
\square \text { literatura faktu ... }\end{array}$ & $\begin{array}{l}\text { Stilistische Charakteristika der Texte } \\
\text { Da diese Werte derzeit nur auf Tschechisch erscheinen, gibt es } \\
\text { auf der nächsten Seite eine kleine sprachliche Hilfe. }\end{array}$ \\
\hline $\begin{array}{l}\text { div.srclang: } \\
\square-(\text { unspezifisch) } \\
\square \text { bg. } \\
\square \text { cs } \ldots\end{array}$ & „Source language“ - Wahl der Originalsprache \\
\hline $\begin{array}{l}\text { div.original: } \\
\square \text { - (unspezifisch) } \\
\square \text { ano/yes } \\
\square \text { ne/no }\end{array}$ & $\begin{array}{l}\text { Originaltexte } \\
\text { Wählen Sie ano, wenn sie nur in (z.B. deutschen) Originaltexten } \\
\text { browsen wollen. }\end{array}$ \\
\hline
\end{tabular}

20 Diese werden im Laufe der Zeit durch leichter identifizierbare Titel ersetzt. 


\section{Übersetzungshilfe:}

\begin{tabular}{|c|c|c|}
\hline $\begin{array}{l}\text { Attribute } \\
\text { (siehe vorherige } \\
\text { Seite) }\end{array}$ & Tschechisch & Deutsch \\
\hline div.group & $\begin{array}{l}\text { jádro/ core } \\
\text { kolekce/ collections }\end{array}$ & $\begin{array}{l}\text { Kernkorpus (manuell alignierte Texte) } \\
\text { Sammlungen (automatisch alignierte Texte) }\end{array}$ \\
\hline div.txtype & $\begin{array}{l}\text { drama } \\
\text { literatura faktu } \\
\text { poezie } \\
\text { právní texty } \\
\text { próza } \\
\text { publicistika - komentáře } \\
\text { publicistika - zprávy } \\
\text { různé } \\
\text { zápis debaty } \\
\text { ano/ yes } \\
\text { ne/ no }\end{array}$ & $\begin{array}{l}\text { dramatische Texte } \\
\text { Sachtexte } \\
\text { Poesie } \\
\text { rechtswissenschaftliche Texte } \\
\text { Prosatexte } \\
\text { publizistische Texte - Kommentare } \\
\text { publ. Texte - Nachrichten u. Berichte } \\
\text { Diverses } \\
\text { Gespräche } \\
\text { ja } \\
\text { nein }\end{array}$ \\
\hline
\end{tabular}

\section{Save}

\section{CSV|XML|TXT|Custom...}

Hier werden die Recherchergebnisse gespeichert. Sowohl Konkordanzen als auch Frequenzlisten lassen sich in verschiedenen Formaten speichern (CSV, XML, TXT): man muss mit der Maus auf die Ikone Save gehen, dann Custom wählen. Es eröffnet sich eine Abfragetabelle:

\begin{tabular}{|ll|}
\hline Custom... & \\
\hline Save concordance as: & $\begin{array}{l}\text { Speicherformat einstellen: Text (txt), XML oder CSV. } \\
\text { Empfehlenswert ist das CSV-Format (einfache Konversion in eine } \\
\text { Excel-Tabelle), denn die KWICs werden in einer separaten Spalte } \\
\text { zwischen dem Kontext links und rechts gespeichert. (Danach folgt } \\
\text { die Arbeit mit Excel: Indexierung, Sortieren etc.) }\end{array}$ \\
\hline Include heading: & $\begin{array}{l}\text { aus-/abwählen (nur bei der Wahl vom TXT- oder XML-Format). } \\
\text { Mitgespeichert werden dabei Informationen über die Abfrage: } \\
\text { Korpus, Eingabe, Abfragemodus etc. }\end{array}$ \\
\hline Include line numbers: & Falls ausgewählt, werden die Konkordanzen nummeriert. \\
\hline Align KWIC: & $\begin{array}{l}\text { Falls ausgewählt, sind die KWICs in der Zeilenmitte und } \\
\text { untereinander geordnet. }\end{array}$ \\
\hline Lines to store: & $\begin{array}{l}\text { In diesem Fensterchen kann die Anzahl der zu speichernden } \\
\text { Konkordanzen eingestellt werden. }\end{array}$ \\
\hline
\end{tabular}


Arbeit mit Konkordanzen: sortieren, mischen, Auswahl der Treffer, Rückkehr zu vorherigen Schritten.

\begin{tabular}{|c|c|}
\hline Current concordance & Schritt zurück zu der zuletzt abgerufenen Konkordanzliste \\
\hline Sorting... & sortiert Konkordanzen nach mehreren Parametern: \\
\hline \multicolumn{2}{|c|}{$\begin{array}{l}\text { Simple Sort: } \\
\text { sortiert Konkordanzen nach dem KWIC oder dessen Kontext rechts oder } \\
\text { links (Sort Key). Die Sortierung erfolgt alphabetisch (automatisch) oder } \\
\text { retrograd (Backward). Sortierungskriterien sind „Attribute“. } \\
\text { Als „Attribute“ gelten: word, lemma, tag (falls getagget) (siehe Query } \\
\text { Type), weiterhin Name des Dokuments, Geschlecht des Autors etc. } \\
\text { Multilevel sort: } \\
\text { ermöglicht die Sortierung nach Kombinationen von mehreren Kriterien. }\end{array}$} \\
\hline Shuffle & mischt die Konkordanzzeilen erneut. \\
\hline Sample & $\begin{array}{l}\text { Nach dem Anklicken kann die Anzahl der Belege reduziert werden } \\
\text { (Zufallsauswahl). }\end{array}$ \\
\hline Query overview... & $\begin{array}{l}\text { Übersicht über die einzelnen Schritte bei der letzten Abfrage. So } \\
\text { gelangt man schnell zu den Ergebnissen, z.B. vor einer } \\
\text { durchgeführten Sortierung (durch Klicken auf View result). }\end{array}$ \\
\hline Undo & \\
\hline
\end{tabular}

\section{Filter}

Positive... | Negative...

Filtern der Konkordanzen: Der Filter ermöglicht, die Belege nach der Umgebung von KWIC zu sortieren. Mit dieser Funktion kann nach sprachlichen Verbindungen gesucht werden. Nach dem Klicken auf Positive... oder Negative... öffnet sich dasselbe Formular:

\begin{tabular}{|ll|}
\hline $\begin{array}{l}\text { Positive... } \\
\text { Concordance Filter } \\
\text { positive: } \\
\text { negative: }\end{array}$ & $\begin{array}{l}\text { zeigt Belege, die den angegebenen Bedingungen entsprechen. } \\
\text { löscht Belege mit der angegebenen Bedingung. }\end{array}$ \\
\hline Selected token: & $\begin{array}{l}\text { Wahlmöglichkeit bei mehrfachem Vorkommen (erstes oder letztes } \\
\text { Vorkommen) desselben Elements im definierten Kontext (Span). } \\
\text { Diese Funktion ist nur für positives Filtern relevant. }\end{array}$ \\
& $\begin{array}{l}\text { Beispiel: } \\
\text { first: } \quad \text { Lbfrage Lemma reden und Suche nach zu in seinem Kontext: } \\
\text { last: } \quad \text { Lukas schien auch keine Lust zu haben , viel zu reden. }\end{array}$ \\
\hline Search Span: & $\begin{array}{l}\text { Einstellung der Spannbreite des Suchkontextes (Abstand links vom } \\
\text { KWIC links: negative Ziffern; rechts vom KWIC: positive Ziffern) }\end{array}$ \\
\hline Query Type: & Basic, Lemma, Phrase etc. (siehe B. Abfragefenster) \\
\hline Query: & Sucheingabe (siehe B. Abfragefenster) \\
\hline
\end{tabular}




\section{Frequency}

Node tags|Node forms|Doc IDs|Text Types|Custom...

Informationen über die Häufigkeiten, Abrufen von Frequenzlisten mit statistischen Angaben über KWICs (Node).

Node tags

Nach dem Anklicken bekommt man die Häufigkeitsliste von Tags der KWICs zur aktuellen Abfrage.

Beispiel: abgefragt wird die Form runde.

Abfrage: Basic: runde $\rightarrow$ Node tags (Abrufen der Frequenzliste - Abb. 71)

\section{Frequenzliste:}

\begin{tabular}{||l|l|l|r||r||}
\hline & & tag & Freq & Freq [\%] \\
\hline \hline 1. & $\underline{\mathrm{p}} / \mathrm{n}$ & $\mathrm{NN}$ & 1,075 & 78.6 \\
\hline \hline 2. & $\underline{\mathrm{p}} / \mathrm{n}$ & ADJA & 280 & 20.5 \\
\hline \hline 3. & $\mathrm{p} / \mathrm{n}$ & VVFIN & 12 & 0.9 \\
\hline
\end{tabular}

Abb. 71: Frequenzliste der Tags zur Abfrage runde (Basic)

Über diesen Weg erfährt man schnell, ob das Substantiv (die Runde) oder das Adjektiv (runde) im Korpus überwiegen.

Aus der Abb. 71 ist ersichtlich, dass etwa 88\% auf das Substantiv Runde und 10\% auf das Adjektiv runde entfallen. Nur $2 \%$ bildet das Verb abrunden mit abgetrenntem $a b-$.

\section{Node forms}

Bei einer Abfrage, die mehr als ein Ergebnis liefert: Lemma, Word Form etc. bekommt man nach dem Anklicken die statistische Übersicht über die einzelnen Formen der Ergebnisse.

Beispiel: abgefragt wird die Grundform des Verbs fragen.

Abfrage: Lemma: fragen $\rightarrow$ Node forms (Abrufen der Frequenzliste)

Frequenzliste $^{21}$ :

\begin{tabular}{|l||l||l||r|r||}
\hline & & word & Freq & Freq [\%] \\
\hline \hline 1. & $\mathrm{p} / \mathrm{n}$ & fragte & 13,646 & 57.4 \\
\hline \hline 2. & $\mathrm{p} / \mathrm{n}$ & fragen & 3,881 & 16.3 \\
\hline \hline 3. & $\mathrm{p} / \mathrm{n}$ & gefragt & 1,961 & 8.2 \\
\hline \hline 4. & $\mathrm{p} / \mathrm{n}$ & fragt & 1,859 & 7.8 \\
\hline
\end{tabular}

Abb. 72: Frequenzliste der Formen zur Abfrage fragen (Lemma)

Diese Funktion ist sehr wichtig für die Erstellung von Frequenzlisten einzelner sprachlicher Erscheinungen (mehr dazu in den Fallbeispielen).

21 In publizistischen Texten kommen die Formen in dieser Reihenfolge der Frequenz vor: fragen, frage, gefragt, fragt, fragte, fragten; in belletristischen Texten: fragte, fragen, fragten, fragst, gefragt. 
Doc IDs

Graphik der Aufteilung von Belegen in einzelnen Dokumenten

Text Types

Graphik der Aufteilung von Belegen in einzelnen Texttypen: sortiert nach Korpus,

Dokument, Stil des Textes, Originalsprache etc.

\section{Custom...}

Benutzerdefinierte Einstellung der Frequenzliste

Diese Applikation ermöglicht die Sortierung der Konkordanzen nach mehreren Kriterien und auf mehreren Ebenen (Level). Als Variablen der Sortierungskriterien gelten das Attribut des zu berücksichtigenden Elements und der Abstand zum KWIC.

\begin{tabular}{|lll|}
\hline Level & Sortierungsebene \\
\hline Attribute & Auswahl der & Charakteristik (Attribut) der Elemente in der Umgebung \\
& word & Wortform (Groß-/Kleinschreibung beachten) \\
& lemma & Lemma (Groß-/Kleinschreibung beachten) \\
& tag & Tag \\
& Ic & Wortform (Groß-/Kleinschreibung ignorieren) \\
& lemma_Ic & Lemma (Groß-/Kleinschreibung ignorieren) \\
\hline Ignore case? & 口 & Groß-/Kleinschreibung beachten/ignorieren \\
\hline Position & 6L & Abstand (vom KWIC) derjenigen Elemente, die in die \\
& Node & Frequenzliste einbezogen werden sollen: von (max.) 6 \\
& 6R & Positionen links bis (max.) 6 Positionen rechts vom KWIC \\
\hline (Node) start at & leftmost KWIC word & Berechnung ab dem linken/ rechten Ende eines \\
& rightmost KWIC word mehrgliedrigen KWICs & \\
\hline
\end{tabular}

\section{Collocations}

\section{Custom...}

Diese Funktion (nach dem Anklicken von Custom...) ermöglicht die automatische Auflistung der häufigsten Kollokationspartner/ Kollokatoren zu KWICs (entspricht der Kookkurrenzanalyse im DeReKo).

Die Berechnung der Kollokationen deckt lockere Verbindungen, die häufig vorkommen, aber auch Phraseme (=feste Verbindungen) auf.

Die Berechnung und Aufstellung von Listen der Kollokationspartner erfolgt nach komplizierten statistischen Formeln. Als Kollokationspartner können Wörter, Lemmata oder Tags berechnet werden.

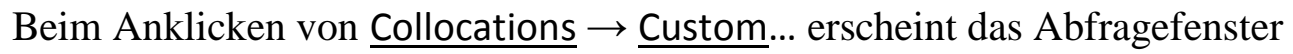
Collocation candidates, in dem folgende Posten einzustellen sind: 


\begin{tabular}{|c|c|}
\hline Attribute: & $\begin{array}{ll}\text { Auswahl der } & \text { Charakteristik (Attribut) des Kollokationspartners: } \\
\text { word } & \text { Wortform (Groß-/Kleinschreibung beachten) } \\
\text { lemma } & \text { Lemma (Groß-/Kleinschreibung beachten) } \\
\text { tag } & \text { Tag } \\
\text { Ic } & \text { Wortform (Groß-/Kleinschreibung ignorieren) } \\
\text { lemma_Ic } & \text { Lemma (Groß-/Kleinschreibung ignorieren) }\end{array}$ \\
\hline In the range from & $\begin{array}{l}-5 \text { to: } 5 \quad \text { Abstand vom KWIC: } \\
\text { links vom KWIC: negative Ziffern (Default }-5 \text { ) } \\
\text { rechts vom KWIC: positive Ziffern (Default 5) }\end{array}$ \\
\hline Minimum frequen & $\begin{array}{l}\text { cy in corpus } 5 \\
\text { In die Liste kommen keine Kollokationspartner, die im Korpus seltener } \\
\text { vorkommen als die angegebene Ziffer (Default 5). }\end{array}$ \\
\hline Minimum frequ & $\begin{array}{l}\text { cy in given range } 3 \\
\text { In die Liste kommen keine Kollokationspartner, die in der Umgebung vom } \\
\text { KWIC seltener vorkommen als die angegebene Ziffer (Default 3). }\end{array}$ \\
\hline Show functions: & 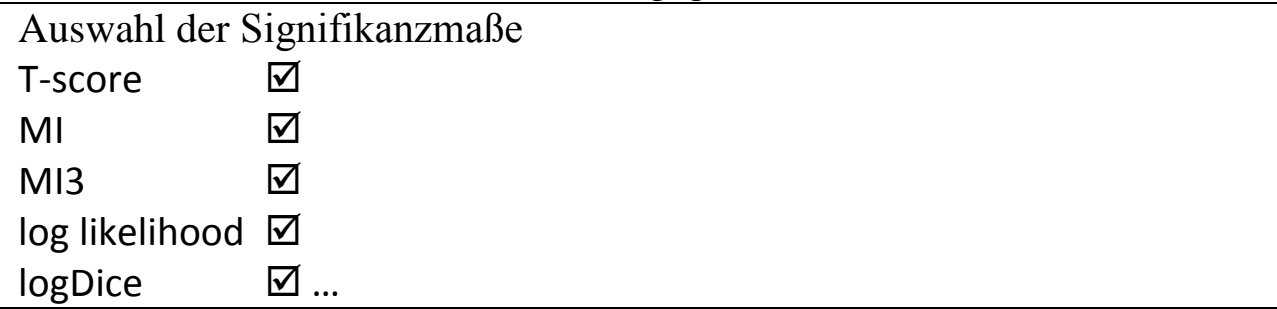 \\
\hline Sort by: & $\begin{array}{ll}\text { ahl der Sortierung } \\
\text { T-score } & 0 \\
\text { MI } & 0 \\
\text { MI3 } & 0 \\
\text { log likelihood } & \odot \\
\text { logDice } & 0\end{array}$ \\
\hline
\end{tabular}

Beispiel: Gesucht wird nach Kollokationen zum Verb fragen.

Korpus: Intercorp de $\rightarrow$ InterCorp en, Version 6 (2013)

\begin{tabular}{|lll|}
\hline $\begin{array}{l}\text { Query Type: } \\
\text { Lemma: }\end{array}$ & $\begin{array}{l}\text { Lemma } \\
\text { fragen }\end{array}$ & $\begin{array}{l}\text { auswählen } \\
\text { eingeben }\end{array}$ \\
& Search anklicken \\
\hline
\end{tabular}

Nachdem die Konkordanzen erscheinen:

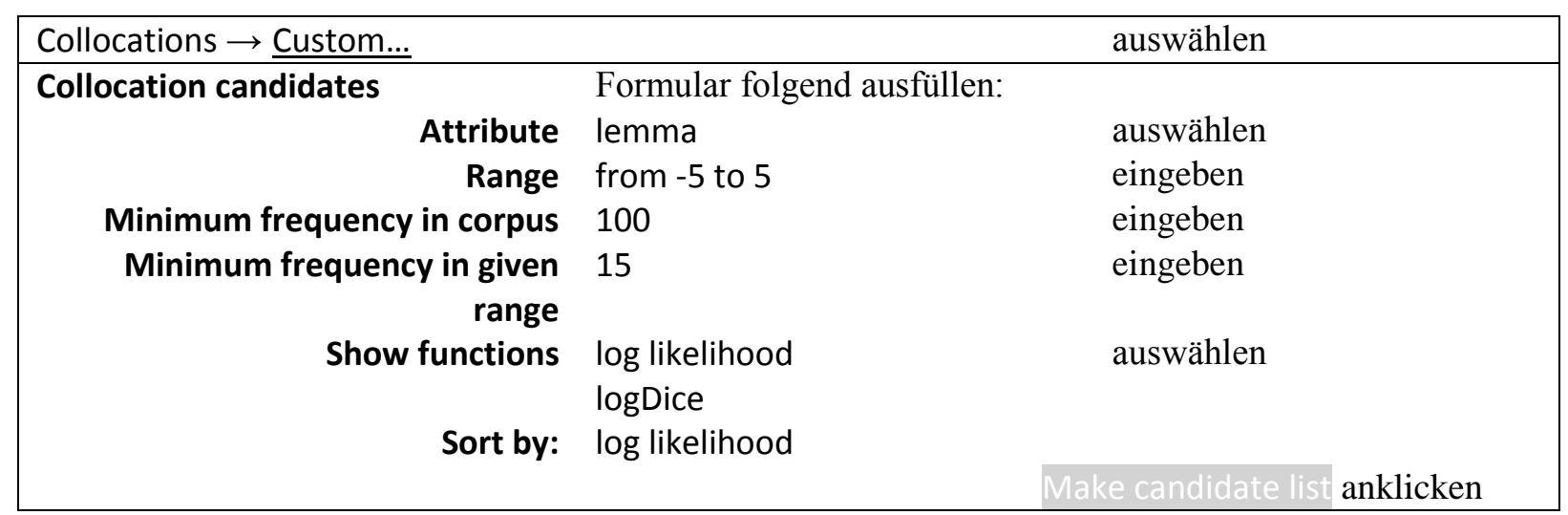


Als Ergebnis erscheint eine Tabelle mit Kollokationskandidaten. (Bemerkenswert ist die Geschwindigkeit, in der die Kandidaten errechnet und die Tabelle erstellt werden.) Die Reihung erfolgt immer nach dem angegebenen Wert absteigend (Abb. 73). Durch das Klicken auf einen anderen Wert (falls seine Berechnung verlangt wurde) reihen sich die Kandidaten nach diesem neu. (Abb. 74).

\begin{tabular}{|c|c|c|c|c|c|}
\hline \multicolumn{3}{|c|}{ Total: 884} & \multirow[b]{2}{*}{ Freq } & \multirow[b]{2}{*}{ log likelihood } & \multirow[b]{2}{*}{ logDice } \\
\hline & Lem & & & & \\
\hline 1. & $\mathrm{p} / \underline{\mathrm{n}}$ & ? & 10714 & 101988.599 & $\mid 11.128$ \\
\hline 2. & $\underline{p} / \underline{n}$ & . & 13560 & 59077.338 & 7.512 \\
\hline 3. & $\underline{p} / \underline{n}$ & , & 14295 & 49574.687 & 6.819 \\
\hline 4. & $\underline{p} / \underline{n}$ & ich & 6868 & 38689.910 & 8.682 \\
\hline 5. & $\underline{p} / \underline{n}$ & « & 5084 & 38402.586 & 9.973 \\
\hline 6. & $\underline{p} / \underline{n}$ & er & 6194 & 35647.718 & 8.783 \\
\hline 7. & $\underline{p} / \underline{n}$ & 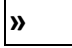 & 3928 & 26931.233 & 9.524 \\
\hline 8. & $\underline{p} / \underline{n}$ & ob & 2879 & 26884.599 & 10.723 \\
\hline 9. & $\underline{p} / \underline{n}$ & $"$ & 3923 & 19828.580 & 8.334 \\
\hline 10. & $\underline{p} / \underline{n}$ & sie & 3462 & 16962.968 & 8.230 \\
\hline 11. & $\mathrm{p} / \underline{\mathrm{n}}$ & was & 2418 & 15984.957 & 9.304 \\
\hline
\end{tabular}

Abb. 73: Kollokatoren zum Lemma fragen: Lemmata, sortiert nach log likelihood (InterCorp_de)

\begin{tabular}{|c|c|c|c|c|c|}
\hline \multicolumn{3}{|c|}{ Total: 884} & \multirow[b]{2}{*}{ Freq } & \multirow[b]{2}{*}{ log likelihood } & \multirow[b]{2}{*}{ logDice } \\
\hline & Wol & & & & \\
\hline 1. & $\mathrm{p} / \underline{\mathrm{n}}$ & $?$ & 10714 & 101988.599 & 11.128 \\
\hline 2. & $\underline{p} / \underline{n}$ & ob & 2879 & 26884.599 & 10.723 \\
\hline 3. & $\underline{p} / \underline{n}$ & $\lll$ & 5084 & 38402.586 & 9.973 \\
\hline 4. & $\underline{p} / \underline{n}$ & warum & 1042 & 9092.661 & 9.783 \\
\hline 5. & $\underline{p} / \underline{n}$ & “ & 2100 & 15011.059 & 9.566 \\
\hline 6. & $\underline{p} / \underline{n}$ & ihn & 3928 & 26931.233 & 9.524 \\
\hline 7. & $\underline{p} / \underline{n}$ & was & 2418 & 15984.957 & 9.304 \\
\hline 8. & $\underline{p} / \underline{n}$ & du & 2185 & 14425.423 & 9.276 \\
\hline 9. & $\underline{p} / \underline{n}$ & mich & 1663 & 11085.375 & 9.232 \\
\hline 10. & $\mathrm{p} / \underline{\mathrm{n}}$ & wer & 625 & 4813.691 & 9.056 \\
\hline 11. & $\underline{p} / \underline{n}$ & danach & 904 & 5792.607 & 8.844 \\
\hline
\end{tabular}

Abb. 74: Kollokatoren zum Lemma fragen: Lemmata, sortiert nach logDice (InterCorp_de)

Diakritische Satzzeichen (Fragezeichen, Anführungszeichen, Punkt, Beistrich etc.) kommen sehr häufig vor, genauso wie Subjekte er, ich und Objekte ihn, mich (Abb. 74), sowie die typischen Rektionsoperatoren (Präposition nach oder einleitende Konjunktion zum Objektsatz $o b)$.

Erklärungen zu den Abb. 73 und 74:

\begin{tabular}{|c|c|}
\hline Total: 884 & Anzahl der errechneten Kollokatoren (hier 884) \\
\hline $\mathrm{p} / \underline{\mathrm{n}}$ & $\begin{array}{l}\text { Positiv/ negativ filtern: } \\
\text { Durch Klicken auf } \mathrm{p} \text { gelangt man zu Konkordanzen mit dem konkreten } \\
\text { Kollokationspartner. Zu ob (8. Kollokationspartner) siehe Abb. } 75 \text {. } \\
\text { Durch Klicken auf } \underline{\mathrm{n}} \text { bekommt man erneut alle Belege der Abfrage, nur } \\
\text { diejenigen mit dem konkreten Kollokationspartner erscheinen nicht. (Ohne } \\
o b \text { siehe Abb. 76). }\end{array}$ \\
\hline $\begin{array}{r}\text { Lemma } \\
\text { Word }\end{array}$ & $\begin{array}{l}\text { Attribut, nach dem die Kollokationspartner gesucht wurden. } \\
\text { Es werden also nur die Grundformen angezeigt, z.B. hier unter ich (4. } \\
\text { Kollokationspartner) wurden auch die Formen meiner }{ }^{22} \text {, mir und mich } \\
\text { gerechnet. Will man konkrete Formen abrufen, muss das Attribut im } \\
\text { Formular Collocation candidates auf word umgestellt werden (Ergebnisse } \\
\text { siehe Abb. 74.) }\end{array}$ \\
\hline Freq & Absolute Frequenz des gemeinsamen Vorkommens. \\
\hline$\frac{\text { log likelihood }}{\underline{\text { logDice }}}$ & Signifikanzmaße (siehe S. 17-18) \\
\hline
\end{tabular}

22 Diese Form allerdings nur theoretisch. Im Korpus gibt es keinen Beleg des Genitivs von ich. 


\section{Beispiele:}

Konkordanzen zum positiven Filter zu $o b$ (8. Kollokationspartner in der Abb. 73); Zufallsauswahl aus 2.879 Belegen (siehe Spalte Freq.):

\begin{tabular}{|rcl|}
\hline $\begin{aligned} \text { Darf ich mir erlauben, Sie zu } \\
\text { Sie }\end{aligned}$ & $\begin{array}{c}\text { fragen } \\
\text { fragte }\end{array}$ & $\begin{array}{l}\text {, ob Sie als Kind allein aufwuchsen } \\
\text { Wolfgang Pie mal mein Tagebuch lesen dürfte . }\end{array}$ \\
Er öffnete die Tür zum Nebenraum und & $\begin{array}{r}\text { gefragt } \\
\text { fragte }\end{array}$ & $\begin{array}{l}\text { ob eine ... Abhandlung ... falsch sei . } \\
\text { Jaynes , ob er eine Minute Zeit hätte }\end{array}$ \\
\hline
\end{tabular}
Abb. 75: Konkordanzen zur Abfrage: Lemma fragen ... ob (InterCorp_de)

Konkordanzen zum negativen Filter zu $o b$ (8. Kollokationspartner in der Abb. 73); Zufallsauswahl aus 6.733 Belegen (Gesamtanzahl der Belege Lemma fragen $=8.271$, ausgeblendet werden alle Belege, in denen $o b$ vorkommt (2.879)):

\begin{tabular}{|rcl|}
\hline und bei der man sich heute & fragt & , wie viele Leute ... der ... Meinung sind . \\
$\ldots$ und ich & $\begin{array}{c}\text { frage } \\
\text { Sie in aller Deutlichkeit ,... }\end{array}$ \\
(...) War mir egal . Ich hab ihn nicht & gefragt & " »Wie lange haben Sie dort gewohnt?» \\
\hline
\end{tabular}

Abb. 76: Konkordanzen zur Abfrage: Lemma fragen ... ohne $o b$ (InterCorp_de)

Konkordanzen zum positiven Filter danach (11. Kollokationspartner in der Abb. 74), Zufallsauswahl.

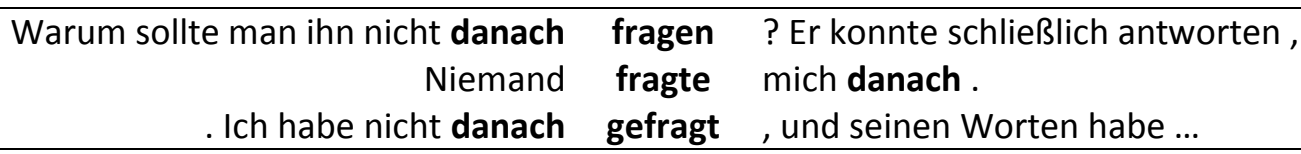

Abb. 77: Konkordanzen zur Abfrage: Lemma fragen ... danach (InterCorp_de)

Über den positiven Filter $\underline{p}$ werden also Konkordanzen aufgerufen, aus denen Chunks mit dem Verb fragen abgeleitet werden können. Die automatische Erstellung von syntagmatischen Mustern, wie es COSMAS II (S. 69) ermöglicht, bietet KonText nicht an.

\section{View options}

KWIC/Sentence|Attributes, structures and references... |General concordance view options...

Hier lässt sich die Ansicht der Konkordanzen nach unterschiedlichen Kriterien einstellen.

\section{KWIC/Sentence:}

Umschalten zwischen der Ansicht der Konkordanzen in Form von KWICs (die KWICs in der Mitte und Konkordanzen zentriert) oder Sätzen (die Konkordanzen sind nach rechts gerückt und umfassen die Länge eines Satzes).

\section{Attributes, structures and references}

\begin{tabular}{ll}
\hline Attributes & Anzeigen von Attributen einzelner Tokens in den Konkordanzzeilen \\
& $\square$ word \\
& $\square$ lemma \\
& $\square$ tag ...
\end{tabular}


und ihre Eigenschaften, welche das Institut vom Tschechischen Nationalkorpus zur Verfügung stellt (Available corpora...)

Das Layout des Helpdesk ist benutzerfreundlich, u.a. auch deswegen, weil es sich an die Form von Wikipedia anlehnt. 


\section{Konkordanzprogramme und korpusähnliche Instrumente}

Konkordanzprogramme (concordancer) sind Softwaretools, die in einem Text oder in einer Textsammlung (Korpus) nach unterschiedlichen sprachlichen Elementen suchen können und diese in Form von Konkordanzzeilen anzeigen. Sie existieren auch unabhängig von Korpora, viele von ihnen werden als Free- oder Shareware ins Internet gestellt.

Korpusähnliche Instrumente sind Software- oder Webtools, die einige Korpuseigenschaften aufweisen, sie können jedoch nicht als Sprachkorpora bezeichnet werden, denn ihnen fehlt eine substanzielle Korpuseigenschaft: entweder basieren sie auf einer $\mathrm{zu}$ kleinen Textsammlung oder sie ermöglichen keine Suche nach sprachlichen Elementen, bzw. können keine statistischen Angaben liefern.

\subsection{TextSTAT}

Aus der Menge der im Internet zugänglichen Konkordanzprogramme, in der sich jede/-r Interessent/-in das Passende für sich aussuchen kann, wird hier ein simples Programm, das keine besonderen Computer-Kenntnisse verlangt, sehr benutzerfreundlich und noch dazu kostenlos ist, vorgestellt: TextSTAT von Mathias Hüning (2014) an der Nederlandistik der FU Berlin. Mit diesem Programm kann man simple Recherchen in beliebigen Texten durchführen. Die Texte werden einfach ohne jegliche vorherige Aufbereitung ins Programm geladen, während für andere Konkordanzprogramme oder Korpora jeder Text von Bildern, Tabellen befreit und konvertiert werden muss. Auch dieser Vorteil macht TextSTAT zum guten Einstiegsinstrument in die Korpuslinguistik.

\section{Arbeit mit TextSTAT ${ }^{24}$}

Es gibt kein ausführliches Manual zu diesem Programm im Internet. Basisinformationen und zwei Quicktours auf Englisch sind von der Seite des TextSTAT aus zugänglich.

\section{TextSTAT-Korpuserstellung:}

1. Internetseite http://neon.niederlandistik.fu-berlin.de/textstat/ besuchen

2. Programm TextSTAT auf die Festplatte herunterladen

3. Programm öffnen - das Arbeitsfeld erscheint (Abb. 78)

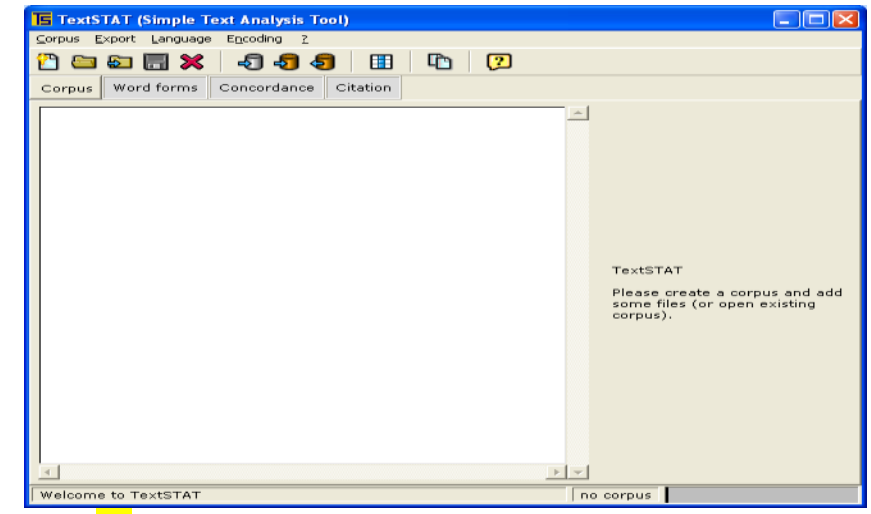

Abb. 78: TextSTAT - Arbeitsfläche

24 Alle TextSTAT-Screenshots mit freundlicher schriftlicher Genehmigung von M. Hüning. 
4. Arbeitssprache einstellen: Language (die letzte Version (2.9) „spricht“ Englisch, Deutsch, Niederländisch, Portugiesisch, Spanisch, Katalanisch, Galizisch, Französisch, Italienisch, Finnisch (Suomi), Polnisch, Tschechisch).

5. Korpus anlegen: Korpus $\rightarrow$ Neues Korpus oder auf die erste Ikone klicken (Abb. 79)

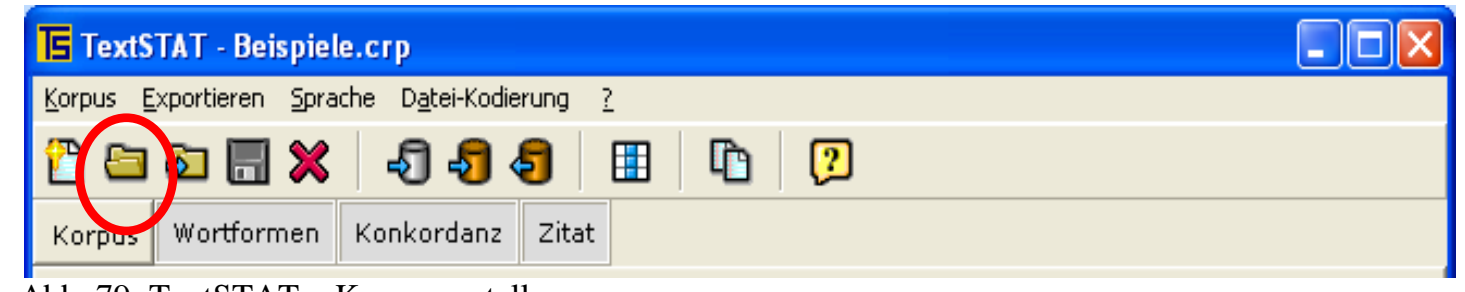

Abb. 79: TextSTAT - Korpus erstellen

6. Datei(en) hinzufügen: Korpus $\rightarrow$ Datei von Festplatte hinzufügen oder Web-Datei hinzufügen oder die „Walzen-Ikonen“ verwenden (Abb. 80)

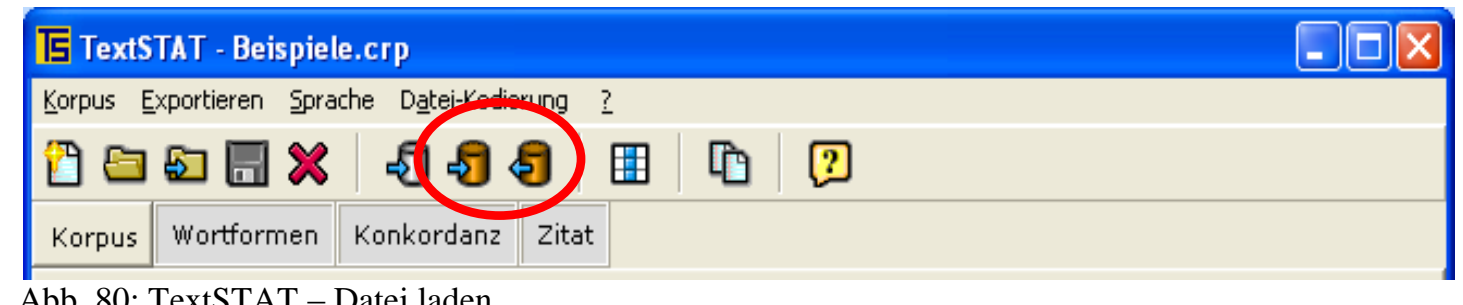

Nachdem das Korpus erstellt ist, beginnt die Arbeit mit den sprachlichen Daten.

\section{Abfragen:}

Zugang über die Taste Konkordanz

\section{Suchmöglichkeiten im TextSTAT:}

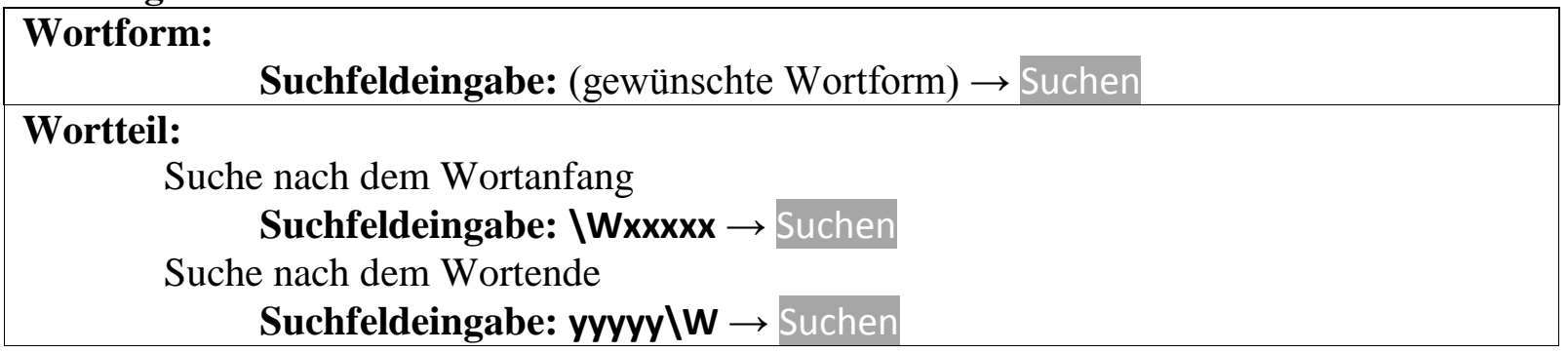

\section{Beispiele:}

\begin{tabular}{|l|l|}
\hline Eingabe & Ergebnis \\
\hline Mannschaft & Mannschaft (nur diese Form) \\
\hline IWhaupt & hauptsächlich, Hauptbahnhof (Wörter mit haupt-) \\
\hline schaft \W & Bekanntschaft, Leidenschaft, Gastwirtschaft (Wörter mit -schaft) \\
\hline
\end{tabular}

\section{Erklärungen und Bemerkungen:}

Das große W steht für ein Leer- oder Satzzeichen, der umgekehrte Schrägstrich hebt die Funktion des Buchstabens $W$ auf.

Lemmasuche (suche nach dem Grundwort) oder nach grammatikalischen Kategorien sind im TextSTAT nicht möglich. 


\section{Funktionen:}

\begin{tabular}{|ll|}
\hline Statistiken: & $\begin{array}{l}\text { Unter der Taste Wortformen werden folgende Angaben abgerufen: } \\
\text { Häufigkeit der Wortformen im Korpus } \\
\text { Liste der Wortformen: } \\
\text { alphabetisch } \\
\text { retrograd }\end{array}$ \\
& $\begin{array}{l}\text { Wörter mit dem String: } \\
\text { Frequenz einer vom User definierten Buchstabenkombination }\end{array}$ \\
& $\begin{array}{l}\text { Frequenzliste anklicken } \\
\text { Speichern: }\end{array}$ \\
& $\begin{array}{l}\text { Zugang über die Taste Exportieren } \\
\text { Frequenzliste: Format xls (csv) wird auf die Festplate gespeichert. } \\
\text { Konkordanzen: Format doc oder txt werden auf die Festplatte gespeichert. }\end{array}$ \\
\hline
\end{tabular}

\section{Einsatz:}

Mit diesem Programm kann man auch schnell (unnötige) Wiederholungen oder begriffliche Inkonsequenzen feststellen. Die Listen können nicht nur nach Frequenz, sondern auch alphabetisch oder retrograd (rückläufig) sortiert werden. Darüber hinaus ist auch die Abfrage nach Wortteilen (,Wörter mit dem String“) möglich.

Das folgende Beispiel der Arbeit mit TextSTAT soll eine Regularität in der Frequenz der Wörter unabhängig von der Länge und vom Stil eines Textes demonstrieren. Die Versuchstexte wurden aus verschiedenen Sprachbereichen und unterschiedlichen stilistischen Niveaus ausgewählt. Beide Romane sind vergleichbar lang, die beiden Sachtexte wesentlich kürzer. Dem statistischen Vergleich wurden folgende Texte unterzogen:

1) Roman 1: Kundera, Milan (1970): Scherz. Ein Roman (113.374 Wörter).

2) Roman 2: Uri, Helene (2008): Nur die Stärksten überleben. Ein Roman (100.069 Wörter).

3) Interview in einer Zeitschrift: profil (2009): Interview: "Hausbesetzer sind konservativ": Blixa Bargeld im Interview mit profil (1.207 Wörter).

4) Fachartikel (Kapitel aus einem Fachbuch): Sorger, Brigitte et al. (2013): Schreiben in mehreren Sprachen. Kapitel Beschreibung einer Handlung (8.249 Wörter).

Jeder Text wurde ins TextSTAT geladen und nach den am häufigsten vorkommenden Wortformen abgefragt. Die Ergebnisse sind in der Tab. 19 zu sehen. Die Ziffern stellen das absolute Vorkommen der Wörter im jeweiligen Text dar.

\begin{tabular}{|c|c|c|c|c|c|c|c|c|}
\hline \multirow[b]{2}{*}{1} & \multicolumn{2}{|c|}{ Roman 1} & \multicolumn{2}{|c|}{ Roman 2} & \multicolumn{2}{|c|}{ Interview } & \multicolumn{2}{|c|}{ Fachartikel } \\
\hline & und & 4033 & und & 3363 & ich & 31 & der & 275 \\
\hline 2 & ich & 3783 & die & 2738 & die & 27 & die & 191 \\
\hline 3 & die & 2736 & er & 2410 & das & 24 & in & 189 \\
\hline 4 & sie & 2518 & sie & 2352 & in & 21 & und & 146 \\
\hline 5 & der & 2488 & der & 1791 & ein & 20 & den & 91 \\
\hline 6 & in & 1603 & in & 1480 & nicht & 20 & auch & 63 \\
\hline 7 & daß & 1503 & das & 1362 & Sie/sie & 20 & sind & 58 \\
\hline 8 & das & 1475 & $\mathrm{zu}$ & 1205 & ist & 18 & im & 57 \\
\hline 9 & $\mathrm{zu}$ & 1411 & sich & 1169 & der & 16 & ist & 57 \\
\hline 10 & nicht & 1344 & ist & 1156 & und & 15 & auf & 56 \\
\hline
\end{tabular}

Tab. 19: Frequenz der Wortformen in vier Texten (TextSTAT) 
Auf den ersten Blick sieht man, dass die Listen der zehn häufigsten Wörter in allen drei Texten fast ident sind. Die Differenzen weisen aber bereits auf typische Merkmale der einzelnen Textsorten hin, die in diese Punkte zusammengefast werden können:

- $\quad i c h$ ist das häufigste Wort in einem Interview sowie in einem in der Ich-Form verfassten Roman (Roman 1);

- Die Wörter er und sie sind stark frequentiert in einem in der Er-Form verfassten Roman (Roman 2).

- $\quad \mathrm{Zu}$ bemerken ist die Absenz des Personalpronomens ich unter den ersten zehn häufigsten Wörtern im Fachartikel. (Der Autor bleibt im „Hintergrund“.)

- Zur Semantik: alle Wörter repräsentieren inhaltlich „leeres“ Wortgut (sich wiederholende Strukturwörter): Deixeis (hier Pronomina), Funktionswörter (Hilfsverb, gram. Partikel), andere Synsemantika (Präpositionen, Konjunktionen), jedoch keine Autosemantika. Dieses Phänomen ist fast in jedem ausformulierten Text zu beobachten. Zum Vergleich: die häufigsten deutschen Wörter in geschriebenen Texten sind: der/die, und, in, von, mit, zu, das/den, im, für, sich (DeReWo 2009).

- Je tiefer man in der Liste gehen würde, desto deutlicher kämen die Konturen der Informationssäulen des Textes (Hauptträger der Informationen auf der Wortebene) zum Vorschein: z.B. im Roman 2 kommen etwa ab der zwanzigsten Stelle (Frequenz um 300 Vorkommen) Namen der Protagonisten vor: Pål, Nanna, Rinkel, Mutter; weiterhin die Schauplätze Institut, Büro, Universität und „Objekte“ des Roman-Plots: Sprache, Projekt, Geschichte. Erst danach kommen die ersten Vollverben: sagen (in der Form sagte), fragen (fragt), stehen (oft in metaphorischer Verwendung).

- In einem Fachtext haben naturgemäß (gleich nach den Funktionswörtern) die Bezeichnungen der „Objekte“, über die im Text geschrieben wird, die höchste Frequenz.

Mit solchen simplen Recherchen können die Lerner/-innen selbst erfahren, wie die Verteilung einzelner sprachlicher Elemente in einem beliebigen Text aussieht. Es empfiehlt sich am Anfang einen kürzeren und vertrauten Text als Versuchskorpus zu wählen. Im Unterricht eignet sich dieses Instrument 1) für die statistische linguistische Arbeit: Häufigkeiten, typisches Umfeld der KWIC (einfache Sortierung der Konkordanzen nach dem linken oder rechten Kontext); 2) für das Erfassen der „Hauptsäulen“ - wichtigsten Träger der Informationen im Text; 3) für das Erschließen von Wortfamilien im gespeicherten Text/ in gespeicherten Texten. Darüber hinaus kann auch gezeigt werden, welche Wörter (Wortarten) in jedem Text gleich sind, welche nicht und warum.

\subsection{Linguee}

Linguee ist eine „Kombination aus einem Wörterbuch und einer Suchmaschine“, wie es die Autoren auf der Homepage (www.lingue.de $\rightarrow$ Über Linguee) nennen. Die Textsammlung besteht aus mehreren Millionen zwei- und mehrsprachigen (fast ausschließlich Internet)Texten. Bisher sind Parallelen Deutsch-Englisch, Deutsch-Französisch, Deutsch-Spanisch und Deutsch-Portugiesisch zugänglich und auch Versionen der Sprachen untereinander (z.B. Spanisch - Englisch, Spanisch - Französisch, Spanisch - Portugiesisch). Die Autoren planen auch Erweiterungen mit anderen Sprachen: Chinesisch, Japanisch und Russisch. 


\section{Zugang und Funktionen:}

Der Zugang ist kostenlos über www.linguee.de.

Einstellung der Sprachen: mit dem Pfeil neben Default Deutsch $\Leftrightarrow$ Englisch

Suchmöglichkeiten im Linguee:

\section{Wortform:}

Wortkombination:

Suchfeldeingabe: "xxxxx" $\rightarrow$ Suchen

Suche nach genauen Wortformen

Suchfeldeingabe: "xxxxx Yyyyy" $\rightarrow$ Suchen

Suche nach allen Formen

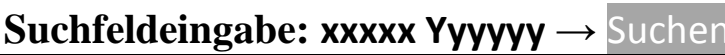

\section{Lemma:}

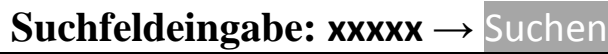

\begin{tabular}{|l|l|}
\hline Eingabe & Ergebnis \\
\hline "blaueres" & blaueres (nur diese Form - siehe Abb. 81) \\
\hline "blauer Himmel" & blauer Himmel (nur diese Formen - siehe Abb. 82) \\
\hline blau & Blau, blaue, blauen (alle Formen - siehe Abb. 83) \\
\hline blau Himmel & $\begin{array}{l}\text { blaue Himmel, blauen Himmel, Blau des Himmels (alle Formen beider } \\
\text { Wörter - siehe Abb. 83) }\end{array}$ \\
\hline
\end{tabular}

Die Suche nach Wortteilen ist in Linguee nicht möglich.

\section{Beispiele:}

Suche nach der genauen Wortform blaueres

- Suchfeldeingabe: "blaueres" $\rightarrow$ Sucher

Die gesuchte genaue Wortform muss (wie bei üblichen Suchmaschinen) in Anführungszeichen gesetzt werden.

Eine "niedrige" Farbtemperatur wie $3200^{\circ} \mathrm{C}$ impliziert zum Beispiel ein wärmeres (gelberes/röteres) Licht, während eine "hohe" Farbtemperatur wie $9300^{\circ} \mathrm{K}$ ein kälteres (blaueres) Licht impliziert.
For example, "low" color temperature, like $3200^{\circ} \mathrm{K}$, implies warmer (more yellow/red) light, while "high" color temperature, like $9300^{\circ} \mathrm{K}$, impliesa cooler (more blue) light.

Abb. 81: Konkordanz zur Abfrage: Wortform blaueres (Linguee 2014)

Suche nach der genauen Wortkombination blauer Himmel

- Suchfeldeingabe: "blauer Himmel" $\rightarrow$ Suchen

Die Maschine zeigt nur die genaue Kombination blauer Himmel an, wie aus der Abb. 82 auf der folgenden Seite ersichtlich ist. 
Im Hintergrund erscheint ein Streifen blauer Himmel mit oben links der Sonne, die auf die Feigen scheint.
A strip of blue sky appears in the background with the sun and its rays on the left at the top, pointing to the figs.

Blue skies, white sandy beaches and tourists in deck chairs with a small magical gadget in their laps.

Blauer Himmel, weißer Sandstrand, Touristen im Liegestuhl mit einem kleinen technischen

Wunderkasten vor sich.

Abb. 82: Konkordanzen zur Abfrage: genaue Phrase blauer Himmel (Linguee 2014)

\section{Suche nach einer Wortkombination mit verschiedenen Formen}

- Suchfeldeingabe: blau Himmel $\rightarrow$ Suchen

Die Maschine gibt die Kombination blau und Himmel in unterschiedlichen Flexions- und sogar Ableitungsformen wieder. Die Texte sind also teilweise und sehr einfach lemmatisiert. Ins Suchfeld muss die Grundform eingegeben werden.

\begin{tabular}{|l|l|}
\hline $\begin{array}{l}\text { Das mittlere Gelbfilter } 022 \text { und das dunkle } 023 \\
\text { schwächen Blau jeweils noch etwas mehr; der blaue } \\
\text { Himmel wird entsprechend stärker abgedunkelt. }\end{array}$ & $\begin{array}{l}\text { The medium yellow filter } 022 \text { and the dark } 023 \\
\text { attenuate blue a little more in each case; the } \\
\text { bluesky is made correspondingly darker. }\end{array}$ \\
\hline $\begin{array}{l}\text { [...] Großstädte wie Salzburg, Wien und Linz und in der } \\
\text { Schweiz das steil in den klaren, blauen } \\
\text { Himmel ragende Matterhorn sowie malerische Dörfer } \\
\text { und Seestädte der Weltklasse besuchen. }\end{array}$ & $\begin{array}{l}\text { [...] great cities: Salzburg, Vienna and Linz, } \\
\text { jutting into the crisp blue sky, its quaint villages } \\
\text { and world-class lakeside cities. }\end{array}$ \\
\hline $\begin{array}{l}\text {...] Weizenfelder sich mit wildem Mohn und Lilien } \\
\text { schmückten und der See das unendliche Blau des } \\
\text { Himmels reflektierte; im Herbst, als der erste Rauche } \\
\text { aus den Kaminen stieg, die [...] }\end{array}$ & $\begin{array}{l}\text { [...] were interspersed with wild poppies and } \\
\text { lilies and the lake reflected the blue of a } \\
\text { cloudless sky;in the fall, when the first smoke } \\
\text { curled out of the chimneys, the [...] }\end{array}$ \\
\hline
\end{tabular}

Abb. 83: Konkordanzer zur Abfrage: Lemmata blau und Himmel (Linguee 2014)

Dieses Instrument eignet sich tatsächlich sehr gut als eine Wörterbuchhilfe und ist zuverlässiger als übliche Übersetzungsprogramme im Internet (etwa google translator). Es ist schnell und - glaubt man der Köllner Mannschaft von Linguee - auch im Angebot der lexikalischen Äquivalente (von Linguee selbst „Redaktionelles Wörterbuch“ genannt) relativ zuverlässig.

Es handelt sich jedoch um kein Korpus (es wird auch nicht als solches deklariert), denn die Texte lassen sich nach keinen Kriterien abgrenzen oder auswählen. Es lässt sich auch nicht feststellen, was die Originalsprache des Textes war, wer die Autoren/-innen waren, aus welchem Bereich der Text stammt etc. Bei jeder Konkordanz ist jedoch ein Link zur Homepage der Texte. Andere Annotationen (Metadaten, Tagging) sind nicht vorhanden.

\section{3 Österreichisches Aussprachewörterbuch, Österreichische Aussprachedatenbank (ADABA)}

Das Österreichische Aussprachewörterbuch und die Österreichische Aussprachedatenbank (ADABA) sind an der Universität Graz unter der Leitung von Rudolf Muhr entstanden und stellen (wie aus dem vollen Namen ersichtlich ist) eine Kombination von einem Wörterbuch und einer Datenbank das. Die ADABA hat viele Eigenschaften üblicher Korpora, es ist aber kein Korpus: Es beinhaltet keine natürlichen Texte, weiterhin 
werden die Ergebnisse nicht in Form von Konkordanzen präsentiert und es sind auch keine statistischen Funktionen vorhanden. Das Ziel dieses Instruments wird deutlich auf der Homepage bestimmt:

„Mit dem Österreichischen Aussprachewörterbuch (ÖAWB) und der damit verbundenen Österreichischen Aussprachedatenbank (ADABA) steht erstmals eine umfassende Dokumentation der Aussprache des Österreichischen Deutsch zur Verfügung. ADABA und ÖAWB dokumentieren die verschiedenen Ausspracheformen und Standardvarianten in Österreich und bieten umfassende Hilfen für die Ausspracheschulung an. Die hier dargestellte Aussprache beschreibt die derzeit in Österreich übliche «Medienpräsentationsnorm», die jener Deutschlands und der Schweiz gegenübergestellt wird. Wörter und Texte liegen transkribiert vor und können beliebig oft nach vielen verschiedenen Kriterien abgehört werden. Umfassende Analysen der Unterschiede sowie eine genaue Beschreibung der Funktionalität der ADABA ergänzen das ÖAWB.“”

(adaba.at: Aussprachewörterbuch $\rightarrow$ 1. Daten im Überblick $\rightarrow$ Ziele. 1.2.2014)

\section{Zugang und Funktionen}

Der Zugang ist kostenlos über http://adaba.at/.

$\underline{\text { ADABA-WEB Auswahl }} \rightarrow$

1. Wörterbuch

2. Texte (Textdatenbank)

3. Hilfe (übersichtliches kurzes Manual

\section{Wörterbuch}

Suchmöglichkeiten im Aussprachewörterbuch:

Orthographische Suche Suche nach der üblichen Orthographie

Phonetische Suche Suche über phonetische Transkriptionscodes. Bei dieser Auswahl öffnet sich das Fenster mit der SAMPA - IPA-Tabelle.

ganzes Wort: $\quad$ Suchfeldeingabe: (gewünschtes Wort) $\rightarrow \rho$

Wortteil:

Suche nach dem Wortanfang: $\quad \square$ anlautend auswählen

Suche nach dem Wortende: $\square$ inlautend auswählen

Suche nach dem Wortende $\square$ auslautend auswählen

Suchfeldeingabe: (Anfang, Mitte oder Ende eines Wortes) $\rightarrow 0$

\section{Beispiele:}

Suche nach der Aussprache von Mathematik

Orthographische Suche

Suchoptionen: Wörterbuch-Auswahl $\rightarrow$ Audiokorpus

Suchfeldeingabe: Mathematik

Das gesuchte Wort (Mathematik) im Kästchen neben den Flaggen

Blauen/ Roten Pfeil neben der gewünschten Varietät zum Abhören

auswählen

auswählen

o anklicken

anklicken

anklicken 
Suche nach der Aussprache von $\mathrm{Ch}$ - am Anfang der entlehnten Substantive

Orthographische Suche

Suchoptionen: Wörterbuch-Auswahl $\rightarrow$ Audiokorpus

auswählen

auswählen

Wortkategorie:

auswählen

Grammatisch $\rightarrow \quad$ Substantiv

Etymologisch $\rightarrow \quad$ Lehnwort

auswählen

Lautumgebung $\rightarrow \quad$ anlautend

auswählen

Suchfeldeingabe: ch

anklicken

Die Ergebnisse der Suche (Cha-Cha-Cha, Chaiselongue bis Chronik) erscheinen im Kästchen neben den Flaggen. Nach dem Anklicken des Wortes kann man die Aussprache anhören (über den blauen/ roten Pfeil neben der jeweiligen Varietät).

\section{Texte (Textdatenbank)}

ADABA beinhaltet auch kohärente (gesprochene) Texte mit Transkripten. In den Texten lässt sich nach Texttyp (biographischer Text, Nachrichtentext etc.) und Sprecher/in (Deutschland männlich/ weiblich, Österreich männlich/ weiblich; Schweiz männlich/ weiblich) recherchieren. Zum gesuchten Text erscheint die genormte und die phonetische (IPA) Transkription. Eine Erstellung von Konkordanzzeilen ist nicht möglich.

Dieses Instrument ist hilfreich für die ,plurizentrische“ Phonetik bzw. auch als Trainingsportal für die Aussprache in einzelnen deutschsprachigen Ländern.

Da die Suche entweder orthographisch oder phonetisch erfolgen kann (phonetisch sind die Texte in IPA $^{25}$-Zeichen transkribiert), eignet sich ADABA auch zu Diskussionen und Übungen in Phonologie.

Es empfiehlt sich ADABA in unterschiedlichen Web-Browsern auszuprobieren (empfohlen: Mozilla Firefox), da einige Browser (Google Chrome) die Transkripte schlecht oder gar nicht wiedergeben können.

\subsection{ParZu}

Ebenfalls kein Korpus, sondern eine Software und ein hilfreiches Instrument zum Demonstrieren, wie die Analyse der Sprache automatisch funktioniert, ist der Parser ParZu, der am Institut für Computerlinguistik der Universität Zürich entwickelt wurde (Sennrich 2009).

\section{Zugang:}

Auf der Adresse http://kitt.cl.uzh.ch/kitt/parzu/ erscheint das ParZu - The Zurich Dependency Parser for German.

Ins Abfragefenster Input text to parse: kann man einen beliebigen Text schreiben oder einfügen. Empfehlenswert (wegen der Übersichtlichkeit) sind maximal zwei einfache Sätze oder ein komplexer Satz.

25 Internationales phonetisches Alphabet 


\section{Beispiel:}

Mit dem Parser wird ein Satz aus der österreichischen Tageszeitung Kurier analysiert:

Stadt Wien weist den Vorwurf der illegalen Parteienfinanzierung zurück (Kurier 2014).

Den Satz: Stadt Wien weist den Vorwurf der illegalen

Parteienfinanzierung zurück.

Output format: Graphical (first sentence only

ins Abfragefenster kopieren

auswählen

anklicken

Das Ergebnis ist eine Graphik (ein Dependenzbaum), aus der die innere Satzstruktur ersichtlich ist - die zugewiesenen Rollen erscheinen als Beschriftungen der Linien. In der Abb. 84 auf der nächsten Seite sind es: subj., obja, avz, app, det, gmod, attr.

Der verbale Teil des Prädikats (weist) ist das Zentrum des Satzes und wird nicht beschriftet.

Die Beschreibung der Abb. 84 von links nach rechts:

subj: Subjekt Stadt mit Apposition (app.) Wien

obja: Akkusativobjekt (den) Vorwurf modifiziert durch eine Genitivergänzung (gmod) (der) illegalen Parteienfinanzierung (genitive modifier (Sennrich et al 2009: 21) mit dem Attribut (attr) illegalen)

avz: abgetrennter Verbzusatz zurück.

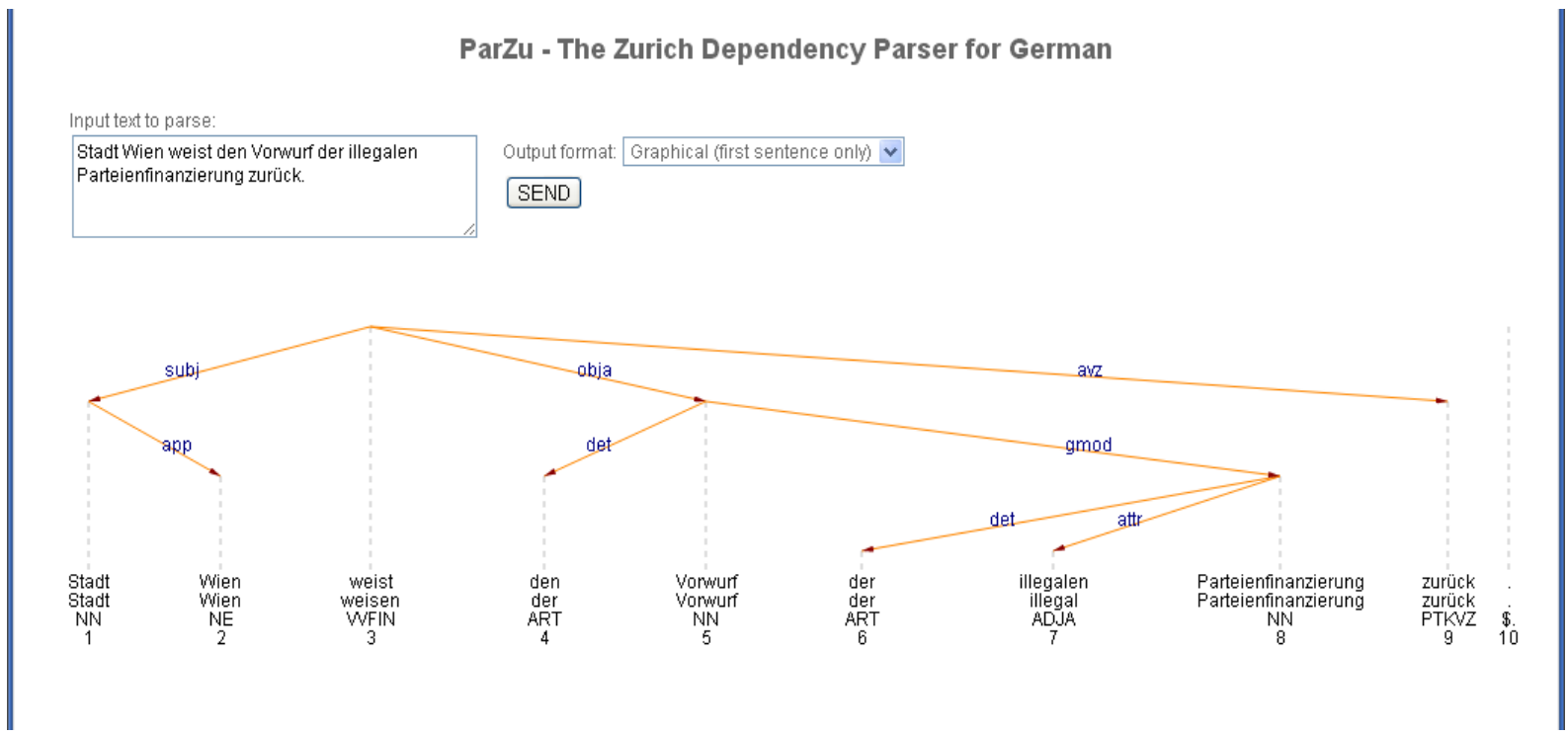

Abb. 84: Geparster Satz: Stadt Wien weist den Vorwurf der illegalen Parteienfinanzierung zurück. (ParZu)

Darüber hinaus sind in der Graphik auch die Lemmatisierung und das Tagging beispielhaft zu sehen:

\begin{tabular}{|l|l|l|l|l|l|l|l|l|l|l|}
\hline & & & & & & & & & \\
\hline Form & Stadt & Wien & weist & den & Vorwurf & der & illegalen & Parteifinanzierung & zurück \\
\hline Lemma & Stadt & Wien & weisen & der & Vorwurf & der & illegal & Parteifinanzierung & zurück \\
\hline Tag & NN & NE & VVFIN & ART & NN & ART & ADJA & NN & PTKVZ \\
\hline
\end{tabular}


Erklärung der Tags:

\begin{tabular}{|l|l|}
\hline NN & (normales) Nomen \\
\hline NE & Nomen - Eigenname \\
\hline VVFIN & Vollverb, finite Form \\
\hline ART & Artikel \\
\hline ADJA & Adjektiv in attributiver Position \\
\hline PTKVZ & abgetrennter Verbzusatz \\
\hline
\end{tabular}

Die Tag-Codes sind ident mit dem Stuttgarter Tagset (siehe Kap. 8.2).

ParZu eignet sich gut als Einstieg zur Erklärung einiger Korpustools und Prozesse: Lemmatisierung, Tagging, Parsing.

Mehr zu geparsten Korpora und zu den zugänglichen Treebanks bringen Jelínek et al. (2012) und Rosen (2012). 


\section{Korpusarbeit - Studien}

Der Einsatz von Korpora und anderen elektronischen Instrumenten im Sprachunterricht, besonders aber in $\mathrm{DaF} / \mathrm{DaZ}$, hängt mit mehreren Überlegungen zusammen (dazu auch Heine 2008: 4), die in folgenden Fragen zusammengefasst werden können:

1) Ist es überhaupt sinnvoll, Korpora und ähnliche Instrumente im (FremdSprachenunterricht einzusetzen?

2) Wenn es sinnvoll ist, wem soll das Korpus/ Instrument dienen - den Lernenden oder den Unterrichtenden?

3) Hat man dafür Zeit?

4) Wenn ja, wie viel Zeit ist man bereit der Korpusarbeit zu widmen?

5) Gibt es dazu auch die entsprechende technische Ausrüstung (Computerraum, Internetzugang)?

6) Wie gewandt sind alle Betroffenen (die Lernenden, aber auch die Lehrenden) im Umgang mit der Technik?

Zum ersten Punkt sind schon einige Publikationen und Artikel erschienen. Bereits die ersten davon, u.a. Leech (1997: 22-23), betonten, dass der Korpuseinsatz den kommunikativen Sprachunterricht positiv beeinflussen kann. Er kann ihn durch gut überlegte Aufgaben unterstützen und um einige Dimensionen erweitern. Im Falle des Fremd- und Zweitsprachenunterrichts ist dies beispielsweise die Interkulturalität, die durch parallele Texte automatisch mittransportiert wird. Die Korpusdaten können nämlich Gemeinsamkeiten und Unterschiede im kulturbedingten Sprachgebrauch aufdecken, neue Informationen über die andere Sprache und über das Zielland bringen. Es muss noch einmal betont werden, dass Texte in Korpora, die hier angesprochen werden, ausschließlich aus der realen Welt kommen. Diese Tatsache mache wiederum die Arbeit mit Korpustexten in den Anfangsphasen des Deutschlernens unmöglich, beklagen viele Elementaristen/-innen, da die authentische Sprache oft zu kompliziert sei. Dies mag immer wieder zutreffen, aber wenn man den Lernenden am Anfang die Rolle eines Beobachters/ einer Beobachterin der Sprache zuweist und zu einfachen Aufgaben motiviert (etwa wie wird (wo) etwas ausgesprochen, geschrieben; welche Formen gibt es von einem Grundwort etc.), könnte es ein guter Einstieg in die spätere selbstständige Arbeit mit einem Korpus bedeuten. Darüber hinaus ist bekannt, dass man authentischen Texten weitere, unerwartete und interessante Informationen entnehmen kann.

Aus organisatorischen, zeitlichen aber auch pragmatischen Gründen ist der Korpuseinsatz für den Unterricht sichtlich bedeutender als im Unterricht selbst, zumindest in den Anfangsphasen. Reflektierte Lehrkräfte sollten zu Korpora greifen. Wie sonst könnten sie einzelne sprachliche Phänomene, Regularitäten oder Ausnahmen den Lernenden erklären und belegen? Das „Bauchgefühl“ funktioniert bei muttersprachlichen und/ oder erfahrenen Lehrern/-innen zwar einwandfrei. Besser ist es jedoch, wenn dieses „Gefühl“, bzw. längst in Vergessenheit geratene Schulformeln, gleich auch durch mehrere Belege objektiviert werden. Das Sprachgefühl kann eigentlich jede/-r mit ausreichend großen Korpora verifizieren (vgl. Studie 9). So gesehen bedeuten plurizentrisch ausgewogene Korpora auch eine große Chance für den (endlich konsequent umgesetzten) plurizentrischen Ansatz in der Deutschvermittlung.

Der Zeitaufwand muss dabei nicht so groß sein, wenn man sich ein Bild über die Korpuslandschaft und Möglichkeiten der Korpusmanager verschafft hat. Zuerst muss man natürlich mit einem (wenn auch minimalen) Zeitaufwand rechnen. Je besser jemand ein Korpus nutzen will, desto mehr Zeit muss er/sie sich für die Recherchen nehmen. 
An Murphys Gesetze darf dabei gar nicht gedacht werden! Dass Korpusmanager auf einmal streiken, Korpus-Server, PC oder Internet abstürzen - immer wieder Pannen auftreten, kann niemand vermeiden. (Nur gegen Stromausfall kann man sich durch Verwendung eines Notebooks rüsten.) Nichtsdestotrotz ist eine Recherche im Korpus des Aufwands wert, wenn man bedenkt, wie lange eine Suche nach einem bestimmten Wort in mehreren Büchern dauern würde.

Die subjektiv-technischen Punkte müssen ad hoc entschieden werden. $\mathrm{Zu}$ bemerken ist, dass es (besonders bei jüngeren Schülerinnen und Schülern) Fälle gibt, in denen ein in den Sprachen eher schlechter Lernende das Interesse an einer Sprache über EDV-Instrumente gewonnen hat. Anfängern wird jedoch empfohlen, sich zuerst ein einfacheres Instrument auszusuchen (Wortschatz, ADABA oder DWDS), einige Abfragen auszuprobieren und so in den Möglichkeiten der Korpusrecherche zu schnuppern.

In den folgenden Studien werden einige Ideen präsentiert, wie man Korpora zur Lösung ausgewählter sprachlicher Fragestellungen nutzen kann. Sie gehen von den erfahrungsgemäß häufigsten Problemen aus, die Lerner/-innen mit unterschiedlichen (europäischen) Erstsprachen beim Erlernen der deutschen Sprache haben. Die Problempunkte verfolgen ungefähr die „klassische“ strukturalistische Linie (in der ich eine gewisse Logik beim ganzheitlichen Er-/ und Begreifen einer Sprache sehe), ausgehend von der kleinsten Einheit. Dies ist übrigens keine originelle Idee. Man findet sie bei Lemnitzer/ Zinsmeister (2010: 124167) für Deutsch, genauso aber auch bei Čermák et al. (2005) für Tschechisch. Während Lemnitzer/ Zinsmeister die theoretische Grundlage für die linguistischen Recherchen präsentieren, in denen dem Leser freie Hand überlassen wird, in welchem Korpus recherchiert werden kann, bieten die Autoren des zweiten Buchs konkrete Beispiele an, wie die Syntax der Abfrage aussieht, wie man Konkordanzen sortiert usw. Das Buch ist als ein Übungsbuch (mit Schlüssel) konzipiert und wird ausschließlich anhand von Daten des Tschechischen Nationalkorpus aufgebaut. Beide Bücher richten sich eher an Linguisten/-innen.

Die Fallstudien hier werden dem DaF/DaZ-Bereich angepasst, betrachten die deutsche Sprache in manchen Punkten plurizentrisch und kontrastiv. Der Vorgang bei den Recherchen wird Schritt für Schritt beschrieben. Jede Studie bringt Ergebnisse, die bisher gar nicht oder zumindest statistisch noch nicht erfasst worden sind. Im Anhang werden weitere statistische Angaben über die deutsche Sprache angeführt, die keinen Kommentar benötigen. Jede der folgenden 13 Studien zeigt einen oder mehrere Lösungswege zu Fragestellungen auf mehreren sprachlichen Ebenen auf. Sie gehen dabei nicht ins letzte Detail, weil sie als Beispiele für die Korpusarbeit und als Inspiration für weitere oder ähnliche Untersuchungen dienen sollen, die in der Erstellung von Lehrmaterialien und Impulsen für den Unterricht münden.

Die Aufgaben und Übungen, welche mithilfe von Korpusdaten erstellt werden können, lassen sich grundsätzlich in diese Typen zusammenfassen:

1) induktive Aufgaben (Feststellen von Regeln)

2) deduktive Aufgaben (Suchen nach Belegen für gewisse Regel)

3) Einsetzübungen

4) Zuordnungsübungen (z.B. Zuordnung passender Überschriften zu Textpassagen)

Die inhaltliche Vielfalt der Aufgaben und Übungen ist jedoch so breit, wie die Sprache reich und bunt ist. 


\section{Phonetik und Orthographie}

Das lautliche und das optische Bild der Sprache wird oft mit der Fassade eines Gebäudes oder mit der Bekleidung eines Menschen verglichen. Wie wichtig oder unwichtig diese „Gewänder“ für die Verständigung und demnach auch für den DaF/DaZ-Unterricht sind, wird hier nicht diskutiert. Dass sie im Fremdsprachenerwerb immer eine gewisse Rolle einnehmen werden, bleibt jedoch unumstritten. Einige Korpora und korpusähnliche Instrumente können helfen, diese Ebene zu beobachten, auf Unterschiede hinzuweisen oder Lücken in Lehrbüchern zu schließen.

\section{Studie 1: Suche nach der „richtigen“ Aussprache}

Eine richtige Aussprache gibt es in keiner Sprache. Die des Deutschen hat dazu drei Varianten, alle drei gelten als Norm. Obwohl die Erstellung elektronischer Instrumente für die Aussprache technisch aufwendig ist, gibt es bereits auch auf diesem Gebiet einige Werkzeuge, die ein einigermaßen plastisches, wenn auch immer noch nicht repräsentatives Bild über die Aussprache des Deutschen darstellen können.

Die „geschulte“ Aussprache (einzelner Wörter) des Deutschen ist aus mehreren elektronischen Quellen abrufbar. Eine Tonspur hat auch fast jedes elektronische Wörterbuch. Die Qualität der Wörterbücher (allgemein) lässt oft zu wünschen übrig, dies gilt auch für die Aussprache. Die Objektivität erreicht man durch Vergleich von mehreren Quellen, deswegen ist es ratsam auch für die Phonetik mehrere Instrumente auszuprobieren.

Die (rein bundesdeutsche) Aussprache findet man im DWDS, für den plurizentrischen Vergleich empfiehlt sich ADABA und Beispiele für die nicht-geschulte, spontane Aussprache in ganzen Texten findet man in der DGD.

Fragestellung 1: Wie ist die Betonung im Wort Kaffee, Sakko, Mathematik? Wie wird im Auslaut -ig ausgesprochen? Gibt es regionale Unterschiede in der Aussprache der Fremdwörter, die auf-ie enden?

Recherchiert wird im DWDS, ADABA und in der DGD. Die Ergebnisse werden dann verglichen.

\section{Recherche im DWDS}

Zuerst wird die Betonung der Wörter Kaffee, Sakko und Mathematik untersucht.

- DWDS $\rightarrow$ Abfragefenster

- Suchfeldeingabe:

$$
\begin{aligned}
& \text { Kaffee } \rightarrow \text { Suche im DWDS } \\
& \text { dann: Sakko } \rightarrow \text { a } \\
& \text { dann: Mathematik } \rightarrow \text { a }
\end{aligned}
$$

- DWDS-Wörterbuch:

- Aussprache: $>$ (Pfeil anklicken)

Die Tonspur ist nicht zu allen Lexemen vorhanden. Die Sprecher sind ausschließlich aus dem deutschsprachigen Raum (allesamt ARD-Mitarbeiter/-innen). 
Obwohl bei einigen wenigen Lexemen mehrere Tonspuren abrufbar sind, handelt es sich stets um eine bundesdeutsche Färbung der Aussprache. Dies erkennt man auch beim Anhören der Wörter Kaffee und Sakko, die jeweils zwei Tonspuren haben:

Kaffee: [dee 'kafe:], [dee ka'fe:]

Der Unterschied im Wortakzent (Kaffe - Kaffe) wird nicht erklärt.

Sakko: [dee 'zako:], [das 'zako:]

Der Wortakzent (nur „,bundesdeutsch“ auf der ersten Silbe: Sakko) bleibt gleich, angedeutet wird das schwankende Genus.

Mathematik: [matema'ti:k ${ }^{\mathrm{h}}$ ]

Hier gibt es nur eine Tonspur mit der bundesdeutschen Standardbetonung auf der letzten Silbe (Mathematik).

Das -ig im Auslaut wurde probeweise an drei Wörtern im DWDS überprüft: schwierig, lustig und zwanzig.

- DWDS $\rightarrow$ Abfragefenster

- Suchfeldeingabe: schwierig $\rightarrow$ a

dann: lustig $\rightarrow$ a

dann: zwanzig $\rightarrow$ a

- DWDS-Wörterbuch:

- Aussprache:

(Pfeil anklicken)

Alle -ig im Auslaut werden nur als ,,ich-Laut“، ausgesprochen:

schwierig: ['Jvi:Riç]

lustig: ['lustiç]

zwanzig: ['tsvantsiç]

Von einer plurizentrischen Abdeckung kann im DWDS kaum gesprochen werden. Dies könnte sogar Absicht sein, denn ein Teil des DWDS wird zum deutschen Teil des plurizentrischen Korpus C4 (siehe dazu Kap. 4.2.1.3).

Die Suche nach den Unterschieden in der Aussprache der Fremdwörter, die auf -ie enden, wäre im DWDS nicht sinnvoll. Im Korpus kann man zwar alle auf -ie endenden Wörter abrufen (Suchfeldeingabe: *ie), die Auswahl der Kandidaten für die weitere Recherche wäre jedoch sehr umständlich, denn das Korpus liefert über 2,7 Mio. Belege (u.a. natürlich auch die Wortformen die, sie, wie, die im Deutschen $\mathrm{zu}$ den am meisten verwendeten Wörtern gehören). Für diese Aufgabe ist es deswegen sinnvoll, ein anderes Instrument zu verwenden.

Ein plurizentrisches Instrument für die Phonetik und Phonologie ist die ADABA (Österreichische Aussprache Datenbank/ Österreichisches Aussprachewörterbuch). Alle Sprecher/-innen sind „geschulte“ professionelle Radiomoderatoren/-innen der öffentlichrechtlichen Anstalten aller drei deutschsprachigen Länder (ARD, ORF und SRG) und decken somit alle Varietäten des Deutschen ab. Das abgerufene Wort ist in jeder Varietät auch mit der IPA-Transkription versehen. Den Lernenden kann vor allem das Aussprachewörterbuch behilflich sein. 


\section{Recherche in der ADABA}

Zuerst wird die Betonung der Wörter Kaffee, Sakko und Mathematik nacheinander abgefragt:

- ADABA $\rightarrow$ ADABA-WEB-Auswahl $\rightarrow \underline{\text { Wörterbuch }} \rightarrow$ Orthographische Suche

- Suchfeldeingabe: Kaffee $\rightarrow$ a

dann: Sakko $\rightarrow$ Q

dann: Mathematik $\rightarrow$ a

Das Wort erscheint im Kästchen neben den Landesflaggen und muss angeklickt werden. Erst dadurch wird die Transkription und Tonspur abgerufen.

Die Aussprachedifferenzen sind in die Tab. 20 zusammengefasst:

\begin{tabular}{|llll|}
\multicolumn{1}{ll}{} & Kaffee & Sakko & Mathematik \\
\hline D: & {$[$ 'kafe:] } & ['zako:] & [matema'ti:k $\left.{ }^{\mathrm{h}}\right]$ \\
A: & {$[$ ka'fe:] } & [sa'ko:] & [mate'ma:tik] \\
Ch: & {$[$ ka'fe:] } & ['sako]/['za..] & [matema'ti:k $\left.{ }^{\mathrm{h}}\right]$ \\
\hline
\end{tabular}

Tab. 20: Regionale Unterschiede in der Aussprache Kaffee, Sakko, Mathematik

Das Instrument hat auch einige „Korpuseigenschaften ${ }^{26 ، ~}$ - es kann z.B. nach Wortteilen gesucht werden (siehe Kap. 5.3). Deswegen kann leicht auch die Aussprache von -ig im Auslaut abgefragt werden (unter Such-Optionen auslautend auswählen. Dazu auf der folgenden Seite die Beschreibung von -ie im Auslaut). Hier werden drei volle Wörter verglichen:

- ADABA $\rightarrow \underline{\text { ADABA-WEB-Auswahl }} \rightarrow \underline{\text { Wörterbuch }} \rightarrow \underline{\text { Orthographische Suche }}$

- Wörterbuch-Auswahl: Audiokorpus auswählen

- Suchfeldeingabe: schwierig $\rightarrow$ a

dann: lustig $\rightarrow$ a

dann: zwanzig $\rightarrow$ Q

Die Ergebnisse sind klar zu sehen und (nach anklicken der Audiofiles) auch zu hören. Zusammengefasst sind sie in der Tab. 21:

\begin{tabular}{|llll|}
\multicolumn{1}{ll}{ schwierig } & lustig & zwanzig \\
\hline D: & ['Jvi:Riç] & ['lustiç] & ['tsvantsiç] \\
A: & ['Jvi:Rik]-[..Riç] & ['lustik]-[..iç] & ['tsvantsik]-[..siç] \\
Ch: & ['Jvi:Rik]-[..Riç] & ['lustik]-[..iç] & ['tsvantsik]-[..siç] \\
\hline
\end{tabular}

Tab. 21: Regionale Unterschiede in der Aussprache -ig im Auslaut: schwierig, lustig, zwanzig

Eine interessante Erweiterung dieser Aufgabe (zum Selbststudium) bietet auch die Sortierung der Wörter, die auf -ig enden (unter Such-Optionen auslautend auswählen, ins Suchfeld wird ig eingegeben). Unter ihnen befinden sich eben auch Bahnsteig, Braunschweig, Bürgersteig, Gehsteig, Steig, Teig und Zweig. Die Lernenden können selbst den Unterschied in der Aussprache zwischen dem Monophthong [i] und dem Diphthong [âe] gefolgt von $g$ erkunden.

26 Eigentlich ist ADABA kein Korpus, weil nicht in Texten gesucht wird, es sind keine Konkordanzen abrufbar, es gibt auch keine statistischen Funktionen. 
Auch für die Erkundung der Aussprache von -ie im Auslaut müssen nur die Such-Optionen eingestellt werden (keine Platzhalter sind notwendig):

- ADABA $\rightarrow$ ADABA-WEB-Auswahl $\rightarrow$ Wörterbuch $\rightarrow$ Orthographische Suche

- Such-Optionen:

$\begin{array}{cc}\text { Grammatisch: Substantiv } & \text { auswählen } \\ \text { Lautumgebung: auslautend } & \text { auswählen } \\ \text { Wörterbuch-Auswahl: Audiokorpus } & \text { auswählen } \\ \text { - Suchfeldeingabe: ie } \rightarrow \text { Q } & \end{array}$

Das Ergebnis der Abfrage sind etwa 130 Substantive (von Aborigine bis Xenie), die man jetzt manuell durchgehen muss. Der Unterschied in der Aussprache ist gleich beim ersten Wort deutlich zu sehen:

Aborigine:

D: [Ebo' лi:d $\int \mathrm{i}:$ ni:]/[..ti:ni:]

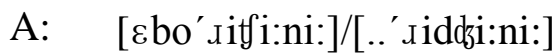

Ch: [abo'ri:fi:ne:]/[...dzi:ne:]

Ein markanter Unterschied ist auch im Wort Serie zu erkennen:
D: ['ze:Ri.e]/[..rji.e]
A: ['se:Rije:]
Ch: [se'ri:]

In der Schweiz wird Serie mit auslautendem [...i:] ausgesprochen, im bundesdeutschen und österreichischen Standard mit [...ije].

Es ist zu bemerken, dass es sich dabei um Standardaussprache handelt. In der schweizerischen Umgangssprache werden z.B. auch das Wort Studie und gelegentlich auch Familie mit [...i:] ausgesprochen.

\section{Recherche in der DGD}

Die Rechercheergebnisse aus Instrumenten, die die Standardaussprache repräsentieren, kann man auch mit Aufnahmen der (z.T. spontan) gesprochenen Sprache in der DGD überprüfen. Zuerst werden wieder die Wörter Kaffee, Sakko und Mathematik abgefragt, die Audiofiles abgehört und die Betonung notiert:

- $\quad$ DGD $\rightarrow \underline{\text { Recherche } \rightarrow \underline{\text { Tokens }}}$

- Korpuswahl: alle einschalten

- Suchfeldeingabe: Lemma: Kaffee $\rightarrow$ Suche starten

dann: Lemma: Sakko $\rightarrow$ Suche starten

dann: Lemma: Mathematik $\rightarrow$ Suche starten

Das Wort Sakko findet man in der DGD (momentan) nicht.

Die meisten Sprechereignisse (gesprochene Texte) bilden Aufnahmen von Sprecher/-innen aus Deutschland. Die Aussprache repräsentiert den bundesdeutschen Standard [matema'ti:k ${ }^{\mathrm{h}}$ ] bis Substandard ['kafe] $]^{27}$.

27 Standardisiert: ['kafe:], [ka' fe:] 
Des Weiteren wurde die Aussprache von -ig im Auslaut abgefragt:

- DGD $\rightarrow \underline{\text { Recherche }} \rightarrow \underline{\text { Tokens }}$

- Korpuswahl: alle einschalten

- Suchfeldeingabe: Normalisiert: \%ig $\rightarrow$ Suche starten

Die Abfrage nach -ig am Ende eines Wortes liefert interessante Ergebnisse: An manchen Stellen ist die Schreibweise nicht „normalisiert“", sondern nur transkribiert (siehe Abb. 85).

\begin{tabular}{|c|c|c|c|}
\hline FOLK_S_00030 & wie & eklich & sagt oma \\
\hline FOLK_S_00026 & skop dran voltmeter dran durschtreten guck isch & fertisch & aus die maus \\
\hline FOLK_S_00026 & net isch isch bin do leischt & linkslastisch & ja \\
\hline FOLK_S_00026 & glaub isch null komma fünf zwo gell & rischtisch & \\
\hline FOLK_S_00026 & wenn s n & richtich & gutes steurgerät wär en grössere teil \\
\hline FOLK_S_00026 & okay awwer & rischtich & was der herr fischer gsaht hot \\
\hline FOLK_S_00026 & mir war & wischtisch & dass mar den hallgeber prüfen \\
\hline
\end{tabular}

Abb. 85: Konkordanzen zur Abfrage \%ig (DGD)

Auch aus diesen Belegen ist ersichtlich, dass die Sprecher/-innen im größten Teil des Korpus eindeutig das bundesdeutsche Idiom sprechen. Auch bei den meisten Belegen, die mit auslautendem -ig transkribiert wurden (richtig, eklig, fertig), ist die Aussprache von -ig eindeutig als ein Ich-Laut zu hören.

Zum Schluss wurde noch die Aussprache von -ie im Auslaut abgefragt:

- DGD $\rightarrow \underline{\text { Recherche }} \rightarrow \underline{\text { Tokens }}$

- Korpuswahl: alle einschalten

- Suchfeldeingabe: Normalisiert: \%ie $\rightarrow$ Suche starten

Eine Abfrage nach allen Wörtern, die auf -ie enden, erwies sich als wenig sinnvoll. Das Korpus liefert nämlich über 38 tausend Belege, darunter auch die, sie, nie und (irgend)wie. Das Filtern ist recht mühsam, daher ist es einfacher, direkt die Wörter, die im ADABA gefunden wurden, zu suchen: Aborigine ist nicht in der DGD vertreten. Das Wort Serie kann man in 25 vertonten Treffern anhören, ausschließlich von Sprechern und Sprecherinnen aus Deutschland, die Aussprache lautet ['ze:Ri.e], bzw. auch ['se:Ri.e].

\section{Fazit:}

Mithilfe elektronischer Instrumente lassen sich Unterschiede in der Aussprache einzelner Wörter auf der segmentalen (hier -ig, -ie) und auch auf suprasegmentalen (hier Wortakzent) Ebene gut demonstrieren. Diese Unterschiede ließen sich auch quantitativ beweisen, wenn es ein regional ausgewogenes Korpus der gesprochenen Sprache mit Transkripten und/oder Tonspuren gäbe.

Fragestellung 2: Kann man mit elektronischen Sprachkorpora auch Suprasegmentalia (z.B. Satzakzent, Satzmelodie) untersuchen?

Vorgang: Wenn Beispiele für die Satzmelodie oder Intonation gesucht werden und die Hörtexte der Lehrwerke nicht ausreichen, bzw. nicht ausreichend ausgewogen sind, kann man sich zweier Instrumente gut bedienen: ganze gesprochene Texte mit abrufbaren Tonspuren sind in der DGD und auch in der ADABA zu finden. 


\section{Recherche in der DGD}

Beispiele der Satzmelodie kann man mit beliebiger Abfrage abrufen und die Tonspur anhören. Als gute Beispiele zeigten sich diese Abfragen: 1) Wort danke; 2) Verbindung Schule und gehen.

Abfrage nach dem Wort danke:

- DGD $\rightarrow \underline{\text { Recherche }} \rightarrow \underline{\text { Tokens }}$

- Korpuswahl: FOLK

- Suchfeldeingabe: Normalisiert: danke $\rightarrow$ Suche starten

Die Ergebnisse erscheinen in Konkordanzzeilen, mit dem KWIC in der Spalte Treffer.

\begin{tabular}{|c|c|c|c|c|c|}
\hline \multirow{2}{*}{\begin{tabular}{|c|} 
Ereignis \\
FOLK_S_00026 \\
\end{tabular}} & \multicolumn{3}{|c|}{ Audio Transkript } & \multirow{2}{*}{\begin{tabular}{|l|} 
Treffer \\
dange \\
\end{tabular}} & \\
\hline & $\nabla$ & & gut & & \\
\hline FOLK_S_00026 & $\nu$ & - & gut & danke & \\
\hline FOLK_S_00027 & $\nabla$ & 亘 & & danke & schön \\
\hline FOLK_S_00027 & $\triangleright$ & 目 & & danke & schön \\
\hline FOLK_S_00027 & $>$ & 国 & & danke & schön sabine \\
\hline FOLK_S_00027 & $>$ & 国 & & danke & schön \\
\hline FOLK_S_00027 & $\nabla$ & 目 & so & danke & schön nina \\
\hline
\end{tabular}

Abb. 86: Konkordanzen zur Abfrage danke (DGD, FOLK)

$\mathrm{Zu}$ jedem Beleg kann man durch Klicken auf den Pfeil $>$ das Audiofile aktivieren und Passagen von je 15 Sekunden anhören. Eigentlich handelt es sich in diesen Beispielen um ganze Sätze.

Abfrage nach der Verbindung Schule und gehen:

Diese Abfrage nach einer Verbindung bzw. nach mehreren Wörtern muss in der DGD in der Volltextsuche getätigt werden:

- $\quad$ DGD $\rightarrow \underline{\text { Recherche }} \rightarrow \underline{\text { Volltext }}$

- Korpuswahl: FOLK

- Suchfeldeingabe: NEAR((\$gehen,Schule),4,false) $\rightarrow$ Suche starten

Die Abfrage bedeutet: Suche nach dem Lemma gehen und der Wortform Schule im Abstand von max. 4 Positionen voneinander, Reihenfolge beliebig.

Auch hier erscheinen die Ergebnisse in Form von Konkordanzzeilen (aussortiert aus 263 Treffern):

\begin{tabular}{|c|c|c|c|}
\hline \# & Transkript-ID & Score & Hörprobe \\
\hline 1 & FOLK_E 0015 f ja sie geht in schule ja ...welche schule geht se & 56 & $>$ \\
\hline 2 & FOLK_E_0018: jetzt hier in die schule gehenden mitschülern zu den & 42 & $>$ \\
\hline 3 & FOLK E 0016: de er damals zur schule gegangen ...zur schule ging also & 29 & $>$ \\
\hline 4 & FOLK_E_0002( genau also es geht um schule und dann sagt er...sie geht in die & 28 & $>$ \\
\hline 5 & FOLK E $0002 \angle$ wie des in der schule ab geht weil sie hat...die schule geht und & 28 & $>$ \\
\hline 6 & FOLK_E $0000<$ zehn jahre in die schule geh oder immer up & 14 & $>$ \\
\hline 7 & FOLK_E_0017 also wenn die schule zeitig anfängt geht dann nach n & 14 & $>$ \\
\hline 8 & FOLK_E_0018: irgendeinem hier in der schule geht & 14 & $>$ \\
\hline 9 & FOLK_E_0002:er s pät v on der schule kommt geht $\mathrm{s}$ halt net & 14 & $>$ \\
\hline 10 & FOLK E 0018! leben di e schule gehen des find ich & 14 & $>$ \\
\hline
\end{tabular}

Abb. 87: Konkordanzen zur Abfrage: Lemma gehen und Schule, Abstand 4 (DGD) 
Die Tonspur wird durch einen Klick auf den Pfeil $>$ in der Spalte Hörprobe abgerufen.

Die Ergebnisse beider Recherchen in der DGD zeigen, dass man sehr schnell zu Ausschnitten vieler gesprochener Texte gelangen kann. Sollten die Konkordanzen mit der Vertonung im Unterricht eingesetzt werden, müssten sie aber sorgfältig aussortiert werden. Viele Tonspuren sind schlecht zu hören, einige falsch mit dem Text aligniert. Und es gibt in der ganzen DGD nur äußerst wenige Texte von Österreichern und Schweizern. Als ein Instrument für die plurizentrische Untersuchung der Satzmelodie ist die DGD nur sehr eingeschränkt geeignet (vgl. Bestand der DGD2 auf ihrer Homepage).

\section{Recherche in der ADABA}

Eine Alternative zur DGD bietet ADABA, in der einige Texte jeweils in drei Formen gespeichert sind: gesprochen (gelesen) auf der Tonspur, „normal“ geschrieben und phonetisch transkribiert. Hier lassen sich die Unterschiede in der Satzmelodie besser demonstrieren.

ADABA:

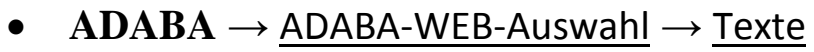

- Optionen: Texttyp (Auswahl: Biographischer Text bis sonstiges)

Sprecher/-in (aus unterschiedlichen deutschsprachigen Regionen)

Durch Klicken auf den Pfeil ( $>$ play) beginnt sich das Audiofile abzuspielen.

Im Feld Ergebnis erscheint der entsprechende Text mit seiner phonetischen Transkription:

Ich bin geboren in Wien, hab meine Kindheit und Jugendzeit zunächst vier Jahre in Vorarlberg verbracht und dann in Maria Enzersdorf - das ist ein kleiner Ort südlich von Wien.

i ç bin gзbợn ì 'vi:n hab maenə' 'ki ndhaet unt 'ju:gønt.tsaet tsunę:çst fì :e ja:Rə in fơarlbeek fə'braxt unt 'dan in maria 'entsesdơef des ìst aen klaene ơet 'sy:dl i ç fơn 'vi:n

Abb. 88: Bibliographischer Text, Sprecher aus Österreich, männlich (ADABA)

Ich bin geboren in Stuttgart. Dort habe ich aber nur sehr kurz gelebt.

iç bin gə'bo:Rən In ' $\int$ tutga:t $\mathrm{t}^{\mathrm{h}}$ dəet ha:b iç ave nu:e se:e kusts gə'le:pt

Abb. 89: Bibliographischer Text, Sprecherin aus Deutschland, weiblich (ADABA)

Die Satzmelodie kann man nach Abrufen unterschiedlicher Sprecher/-innen vergleichen. Sie ist jedoch nicht im Text gekennzeichnet, wie aus den Abb. 88 u. 89 ersichtlich ist.

\section{Fazit:}

Für einen plurizentrisch ausgewogenen DaF-Unterricht eignet sich also momentan nur die ADABA. DaZ in Deutschland kann sehr gut auch mit Tonspuren des DWDS und der DGD ${ }^{28}$ arbeiten. Die DGD ist eine hervorragende Datenbank gesprochener Texte aus vielen Regionen v.a. Deutschlands, ihre Daten sind daher in erster Linie für die Dialektologie interessant.

\section{Schlussbemerkung zur Studie 1:}

Zum Schluss des phonetischen Teils der Korpusarbeit ist festzustellen, dass der Phonetik v.a. an Auslandsgermanistiken, die von der Tradition der russischen Fremdsprachendidaktik beeinflusst worden sind, viel Zeit gewidmet wird (vgl. Sorger 2012: 93). Trotzdem können die Studierenden am Ende ihres Studiums die Unterschiede in der Aussprache der drei Standardvarietäten kaum erkennen: Über Jahre hinweg habe ich Studenten/-innen der Brünner

28 Ein sinnvoller Einsatz der DGD im DaZ muss wegen schlechter Qualität einiger Tonspuren und zum Teil starker Dialektfärbung einzelner Sprecher sehr gut überlegt werden. 
Germanistik (Niveau C1/2) an drei kurzen Ansagen entscheiden lassen, aus welchem Land der Sprecher oder die Sprecherin kommt. Regional einzuordnen waren zwei Programmansagen (Karin Eppler, SAT1 und Chris Lohner, ORF) und ein kurzer Tonausschnitt aus der Themenübersicht der SRG-Nachrichtensendung „10 vor 10“ (Stefan Klapproth). Obwohl sich alle drei Aufnahmen durch „höchstes“ Deutsch, allerdings jeweils mit einem deutlich erkennbaren Regionalanschlag auszeichnen, konnten nur die wenigsten Studierenden (C1-Niveau) die richtige Varietät mit Sicherheit bezeichnen. Diesbezügliche mündliche Befragungen meiner Kolleg/-innen aus anderen tschechischen und slowakischen Universitäten bestätigen die Annahme, dass auf diesem Gebiet noch viel Arbeit (zumindest im DaF-Bereich in Tschechien und in der Slowakei) geleistet werden muss. Die Phonetik ist auf der plurizentrischen Seite offensichtlich noch unzureichend abgedeckt und ein gezieltes Sensibilisieren für diese Problematik wäre notwendig. Einfache Hinweise über das Ursprungland der Hörtexte in plurizentrisch halbwegs gut ausgebauten Lehrwerken reichen dazu offenbar nicht. 


\section{Studie 2: Suche nach dem „richtigen“ Schriftbild}

Die Besonderheiten, Irregularitäten und Zweifelsfälle der deutschen Orthographie oder einfach auch die Tatsache, dass man sich nicht sicher ist, wie etwas üblicherweise geschrieben wird, erkundet man am schnellsten an Daten des größten Korpus für die deutsche geschriebene Sprache - DeReKo.

Fragestellung 1: Welche Schreibweise überwiegt: soft drink, soft Drink, Soft drink oder Softdrink? Gibt es auch regionale Unterschiede?

Da das Archiv der geschriebenen Sprache des DeReKo stilistisch und regional ausgewogen ist, wird jetzt hier recherchiert, um gegebenenfalls auch regionale Unterschiede beobachten zu können. Bei den Abfragen muss besonders die Einstellung von Groß-/Kleinschreibung in den Optionen beachtet werden.

- Cosmas $\mathbf{I I}_{\mathbf{w e b}} \rightarrow \underline{\text { Recherche }}$

- Archiv: W-Archiv der geschriebenen Sprache

- Korpus: W-öffentlich - alle öffentlichen Korpora des Archivs W

- Optionen: Suchmodalitäten:

Groß- / Kleinschreibung beachten für 1 . Zeichen

auswählen

Groß- / Kleinschreibung beachten für andere Zeichen

Expansionslisten:

auswählen

Übernehmen

Ergebnispräsentation: Länderansicht

abwählen

auswählen

- Suchanfrage (Eingabe ins Suchfeld):

dann: soft·Drink $\rightarrow$ Sucher

dann: Soft-drink $\rightarrow$ Sucher

dann: Softdrink $\rightarrow$ Suchen

Die Ergebnisse der einzelnen Abfragen sind in der folgenden Tabelle zusammengefasst:

\begin{tabular}{|l|l|l|}
\hline Form & rel. Häuf. $\mathbf{p M W} \mathbf{2 0}^{\mathbf{2 9}}$ & Land \\
\hline soft drink & & \\
\hline & 0.0029 & $\mathrm{~A}$ \\
\hline & 0.0013 & $\mathrm{D}$ \\
\hline soft Drink & & \\
\hline & 0 & - \\
\hline Soft Drink & & \\
\hline & 0.0132 & $\mathrm{~A}$ \\
\hline & 0.0057 & $\mathrm{D}$ \\
\hline & 0.0043 & $\mathrm{CH}$ \\
\hline Softdrink & & \\
\hline & 0.0651 & $\mathrm{D}$ \\
\hline & 0.0496 & $\mathrm{CH}$ \\
\hline & 0.0396 & $\mathrm{~A}$ \\
\hline
\end{tabular}

Tab. 22: Schreibweise Softdrink/ Soft Drink/ soft Drink/ soft drink

Fazit:

Allgemein gesehen ist das Lexem Softdrink in der geschriebenen deutschen Sprache relativ selten. Dies erkennt man an der relativen Häufigkeit pro Mio. Wörter (mittlere Spalte).

29 Relative Häufigkeit pro eine Million Worte. 
Dennoch lässt sich Folgendes feststellen: diese Bezeichnung kommt in der ursprünglichen Form soft drink nur marginal, als soft Drink gar nicht vor. Alle Belege soft drink sind jeweils in einem englischen Satz eingebaut, z.B. Take a soft drink.

Die üblichste Schreibweise ist Softdrink, die Form Soft Drink kann gelegentlich, am ehesten in Österreich, vorkommen.

Ein ähnliches Problem stellen „Doppelnamen“ (Allonyme) von Eigennamen dar.

Fragestellung 2: Welche Form überwiegt: Peking oder Beijing? Gibt es auch regionale Unterschiede? Seit wann ist die Form Beijing belegt?

Recherchiert wird wieder im DeReKo, angezeigt wird die Jahrzehnt- und Länderansicht.

- Cosmas $\mathbf{I I}_{\text {web }} \rightarrow \underline{\text { Recherche }}$

- Archiv: W-Archiv der geschriebenen Sprache

- Korpus: W-öffentlich - alle öffentlichen Korpora des Archivs W

- Optionen: Suchmodalitäten:

Groß- / Kleinschreibung beachten für 1. Zeichen

Groß- / Kleinschreibung beachten für andere Zeichen

Expansionslisten anzeigen

mit Häufigkeiten

Sortierung: nach Häufig. absteigend

Übernehmen

Ergebnispräsentation: Länderansicht

- Suchanfrage (Eingabe ins Suchfeld): $\quad$ Peking $\rightarrow$ Suchen

auswählen

auswählen

abwählen

auswählen

auswählen

auswählen

dann: Beijing $\rightarrow$ Suchen

Die Ergebnisse der Recherchen sind in der folgenden Tabelle (Tab. 23) zusammengefasst.

\begin{tabular}{|l|l|l|l|l|}
\hline Form & rel. Häufigkeit pMW & von & bis & Land \\
\hline Peking & & & & \\
\hline & 26.38 & 1996 & 2013 & CH \\
\hline & 21.11 & 1991 & 2013 & A \\
\hline & 17.92 & 1949 & 2013 & D \\
\hline & & & & \\
\hline & 1.340 & 1985 & 2013 & D \\
\hline & 0.475 & 1996 & 2013 & CH \\
\hline & 0.370 & 1991 & 2013 & A \\
\hline
\end{tabular}

Tab. 23: Formen Peking und Beijing in einzelnen Ländern und Jahrzehnten

Aus der Ergebnistabelle ist ersichtlich, dass die Form Beijing sich in erster Linie in den Texten aus Deutschland eingenistet hat, und das bereits seit 1985. Die Belege aus dem Jahr 1985 sind aber eine Ausnahme: alle (insgesamt 8) sind in demselben Artikel der Wochenzeitung DIE ZEIT ${ }^{30}$ zu finden. Bis 1991 ist Beijing nicht belegt. Ein signifikantes Vorkommen dieser Form erscheint erst ab 1995 (mit sehr wenigen Belegen auch zw. 1991 und 1994).

Beim Abrufen der Themenansicht bekommt man ein Bild über die Verteilung der Belege in einzelnen Textsorten. Die meisten Belege sind naturgemäß aus Zeitungstexten.

30 Die Zeit, 05.04.1985, S. 09; Das größte Experiment der Geschichte. Quelle: DeReKo. 
Über österreichische Texte vor 1991, bzw. schweizerische vor 1996 lassen sich keine Schlussfolgerungen ziehen, weil die ältesten Texte im Korpus eben aus den Jahren 1991 bzw. 1996 kommen.

\section{Fazit:}

Beijing kommt häufiger in bundesdeutschen Texten vor, heutzutage ist es eine gelegentliche Form auch in der Schweiz und in Österreich. Peking lebt auch heute noch in allen Varietäten.

\section{Schlussbemerkungen zur Studie 2:}

Analog zu den Recherchen können auch andere Lexeme mit schwankender Schreibweise untersucht werden. Es handelt sich um direkte Entlehnungen aus fremden Sprachen, deren Schreibweise zwar reglementiert wird, die tägliche Praxis kann davon jedoch (stark) abweichen. Dies gilt übrigens auch für zwanghafte Umsetzungen von Rechtschreibreformen. Korpora können Rückmeldungen ob der Akzeptanz der Rechtschreibreform liefern. Zum Beispiel die Wörter

Schluss und dass werden häufig auch nach $2005^{31}$ Schlu $\beta$ bzw. daß geschrieben. Interessant wäre die Aufdeckung der Quellen, in denen die ,alte“ Schreibweise beibehalten wird.

Im DeReKo wurden Schriftbilder einiger Lexeme gefunden, wie sie in der Tab. 24 angeführt werden. die im Duden (2006) maximal als Dubletten oder gar (noch) nicht vorkommen:

\begin{tabular}{|l|l|}
\hline DeReKo & Duden (2006) \\
\hline E-Mail, e-mail, E-mail & E-Mail \\
\hline Hot Dog, Hotdog, Hot-Dog, hot dog, Hot-dog, HotDog & Hotdog, Hot Dog \\
\hline $\begin{array}{l}\text { iPhone, IPhone, Iphone, iphone } \\
\text { i-Phone, I-Phone, I-phone, i-phone }\end{array}$ & keine Angabe \\
\hline Lavazza (ohne Kaffee), Lavazza-Kaffee, Lavazza-Caffè & keine Angabe \\
\hline
\end{tabular}

Tab. 24: Schriftbilder der Lexeme E-Mail, Hot Dog, iPhone und Lavazza-Kaffee

Die einzelnen Formen im DeReKo sind nach der Häufigkeit absteigend geordnet. Die Ergebnisse der Abfragen liefern auch andere interessante Informationen über die Lexeme, die hier nicht weiter ausgeführt werden können. Es sei nur bemerkt, dass $e$-mail die üblichere Schreibform bis etwa 2000 war und PPhone $^{32}$ offensichtlich die beliebteste Form für die Bezeichnung der entsprechenden Geräte ist, denn diese Schreibweise übertrifft alle anderen fast hundertfach.

Analog zur Fragestellung 2 ist auch der Wandel des Namens Bombay (seit 1996 offiziell) Mumbai zu beobachten. Im Korpus DeReKo kann man diesen Bruch deutlich beobachten: in deutschen und österreichischen Texten kommt Bombay vor 1996 (und auch danach) vor, Mumbai lediglich nach 1996 mit jährlich zunehmender Häufigkeit. (In schweizerischen Texten kann nur die zunehmende Häufigkeit von Mumbai nach 1996 verfolgt werden, denn die Texte aus der Schweiz sind im DeReKo nicht älter als 1996.)

Eine Studie, die zeigt, wie der inhaltliche Wandel mithilfe von Korpusdaten erkannt werden kann, ist in der Studie 12.

31 Die letzte Rechtschreibreform ist seit dem 1. 8. 2005 verbindlich in Kraft.

32 Nach Angaben einiger US-Amerikaner und Australier wird übrigens iphone langsam appellativiert (zur allgemeinen Bezeichnung wie Tempo für Papiertaschentuch) und zum Synonym von mobile (phone) oder cell phone. 


\section{Grammatik, Morphologie und Syntax}

Der einzige „feste“ Punkt in der Sprache ist die Grammatik. Im modernen Fremdsprachenunterricht wird sie (aus verschiedenen Gründen) aus den Kontaktstunden in die individuelle Phase des Fremdsprachenerwerbs verlagert. Dennoch bleibt sie ein wichtiger Teil der Sprachkompetenz und muss trainiert werden.

Für die Erstellung (auch online) grammatikalischer Übungen gibt es kaum eine bessere Quelle als ein Korpus, weil die Beispiele aus dem sprachlichen „Alltag“ kommen, deswegen wirken (und sind) sie natürlich, man kann aus unzähligen Belegen den passenden wählen und darüber hinaus kann man - wenn mit einem Parallelkorpus gearbeitet wird - die Phänomene mit der Erstsprache der Lernenden vergleichen und ihnen so helfen, die Unterschiede besser zu verstehen.

Da die Rechercheergebnisse aus allen Korpora elektronisch in editierbarer Form dargestellt werden (doc-, rtf-, oder xls-Datei), können sie schnell in E-Learning-Übungen umgewandelt werden. Manchmal muss man natürlich die Belege modifizieren (kürzen) und/ oder solche auswählen, die dem sprachlichen Niveau der Lernenden entsprechen. Die mühevolle Suche nach passenden Beispielen einzelner grammatischer Phänomene in Büchern, Zeitungen, Zeitschriften oder im Internet fällt aber weg.

Im Folgenden werden exemplarisch einige korpusbasierte Übungsbeispiele zur deutschen Grammatik und Lexik vorgestellt, um zu demonstrieren, dass ein sicherer und zielführender Umgang mit Korpora viel Mühe und Zeit sparen kann und dass eine maßgeschneiderte Übung oder Prüfung mit authentischen Texten nicht besonders schwierig zu erstellen ist.

\section{Studie 3: Grammatik auf einen Klick}

Die folgenden Fragestellungen repräsentieren nur einen kleinen Ausschnitt aus dem Grammatikkomplex der deutschen Sprache. Sie beschränken sich auf einige wenige morphosyntaktische Phänomene, die absolut feste Regeln haben, dadurch können sie auch am PC trainiert und geprüft werden ${ }^{33}$.

Fragestellung 1: Wie erstellt man eine Übung zu Richtungsangaben?

Gesucht wird nach dem Prädikatsverb stellen mit der Richtungsangabe auf (weiter auch in, unter, über, hinter): Satzmuster: stellen etw. auf (in/unter) etw.

Recherchiert wird im InterCorp, da dieses auch den Vergleich mit anderen Sprachen ermöglicht. Darüber hinaus ist die Abfrage im InterCorp einfacher als im DeReKo, obwohl es auf den ersten Blick vielleicht nicht so aussieht. Für diejenigen, die sich mit der Syntax der Abfrage vertraut gemacht haben, ist das Generieren der Abfrage simpel. Ihre Bedeutung wird anschließend erklärt.

- $\quad$ korpus.cz $\rightarrow$ Login $\rightarrow \underline{\text { KonText }} \rightarrow$ Parallel corpus InterCorp $\rightarrow$ intercorp de (ggfs. noch (eine) andere Parallelsprache(n) dazu)

- Query Type: CQL auswählen

- CQL (Suchfeldeingabe): [lemma="stellen"] []\{0,2\} [word="auf"] []\{0,2\} [tag="NN"] $\rightarrow$ Search

33 Vgl. Grammatikkurse (B1) am Lehrstuhl für deutsche Sprache, Masaryk-Universität oder Káňa 2012. 
Die Abfrage bedeutet: Suche nach dem Lemma stellen, Wortform auf und allen normalen Substantiven, dazwischen jeweils der Abstand von 2 Positionen.

Die Auswahl der Ergebnisse (in Konkordanzzeilen) präsentiert Abb. 90:

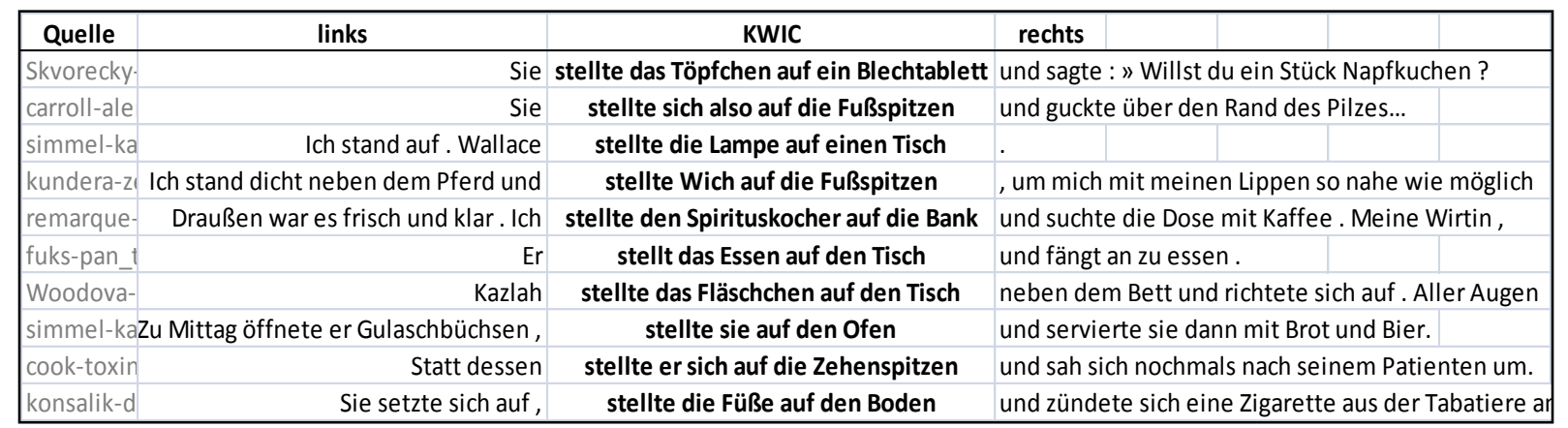

Abb. 90: Konkordanzen zur Abfrage: stellen (...) auf (...) (InterCorp_de)

Abfragen nach anderen Lokalpräpositionen mit dem Verb stellen sehen gleich aus, lediglich die Präposition in der Klammer [word="auf"] wird geändert: [word="in"] bzw. [word="unter"]. Entsprechend kann auch der Abstand geändert werden: []\{0,2\}heißt Abstand von zwei Positionen, []\{0,3\} heißt Abstand von drei Positionen.

- korpus.cz $\rightarrow \underline{\text { Login }} \rightarrow \underline{\text { KonText }} \rightarrow \underline{\text { Parallel corpus InterCorp }} \rightarrow \underline{\text { intercorp de }}$

- Query Type: CQL auswählen

- CQL (Suchfeldeingabe):

$$
\begin{aligned}
& {[\text { lemma="stellen"] []\{0,2\} [word="in"] []\{0,2\} [tag="NN"] } \rightarrow \text { Search }} \\
& \text { dann: }[\text { lemma="stellen"] [] }\{0,2\}[\text { word="unter"] [] }\{0,2\}[\text { tag="NN"] } \rightarrow \text { Search }
\end{aligned}
$$

etc.

$\mathrm{Zu}$ jeder Abfrage bekommt man mehrere tausend Konkordanzzeilen. Es ist empfehlenswert, diese zu mischen (auf dem waagrechten Menübalken: Concordance $\rightarrow$ Shuffle), dadurch bekommt man Belege aus unterschiedlichen Quellen (siehe in der Abb. 90 die Spalte Quelle). Dann müssen nur diejenigen ausgewählt werden, die wirklich eine Lokalpräposition darstellen. Die meisten Belege repräsentieren nämlich Phraseme: unter Verdacht stellen, unter (...) Willen stellen, in Frage stellen, in Rechnung stellen und viele andere mehr.

Gut gewählte Konkordanzen können als Übungen zum Trainieren von gleich mehreren grammatikalischen Aspekten verwendet werden:

Ergänzungen von 1) Artikel; 2) (Personal-)Pronomen; 3) Präpositionen, wie die folgenden Beispiele zeigen:

\section{1) Artikelergänzung:}

Ergänzen Sie den passenden Artikel:

Ich packte die Wiege und stellte sie (...) neben Kopfende meines Bettes. (das)

Die Herren stellen sich in Ecke und politisieren. (eine)

Kalmat stellte sich innen in Weg. (den)

Spann deinen Schirm auf und stell ihn in Ausguß, da kann er trocknen. (den)

\section{2) Ergänzung von Pronomen}

Ergänzen Sie das passende Personal- oder Possessivpronomen:

Eines Abends stellte er sich unter Fenster, als sie gerade mit ein paar Freundinnen Tee trank. (ihr)

Stellen Sie sich hinter Rücken an Rücken! (mich, ihn, sie) 
3) Ergänzung von Präpositionen:

Ergänzen Sie die passende Präposition: Ich packte die Wiege und stellte sie (...) __ das Kopfende meines Bettes. (neben, an,
(auf))

Die Herren stellen sich

Kalmat stellte sich innen eine Ecke und politisieren. (in) den Weg. (in)

Spann deinen Schirm auf und stell ihn den Ausguß, da kann er trocknen. (in)

Eines Abends stellte er sich ihr Fenster, als sie gerade mit ein paar Freundinnen Tee trank. (unter)

Stellen Sie sich mich, Rücken an Rücken! (hinter)

Die Leerstellen können im elektronischen Übungsportal durch ein Eingabefeld oder Auswahlfensterchen ersetzt werden.

Stellen Sie sich mich, Rücken an Rücken!

Stellen Sie sich hinter mich, Rücken an Rücken!

zu

bei

Fragestellung 2: Wie erstellt man eine Übung zum Gebrauch von Dativ und Akkusativ nach einer Präposition?

Die Recherche kann wieder im InterCorp durchgeführt werden, wegen Absenz einiger Sprachen im InterCorp wird auch im OPUS-Corpus recherchiert.

\section{Recherche im InterCorp}

- korpus.cz $\rightarrow$ Login $\rightarrow$ KonText $\rightarrow$ Parallel corpus InterCorp $\rightarrow$ intercorp de (ggfs. noch (eine) andere Parallelsprache(n) dazu)

- Query Type: CQL

- CQL (Suchfeldeingabe): [word="auf"] []\{0,2\} [tag="NN"] $\rightarrow$ Search

Die Abfrage bedeutet: Suche nach der Wortform auf und allen normalen Nomina im Abstand von 2 Positionen.

Nach dem die Konkordanzen erschienen sind:

- Frequency $\rightarrow$ Node forms.

Als Ergebnis bekommt man die Statistik einzelner Kombinationen nach der absoluten Häufigkeit des Vorkommens im Korpus. Die 50 häufigsten Verbindungen im InterCorp sind auf der folgenden Seite in der Abb. 91 aufgelistet. 
Frequenzliste der Formen auf + Substantiv (gekürzt)

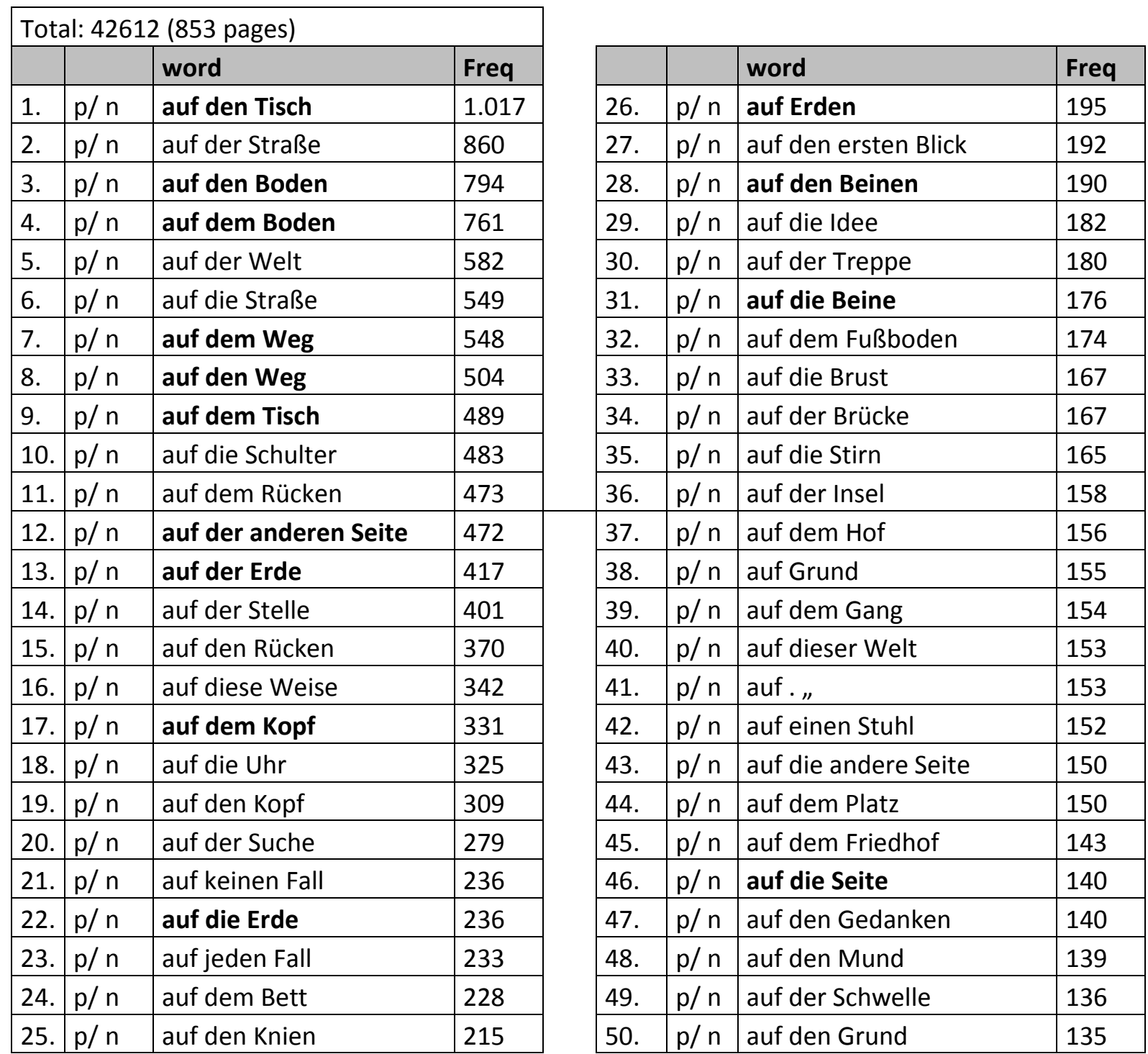

Abb. 91: Die 50 häufigsten Kombinationen von auf + Substantiv im Abstand von 2 Positionen (InterCorp_de)

$\mathrm{Zu}$ beobachten ist ein interessantes Phänomen: einige Verbindungen mit demselben Substantiv (in Fettschrift) weisen (sehr grob gesehen) ähnliche Frequenz auf: auf den/dem Tisch, auf den/dem Boden, auf den/dem Weg. Dieses Phänomen muss noch mit Einbeziehung der dazugehörigen Verben untersucht werden. Dies erfolgt am einfachsten über eine Kollokationsanalyse.

Als Beispiel werden die Verbindungen auf den/dem Tisch gewählt:

Beim Klicken auf $\underline{p}$ (positiver Filter) bei der Akkusativverbindung auf den Tisch öffnen sich die (parallelen) Konkordanzen. Jetzt kann man die Kollokationsanalyse durchführen:

- $\quad \underline{p}$ (positiver Filter) anklicken

Nach dem die Konkordanzen erschienen sind:

- Collocations $\rightarrow$ Custom... (Standardeinstellung) $\rightarrow$ Make Canditate List

Denselben Vorgang unternimmt man mit der Dativverbindung. 
Die Berechnung der Kollokationen zeigt folgende signifikante Kollokationspartner:

Akkusativverbindung: auf den Tisch stellen, bringen, schlagen, hauen ...

Dativverbindung: auf dem Tisch

liegen, stehen, ausbreiten, sitzen, haben ...

Die Analyse zeigt hochfrequentierte Chunks in der geschriebenen Sprache. Sie sind auch in der gesprochenen Sprache üblich - dies belegen auch Beispiele in den Abb. 92 und 93 in der direkten Rede. Aus diesen Gründen sollte solchen Verbindungen im Unterricht Aufmerksamkeit gewidmet werden.

\begin{tabular}{|r|l|l|}
\hline Er stellte sein Glas & auf den Tisch & und sagte: \\
\hline Jonas brachte Kürbisse & auf den Tisch &,$\ldots$ \\
\hline Der Erste Offizier schlägt & auf den Tisch & und erklärt ihm ... \\
\hline Da hab' ich & auf den Tisch & gehauen und staunte zugleich über mich: (...) \\
\hline
\end{tabular}

Abb. 92: Auswahl aus Konkordanzzeilen zur Abfrage auf den Tisch (InterCorp_de)

\begin{tabular}{|r|l|l|}
\hline „Die Blumen für dich liegen im Wohnzimmer & auf dem Tisch & “, sagte der Vater. \\
\hline Wie viele Weingläser stehen & auf dem Tisch & ? \\
\hline ... und nahm eine (...) Karte heraus, die er & auf dem Tisch & ausbreitete. \\
\hline Der Feldkurat saß recht bequem & auf dem Tisch & und drehte sich eine Zigarette. \\
\hline „Wir haben nichts & auf dem Tisch & $($....)“, erklärt der Senatspräsident ... \\
\hline
\end{tabular}

Abb. 93: Auswahl aus Konkordanzzeilen zur Abfrage auf dem Tisch (InterCorp_de)

Ausgewählte Konkordanzzeilen (Sätze oder Textpassagen) bieten wieder mehrere Möglichkeiten an, wie sie in Übungen umgewandelt werden können - Artikelergänzung oder Ergänzung von Verben, um nur zwei zu nennen:

1) Artikelergänzung:

Ergänzen Sie den passenden Artikel:

Er stellte sein Glas auf __ Tisch und sagte: (den)

... zog eine Schublade auf und nahm eine zusammengerollte Karte heraus, die er auf Tisch ausbreitete. (dem)

etc.

\section{2) Ergänzung von Verben}

Ergänzen Sie das passende Verb:

Der Feldkurat recht bequem auf dem Tisch und drehte sich eine Zigarette. (saß)

Da hab' ich auf den Tisch und staunte zugleich über mich: Mein Gott, so energisch bin ich doch sonst nicht. (gehauen)

etc.

Die Leerstellen können in einem Übungsportal durch Einsetzfelder oder Multiple-ChoiceAuswahl ersetzt werden.

Falls am Anfang der Abfrage eine Parallelsprache ausgewählt wurde, werden zu den Konkordanzen die Parallelpassagen immer automatisch abgerufen. 


\section{Recherche im OPUS-Corpus}

Ist die gewünschte Sprache im InterCorp (noch) nicht vertreten, kann man zum OPUS-Corpus ausweichen, wo auch einige außereuropäische Sprachen recherchierbar sind. InterCorp verfügt d.Z. über keine türkische Parallele, dennoch können Lernende mit dieser Erstsprache bzw. deutschsprachige Türkischlernende auch das hier betrachtete Phänomen (auf + Tisch im Dativ oder Akkusativ) mit dem Deutschen vergleichen.

- OPUS-Corpus $\rightarrow$ Search \& Browse $\rightarrow$ OpenSubtitles search interface

- lang = de (ganz rechts)

- CQP Query (Suchfeldeingabe):

im Suchfeld "viol.*" löschen, statt dessen: "auf"•".*"•"Tisch" eingeben.

- Display: context = sentence (zur Auswahl 5 Wörter, 2 Sätze etc.) auswählen

- Alignments: tr (Türkisch)

auswählen $\rightarrow$ Run Query

Beim Eingeben der Suchanfrage Achtung auf den richtigen Abstand und auf die Anführungszeichen!

Die Ergebnisse erscheinen in Form einer Tabelle, wie die Abb. 94 zeigt:

\begin{tabular}{|l|l|}
\hline de & tr \\
\hline $\begin{array}{l}\text { Pack die Scheine wieder auf den Tisch, ich bin } \\
\text { dran mit Geben. }\end{array}$ & $\begin{array}{l}\text { - Senin için sevindim ama burası benim garajım } \\
\text { evlat ! }\end{array}$ \\
\hline Alles auf diesem Tisch wurde hier geerntet. & Masadaki her şey burada yetiştirildi . \\
\hline $\begin{array}{l}\text { Du schüttest ihn runter und haust das leere } \\
\text { Glas auf den Tisch. }\end{array}$ & $\begin{array}{l}\text { Önce fondip yapıp bardağı masaya vuracaksın, } \\
\text { tamam mı ? }\end{array}$ \\
\hline Und die Leute, die auf einen Tisch warten? & - Masa bekleyenleri nereye alacağım ? \\
\hline $\begin{array}{l}\text { Leg die Ellenbogen auf den Tisch, beug dich } \\
\text { über den Brief und lies ihn vor. }\end{array}$ & $\begin{array}{l}\text { Dirseklerini masaya koy ... eğil ... yüzünü } \\
\text { mektuba yaklaştır ve yüksek sesle oku . }\end{array}$ \\
\hline Leg die Hände auf den Tisch. & $\begin{array}{l}\text { Avuçların aşağıda kalacak şekilde , ellerini } \\
\text { masaya koy. }\end{array}$ \\
\hline Ich habe sie Ihnen auf den Tisch gelegt. & $\begin{array}{l}\text { - Istersiniz diye düşündüm , masanızın üzerine } \\
\text { koydum }\end{array}$ \\
\hline
\end{tabular}

Abb. 94: Konkordanzen zur Abfrage auf ... Tisch (OPUS-Corpus, de - tr)

Aufgaben und Übungen können analog zu den oben angeführten erstellt werden, dabei kann

(!) die parallele Sprache die Übung entlasten:

Alles auf diesem Tisch wurde hier geerntet.

Masadaki her şey burada yetiştirildi.

Die Betonung von können ist hier wichtig. Man muss immer davon ausgehen, dass es sich um parallele Texte handelt, die „funktional-äquivalent“ (Knittlová 2009:7), nicht strukturäquivalent sind. Die parallelen Belege sollen entlasten, nicht Fragezeichen über die lexikalische und grammatikalische Äquivalenz aufkommen lassen. Deswegen müssen die Belege zur Übung von grammatikalischen Erscheinungen sorgfältig ausgesucht werden. Darüber hinaus muss man auch bedenken, dass es sich in den Korpustexten oft um keine Übersetzungen zwischen den zwei ausgewählten Sprachen handelt, sondern, dass die Texte vielleicht aus einer dritten Sprache übersetzt wurden, wie es auch hier in der Abb. 94 der Fall ist. Die Texte sind aus dem Englischen ins Deutsche und ins Türkische übersetzt worden, es handelt sich um Untertitel zu amerikanischen Filmen. Dadurch dürfen die Entsprechungen nicht nur formal, sondern auch semantisch etwas weiter voneinander liegen. 
Fragestellung 3: Wie erstellt man eine Übung zur Deklination von Artikel und Adjektiv nach einer Präposition für Lernende mit ukrainischer oder türkischer Erstsprache?

Wenn der Kontrast zu einer anderen Sprache benötigt wird, ist für die Übungserstellung das InterCorp das geeignete Instrument.

- $\quad$ korpus.cz $\rightarrow$ Login $\rightarrow \underline{\text { KonText }} \rightarrow$ Parallel corpus InterCorp $\rightarrow$ intercorp de (ggfs. noch (eine) andere Parallelsprache(n) dazu)

- Query Type: CQL

- CQL (Suchfeldeingabe): [tag="APPR"] [tag="ART"] [tag="ADJA"] [tag="NN"] $\rightarrow$ Search

Diese Suchanfrage ruft aus dem Korpus alle Wortketten ab, in denen Präposition - Artikel Adjektiv - Nomen (ohne Eigennamen) nacheinander vorkommen:

\begin{tabular}{|c|c|c|}
\hline der (...) gekrümmt in der Ecke & unter einer kleinen Palme & stand. \\
\hline \multirow[t]{2}{*}{ Hermine hatte das Bild dann } & mit einem kleinen Zaubertrick & (...) zum Leuchten gebracht. \\
\hline & In der kleinen Stadt & wurde es bald allgemein bekannt ... \\
\hline Dann trocknet er sich die Stirn & \multicolumn{2}{|c|}{ mit einem rotkariertem Taschentuch } \\
\hline Zuerst wollte er & mit dem unteren Teil & seines Körpers aus dem Bett... \\
\hline ... und sie hielten sich & in den tiefsten Löchern & verborgen. \\
\hline .., so daß ich & wegen einem dummen Faß & Benzin nicht auslernen hab können. \\
\hline
\end{tabular}

Abb. 95: Konkordanzen zur Abfrage: Präposition - Artikel - Adjektiv - (normales) Nomen (InterCorp)

Die Konkordanzen können in Ergänzungsübungen umgewandelt werden:

... der linkisch gekrümmt in der Ecke unter ein__ klein__ Palme stand. das Bild mit ein__ klein__ Zaubertrick zum Leuchten bringen In d__ klein__ Stadt wurde es bald allgemein bekannt, dass ...

Und natürlich kann man nach Bedarf entsprechende Parallelen in einer anderen Sprache zur Verfügung stellen (hier Ukrainisch):

... der unter ein__klein__Palme stand

... стояв , втягнувши голову в плечі , під карликовою пальмою .

$-$

Hermine hatte das Bild dann mit ein_ klein__ Zaubertrick in verschiedenen Farben zum Leuchten gebracht.

А Герміона з допомогою одного заклинання зробила так, що фарба на плакаті стала мінитися різними барвами .

$--$

In d__ klein__ Stadt wurde es bald allgemein bekannt, dass ...

У малесенькому містечку швидко поширилася чутка , що ...

\section{Fazit und Schlussbemerkung zur Studie 3:}

Mit einer klug gestellten Abfrage können viele grammatikalische Phänomene abgefragt werden. Einige Beispiele wurden hier ausführlicher angeführt. Weitere Abfragen ergeben sich aus den kommenden Studien, viele bleiben jedoch offen. Es liegt an den Korpusbenutzern und -benutzerinnen selbst, wie kreativ sie mit Korpusmanagern umgehen (können). Aus den Aufgaben ist jedoch ersichtlich, dass es sinnvoll ist, die morphologische Annotation (Tags) zumindest teilweise zu beherrschen.

Abschließend muss noch betont werden, dass die Möglichkeiten, die Korpora für die einfache, aber auch kontrastive Grammatikvermittlung anbieten, fast berauschend ist. Diese Tatsache 
darf aber natürlich nicht zur Wiederkehr der Grammatik-Übersetzungsmethode führen und verführen.

\section{Morphologie und Syntax}

Die Grammatik deckt sich teilweise mit den linguistischen Disziplinen Morphologie und Syntax. Bei der systematischen Analyse der Korpusdaten überschneiden sich auch diese beiden Disziplinen in vielen Punkten. Die Morphologie befasst sich mit den kleinsten sprachlichen Einheiten, die eine „Bedeutung ${ }^{34 ، ~ h a b e n . ~ G r a m m a t i k a l i s c h e ~ M o r p h e m e ~ s i n d ~}$ „Biegungselemente“, die einzelne morphologische Kategorien (grammatikalisches Geschlecht, Zahl, Steigerungsform, Tempusform u.a.) repräsentieren.

Die Syntax befasst sich wiederum damit, wie die einzelnen Elemente der Sprache (morphologisch richtig) in sinnvollen Sätzen gebildet werden. Da aber die morphologischen Kategorien unterschiedlich realisiert werden - blieb (ist eine rein morphologisch gebildete Tempusform) vs. bin geblieben (ist eine morphosyntaktisch gebildete Tempusform) - scheint es sinnvoll, die beiden Ebenen zu verbinden. Dieser Schritt hat mehrere Gründe: 1) Die Grenze zwischen der Morphologie und Syntax ist fließend (vgl. Skalička 1957). Betrachtet man rigide die Morphologie als eine Disziplin, die sich mit sprachlichen Elementen bis zur Wortgrenze befasst, dann ist Deutsch morphologisch ziemlich arm (Englisch und Spanisch hätten demnach fast keine morphologischen Formen); 2) Würde man typologisch unterschiedliche morphologische Systeme von Sprachen strikt vergleichen, dann gäbe es viele Null-Äquivalente (Divergenzen), weil es grammatikalische Kategorien gibt, die sich in einer Sprache morphologisch demonstrieren, während sie in einer anderen Sprache syntaktisch gebildet werden; 3) Im modernen Fremdsprachenunterricht spielt die Aufteilung in Morphologie und Syntax eher eine geringere Rolle. Die ,grammatikalische ${ }^{35}$ Richtigkeit“ tritt zugunsten der kommunikativen Kompetenzen in den Hintergrund. Diese Kompetenzen zeigen sich in erster Linie auf der lexikalisch-semantischen und pragmatischen Ebene. Die Lernenden werden im heutigen Unterricht oft aufgefordert, selbst morphologische und syntaktische Regularitäten aufzudecken und so ihre produktiven Fertigkeiten auf Richtigkeit zu prüfen. Dazu können ihnen auch Korpora behilflich sein.

Im Folgenden werden einige wenige Strukturen anhand von Korpusdaten exemplarisch erforscht. Die Aufgaben zu einzelnen morphosyntaktischen Phänomenen können in Grammatikübungen umgewandelt werden.

\section{Studie 4: Entdeckung der Flexionsformen}

Wie die einzelnen Flexionsformen/ Biegungsformen eines beliebigen deutschen Wortes aussehen, können erfahrene Lehrer/-innen ohne Recherchen aufzählen. Lernende können sie in (guten) Wörterbüchern nachschlagen, aber es gibt auch Dubletten/ Doppelformen, bei denen man sich oft nicht sicher ist, wann welche gebraucht wird. In Wörterbüchern ist wenig Platz für alle plausiblen Beispiele in aufschlussreichem Kontext. Auch Informationen darüber, ob ein Wort, das mehrere syntaktisch-semantische Funktionen hat, mehrheitlich die eine oder

34 Die Bedeutung kann grammatikalischer oder lexikalischer Natur sein. Da die grammatikalische „Bedeutung“ nicht ganz dem üblichen Sinn des Wortes „Bedeutung“ entspricht, ist dieses hier in Anführungszeichen gesetzt.

35 Die Grammatik deckt sich nur teilweise mit den Begriffen Morphologie und Syntax. Dazu auch Fachlexikon (2010: 106-107). 
die andere Funktion vertritt, werden selten angegeben. Eine Korpusstudie zu diesem Thema dauert zwar länger als das Nachschlagen in einem Wörterbuch oder einer Grammatik, liefert aber ziemlich sichere und realitätsnahe Ergebnisse.

Fragestellung 1. Welche Formen hat das Verb werden? Kommt es in der Sprache öfter als Vollverb oder als Hilfsverb/ Auxiliar vor?

Diese Aufgabe lässt sich einfach auch „manuell“ an einem beliebigen Text lösen, denn das Verb werden ist im Deutschen (nach sein) das zweithäufigste Verb überhaupt (vgl. DeReWo 2009). Schneller und effektiver geht die Recherche in einem Korpus. Das Ergebnis ist auch objektiver, wenn mehrere unterschiedliche Texte einbezogen werden.

Für die Recherche eignet sich jedes getaggte Korpus. Das Tagging braucht man zur automatischen Analyse der syntaktisch-semantischen Funktionen des Verbs (werden als Auxiliar oder als Vollverb). Einfach und schnell geht es mit InterCorp.

- korpus.cz $\rightarrow \underline{\text { Login }} \rightarrow \underline{\text { KonText }} \rightarrow \underline{\text { Parallel corpus InterCorp }} \rightarrow \underline{\text { intercorp de }}$

- Query Type: Lemma auswählen

- Lemma (Suchfeldeingabe): werden $\rightarrow$ Search

Nach dem die Konkordanzen erschienen sind:

- Frequency $\rightarrow$ Node forms.

dann

- Frequency $\rightarrow$ Node tags.

Die Ergebnisse erscheinen in automatisch erstellten Tabellen (Abb. 96, 97). In der Abb. 96 werden die Formen (Spalte word) des Verbs nach der Häufigkeit (Freq.) angeführt, in der Abb. 97 sind seine Funktionen (Spalte tag) auch nach der Frequenz geordnet zu sehen. Um die syntaktisch-semantischen Funktionen richtig interpretieren zu können, muss man die Kürzel des Tagsets entschlüsseln können (siehe dazu Kap. 8.2).

\begin{tabular}{|l|l|l|l|l|}
\hline \multicolumn{3}{|l|}{ Total: 39 (1 pages) } \\
\hline \hline & & word & Freq & Freq [\%] \\
\hline 1. & $\underline{\mathrm{p}} / \mathrm{n}$ & werden & 310,128 & 40.6 \\
\hline \hline 2. & $\underline{\mathrm{p}} \mathrm{n}$ & wird & 201,263 & 26.4 \\
\hline 3. & $\mathrm{p} / \mathrm{n}$ & wurde & 82,681 & 10.8 \\
\hline \hline 4. & $\underline{\mathrm{p}} \mathrm{n}$ & wurden & 49,964 & 6.5 \\
\hline \hline 5. & $\underline{\mathrm{p}} \mathrm{n}$ & würde & 37,813 & 5.0 \\
\hline 6. & $\underline{\mathrm{p}} \mathrm{n}$ & worden & 30,638 & 4.0 \\
\hline \hline 7. & $\underline{\mathrm{p}} \mathrm{n}$ & werde & 14,520 & 1.9 \\
\hline 8. & $\underline{\mathrm{p}} \mathrm{n}$ & würden & 13,966 & 1.8 \\
\hline 9. & $\underline{\mathrm{p}} \mathrm{n}$ & geworden & 8,639 & 1.1 \\
\hline \hline 10. & $\underline{\mathrm{p}} \mathrm{n}$ & wirst & 2,619 & 0.3 \\
\hline
\end{tabular}

Abb. 96: Frequenzliste der Formen des Verbs werden

\begin{tabular}{|l||l|l||l|l|}
\hline \multicolumn{2}{|l|}{ Total: 7 (1 page) } \\
\hline \hline & & tag & Freq & Freq [\%] \\
\hline \hline 1. & $\mathrm{p} / \mathrm{n}$ & VAFIN & 562,707 & 73.7 \\
\hline 2. & $\mathrm{p} / \mathrm{n}$ & VAINF & 160,726 & 21.0 \\
\hline 3. & $\mathrm{p} / \mathrm{n}$ & VAPP & 39,085 & 5.1 \\
\hline 4. & $\mathrm{p} / \mathrm{n}$ & VVFIN & 894 & 0.1 \\
\hline 5. & $\mathrm{p} / \mathrm{n}$ & VVPP & 192 & 0.0 \\
\hline 6. & $\mathrm{p} / \mathrm{n}$ & VVINF & 95 & 0.0 \\
\hline 7. & $\mathrm{p} / \mathrm{n}$ & VAIMP & 3 & 0.0 \\
\hline
\end{tabular}

Abb. 97: Frequenzliste der Funktionen des Verbs werden 


\section{Fazit:}

In der Abb. 96 sind die ersten 10 üblichsten Konjugationsformen des Verbs werden angeführt. Markant ist die extrem hohe Frequenz der „Grundform“ werden (40\% aller Belege). Hier spielt natürlich auch die Homonymie der Formen eine wichtige Rolle, denn der Infinitiv und die 1. u. 3. Person P1. Präsens sind formal ident. Wie die Verteilung unter diesen Kategorien aussieht, lässt sich grob durch weitere Schritte erkennen: positiver Filter $\underline{p} \rightarrow$ Node Tags. Dies ergibt, dass es sich ausschließlich um Hilfsverben ${ }^{36}$ handelt.

Aus der Abb. 97 ist ersichtlich, dass das Verb in erster Linie als ein Hilfsverb fungiert: über $73 \%$ (fast $3 / 4$ ) aller Vorkommen des Verbs werden entfallen auf seine finite Form ${ }^{37}$ (VAFIN = Auxiliarverb, finite Form) als Auxiliar. Weitere 21\% der Belege bilden infinitive Formen des Auxiliars, insgesamt sind also fast $95 \%$ der Wörter werden Hilfsverben. Seine Funktion als Vollverb (Kürzel beginnend mit VV) ist vergleichsmäßig marginal, trotzdem beträgt sie über 1.000 Belege im Korpus. Diese Belege müssten jedoch noch sorgfältig aussortiert werden. Mehr als die Hälfte von ihnen wurde vom automatischen Tagger dort falsch erkannt (und annotiert), wo das Verb werden in einer für ein Auxiliar vergleichsweise eher untypischen Position steht (z.B. am Satzanfang) und das Vollverb sehr weit davon, weshalb es vom Tagger unerkannt blieb. Es ist darauf hinzuweisen, dass die automatische Annotation sich sehr stark auf die prototypische Verbindung der Wortform und ihrer Funktion stützt - in diesem Fall wird beim Tagging zu jeder Form von werden in demselben Satz nach einem zweiten Verb im Infinitiv oder Partizip Perfekt gesucht. Wird der Infinitiv, das Partizip Perfekt oder gar die Satzgrenze (vom ersten Großbuchstaben bis zum ersten Satzendzeichen) falsch erkannt, entsteht ein Annotationsfehler. Damit ist immer zu rechnen. Je eindeutiger die Funktion einer Wortform in der Sprache ist, desto niedriger ist die Wahrscheinlichkeit, dass die Wortform einen falschen Tag bekommt.

Zum Schluss dieser Recherche muss festgehalten werden, dass das präsentierte Ergebnis über die syntaktisch-semantische Verteilung der Funktionen des Verbs werden zwar naheliegend ist und dem linguistischen Bauernvernunft vollkommen entspricht, durch viele Korpusdaten ist diese These nun aber auch mehrfach (an verschiedenen Texten) bewiesen. Beispiele können sofort durch Klicken auf $\underline{p}$ (in der 2. Spalte) präsentiert werden.

\section{Fragestellung 2. Welche Formen hat das Verb backen?}

Die Konkurrenz der schwachen und starken Deklinations- und Konjugationsformen ist ein Evergreen in der deutschen Morphologie sowie eine Qual im traditionellen Unterricht. In der Sprache gibt es aber (pragmatisch gesehen) kaum etwas Unwichtigeres für eine erfolgreiche Kommunikation. Trotzdem wird nach der typischen Verwendung der sich konkurrierenden Formen oft gesucht. In diesem Falle können sie im InterCorp leicht gefunden werden. Die Abfrage ist gleich wie die in der vorherigen Fragestellung.

- $\quad$ korpus.cz $\rightarrow \underline{\text { Login }} \rightarrow \underline{\text { KonText }} \rightarrow \underline{\text { Parallel corpus InterCorp }} \rightarrow \underline{\text { intercorp de }}$

- Query Type: Lemma auswählen

- Lemma (Suchfeldeingabe): backen $\rightarrow$ Search

Nach dem die Konkordanzen erschienen sind:

- Frequency $\rightarrow$ Node forms.

36 In der nächsten Phase des Korpustaggings wird auch das Abrufen anderer morphologischen Kategorien möglich (Person, Numerus, Tempus, Modus, Genus Verbi).

37 Die häufigste finite Form ist die 3. Pers. Singular. 
Die häufigsten Formen des Verbs backen sind aus der Abb. 98 ersichtlich.

Frequenzliste der Formen: Lemma backen

\begin{tabular}{|l|l|l|l|l|l|}
\hline & & word & Freq & Freq [\%] \\
\hline 1. & $\mathrm{p} / \mathrm{n}$ & gebacken & 88 & 36.1 & \\
\hline 2. & $\mathrm{p} / \mathrm{n}$ & backen & 49 & 20.1 & \\
\hline 3. & $\mathrm{p} / \mathrm{n}$ & Back & 25 & 10.2 & \\
\hline 4. & $\mathrm{p} / \mathrm{n}$ & back & 24 & 9.8 & \\
\hline 5. & $\mathrm{p} / \mathrm{n}$ & bäckt & 11 & 4.5 & \\
\hline 6. & $\mathrm{p} / \mathrm{n}$ & backte & 11 & 4.5 & \\
\hline
\end{tabular}

\begin{tabular}{|l|l|l|l|l|}
\hline & & word & Freq & Freq [\%] \\
\hline 7. & $\mathrm{p} / \mathrm{n}$ & backe & 11 & 4.5 \\
\hline 8. & $\mathrm{p} / \mathrm{n}$ & buk & 10 & 4.1 \\
\hline 9. & $\mathrm{p} / \mathrm{n}$ & buken & 8 & 3.3 \\
\hline 10. & $\mathrm{p} / \mathrm{n}$ & backten & 3 & 1.2 \\
\hline 11. & $\mathrm{p} / \mathrm{n}$ & backt & 3 & 1.2 \\
\hline 12. & $\mathrm{p} / \mathrm{n}$ & gebacken & 1 & 0.4 \\
\hline
\end{tabular}

Abb. 98: Formen zum Lemma backen (InterCorp)

Die einzelnen Formen wurden weiter in einer Seminararbeit (Mair 2013) nach Texten und Zeit ihrer Entstehung untersucht und noch mit Daten des DeReKo verglichen. Die Ergebnisse zeigten deutlich, dass die schwachen Formen backtel gebackt, ,im Vormarsch“ sind. Die Form backte kommt allgemein sogar wesentlich häufiger vor als die starke Form buk. Sie kommt signifikant häufiger in bundesdeutschen und schweizerischen Texten vor, und zwar sowohl in der direkten als auch in der indirekten Rede. Die starke Form buk ist über die Länder gleichmäßig verteilt, markant ist jedoch der historische Touch dieser Form, denn in modernen deutschen Texten kommt sie vor allem in Verbindung mit Till Eugenspiegels Streich vor, wie die automatisch berechneten syntagmatischen Muster in der Abb. 99 zeigen (grau unterlegt), während die Form backte in Verbindungen mit semantisch relevanten Lexemen gleichmäßig verteilt ist. (Für den allgemeinen Sprachgebrauch „,irrelevante“ Verbindungen: homonyme Formen aus slawischen Sprachen (Abb. 100, Zeilen 1, 3, 4, 6) und linguistische Behandlungen der schwachen und starken Konkurrenzformen (Abb. 99, Zeile 3; Abb. 100, Zeilen 3, 9, 21) sind durchgestrichen.)

Kookkurrenzen zu buk (gekürzt)

\begin{tabular}{|c|c|c|c|c|c|}
\hline \multicolumn{6}{|c|}{ \# Total Anzahl LLR Kookkurrenzen } \\
\hline 1 & 7 & 7 & 107 & Skradinski & $100 \%$ Skradinski buk \\
\hline \multirow[t]{2}{*}{2} & 16 & 9 & 94 & Meerkatzen Eul & 77\% er die Eulen und Meerkatzen buk \\
\hline & 18 & 2 & & Meerkatzen & $100 \%$ Meerkatzen [...] buk \\
\hline 3 & 27 & 9 & 81 & backte & 14: buk... backte \\
\hline 4 & 30 & 3 & 41 & slaw & $100 \%$ slaw $[\ldots]$ buk ... Buche \\
\hline \multirow[t]{2}{*}{5} & 31 & 1 & 34 & Brot Eulen & $100 \%$ buk Eulen ... Brot \\
\hline & 62 & 31 & & Brot & $58 \%$ buk $[\ldots]$ Brot \\
\hline 6 & 63 & 1 & 30 & slap & $100 \%$ slap... buk \\
\hline 7 & 64 & 1 & 28 & Eulen & $100 \%$ buk ... Eulen \\
\hline 8 & 70 & 6 & 25 & Reibekuchen & $100 \%$ buk [... leckere] Reibekuchen und \\
\hline 9 & 80 & 10 & 23 & Waffeln & 90\% buk [leckerelfrische] Waffeln \\
\hline 10 & 87 & 7 & 21 & Backofen & 57\% im Backofen [...] buk \\
\hline 11 & 102 & 15 & 21 & Brötchen & $80 \%$ buk $[\ldots$ kleinelkleinere $]$ Brötchen \\
\hline 12 & 109 & 7 & 20 & kochte & $71 \%$ kochte und buk \\
\hline 13 & 115 & 6 & 19 & Brote & 83\% buk ... Brote \\
\hline 14 & 122 & 7 & 17 & Kekse & $85 \%$ buk $[\ldots]$ Kekse \\
\hline 15 & 133 & 11 & 15 & Bäcker & Ein findiger Bäcker [...] buk daraufhin Dreck \\
\hline 17 & 139 & 3 & 8 & Torten & $66 \%$ buk ... Torten \\
\hline 18 & 142 & 3 & 7 & Pfannkuchen & $100 \%$ buk $[\ldots]$ Pfannkuchen \\
\hline 19 & 143 & 1 & 7 & Eulenspiegel & $100 \%$ buk ... Eulenspiegel \\
\hline 20 & 146 & 3 & 6 & Backstube & $100 \%$ Backstube ... und buk \\
\hline 21 & 150 & 4 & 6 & Bäckermeister & $50 \%$ buk Bäckermeister \\
\hline
\end{tabular}

Abb. 99: Kookkurrenzen und syntagmatische Muster zur Abfrage: Wortform buk (DeReKo) 
Kookkurrenzen zu backte (gekürzt)

\begin{tabular}{|c|c|c|c|c|c|}
\hline \multicolumn{5}{|c|}{ \# Total Anzahl LLR Kookkurrenzen } & syntagmatische Muster \\
\hline 1 & 47 & 47 & 197 & Waffeln & 85\% backte [...] Waffeln und \\
\hline 2 & 72 & 25 & 137 & kochte & 76\% kochte [und] backte \\
\hline 3 & 81 & 9 & 81 & buk & $44 \%$ backte ... buk \\
\hline 4 & 112 & 31 & 67 & Brötchen & 74\% backte [... kleine] Brötchen und \\
\hline 5 & 135 & 23 & 50 & Plätzchen & 56\% backte $[\ldots]$ Plätzchen und \\
\hline 6 & 138 & 3 & 41 & Konditor-Meisterin & $100 \%$ Konditor-Meisterin backte auf dem \\
\hline 7 & 153 & 15 & 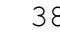 & leckere & $86 \%$ backte $[\ldots]$ leckere \\
\hline 8 & 159 & 6 & 33 & Weihnachtsplätzchen & 50\% backte der ... Weihnachtsplätzchen \\
\hline 9 & 162 & 3 & 33 & gebackt & $100 \%$ - backte - gebackt \\
\hline 10 & 213 & 51 & 27 & Kuchen & 76\% backte $[\ldots$ einen $]$ Kuchen fürlund \\
\hline 12 & 251 & 32 & 21 & Brot & 71\% und backte $[\ldots]$ Brot \\
\hline 14 & 256 & 3 & 15 & Meerkatzen & $66 \%$ backte ... Eulen und Meerkatzen \\
\hline 15 & 262 & 6 & 13 & Kekse & 83\% backte ... Kekse \\
\hline 16 & 265 & 3 & 12 & Streuselkuchen & $100 \%$ backte [leckeren] Streuselkuchen \\
\hline 17 & 268 & 3 & 11 & Reibekuchen & $100 \%$ backte $[\ldots]$ Reibekuchen \\
\hline 18 & 274 & 6 & 10 & Bäckermeister & 66\% backte [...] Bäckermeister \\
\hline 19 & 276 & 2 & 9 & Crêpes & 100\% backte $[\ldots]$ Crêpes \\
\hline 20 & 279 & 3 & 9 & Kartoffelpuffer & $100 \%$ backte die ... Kartoffelpuffer \\
\hline 21 & 281 & 2 & s & backt & $50 \%$ backte... backt \\
\hline 23 & 285 & 3 & $\varepsilon$ & Konditor & $66 \%$ backte $[\ldots]$ Konditor \\
\hline 24 & 288 & 3 & $\varepsilon$ & Muffins & $100 \%$ backte [...] Muffins \\
\hline 25 & 292 & 4 & 7 & Brote & $50 \%$ backte ... Brote \\
\hline 26 & 296 & 4 & 6 & Torten & 75\% backte die... Torten \\
\hline
\end{tabular}

Abb. 100: Kookkurrenzen und syntagmatische Muster zur Abfrage: Wortform backte (DeReKo)

Die Form des Partizips II von backen lautet in den meisten deutschsprachigen Texten gebacken. Nur gelegentlich kommt auch die schwache Form gebackt vor. Diese ist jedoch ausschließlich in bundesdeutschen Texten und meistens nur in der Wiedergabe der direkten Rede zu finden, wie die Konkordanzen in der Abb. 101 zeigen.

Konkordanzen zu gebackt (Auswahl)

\begin{tabular}{|cc|}
\hline RHZ01 & kurze Zeit "gehen", bevor die Waffeln gebackt werden können. \\
RHZ01 & Beitrag geleistet. Plätzchen wurden gebackt, kleine Geschenke \\
RHZ06 & Erst wurde eifrig gebackt, und dann verzehrt \\
RHZ12 & wollen. "Da haben wir kleine Brötchen gebackt. Wir waren uns \\
RHZ12 & meiner Mutter: "Ich honn noch enn Sort gebackt", und nannte noch \\
WDD11 & da 2 Euro." "Die Faasekichelcha genn gebackt. "Rheinfränkisch: \\
WDD11 & gebackt." Rheinfränkisch: ". . werre gebackt." --Speifensender \\
WDD11 & dies da ich die jahrelang als Bäcker gebackt habe. Heute gibt \\
\hline
\end{tabular}

Abb. 101: Konkordanzen zur Abfrage: Wortform gebackt (DeReKo)

\section{Fazit:}

In Anbetracht der Tatsache, dass die Recherchen in Texten der geschriebenen Sprache durchgeführt wurden (die gesprochene Sprache ist in der Entwicklung der geschriebenen voran), könnte eine Tendenz zu den schwachen Formen betrachten werden. Die Standardform des Imperfektums von backen ist backte, die Standardform des Partizips II ist immer noch gebacken, es ist vielleicht nur eine Frage der Zeit, dass auch diese mehrheitlich „schwach“ gebildet wird. Eine regionale Differenzierung ist dabei nicht ausgeschlossen.

\section{Schlussbemerkung zur Studie 4:}

Die Konjugations- bzw. Deklinationsformen lassen sich in den meisten Korpora über die Funktion Lemma abrufen. Im DeReKo muss man die Einstellungen der Optionen beachten (Optionen $\rightarrow$ Lemmatisierung, es sollen nur Flexionsformen ausgewählt bleiben.) Man muss bedenken, dass die Korpora automatisch lemmatisiert sind, es können also viele Ballastformen mitabgerufen werden (z.B. zum Lemma des Verbs fließen sind es auch das Substantiv Floss/ Floß). Dennoch kann man sich darauf verlassen, dass gut lemmatisierte Korpora auch suppletive Formen sicher erkennen (gut, besser, beste) können. 
Die Erweiterung von morphologischen (Deklinations- bzw. Konjugations-)Formen stellen Wortbildungsformen dar. Auch diese lassen sich gut erschließen, wie die folgende Studie zeigt.

\section{Studie 5: Ermittlung einer Wortfamilie}

Die lexikalische Kompetenz der Lernenden wird dadurch gefördert, dass auf formale und semantische Zusammenhänge aufmerksam gemacht wird. Die formalen Zusammenhänge im Lexikon sind an einzelnen Gliedern einer Wortfamilie deutlich zu sehen. Die Glieder einer Wortfamilie lassen sich schnell abrufen, dabei kann man auch ihre Frequenz beobachten und vergleichen.

Fragestellung: Welche Wörter haben die gemeinsame Wortwurzel -grund-?

Die Recherche kann in jedem Korpus durchgeführt werden, solange die Suchanfrage nach einem Intervall (Zeichenkette) möglich ist. Hier werden zwei Recherchen durchgeführt: im InterCorp, denn hier können auch Angaben über die vertretenen Wortarten abgerufen werden, und dann im DeReKo, das aufgrund seiner Größe wesentlich mehr Formen liefern kann.

\section{Recherche im InterCorp}

- $\quad$ korpus.cz $\rightarrow \underline{\text { Login }} \rightarrow \underline{\text { KonText }} \rightarrow \underline{\text { Parallel corpus InterCorp }} \rightarrow \underline{\text { intercorp de }}$

- Query Type: Character auswählen

- Character (Suchfeldeingabe): grund $\rightarrow$ Search

Alternative:

- Query Type: Word Form

- Word Form (Suchfeldeingabe): .*grund.* $\rightarrow$ Search

Nach dem die Konkordanzen erschienen sind:

- $\quad$ Frequency $\rightarrow$ Node forms (Ergebnisse siehe Abb. 102)

dann

- $\quad \underline{\text { Frequency }} \rightarrow \underline{\text { Node tags }}$ (Ergebnisse siehe Abb. 103)

Die Ergebnisse erscheinen in Form einer Liste, gereiht nach relativer Frequenz der KWICFormen (Abb. 102) oder nach der morphosyntaktischen Charakteristik der KWIC (Tag) (Abb. 103).

Frequenzliste der Formen (gekürzt)

\begin{tabular}{|l|l|l|l|l|}
\hline & Filter & Tag & Abs. & $\%$ \\
\hline 1. & $\mathrm{p} / \mathrm{n}$ & aufgrund & 16,283 & 35.9 \\
\hline 2. & $\mathrm{p} / \mathrm{n}$ & zugrunde & 3,642 & 8.0 \\
\hline 3. & $\mathrm{p} / \mathrm{n}$ & grundlegend(e) & 3,400 & 7.1 \\
\hline 4. & $\mathrm{p} / \mathrm{n}$ & Aufgrund & 2,814 & 6.2 \\
\hline 5. & $\mathrm{p} / \mathrm{n}$ & Hintergrund & 2,797 & 6.2 \\
\hline 6. & $\mathrm{p} / \mathrm{n}$ & Rechtsgrundlage & 2,330 & 5.1 \\
\hline 7. & $\mathrm{p} / \mathrm{n}$ & grundsätzlich & 1,824 & 4.0 \\
\hline 8. & $\mathrm{p} / \mathrm{n}$ & Rechtsgrundlagen & 786 & 1.7 \\
\hline 9. & $\mathrm{p} / \mathrm{n}$ & Erwägungsgrund & 714 & 1.6 \\
\hline 10. & $\mathrm{p} / \mathrm{n}$ & Vordergrund & 580 & 1.3 \\
\hline
\end{tabular}

Abb. 102: Abfrage -grund-, Node forms (alle Texte)
Frequenzliste der Tags

\begin{tabular}{|l|l|l|r|r|}
\hline & Filter & Tag & Abs. & \multicolumn{1}{l|}{$\%$} \\
\hline 1. & $\mathrm{p} / \mathrm{n}$ & APPR & 19,097 & 42.1 \\
\hline 2. & $\mathrm{p} / \mathrm{n}$ & $\mathrm{NN}$ & 12,524 & 27.6 \\
\hline 3. & $\mathrm{p} / \mathrm{n}$ & ADJA & 7,323 & 16.1 \\
\hline 4. & $\mathrm{p} / \mathrm{n}$ & ADV & 3,338 & 7.4 \\
\hline 5. & $\mathrm{p} / \mathrm{n}$ & ADJD & 2,695 & 5.9 \\
\hline 6. & $\mathrm{p} / \mathrm{n}$ & PTKVZ & 298 & 0.7 \\
\hline
\end{tabular}

Abb. 103: Abfrage -grund-, Node tags 
Aus den Kürzeln der Tags in der Abb. 103 (Erklärung siehe im Kap. 8.2) ist ersichtlich, dass die Wortwurzel -grund- überraschenderweise am häufigsten in der Präposition aufgrund vorkommt. Erst danach haben in diesem Korpus die zweit höchste Frequenz Substantive (Komposita Hintergrund, Rechtsgrundlage, Erwägungsgrund und Vordergrund), Adjektive (konvertiertes Partizipium grundlegend, Ableitung vom Substantiv grundsätzlich). Man muss jedoch immer im Auge behalten, in welchen Texten recherchiert wurde: Hier waren es verschiedene Texte der geschriebenen Sprache, noch dazu bilden den größten Teil Gesetzestexte der EU und Schriften aus dem EU-Parlament, publizistische Texte und andere Sachtexte.

In vorwiegend belletristischen Texten (InterCorp: Kernkorpus) sieht die Reihenfolge etwas anders aus. Die nachfolgende Recherche deutet darauf hin, dass die Präposition aufgrund eigentlich typisch für Sachtexte ist und in belletristischen Texten nicht so häufig vorkommt. Immerhin nähern sich belletristische Texte der gesprochenen Sprache stärker an als Sachtexte, beispielswiese in der direkten Rede. Dies bestätigte auch eine Recherche in der DGD (Korpus nur mit gesprochener Sprache): Die Präposition aufgrund kommt etwa 10 Mal seltener vor als das konkurrierende wegen.

Frequenzliste der Formen (gekürzt)

\begin{tabular}{|l|l|l|l|l|}
\hline & Filter & Tag & Abs. & $\%$ \\
\hline 1. & $\mathrm{p} / \mathrm{n}$ & Hintergrund & 668 & 20.5 \\
\hline 2. & $\mathrm{p} / \mathrm{n}$ & zugrunde & 324 & 10.0 \\
\hline 3. & $\mathrm{p} / \mathrm{n}$ & Abgrund & 309 & 9.5 \\
\hline 4. & $\mathrm{p} / \mathrm{n}$ & aufgrund & 280 & 8.6 \\
\hline 5. & $\mathrm{p} / \mathrm{n}$ & grundsätzlich & 192 & 5.9 \\
\hline 6. & $\mathrm{p} / \mathrm{n}$ & grundlegende & 124 & 3.8 \\
\hline 7. & $\mathrm{p} / \mathrm{n}$ & grundlegenden & 117 & 3.6 \\
\hline 8. & $\mathrm{p} / \mathrm{n}$ & Vordergrund & 107 & 3.3 \\
\hline 9. & $\mathrm{p} / \mathrm{n}$ & Untergrund & 88 & 2.7 \\
\hline 10. & $\mathrm{p} / \mathrm{n}$ & grundlegend & 70 & 2.2 \\
\hline Abb. $104:$ Abfrage - grund-, Node forms (Belletristik)
\end{tabular}

\section{Frequennzliste der Tags}

\begin{tabular}{|l|l|l|l|r|}
\hline & Filter & Tag & Abs. & $\%$ \\
\hline 1. & $\mathrm{p} / \mathrm{n}$ & $\mathrm{NN}$ & 1,697 & 52.2 \\
\hline 2. & $\mathrm{p} / \mathrm{n}$ & ADJA & 511 & 15.7 \\
\hline 3. & $\mathrm{p} / \mathrm{n}$ & ADJD & 378 & 11.6 \\
\hline 4. & $\mathrm{p} / \mathrm{n}$ & APPR & 327 & 10.1 \\
\hline 5. & $\mathrm{p} / \mathrm{n}$ & ADV & 271 & 8.3 \\
\hline 6. & $\mathrm{p} / \mathrm{n}$ & PTKVZ & 51 & 1.6 \\
\hline
\end{tabular}

Wenn man die Abb. 102 und 104 vergleicht, sieht man die Verschiebung der Reihenfolge in der Frequenz einzelner Substantive.

Diese Ergebnisse können an Texten des größten Korpus des Deutschen DeReKo überprüft werden.

\section{Recherche im DeReKo}

- Cosmas $\mathbf{I I}_{\text {web }} \rightarrow \underline{\text { Recherche }}$

- Archiv: W-Archiv der geschriebenen Sprache

- Korpus: W-öffentlich - alle öffentlichen Korpora des Archivs W

- Optionen: Suchmodalitäten:

Groß- / Kleinschreibung beachten für 1. Zeichen

Groß- / Kleinschreibung beachten für andere Zeichen

Expansionslisten anzeigen:

Expansionslisten mit Häufigkeiten (langsam)

Sortierung nach Häufig. absteigend

auswählen

abwählen

auswählen

auswählen

auswählen 


\section{Lemmatisierung: \\ Komposita \\ Sonstige Wortbildungsformen \\ Spezialfälle}

abwählen

auswählen

abwählen $\rightarrow$ Übernehmen

- Suchanfrage (Eingabe ins Suchfeld): \&Grund $\rightarrow$ Suchen

Die Abfrage bedeutet: Suche alle Formen zur Grundform Grund.

In der Liste der Ergebnisse (Abb. 106) ist auch das Basissubstantiv Grund aufgelistet (im Unterschied zum InterCorp, wo dieses ausgeblendet wird). Die Wörter in den Listen in Abb. 102, 104 und 106 sind fast gleich (Differenzen sind auf geringe Frequenzunterschiede zurückzuführen).

Formenliste zur Abfrage Lemma: Grund (gekürzt)

\begin{tabular}{|c|c|c|}
\hline \multicolumn{3}{|c|}{ Suchbegriff-Expansionslisten (nach Häufig. absteigend) } \\
\hline Grund & $: 877.086$ & $(41.20 \%)$ \\
\hline Hintergrund & $: 318.064$ & $(14.94 \%)$ \\
\hline Gründen & : 295.670 & $(13.89 \%)$ \\
\hline Gründe & : 199.128 & $(9.35 \%)$ \\
\hline Aufgrund & $: 170.052$ & $(7.99 \%)$ \\
\hline Grunde & : $\quad 94.000$ & $(4.42 \%)$ \\
\hline Hintergründe & 39.966 & $(1.88 \%)$ \\
\hline Untergrund & 33.907 & $(1.59 \%)$ \\
\hline Hauptgrund & 19.510 & $(0.92 \%)$ \\
\hline Abgrund & 14.356 & $(0.67 \%)$ \\
\hline HINTERGRUND & 9.324 & $(0.44 \%)$ \\
\hline Grundig & 9.188 & $(0.43 \%)$ \\
\hline Abgründe & 7.076 & $(0.33 \%)$ \\
\hline grundlos & 6.939 & $(0.33 \%)$ \\
\hline Hauptgründe & 5.422 & $(0.25 \%)$ \\
\hline
\end{tabular}

Abb. 106: Formenliste (Expansionsliste) der Wortfamilie -grund- (DeReKo)

In die Expansionsliste (Abb. 106) ist auch ein „,blinder Passagier“ mittransportiert worden: die Marke Grundig. An diesem Fehler sieht man, wie das Korpus funktioniert: es sucht nach entsprechenden Graphemketten und vergleicht sie mit einem Wörterbuch bzw. einer Liste der Lemmata, über die das Korpus lemmatisiert wurde. In diesem Wörterbuch fehlte offensichtlich der Name dieser Firma.

Auch DeReKo ermöglicht die Abfrage nach einem offenen Intervall, also nach Wortformen, in denen die Buchstabenkombination grund vorkommt:

Einstellung wie vorher

\section{- Suchanfrage (Eingabe ins Suchfeld): *grund* $\rightarrow$ Suchen}

Diese Abfrage bedeutet: Suche nach allen Wörtern, die die Buchstabenkombination grund beinhalten. Die Errechnung der Ergebnisse dauert im DeReKo sehr lange. Im Prinzip kann nur die Expansionsliste (wie Abb. 106) abgerufen werden - sie zeigt mehr als 68.000 Formen. Ihr Inhalt entspricht den Ergebnissen in der Abb. 104 (nach Frequenz absteigend: aufgrund, Hintergrund, gegründet, grundsätzlich, Vordergrund, gründlich etc.)

Es ist nicht sinnvoll, die Ergebnisse (Konkordanzen) abzurufen, nachdem die Expansionsliste erschienen ist, weil die Dauer der Erstellung der gesamten KWIC-Liste das erlaubte Limit 
überschreitet, der Korpusmanager COSMAS II liefert eine Fehlermeldung. Darüber hinaus kann man mit Konkordanzen, in denen gemischt alle Wörter einer Wortfamilie vorkommen, wenig anfangen. So gesehen erweist sich die Arbeit mit InterCorp als effektiver.

\section{Fazit:}

Vergleicht man die Ergebnisse der Recherche im InterCorp mit DeReKo, stellt man fest, dass neben dem Stamm Grund (mit allen seinen Deklinationsformen) seine folgenden Ableitungen sehr häufig (daher für die Lernenden auch wichtig) sind: aufgrund; grundlegend(e/en/er); grundsätzlich; Hintergrund; zugrunde. Beim letzten Wort handelt es sich um einen Verbzusatz zu gehen/ liegen/ richten. Verbzusätze werden in der Studie 7 behandelt.

\section{Schlussbemerkung zur Studie 5}

Für die Ermittlung der Wortformen ist InterCorp bisher die beste Wahl. Ohne komplizierte Einstellungen kann man alle Formen abrufen und dann auf einen Klick ihre Frequenzen berechnen lassen. Der Vorteil liegt auch darin, dass die Frequenzliste sich durch Klicken auf word in eine alphabetische Liste umwandeln lässt.

Die zentralen Glieder der Wortfamilie grund (zwanzig mit der höchsten Frequenz) sind: abgrund, abgrundtief, aufgrund, beweggrund, großgrundbesitz, großgrundbesitzer, grundlegend, grundlos, grundsätzlich, grundverschieden, hauptgrund, hintergrund, lebensgrundlage, meeresgrund, untergrund, untergrundbahn, urgrund, vordergrund, zugrunde, zugrundeliegend

Groß-/Kleinschreibung wurde hier ignoriert. Dies könnte jetzt im Unterricht ein guter Anlass sein, in einer Grammatikarbeit die Lernenden entscheiden zu lassen, zu welcher Wortart die einzelnen Wörter gehören.

Im akademischen Bereich können an solchen Wortfamilien die einzelnen (wichtigsten) Wortbildungsarten des Deutschen gezeigt werden:

Komposition: Grund $\rightarrow$ als Bestimmung Grundbesitz, grundlegend $\rightarrow$ als Basis $\quad$ Beweggrund, Meeresgrund; (weiter Hauptgrund, Hintergrund, Untergrund, Vordergrund als Übergangsbereich zur Derivation)

Derivation: $\quad$ Grund $\rightarrow$ Abgrund, Urgrund, grundlos

Konversion: auf Grund, zu Grunde (Präpositionalverbindungen) $\rightarrow$ aufgrund (Präp.); zugrunde (Part. (Verbzusatz)); Grund (Subst.) $\rightarrow$ gründen (Verb)

$\mathrm{Zu}$ jedem Wort können mehrere Sätze gezeigt werden, die die Bedeutungsunterschiede beleuchten. 


\section{Studie 6: Wortbildung}

Die Wortbildung stellt einen Bereich der Sprache dar, der mithilfe von Computerprogrammen bestens erforscht werden kann. Jeder Texteditor und fast jedes Internetformular oder jede Suchmaschine kann nach genauen Wörtern oder nach Wortteilen suchen ${ }^{38}$. Versteht man die Wortbildung als „Bildung eines Lexems bis zur Wortgrenze“, dann bieten Korpora unzählige Möglichkeiten an, Komposita und Ableitungsformen zu untersuchen. Mit einer gut formulierten Abfrage kann man sehr schnell einen Überblick bekommen, wie sich Wortstämme, Vor- und Nachsilben (Affixe) verhalten, welche Wörter zusammengesetzt werden können, welche Wortkombinationen üblich sind.

Erfahrungsgemäß ist für Deutschlerner/-innen oft die Wahl des richtigen adjektivischen Suffixes problematisch: „Wie heißt es eigentlich: lustig, lustich, lustlich oder gar lustisch?“ Als ein Fallbeispiel der Wortbildung werden die Adjektivableitungen untersucht.

Fragestellung: Welche Grundwörter verbinden sich mit den adjektivischen Suffixen -ig, -lich und -isch?

Die Suche kann in jedem Korpus erfolgen. Wahrscheinlich am schnellsten und einfachsten geht sie im InterCorp.

- $\quad$ korpus.cz $\rightarrow \underline{\text { Login }} \rightarrow \underline{\text { KonText }} \rightarrow \underline{\text { Parallel corpus InterCorp }} \rightarrow \underline{\text { intercorp de }}$

- Query Type: CQL auswählen

- CQL (Suchfeldeingabe): [tag="ADJ.*"\&lemma=".*[ig|lich|isch]"] $\rightarrow$

Die Abfrage bedeutet: „Suche alle Adjektive, die auf -ig, -lich oder -isch enden“. Sie liefert fast 2 Mio. Konkordanzen, in denen viele KWICs gleich sind, weil die Wörter mit Ableitungen auf -ig, -lich oder -isch ziemlich häufig sind: wirklich (zu wirken), plötzlich (zu Plotz ${ }^{39}$ ), völlig (zu voll), möglich (zu mögen), um nur die häufigsten vier zu nennen. Man braucht jedoch jedes Wort, besser sogar nur seine Grundform (Lemma), nur einmal, um festzustellen, mit welchem Suffix (Nachsilbe) es vorkommt. Dies erreicht man über die Frequenzliste:

- Frequency $\rightarrow$ Custom $\rightarrow$ Level $1 \rightarrow$ Attribute: Lemma auswählen $\rightarrow$ ignore case auswählen $\rightarrow$ Position: Node auswählen $\rightarrow$

Die Ergebnisse können in eine Excel-Tabelle übertragen und alphabetisch sortiert werden. In der alphabetischen Liste wurden alle Ableitungen mit gleichem Wortstamm markiert und diese in die Tab. 25 (auf der folgenden Seite) übertragen.

38 Einige Suchmaschinen können auch nach Lemmata suchen (Google), manche sogar nach Allonymen (Eigennamen, die in verschiedenen Sprachen anders aussehen): Auf dem Portal der Österreichischen Bundesbahnen (www.oebb.at) kann man z.B. die Suche nach der Verbindung Brünn Hbf. - PreßburgEngerau eingeben und bekommt automatisch den Fahrplan zwischen Brno hl.n. und Bratislava-Petržalka.

39 Bedeutete „Aufprall“ (Kluge 2002:709). 
Adjektivische Ableitungen mit derselben Basis

\begin{tabular}{|l|l|}
\hline Lemma & abs. Freq. \\
\hline geistig & 2413 \\
\hline geistlich & 208 \\
\hline geschäftig & 114 \\
\hline geschäftlich & 690 \\
\hline gütig & 209 \\
\hline gütlich & 118 \\
\hline heimisch & 505 \\
\hline heimlich & 1170 \\
\hline herrisch & 88 \\
\hline herrlich & 1010 \\
\hline
\end{tabular}

\begin{tabular}{|l|l|}
\hline Lemma & abs. Freq. \\
\hline herzigen $^{1)}$ & 5 \\
\hline herzlich & 1768 \\
\hline höfischen & 21 \\
\hline höflich & 892 \\
\hline jährlich & 9101 \\
\hline -jährig $^{2)}$ & 5450 \\
\hline kindisch & 143 \\
\hline kindlich & 358 \\
\hline launig & 20 \\
\hline launisch & 53 \\
\hline
\end{tabular}

\begin{tabular}{|l|l|}
\hline Lemma & abs. Freq. \\
\hline mündig & 48 \\
\hline mündlich & 1615 \\
\hline ungläubig & 440 \\
\hline unglaublich & 1232 \\
\hline verständig & 77 \\
\hline verständlich & 1295 \\
\hline zeitig & 140 \\
\hline zeitlich & 1515 \\
\hline & \\
\hline & \\
\hline
\end{tabular}

Tab. 25: Adjektivische Ableitungen (InterCorp)

\section{Anmerkungen:}

${ }^{1)}$ Signifikante Frequenz hat nur halbherzig, (un)barmherzig.

${ }^{2)}$ Kommt nur als Basis eines Kompositums vor: mehrjährig, langjährig, diesjährig, dreijährig, minderjährig, fünfjährig sind die häufigsten aus insgesamt 203 Komposita im InterCorp.

Die 28 Lexeme in der Tabelle 24 wurden aus 3.400 Lemmata (Grundformen) von Adjektiven auf -ig, -lich oder -isch aussortiert. Ihre Frequenz im Korpus InterCorp_de (Gesamtkorpus) ist (bis auf herzig) höher als 20 Belege in absoluten Zahlen.

\section{Fazit:}

Kein Grundwort bildet frequentierte Ableitungen mit allen drei Suffixen. Es gibt auch sehr wenige Paare (14), die zwei von diesen Suffixen haben, wobei beide suffigierten Wörter einigermaßen häufig vorkommen.

Aus der Tab. 24 geht auch hervor, dass jedes von diesen Suffixen das jeweilige Wort so weit spezifiziert, dass es zwischen zwei Ableitungen von derselben Basis gravierende semantische Unterschiede gibt: geistig - geistlich; höflich - höfisch; ... zeitig - zeitlich. Der minimalste Unterschied besteht vielleicht noch zwischen launig und launisch.

Unter den aufgelisteten Ableitungen fehlt z.B. neidig x neidisch. Neidisch hat (im InterCorp) relativ niedrige Frequenz (nur 52 Belege in absoluten Zahlen), neidig kommt gar nicht vor. Im DeReKo ist das Verhältnis neidig zu neidisch etwa $1 \mathrm{zu}$ 4. Neidisch ist also - allgemein gesehen - die üblichere Form in geschriebenem Deutsch. Die Länderansicht im DeReKo zeigt jedoch deutlich, dass die Form neidig eindeutig in österreichischen Texten überwiegt. Während neidisch in deutschen und schweizerischen Texten gleich vertreten ist, kommt diese Form in österreichischen Texten signifikant seltener vor.

\section{Schlussbemerkung zur Studie 6:}

Diese Unterschiede zwischen den einzelnen Paaren in dieser Studie könnte man in einem weiteren Rechercheschritt erkunden: über die Kollokationsanalyse bzw. über den Kontrast zu einer anderen Sprache. (Mehr zu Wortbildung im Kontrast in Káňa 2012). 


\section{Studie 7: Verben mit Zusatz}

Diese Problematik liegt zwischen den Fachgebieten der Morphologie, Syntax und Lexikologie. Als Zusatz zum Verb wird hier der trennbare Teil des Verbalkompositums verstanden, der traditionell oft als „trennbares Präfix“ (in Opposition zum ,untrennbaren Präfix " $)^{40}$ bezeichnet wird. Dieses Phänomen bereitet den Deutschlernenden ziemliche Schwierigkeiten und ,sollte im DaF/DaZ-Unterricht dementsprechend berücksichtigt werden“ (Fachlexikon DaF/DaZ 2010: 344). Schwierig empfinden die Lernenden vor allem homographische Verben (mit gleichem Schriftbild, jedoch unterschiedlich betont): umstellen vs. umstellen, übersetzen vs. übersetzen, aber auch andere Verben, bei denen (oft auch Muttersprachler/-innen) zweifeln, ob der Zusatz abgetrennt werden soll oder nicht (z.B. (sich) widerspiegeln).

Die Verbzusätze lassen sich in Korpora leicht abfragen, wenn man das Tagging in morphosyntaktisch annotierten Korpora zur Hand hat. Da die meisten Korpora für Deutsch mit dem gleichen Tagset annotiert sind, reicht es, wenn man sich merkt, dass Verbzusätze das Kürzel PTKVZ haben.

Fragestellung 1: Was sind die häufigsten Verbzusätze im Deutschen?

Recherchiert wird in mehreren Korpora: DWDS, DeReKo und InterCorp.

\section{Recherche im DWDS}

- DWDS $\rightarrow$ Abfragefenster

- Suchfeldeingabe: $\quad \$ p=P T K V Z \rightarrow \underline{\text { oder }}$

Das Ergebnis sind Konkordanzen der einzelnen Korpora des DWDS. In der Abb. 107 sind lediglich 4 Beispiele aus insgesamt 721.351 Treffern im Kernkorpus angeführt:

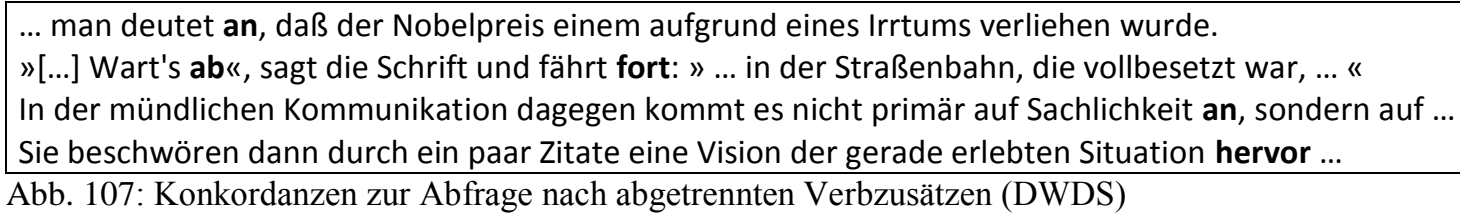

Es ist im DWDS nicht möglich, die Treffer auf nur einen Beleg pro Form zu reduzieren, um festzustellen, welche Zusätze es gibt. Eine Statistik der Frequenzen einzelner Zusätze lässt sich im DWDS auch nicht erstellen. Aus diesen Gründen wird in weiteren Korpora recherchiert.

40 Vgl. Engel 1988: 440 oder Zapletal et al. 1980: 128. 


\section{Recherche im DeReKo}

Dieselbe Recherche kann man auch im DeReKo durchführen. Dazu wird COSMAS II $_{\text {win }}$ dringend empfohlen, denn nur hier kann auch die Statistik der Häufigkeiten von Verbzusätzen abgerufen werden.

- Cosmas II $_{\text {win }} \rightarrow \underline{\text { Recherche }} \rightarrow$

- Archiv: TAGGED-T - Archiv morphosyntakt. annotierter Korpora (TreeTagger)

- Korpus: TAGGED-T-öffentlich - alle öffentlichen Korpora des Archivs TAGGED-T

- Suchanfrage (Eingabe ins Suchfeld): MORPH(PTK vz) $\rightarrow$

Die Abfrage liefert 6,5 Mio. Treffer, viele von ihnen haben dieselbe Form, und diese Form (Wort-Type) will man nur einmal ansehen. Dazu dient der Befehl:

- $\quad$ Ansicht $\rightarrow \underline{\S A n s i c h t ~ n a c h ~ W o r t-T y p e s ~(n u r ~ i m ~ C O S M A S ~ I I ~}{ }_{\text {win }}$ )

Das Ergebnis - 405 Wort-Types - ist in der Abb. 108 zu sehen. Sie sind nach der relativen Frequenz (rel [\%]) absteigend sortiert, können jedoch auch nach der absoluten Frequenz oder alphabetisch neu sortiert werden, wenn man die entsprechende Überschrift im Kopf der Tabelle anklickt.

\section{Wort-Types zur Abfrage Verbzusätze (gekürzt)}

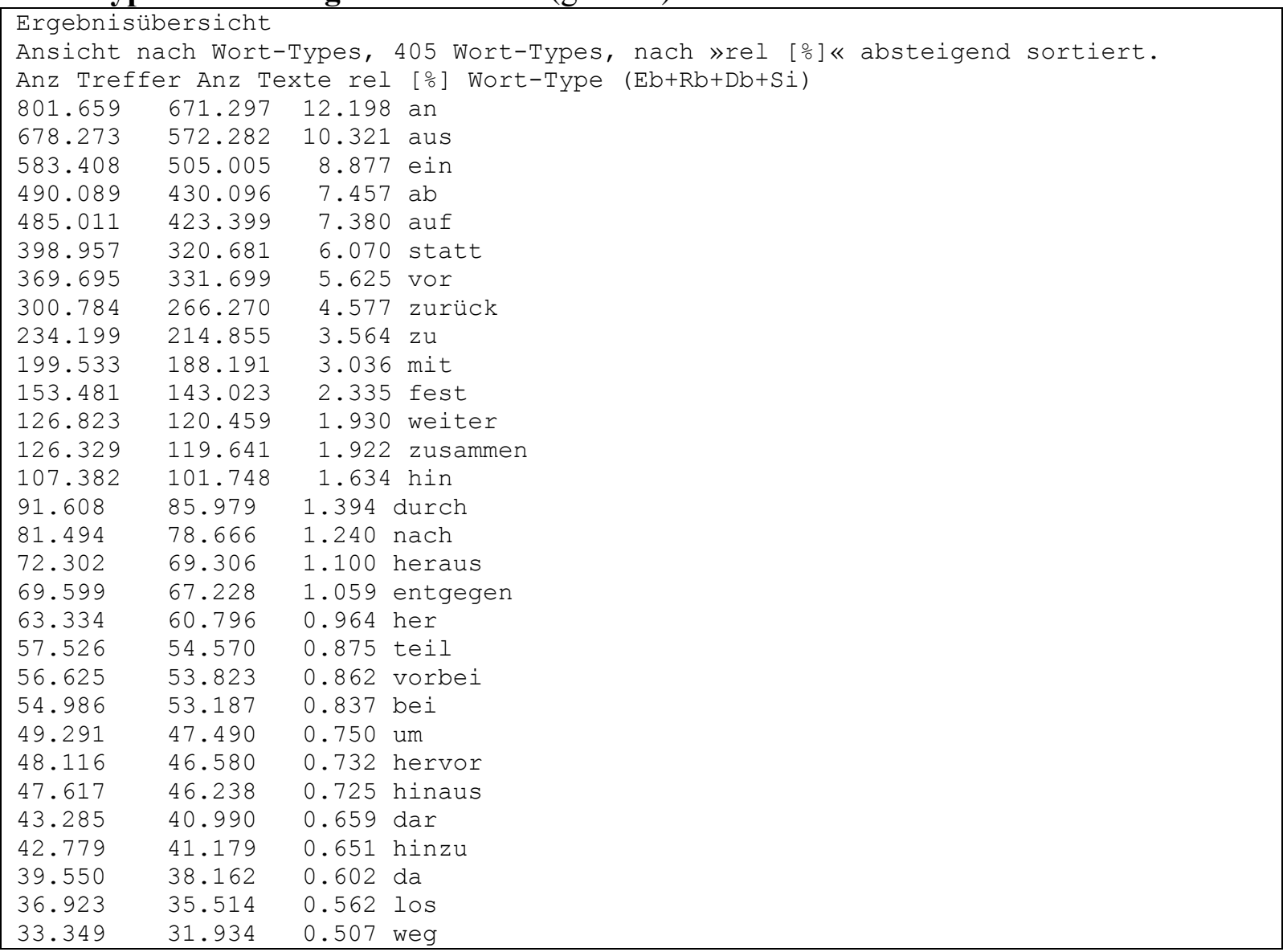

Abb. 108: Frequenz der Formen zur Abfrage: Verbzusätze (DeReKo, Archiv TAGGED)

Die Verben zu einzelnen Zusätzen findet man wie folgt: mit der Maus auf die Zeile mit dem gewünschten Zusatz fahren, mit einem Doppelklick werden die Konkordanzen abgerufen, in denen als KWIC der Verbzusatz dargestellt wird (in der Abb. 109 ist es fort). Nach dem Verb, 
zu dem der Verbzusatz gehört, muss entweder in der Konkordanzzeile gesucht werden oder es kann über die Kookkurrenzanalyse ermittelt werden (siehe weiter).

\begin{tabular}{|ll|}
\hline A09 in Bleichi und setzte seine Fahrt & fort, ohne sich um den Schaden zu kümmern. \\
A09 dennoch nicht abwenden», fährt er & fort. Zu Hause in den warmen Winterstuben \\
A09 hnson setzt 1963 ein, schreitet & fort bis ins Jahr 1970 und endet mit einem \\
A09 Damit setzte sich der Strukturwandel (...) & fort. Seit 1999 hörten 402 Bauern \\
A09 hübsch und sehr gescheit», fährt Leonor & fort. Sie bricht in Tränen aus. \\
\hline
\end{tabular}
Abb. 109: Konkordanzen zum Verbzusatz fort (Auswahl aus 28.671 Treffern)

Mit nur kursorischem Lesen dieser Konkordanzzeilen fällt auf, dass das häufigste Verb mit dem Zusatz fort vermutlich fortsetzen sein wird, gefolgt von fortfahren und fortschreiten.

\section{Recherche im InterCorp}

Noch einmal werden die Verbzusätze im InterCorp abgerufen.

- $\quad$ korpus.cz $\rightarrow \underline{\text { Login }} \rightarrow \underline{\text { KonText }} \rightarrow \underline{\text { Parallel corpus InterCorp }} \rightarrow \underline{\text { intercorp de }}$

- Query type: CQL

- CQL (Suchfenstereingabe): [tag=“PTKVZ“] $\rightarrow$ Search

Nachdem die Konkordanzen erschienen sind:

- Frequency $\rightarrow$ Node forms

Als Ergebnis bekommt man eine Frequenzliste der Verbzusätze nach Frequenz absteigend (gekürzt):

\begin{tabular}{|l|l|l|r|r|}
\hline & & \multicolumn{1}{c|}{ word } & \multicolumn{1}{c|}{ Freq } & Freq [\%] \\
\hline 1. & $\mathrm{p} / \mathrm{n}$ & an & 38,280 & 10.8 \\
\hline 2. & $\mathrm{p} / \mathrm{n}$ & auf & 34,032 & 9.6 \\
\hline 3. & $\mathrm{p} / \mathrm{n}$ & aus & 27,689 & 7.8 \\
\hline 4. & $\mathrm{p} / \mathrm{n}$ & vor & 24,259 & 6.8 \\
\hline 5. & $\mathrm{p} / \mathrm{n}$ & zu & 20,923 & 5.9 \\
\hline 6. & $\mathrm{p} / \mathrm{n}$ & ein & 19,950 & 5.6 \\
\hline 7. & $\mathrm{p} / \mathrm{n}$ & ab & 18,122 & 5.1 \\
\hline 8. & $\mathrm{p} / \mathrm{n}$ & zurück & 14,344 & 4.0 \\
\hline 9. & $\mathrm{p} / \mathrm{n}$ & mit & 11,035 & 3.1 \\
\hline 10. & $\mathrm{p} / \mathrm{n}$ & fest & 9,764 & 2.7 \\
\hline 11. & $\mathrm{p} / \mathrm{n}$ & hin & 9,270 & 2.6 \\
\hline 12. & $\mathrm{p} / \mathrm{n}$ & zusammen & 6,715 & 1.9 \\
\hline 13. & $\mathrm{p} / \mathrm{n}$ & nach & 6,215 & 1.7 \\
\hline 14. & $\mathrm{p} / \mathrm{n}$ & dar & 5,934 & 1.7 \\
\hline 15. & $\mathrm{p} / \mathrm{n}$ & um & 5,532 & 1.6 \\
\hline
\end{tabular}

\begin{tabular}{|l|l|l|r|r|}
\hline & & \multicolumn{1}{|c|}{ word } & Freq & Freq [\%] \\
\hline 16. & $\mathrm{p} / \mathrm{n}$ & weiter & 5,140 & 1.4 \\
\hline 17. & $\mathrm{p} / \mathrm{n}$ & heraus & 4,071 & 1.1 \\
\hline 18. & $\mathrm{p} / \mathrm{n}$ & fort & 3,846 & 1.1 \\
\hline 19. & $\mathrm{p} / \mathrm{n}$ & hervor & 3,808 & 1.1 \\
\hline 20. & $\mathrm{p} / \mathrm{n}$ & hinaus & 3,667 & 1.0 \\
\hline 21. & $\mathrm{p} / \mathrm{n}$ & $\mathrm{da}$ & 3,575 & 1.0 \\
\hline 22. & $\mathrm{p} / \mathrm{n}$ & her & 3,559 & 1.0 \\
\hline 23. & $\mathrm{p} / \mathrm{n}$ & durch & 3,485 & 1.0 \\
\hline 24. & $\mathrm{p} / \mathrm{n}$ & herum & 3,446 & 1.0 \\
\hline 25. & $\mathrm{p} / \mathrm{n}$ & bei & 3,305 & 0.9 \\
\hline 26. & $\mathrm{p} / \mathrm{n}$ & statt & 3,285 & 0.9 \\
\hline 27. & $\mathrm{p} / \mathrm{n}$ & entgegen & 2,507 & 0.7 \\
\hline 28. & $\mathrm{p} / \mathrm{n}$ & hinzu & 2,466 & 0.7 \\
\hline 29. & $\mathrm{p} / \mathrm{n}$ & vorbei & 2,321 & 0.7 \\
\hline 30. & $\mathrm{p} / \mathrm{n}$ & hinein & 2,124 & 0.6 \\
\hline
\end{tabular}

Abb. 110: Frequenzliste der Verbzusätze (InterCorp)

Die einzelnen Posten im Kopf der Tabelle kann man anklicken und so nach diesem Argument neu sortieren. Nach dem Klicken auf word werden die Treffer alphabetisch sortiert. 


\section{Fazit:}

Die Rechercheergebnisse aus DeReKo und InterCorp sind durchaus vergleichbar: In den Abb. 108 und 110 sind die 30 häufigsten Verbzusätze im jeweiligen Korpus dargestellt. Die Verbzusätze fort-, herum-, hinein-, los-, teil-, und weg-fehlen zwar in einer der beiden Ergebnislisten. Sie kommen jedoch jeweils in den unmittelbar nachkommenden Positionen (31 bis 60) vor. Vergleicht man also die häufigsten 60 Verbzusätze in beiden Korpora, ergibt sich das Bild über die häufigsten Verbzusätze in geschriebenem Deutsch:

ab-, an-, auf-, aus-, bei-, da-, dar-, durch-, ein-, entgegen-, fest-, fort-, her-, heraus-, herum-, hervor-, hin-, hinaus-, hinein-, hinzu-, los-, mit-, nach-, statt-, teil-, um-, vor-, vorbei-, weg-, weiter-, zu-, zurück- und zusammen-.

In einem weiteren Schritt wird festgestellt, welche Verben diese häufigsten Verbzusätze haben.

Fragestellung 2: Zu welchen Grundverben tritt der häufigste Verbzusatz (an) zu?

Der Anfang der Recherche hat denselben Ablauf wie in der vorherigen Fragestellung. Die Fortsetzung wird jedoch nur im InterCorp durchgeführt.

Nachdem die Liste mit den häufigsten Verbzusätzen abgerufen worden ist (Abb. 110), klickt man in der zweiten Spalte auf p (= positiver Filter), wie der Ausschnitt aus der Liste (Abb. 110a) zeigt:

\begin{tabular}{|l|l|l|l|l|}
\hline & & word & Freq & Freq [\%] \\
\hline 1. & $\underline{\mathrm{p}} / \underline{\mathrm{n}}$ & an & 38,280 & 10.8 \\
\hline 2. & $\underline{\mathrm{p}} / \underline{\mathrm{n}}$ & auf & 34,032 & 9.6 \\
\hline 3. & $\underline{\mathrm{p}} / \underline{\mathrm{n}}$ & aus & 27,689 & 7.8 \\
\hline 4. & $\underline{\mathbf{p}} / \underline{\mathrm{n}}$ & vor & 24,259 & 6.8 \\
\hline
\end{tabular}

Abb. 110a: Ausschnitt aus der Frequenzliste der Verbzusätze (Abb. 110, vorherige Seite)

Danach erscheinen unsortierte Konkordanzen, in denen an als KWIC steht (am Ende des Satzes). Die Verben zum Verbzusatz an lassen sich am effektivsten über die Kollokationsberechnung ermitteln:

- Collocation $\rightarrow \underline{\text { Custom... }}$

Einstellung der Kollokationsanalyse (Collocations candidates):

- Attribute: Lemma

- In the range from: -10 to

- Minimum frequency in corpus:

- Minimum frequency in given range: Make Candidate List

(spielt keine Rolle für diese Abfrage) (spielt keine Rolle für diese Abfrage)

Aus der Liste der insgesamt 5.396 automatisch berechneten Kollokatoren (in der Abb. 111 auf der folgenden Seite siehe links oben die Angabe Total) müssen alle Nicht-Verben manuell aussortiert werden (in der Abb. 111 sind sie durchgestrichen). 
Kollokationspartner zum Verbzusatz an (gekürzt)

\begin{tabular}{|c|c|c|c|c|c|}
\hline \multicolumn{3}{|c|}{ Total: 5396} & \multirow[b]{2}{*}{ Freq } & \multirow[b]{2}{*}{ log likelihood } & \multirow[b]{2}{*}{ logDice } \\
\hline & & & & & \\
\hline 1. & $\mathrm{p} / \underline{\mathrm{n}}$ & sehen & 5249 & 42336.240 & 10.649 \\
\hline 2. & $\mathrm{p} / \underline{\mathrm{n}}$ & fangen & 2028 & 25391.592 & 10.628 \\
\hline 3. & $\mathrm{p} / \underline{\mathrm{n}}$ & nehmen & 2637 & 20227.721 & 10.100 \\
\hline 4. & $\mathrm{p} / \underline{\mathrm{n}}$ & starren & 1261 & 14800.071 & 9.966 \\
\hline 5. & $\mathrm{p} / \underline{\mathrm{n}}$ & schauen & 1001 & 9907.180 & 9.557 \\
\hline 6. & $\underline{p} / \underline{n}$ & blicken & 954 & 9084.744 & 9.465 \\
\hline 7. & $\underline{p} / \underline{n}$ & rufen & 1005 & 8173.972 & 9.353 \\
\hline 8. & $\mathrm{p} / \underline{\mathrm{n}}$ & erkennen & 922 & 7679.240 & 9.288 \\
\hline 9. & $\mathrm{p} / \underline{\mathrm{n}}$ & wenden & 841 & 7341.082 & 9.231 \\
\hline 10. & $\underline{\mathrm{p}} / \underline{\mathrm{n}}$ & bieten & 825 & 6780.409 & 9.145 \\
\hline 11. & $\underline{p} / \underline{n}$ & ef & 7774 & 40474.744 & 9.066 \\
\hline 12. & $\underline{p} / \underline{n}$ & 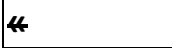 & 2839 & 15050.463 & 9.011 \\
\hline 13. & $\underline{p} / \underline{n}$ & kommen & 1600 & 8943.576 & 8.946 \\
\hline 14. & $\underline{p} / \underline{n}$ & Sie|sie & 3123 & 15952.103 & 8.920 \\
\hline 15. & $\mathrm{p} / \underline{\mathrm{n}}$ & schließen & 718 & 5279.247 & 8.854 \\
\hline
\end{tabular}

\begin{tabular}{|c|c|c|c|c|c|}
\hline & & & Freq & log likelihood & logDice \\
\hline 16. & $\mathrm{p} / \underline{\mathrm{n}}$ & ziehen & 735 & |5007.362 & 8.773 \\
\hline 17. & $\mathrm{p} / \underline{\mathrm{n}}$ & halten & 930 & |5589.093 & 8.766 \\
\hline 18. & $\mathrm{p} / \underline{\mathrm{n}}$ & hören & 673 & 4390.259 & 8.622 \\
\hline 19. & $\underline{p} / \underline{\mathrm{n}}$ & sie & 4343 & 19057.474 & 8.503 \\
\hline 20. & $\mathrm{p} / \underline{\mathrm{n}}$ & ich & 6214 & 27162.864 & 8.500 \\
\hline 21. & $\mathrm{p} / \underline{\mathrm{n}}$ & kündigen & 431 & 5003.835 & 8.487 \\
\hline 22. & $\underline{p} / \underline{n}$ & sich & 4075 & 17745.623 & 8.476 \\
\hline 23. & $\mathrm{p} / \underline{\mathrm{n}}$ & zünden & 419 & 5932.286 & 8.469 \\
\hline 24. & $\underline{p} / \underline{n}$ & Auge & 645 & 3900.646 & 8.465 \\
\hline 25. & $\mathrm{p} / \mathrm{n}$ & darauf & 653 & 3664.029 & 8.353 \\
\hline 26. & $\underline{p} \underline{\mathrm{n}}$ & du & 1274 & 5797.522 & 8.327 \\
\hline 27. & $\underline{p} / \underline{n}$ & $?$ & 1640 & 7098.896 & 8.293 \\
\hline 28. & $\underline{p} / \underline{n}$ & lächeln & 406 & 3423.408 & 8.291 \\
\hline 29. & $\underline{p} / \underline{n}$ & 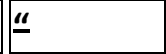 & 975 & 4517.483 & 8.245 \\
\hline 30. & $\underline{p} / \underline{\mathrm{n}}$ & wieder & 785 & 3858.788 & 8.244 \\
\hline
\end{tabular}

Abb. 111: Frequenzliste der Kollokatoren zum Verbzusatz an (InterCorp)

Als Beispiel wird das zweit häufigste Verb in der Liste abgerufen: anfangen. Durch Klicken auf $\underline{p}$ (=positiver Filter) neben einem Verb (fangen) kann man Konkordanzzeilen mit dem Verb anfangen mit abgetrenntem an abrufen:

\begin{tabular}{|c|c|c|}
\hline $\begin{array}{r}\text {... fängt er endlich } \\
\ldots \text {, sie ließ sich ihre Körbe bringen und fing } \\
\text { Dies fängt bereits bei der Wortwahl (...) } \\
\ldots \text {, zeigte in die Ferne und fing } \\
\text { Dann fing ich } \\
\text { Wir fangen immer damit } \\
\text { und sofort fing sie }\end{array}$ & $\begin{array}{l}\text { an } \\
\text { an } \\
\text { an } \\
\text { an } \\
\text { an } \\
\text { an } \\
\text { an }\end{array}$ & $\begin{array}{l}\text {, die Notwendigkeit (...) zu betonen . } \\
\text { wie eine Gemüsefrau auf dem Markt ... } \\
\text {, wobei bekanntlich schon die ... } \\
\text {, mir (...) etwas zu erklären . } \\
\text {, es wirklich zu genießen } \\
\text {, öffentliche Ausgaben zu beschneiden } \\
\text { zu dichten : }\end{array}$ \\
\hline
\end{tabular}

Abb. 112: Auswahl aus Konkordanzen zum positiven Filter des Kollokators fangen in der Abb. 111 (InterCorp)

\section{Fazit:}

Der Verbzusatz an kommt am häufigsten mit diesen Verben vor: sehen (ansehen), fangen (anfangen), nehmen (annehmen), starren (anstarren), schauen (anschauen), blicken (anblicken), rufen (anrufen), erkennen (anerkennen), wenden (anwenden), bieten (anbieten) und kommen (ankommen).

Analog können auch Verben mit anderen Zusätzen festgestellt werden. (Verben, zu denen die zehn häufigsten Verbzusätze zutreten, sind in der im Kap. 7.2 aufgelistet.) Zu allen können auch parallele Passagen in einer anderen Sprache angezeigt werden, falls dies am Anfang der Recherche eingestellt wurde, wie die folgende Fragestellung 3 zeigt. 
Fragestellung 3: Was sind die häufigsten Verben mit dem Zusatz fest? Was entspricht dem deutschen Verb festhalten im Englischen?

Recherchiert wird im InterCorp, es gibt mehrere Wege, wie man das ganze Konjugationsparadigma des Verbs festhalten (analog aller anderen Verben mit trennbarem Zusatz) abrufen kann:

- $\quad$ korpus.cz $\rightarrow \underline{\text { Login }} \rightarrow \underline{\text { KonText }} \rightarrow \underline{\text { Parallel corpus InterCorp }} \rightarrow \underline{\text { intercorp de }}$

- Aligned corpora $\rightarrow$ intercorp_en $\rightarrow$ Add Query type: CQL

- CQL (Suchfenstereingabe): [tag=“PTKVZ"\&word=“fest"] $\rightarrow$ Search

Nachdem die Konkordanzen erschienen sind:

- Collocations $\rightarrow$ Custom...

Einstellung der Kollokationsanalyse (Collocations candidates):

- Attribute: Lemma

- Range: -10 to $0 \rightarrow$ Make Candidate List

Das Ergebnis dieser Abfrage ist eine automatisch erstellte Liste, in der auch andere Kollokationspartner erscheinen - Wörter, die signifikant oft mit dem Verbzusatz fest- vorkommen. Diese müssen ausgeblendet werden, denn für die Auswahl sind nur Verben relevant. Die manuell ausgewählten Verben sind in der Abb. 113 aufgelistet und nach der Wahrscheinlichkeit des Vorkommens mit fest (Signifikanzmaß logDice) sortiert.

\begin{tabular}{|l||l||l||l|l|}
\hline & & & Freq & logDice \\
\hline 1. & $\mathrm{p} / \mathrm{n}$ & stellen & 4043 & 11.305 \\
\hline \hline 2. & $\mathrm{p} / \mathrm{n}$ & legen & 1528 & 10.727 \\
\hline 3. & $\mathrm{p} / \mathrm{n}$ & halten & 921 & 9.506 \\
\hline \hline. & $\mathrm{p} / \mathrm{n}$ & setzen & 319 & 8.309 \\
\hline \hline 5. & $\mathrm{p} / \mathrm{n}$ & stehen & 543 & 8.230 \\
\hline
\end{tabular}

\begin{tabular}{|l||l||l||l||l|}
\hline & & & Freq & logDice \\
\hline \hline 12. & $\mathrm{p} / \mathrm{n}$ & klammern & 48 & 7.264 \\
\hline
\end{tabular}

$\cdots$

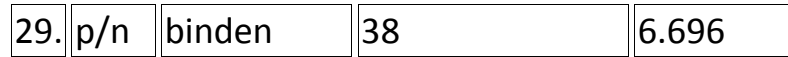

$\ldots$

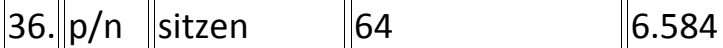

Abb. 113: Basisverben mit dem Verbzusatz fest (Auswahl)

Aus dieser Tabelle lassen sich die häufigsten Verben mit dem Zusatz fest ableiten: feststellen, festlegen, festhalten, festsetzen, feststehen, festklammern, festbinden, festsitzen.

Es muss betont werden, dass es sich in den Kollokationstabellen (auch Abb. 113) um potentielle Kandidaten handelt, die automatisch berechnet worden sind. Aus diesem Grund sollten immer die Konkordanzzeilen stichpunktartig durchgesehen werden, ob die Ergebnisse den Erwartungen entsprechen. Die folgenden Konkordanzzeilen zeigen, welche Ergebnisse zu erwarten sind, wenn man sich auf das Verb sitzen (Abb. 113) konzentriert und die Konkordanzen durch Klicken auf $\underline{p}$ abruft:

(1) wir saßen in der sowjetisch besetzten Zone fest, Mutter sogar freiwillig ,

(2) Im Augenblick sitzen Sie hier fest, weil wir Sie nicht auf Kaution

(3) eine Tatsache ", sagte Kaninchen . " Du sitzt fest . "

(4) Er saß oft auf seiner Veranda und stellte fest, ob die Züge pünktlich vorbeifuhren. 
Die meisten Konkordanzen beinhalten tatsächlich das verbale Kompositum festhalten (1), (2), (3). Es können aber auch „Fehler“ vorkommen, wie das Beispiel (4) zeigt. Dennoch sind die Ergebnisse überzeugend, die absolut überwiegende Menge ist richtig berechnet, man kann sie als tragfähig für eine weitere Forschung betrachten.

\section{Fazit:}

Die Verben feststellen, festlegen, festhalten, festsetzen und feststehen sind die häufigsten mit dem Zusatz fest-. Ein wenig geringer, wenn immer noch hochfrequentiert sind festklammern, festbinden, und festsitzen. Vergleichsweise seltener sind die Verben feststecken, festmachen, festnageln, festkrallen, festbeißen, festdrücken und festsaugen (in der Verbindung sich an jmdn. festsaugen). Andere Verben mit fest- haben verhältnismäßig geringe Frequenz in der geschriebenen Sprache.

Konkordanzzeilen werden wieder durch Anklicken des positiven Filters (p) abgerufen, so auch die Konkordanzen, in denen festhalten eine Klammer (...hält ... fest.) bildet.

Jetzt wird nach den englischen Entsprechungen zum Verb festhalten gesucht. Zuerst muss das ganze Konjugationsparadigma des Verbs abgerufen werden. Dies muss wegen der Trennbarkeit des Zusatzes fest in zwei Schritten erfolgen:

\section{Schritt 1:}

- Query type: CQL

- CQL (Suchfenstereingabe): [lemma="halten"] []* [word="fest"] within <s id=".*"/> $\rightarrow$ Search

Die Abfrage bedeutet: Suche alle Formen von halten, davon rechts im beliebigen Abstand das Wort fest, allerdings nur bis zur Grenze des Satzes. (Eine Liste der Abfragemöglichkeiten im Modus CQL ist im Kap.VII angeführt.) Diese Abfrage ist eine Erweiterung der vorherigen Abfrage um das Argument within (Suche innerhalb eines definierten Korpusabschnittes, in diesem Fall Satz).

\section{Schritt 2:}

- Query type: CQL

- CQL (Suchfenstereingabe): [lemma="festhalten"] $\rightarrow$ Search

Diesen zweiten Schritt kann man als Standard bezeichnen. So werden üblicherweise alle Formen einer Grundform abgerufen (vgl. dazu auch Studie 4). Als Ergebnisse bekommt man Konkordanzen mit allen Formen von festhalten, die als eine ununterbrochene Zeichenkette (ein Wort) im Korpus vorkommen. Ein Ausschnitt davon ist in der Abb. $115 \mathrm{zu}$ sehen.

Die Konkordanzzeilen zu jeder Abfrage erscheinen gleich mit einer Parallelpassage im Englischen (Abb. 114 und 115 auf der nächsten Seite). Diese Sprache wurde eben am Anfang der Recherche ausgewählt (siehe vorherige Seite oben). 


\section{Parallelpassagen Deutsch - Englisch}

\begin{tabular}{|c|c|c|}
\hline & intercorp_de & intercorp_en \\
\hline (1) & $\begin{array}{l}\text { Sie drückte ihn an sich und hielt ihn eine } \\
\text { lange Minute ganz fest. }\end{array}$ & She squeezed him, and held him tight. \\
\hline (2) & $\begin{array}{l}\text { Auch wenn mir das nicht gelingt, halte ich } \\
\text { an meiner eigenen Meinung und meinem } \\
\text { Urteil fest. }\end{array}$ & $\begin{array}{l}\text { And if that doesn't work, I 'll have to stick with my } \\
\text { own opinions and judgment . }\end{array}$ \\
\hline (3) & $\begin{array}{l}\text { Der Blick seiner blauen Augen hielt sie fest } \\
\text { während er allmählich wieder zu Atem kam . }\end{array}$ & $\begin{array}{l}\text { Langdon caught his breath as his blue eyes held her } \\
\text { firmly. }\end{array}$ \\
\hline (4) & $\begin{array}{l}\text { Sie hielt sich einfach nur an ihm fest, und } \\
\text { das war bei Gott ein fantastisches Gefühl. }\end{array}$ & All she did was hang on, and, God, it felt great \\
\hline (5) & $\begin{array}{l}\text { Trotzdem halte ich an ihnen fest, trotz allem } \\
\text {, weil ich noch immer an das innere Gute im } \\
\text { Menschen glaube. }\end{array}$ & $\begin{array}{l}\text { Yet I cling to them because I still believe, in spite of } \\
\text { everything, that people are truly good at heart. }\end{array}$ \\
\hline (6) & $\begin{array}{l}\text { Ich hielt sie fest ; hätte ich das nicht getan } \\
\text { dann wäre sie von der Schaukel gefallen. }\end{array}$ & $\begin{array}{l}\text { I held her firmly; if not, she would 've fallen onto } \\
\text { the porch. }\end{array}$ \\
\hline (7) & $\begin{array}{l}\text { Sie verschränkte die Arme vor der Brust } \\
\text { und hielt sich an den Ellbogen fest. }\end{array}$ & $\begin{array}{l}\text { She crossed her arms over her middle and hugged } \\
\text { her elbows. }\end{array}$ \\
\hline (8) & $\begin{array}{l}\text { Isebel war eine Konservative und hielt am } \\
\text { alten Glauben fest. }\end{array}$ & $\begin{array}{l}\text { Jezebel was a conservative, sticking to the old } \\
\text { beliefs against the new ones. }\end{array}$ \\
\hline (9) & $\begin{array}{l}\text { Arthur rappelte sich hoch und hielt sich } \\
\text { ängstlich fest. }\end{array}$ & $\begin{array}{l}\text { Arthur struggled to his feet and hugged himself } \\
\text { apprehensively. }\end{array}$ \\
\hline
\end{tabular}

Abb. 114: Auswahl aus den Konkordanzzeilen zur Abfrage festhalten (abgetrennt) und ihre Entsprechungen im Englischen (InterCorp_de-en)

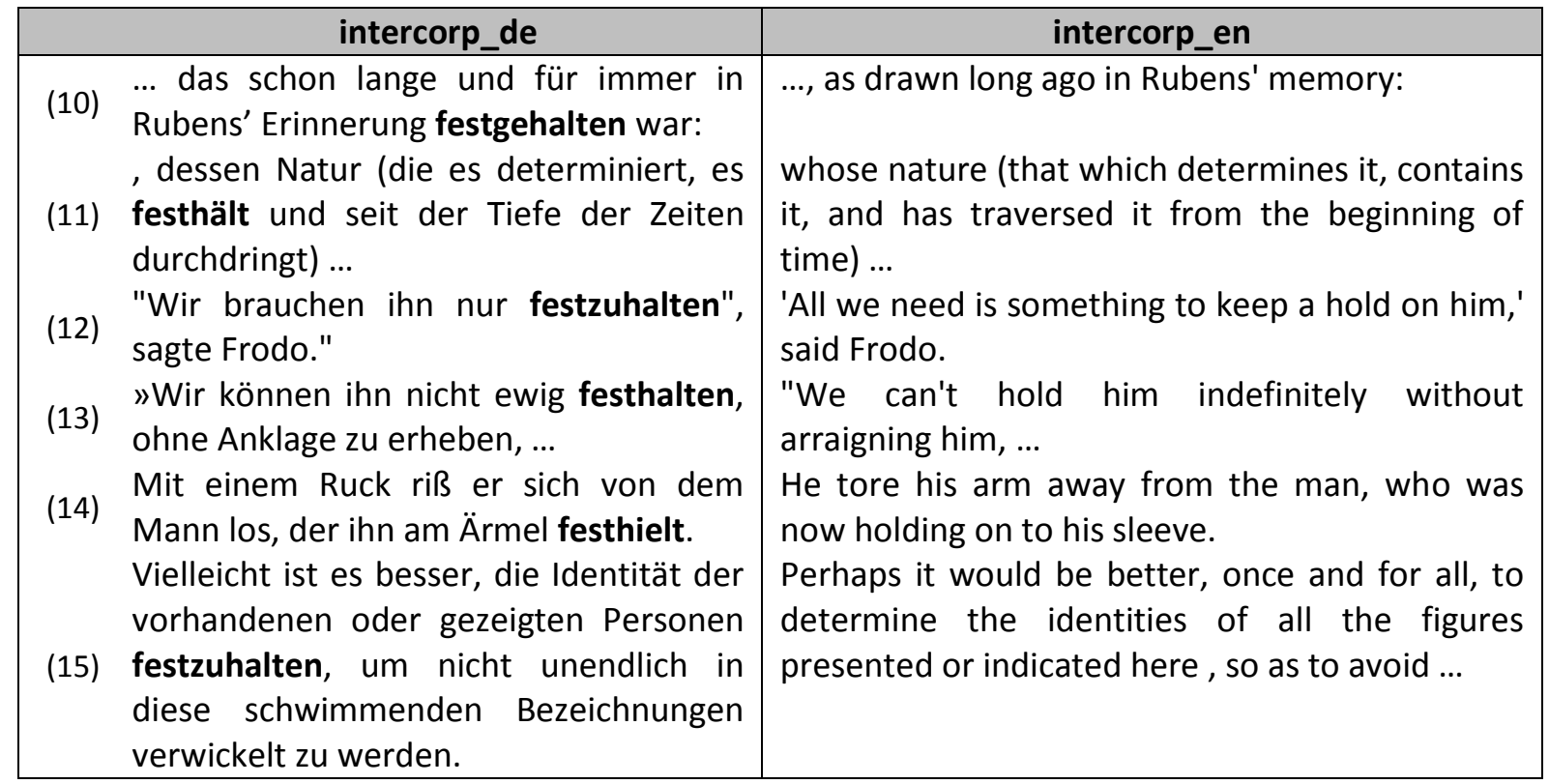

Abb. 115: Auswahl aus Konkordanzzeilen zur Abfrage festhalten (lemmatisiert) und ihre Entsprechungen im Englischen (InterCorp_de-en)

\section{Fazit:}

Aus den Parallelpassagen in den Abb. 114 und 115 ist ersichtlich, wie sich das deutsche Wort festhalten verhält. Im Kontext ist es ein Bestandteil der folgenden Phraseme und Chunks: sich festhalten (9), 
(sich) an jmdm./ etw. festhalten $(2,4,5,8,14)$,

jmdn. /sich/ etw. an etw. festhalten (7),

jmdn. letw. festhalten $(1,3,6,11,12,13,15)$

in ... festgehalten sein (10).

Die englischen Entsprechungen decken interessante Facetten im sprachlichen Kontrast auf:

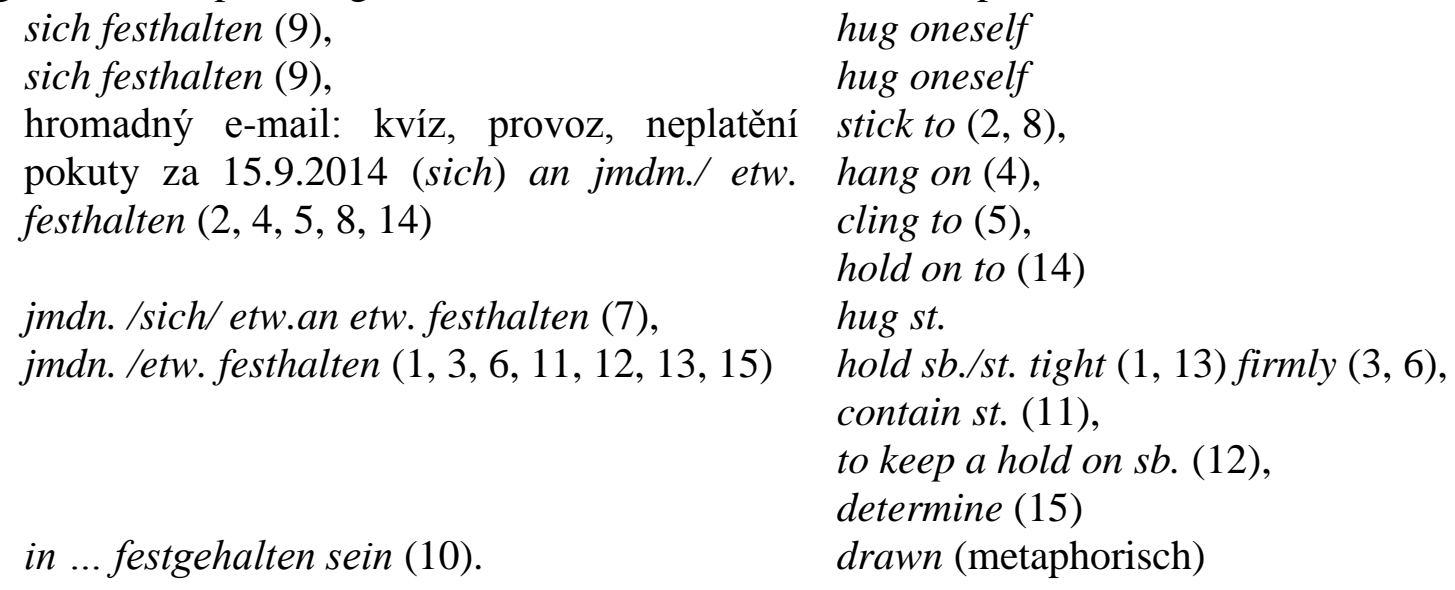

Für lexikographische Zwecke müsste jedes intersprachliche Paar noch gründlich untersucht und statistisch ausgewertet werden. Dennoch umreißen auch diese wenigen Pendants im Englischen ein Bild über die Entsprechungen, die ein herkömmliches Wörterbuch kaum liefern könnte. Diesen Vorteil nutzen eher erfahrene Lehrer/-innen, Linguist/-innen oder Student/-innen. Anfänger/-innen könnten mit diesem Überangebot an unterschiedlichen Entsprechungen natürlich überfordert sein. 


\section{Studie 8: Verbalkomplexe}

Die Problematik der deutschen Verbalkomplexe wird in jeder Grammatik behandelt, in Lehrbüchern wird ihr auch relativ viel Platz gewidmet. Selten wird jedoch auf (regionale) Unterschiede in der Reihenfolge innerhalb der Verbalkomplexe hingewiesen.

Das folgende Phänomen ist zwar allgemein bekannt, in gängigen Grammatiken wird es allerdings nicht ,plurizentrisch“ behandelt und (meines Wissens) wurde es an einer repräsentativen Anzahl von Beispielen bisher noch nicht belegt. Das folgende Beispiel für die Korpusarbeit behandelt dreiteilige Verbalkomplexe am Ende eines Nebensatzes.

Nach Duden - Grammatik (2005: 480-482), Zifonun (1997: 1285-1287) oder Engel (1988: 445-447) ist die ,grammatikalische“" Reihenfolge eines dreiteiligen Verbalkomplexes wie folgt:

finites Auxiliar - Infinitiv Vollverb - Infinitiv des Modal-/oder AcI-Verbs ${ }^{41}$, also prinzipiell wie in (1).

(1) Das hätte bedeutet, dass die Bauarbeiten spätestens 2009 hätten beginnen müssen. ${ }^{42}$

Andere Reihenfolgen, auch die in (2), wurden von Lehrern/-innen oft als ,falsch“ gekennzeichnet. Die österreichische sprachliche Realität kennt aber eben diese Abfolge:

Infinitiv Vollverb - finites Auxiliar - Infinitiv Modalverb:

(2) »Nur das Geschwätz, das er halt schreiben hat müssen, sagt der Doktor Forster«, erklärte Valerie nervös... ${ }^{43}$

Ob diese (2) Reihenfolge in Österreich zur Standard- oder Randerscheinung gehört, kann mit Korpusdaten belegt werden. Für die Recherche eignet sich besser das DeReKo als andere Korpora, denn hier sind die Ursprungsländer der Texte sehr einfach abrufbar und die Ergebnisse äußerst übersichtlich.

\section{Recherche im InterCorp}

Zuerst wird die Reihenfolge wie in (1) abgerufen (Abfrage 1), dann die Reihenfolge wie in (2) (Abfrage 2).

Abfrage 1: finites Auxiliar - Infinitiv Vollverb - Infinitiv des Modal-/oder AcI-Verbs

- Cosmas $\mathbf{I I}_{\text {web }} \rightarrow \underline{\text { Recherche }}$

- Archiv: TAGGED-T - Archiv morphosyntakt. annotierter Korpora (TreeTagger)

- Korpus: TAGGED-T-öffentlich - alle öffentlichen Korpora des Archivs TAGGED-T

- Optionen:

Expansionslisten abwählen $\rightarrow$ Übernehmen

Ergebnispräsentation

Länderansicht auswählen $\rightarrow$ Übernehmen

- Suchanfrage (Eingabe ins Suchfeld): $\underline{\text { MORPH-Assistent }} \rightarrow$ Verben: finit, ohne Imperativ $\rightarrow$ Klasse: auxiliar auswählen $\rightarrow$ Übernehmen

${ }^{41}$ AcI = lat. Accusativ cum Infinitivo, grundsätzlich handelt es sich im Deutschen um Verben mit Infinitiv ohne $z u$ (Wahrnehmungsverben, lassen etc.).

42 St. Galler Tagblatt, 3.1.2009, S. 31 (DeReKo).

43 Simmel, Johannes Mario: Und Jimmy ging zum Regenbogen (InterCorp). 
Im Suchfeld erscheint: MORPH(VRB fin a), dann den Cursor hinter die Klammer positionieren und mithilfe des morphologischen Assistenten das nächste Verb definieren:

MORPH-Assistent $\rightarrow$ Verben: Infinitiv $\rightarrow$ Klasse: voll auswählen $\rightarrow$ Übernehmen

Dieser Schritt ergibt im Suchfeld: MORPH(VRB fin a)MORPH(VRB inf v). Jetzt den Cursor wieder hinter der Klammer positionieren und das letzte Verb definieren:

MORPH-Assistent $\rightarrow$ Verben: Infinitiv $\rightarrow$ Klasse: modal auswählen $\rightarrow$ Übernehmen

Nach diesem Schritt sind alle Verben im Suchfeld definiert, die Abfrage sieht folgend aus:

MORPH(VRB fin a)MORPH(VRB inf v)MORPH(VRB inf $\mathrm{m}$ )

Nun fehlt die rechte Satzgrenze zu markieren, wo sich der Verbalkomplexe befinden sollen:

$\# I N(R)<s>$

Dieser Schritt eliminiert aus den Konkordanzen zufällige Anhäufungen von Verben.

Die ganze Eingabe lautet jetzt (Achtung! Leerzeichen an den richtigen Stellen hinzufügen!):

MORPH(VRB·fin·a) MORPH(VRB·inf·v)·MORPH(VRB·inf·m) \#IN(R) $<$ s $>\rightarrow$ Suchen

Die Abfrage bedeutet: Suche finites Hilfsverb (VRB fin a), Infinitiv eines Vollverbs (VRB inf v) und Infinitiv eines Modalverbs (VRB inf $m$ ) in dieser Reihenfolge und am Ende (\#IN(R) $=$, rechtes Ende“) eines Satzes $(<s>)$.

Das Ergebnis sind über 20.000 Treffer, aus denen in die Abb. 116 per Zufall 10 Konkordanzzeilen ausgewählt wurden.

Verbalkomplexe im (DeReKo, Tagged-T)

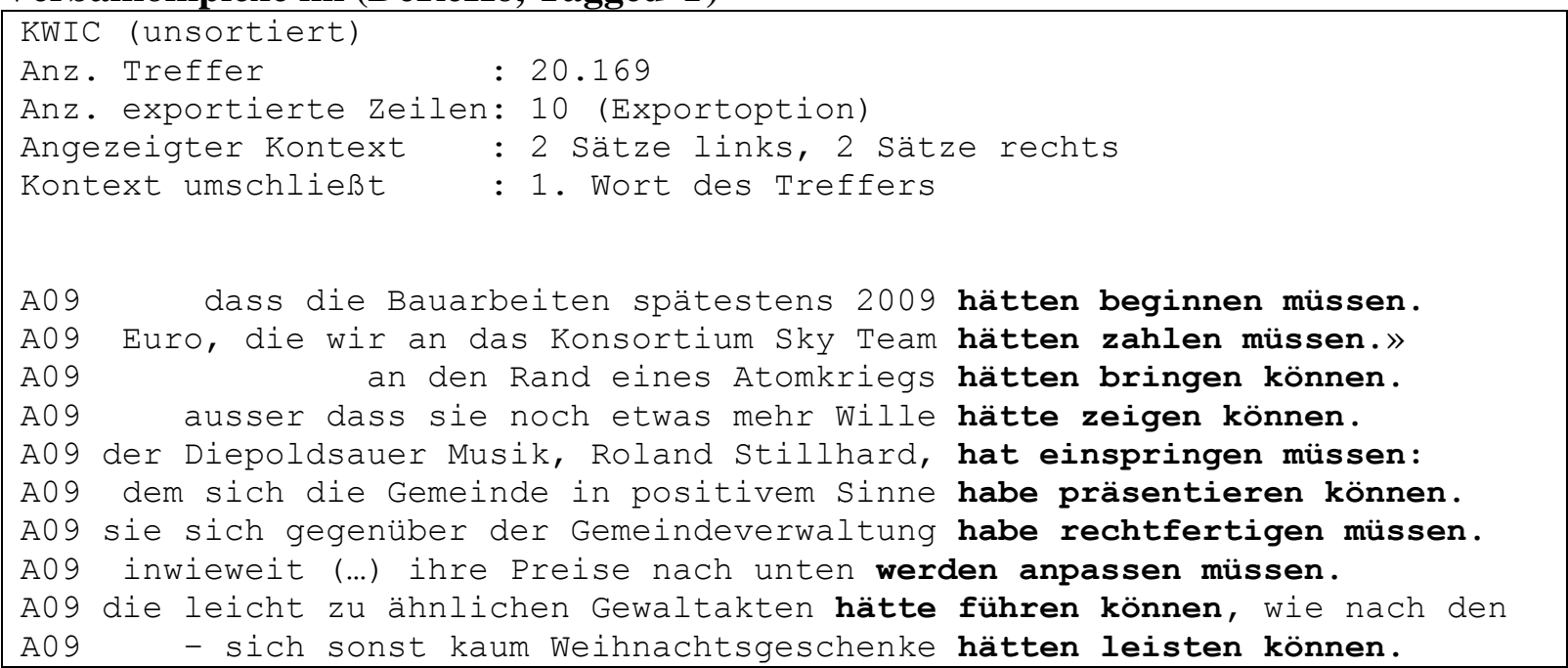

Abb. 116: Auswahl aus den Konkordanzzeilen zur Abfrage Verbalkomplex: fin. Auxiliar - Inf. Vollverb - Inf. Modalverb (DeReKo)

Die Konkordanzen zeigen ein zufriedenstellendes Bild. Es handelt sich tatsächlich um Verbalkomplexe am Ende von Nebensätzen. Die Fehlerquote ist sehr gering.

Um die Verteilung dieser Verbalkomplexe in Texten einzelner Länder zu beobachten, muss jetzt die Länderansicht aktiviert werden. 
Die Länderansicht erreicht man über den Befehl Ergebnisse $\rightarrow$ Länderansicht. Die Verteilung dieser Verbalkomplexe in deutschen (D), österreichischen (A) und schweizerischen $(\mathrm{CH})$ Texten zeigt die Abb. 117. Die Ergebnisse sind nach der relativen Häufigkeit (berechnet pro eine Mio. Worte) gereiht.

\begin{tabular}{|c|c|c|c|c|c|c|}
\hline & Treffer & rel. Häuf. & Texte & von & $\underline{\text { bis }}$ & Land \\
\hline 田 & 331 & $1.385 \mathrm{pMW}$ & 331 & 1997 & 2009 & $\mathrm{CH}$ \\
\hline 田 & 612 & $0.945 \mathrm{pMW}$ & 612 & 1998 & 2009 & D \\
\hline 田 & 57 & $0.427 \mathrm{pMW}$ & 57 & 1999 & 2009 & A \\
\hline & 1.000 & $0.980 \mathrm{pMW}$ & 1.000 & 1997 & 2009 & 3 Lände \\
\hline
\end{tabular}

Abb. 117: Länderansicht zur Abfrage Verbalkomplex: fin. Auxiliar-Inf. Vollverb-Inf. Modalverb (DeReKo)

Dieses Rechercheergebnis (Abb. 117) wird mit dem Ergebnis in der Abfrage 2 (Abb. 118 auf der folgenden Seite) verglichen.

\section{Abfrage 2:}

In dieser Abfrage wird nach der „österreichischen“ Reihenfolge der Verben im Verbalkomplex gesucht, wie sie im Beispielsatz (2) auf Seite 155 erscheint. Der Anfang der Abfrage (die Archiv- und Korpuswahl sowie die Einstellung der Optionen) ist gleich wie in der Abfrage 1.

- Suchanfrage (Eingabe ins Suchfeld):

Es wäre logisch die Suchanfrage an den Korpusmanager COSMAS II so zu formulieren, wie es in der Abfrage 1 der Fall war, nur mit getauschten Positionen. Also: Infinitiv des Vollverbs - Finites Hilfsverb - Infinitiv des Modalverbs am Satzende. „Übersetzt“ in die COSMAS II-Sprache:

MORPH(VRB inf v)·MORPH(VRB fin a) $\cdot M O R P H(V R B$ inf $m) \cdot \# I N(R)<s>$

Diese Anfrage ergibt jedoch keine Ergebnisse. Der Grund ist naheliegend: die Reihenfolge wird eben als „ungrammatisch“ betrachtet und dies wirkt sich auch auf die Annotation des Korpus aus. Bei der Annotation wird nämlich jedem Wort sein Tag u.a. nach seiner Umgebung zugewiesen ${ }^{44}$. Deswegen muss man auf eine einfachere Eingabe ausweichen und statt Infinitiv modal (die letzte Position im Verbalkomplex) Infinitiv „,beliebig“ eingeben.

Die Eingabe lautet jetzt (Achtung! Leerzeichen an den richtigen Stellen hinzufügen!)

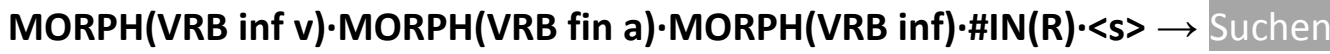

Die Abfrage bedeutet: Suche Infinitiv eines Vollverbs (VRB inf v), finites Hilfsverb (VRB fin a) und Infinitiv eines beliebigen Verbs (VRB inf) in dieser Reihenfolge und am Ende $(\# I N(R)=$ „rechtes Ende“) eines Satzes (<s>).

Nachdem die Treffer geliefert worden sind, kann man die Länderansicht betrachten. Sie ist in der Abb. 118 auf der nächsten Seite zu sehen.

44 Fein annotierte Korpora (z.B. deTenTen) können diese Abfrage beantworten und liefern zufriedenstellende Ergebnisse. Ihr Nachteil ist wiederum, dass sie oft keine Länderansicht ermöglichen. 


\begin{tabular}{|c|c|c|c|c|c|c|}
\hline & Treffer & rel. Häuf. & Texte & von & bis & Land \\
\hline$\boxplus$ & 184 & $1.377 \mathrm{pMW}$ & 184 & 1999 & \begin{tabular}{|l|}
2009 \\
\end{tabular} & A \\
\hline 田 & 81 & $0.339 \mathrm{pMW}$ & 81 & 1997 & 2009 & $\mathrm{CH}$ \\
\hline 田 & 165 & $0.255 \mathrm{pMW}$ & 165 & 2002 & 2010 & D \\
\hline & 430 & $0.421 \mathrm{pMW}$ & 430 & 1997 & 2010 & 3 Länder \\
\hline
\end{tabular}

Abb. 118: Länderansicht zur Abfrage Verbalkomplex: Inf. Vollverb - fin. Auxiliar - beliebiger Infinitiv

Die Ergebnisse der Verteilung dieser Verbalkomplexe in deutschen (D), österreichischen (A) und schweizerischen $(\mathrm{CH})$ Texten sind in der Abb. 118 nach der relativen Häufigkeit (berechnet pro eine Mio. Worte) gereiht. Es geht deutlich hervor, dass die Mehrheit dieser Verbalkomplexe (sowohl absolut, als auch relativ gerechnet) in österreichischen Texten vorkommt.

Die KWICs bzw. Volltexte sollten nun näher nach einzelnen Ländern betrachtet werden. Ruft man die deutschen (Abb. 119) oder schweizerischen (Abb. 120) Konkordanzen ab, sieht man überwiegend solche Belege, die nicht in diese Problematik gehören, denn bei der Abfrage werden alle Abfolgen von drei Verben (mit hier definierten Eigenschaften) abgerufen, also auch diejenigen, die zwar hintereinander stehen, jedoch keinen Verbalkomplex bilden. Sie stehen zwar alle am Ende eines komplexen Satzes, jeweils eines oder zwei von diesen Verben gehören jedoch zum eingeschobenen Satz.

\begin{tabular}{|lrll|}
\hline BRZ09 & solch einer Tat zu & vermindern, ist zuzuhören. & Den Opfern, \\
HAZ09 & dem künftigen Pensionär zu & tun hatten, sagen, & er hätte immer ein \\
HAZ09 & lohnt. „Ich würde gern & mithelfen, würde mitgehen & und ihnen \\
HMP09 & können gar nicht auf ein 0:0 & spielen, werden angreifen & - mit \\
HMP09 & auf den Turniersieg zu & stürzen, war abzusehen. & "Ich habe \\
RHZ09 & wir Anfang nächsten Jahres & veröffentlichen werden, sagen & 95,5 \\
\hline
\end{tabular}

Abb. 119: Auswahl fehlerhafter Konkordanzen zur Abfrage Infinitiv Vollverb - finites Hilfsverb - beliebiger Infinitiv, Texte aus Deutschland

\begin{tabular}{|lrl|}
\hline A08 & nur noch, den Käfig, den ich jetzt wohl & verschenken würde, auszumisten. \\
\hline A99 & dämmerigen Kirche, sofern man sie dort & antreffen würde, erkennen. \\
\hline A08 & von Markus Sprenger (CVP), der in Pension & gehen wird, antreten. \\
\hline
\end{tabular}

Abb. 120: Auswahl fehlerhafter Konkordanzen zur Abfrage Infinitiv Vollverb - finites Hilfsverb - beliebiger Infinitiv, Texte aus der Schweiz

Das Problem der Überlappung der Sätze kann man damit umgehen, dass die Beistriche in der Abfrage negiert werden (Negationsoperator ist \%). Dabei müssen auch die Klammern entsprechend eingesetzt werden.

Die Suchfeldeingabe sieht dann folgendermaßen aus (Achtung! Leerzeichen an den richtigen Stellen hinzufügen!):

((MORPH(VRB inf v) \%+w1,) MORPH(VRB fin a) \%+w1,) MORPH(VRB inf) \#IN(R) <s> 
Die Ergebnisse dieser Abfrage sind ziemlich eindeutig: Wenn in einem deutschen oder schweizerischen Text das Auxiliar an zweiter Stelle steht, dann nur in Verbalkomplexen mit dem Verb lassen, wie die Abb. 121 zeigt.

Deutsche und schweizerische Texte

\begin{tabular}{|l|r|l|}
\hline (D) & sich in der Region etwas zu Schulden & kommen haben lassen. \\
\hline (D) & der das „Apollo“ (...) zwölf Jahre lang & leerstehen hat lassen. \\
\hline (D) & die Ablehnung einiger kleiner Parteien (...) & aufhorchen hätte lassen. \\
\hline (Ch) & Emotionalität setzende Zeichensprache & hineinfliessen hat lassen. \\
\hline (Ch) & und Flurin Caviezel die Fränzlis wieder & aufleben haben lassen. \\
\hline (Ch) & von der Schweizerischen Gesellschaft für Hotelkredite & prüfen hatte lassen. \\
\hline
\end{tabular}

Abb. 121: Auswahl der Konkordanzen zur Abfrage Infinitiv Vollverb - finites Hilfsverb - beliebiger Infinitiv, ohne Beistriche (DeReKo, Tagged-T)

Die Belege aus österreichischen Texten (A) liefern ein anderes Bild: das Auxiliar an zweiter Stelle erscheint in den Verbalkomplexen nicht nur, wenn am Ende das Verb lassen steht, sondern auch wenn diese Position Modalverben einnehmen. Wahrnehmungsverben und andere Verben mit Infinitiv ohne $z u$ (AcI-Verben) kommen aber äußerst selten vor. Es scheint, dass sich diese „österreichische“ Reihenfolge auf Verbalkomplexe mit einem Modalverb oder mit dem Verb lassen beschränkt. Zumindest in gesprochenen Texten sind andere AcI-Verben rar, dennoch belegt, wie der Abb. 122 zu entnehmen ist.

\section{Österreichische Texte}

\begin{tabular}{|llll|}
\hline NON09 & hat man die Gewissheit, dass man & weichen wird müssen. & "Wenn der Tunnel \\
X99 & Schilling, die man über erhöhte Beiträge & finanzieren habe müssen. \\
BVZ09 & Mannschaft, die jederzeit noch weiter & zuschlagen hätte können. \\
BVZ09 & weil er sein Kind (...) in die zweisprachige Volksschule schicken hätte können. \\
NON09 & Bürger, die nur gemeinsam etwas & bewegen hätten können. Gewonnen hat \\
NON09 & , da der Verein den Traktor gar nicht & verkaufen hätte dürfen. \\
BVZ08 & , wer (...) in Traiskirchen um den heurigen Titel kämpfen wird dürfen. \\
NON09 & Kindergarten, dessen Bau schon längst & beginnen hätte sollen. "Wir haben einen \\
NON09 & gemacht werden, die das Buntmetall & verkaufen hätte sollen. Dort erfuhren \\
BVZ08 & und Ist. Links das iV-Center, wie es & aussehen hätte sollen. Rechts der \\
NON09 & , dass der Schiri die beiden Streithanseln & austauschen hätte lassen. \\
NON09 & jene Person, die der Unternehmerin das Heroin zukommen hat lassen. \\
X99 & Spiele, die sich der Veranstalter für die jungen Gäste einfallen hatte lassen. \\
NON08 & dass sie einen (...) Mann mit "Kapuze und weißer Unterhose" weglaufen hat sehen. \\
\hline
\end{tabular}

Abb.. 122: Auswahl der Konkordanzen zur Abfrage Infinitiv Vollverb - finites Hilfsverb - beliebiger Infinitiv, Texte aus Österreich

Vergleicht man die Abb. 119, 120 und 121 mit der Abb. 122, dann kommt klar zum Vorschein, dass die Ergebnisse der Länderansichten (Abb. 117, Seite 157 und 118, Seite 159) nicht trügen. Die Topographie der dreiteiligen Verbalkomplexe weist länderspezifische Abweichungen auf.

Ergänzend wird nun das Phänomen auch noch im InterCorp abgefragt. 


\section{Recherche im InterCorp}

Die Abfrage lässt sich über den Abfragemodus CQL erstellen, allerdings ohne die Möglichkeit, die Ansicht nach Ursprungsland der Texte abzurufen ${ }^{45}$.

- $\quad$ korpus.cz $\rightarrow \underline{\text { Login }} \rightarrow \underline{\text { KonText }} \rightarrow \underline{\text { Parallel corpus InterCorp }} \rightarrow \underline{\text { intercorp de }}$

- Query type: CQL

Abfrage 1: „Deutsche“ Reihenfolge: finites Hilfsverb - Infinitiv Vollverb - Infinitiv Modalverb

- CQL (Suchfenstereingabe):

[tag="VAFIN.*"] [tag="VVINF.*"] [tag="VMINF.*"] [word="[;,I.।!]"] $\rightarrow$ Search

Diese Abfrage ist sowohl struktur- als auch zeichenmäßig eigentlich ident mit der Abfrage 1 im DeReKo (Seite 156). Sie bedeutet: Suche finites Hilfsverb (VAFIN), Infinitiv Vollverb (VVINF), Infinitiv Modalverben (VMINF) gefolgt von einem Satzendezeichen [;, \.।!].

Die Abfrage liefert fast 4.000 Belege.

Abfrage 2: „Österreichische“ Reihenfolge: Infinitiv Vollverb - finites Hilfsverb - Infinitiv Modalverb. Im InterCorp kann (im Unterschied zum DeReKo) diese Abfrage ohne Einschränkung durchgeführt werden.

- $\quad$ CQL (Suchfenstereingabe):

[tag="VVINF.*"] [tag="VAFIN.*"] [tag="VMINF.*"] [word="[;,I.I!]"] $\rightarrow$ Search

Das Ergebnis der Abfrage beträgt lediglich 27 Belege. Sie bestätigen jedoch die Ergebnisse der Recherche im DeReKo, wenn die Quellen näher angesehen werden: In der ersten Spalte ist der ,technische“ Titel (deswegen auf Tschechisch) der Datei angeführt, in dem der Beleg vorkommt.

\begin{tabular}{|cl|cc|}
\hline \multicolumn{1}{|c|}{ opus } & \multicolumn{1}{c|}{ links } \\
\hline$(1)$ & jelinek-pianistka & Er prophezeit, daß er sicher drei Tage nicht & gehen wird können. \\
$(2)$ & Bachmannova-Povidky & diese Person, die auch noch Todesurteile & unterzeichnen hatte müssen, \\
$(3)$ & simmel-a_jimmy_sel_za & Nur das Geschwätz, das er halt & schreiben hat müssen, \\
$(4)$ & Frankova-DenikA_Franko & , weil er weiß, welche Opfer Mutter & bringen hat müssen . \\
$(5)$ & -EUROPARL & dass man die (...) Katastrophe etwas stärker & hervorheben hätte müssen. \\
$(6)$ & Grass-Sire_pole & " bei seinem Vortrag, den er ja doch nich & halten wird können, (...) « \\
\hline
\end{tabular}

Abb. 123: Konkordanzzeilen zur Abfrage Infinitiv Vollverb - finites Hilfsverb - Infinitiv Modalverb (InterCorp)

Die hier ausgewählten Beispiele sind aus Büchern österreichischer Autor/-innen (1-3), aus einer Übersetzung einer in München lebenden Übersetzerin ${ }^{46}$ (4) und aus festgeschriebenen Reden im EU-Parlament (5), wo die Identität des Autors nicht feststellbar ist. Ein Beispiel (6) stammt aus einem Werk von Günter Grass.

45 Die Option „Suche nach Ursprungsland der Texte“ ist im InterCorp erst in Vorbereitung.

46 Mirjam Pressler: Anne Frank Tagebuch http://www.mirjampressler.de/about/ 


\section{Fazit:}

Die Verbalkomplexe mit drei Verben haben in den österreichischen Texten eine andere Topographie (Reihenfolge) als in deutschen und schweizerischen Texten. Das Auxiliar erscheint in österreichischen Texten mehrheitlich in der mittleren Position. Dies gilt für Verbalkomplexe mit Modalverben, bedingt auch für andere AcI-Verben hören (7) und sehen $(8)^{47}$ :

(7) Ich fand es sehr heftig, ja schon, aber hatte es mir noch brutaler vorgestellt, so wie man die Leute reden hat hören.

(8) Frau Präsidentin, wir sind der Auffassung, dass die im Entwurf vorliegenden Schlussfolgerungen des Rates, die wir alle diese Woche durchsickern haben sehen, eine sehr reale Gefahr für die Europäische Union bedeuten könnten .

Verbalkomplexe in deutschen und schweizerischen Texten haben gelegentlich auch die „österreichische“ Reihenfolge, wenn an ihrem Ende das Verb lassen steht (9), (10):

(9) ..., der sich selbst als einer der ersten impfen hat lassen.

(10) ..., die bei mir die Alarmglocken schrillen haben lassen.

Wie sich die Verbalkomplexe mit anderen AcI-Verben verhalten, muss noch recherchiert werden.

\section{Schlussbemerkung zur Studie 8}

Mit dieser Studie entstehen (mindestens) zwei Fragen: 1) Ist die bestehende Annotation der Korpora ausreichend? 2) Warum findet ein so häufiges Phänomen, welches hier behandelt wurde, keine Resonanz in Grammatiken? Es ist nur zu hoffen, dass diese (und andere) Lücken in der Beschreibung der deutschen Sprache mit dem internationalen Projekt „Variantengrammatik des Standarddeutschen“ (Dürscheid/ Elspaß/ Ziegler 2014) geschlossen werden.

47 Belege aus dem deTenTen-Corpus (Web-Corpus an der Masaryk-Universität); Abfrage: CQL: [tag="V.*"] [lemma="haben"] [lemma="hören"] [word="[;,।..!]"], bzw. [tag="V.*"] [lemma="haben"] [lemma="sehen"] [word="[;,I.!!]"] 


\section{Studie 9: Präposition pro}

Auch diese Studie bleibt auf der syntaktischen Ebene und betrachtet dabei die deutsche Sprache aus plurizentrischer Sicht. Der Anlass dazu war ein Disput mit einem Kollegen über die Formulierung eines Prüfungstests. In der Punktevergabe hieß es: pro richtiger Antwort 5 Punkte. Diese Angabe hat er als grammatikalisch falsch angezeigt, mit dem Hinweis, es solle heißen pro richtige Antwort, ich möge den Fehler korrigieren und das Testformular neu ausdrucken. Bauchgefühle täuschen, deswegen gibt es Nachschlagewerke - und Korpora.

Im Duden Universalwörterbuch (2006) steht folgender Eintrag:

pro $<$ Präp. mit Akk. $>$ [lat. pro $=$ vor, für, anstatt]:

1. jeweils, je, für (jede einzelne Person od. Sache): p. Person [und Jahr]; $100 \mathrm{~km}$ p. Stunde; er rasiert sich einmal p. Tag.

2. ${ }^{1}$ für $(1 \mathrm{~b})$.

Im Österreichischen Wörterbuch (2001: 459) findet man keine explizite Angabe über den Kasus, den die Präposition pro regiert. Die Schwankung zwischen dem Akkusativ und Dativ wird jedoch am Verwendungsbeispiel angedeutet:

pro; pro (per) Stück: für jedes Stück; pro anwesende(r) Dame ...

Im Variantenwörterbuch (2004) gibt es dazu keinen Eintrag.

$\mathrm{Ob}$ es sich um einen regionalen Unterschied oder um eine gesamtdeutsche Schwankung handelt, kann man an Daten solcher Korpora überprüfen, in denen die Markierung (Metadaten) über den Ursprung der Texte abrufbar ist. Dies ist der Fall im DWDS und im DeReKo. Alle modernen Texte (nach 1945) im DWDS stammen ausschließlich aus Deutschland, deswegen scheidet dieses Korpus für eine plurizentrische Recherche aus. Ob die Kasusschwankung auf regionaler Ebene betrachtet werden kann, überprüft man am besten im DeReKo. Gesucht wird nach der Präposition pro, die von einem Adjektiv in attributiver Funktion gefolgt wird.

- Cosmas II $_{\text {web }} \rightarrow \underline{\text { Recherche }} \rightarrow$

- Archiv: TAGGED-T - Archiv morphosyntakt. annotierter Korpora (TreeTagger) $\rightarrow$

- Korpus: TAGGED-T-öffentlich - alle öffentlichen Korpora des Archivs TAGGED-T

- Optionen: Suchmodalitäten:

Groß- / Kleinschreibung beachten für 1. Zeichen

abwählen

Groß- / Kleinschreibung beachten für andere Zeichen abwählen

Expansionslisten anzeigen: abwählen

Übernehmen

Ergebnispräsentation:

Übernehmen

Länderansicht auswählen

- Suchanfrage (Eingabe ins Suchfeld): $\underline{\text { MORPH-Assistent }} \rightarrow \underline{\text { Adj. }} \rightarrow$ attributiv auswählen

Im Suchfeld erscheint: MORPH(ADJ at), dann den Cursor vor MORPH positionieren und davor schreiben: pro $/+w 1 \cdot$. 
Die Abfrage sieht folgendermaßen aus (Achtung! Leerzeichen an den richtigen Stellen hinzufügen!):

$$
\text { pro·/+w1·MORPH(ADJ·at) } \rightarrow \text { Suchen }
$$

Die Abfrage bedeutet: Suche alle Formen pro und im Abstand von einem Wort rechts alle Adjektive in attributiver Position. Die Ergebnisse werden in Form einer Tabelle mit der Verteilung in Texten in einzelnen Ländern ausgegeben(Abb. 124). Man kann erkennen, dass die Kombination pro mit Adjektiv am häufigsten in schweizerischen Texten vorkommt.

\begin{tabular}{||l|l|l||l||l||l|l||}
\hline \hline & Treffer & rel. Häuf. & Texte & von & bis & Land \\
\hline \hline$⿴$ & 491 & $3.675 \mathrm{pMW}$ & 457 & 1999 & 2009 & A \\
\hline \hline$⿴$ & 996 & $4.168 \mathrm{pMW}$ & 926 & 1997 & 2009 & CH \\
\hline \hline & 1.775 & $2.741 \mathrm{pMW}$ & 1.673 & 1998 & 2010 & D \\
\hline \hline
\end{tabular}

Abb. 124: Ergebnisse pro+Adj. attributiv, Länderansicht (DeReKo)

Zu erkennen ist dies an der Spalte ,relative Häufigkeit“ ( eine Mio. Worte angibt.

Eine direkte Ermittlung des Kasus ist im Archiv TAGGED-T nicht möglich ${ }^{48}$, daher muss man die Kookkurrenzanalyse durchführen, erst dann aus den syntagmatischen Mustern die Kasusformen ableiten und von ihnen die Frequenzen in einzelnen Ländern abrufen.

- Kookkurrenzanalyse

Einstellungen: Kontext:

0 Wörter links 2 Wörter rechts

Die restliche Einstellung kann in der Standardeinstellung bleiben $\rightarrow$ Starten.

Die Berechnung der Kookkurrenzen (Belica 1995) liefert folgende syntagmatische Muster:

\begin{tabular}{|cccc|}
\hline \multicolumn{2}{|c|}{ \# Total Anzahl LLR Kookkurrenzen } & syntagmatische Muster \\
1 & 295 & 2952626 Kilometer & $81 \%$ pro gefahrenem | gefahrenen Kilometer \\
2 & 392 & 972031 verkauftem & $83 \%$ pro verkauftem \\
3 & 486 & 942024 mente & $85 \%$ pro mente " \\
4 & 493 & 71629 gefahrenem & $100 \%$ pro gefahrenem \\
5 & 569 & 761324 verkaufter & $73 \%$ pro verkaufter Karte \\
6 & 629 & 601263 gelaufener & $81 \%$ pro gelaufener Runde \\
7 & 634 & 51191 gefahrenen & $60 \%$ pro gefahrenen \\
8 & 761 & 1271093 Person & $85 \%$ pro erwachsene Person \\
9 & 762 & 11068 gelaufenem & $100 \%$ pro gelaufenem \\
10 & 818 & 56960 angefangene & $87 \%$ pro angefangene Stunde \\
11 & 875 & 57898 laufendem & $84 \%$ pro laufendem Meter \\
12 & 930 & 55875 gelaufene & $83 \%$ pro gelaufene Runde \\
\hline
\end{tabular}

Abb. 125: Auswahl der Kookkurrenzen und syntagmatischer Muster zur Abfrage Präp. pro und Adjektiv (DeReKo, TAGGED-T)

48 Die Abfrage nach Kasus über Tag ist nur im DeReKo-Korpus Tagged-M möglich. Dieses Korpus besteht allerdings nur aus bundesdeutschen Texten (vor allem Mannheimer Morgen und Der Spiegel - siehe Textorganisation unter COSMAS II $\rightarrow$ Korpora). 
Gleich das erste Syntagma (pro gefahrenem/ gefahrenen Kilometer) deutet auf die Kasusschwankung hin. Ob es signifikante Unterschiede im regionalen Bereich gibt, lässt sich mit der nächsten Abfrage feststellen.

Es ist ratsam diese im Archiv W - Archiv der geschriebenen Sprache durchzuführen, da dieses wesentlich größer ist:

- Archiv: W - Archiv der geschriebenen Sprache $\rightarrow$

- Korpus: W-öffentlich - alle öffentlichen Korpora des Archivs W

- Optionen: (keine Änderung notwendig)

- Suchanfrage (Eingabe ins Suchfeld): $\quad$ pro-gefahrenem·Kilometer $\rightarrow$ Suchen dann pro-gefahrenem $\cdot$ Kilometer $\rightarrow$ Suchen

Auch die weitere(n) Suchanfrage(n) nach den syntagmatischen Mustern aus der Abb. 125 sollte(n) mit derselben Einstellung erfolgen, um die Validität der Daten zu gewährleisten.

Die Ergebnisse der Abfragen in den Abb. 126 und 127 deuten darauf hin, dass die Kasusschwankung der Präposition pro tatsächlich regional bedingt sein kann:

\begin{tabular}{|l|r|l|r|l|l|l|}
\hline & Treffer & rel. Häuf. & Texte & $\underline{\text { von }}$ & bis & Land \\
\hline \pm & 64 & $0.0939 \mathrm{pMW}$ & 57 & 1992 & 2013 & A \\
\hline \pm & 158 & $\mathbf{0 . 0 5 0 4} \mathrm{pMW}$ & 152 & 1987 & 2013 & D \\
\hline \pm & 10 & $0.0216 \mathrm{pMW}$ & 10 & 1997 & 2013 & $\mathrm{CH}$ \\
\hline & & & & & & \\
\hline & 232 & $0.0542 \mathrm{pMW}$ & 219 & 1987 & 2013 & 3 Länder \\
\hline
\end{tabular}

Abb. 126: Ergebnis der Abfrage pro gefahrenem Kilometer, Länderansicht, sortiert nach rel. Häufigkeit absteigend (DeReKo)

\begin{tabular}{|l|r|l|r|r|r|l|}
\hline & \multicolumn{1}{|l|}{ Treffer } & rel. Häuf. & Texte & von & bis & $\underline{\text { Land }}$ \\
\hline$\boxplus$ & 45 & $0.0971 \mathrm{pM}$ & 43 & 1996 & 2013 & $\mathrm{CH}$ \\
\hline$\boxplus$ & 125 & $0.0399 \mathrm{pM}$ & 119 & 1994 & 2012 & D \\
\hline$\boxplus$ & 25 & $0.0367 \mathrm{pM}$ & 24 & 1992 & 2013 & A \\
\hline & & & & & & \\
\hline & 195 & $0.0456 \mathrm{pM}$ & 186 & 1992 & 2013 & 3 Länder \\
\hline
\end{tabular}

Abb. 127: Ergebnis der Abfrage pro gefahrenen Kilometer, Länderansicht, sortiert nach rel. Häufigkeit absteigend (DeReKo)

Als Kontrollbeispiel wurde noch das syntagmatische Muster pro gelaufener Rundel pro gelaufene Runde abgefragt. Auch diese Ergebnisse zeigen dieselben Verhältnisse der Aufteilung von Treffern:

\begin{tabular}{|r|r|l|r|l|l|l|}
\hline & Treffer & rel. Häuf. & Texte & von & bis & Land \\
\hline \pm & 29 & $0.0426 \mathrm{pMV}$ & 28 & 2000 & 2013 & A \\
\hline \pm & 70 & $0.0223 \mathrm{pMV}$ & 70 & 2001 & 2012 & D \\
\hline \pm & 2 & $0.0043 \mathrm{pMV}$ & 2 & 2007 & 2011 & $\mathrm{CH}$ \\
\hline & & & & & & \\
\hline & 101 & $0.0236 \mathrm{pMV}$ & 100 & 2000 & 2013 & 3 Länder \\
\hline
\end{tabular}

Abb. 128: Ergebnis der Abfrage pro gelaufener Runde, Länderansicht, sortiert nach rel. Häufigkeit absteigend (DeReKo)

\begin{tabular}{|c|c|c|c|c|c|c|}
\hline & Treffer & rel. Häuf. & Texte & von & bis & Land \\
\hline$\Phi$ & 21 & $0.0453 \mathrm{pM}$ & 18 & 1998 & 2013 & $\mathrm{CH}$ \\
\hline$\boxplus$ & 48 & $0.0153 \mathrm{pM}$ & 48 & 2004 & 2013 & $D$ \\
\hline \pm & 4 & $0.0059 \mathrm{pM}$ & 4 & 2008 & 2013 & A \\
\hline & 73 & $0.0171 \mathrm{pM}$ & 70 & 1998 & 2013 & 3 Länder \\
\hline
\end{tabular}

Abb. 129: Ergebnis der Abfrage pro gelaufene Runde, Länderansicht, sortiert nach rel. Häufigkeit absteigend (DeReKo)

$\mathrm{Zu}$ diesen Ergebnissen gelangt man relativ rasch. (Man bedenke, wie viele Arbeitstage ein Mensch nur für eine dieser Recherchen brauchen würde.) In weiterer Folge wurden noch diese Abfragen durchgeführt: pro angefangenerl pro angefangene, pro verkaufterl pro verkaufte, pro verkauftem, pro verkauftes. Sie alle bestätigten die Ergebnisse in den Abb. 126-129. 


\section{Fazit:}

Die Ergebnisse zeigen ein plastisches Bild über den Gebrauch von pro + Dativ/ Akkusativ: In schweizerischen Texten wird fast ausschließlich Akkusativ verwendet, in österreichischen Texten wiederum in den meisten Fällen Dativ, die Akkusativform ist eine Seltenheit. Und in den deutschen Texten scheint dieses Phänomen als eine regelrechte Doppelform mit leichter Tendenz zum Dativ zu sein. Ob dies auch (innerhalb Deutschlands) regionalbeding ist oder ob textsortenspezifische Faktoren auch eine Rolle spielen, muss für weitere Untersuchungen offen bleiben. Auf jeden Fall ist mit dieser Studie ein weiteres Steinchen in das plurizentrische Mosaikbild der deutschen Sprache gelegt worden. 


\section{Kollokationen, Phraseme und Lexik}

Ein reflektierter Umgang mit einer (Fremd-)Sprache soll immer die Frage aufkommen lassen: was ist in der Sprache üblich, was ist eher selten; was ist wichtig zu beherrschen, was ist weniger wichtig; welche Mittel braucht man um das Kommunikationsziel zu erreichen. Diese Mittel können im Kopf grundsätzlich über zwei Wege abgerufen werden: 1) als Ergebnis eines grammatisch-lexikalischen Prozesses, oder 2) als „Fertigteile“ (Chunks), die als modifizierbare Einheiten gespeichert sind. Im Fremdsprachenunterricht scheint es sinnvoll, beide Wege zu kombinieren, wie Swan (2006) betonte. Die Überlegungen, welche Aspekte der Grammatik hervorgehoben werden sollen, welche Lexik und welche Fertigteile (Chunks) primär vermittelt werden sollen, hängen eng mit dem Ziel des Unterrichts zusammen. Wegen ihrer Breite kann auf sie nicht im Einzelnen eingegangen werden.

In diesem Kapitel werden einige wenige Fallbeispiele präsentiert, in denen sich mehrere sprachliche Ebenen und Aspekte verbinden: paradigmatische und syntagmatische Beziehungen, feste und freie Verbindungen, diachrone und synchrone Betrachtung der Lexik. Die ersten zwei Dichotomien sind stark mit der Phraseologie verbunden. Ohne an dem „Terminologiekrieg um die Besetzung des linguistischen Terminus Kollokation“ (Ďurčo et al. 2010: 7) teilzunehmen ${ }^{49}$, werden hier Beispiele gezeigt, wie Wortverbindungen mithilfe Korpora aufgedeckt werden können.

\section{Studie 10: Kollokationen, syntagmatische Muster, Chunks}

Die Frage danach, was in der Sprache typisch ist, was eher nicht, betrifft im nicht geringeren Maße die Wortverbindungen. Diese können mithilfe der Korpusmanager errechnet und elegant mit einer anderen Sprache verglichen werden, wie es z.B. für feste Wortverbindungen im deutsch-slowakischen Kontrast Ďurčo et al. (2010) gemacht haben.

Die Frage ist nun: Wie kann man die Wortverbindungen aus dem Korpus abrufen und so aufdecken?

Fragestellung 1: Was sind die am meisten frequentierten Wörter in der Sprache und wie ermittelt man die häufigsten Verbindungen, in denen sie vorkommen?

Fürs Deutsche wurde eine Liste der 100.000 häufigsten Wortformen und 40.000 Grundformen (DeReWo 2009) erstellt. Diese Liste wird folgend interpretiert: diejenigen Wörter, die oben stehen haben die höchste Frequenz, sie waren am häufigsten in Texten des DeReKo 2009 vertreten. Es ist daher sehr wahrscheinlich, dass sie allgemein in den meisten deutschen Texten vorkommen. Am Anfang dieser Liste stehen synsemantische Relationswörter, die in fast jedem Text vorkommen (Artikel, Präpositionen und Konjunktionen, Hilfsverben). Vergleicht man die jeweils zwanzig häufigsten Wörter in der Liste DeReWo (2009) mit der Word List aus InterCorp_de, Kernkorpus (Dovalil/ Káňa/ Peloušková et al. 2013), stellt man fest, dass sich diese grob entsprechen:

49 Dazu im Detail: Hausmann 2003: 320-321. 
Die zwanzig häufigsten Wortformen nach DeReWo (2009), alphabetisch:

auf, auch, das, dem, den, der, des, die, ein, eine, für, im, in, ist, mit, nicht, sich, und, von, $z u$

Die zwanzig häufigsten Wortformen im InterCorp_de (core) (2013), alphabetisch:

auf, das, dem, den, der, die, ein, er, es, ich, in, ist, mit, nicht, sie, sich, und, von, war, $z u$

Es sind (wenig überraschend) alles Synsemantika („Systemwörter“). Erst auf weiteren Frequenzpositionen stehen autosemantische Wörter ${ }^{50}$. Diese „entsprechen“ dem Inhalt des Korpus. (Autosemantika werden nämlich nach Thema des Textes häufig/ weniger häufig/gar nicht verwendet.) Auch hier gibt es Übereinstimmungen. Vergleicht man die ersten 200 häufigsten Lemmata im DeReWo und Intercorp_de (core), stellt man fest, dass die folgenden Wörter in beiden Korpora (obwohl in jedem Korpus andere Texte gespeichert sind und die Korpora unterschiedlich aufgebaut werden) am häufigsten vorkommen:

beginnen, bleiben, bringen, Ende, fahren, fragen, Frau, geben, gehen, groß, gut, halten, Haus, heißen, Jahr, jung, Kind, klein, kommen, kurz, lang, leben, liegen, legen, Mann, Mensch, sagen, sehen, Seite, schön, stehen, stellen, Tag, Weg, weit, Welt, zeigen, Zeit.

Diese Wörter bilden den „härtesten“ Kern des deutschen Wortschatzes ${ }^{51}$.

Als Beispiel wird in einem nächsten Schritt das Wort Jahr in seinen typischen Chunks ermittelt. Es ist übrigens das allerhäufigste autosemantische Wort gleich in mehreren Korpora: DeReKo, deTenTen, deWaC. Allgemein ist es im geschriebenen Deutsch also sehr gebräuchlich. Um auch die gesprochene Sprache annähernd einzubeziehen, wurde die Abfrage im DeReKo auf belletristische Texte eingeschränkt (Belletristik liegt am nähersten der gesprochenen Sprache wegen deren Nachahmung in der direkten, teilweise auch indirekten Rede). Dazu wurde ein Sub-Korpus aus zugänglichen belletristischen Texten (über 16 Mio. laufender Wörter) erstellt.

- Cosmas $\mathbf{I I}_{\mathbf{w e b}} \rightarrow \underline{\text { Recherche }}$

- Archiv: W-Archiv der geschriebenen Sprache $\rightarrow$ Subkorpus erstellen

- $\quad \underline{\text { Korpus }} \rightarrow \underline{\text { Korpusverwaltung }} \rightarrow$ Benutzerdefinierte Korpora $\rightarrow$ Definieren gewünschte Texte in die rechte Spalte verschieben Name (Korpus benennen) $\rightarrow$ übernehmen $\rightarrow$ Speichern

- Optionen: Standardeinstellung

- Suchanfrage (Eingabe ins Suchfeld): \&Jahr $\rightarrow$ Suchen

Nachdem die Ergebnisse erschienen sind, wird die Kookkurrenzanalyse durchgeführt:

- Kookkurrenzanalyse

Einstellungen: $\rightarrow$ Zurücksetzen (Standardeinstellung)

Funktionswörter ignorieren abwählen $\rightarrow$ Starten

In Kürze erscheint ein Bild (Abb. 110) mit Kookkurrenzpartnern/ Kollokatoren (hier Kookkurrenzen genannt) und entsprechenden syntagmatischen Mustern, in denen sie vorkommen. Es zahlt sich aus, die syntagmatischen Muster durchzugehen. Jede/-r, der/die in

50 Zur Definition von Auto- und Synsemantika siehe Fachlexikon DaF/ DaZ (2010: 22, 328).

51 Die Ergebnisse wurden auch an Daten anderer großer Korpora überprüft (deTenTen und deWac). 
der deutschen Sprache täglich lebt, stellt fest, dass viele von diesen Mustern tatsächlich ,unglaublich vertraut" klingen.

\begin{tabular}{|c|c|c|c|c|c|c|c|}
\hline \multicolumn{7}{|c|}{$\begin{array}{c}\text { \# Total Anzahl Autofokus LLR Kookkurrenzen } \\
\text { von bis }\end{array}$} & syntagmatische Muster \\
\hline 1 & 3 & 3 & 1 & 1 & 94 & alt dreißig & $100 \%$ dreißig Jahre alt und ... \\
\hline 2 & 71 & 3 & -1 & -1 & 71 & dreißig lang & 100\% dreißig Jahre lang \\
\hline 3 & 110 & 22 & -1 & -1 & 69 & zwanzig & 59\% vor zwanzig Jahren \\
\hline 4 & 117 & 7 & -1 & -1 & 41 & siebzehn & 57\% siebzehn Jahre \\
\hline 5 & 123 & 6 & -1 & -1 & 38 & fünfundzwanzig & $50 \%$ fünfundzwanzig Jahre \\
\hline 6 & 139 & 16 & -1 & -1 & 34 & halbes & 93\% ein halbes Jahr \\
\hline 7 & 142 & 3 & -1 & -1 & 30 & sechsundzwanzig & $100 \%$ sechsundzwanzig Jahre \\
\hline 8 & 152 & 10 & -1 & -1 & 26 & vierzig & $50 \%$ vierzig $[\ldots]$ Jahre \\
\hline 9 & 157 & 5 & -1 & -1 & 25 & achtzehn & $80 \%$ achtzehn Jahren \\
\hline 10 & 160 & 3 & -1 & -1 & 24 & einundzwanzig & 100\% einundzwanzig Jahre \\
\hline 11 & 165 & 5 & -1 & -1 & 19 & sechzig & $100 \%$ sechzig Jahre \\
\hline 12 & 177 & 12 & 1 & 1 & 18 & älter & 75\% Jahre älter als \\
\hline 13 & 185 & 8 & 1 & 1 & 18 & jünger & $50 \%$ Jahre jünger \\
\hline 14 & 187 & 2 & 1 & 3 & 17 & PGN & $100 \%$ Jahr [zu Jahr $]$ PGN \\
\hline 15 & 192 & 5 & -1 & -1 & 15 & dreizehn & $100 \%$ dreizehn Jahre alt \\
\hline 16 & 196 & 4 & -1 & -1 & 15 & zwanziger & 50\% zwanziger Jahre \\
\hline 17 & 198 & 2 & -5 & -5 & 14 & Kontaktanzeige & $100 \%$ Kontaktanzeige im folgenden J. \\
\hline 18 & 211 & 13 & -1 & -1 & 12 & halben & $100 \%$ seit|vor einem halben Jahr \\
\hline 19 & 215 & 4 & -1 & -1 & 10 & vierzehn & 50\% vierzehn Jahre \\
\hline 20 & 217 & 2 & 2 & 4 & 10 & Seydlitz & $50 \%$ Jahre ... Seydlitz \\
\hline 21 & 219 & 2 & -1 & -1 & 10 & vierhundert & 50\% vierhundert Jahre \\
\hline 22 & 221 & 2 & -5 & 4 & 10 & Mäsjuh & 50\% Jahres ... Mäsjuh \\
\hline 23 & 223 & 2 & -1 & -1 & 9 & Fünfzehn & $100 \%$ Fünfzehn Jahre \\
\hline 24 & 228 & 5 & -1 & -1 & 9 & fünfzehn & $80 \%$ fünfzehn $[\ldots]$ Jahre \\
\hline 25 & 231 & 3 & 2 & 4 & 8 & Sils & 66\% Jahr nach Sils \\
\hline 26 & 235 & 4 & -1 & -1 & 8 & sechziger & $50 \%$ sechziger Jahre \\
\hline 27 & 240 & 5 & -1 & -1 & 8 & fünfzig & $80 \%$ fünfzig $[\ldots]$ Jahre \\
\hline 28 & 242 & 2 & 3 & 4 & 7 & zugebracht & $100 \%$ Jahre im ... zugebracht \\
\hline 29 & 253 & 11 & -2 & -2 & 6 & Laufe & 90\% im Laufe der Jahre \\
\hline 30 & 256 & 3 & -2 & -1 & 6 & sechzehn & $100 \%$ sechzehn $[\ldots]$ Jahre alt \\
\hline 31 & 259 & 3 & -1 & -1 & 6 & fünfziger & $100 \%$ fünfziger Jahren \\
\hline 32 & 263 & 4 & -4 & -4 & 6 & zarten & 75\% im zarten Alter von \\
\hline 33 & 266 & 3 & -1 & -1 & 5 & Letztes & $100 \%$ Letztes Jahr \\
\hline 34 & 269 & 3 & -3 & -1 & 5 & siebzig & $100 \%$ siebzig [oder .... Jahre \\
\hline 35 & 271 & 2 & -2 & -2 & 5 & hab's & $100 \%$ hab's ... Jahre \\
\hline 36 & 273 & 2 & 2 & 2 & 4 & aufgebraucht & $50 \%$ Jahre ... aufgebraucht \\
\hline 37 & 306 & 33 & 1 & 1 & 4 & lang & $60 \%$ Jahre lang \\
\hline 38 & 312 & 6 & 1 & 1 & 3 & vergangen & 66\% Jahre [waren] vergangen \\
\hline 39 & 314 & 2 & -2 & -1 & 3 & tausenden & $100 \%$ tausenden $[\ldots]$ Jahren \\
\hline 40 & 316 & 2 & -1 & 3 & 3 & unbeschwerten & 50\% Jahre ... unbeschwerten \\
\hline 41 & 325 & 9 & -1 & -1 & 2 & hundert & 55\% hundert Jahren \\
\hline 42 & 327 & 2 & 4 & 4 & 2 & solltest & $100 \%$ Jahre vor dir und solltest \\
\hline 43 & 346 & 19 & -1 & -1 & 2 & jedes & $84 \%$ jedes Jahr \\
\hline 44 & 348 & 2 & -1 & -1 & 2 & $1 / 2$ & $50 \% 1 / 2$ Jahren \\
\hline 45 & 350 & 2 & 4 & 4 & 2 & wunderschöne & $100 \%$ Jahren ... wunderschöne \\
\hline 46 & 352 & 2 & 2 & 2 & 2 & aufbewahrt & 50\% Jahre ... aufbewahrt \\
\hline 47 & 356 & 4 & -2 & -2 & 2 & deinen & 75\% deinen ... Jahren \\
\hline 48 & 361 & 5 & -1 & -1 & 2 & ganzes & 80\% ein ganzes Jahr \\
\hline 49 & 363 & 2 & 4 & 5 & 2 & Feinden & $100 \%$ Jahre ... Welt ... Feinden \\
\hline 50 & 365 & 2 & -3 & -3 & 1 & litt & $100 \%$ litt ... Jahren \\
\hline 51 & 367 & 2 & 2 & 3 & 1 & Voraus & $100 \%$ Jahr im Voraus \\
\hline 52 & 373 & 6 & 4 & 4 & 1 & gelebt & 83\% Jahre $[\ldots]$ gelebt \\
\hline 53 & 375 & 2 & -2 & 2 & 1 & einhundert & $50 \%$ Jahr ... einhundert \\
\hline 54 & 377 & 2 & 2 & 5 & 1 & siebenmal & $100 \%$ Jahre ... oder ... siebenmal \\
\hline 55 & 379 & 2 & 1 & 1 & 1 & gedauert & $100 \%$ Jahre gedauert \\
\hline 56 & 383 & 4 & -1 & -1 & 1 & nächstes & $100 \%$ nächstes Jahr \\
\hline 57 & 385 & 2 & -1 & -1 & 1 & achtziger & 100\% achtziger Jahre \\
\hline 58 & 388 & 3 & -1 & -1 & 1 & Nächstes & $100 \%$ Nächstes $[\ldots]$ Jahr \\
\hline 59 & 391 & 3 & -3 & -2 & 1 & mochte & 66\% mochte ... Jahre \\
\hline 60 & 393 & 2 & -1 & -1 & 1 & siebziger & 50\% siebziger Jahre \\
\hline
\end{tabular}

Abb. 130: Kookkurrenzen und syntagmatische Muster zu Jahr (DeReKo) 
Aus den syntagmatischen Mustern kann man einige Chunks ableiten (Tab. 26), die gefühlsmäßig auch in der gesprochenen Sprache besonders häufig vorkommen. Sie sollten auch vorrangig vermittelt werden.

\begin{tabular}{|cc|}
\hline X Jahre alt/lang & seit $\mid$ vor einem halben Jahr \\
vor X Jahren & im vergangenen Jahr \\
ein halbes Jahr & jedes Jahr \\
Jahre älter als & nächstes Jahr \\
\hline
\end{tabular}

Tab. 26: Chunks aus syntagmatischen Mustern mit Lemma Jahr

In den syntagmatischen Mustern sind natürlich auch phraseologische Einheiten zu finden: im Laufe der Jahre im zarten Alter von ... Jahren

Die Konkordanzen zu jedem berechneten Muster sind durch den Klick auf die entsprechende Zeile abrufbar.

Würde man im ganzen öffentlichen Korpus recherchieren, wären auch diese syntagmatischen Muster relevant:

$$
\begin{aligned}
& \text { freiwilliges (soziales/ ökologisches) Jahr } \\
& \text { zu ... Jahren Haft verurteilt }
\end{aligned}
$$

Sie sind jedoch nur für Sachtexte typisch. Ihre Frequenz in der gesprochenen Sprache kann mithilfe bestehender Korpora nicht überprüft werden.

\section{Fazit:}

Die häufigsten Wörter im Deutschen sind Synsemantika: Artikelwörter der, die, das, den, dem, des, ein(e), gefolgt von Präpositionen (in, für etc.), dem grammatikalischen Partikel zu u.a.

Aus den autosemantischen Wörtern sind es:

Substantive: Ende, Frau, Haus, Jahr, Kind, Mann, Mensch, Seite, Tag, Weg, Welt, Zeit;

Adjektive: groß, gut, jung, klein, kurz, lang, schön, weit;

Verben: beginnen, bleiben, bringen, fahren, fragen, geben, gehen, halten, heißen, kommen, leben, legen, liegen, sagen, sehen, stehen, stellen, zeigen. (Es ist jedoch anzunehmen, dass die meisten dieser Verben desemantisiert in verbo-nominalen Phrasen vorkommen, deswegen haben sie sehr hohe Frequenz.)

Auf der Frequenzliste der häufigsten deutschen Wörter stehen zwischen den synsemantischen und autosemantischen Wörtern eine Reihe deiktischer Wörter (er, heute, hier, ich, Sie/sie, mein, $d a$ ) und alle Hilfs- und Modalverben.

Autosemantische Wörter mit der höchsten Frequenz sollten als erste vermittelt werden. Die häufigsten Phrasen und Chunks, in denen sie vorkommen, deckt die Kookkurrenz/Kollokationsanalyse auf.

Man kann die Abfrage um ein Element erweitern (Fragestellung 2) oder gleich nach einem komplexeren Element wie Phrase, Chunk, mehrgliedriges Satzglied (Fragestellung 3) suchen. Beide Abfragen werden ähnlich durchgeführt. 
Fragestellung 2: In welchen semantischen Korrelationen stehen die Wörter Kind und Schule? Für Lernende kann die Frage auch einfacher formuliert werden: Was können Kinder mit Schule ,tun"?

Einige Phrasen oder Chunks können direkt aus den Konkordanzen festgestellt werden. Sogar wenig fortgeschrittene Lerner/-innen können aus vorsichtig ausgewählten Konkordanzzeilen eine induktive Aufgabe selbst lösen.

Die Recherche kann im beliebigen Korpus durchgeführt werden. Im Folgenden werden wieder belletristische Texte des DeReKo verwendet.

- Cosmas $\mathbf{I I}_{\text {web }} \rightarrow \underline{\text { Recherche }}$

- Archiv: W-Archiv der geschriebenen Sprache $\rightarrow$ Subkorpus Belletristik (Erstellung siehe Fragestellung 1)

- Optionen: Standardeinstellung

- Suchanfrage (Eingabe ins Suchfeld): \&Schule /s0 \&Kind $\rightarrow$ Suchen

Die Abfrage bedeutet: Suche Lemmata Schule und Kind in einem Satz, die Reihenfolge im Satz ist beliebig. Als Ergebnis bekommt man ein paar Dutzend Konkordanzen, wie die Abb. 131 zeigt.

\begin{tabular}{|lr|}
\hline DIV & Frühe wieder zur Arbeit, während die Kinder der reichen Eltern zur Schule gehen \\
DIV & Kilometer entfernte Nachbardorf, dessen Kinder die neue Schule nicht besuchen sollen, \\
DIV & das Leben in der Dorfgemeinschaft: Die Kinder gehen von morgens 6 Uhr bis zum \\
DIV & warteten vor der Tür. Man wollte die Kinder schließlich daran gewöhnen, dass sie \\
DIV & ihre Tochter davon überzeugte, dass Kinder in die Schule gehen müssen. Wie sie das \\
DIV & Karla davon überzeugt, dass jedes Kind, was sie natürlich mit einschloss, zur \\
DIV & das war dann bitter. Früher mussten die Kinder von Möllersgrund und Springen bis nach \\
DIV & ich nicht. Zu der Zeit spielten wir Kinder nach der Schule oft am Waldrand, dort \\
DIV & werden, es war halt Krieg. Unsere Schule bekam dann auch öfters fremde Kinder, \\
DIV & zu trinken, und eines Tages wurden die Kinder von der Schule weg abgeholt und ins \\
DIV & ruhigen Augen stillstehend wie ein Kind in der Schule, aller Hohn und Spott wird \\
WAM war. Also wahrscheinlich ein Dentist. Die Kinder promovierter Ärzte hatten sich in der \\
\hline
\end{tabular}

Abb. 131: Auswahl der Konkordanzen zur Abfrage Lemma Schule und Lemma Kind in einem Satz (DeReKo)

Aus diesen (nur einigen wenigen ausgewählten) Konkordanzen können die Lerner folgende Schlussfolgerungen ziehen: was im Satz wichtig ist, welche Konstruktionen gebräuchlich sind, welche „Korrelationen“ es zwischen Schule - Kind geben kann.

\begin{tabular}{|l|l|}
\hline Kind(er) in der Subjektrolle & Kind(er) in Rolle eines Objektes \\
\hline Kinder gehen zur/ in die Schule & Unsere Schule bekam dann auch öfters fremde Kinder \\
Kinder müssen in die Schule gehen & Kinder werden abgeholt \\
Kinder besuchen die Schule & \\
Kinder machen sich lustig über.. & \\
\hline
\end{tabular}

Tab. 27: Das Wort Kind und seine Rollen im Satz mit Schule

Am häufigsten steht Kind (meistens im Plural) in der Rolle eines Subjekts/ Agens (Tab. 27 links) oder Objekts des Geschehens (Tab. 27 rechts). 
Fragestellung 3: Wie sucht man nach mehrteiligen Prädikaten und entdeckt die Rollen um das Prädikat herum?

Im syntaktischen Zentrum des Satzes stehen einfache oder komplexe ${ }^{52}$ Verben, die den Kern des Prädikats bilden. Prädikate sind auch entweder (1) einfach (nur Vollverben in finiter Form) oder (2) komplex (finite Form eines ,entleerten“ Hilfsverbs ${ }^{53}$ in Verbindung mit einem „lexikalischen Kern“ (Duden - Grammatik 2005: 422) des Prädikats).

(1) Die Abfrage nach einfachen Prädikaten erfolgt in jedem Korpus über die Funktion Lemma (siehe Fallbeispiel Jahr auf Seite 167). Besteht das einfache Prädikat aus einem Verb mit Zusatz, dann entspricht die Abfrage dem Vorgang auf der Seite 151 und 152.

(2) Komplexe Prädikate (hier das Beispiel bekannt sein) können in DeReKo und in InterCorp wie folgt abgefragt werden.

\section{Recherche im DeReKo}

- Cosmas II $\rightarrow$ Recherche

- Archiv: W-Archiv der geschriebenen Sprache

- Korpus: W-öffentlich - alle öffentlichen Korpora des Archivs W

- Optionen: Standardeinstellung

- Suchanfrage (Eingabe ins Suchfeld): \&sein /s0 bekannt $\rightarrow$ Suchen

Die Abfrage bedeutet: Suche Lemma sein und das Wort bekannt in einem Satz.

\section{Recherche im InterCorp}

- korpus.cz $\rightarrow \underline{\text { Login }} \rightarrow \underline{\text { KonText }} \rightarrow \underline{\text { Parallel corpus InterCorp }} \rightarrow \underline{\text { intercorp de }}$

- Query type: CQL

- $\mathrm{CQL}$ (Suchfeldeingabe):

$<$ /> containing [lemma="sein"] containing [word="bekannt"] $\rightarrow$ Search

Die Abfrage bedeutet: Suche Sätze, die das Lemma sein und das Wort bekannt beinhalten.

Die Konkordanzen können recht unübersichtlich sein, denn der Korpusmanager gibt alle Sätze wieder, in denen diese zwei Wörter vorkommen: man sieht also (auf dem Bildschirm) rot! Es besteht aber eine alternative Abfrage:

- $\quad$ korpus.cz $\rightarrow \underline{\text { Login }} \rightarrow \underline{\text { KonText }} \rightarrow \underline{\text { Parallel corpus InterCorp }} \rightarrow \underline{\text { intercorp de }}$

- Query type: Word Form (Match case auswählen empfohlen)

- Word Form (Suchfeldeingabe): bekannt

Nachdem die Konkordanzzeilen erschienen sind, führt man die Kollokationsanalyse durch:

- Collocation $\rightarrow \underline{\text { Custom... }}$

52 Einfache Verben werden durch lexikalische Simplizia (d.h. lexikalisch nicht weiter zerlegbare Worte) realisiert (gehen, laufen, schreiben ...).

Komplexe Verben sind entweder verbale Ableitungen (beschreiben, verlaufen, zerkochen ...) oder verbale Komposita/ Verben mit Zusätzen (abschreiben, weglaufen, kennenlernen ... ).

53 Hilfsverb oder Auxiliar wird hier als Sammelbegriff für Hilfsverben im traditionellen Sinne (haben, sein, werden) verwendet, weiterhin für Modalverben, Prädikativ(Kopula)- und Funktionsverben. (Hierzu: Duden Grammatik 2005: 420-423.) 
Einstellung der Kollokationsanalyse (Collocations candidates):

- Attribute: Lemma

- In the range from: -5 to

- Minimum frequency in corpus:

- Minimum frequency in given range: Make Candidate List

(spielt keine Rolle für diese Abfrage) (spielt keine Rolle für diese Abfrage)

Unter den signifikanten Kollokationspartnern findet man die Verben geben, werden, machen und auch das gesuchte sein. Durch einen Klick auf $p$ (positiver Filter) erscheinen die Konkordanzen mit den automatisch errechneten Kollokationspartnern (Abb. 132). Unter ihnen sind auch fehlerhaft errechnete Kollokationen wie diese:

"Das (...) Fischereiministerium gibt heute bekannt, dass -« »lst doch nicht zu fassen! ... «

Valerie hatte die Männer miteinander bekannt gemacht, sie waren nun sehr häufig zusammengetroffen -

Die Fehlerquote liegt jedoch unter 5\%.

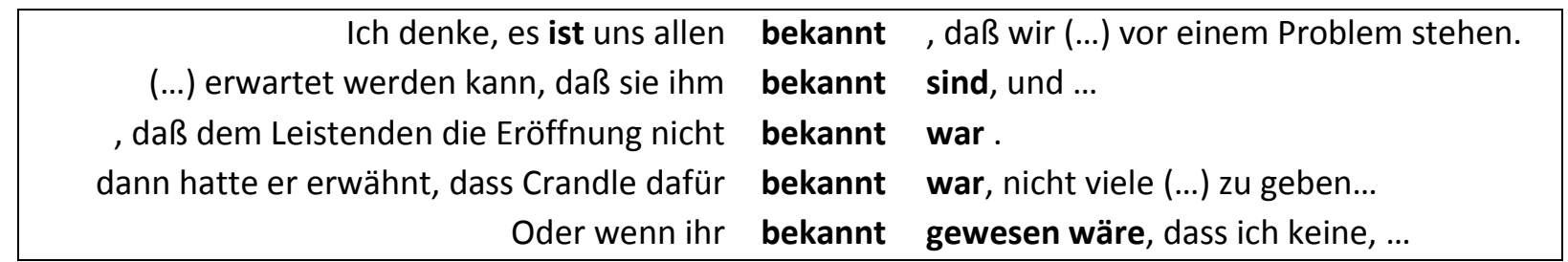

Abb. 132: Auswahl der Konkordanzen zur Abfrage bekannt sein in einem Satz (InterCorp)

Nach diesem Vorgang lassen sich alle mehrteiligen Prädikate abrufen. Aus den Kollokationsanalysen könne die typischen Prädikationen (= Basisgerüst jeder minimalen sinnvollen Aussage) aufgedeckt werden, wie auch der folgende Schritt zeigt.

Als Prädikat steht ein Verb mit einen Zusatz: anziehen.

Sucht man nach dem typischen Umfeld von einem Verb mit Zusatz, ist der Vorgang ähnlich der vorherigen Fragestellung 2. Man soll jedoch das ganze Paradigma des Verbs anziehen abfragen. Dementsprechend müssen auch alle Formen mit dem abgetrennten Zusatz abgerufen werden (vgl. auch Studie 7)

\section{Recherche im DeReKo}

- Cosmas $\mathbf{I I}_{\text {web }} \rightarrow \underline{\text { Recherche }}$

- Archiv: W-Archiv der geschriebenen Sprache

- Korpus: W-öffentlich - alle öffentlichen Korpora des Archivs W

- Optionen: Standardeinstellung

- Suchanfrage (Eingabe ins Suchfeld):

$$
\text { \&anziehen·oder·(\&ziehen·/s0·an·\#IN(R) }<\mathrm{s}>) \rightarrow \text { Suchen }
$$

Die Abfrage bedeutet: Suche alle Formen des Verbs anziehen (\&anziehen) oder (oder) alle Formen des Verbs ziehen, wobei am Ende (\#IN(R)) desselben Satzes (/s0) das Element an (an) vorkommt. 
An den Ergebnissen (KWICs) wird wieder die Kookkurrenzanalyse durchgeführt:

- Kookkurrenzanalyse

Einstellungen: $\rightarrow$ Zurücksetzen (Standardeinstellung) $\rightarrow$ Starten

Das Ergebnis der Berechnung wird wieder in Form einer Tabelle (Abb. 133) mit syntagmatischen Mustern präsentiert.

\begin{tabular}{|c|c|c|c|c|c|}
\hline \# & Total & Anzahl & LLR & Kookkurrenzen & syntagmatische Muster \\
\hline 1 & 1077 & 1077 & 9649 & warm & 80\% sich warm [...] anziehen \\
\hline 2 & 1608 & 531 & 6867 & magisch & $29 \%$ magisch $[\ldots]$ angezogen \\
\hline 3 & 3118 & 1510 & 6776 & Besucher & 19\% zog [viele] Besucher an \\
\hline 4 & 3382 & 264 & 2644 & Zügel & 29\% die Zügel [...] angezogen \\
\hline 5 & 4187 & 805 & 2509 & viele & $20 \%$ zog $[\ldots]$ viele $\ldots$ an \\
\hline 6 & 4456 & 269 & 2182 & Massen & 27\% zieht [die] Massen an \\
\hline 7 & 4457 & 1 & 2141 & Handbremse vergessen & $100 \%$ vergessen Handbremse anzuziehen \\
\hline 8 & 4911 & 256 & 1693 & Konjunktur & $32 \%$ wenn die Konjunktur [wieder] anzieht \\
\hline 9 & 5397 & 486 & 1436 & Publikum & 26\% Publikum [...] anziehen \\
\hline 10 & 5628 & 231 & 1421 & Touristen & 27\% und zieht Touristen an \\
\hline 11 & 5645 & 17 & 1328 & Schuhe feste & 70\% feste Schuhe [...] anziehen \\
\hline 14 & 6388 & 103 & 947 & Schraube & 41\% die Schraube [...] angezogen \\
\hline 15 & 6463 & 75 & 914 & Daumenschrauben & die Daumenschrauben [...] angezogen \\
\hline 16 & 6580 & 117 & 903 & Handschuhe & 35\% Handschuhe [...] anziehen \\
\hline 19 & 6938 & 3 & 841 & Kleidung bequeme warme & 100\% warme bequeme Kleidung a. \\
\hline 34 & 8860 & 66 & 487 & Schrauben & $42 \%$ die Schrauben [...] angezogen \\
\hline 37 & 9058 & 77 & 453 & Jacke & $37 \%$ eineldie Jacke $[\ldots]$ anziehen \\
\hline 43 & 10212 & 55 & 358 & Pullover & 32\% einen ... Pullover anziehen \\
\hline
\end{tabular}

Abb. 133: Auswahl der Kookkurrenzen zu anziehen (DeReKo)

Aus den syntagmatischen Mustern geht deutlich die Diversität der Lesarten dieses Verbs hervor: sich warm anziehen, Schuhe/ Handschuhe/Kleider/ (warme, bequeme) Kleidung a , Handbremse a ; in übertragenem Sinne: Besucher, Massen, Preise, Schrauben, (hoffentlich auch nur metaphorisch) Daumenschrauben a ; jmd/etw. zieht (jmdn./etw.) an (ein Künstler zieht viele Besucher/ großes Publikum an, die Konjunktur zieht (wieder) an).

Dieselbe Abfrage kann auch im InterCorp durchgeführt werden.

\section{Recherche im InterCorp}

- korpus.cz $\rightarrow \underline{\text { Login }} \rightarrow \underline{\text { KonText }} \rightarrow \underline{\text { Parallel corpus InterCorp }} \rightarrow \underline{\text { intercorp de }}$

- Query type: CQL

- CQL (Suchfeldeingabe):

[lemma="anziehen"]|[lemma="ziehen"] []* [word="an"] within $<$ id=".*" / > $\rightarrow$ Search

Die Abfrage bedeutet: Suche alle Formen von anziehen oder $(\mid)$ ziehen, davon rechts im beliebigen Abstand das Element an, allerdings nur bis zur Grenze des Satzes.

Auch im InterCorp wird jetzt die Kollokationsanalyse durchgeführt:

- Collocation $\rightarrow \underline{\text { Custom... }}$

Einstellung der Kollokationsanalyse (Collocations candidates):

- Attribute: Lemma

- In the range from: - to

- Minimum frequency in corpus:

(empfohlen)

- Minimum frequency in given range:

(empfohlen) Make Candidate List 
Das Ergebnis bekommt man nicht in Form der syntagmatischen Muster (im Unterschied zum COSMAS II), sondern als einzelne Lemmata oder Wortformen (je nach Einstellung). Signifikante Kollokationspartner bzw. Nachbarn des Wortes sind in der Abb. 134 angeführt.

\begin{tabular}{|l|l|l|r|r|}
\hline \multicolumn{9}{|c|}{ Freq } & \multicolumn{1}{l|}{ logDice } \\
\hline 1. & $\underline{\mathrm{p}} / \underline{\mathrm{n}}$ & Kleid & 49 & 8.211 \\
\hline 2. & $\underline{\mathrm{p}} / \underline{\mathrm{n}}$ & vorbei & 43 & 7.615 \\
\hline 3. & $\underline{\mathrm{p}} / \underline{\mathrm{n}}$ & heran & 28 & 7.595 \\
\hline 4. & $\underline{\mathrm{p}} \underline{\mathrm{n}}$ & Hemd & 25 & 7.540 \\
\hline 5. & $\underline{\mathrm{p}} / \underline{\mathrm{n}}$ & Schuh & 28 & 7.529 \\
\hline 6. & $\mathrm{p} / \underline{\mathrm{n}}$ & Brust & 32 & 7.458 \\
\hline 7. & $\underline{\mathrm{p}} / \underline{\mathrm{n}}$ & Uniform & 22 & 7.403 \\
\hline 8. & $\underline{\mathrm{p}} / \underline{\mathrm{n}}$ & waschen & 22 & 7.374 \\
\hline 9. & $\underline{\mathrm{p}} / \underline{\mathrm{n}}$ & Bein & 37 & 7.336 \\
\hline 10. & $\mathrm{p} / \underline{\mathrm{n}}$ & Hose & 21 & 7.274 \\
\hline
\end{tabular}

\begin{tabular}{|c|c|c|c|c|}
\hline \multicolumn{4}{|c|}{ Freq } & logDice \\
\hline 11. & $\mathrm{p} / \underline{\mathrm{n}}$ & Mantel & 20 & 7.189 \\
\hline 12. & $\mathrm{p} / \underline{\mathrm{n}}$ & Stiefel & 16 & 7.094 \\
\hline 13. & $p / n$ & Betracht & 23 & 7.044 \\
\hline 14. & $\mathrm{p} / \mathrm{n}$ & Haar & 32 & 7.043 \\
\hline 15. & $p / n$ & Schluß & 35 & 7.034 \\
\hline 16. & $\mathrm{p} / \underline{\mathrm{n}}$ & duschen & 13 & 6.980 \\
\hline 17. & $\mathrm{p} / \underline{\mathrm{n}}$ & ausländisch & 22 & 6.979 \\
\hline 18. & $\mathrm{p} / \mathrm{n}$ & Bett & 32 & 6.938 \\
\hline 19. & $\underline{p} / \underline{n}$ & Rock & 15 & 6.876 \\
\hline 20. & $\mathrm{p} / \underline{\mathrm{n}}$ & Knie & 17 & 6.867 \\
\hline
\end{tabular}

Abb. 134: Auswahl aus signifikanten Kollokationspartnern zum Verb anziehen (InterCorp)

Über den positiven Filter (klicken auf p) gelangt man zu den Konkordanzen, in denen das Verb anziehen und der entsprechende Kollokationspartner in einem Satz stehen. Diese decken interessante Prädikationen auf. Einige von ihnen wurden hier ausgewählt. Die eher „unerwarteten“ Kollokationspartner sind unterstrichen (2-9).

(1) Zwei Stunden lang zog ich an der TV-Kamera einen Drahtzaun vorbei , um die Illusion der Fahrt vollkommen zu machen

(2) ... und zog die Faust mit ganzer Kraft an die Brust, ...

(3) Instinktiv zog sie die Decke an die Brust.

(4) aber sie trank rasch einen Schluck, während sie sich eilig wusch und anzog.

(5) Morgens war er lange vor mir wach, gewaschen und angezogen.

(6) In der Hoffnung ausländische Investoren anzuziehen halten lateinamerikanische Regierungen hartnäckig daran fest, ...

(7) Er zog den Stuhl näher an das Bett ...

(8) Ich duschte und zog mehrere Schichten Kleider an.

(9) Das Nachthemd (...) war zum braungebrannten Bauch gerutscht, weil sie die Knie bis fast zum Kinn angezogen hatte.

Die Kollokationsanalyse sortiert nach logDice bezeugt auch die hohe Frequenz einiger verbonominalen Verbindungen und damit auch die Wichtigkeit, diese im Unterricht zu vermitteln:

(10) Noch ein weiterer ermutigender Aspekt ist in Betracht zu ziehen, ...

(11) Daraus muss der traurige Schluss gezogen werden, dass dem mangelnden Interesse an Fragen der Gleichstellung der Geschlechter ...

Die Konkordanzen erscheinen auch mit parallelen Passagen in einer anderen Sprache, falls diese am Anfang der Recherche ausgewählt wurde.

\section{Fazit:}

Aus beiden Recherchen ist ersichtlich, in welchen Verbindungen das Verb anziehen in deutschen geschriebenen Texten vorkommen kann. Die signifikanten Kollokationspartner in den Abb. 133 und 134 bieten Ausgangspunkte für weiterführende Arbeiten auf der syntaktischen und lexikalischen Ebene an, die sich auf die textuelle Ebene erweitern lassen (vgl. Punkt 3 auf der folgenden Seite). 
Die Recherchen zeigten:

1) syntaktisch-semantische Rollen, die sich um das Prädikat anziehen verbreiten:

[eine Person] zieht ([sich/ etwas]) an

[etwas] anziehen

[ein Ereignis] zieht [Personen] an etc.

2) semantische Gruppen von Substantiven, mit denen das Verb anziehen häufig vorkommt (hier in Rolle des Patiens):

a) Besucher/Massen/Scharen/Publikum/Touristen anziehen

b) Zügel/ Handbremse/ Schraube anziehen

c) Kleider, [warme, bequeme] Kleidung anziehen

3) Prädikatsverben, die häufig in der Umgebung des Verbs anziehen vorkommen (waschen, duschen), sind Zentren benachbarter Prädikationen. Gemeinsam mit der Prädikation mit anziehen gewährleisten sie die textuelle Kohärenz. Eine solche ikonographische Schilderung (sich waschen, dann [etwas] anziehen) ist in den Texten offensichtlich nicht selten.

Diese Schlussfolgerungen müssten noch nach Wahl der Korpora, in denen recherchiert wurde, differenziert werden. Sie zeigen aber deutlich die Möglichkeiten von Interpretationen, die aus Ergebnissen der automatisch berechneten Kookkurrenzen/ Kollokationen gezogen werden können.

\section{Bemerkung zur Studie 10}

Zum Schluss dieser Studie muss nochmals die Kookkurrenz-/ bzw. Kollokationsanalyse hervorgehoben werden. Sie stellt eine wichtige, sogar revolutionäre Funktion für die Bereiche der Erforschung der Sprache(n) in gleich mehreren Aspekten dar:

Im (Fremd-)Sprachenunterricht ist sie grundlegend für die Ermittlung von „wahrscheinlichen Wortkombinationen“" in der Sprache (Westhoff 1991: 16), derer Kenntnis das Westhoffsche (1987: 41) „,dritte Redundanzfeld“ bilden.

Des Weiteren beschränken sich die Möglichkeiten der Kollokations-/ Kookkurrenzanalyse nicht nur auf die (in dieser Studie und in diesem Buch betonte) linguistische, bzw. linguodidaktische Ebene. Sie hilft unter anderem auch, die metaphorische Sprache der Börsianer (vgl. Lišková 2010: 25) aufzudecken und besser zu verstehen. Eine Kookkurrenzanalyse zu Aktienkurs im DeReKo deckt (auch für einen Wirtschaftslaien) interessante syntagmatische Muster auf. Hier werden nur die bildhaftesten genannt, weitere siehe Lišková 2010.

der Aktienkurs sackte (ab/ein)

dümpelt (vor sich hin)

steigt/ schnellt (in die Höhe/ nach oben)

bricht ein

beflügelt jmdn..

Durch die Berechnung der typischen Kollokationen aus Zeitschriften- und Zeitungstexten lassen sich auch politische, soziale und kulturelle Einstellungen der sprachlichen Kommunität ableiten. Ein Vergleich der signifikanten Kollokationspartner zum Wort anpassungsfähig (anpassungsunwillig) in drei Korpora mit Zeitungstexten in drei Sprachen ist verblüffend:

Während in deutschen Texten (Subkorpus Deutscher Zeitungen und Zeitschriften aus den Jahren 2004-2013) diese Wörter relativ selten und am häufigsten mit dem Substantiv Wesen (anpassungsfähiges/ anpassungsunwilliges Wesen) verbunden sind, haben seine tschechischen 
und slowakischen lexikalischen Entsprechungen (nepřizpůsobivý, bzw. nepriespôsobivý) in publizistischen Texten eine hohe Frequenz und diese signifikanten Partner:

sociálně, neplatič ... spoluobčan, občan ... Rom ... problémový ...ghetto

neplatič, sociálne ... asociál ... bezdomovec, občan, cigán ... etnikum

Anpassungsunfähig/-unwillig sind in tschechischen und slowakischen publizistischen Texten also in erster Linie Menschen, sogar konkret die Roma-Minderheit.

Wenn man die Analyse „umdreht“ und in den deutschen Texten das Lemma Türke, also die Bezeichnung der größten Minderheit in Deutschland abfragt, bekommt man syntagmatische Muster, die in der Abb. $135 \mathrm{zu}$ sehen sind.

\begin{tabular}{|c|c|c|c|c|c|}
\hline \multicolumn{2}{|c|}{ \# Total } & \multicolumn{4}{|r|}{ syntagmatische Muster } \\
\hline 3 & 425 & 255 & 111 & Griechen & 64\% Griechen [und] Türken \\
\hline 4 & 612 & 187 & 88 & Kurden & 66\% zwischen Türken [und] Kurden \\
\hline 6 & 744 & 117 & 72 & Araber & 77\% Türken [und] Araber \\
\hline 7 & 1100 & 356 & 72 & lebenden & 99\% Deutschland|hier lebenden [...] Türken \\
\hline 8 & 1111 & 11 & 60 & Türkischstämmigen & 100\% Prozent der Türken und Türkischstämmigen \\
\hline 13 & 1246 & 26 & 49 & eingebürgerten & 100\% von eingebürgerten [...] Türken \\
\hline 14 & 1270 & 24 & 48 & Türkinnen & 75\% Türkinnen und Türken \\
\hline 15 & 1291 & 21 & 41 & bewohntes & 100\% ein von Türken bewohntes Haus \\
\hline 16 & 1299 & 8 & 41 & niederbayrische & 100\% der niederbayrische Türke mit ... \\
\hline 17 & 1324 & 25 & 37 & eingebürgerte & $100 \%$ eingebürgerte Türken \\
\hline 41 & 1589 & 15 & 14 & aufgewachsenen & $100 \%$ Deutschland/und aufgewachsenen [...] Türken \\
\hline 42 & 1619 & 30 & 13 & geborener & $90 \%$ Deutschland geborener [...] Türke und ein \\
\hline
\end{tabular}

Abb. 135: Kookkurrenzen und syntagmatische Muster zum Lemma Türke (DeReKo)

Diese Beispiele zeigen, dass Korpusdaten auch für Politologen und Soziologen eine wichtige Forschungsquelle darstellen können, denn die Sprache ist bekanntlich ein Spiegel der Gesellschaft. 


\section{Studie 11: Quasi-Anglizismen}

Der Vergleich von Sprachen führt zu einer höheren Sprachbewusstheit, diese ,stellt den ersten wichtigen Schritt zum eigenverantwortlichen Umgang mit Textprodukten und Lernprozessen dar" (Sorger et al. 2013: 290), dadurch werden die Sprachkompetenzen der Lerner/-innen erhöht. Der Vergleich von mehreren Sprachen auf der lexikalischen Ebene kann kulturelle und soziale Kontakte, bzw. gegenseitige Beeinflussungen und oder Irrtümer zeigen.

Zum Vergleich von Lexemen reichen oft gängige zweisprachige Wörterbücher ${ }^{54}$. Nun können diese aber erstens nie alle Lexeme der Sprache beinhalten, zweitens können sie auch nicht genug Kontext liefern. Dieses Manko lässt sich durch Korpusrecherchen ausgleichen, wie diese Studie über scheinbare Anglizismen zeigt.

In den mitteleuropäischen Sprachen gibt es Modewörter, die das „modische“ Englisch bloß nachahmen, bzw. die gängigen englischen Formen (leicht) verändern. Solche Wörter bilden sog. Faux amis, weil sie im Englischen in der Form, die den Englischlernenden „vertraut“" ist, gar nicht existieren. $\mathrm{Zu}$ ihnen gehören im Deutschen Aircondition (weniger üblich Air Condition), Happy End (oder weniger üblich Happyend) ${ }^{55}$, Oldtimer, Showmaster oder das allgegenwärtige und fast notorische Handy. Bis auf Handy handelt es sich tatsächlich um „Mitteleuropäismen“, denn die meisten von ihnen sind in Nationalen Korpora einiger mitteleuropäischer Sprachen belegt, wie aus der Tab. 28 ersichtlich ist. Die Schreibweise wurde im originalgetreuen Bild der jeweiligen Sprache beibehalten. In Klammern sind die absoluten Anzahlen der Belege angegeben. Sie sollen hier nur die Existenz der Formen bestätigen, sagen jedoch wenig über die Frequenz der Wörter in der jeweiligen Sprache aus.

\begin{tabular}{|l|l|l|l|l|}
\hline \multicolumn{1}{|c|}{$\begin{array}{c}\text { Slowenisch } \\
\text { FidaPLUS }\end{array}$} & $\begin{array}{c}\text { Polnisch } \\
\text { Nacijonalnyj korpus } \\
\text { Jezyka Polskeigeo }\end{array}$ & \multicolumn{1}{|c|}{$\begin{array}{c}\text { Ungarisch } \\
\text { Magyar Nemzeti } \\
\text { Szövegtár }\end{array}$} & $\begin{array}{c}\text { Tschechisch } \\
\text { Český národní koprus }\end{array}$ & $\begin{array}{c}\text { Slowakisch } \\
\text { Slovenský národný } \\
\text { korpus }\end{array}$ \\
\hline $\begin{array}{l}\text { happy end } \\
\text { happyend (2) }\end{array}$ & happy end (133) & happyend (2) & $\begin{array}{l}\text { happy end (1450) } \\
\text { happyend (522) }\end{array}$ & $\begin{array}{l}\text { happy end (283) } \\
\text { happyend (204) }\end{array}$ \\
\hline oldtimer (42) & oldtimer (3) & oldtimer (18) & oldtimer (322) & oldtimer (19) \\
\hline air condition (37) & air condition (4) & air kondi (2) & aircondition (5) & aircondition \\
\hline showmaster (1) & - & - & showmaster (13) & $\begin{array}{l}\text { show master (2) } \\
\text { šoumáster (10) }\end{array}$ \\
\hline
\end{tabular}

Tab. 27: Quasi-Anglizismen in einigen Korpora mitteleuropäischer Sprachen.

Die Quasi-Anglizismen happy end und air condition beschränken sich nicht nur auf die hier betrachteten Sprachen. Sie sind auch im Türkischen Nationalkorpus belegt, wie einige Konkordanzzeilen in den Abb. 136 und 137 bezeugen.

\begin{tabular}{|llll|}
\hline No & Text & Query Results \\
1 & AG03A1B-3172 & genellikle mutlu bir sonla noktalanır (happy end). Aslında herşeyin planlı, \\
2 & HG09C2A-0593 & perdenin ardından gözlerindeki yaşı silecektir... Happy End!... Film, \\
3 & DG03C4A-1508 & (yanlış) aşılama yöntemi, sanıldığı gibi "HAPPY END" ile sonuçlanmıyor. \\
\hline
\end{tabular}

Abb. 136: Konkordanzen zur Abfrage happy end (Türkçe Ulusal Derlemi)

54 Bis auf Aircondition sind die hier analysierten im Pons Globalwörterbuch Deutsch-Englisch (1998) aufgelistet.

55 Die Schreibweisen Aircondition/ Air Condition, Happy End/ Happyend wurden an Daten von DeReKo überprüft. 


\begin{tabular}{|lll|}
\hline No & Text & Query Results \\
1 & NG24D1B-2301 & Yazları sıcaktan bunalınca air condition cenneti kütüphanelere takılın. \\
2 & GD02A3A-1321 & , kuru temizlemeden, elektrikli battaniye, air condition ve aya insan ay \\
3 & BD02A3A-2967 & ... distribütörün yanından geçirip air condition kompresörüne bağlayın \\
4 & MD30D1B-2199 & gün toplanan MGK'daki havayı, "Air condition çalışıyordu, hiç üşümedik" \\
5 & ED02A2A-1023 & mutfak, tuvalet, soğukhava tertibatı "air condition" gibi günlük ihtiyaçları \\
6 & MD30D1B-2199 & Toplantıda hava gayet iyiydi. Air condition bile çalışıyordu. \\
7 & EA16B1A-1745 & bir koltuk. Dolby stereo sistem. Air condition. İşte camlı bölmede ve \\
\hline
\end{tabular}

Abb. 137: Konkordanzen zur Abfrage air condition (Türkçe Ulusal Derlemi)

Wie die Entsprechungen im gleichen Text, jedoch in einer anderen Sprache aussehen können, lässt sich entweder aus einem Parallelkorpus oder aus einem Instrument, das auf parallelen Texten aufgebaut ist (z.B. linguee.de), feststellen.

Fragestellung: Was entspricht dem deutschen Wort Oldtimer im Englischen und im Tschechischen?

Die Suche wird im InterCorp durchgeführt (im Opus-Corpus gibt es keine Belege zu Oldtimer.)

- $\quad$ korpus.cz $\rightarrow \underline{\text { Login }} \rightarrow \underline{\text { KonText }} \rightarrow \underline{\text { Parallel corpus InterCorp }} \rightarrow \underline{\text { intercorp de }}$

- Aligned corpora $\rightarrow$ intercorp_en $\rightarrow$ Add

- Aligned corpora $\rightarrow$ intercorp_cs $\rightarrow$ Add

- Query type: Basic

- Basic (Suchfenstereingabe): Oldtimer $\rightarrow$ Search

Die Ergebnisse erscheinen in Form einer Tabelle mit den ausgewählten Sprachen. Die KWICs sind in der Ausgangssprache rot markiert, in den anderen Sprachen müssen sie gesucht werden. In der Abb. 138 sind sie mit Fettschrift hervorgehoben.

\begin{tabular}{|l|l|l|}
\hline \multicolumn{1}{|c|}{ de } & \multicolumn{1}{|c|}{ cs } & \multicolumn{1}{c|}{ en } \\
\hline $\begin{array}{l}\text { [Er] hatte die Boxen zu } \\
\begin{array}{l}\text { Unterstellplätzen für nagelneue } \\
\text { Oldtimer umgebaut. }\end{array}\end{array}$ & $\begin{array}{l}\text { Stáj byla přeměněna v } \\
\text { impozantní garáž a zaparkované } \\
\text { vozy tvořily opravdu sbírku } \\
\text { prvotř́dních značek: černé } \\
\text { ferrari, (...) - a historické } \\
\text { Porsche 356. }\end{array}$ & $\begin{array}{l}\text { The stalls had been converted into } \\
\text { an impressive automotive parking } \\
\text { facility. The collection was } \\
\text { astonishing - a black Ferrari (...), a } \\
\text { vintage Porsche 356. }\end{array}$ \\
\hline $\begin{array}{l}\text {... zur Verwendung in älteren, } \\
\text { besonders beschaffenen Fahrzeugen } \\
\text { (Oldtimer) }\end{array}$ & $\begin{array}{l}\text { [prodej] pro použití ve starých } \\
\text { vozidlech zvláštních vlastností }\end{array}$ & $\begin{array}{l}\text {... sales to be used by old vehicles } \\
\text { of a characteristic nature }\end{array}$ \\
\hline $\begin{array}{l}\text { (10) Oldtimer, d. h. historische } \\
\text { Samrzeuge, Fahrzeuge mit }\end{array}$ & $\begin{array}{l}\text { (10) Dobová vozidla, to } \\
\text { znamená historická vozidla nebo } \\
\text { vozidla se sběratelskou } \\
\text { hodnotou nebo }\end{array}$ & $\begin{array}{l}\text { (10) Vintage vehicles, meaning } \\
\text { historic vehicles or vehicles of } \\
\text { value to collectors }\end{array}$ \\
\hline $\begin{array}{l}\text { Und selbst als Oldtimer für kleine } \\
\text { Ausflüge am Sonntag taugt der Käfer } \\
\text { von Anfang der 70er ... }\end{array}$ & $\begin{array}{l}\text { Brouk ze začátku 70 . let se moc modí ani jako veterán na } \\
\text { nehoder } \\
\text { krátké nedělní výlety ... }\end{array}$ & $\begin{array}{l}\text { And even for Sunday drives the } \\
\text { Beetle of the early 1970s can } \\
\text { hardly be regarded as a vintage } \\
\text { car ... }\end{array}$ \\
\hline $\begin{array}{l}\text { Obwohl eine kleine Gruppe von } \\
\text { Sammlern spekulativ in Oldtimer } \\
\text { oder Automobilraritäten investiert ... }\end{array}$ & $\begin{array}{l}\text { Trebaže malá skupina sběratelů } \\
\text { spekulativně investuje do } \\
\text { veteránů a nevšedních vozů ... }\end{array}$ & $\begin{array}{l}\text { Though a small group of collectors } \\
\text { invests speculatively in antique or } \\
\text { specialty cars ... }\end{array}$ \\
\hline
\end{tabular}

Abb. 138: Parallele Textpassagen zur Abfrage Oldtimer (InterCorp de - cs - en. Hervorhebungen Autor) 
Analog zu dieser Recherche erfolgt auch die Suche nach andern Quasi-Anglizismen. Es können auch mehrere Sprachen hinzugefügt werden.

\section{Fazit:}

Auch ein/e wenig erfahrene/r Schüler/in schlussfolgert aus den parallelen Passagen, dass der „mitteleuropäische Anglizismus“ Oldtimer im Englischen nicht existiert, sondern dass ihm die Verbindung mit dem Attribut vintage oder antique am ehesten entspricht.

Im Tschechischen findet man hier als Entsprechung zum deutschen Oldtimer nur die Bezeichnung veterán. Das Wort oldtimer wird im Tschechischen zwar (fast) in demselben Sinn, vielleicht aber seltener verwendet, als sein deutsches Pendant. Wie die prozentuelle Verteilung der Entsprechungen in einzelnen Texten zueinander stehen, muss aufgrund der zu dieser Recherche immer noch geringen Korpusgröße vorerst unbeantwortet bleiben. 


\section{Studie 12: Ein Blick in die Geschichte vom Hit}

An Korpusdaten lassen sich sogar einige Änderungen in der Sprache beobachten. Als Beispiel für solche Recherchen wird die Änderung im Gebrauch der Wörter Schlager und Hit gezeigt.

Forschungsfrage: Wann hat eigentlich der Hit den Schlager abgelöst?

Die Antwort auf diese Frage zu Diachronie der Sprache kann natürlich niemand auf das Jahr oder sogar den Tag genau sagen. Die Änderungen im Wortschatz verlaufen auf verschiedenen Wegen: der Hit ist zu einem Modewort geworden, hat den Schlager aus seiner Position geschlagen, aber der Schlager konnte sich behaupten und mit einer leicht veränderten Bedeutung ist er auch im 21. Jh. ein fester Bestandteil der deutschen Sprache (vgl. Abb. 143 auf Seite 182).

Einen groben Überblick in die Geschichte der Verwendung einzelner Wörter verschaffen am besten die Korpora DWDS und DeReKo.

\section{Recherche im DWDS}

- DWDS $\rightarrow$ Abfragefenster

- Suchfeldeingabe: Hit $\rightarrow$ Suche im DWDS

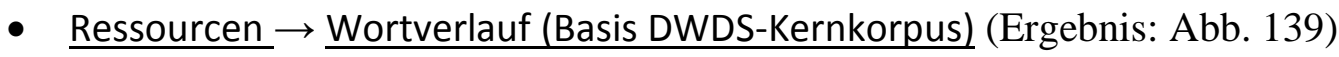

dann

- Suchfeldeingabe: Schlager $\rightarrow$ a

- Ressourcen $\rightarrow$ Wortverlauf (Basis DWDS-Kernkorpus) (Ergebnis: Abb. 140)

$\mathrm{Zu}$ beiden Abfragen liefert das DWDS eine graphische Übersicht über die Streuung der Wörter in Dekaden über das 20. Jahrhundert.

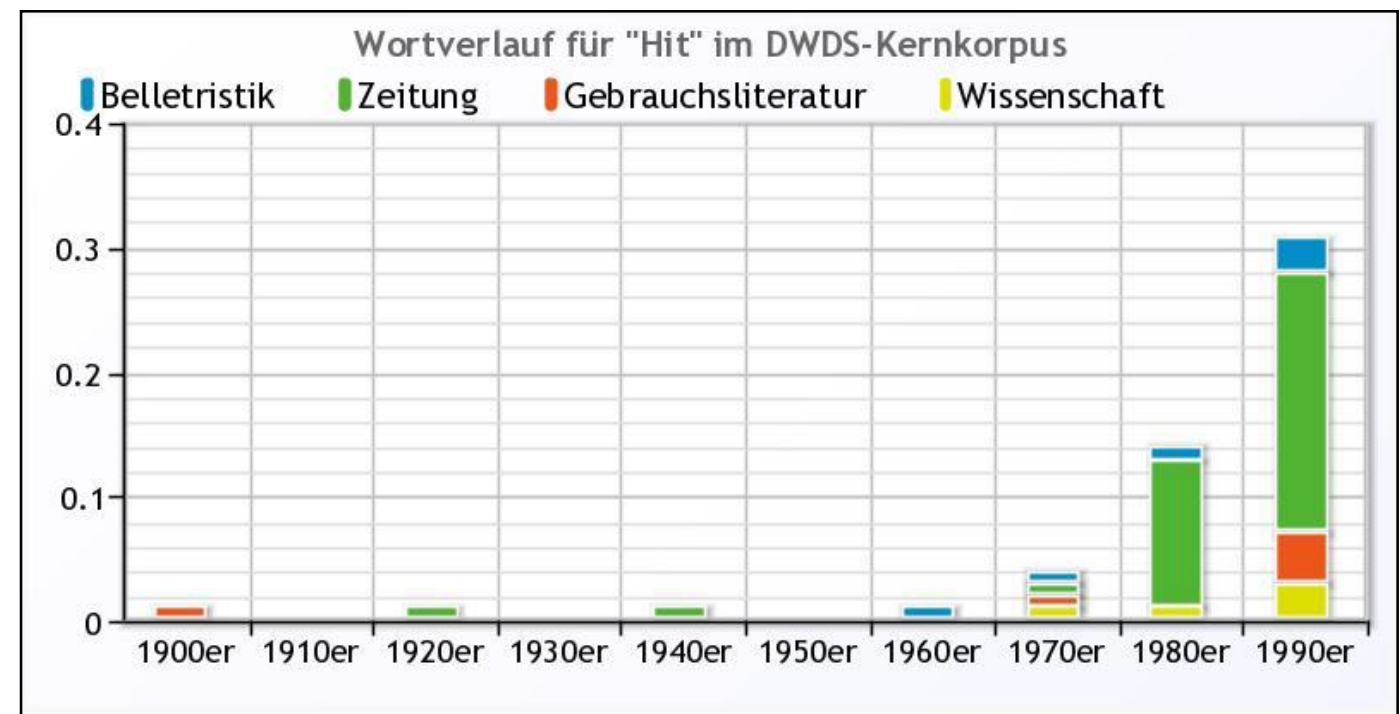

Abb. 139: Graphik: Wortverlauf von Hit im 20. Jh. (DWDS)

Die Belege in den 0-er, 20-er und 40-er Jahren sind Tippfehler (jeweils ein Beleg), die das Korpus falsch erkannt hat. 


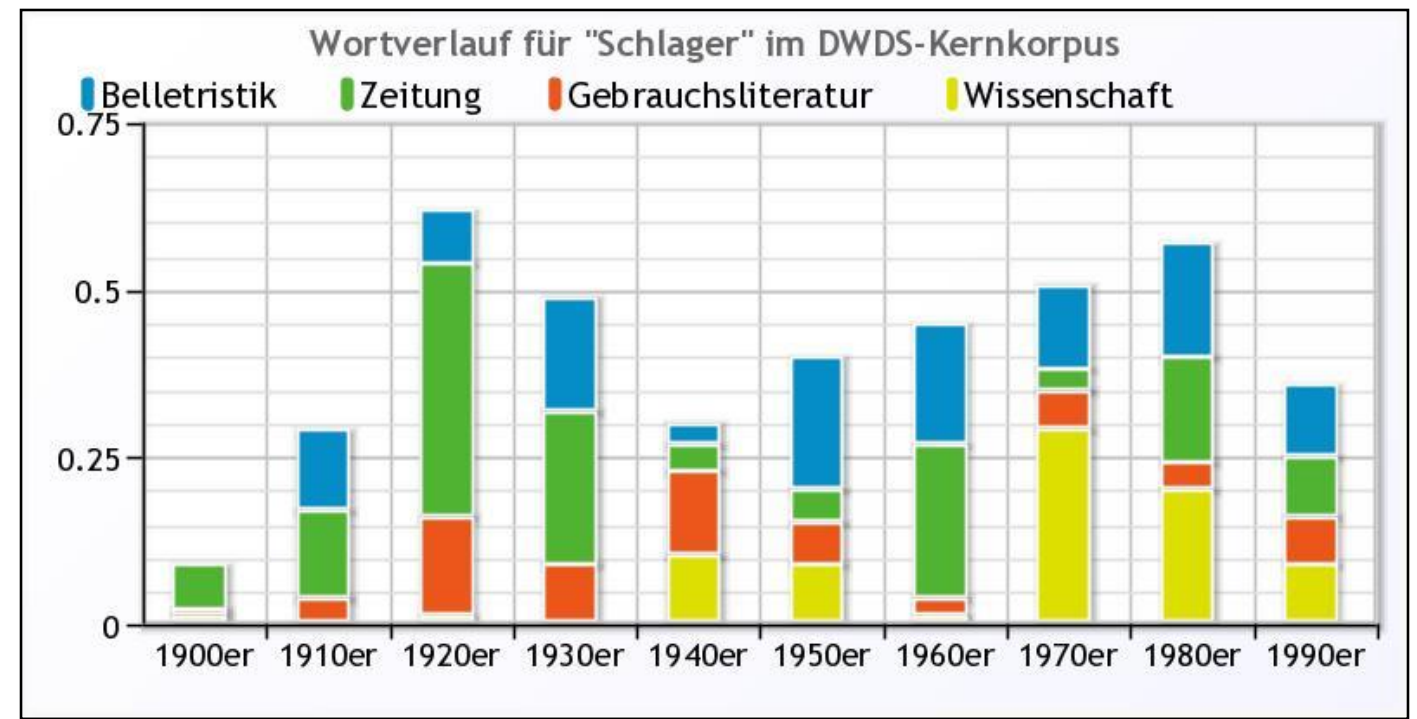

Abb. 140: Graphik: Wortverlauf von Schlager im 20. Jh. (DWDS)

Die Streuung des Wortes Schlager im 20. Jh. erinnert an eine Sinuskurve. Bei der Betrachtung der Belege stellt man Folgendes fest: In den ersten Jahrzehnten war mit Schlager allgemein ein künstlerisches Erfolgswerk gemeint. Dies lässt sich aus den ausgewählten Textpassagen in der Abb. 141 ableiten. In Klammern sind die Erscheinungsjahre der Texte angegeben.

(1903) Eine "Elf Scharfrichter" - Nummer mit einem Titelbild von Th. Th. Heine ist der neueste Schlager , mit dem der Verlag Bühne und Brettl (...), soeben auf dem Plane erscheint.

(1914) Um diesen Schlager wird die Operette gebaut, wölbt sie sich, wie die Glasglocke um den Kuhkäse.

(1922) Dem Schauspieler wurde es immer flauer ums Herz. Vor diesen Leuten sollte er sich nun produzieren, sollte lustige Schlager zum besten geben. In einem Kaffeehaus...

(1922) Der Schlager des Tages aber sollte die Ausstellung des damals eben gezüchteten Rassepferdes "Westfälisches Edelblut" sein.

Abb. 141: Auswahl der Konkordanzen zur Abfrage Schlager (DWDS, Kernkorpus)

Die Abfragen werden zum Vergleich im DeReKo durchgeführt.

\section{Recherche im DeReKo}

Der Korpusmanager Cosmas II ermöglicht nämlich auch die Jahrzehntansicht, darüber hinaus aber auch die Ansicht nach Jahren (Jahresansicht), Monaten (Monatsansicht), sogar Tagen (Tagesansicht).

- Cosmas $\mathbf{I I}_{\text {web }} \rightarrow$ Recherche

- Archiv: W-Archiv der geschriebenen Sprache

- Korpus: W-öffentlich - alle öffentlichen Korpora des Archivs W

- Optionen: Suchmodalitäten:

Groß- / Kleinschreibung beachten für 1. Zeichen

Expansionslisten:

auswählen

Übernehmen

Ergebnispräsentation: Jahrzehntansicht abwählen auswählen

- Suchanfrage (Eingabe ins Suchfeld): Hit $\rightarrow$ Suchen (Ergebnis: Abb. 142) dann: Schlager $\rightarrow$ 


\begin{tabular}{|l|r|l|r|l|}
\hline & Treffer & rel. Häuf. & Texte & Jahrzehnt \\
\hline$\boxplus$ & 4 & $1.41 \mathrm{pMW}$ & 4 & $1960-1969$ \\
\hline$\boxplus$ & 1 & $0.51 \mathrm{pMW}$ & 1 & $1970-1979$ \\
\hline$\boxplus$ & 28 & $2.36 \mathrm{pMW}$ & 27 & $1980-1989$ \\
\hline$\boxplus$ & 9.915 & $0.19 \mathrm{pMW}$ & 9.105 & $1990-1999$ \\
\hline$\boxplus$ & 13.855 & $7.75 \mathrm{pMW}$ & 12.564 & $2000-2009$ \\
\hline$\boxplus$ & 15.449 & $0.33 \mathrm{pMW}$ & 12.224 & $2010-2019$ \\
\hline
\end{tabular}

Abb. 142: Wortverlauf von Hit (DeReKo)

\begin{tabular}{|l|r|l|r|l|}
\hline & Treffer & rel. Häuf. & Texte & Jahrzehnt \\
\hline$\boxplus$ & 3 & $6.89 \mathrm{pMW}$ & 3 & $1940-1949$ \\
\hline$\boxplus$ & 5 & $2.82 \mathrm{pMW}$ & 3 & $1950-1959$ \\
\hline$\boxplus$ & 28 & $9.85 \mathrm{pMW}$ & 21 & $1960-1969$ \\
\hline$\boxplus$ & 14 & $7.09 \mathrm{pMW}$ & 13 & $1970-1979$ \\
\hline$\boxplus$ & 39 & $3.29 \mathrm{pMW}$ & 35 & $1980-1989$ \\
\hline$\boxplus$ & 15.122 & $5.54 \mathrm{pMW}$ & 12.096 & $1990-1999$ \\
\hline$\boxplus$ & 16.047 & $8.97 \mathrm{pMW}$ & 12.919 & $2000-2009$ \\
\hline$\boxplus$ & 10.431 & $6.97 \mathrm{pMW}$ & 8.064 & $2010-2019$ \\
\hline & & & & \\
\hline & $\mathbf{4 1 . 6 8 9}$ & $\mathbf{9 . 7 4} \mathrm{pMW}$ & $\mathbf{3 3 . 1 5 4}$ & 8 Jahrzehnte \\
\hline
\end{tabular}

Abb. 143: Wortverlauf von Schlager (DeReKo)

Zur Ansicht nach Jahren wechselt man durch Klicken auf Ergebnisse $\rightarrow$ Jahresansicht am linken Rand.

\section{Fazit}

Die Daten in den Abb. 142 und 143 bestätigen die grobe Darstellung der Abb. 139 und 140: es ist deutlich zu sehen, dass der Hit etwa Anfang der 1970er Jahre den Schlager abgelöst hat. (Konkordanzen aus den einzelnen Jahrzehnten werden durch einen Klick auf $\boxplus$ in der entsprechenden Zeile abgerufen.) Interessant wäre noch der Vergleich von Kookkurrenzen von Schlager in den 1940ern bis 1960ern und Hit ab den 1970er Jahren. Diese Untersuchung bleibt vorerst ein Desiderat. 


\section{Pragmatik}

Mithilfe von Korpora lassen sich relativ gut auch pragmatische Elemente der Sprache abrufen, solange diese Elemente irgendwie fest an der sprachlichen Oberfläche verankert sind.

\section{Studie 13: Illokutionsverben}

Zur Manifestation eines pragmatischen Markers zählen Prädikate in Einleitungssätzen zu direkter oder indirekten Rede. Diese können nur in morphosyntaktisch annotierten Korpora recherchiert werden (hier DeReKo), indem das Prädikatsverb abgerufen wird. Die Fragestellung nach den Realisierungsmöglichkeiten dieser Verben eröffnet auch die Hypothese, dass diese Verben nicht alle dem Wortfeld/ der semantischen Gruppe der Verben des Sprechens (verba dicendi) angehören müssen (vgl. Hirschová 2013: 172-174).

- Cosmas $\mathbf{I I}_{\text {web }} \rightarrow \underline{\text { Recherche }}$

- Archiv: TAGGED-T - Archiv morphosyntakt. annotierter Korpora (TreeTagger)

- Korpus: TAGGED-T-öffentlich - alle öffentlichen Korpora des Archivs TAGGED-T

- Optionen: Standardeinstellungen

- Suchanfrage (Eingabe ins Suchfeld): MORPH(VRB·fin $\cdot v) \cdot \because \cdot 1 " \rightarrow$ dann:

- Suchanfrage (Eingabe ins Suchfeld):

$$
\text { MORPH(VRB·fin·v) } \cdot \cdot /+ \text { w1.dass·oder } \cdot \text { daß } \rightarrow
$$

Die erste Abfrage bedeutet: Suche alle finiten Verben gefolgt von einem Doppelpunkt und Anführungszeichen. Das Ergebnis (Auswahl) ist in der Abb. 144 zu sehen.

Die zweite Abfrage bedeutet: Suche alle finiten Verben gefolgt von einem Beistrich und dass, bzw. daß. Einige Ergebnisse dieser Abfrage sind in der Abb. $145 \mathrm{zu}$ sehen.

Die Einleitungen bzw. „Ausleitungen“ der direkten Rede werden u.a. durch diese Prädikate realisiert:

\begin{tabular}{|lrll|}
\hline HMP09 & Und auch Sturm-Partner Klose & bemerkte erleichtert: "Ich & bin froh, \\
NON09 & Truppe gibt. An die Kritiker & appelliert er: "Wir & befinden uns mit \\
RHZ09 & zu führen. Der CDU-Politiker & betonte zugleich: "Die & Straße ist fast \\
NON08 & und Ziele für Peking & sagt er: "Ich & erwarte nicht, denn \\
RHZ08 & zum Koblenzer Stadtrand, dann & sagt sie: "Auf & geht's. Du läufst heute \\
M07 & der Radsport Doping-verseucht?" & antwortete Ullrich: "Das & wüsste ich \\
NON07 & Leopold Schmölz & ist zuversichtlich: "Mit & der Gemeinde \\
RHZ07 & erklärt Klute-Wetterauer und & fügt hinzu: "Wir & wollen dem Nachwuchs \\
HMP06 & Corinna Berghoff - selbst unverheiratet - lächelt vieldeutig: "Darauf warte ich \\
RHZ06 & Elsbeth Schmidt & zog Erfolgsbilanz: "Wir & haben den \\
RHZ06 & herum. Trainer Burkhard Lau & meint aber: "Abgerechnet & wird am \\
RHZ06 & Trainer Stefan Liesenfeld & ärgerte sich: "Von & den Chancen her \\
\hline
\end{tabular}

Abb. 144: Auswahl der Konkordanzen zur Abfrage: finites Verb - Doppelpunkt - Anführungszeichen (DeReKo)

Diese Prädikate können mit jenen verglichen, die die indirekte Rede einleiten.

Die indirekte Rede kann im Deutschen durch die Prädikate in der Abb. 145 (nächste Seite) ein- oder ausgeleitet werden. 


\begin{tabular}{|lcl|}
\hline NUN9 & \multicolumn{1}{c|}{ Die Autoren } & \multicolumn{2}{c|}{ bemerken kritisch, dass der (westlichen) melancholischen Sehnsucht } \\
M08 & ...alle (...) & sagen uns, dass wir viel zu streng sind. \\
A09 & Gemeindepräsident Hans Bütikofer & hielt fest, dass er und seine Amtskollegen die \\
A09 & Remer und seine Kollegen sagen (...)und & warnen zugleich, dass in bestimmten Regionen - \\
A09 & Konkordanzregierung mitzumachen. Ich & denke nicht, dass die SVP irgendjemandem, schon \\
A09 & Militärsprecher behauptet habe. Weiter & erzählte Sanur, dass er Material und Werkzeug \\
A09 und Maximalsteuerfüsse vorschreibe. Zudem & bemängelten sie, dass die Regelung lediglich \\
A09 & die uns gar nicht passen», sagte Grau, & betonte aber, dass Veränderungen zu jedem Leben \\
A09 & Gemeindepräsident Hans Bütikofer & hielt fest, dass er und seine Amtskollegen die \\
A09 & nicht als Frühaufsteherin bezeichnet, & erklärte sie, dass man einmal im Jahr eine \\
A09 & muss aufrechterhalten werden.» Daneben & betonte er, dass das frühe Aufstehen für ihn \\
\hline
\end{tabular}

Abb. 145: Auswahl der Konkordanzen zur Abfrage: finites Verb - Doppelpunkt - dass/daß (DeReKo)

Die Abfragen in Cosmas II liefern über 62.000 Treffer für die direkte Rede (eine Auswahl davon ist in der Abb. 144 abgedruckt) und insgesamt über eine halbe Mio. Treffer für die indirekte Rede (Auswahl in der Abb. 145). Unter ihnen sind auch etliche Belege, die der gewünschten Abfrage nicht entsprechen, etwa:

Sein Ziel ist es, dass es künftig weniger

für Gebäude im Mittelpunkt stehen werde: "Was bedeutet der

Das Aussortieren solcher Fehlbelege dauert jedoch wesentlich kürzer als eine „manuelle“ Recherche mittels Durchlesen von Büchern, Zeitschriften, Zeitungen und anderen Texten.

\section{Fazit:}

Die Prädikate der einleitenden Sätze entsprechen der Intention der Aussage, die sie einleiten (sie entsprechen der illokutiven Kraft der Aussage). Sie spiegeln gewissermaßen die Einstellung der Sprechenden zum Sachverhalt der Aussage wider. Diese Einstellung ist aus den Prädikaten der Einleitungssätze ersichtlich. In der Tab. 29 werden sie alle aus den Abb. 144 und 145 zusammengefasst und alphabetisch aufgelistet

\begin{tabular}{|l|l|}
\hline Prädikat & Illokution \\
\hline Ullrich antwortete & ANTWORTEN \\
\hline er appelliert & APPELLIEREN \\
\hline Trainer ärgerte sich & (sich) ärgern (= SAGEN VERÄRGERT) \\
\hline sie bemängelten & BEMÄNGELN \\
\hline Partner, Autoren bemerkte(n) (erleichtert, kritisch) & BEMERKEN \\
\hline (Politiker) betonte & BETONEN \\
\hline denkt (nicht) & DENKEN/ NICHT DENKEN \\
\hline Schmidt zog Erfolgsbilanz & $\begin{array}{l}\text { Erfolgsbilanz ziehen } \\
=\text { ERKLÄREN STOLZ, PRAHLEN }\end{array}$ \\
\hline sie erklärt & ERKLÄREN \\
\hline Sanur erzählt & ERZÄHLEN \\
\hline Gemeindepräsident hielt fest & FESTHALTEN \\
\hline Klutte-W. fügt hinzu & HINZUFÜGEN \\
\hline Corinna Berghoff lächelt & lächeln = (Implikatur) SAGEN FRÖHLICH \\
\hline Trainer meint & MEINEN \\
\hline sie sagt & SAGEN \\
\hline Remer und seine Kollegen warnen & WARNEN \\
\hline L. Schmölz ist zuversichtlich. & zuversichtlich sein \\
& $=$ SAGEN ZUVERSICHTLICH, OPTIMISTISCH \\
\hline
\end{tabular}

Tab. 29: Auswahl der Prädikate in den Einleitungssätzen zur direkten und indirekten Rede 
Vergleicht man die Prädikate, die die direkte und indirekte Rede einleiten, stellt man fest, dass sie sich oft entsprechen: bemerken, betonen, sagen um nur einige, die für die Abb. 144 und 145 ausgewählt worden sind, zu nennen.

Es gibt natürlich weit mehr Möglichkeiten, die Intention der Sprechenden im Einleitungssatz darzustellen. Wie diese in Rundfunknachrichten realisiert werden, beschreibt Káňa (2007). Für die Analyse der Nachrichten musste ein eigenes Korpus erstellt werden, da in keinem elektronischen Korpus Radiokurznachrichten vorhanden sind. Für den allgemeinen Sprachgebrauch im DaF/DaZ-Unterricht sind die üblichen Korpora jedoch völlig ausreichend und die Lernenden können die Sprache aus diesem pragmatischen Gesichtspunkt erfassen. 


\section{Statistiken}

Statistische Angaben über eine Sprache umreißen die Konturen dieser Sprache. Aus ihnen können wichtige Elemente für die Vermittlung der Sprache abgeleitet werden. Bisherige Werke, die sich in irgendeiner Hinsicht mit Häufigkeiten befassten (Wörterbücher, (Lerner)Grammatiken, Frequenzwörterbücher), sind veraltet und „viele davon wenig repräsentativ für die deutsche Sprache in ihrer Gesamtheit im gesamten deutschsprachigen Raum“ (Tschirner: 2005: 136). Die folgenden Angaben betreffen nur einige wenige Aspekte des Deutschen, beschränken sich mehrheitlich auf die Lexik und (teilweise) ihre Morphologie. Andere Aspekte müssen vorerst ein Desiderat bleiben, mit der Hoffnung, dass sie auch bald statistisch erfasst werden (können).

Die Angaben über die deutsche Sprache in den kommenden Tabellen basieren auf Daten des Korpus InterCorp_de (Gesamtkorpus) sowie auf Daten des deTenTen-Korpus. (Nur im letzteren lassen sich einige Kategorien sinnvoll abfragen.) Die Ergebnisse wurden teilweise (falls es die Eigenschaften des COSMAS II ermöglichen) mit Rechercheergebnissen aus DeReKo verglichen. So sollen die Daten objektiviert werden und einen Leitfaden anbieten, welche Elemente im Deutschunterricht auf keinen Fall fehlen sollten.

Die Tabellen sind entweder nach der Häufigkeit oder alphabetisch absteigend gereiht. Die Frequenzangaben beziehen sich auf die relative Häufigkeit umgerechnet auf 1 Mio. Wörter (in der Sprache des InterCorp ,i.p.m. - instances per million“, im DeReKo „p.M.W. - pro Million Worte").

\subsection{Morphologische Kategorien}

\section{Genus der Substantive}

\begin{tabular}{|l|l|}
\hline Feminina & 76562 p.M.W. \\
\hline Maskulina & 64479 p.M.W. \\
\hline Neutra & 42428 p.M.W. \\
\hline
\end{tabular}

\section{Kasus der Substantive (alle Genera)}

\begin{tabular}{|l|r|}
\hline Nominativ & 59542 p.M.W. \\
\hline Dativ & 53000 p.M.W. \\
\hline Akkusativ & 51913 p.M.W. \\
\hline Genitiv & 18900 p.M.W. \\
\hline
\end{tabular}

\section{Numerus der Substantive (alle Genera)}

\begin{tabular}{|l|r|}
\hline Singular & 131153 p.M.W. \\
\hline Plural & 52718 p.M.W. \\
\hline
\end{tabular}

Die häufigste morphologische Form der Substantive ist Nominativ Singular Maskulinum, gefolgt von Nominativ Singular Femininum.

Die vergleichsmäßig ,,seltensten“ Formen sind Genitive Plural (feminin, maskulin, neutrum). 
Komparationsformen der Adjektive

\begin{tabular}{|l|r|}
\hline Positiv & 46559 p.M.W. \\
\hline Komparativ & 1523 p.M.W. \\
\hline Superlativ & 1522 p.M.W. \\
\hline
\end{tabular}

\section{Artikel}

\begin{tabular}{|l|l|}
\hline Bestimmte Artikel & 70016 p.M.W. \\
\hline Unbestimmte Artikel & 17049 p.M.W. \\
\hline
\end{tabular}

\section{Frequenzen der Verben nach ihrer Form und Funktion:}

\begin{tabular}{|l|r|}
\hline Finite Vollverben & etwa $43 \%$ \\
\hline Finite Hilfsverben & etwa $22 \%$ \\
\hline Partizip Perfekt von Vollverben & etwa $11 \%$ \\
\hline Infinite Vollverben & etwa $11 \%$ \\
\hline Finite Modalverben & etwa $6 \%$ \\
\hline Infinite Hilfsverben & etwa $2 \%$ \\
\hline (Fehler & etwa 5\%) \\
\hline
\end{tabular}




\subsection{Lexikalische Realisierungen}

Substantive mit der höchsten Frequenz (alphabetisch):
1. Abend
34. Herz
67. Raum
2. Anfang
35. Hilfe
68. Recht
3. Arbeit
36. Idee
69. Richtung
4. Art
37. Jahr
70. Sache
5. Arzt
6. Aufgabe
38. Jahrhundert
71. Seite
39. Junge
72. Schritt
7. Auge
40. Kampf
73. Schule
8. Auto
41. Kind
74. Sinn
9. Beispiel
42. Kirche
75. Sohn
10. Bild
43. Kopf
76. Spiel
11. Blick
44. Kraft
77. Staat
12. Buch
45. Krieg
78. Stadt
13. Deutsch/e
46. Land
79. Stelle
14. Eltern
47. Leben
80. Stimme
15. Ende
48. Leute
81. Straße
16. Fall
49. Mädchen
82. Stück
17. Familie
50. Mal
83. Stunde
18. Form
51. Mann
84. Tag
19. Frage
52. Mensch
85. Teil
20. Frau
53. Meter
86. Tier
21. Freund
54. Minute
87. Tod
22. Geld
55. Mitte
88. Tochter
23. Gesellschaft
56. Möglichkeit
89. Uhr
24. Gesetz
57. Monat
90. Vater
25. Geschichte
58. Mutter
91. Wasser
26. Gesicht
59. Nacht
92. Weg
27. Gespräch
60. Name
93. Welt
28. Grenze
61. Ort
94. Wohnung
29. Grund
62. Partei
95. Woche
30. Gruppe
63. Person
96. Wort
31. Hand
64. Platz
97. Zeit
32. Haus
65. Problem
98. Zeitung
33. Herr
66. Punkt
99. Ziel
100. Zug 


\section{Adjektive mit der höchsten Frequenz (alphabetisch):}

\begin{tabular}{|c|c|c|c|}
\hline 1. absolut & 34. bunt & 67. & entfernt \\
\hline 2. ähnlich & 35. dankbar & 68. & entsetzlich \\
\hline 3. allgemein & 36. dauernd & 69. & entscheidend \\
\hline 4. allmählich & 37. deutlich & 70. & entsprechend \\
\hline 5. alt & 38. deutsch & 71. & erfolgreich \\
\hline 6. angeblich & 39. dick & 72. & genau \\
\hline 7. angenehm & 40. dicht & 73. & gleich \\
\hline 8. ängstlich & 41. direkt & 74. & groß \\
\hline 9. anscheinend & 42. doppelt & 75. & gut \\
\hline 10. anständig & 43. dringend & 76. & hoch \\
\hline 11. arm & 44. dritt & 77. & jung \\
\hline 12. aufmerksam & 45. dumm & 78. & kalt \\
\hline 13. ausgerechnet & 46. dumpf & 79. & klar \\
\hline 14. bekannt & 47. dunkel & 80. & klein \\
\hline 15. bequem & 48. dünn & 81. & kurz \\
\hline 16. bereit & 49. düster & 82. & lang \\
\hline 17. berühmt & 50. egal & 83. & leer \\
\hline 18. bescheiden & 51. ehrlich & 84. & leicht \\
\hline 19. besorgt & 52. echt & 85. & letzt \\
\hline 20. bestimmt & 53. eifrig & 86. & möglich \\
\hline 21. bewuss/ßt & 54. eigen & 87. & nah \\
\hline 22. billig & 55. eilig & 88. & neu \\
\hline 23. bitter & 56. eindeutig & 89. & offen \\
\hline 24. blass $/ \AA$ & 57. einfach & 90. & öffentlich \\
\hline 25. blau & 58. einsam & 91. & politisch \\
\hline 26. bleich & 59. einzeln & 92. & richtig \\
\hline 27. blöd & 60. einzig & 93. & rund \\
\hline 28. blond & 61. elegant & 94. & schnell \\
\hline 29. bloß & 62. elend & 95. & schön \\
\hline 30. böse & 63. endgültig & 96. & schwer \\
\hline 31. braun & 64. endlos & 97. & stark \\
\hline 32. brav & 65. eng & 98. & vergangen \\
\hline 33. breit & 66. englisch & 99. & weiß \\
\hline & & 100. & wichtig \\
\hline
\end{tabular}




\section{Vollverben mit der höchsten Frequenz (alphabetisch)}

1. ändern

2. arbeiten

3. aufnehmen

4. aussehen

5. bauen

6. befinden

7. beginnen

8. bekommen

9. berichten

10. bestehen

11. bieten

12. bleiben

13. brauchen

14. bringen

15. denken

16. entscheiden

17. entstehen

18. erfahren

19. erhalten

20. erinnern

21. erklären

22. erreichen

23. erscheinen

24. erwarten

25. erzählen

26. fahren

27. fallen

28. fehlen

29. finden

30. folgen

31. fordern

32. fragen

33. freuen
34. fühlen

35. führen

36. geben

37. gehen

38. gehören

39. gelten

40. geraten

41. gewinnen

42. glauben

43. halten

44. handeln

45. heißen

46. helfen

47. hoffen

48. holen

49. hören

50. kennen

51. kommen

52. lassen

53. laufen

54. leben

55. legen

56. leisten

57. lernen

58. lesen

59. liegen

60. machen

61. meinen

62. melden

63. nehmen

64. nennen

65. öffnen

66. reden
67. rechnen

68. sagen

69. sehen

70. setzen

71. schaffen

72. scheinen

73. schlagen

74. schließen

75. schreiben

76. sitzen

77. sorgen

78. spielen

79. sprechen

80. stehen

81. steigen

82. stellen

83. sterben

84. suchen

85. tragen

86. treffen

87. tun

88. übernehmen

89. verlassen

90. verlieren

91. verstehen

92. versuchen

93. vorstellen

94. wählen

95. warten

96. wirken

97. wissen

98. zählen

99. zeigen

100. 100. ziehen

Die Verben haben und sein als Vollverben befinden sich erst unter den ersten 120 häufigsten deutschen Verben.

\section{Modalverben}

(Anteil am Gesamtvorkommen der Modalverben)
1. können $37 \%$
2. müssen $25 \%$
3. sollen $18 \%$
4. wollen $8 \%$
5. mögen $7 \%$
6. dürfen $5 \%$ 


\section{Partizipien Perfekt mit der höchsten Frequenz (nach Frequenz):}
1. gesagt
2. gesehen
3. gekommen
4. gemacht
5. gegeben
6. getan
7. gefunden
8. gebracht
9. gegangen
10. verloren
11. gedacht
12. gesprochen
13. genommen
14. vergessen
15. geschrieben
16. verlassen
17. geblieben
18. gehört
19. verschwunden
20. gefallen
21. erfahren
22. geschehen
23. passiert
24. gehalten
25. genannt
26. gefragt
27. erreicht
28. gelesen
29. geboren
30. versucht
31. gestellt
32. gelernt
33. gestorben

34. geführt

67. gezwungen

35. verstanden

68. geschickt

36. erwartet

69. gekauft

37. gesetzt

70. erfüllt

38. gelegt

71. geworfen

39. getroffen

72. verändert

40. gezogen

73. erkannt

41. entdeckt

74. gelebt

42. gewuss/ßt

75. erschrocken

43. geschlossen

76. überrascht

44. erklärt

77. entschlossen

45. begonnen

78. gelegen

46. verrückt

79. versteckt

47. beschäftigt?

80. angenommen

48. geschlagen

81. gemeint

49. erschienen

82. begriffen

50. erzählt

51. gelungen

83. getragen

52. entfernt

84. gespielt

53. bestimmt

85. geschafft

54. gefahren

86. gewartet

55. geraten

56. bemerkt

87. geliebt

57. gerichtet

88. gewonnen

89. verbunden

58. geglaubt

90. gerufen

91. bekommen

59. gezeigt

92. verboten

60. gewöhnt

93. geschlafen

61. erhalten

94. gebaut

62. angefangen

95. versprochen

63. gelassen

96. bekannt

64. geöffnet

97. gesucht

65. aufgenommen

98. gebeten

66. gestanden

99. vorbereitet

100. gerettet

Infinitive zu diesen häufigsten Partizipien der deutschen Verben sind auf der folgenden Seite alphabetisch aufgelistet. 


\section{Infinitive zu den häufigsten Partizipien (alphabetisch):}

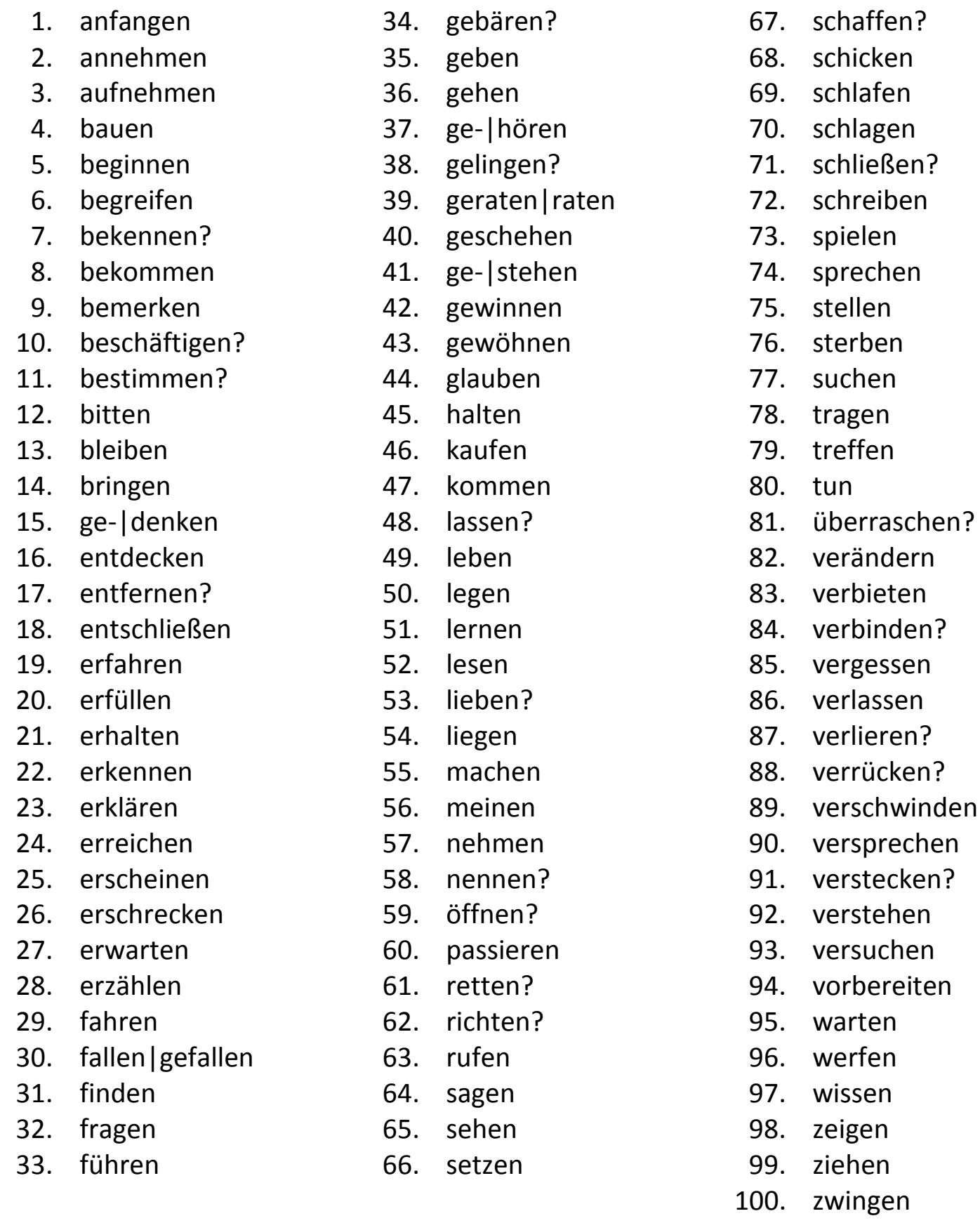

\section{Bemerkung:}

Bei den meisten deutschen Verben gilt Folgendes: das Vorkommen vom Infinitiv zusammengerechnet mit allen finiten Formen desselben Verbs hat eine höhere Frequenz als sein Partizip Perfekt (inkl. fehlerhaft annotierte deverbative Adjektive). Umgekehrt ist es bei den Verben, die mit einem Fragezeichen gekennzeichnet sind. 


\section{Verbzusätze mit der höchsten Frequenz}

\section{Nach Frequenz}

1. an

2. auf

3. aus

4. zu

5. ein

6. $a b$

7. zurück

8. vor

9. hin

10. um

11. zusammen

12. nach

13. weiter

14. heraus

15. fort

16. mit

17. herum

18. da

19. her

20. fest

21. hinaus

22. hervor

23. vorbei

24. hinein

25. los
26. hoch

27. hinzu

28. hinunter

29. hinauf

30. weg

31. entgegen

32. durch

33. heran

34. nieder

35. nahe

36. herein

37. gegenüber

38. hinüber

39. herunter

40. herab

41. über

42. hinab

43. bei

44. empor

45. voraus

46. inne

47. raus

48. umher

49. statt

50. auseinander

\section{Alphabetisch}

1. $a b$

26. hinaus

2. an

27. hinein

3. auf

28. hinüber

4. aus

29. hinunter

5. auseinander

6. bei

30. hinzu

31. hoch

7. da

32. inne

8. durch

33. los

9. ein

34. mit

10. empor

35. nahe

11. entgegen

36. nach

12. fest

37. nieder

13. fort

38. raus

14. gegenüber

39. statt

15. her

16. herab

40. über

41. um

17. heran

18. heraus

42. umher

43. vor

19. herein

44. voraus

20. herum

45. vorbei

21. herunter

46. weg

22. hervor

23. hin

47. weiter

24. hinab

48. zu

49. zurück

25. hinauf

50. zusammen 


\section{Verben mit den häufigsten Verbzusätzen}

Die folgenden alphabetischen Listen der häufigsten Verben mit einzelnen Verbzusätzen wurden als ein Querschnitt von komplexen Abfrageergebnissen (siehe Studie 7) im DeReKo und InterCorp_de zusammengestellt. Die Kriterien für die Auswahl der Lexeme waren signifikante Frequenzen in beiden Korpora und bei allen Abfragen. Die Vorgangsweise bei der Recherche ermöglicht auch den Vergleich, wie produktiv einzelne Verbzusätze sind. Aus diesem Grund variiert die Anzahl der Lexeme mit einzelnen Zusätzen. Die Listen wurden für die häufigsten zehn Verbzusätze zusammengestellt, des Weiteren auch für hinüber- und inne- um die Schwankungen in der Produktivität zu verdeutlichen.

\section{Die häufigsten Verben mit dem Zusatz an (alphabetisch):}

$\begin{array}{lll}\text { anbieten } & \text { anhören } & \text { anschauen } \\ \text { anblicken } & \text { ankommen } & \text { anschließen } \\ \text { anfahren } & \text { anlächeln } & \text { anschreien } \\ \text { anfangen } & \text { annehmen } & \text { ansprechen } \\ \text { angehen } & \text { anrufen } & \text { anstarren } \\ \text { angreifen } & \text { ansagen } & \text { anstoßen } \\ \text { anhalten } & \text { ansehen } & \text { anziehen } \\ \text { anheben } & \text { ansetzen } & \text { anzünden }\end{array}$

\section{Die häufigsten Verben mit dem Zusatz auf (alphabetisch):}

$\begin{array}{ll}\begin{array}{l}\text { aufatmen } \\ \text { aufblicken } \\ \text { aufbrechen } \\ \text { auffallen }\end{array} & \text { auflachen } \\ \text { auflegen } \\ \text { auffangen } & \text { aufleuchten } \\ \text { auffliegen } & \text { auflösen } \\ \text { auffordern } & \text { aufnehmen } \\ \text { aufgehen } & \text { aufpassen } \\ \text { aufhalten } & \text { aufreißen } \\ \text { aufheben } & \text { aufrichten } \\ \text { aufhören } & \text { aufsehen } \\ \end{array}$

aufschlagen

aufschließen

aufschreien

aufspringen

aufstehen

aufsteigen

aufstoßen

auftauchen

aufwachen

\section{Die häufigsten Verben mit dem Zusatz aus (alphabetisch):}

\begin{abstract}
ausbrechen
ausbreiten

ausdrücken

ausgehen

aushalten

ausholen

auskennen

auslachen

auslösen
\end{abstract}
ausmachen
auspacken
ausreichen
ausrufen
ausruhen
aussehen
ausschalten
aussprechen
ausspucken

\begin{abstract}
aussteigen
ausstoßen

ausstrahlen

ausstrecken

aussuchen

austauschen

austrinken

ausweichen

ausziehen
\end{abstract}


Die häufigsten Verben mit dem Zusatz zu (alphabetisch):

zudecken
zudrehen
zuflüstern
zugehen
zuhören
zuklappen
zukommen
zulächeln
zulassen

zulaufen

zuschreien

zunehmen

zunicken

zusteuern

zurennen

zustimmen

zurufen

zutrauen

zusehen

zuschauen

zuwenden

zuwerfen

zuschieben

zuwinken

zuschlagen

zuzwinkern

Die häufigsten Verben mit dem Zusatz ein (alphabetisch):

$\begin{array}{lll}\text { einatmen } & \text { einleuchten } & \text { einschlafen } \\ \text { einbiegen } & \text { einmischen } & \text { einschlagen } \\ \text { einbilden } & \text { einnehmen } & \text { einschließen } \\ \text { eindringen } & \text { einräumen } & \text { einsperren } \\ \text { einfallen } & \text { einreden } & \text { einstecken } \\ \text { eingehen } & \text { einrichten } & \text { einsteigen } \\ \text { eingestehen } & \text { einsammeln } & \text { einstellen } \\ \text { eingießen } & \text { einsaugen } & \text { eintreffen } \\ \text { eingreifen } & \text { einsehen } & \text { eintreten } \\ \text { einholen } & \text { einsetzen } & \text { einwenden } \\ \text { einhüllen } & \text { einschalten } & \text { einwerfen } \\ \text { einladen } & \text { einschenken } & \text { einziehen }\end{array}$

Die häufigsten Verben mit dem Zusatz ab (alphabetisch):

abbiegen
abbrechen
abdrücken
abfahren
abfallen
abhängen
abhauen
abheben
abholen
ablaufen
ablegen
ablehnen

ablösen

abnehmen

abreisen

abreißen

absetzen

abschalten

abschließen

abschneiden

abschütteln

abspielen

abspringen

abstoßen

abstreifen

absuchen

abtasten

abtrocknen

abwarten

abwehren

abwenden

abwinken

abwischen

absteigen

abzeichnen 


\title{
Die häufigsten Verben mit dem Zusatz zurück (alphabetisch):
}

\author{
zurückbleiben \\ zurückblicken \\ zurückfahren \\ zurückfallen \\ zurückgeben \\ zurückgehen \\ zurückhalten \\ zurückkehren
}

\author{
zurückkommen \\ zurücklassen \\ zurücklaufen \\ zurücklegen \\ zurücklehnen \\ zurückrennen \\ zurückrufen \\ zurückschieben
}

zurückschreien

zurückspringen

zurückstoßen

zurücktreten

zurückweisen

zurückwerfen

zurückwinken

zurückziehen

\section{Die häufigsten Verben mit dem Zusatz vor (alphabetisch):}

$\begin{array}{lll}\text { vorbereiten } & \text { vorlegen } & \text { vorschlagen } \\ \text { vorbeugen } & \text { vorlesen } & \text { vorstellen } \\ \text { vordringen } & \text { vorliegen } & \text { vorstrecken } \\ \text { vorfahren } & \text { vornehmen } & \text { vortäuschen } \\ \text { vorfinden } & \text { vorrücken } & \text { vortreten } \\ \text { vorgehen } & \text { vorsehen } & \text { vorwerfen } \\ \text { vorkommen } & \text { vorschieben } & \text { vorziehen }\end{array}$

Die häufigsten Verben mit dem Zusatz hin (alphabetisch):

$\begin{array}{lll}\text { hinbewegen } & \text { hinkriegen } & \text { hinschieben } \\ \text { hinblicken } & \text { hinlaufen } & \text { hinschwanken } \\ \text { hindeuten } & \text { hinlegen } & \text { hinsingen } \\ \text { hindrehen } & \text { hinmurmeln } & \text { hinstarren } \\ \text { hinfahren } & \text { hinnehmen } & \text { hinstrecken } \\ \text { hinfliegen } & \text { hinrennen } & \text { hinsummen } \\ \text { hingehen } & \text { hinrutschen } & \text { hinwandern } \\ \text { hinhalten } & \text { hinsehen } & \text { hinweisen } \\ \text { hinhocken } & \text { hinsetzen } & \text { hinwerfen } \\ \text { hinknien } & \text { hinschauen } & \text { hinziehen }\end{array}$

\section{Die häufigsten Verben mit dem Zusatz um (alphabetisch):}

$\begin{array}{lll}\text { umbinden } & \text { umhauen } & \text { umschnallen } \\ \text { umblättern } & \text { umkehren } & \text { umsteigen } \\ \text { umblicken } & \text { umkippen } & \text { umstoßen } \\ \text { umbringen } & \text { umreißen } & \text { umwenden } \\ \text { umdrehen } & \text { umsehen } & \text { umwerfen } \\ \text { umfallen } & \text { umschauen } & \text { umziehen } \\ \text { umgehen } & \text { umschlagen } & \end{array}$




\section{Die häufigsten Verben mit dem Zusatz hinüber (alphabetisch):}

\author{
hinüberbeugen \\ hinüberblicken \\ hinüberfahren \\ hinübergehen \\ hinüberkommen \\ hinüberlaufen \\ hinübernicken
}

hinüberrufen

hinübersehen

hinüberschauen

hinüberschieben

hinüberschielen

hinüberspringen

hinüberstarren hinüberstehen

hinübertragen

hinüberwerfen

hinüberwinken

hinüberzeigen

hinüberziehen

\section{Die häufigsten Verben mit dem Zusatz inne (alphabetisch):}

innehaben

innehalten

Verben, die die meisten Zusätze (aus den 10 häufigsten: an, auf, aus, zu, ein, ab, zurück, vor, hin, um) an sich binden sind auf der folgenden Seite aufgelistet.

(Wegen der Übersichtlichkeit werden die Nachbarverben abwechselnd in Fett- und Normalschrift gesetzt.) 


\section{Hochfrequentierte Verben mit den häufigsten zehn}

\section{Verbzusätzen:}

$$
\begin{array}{r}
\text { zurück blicken } \\
\text { um blicken } \\
\text { hin blicken } \\
\text { hinüber blicken } \\
\text { auf blicken } \\
\text { an blicken } \\
\text { ab brechen } \\
\text { aus brechen } \\
\text { auf brechen } \\
\text { um drehen } \\
\text { hin drehen } \\
\text { zu drehen } \\
\text { zurück fahren } \\
\text { vor fahren } \\
\text { hin fahren } \\
\text { ab fahren } \\
\text { hinüber fahren } \\
\text { an fahren } \\
\text { zurück fallen } \\
\text { um fallen } \\
\text { ein fallen } \\
\text { ab fallen } \\
\text { auf fallen } \\
\text { zurück gehen } \\
\text { vor gehen } \\
\text { um gehen } \\
\text { hin gehen } \\
\text { ein gehen } \\
\text { ab heben }
\end{array}
$$

ein holen
ab holen
aus holen
zu hören
auf hören
an hören
zurück kommen
vor kommen
hinüber kommen
zu kommen
an kommen
hinüber laufen
zurück laufen
hin laufen
ab laufen
zu laufen
zurück legen
vor legen
hin legen
ab legen
auf legen
ab lösen
aus lösen
auf lösen
vor nehmen
hin nehmen
ein nehmen
ab nehmen
zu nehmen
auf nehmen
an nehmen
hinüber nicken
um reißen
ab reißen
auf reißen
zurück rennen

zurück rufen

hinüber rufen

zu rufen

aus rufen

an rufen

vor sehen

um sehen

hin sehen

ein sehen

hinüber sehen

zu sehen

aus sehen

auf sehen

an sehen

hin setzen

ein setzen

ab setzen

auf setzen

an setzen

ein schalten

ab schalten

aus schalten

hinüber schauen

um schauen

hin schauen

zu schauen

an schauen

hinüber schieben

zurück schieben

vor schieben

hin schieben

zu schieben

hinüber schielen

vor schlagen

um schlagen

ein schlagen

zu schlagen

auf schlagen

ein schließen

ab schließen

auf schließen

an schließen

Fortsetzung auf nächster Seite 


$\begin{array}{llllll}\text { zurück } & \text { schreien } & \text { vor } & \text { strecken } & \text { hinüber } & \text { winken } \\ \text { zu } & \text { schreien } & \text { hin } & \text { strecken } & \text { zurück } & \text { winken } \\ \text { auf } & \text { schreien } & \text { aus } & \text { strecken } & \text { ab } & \text { winken } \\ \text { an } & \text { schreien } & \text { hinüber } & \text { tragen } & \text { zu } & \text { winken } \\ \text { hinüber } & \text { springen } & \text { zurück } & \text { treten } & \text { hinüber } & \text { zeigen } \\ \text { zurück } & \text { springen } & \text { vor } & \text { treten } & \text { zurück } & \text { ziehen } \\ \text { ab } & \text { springen } & \text { ein } & \text { treten } & \text { vor } & \text { ziehen } \\ \text { auf } & \text { springen } & \text { um } & \text { wenden } & \text { um } & \text { ziehen } \\ \text { hinüber } & \text { starren } & \text { ein } & \text { wenden } & \text { hin } & \text { ziehen } \\ \text { hinüber } & \text { stehen } & \text { ab } & \text { wenden } & \text { ein } & \text { ziehen } \\ \text { um } & \text { steigen } & \text { zu } & \text { wenden } & \text { aus } & \text { ziehen } \\ \text { ein } & \text { steigen } & \text { zurück } & \text { werfen } & \text { hinüber } & \text { ziehen } \\ \text { ab } & \text { steigen } & \text { vor } & \text { werfen } & \text { an } & \text { ziehen } \\ \text { aus } & \text { steigen } & \text { um } & \text { werfen } & & \\ \text { auf } & \text { steigen } & \text { hin } & \text { werfen } & & \\ \text { zurück } & \text { stoßen } & \text { ein } & \text { werfen } & & \\ \text { um } & \text { stoßen } & \text { hinüber } & \text { werfen } & & \\ \text { ab } & \text { stoßen } & \text { zu } & \text { werfen } & & \\ \text { aus } & \text { stoßen } & & & & \\ \text { auf } & \text { stoßen } & & & & \\ \text { an } & \text { stoßen } & & & \end{array}$




\section{Adverbien mit der höchsten Frequenz (alphabetisch):}

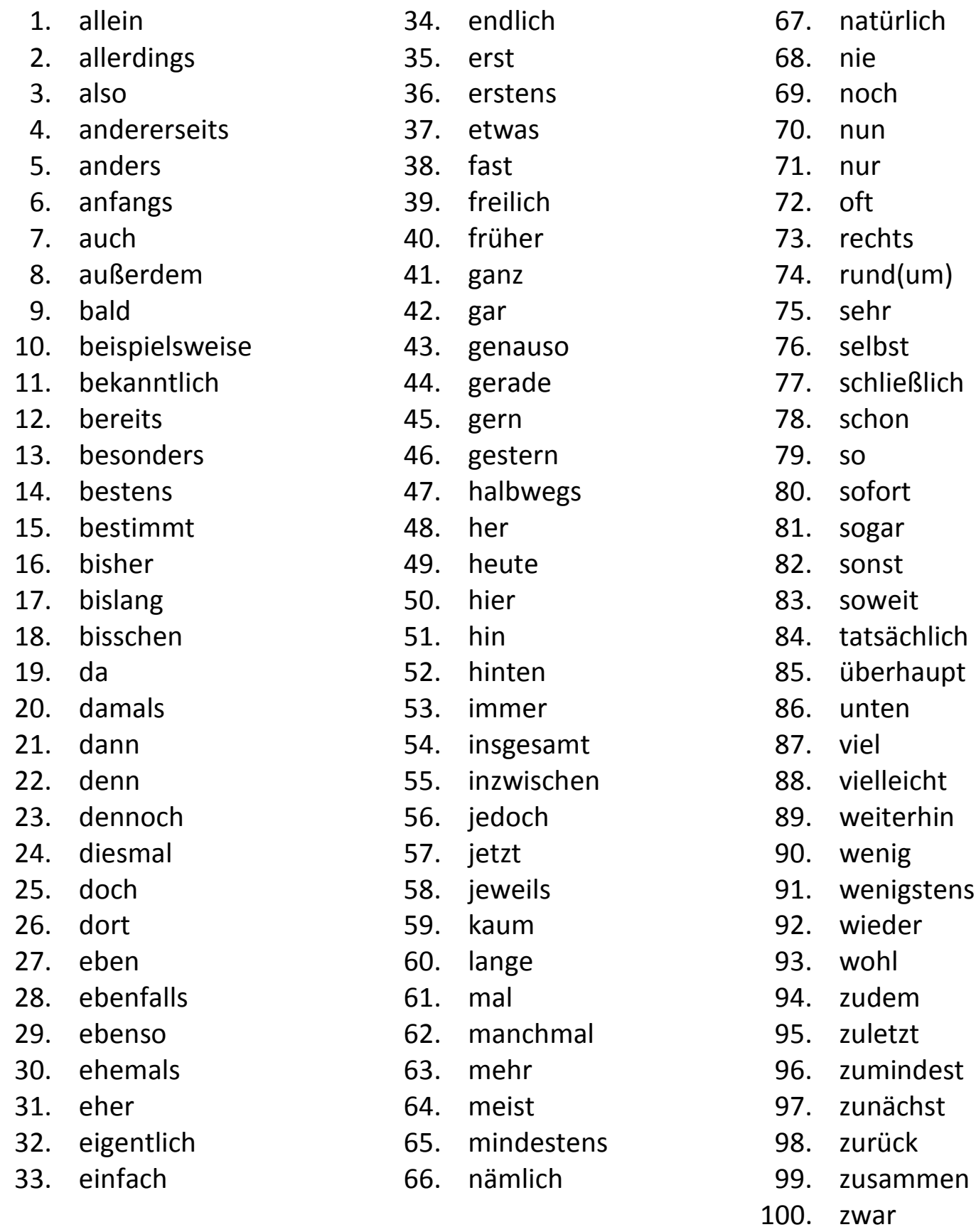

Obwohl diese Liste ein Ergebnis des Durchschnittsvorkommens der häufigsten Adverbien in gleich drei Korpora (DeReWo = DeReKo, InterCorp (Kernkorpus) und deTenTen) darstellt, ist ein Zweifel an der Richtigkeit der Berechnung angebracht: viele Adverbien sind homonym zu Präpositionen, Konjunktionen oder Partikel (z.B. denn). 


\section{Präpositionen (nach Frequenz):}

1. in

2. mit

3. auf

4. von

5. an

6. zu

7. für
8. aus

9. nach

10. über

11. vor

12. bei

13. durch

14. um
15. unter

16. gegen

17. ohne

18. zwischen

19. hinter

20. seit

\section{Die häufigsten Präpositionen (alphabetisch):}

ab
an
angesichts
auf
aufgrund
aus
außer
außerhalb
bei
bis
dank
durch
einschließlich
entlang
für
gegen
gegenüber

gleich
hinter
in
infolge
inmitten
innerhalb
je
jenseits
laut
mit
mitsamt
nahe
nach
namens
neben
oberhalb
ohne

per

pro

samt

seit

statt

trotz

über

um

unter

unterhalb

von

vor

während

wegen

zu

zugunsten

zwischen

\section{Interjektionen (nach Frequenz):}

Internettexte: Korpus deTenTen
1. ja
13. Aha
25. schwupp
2. $\mathrm{Na}$
14. ha
26. Gell
3. naja
15. Bravo
27. Ätsch
4. Ach/ach
16. Huch
28. tschüß
5. hallo
17. stop
29. Hoho
6. Oh
18. Basta
30. prost
7. Tja
19. ade
31. schwupps
8. nu
20. zack
32. autsch
9. Ah
21. au
33. uh
10. äh
22. hurra
34. marsch
11. $\mathrm{He}$
23. hopp
35. ahoi
12. Mann
24. piep 
Belletristische Texte: Korpus InterCorp_de
1. $\mathrm{Na}$
8. nu
15. ha
2. Ach
9. he
16. Bravo
3. Oh
10. marsch
17. ade
4. $\mathrm{au}$
11. basta
18. hurra
5. Hallo
12. hopp
19. Uh
6. Ah
13. Menschenskind
20. Prost
7. Aha
14. stop
21. Bah

\section{Die häufigsten Interjektionen (alphabetisch):}

Geschriebenes Deutschen (allgemein) ade

Ah

Gell

$\mathrm{Na}$

äh

ha

naja

Aha

Hallo

nu

ahoi

he

Oh

Ach

Hoho

piep

Ätsch

hopp

Prost

au

Huch

schwupp(s)

hurra

autsch

Bah

ja

stop

Tja

Mann

tschüß

basta

marsch

Uh

Bravo

Menschenskind

zack

\section{Bemerkung:}

Prinzipiell werden Interjektionen kleingeschrieben. Die Groß-/Kleinschreibung in den vorliegenden Listen bezeichnet die häufigere Form in Korpora und deutet darauf hin, ob die Präposition einen selbstständigen Satz bildet (bzw. am Anfang eines Satzes steht) oder ob sie in einem Satz integriert ist.

\subsection{Satz- und Textdiakritika (nach Häufigkeit):}

1.

2. .

3. ()

4. "

5. -

6. :
7. ;

8. " "

9. ?

10. !

11. []

12.
13.

14. /

15. $=$

$16 .--$

17.

18. \{\} 


\section{Korpusabfragehilfen}

\subsection{Abfragbare Affixe}

\section{Abfrage im DeReKo}

Im DeReKo können Affixe folgendermaßen ausgesucht werden:

- Einstellung der Suchoptionen: Lemmatisierung $\rightarrow$ sonstige Wortformen auswählen

- Suchfeldeingabe Präfixe: \&ab- $\rightarrow$

- Suchfeldeingabe Suffixe: $\&$-abel $\rightarrow$

- Ergebnisse $\rightarrow$ Export

- Export der Wortformen: Expansionsliste auswählen

Die Liste suchbarer Affixe in COSMAS II ${ }_{\text {win, web }}(2012)$ ist über das Hilfeportal ${ }^{56}$ abrufbar.

\begin{tabular}{|c|c|}
\hline Präfixe: & $\begin{array}{l}\text { ab-, abwärts-, agrar-, agro-, all-, alt-, an-, anti-, aqua-, äqui-, ar-, archä-, auf-, } \\
\text { aus-, be-, bei-, bi-, co-, d-1, da-, dar-, de-, dein-, di-, ein-, ent-, epi-, ex-, gar-, ge-, } \\
\text { gegen-, geo-, gut-, häm-, heim-, hell-, hemi-, her-, hin-, irr-, jen-, ko-, los-, mit-, } \\
\text { neo-, non-, ober-, para-, per-, phen-, post-, prä-, pre-, re-, rück-, rum-, semi-, sub-, } \\
\text { supra-, teil-, über-, um-, un-, unter-, ur-, ver-, vor-, weg-, wider-, wieder-, zer-, zu- } \\
\text { (insgesamt 72) }\end{array}$ \\
\hline Suffixe: & $\begin{array}{l}\text {-abel, -achsig, -al, -artig, -bar, -er, -fach, -förmig, -getreu, -haft, -haltig, -heit, -ig, } \\
\text {-in, -isch, -isierung, -ismus, -ist, -istin, -ität, -itis, -jährig, -kalibrig, -keit, -lagig, - } \\
\text { ler, -lich, -los, -mal, -maschig, -mässig/-mäßig, -phasig, -reihig, -sam, -schaft, - } \\
\text { schaftlich, -seitig, -strängig, -teilig, -tum, -ung, -volumig, -weise, -wertig, - } \\
\text { zylindrig (insgesamt 45) } \\
\text {-chen, -lein (Diminutivsuffixe) }\end{array}$ \\
\hline
\end{tabular}

\footnotetext{
Anmerkungen

${ }^{1}$ Artikel und Artikelwörter beginnend auf $d$ -

${ }^{2}$ Für Recherchen v.a. in schweizerischen Texten
}

\section{Abfrage im InterCorp}

Im InterCorp_de können Affixe nur einzeln ausgesucht werden.

Beispiel: Präfix $a b-$ :

- Query Type: CQL $\rightarrow$ CQL: [lemma="ab.*"] $\rightarrow$ Frequency $\rightarrow$ Node forms

Dann müssen einzelne Formen aussortiert werden.

56 http://www.ids-mannheim.de/cosmas2/web-app/hilfe/suchanfrage/affixe.html 


\subsection{Tag-Kürzel}

Die folgenden Kürzel der morphosyntaktischen Annotation werden in den meisten Korpora mit Deutsch verwendet, also auch im DWDS, DeReKo (Archiv TAGGED-T) und im InterCorp_de.

Das Original der folgenden Tabelle ist als STTS Tag Table (1995/1999) (Schmid 1995) auf der Homepage des Instituts für Maschinelle Sprachverarbeitung, Universität Stuttgart zugänglich (siehe IMS (2013) im Literaturverzeichnis). Hier ist sie leicht modifiziert und ergänzt. Beispiele der KWICs sind in Fettschrift gesetzt.

\section{Alphabetische Auflistung des Tag-Sets (STTS)}

\begin{tabular}{|c|c|c|}
\hline Tag & Beschreibung & Beispiel \\
\hline ADJA & attributives Adjektiv & das große Haus \\
\hline ADJD & adverbiales oder prädikatives Adjektiv & er fährt schnell, er ist schnell \\
\hline ADV & Adverb & schon, bald, doch \\
\hline APPO & Postposition & ihm zufolge, der Sache wegen \\
\hline APPR & Präposition; Zirkumposition links & in der Stadt, ohne mich \\
\hline APPRART & Präposition mit Artikel & im Haus, zur Sache \\
\hline APZR & Zirkumposition rechts & von jetzt an \\
\hline ART & bestimmter oder unbestimmter Artikel & der, die, das, ein, eine \\
\hline CARD & Kardinalzahl & zwei Männer, im Jahre 1994 \\
\hline FM & fremdsprachliches Material & Er hat das mit "A big fish" übersetzt \\
\hline ITJ & Interjektion & $\mathrm{mhm}, \mathrm{ach}, \mathrm{tja}$ \\
\hline KOKOM & Vergleichskonjunktion & als, wie \\
\hline KON & nebenordnende Konjunktion & und, oder, aber \\
\hline KOUI & $\begin{array}{l}\text { unterordnende Konjunktion mit "zu" und } \\
\text { Infinitiv }\end{array}$ & um zu leben, anstatt zu fragen \\
\hline KOUS & unterordnende Konjunktion mit Satz & weil, dass, damit, wenn, ob \\
\hline NE & Eigennamen & Hans, Hamburg, HSV \\
\hline NN & normales Nomen & Tisch, Herr, das Reisen \\
\hline PAV & Pronominaladverb & dafür, dabei, deswegen, trotzdem \\
\hline PDAT & attribuierendes Demonstrativpronomen & jener Mensch \\
\hline PDS & substituierendes Demonstrativpronomen & dieser, jener \\
\hline PIAT & $\begin{array}{l}\text { attribuierendes Indefinitpronomen ohne } \\
\text { Determiner }\end{array}$ & kein Mensch, irgendein Glas \\
\hline PIDAT $^{1}$ & $\begin{array}{l}\text { attribuierendes Indefinitpronomen mit } \\
\text { Determiner }\end{array}$ & einwenig Wasser, die beiden Brüder ${ }^{1}$ \\
\hline PIS & substituierendes Indefinitpronomen & keiner, viele, man, niemand \\
\hline PPER & irreflexives Personalpronomen & ich, er, ihm, mich, dir \\
\hline PPOSAT & attribuierendes Possessivpronomen & mein Buch, deine Mutter \\
\hline PPOSS & substituierendes Possessivpronomen & meins, meinige, deiner, ihrs \\
\hline PRELAT & attribuierendes Relativpronomen & der Mann, dessen Hund \\
\hline
\end{tabular}




\begin{tabular}{|c|c|c|}
\hline PRELS & substituierendes Relativpronomen & der Hund, der \\
\hline PRF & reflexives Personalpronomen & sich, einander, dich, mir \\
\hline PTKA & Partikel bei Adjektiv oder Adverb & am schönsten, zu schnell \\
\hline PTKANT & Antwortpartikel & ja, nein, danke, bitte \\
\hline PTKNEG & Negationspartikel & nicht \\
\hline PTKVZ & abgetrennter Verbzusatz & er kommt an, er fährt rad \\
\hline PTKZU & "zu" vor Infinitiv & zu gehen \\
\hline PWAT & attribuierendes Interrogativpronomen & welche Farbe, wessen Hut \\
\hline PWAV & $\begin{array}{l}\text { adverbiales Interrogativ- oder } \\
\text { Relativpronomen }\end{array}$ & warum, wo, wann, worüber, wobei \\
\hline PWS & substituierendes Interrogativpronomen & wer, was \\
\hline TRUNC & Kompositions-Erstglied & Sumpf- und Seelandschaft \\
\hline VAFIN & finites Hilfsverb (auxiliar) & du bist, wir werden \\
\hline VAIMP & Imperativ eines Hilfsverbs $^{2}$ & sei ruhig! \\
\hline VAINF & Infinitiv eines Hilfsverbs & werden, sein, haben \\
\hline VAPP & Partizip Perfekt eines Hilfsverbs & gewesen, worden \\
\hline VMFIN & finites Modalverb & kann, sollte, mag \\
\hline VMINF & Infinitiv eines Modalverbs & $\begin{array}{l}\text { können, müssen, wollen, sollen, } \\
\text { dürfen, mögen }{ }^{3}\end{array}$ \\
\hline VMPP & Partizip Perfekt eines Modalverbs & gekonnt, er hat gehen können \\
\hline VVFIN & finites Vollverb & du gehst, wir kommen an \\
\hline VVIMP & Imperativ eines Vollverbs & komm ! \\
\hline VVINF & Infinitiv eines Vollverbs & gehen, ankommen \\
\hline VVIZU & Infinitiv eines Vollverbs mit "zu" & anzukommen, loszulassen \\
\hline VVPP & Partizip Perfekt eines Vollverbs & gegangen, angekommen \\
\hline $\mathbf{X Y}$ & Nichtwort, Sonderzeichen enthaltend & 3:7, H2O, D2XW3 \\
\hline$\$$ & sonstige Satzzeichen &,-() \\
\hline$\$$, & Beistrich/ Komma & , \\
\hline$\$$. & satzbeendende Interpunktion & .?!;: \\
\hline
\end{tabular}

\section{Anmerkungen}

1 Kürzel nur im DWDS vorhanden und liefert Ergebnisse: manch (ein), welch (ein), solch (ein), all (die).

2 Imperative von sein und werden in der Rolle des Kopulaverbs. Sinnvolle Ergebnisse liefert nur das DWDS.

3 Frequenz in dieser Reihenfolge 


\section{Thematische Auflistung der morphosyntaktischen Tags}

Die folgende Tabelle bringt die Übersicht über einzelne Kategorien, die in drei morphosyntaktisch annotierten Korpora abgefragt werden können: DeReKo (TAGGED-M, TAGGED-T) und InterCorp_de. (In normaler Schrift angeführte Tags in der Spalte von InterCorp gelten natürlich auch für das DWDS.)

\section{Abfrage im DeReKo}

Die Abfrageerstellung erfolgt über die sog. „graphische Suche“: aus dem Menüangebot werden die Kategorien hierarchisch ausgewählt. Übergeordnete Ebenen („Oberklassen“) sind in Fettschrift gesetzt.

\section{Abfrage im InterCorp}

Die Abfrageerstellung erfolgt über

- Query Type: CQL $\rightarrow$ CQL: [tag="..."].

Die Abfrage muss gewöhnlich durch die Platzhalter (.*) ergänzt werden, um alle zutreffenden Belege abzurufen (z.B. [tag="ADJ.*"]).

\begin{tabular}{|c|c|c|c|}
\hline \multicolumn{3}{|l|}{ DeReKo } & \multirow{2}{*}{$\begin{array}{c}\text { InterCorp } \\
\text { InterCorp_de } \\
\text { TreeTagger/RFTagger }\end{array}$} \\
\hline Archiv: $\mathbf{T} A$ & GGED-M (MECOLB) & $\begin{array}{c}\text { Archiv: TAGGED-T } \\
\text { (TreeTagger) }\end{array}$ & \\
\hline Substantiv & $\begin{array}{l}\text { Unterklasse: } \\
\quad \text { Gattungsbez. } \\
\quad \text { Eigenname } \\
\text { Kasus } \\
\text { Numerus } \\
\text { Genus }\end{array}$ & $\begin{array}{l}\text { Nomina: } \\
\text { normale } \\
\text { Eigennamen }\end{array}$ & $\begin{array}{l}\text { N } \\
\text { NN N.Reg. } \\
\text { NE N.Name } \\
\text { Nom., Gen., Dat., Acc. } \\
\text { Sg., Pl. } \\
\text { Masc, Fem, Neut }\end{array}$ \\
\hline Adjektiv & $\begin{array}{l}\text { Kasus } \\
\text { Numerus } \\
\text { Genus } \\
\text { Komparation } \\
\text { Form } \\
\end{array}$ & $\begin{array}{l}\text { Adj.: } \\
\text { attributiv } \\
\text { prädikativ oder adverbial }\end{array}$ & $\begin{array}{l}\text { ADJ } \\
\text { ADJA } \\
\text { ADJD } \\
\text { Nom., Gen., Dat., Acc. } \\
\text { Sg., Pl. } \\
\text { Masc, Fem, Neut } \\
\text { Pos., Comp., Sup. } \\
-\end{array}$ \\
\hline Pronomen & $\begin{array}{l}\text { Art: } \\
\quad \text { demonstrativ } \\
\quad- \\
\quad \text { interrogativ } \\
\quad \text { personal } \\
\quad- \\
\quad \text { reflexiv } \\
\quad \text { relativ } \\
\text { Kasus: } \\
\text { Numerus: } \\
\text { Genus: }\end{array}$ & $\begin{array}{l}\text { Pron.: } \\
\text { Demonstrativpronomen } \\
\text { Indefinitpronomen } \\
\text { Interrogativpronomen } \\
\text { Personalpronomen } \\
\text { Possessivpronomen } \\
\text { Reflexivpronomen } \\
\text { Relativpronomen }\end{array}$ & $\begin{array}{l}\text { PRO } \\
\text { Dem. } \\
\text { Indef. } \\
\text { Inter. } \\
\text { Pers. } \\
\text { Poss. } \\
\text { Refl. } \\
\text { Rel. } \\
\text { Nom., Gen., Dat., Acc. } \\
\text { Sg., Pl. } \\
\text { Masc, Fem, Neut }\end{array}$ \\
\hline
\end{tabular}

Fortsetzung auf nächster Seite

57 Schmid/ Laws 2008. Tags in Kursivschrift ab der Version 7. 


\begin{tabular}{|c|c|c|c|}
\hline \multicolumn{3}{|l|}{ DeReKo } & \multirow[b]{2}{*}{$\begin{array}{l}\text { InterCorp } \\
\text { ART } \\
\text { Def. } \\
\text { Indef. } \\
\text { Nom., Gen., Dat., Acc. } \\
\text { Sg., Pl. } \\
\text { Masc, Fem, Neut }\end{array}$} \\
\hline Artikel & $\begin{array}{l}\text { Form } \\
\text { bestimmt } \\
\text { unbestimmt } \\
\text { Kasus } \\
\text { Numerus } \\
\text { Genus } \\
\end{array}$ & Art. & \\
\hline Numerale & $\begin{array}{l}\text { Typ } \\
\text { Kardinale } \\
\text { Ordinale } \\
\text { Bruchzahl } \\
\text { Kasus } \\
\text { Numerus } \\
\text { Genus }\end{array}$ & Zahlen & $\begin{array}{l}\text { CARD } \\
- \\
- \\
- \\
- \\
-\end{array}$ \\
\hline Verb & & $\begin{array}{l}\text { Verb } \\
\text { finit ohne Imperativ } \\
\quad \text { auxiliar } \\
\text { voll } \\
\text { modal } \\
\text { Infinitiv } \\
\quad \text { auxiliar } \\
\text { voll } \\
\text { modal } \\
\text { mit zu } \\
\text { Partizip Perfekt } \\
\quad \text { auxiliar } \\
\quad \text { voll } \\
\quad \text { modal } \\
\end{array}$ & $\begin{array}{l}\text { V } \\
\text { VFIN } \\
\text { VAFIN VFIN.Aux } \\
\text { VVFIN VFIN.Full } \\
\text { VMFIN VFIN.Mod } \\
\text { VINF } \\
\text { VAINF VINF.Aux. } \\
\text { VVINF VINF.Full } \\
\text { VMINF VINF.Mod. } \\
\text { VVIZU VINF.Full.zu } \\
\text { VPP } \\
\text { VAPP VPP.Aux } \\
\text { VVPP VPP.Full } \\
\text { VMPP VPP.Mod. } \\
\end{array}$ \\
\hline & $\begin{array}{l}\text { Genus Verbi } \\
\text { Tempus } \\
\text { (Präsens) } \\
\text { (Präteritum) } \\
\text { Modus } \\
\text { (Indikativ) } \\
\text { (Konjunktiv) } \\
\text { (Imperativ) } \\
\text { Numerus } \\
\text { (Singular) } \\
\text { (Plural) } \\
\text { Person } \\
\text { (1.) } \\
\text { (2.) } \\
\text { (3.) }\end{array}$ & & $\begin{array}{l}- \\
\text { V } \\
\text { Pres. } \\
\text { Past. } \\
\text { V } \\
\text { Ind. } \\
\text { Subj. } \\
\text { VIMP } \\
\text { V } \\
\text { Sg. } \\
\text { Pl. } \\
\text { V } \\
1 . \\
2 . \\
3 . \\
\end{array}$ \\
\hline Verbzusatz & & (siehe Partikel) & PTKVZ \\
\hline Adverb & & Adv. & $\mathrm{ADV}$ \\
\hline Konjunktion & $\begin{array}{l}\text { Typ } \\
\text { subordinierend } \\
\text { koordinierend }\end{array}$ & $\begin{array}{l}\text { Konj. } \\
\text { unterordnende } \\
\text { mit Infinitiv } \\
\text { mit Satz } \\
\text { nebenordnende } \\
\text { Vergleichspartikel (als, wie) }\end{array}$ & $\begin{array}{l}\text { K } \\
\text { KOU } \\
\text { KOUI } \\
\text { KOUS } \\
\text { KON } \\
\text { KOKOM } \\
\end{array}$ \\
\hline
\end{tabular}




\begin{tabular}{|c|c|c|c|}
\hline Präposition & $\begin{array}{l}\text { Position } \\
\text { Präposition } \\
\text { Postposition } \\
\text { Kasus } \\
\text { Artikelverschmelzung }\end{array}$ & $\begin{array}{l}\text { Adp. } \\
\quad \text { Präpositionen } \\
\quad \text { Postposition } \\
\text { Zirkumposition rechts (auf ... hin) } \\
\text { Präpositionen mit Artikel }\end{array}$ & $\begin{array}{l}\text { AP } \\
\text { APPR } \\
\text { APPO } \\
\text { Gen., Dat., Acc. } \\
\text { APZR } \\
\text { APPRART }\end{array}$ \\
\hline (Partikel) & & $\begin{array}{l}\text { Partikel } \\
z u \text { vor Infinitiv } \\
\text { Negationspartikel } \\
\text { abgetrennter Verbzusatz } \\
\text { (all)zu bei Adj. oder Adverb } \\
\text { Antwortpartikel }\end{array}$ & $\begin{array}{l}\text { PTK } \\
\text { PTKZU } \\
\text { PTKNEG } \\
\text { PTKVZ } \\
\text { PTKA } \\
\text { PTKANT PART.Ans }\end{array}$ \\
\hline (Sonstiges) & & $\begin{array}{l}\text { Sonst. } \\
\text { Interjektionen } \\
\text { Kompositions-Erstglied } \\
\text { Nichtwörter } \\
\text { fremdsprachliches Material } \\
\text { Satzzeichen, Kürzel }\end{array}$ & $\begin{array}{l}\text { ITJ } \\
\text { TRUNC Adj., Noun., Verb } \\
\text { XY } \\
\text { FM } \\
\text { SYM }\end{array}$ \\
\hline
\end{tabular}




\subsection{Tastenkürzel, Shortcuts}

Die Tastenkürzel funktionieren zuverlässig im MS-Office-Paket auf den deutschen Standardtastaturen (de) für Deutsch (Deutschland), (Österreich), (Schweiz), (Liechtenstein) und (Luxemburg) bzw. auf der tschechischen und größtenteils auch auf der slowakischen Standardtastatur.

Die Tastenkombinationen (shortcuts) gelten allgemein.

\begin{tabular}{|c|c|c|c|c|}
\hline \multirow[b]{2}{*}{ Zeichen } & \multirow[b]{2}{*}{ Beschreibung } & \multicolumn{2}{|c|}{ Tastenkürzel } & \multirow[b]{2}{*}{$\begin{array}{c}\text { Tastenkombination } \\
\text { (shortcut) }\end{array}$} \\
\hline & & $\begin{array}{c}\text { deutsche } \\
\text { (de) }\end{array}$ & \begin{tabular}{|l} 
astatur \\
tschechische \\
(cs)
\end{tabular} & \\
\hline 1 & runde Klammer auf & shift+8 & shift+) & Alt +40 \\
\hline 1 & runde Klammer zu & shift+9 & 1 & Alt +41 \\
\hline\{ & geschwungene/ geschweifte Klammer auf & AltGr+7 & AltGr+b & Alt+123 \\
\hline$\}$ & geschwungene/ geschweifte Klammer zu & AltGr+0 & AltGr+n & Alt +125 \\
\hline 1 & senkrechter Abgrenzungsbalken & AltGr+> & AltGr+w & Alt+0124 \\
\hline[ & eckige Klammer auf & AltGr+8 & AltGr+f & Alt+91 \\
\hline ] & eckige Klammer zu & AltGr+9 & AltGr+g & Alt+93 \\
\hline I & Schrägstrich/ Querstrich/slash & shift+7 & shift+ů & Alt+47 \\
\hline 1 & back slash/ umgekehrter Schrägstrich & AltGr+ß & AltGr+q & Alt+92 \\
\hline$"$ & Anführungszeichen ("Gänsefüßchen") & shift+2 & shift+ů & Alt+34 \\
\hline « & Anführungszeichen "Guillemets" & & & Alt+0171 \\
\hline$\|$ & Anführungszeichen "Guillemets" & & & Alt+0187 \\
\hline 8 & Anführungszeichen "Guillemets" (einfach) & & & Alt+0139 \\
\hline , & Anführungszeichen "Guillemets" (einfach) & & & Alt+0155 \\
\hline$\hat{n}$ & Zirkumflex & $\wedge$ & AltGr+3+space & Alt+94 \\
\hline$<$ & größer als & $<$ & AltGr+. & Alt +60 \\
\hline$>$ & kleiner als & $>$ & AltGr+, & Alt+62 \\
\hline * & Asterisk/ Sternchen & shift++ & AltGr+- & Alt +42 \\
\hline+ & Pluszeichen & + & + & Alt +43 \\
\hline- & Minuszeichen & - & - & Alt +45 \\
\hline$\#$ & Raute & $\#$ & AltGr+x & Alt +35 \\
\hline$\%$ & Prozent & shift+4 & shift $+=$ & Alt +37 \\
\hline$@$ & At-Zeichen & AltGr+q & AltGr+v & Alt+64 \\
\hline$!$ & Rufzeichen & shift+1 & shift $+\S$ & Alt +33 \\
\hline$?$ & Fragezeichen & shift $+\beta$ & shift+, & Alt+63 \\
\hline$\&$ & Ampersand & shift+6 & AltGr+c & Alt +38 \\
\hline$\$$ & Dollarzeichen & shift+4 & AltGr+ů & Alt +36 \\
\hline 及 & scharfes S & B & AltGr+§ & Alt +225 \\
\hline ä & a-Umlaut & ä & $\ddot{+}+a$ & Alt+132 \\
\hline$\ddot{A}$ & A-Umlaut & $\ddot{A}$ & " + shift+a & Alt+142 \\
\hline$\ddot{~ o ̈ ~}$ & o-Umlaut & ö & $"+0$ & Alt+148 \\
\hline ö & O-Umlaut & Ö & +shift+o & Alt+153 \\
\hline$\ddot{\mathrm{u}}$ & u-Umlaut & ü & 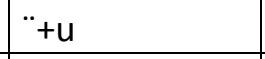 & Alt+129 \\
\hline$\ddot{U}$ & U-Umlaut & $\ddot{U}$ & $"+$ shift+u & Alt+154 \\
\hline
\end{tabular}




\subsection{InterCorp: CQL-Abfragen}

In den folgenden Tabellen sind Syntaxhilfen für einige Abfragen im InterCorp angeführt. Alle Abfragen gelten nur für den Abfragemodus CQL (Query Type: CQL). Die Reihung in der ersten Tabelle (Reihung nach Abfrage: S. 211-214) geht von den einfachsten Abfragen nach Wort und Wortteilen zu den kompliziertesten Abfragen nach Kombinationen von Elementen im Satz.

Die zweite Tabelle (Alphabetische Reihung: S. 215-218) entspricht der ersten, die Reihung der Abfragen ist jedoch nach der Bedeutung der Abfrage angeordnet und geht alphabetisch von Abstand bis zu Wortform. Die offenbare Mischung von grammatikalischen Kategorien (z.B. Partizipialphrase) und korpuslinguistischen Abfragebegriffen (z.B. Abstand) darf nicht verwirren. Sie ist durch die Abfragemöglichkeiten des Korpusmanagers verursacht. Die grammatikalischen und sonstigen linguistischen Kategorien können nämlich größtenteils nicht direkt abgefragt werden, sondern sie müssen über gut überlegte Kombinationen von möglichen Suchfeldeingaben abgefragt werden (z.B. die Abfrage nach einer Partizipphrase mit Partizip I kann über eine Kombination von einem Tag für Adjektive in der Attributposition (ADJA) und einem Lemma, das auf -end endet, erfolgen).

Eine vollständige Tabelle für die Abfragen kann es wegen den unzähligen Kombinationsmöglichkeiten der abzufragenden Elemente nicht geben. Die folgenden Tabellen sind ein Bruchteil aller Abfragemöglichkeiten. Sie sind als erste Hilfe für die üblichsten Abfragen konzipiert. 
CQL-Abfragen (InterCorp): Reihung nach Abfrage

\begin{tabular}{|c|c|c|}
\hline Abfrage & Bedeutung & $\begin{array}{l}\text { Ergebnis in Fettschrift, Reihenfolge nach Häufigkeit } \\
\text { (wenn sinnvoll) }\end{array}$ \\
\hline [word="rund"] & Wortform & rund \\
\hline [word="rund.*"] & Wortanfang & rund, runde, rundherum, Runde, rundlich \\
\hline [word=".*rund"] & Wortende & aufgrund, Grund, rund, Hintergrund \\
\hline [word="Schlu(ß|ss)"] & Alternative $\beta$ oder ss im Wort & Schluss, Schluß \\
\hline [word="gr[^ü]ße"] & Alternative (ü ignorieren) & große, Größe \\
\hline [wort="m[^a|ü]de"] & Alternative ( $a$ oder $\ddot{u}$ ignorieren) & Mode \\
\hline [lemma="Grund"] & Grundform/ Lemma & Gründe, Grund, Grunde, Grundes \\
\hline [lemma=".*schaft"\&!lemma="Gemeinschaft"] & $\begin{array}{l}\text { Wort auf -schaft, jedoch NICHT } \\
\text { Gemeinschaft }\end{array}$ & Wirtschaft, Gesellschaft, Landwirtschaft \\
\hline [tag="VVINF.*"] [word="hätte"] [tag="VMINF.*"] & $\begin{array}{l}\text { Verbalkomplex („österreichische“ } \\
\text { Reihenfolge) }\end{array}$ & $\begin{array}{l}\text { wie ich sie auffassen hätte können; Mehrwert, den er } \\
\text { erbringen hätte können; etwas stärker hervorheben } \\
\text { hätte müssen; einer wirklichen Selbstkritik unterziehen } \\
\text { hätte sollen }\end{array}$ \\
\hline [word="hätte"] [tag="VVINF.*"] [tag="VMINF.*"] & $\begin{array}{l}\text { Verbalkomplex („,deutsche“ } \\
\text { Reihenfolge) }\end{array}$ & $\begin{array}{l}\text {..., daß sie seine Mutter hätte sein können.; , der genau } \\
\text { hätte sagen können,; ... die Preise, die ich ihnen hätte } \\
\text { zahlen müssen }\end{array}$ \\
\hline [tag="N.*"\&lemma=".*el"] & Substantiv auf-el & Artikel, Mittel, Ziel, Beispiel... \\
\hline [tag="N.*"\&lemma=".*el"\&!lemma="Artikel"] & $\begin{array}{l}\text { Substantiv auf-el, außer Artikel (Fast } \\
50 \% \text { aller Treffer der Subst. auf -el } \\
\text { fallen auf das Wort Artikel.) }\end{array}$ & $\begin{array}{l}\text { Mittel, Ziel, Beispiel, Handel, Kapitel ... aber auch } \\
\text { Desubstantive auf -el: Geflügel, Flügel, Gürtel, Rätsel, } \\
\text { Bündel ... }\end{array}$ \\
\hline [tag="APPR"\&word="auf"] & Präposition auf & auf dem Bauch; auf dem Boden; auf den Boden \\
\hline [tag="PTKVZ"\&word="auf"] [word="\?"] & $\begin{array}{l}\text { Verbzusatz auf am Ende eines } \\
\text { Fragesatzes }\end{array}$ & Nehmen Sie das auf ?; Warum regst du dich denn so auf ? \\
\hline [lemma="fordern"][]*[word="auf"] within $<s />$ & Verb mit abgetrenntem Zusatz & $\begin{array}{l}\text { fordert die Kommission/ den Rat (nachdrücklich) auf; } \\
\text { fordere Sie auf, forderte ihn auf, fordert dazu auf }\end{array}$ \\
\hline
\end{tabular}




\begin{tabular}{|c|c|c|}
\hline Abfrage & Bedeutung & $\begin{array}{l}\text { Ergebnis in Fettschrift, Reihenfolge nach } \\
\text { Häufigkeit (wenn sinnvoll) }\end{array}$ \\
\hline [lemma="auffordern"] & Verb mit Zusatz & $\begin{array}{l}\text { auffordern, aufzufordern, auffordert, } \\
\text { aufforderte, aufforderten, auffordere }\end{array}$ \\
\hline [word="frage"] []* [word="ich"] within $<s />$ & Satzelemente: inverse Wortstellung (frage ... ich) & $\begin{array}{l}\text { frage ich; frage mich, ob ich; frage ich mich, ob } \\
\text { ich }\end{array}$ \\
\hline [word="ich"][][word="frage"] & $\begin{array}{l}\text { Satzelemente: normale Wortstellung (ich ... } \\
\text { frage; dazwischen } 1 \text { Wort) }\end{array}$ & ich mich/ Sie/ ihn frage; ich freundlich frage \\
\hline [lemma=".*park"]within <div·author="Bachmann, Ingeborg"/> & $\begin{array}{l}\text { Grundformen/ Lemma aller Wörter auf -park in } \\
\text { Werken von Bachmann, Ingeborg }\end{array}$ & Stadtpark, Stadtparks, Resselpark \\
\hline [lemma="lieb.*"]within <div·title="Scherz"/> & $\begin{array}{l}\text { Grundformen/ Lemmata beginnend mit lieb- im } \\
\text { Werk Scherz (von Milan Kundera) }\end{array}$ & liebte, geliebt, lieben, lieber, liebste, Liebe \\
\hline [lemma="[Ww]ien.*"]within <div title="Malina" /> & $\begin{array}{l}\text { Grundformen/ Lemmata beginnend mit Wien- } \\
\text { oder wien- im Werk Malina (von Ingeborg } \\
\text { Bachmann) }\end{array}$ & $\begin{array}{l}\text { Wien; Wiener; wienerisches; Wienerwald; } \\
\text { Wienerinnen }\end{array}$ \\
\hline [word="allein"][word="\."] & Satzende: Wort allein am Satzende & allein. \\
\hline [word=".*lein"\&!word="[k|K]lein |[a|A]llein"] & $\begin{array}{l}\text { Negation: alle Wörter auf -lein, jedoch nicht } \\
\text { Klein/klein und Allein/allein }\end{array}$ & $\begin{array}{l}\text { Fräulein, Häuflein, Büchlein, Männlein, } \\
\text { Henlein, Äuglein, Bäuchlein, Bächlein... }\end{array}$ \\
\hline$<s>$ [word="Dich"] & Satzanfang: Dich am Satzanfang & $\begin{array}{l}\text { Dich aber beneide ich; Dich kann nichts rühren; } \\
\text { Dich so zu erschrecken; Dich zu lieben bedeutet }\end{array}$ \\
\hline$<s>[$ tag="VMFIN"] & $\begin{array}{l}\text { Satzanfang: Modalverb, finite Form am } \\
\text { Satzanfang }\end{array}$ & $\begin{array}{l}\text { Kann ich Ihnen helfen?; Sollte er das erzählen?; } \\
\text { Können Sie bitte erklären, }\end{array}$ \\
\hline$<\mathrm{s}>$ [tag="VMFIN"\&lemma="mögen"] & Satzanfang: Modalverb mögen am Satzanfang & Mag, Möge, Mögen, Möchten... \\
\hline [lemma="Kopf"][]\{0,5\}[lemma="Hand"] & $\begin{array}{l}\text { Abstand: Lemma Kopf und Lemma Hand im } \\
\text { Abstand von maximal } 5 \text { Positionen }\end{array}$ & $\begin{array}{l}\text { Kopf in die Hände; Kopf in beide Hände; Kopf } \\
\text { mit beiden Händen; Kopf und die Hände }\end{array}$ \\
\hline [lemma="Ku(ss|ß)"][]*[lemma="Liebe"] within $<p />$ & $\begin{array}{l}\text { Abstand: Lemma Kuss oder Kuß und Lemma } \\
\text { Liebe in einem Absatz }\end{array}$ & $\begin{array}{l}\text { stoßweise gehender Atem und seine } \\
\text { wilden Küsse? Was war das alles, wenn es } \\
\text { keine Liebe war? }\end{array}$ \\
\hline
\end{tabular}




\begin{tabular}{|c|c|c|}
\hline Abfrage & Bedeutung & $\begin{array}{l}\text { Ergebnis in Fettschrift, Reihenfolge nach } \\
\text { Häufigkeit (wenn sinnvoll) }\end{array}$ \\
\hline [lemma="essen"][]*[word="gern"] within $<s />$ & $\begin{array}{l}\text { Abstand: Lemma essen und Wort gern in einem } \\
\text { Satz }\end{array}$ & Essen Sie gern Gorgonzola? \\
\hline [lemma="halten"] []* [word="fest"] within <s id=".*"/> & Verb mit abgetrenntem Zusatz & $\begin{array}{l}\text { ich hielt sie an den Schultern fest; Er blieb } \\
\text { stehen und hielt sich am Geländer fest. }\end{array}$ \\
\hline [lemma="halten"] []* [word="fest"] [tag="\\
$."] & $\begin{array}{l}\text { Abstand: Lemma halten im beliebigem Abstand } \\
\text { vom Wort fest am Satzende }\end{array}$ & hält die Kommission fest. \\
\hline [lemma="halten"] []* [word="fest"] [tag="\\
$."] within <s id=".*"/> & $\begin{array}{l}\text { Verb mit abgetrenntem Zusatz (in einem Satz); } \\
\text { Zusatz am Satzende }\end{array}$ & $\begin{array}{l}\text { aber kaum hielt er sich derart fest,; Für einen } \\
\text { kurzen Augenblick hielt ihr Blick den seinen fest. }\end{array}$ \\
\hline [word="es"] []*[lemma="verlangen"] []\{0,3\} [word="nach"] within $<s />$ & $\begin{array}{l}\text { Phrase: jmdn. verlangt es nach etw. } \\
\text { (Reihenfolge im Satz: es - verlangen - nach) }\end{array}$ & $\begin{array}{l}\text { es hat mich so verlangt nach; es satt, und ihr } \\
\text { Geist verlangte nach }\end{array}$ \\
\hline [lemma="verlangen"][]*[word="es"] []\{0,3\}[word="nach"] within $<s />$ & $\begin{array}{l}\text { Phrase: jmdn. verlangt es nach etw. } \\
\text { (Reihenfolge im Satz: verlangen - es - nach) }\end{array}$ & $\begin{array}{l}\text { Die Männer verlangte es nach weiblicher } \\
\text { Gesellschaft; verlangt es den Menschen } \\
\text { mitunter nach dem monotonen Rhythmus }\end{array}$ \\
\hline$<s />$ containing [lemma="fest"] containing [lemma="halten"] & $\begin{array}{l}\text { Satz mit Lemmata fest und halten (Als KWIC } \\
\text { erscheint der ganze Satz.) }\end{array}$ & $\begin{array}{l}\text { Sie hielten mich erst in Bagram fest, dann in } \\
\text { Kandahar und schließlich in Guantánamo Bay. } \\
\text { Er hob die Hände, ertastete sich eine Stuhllehne } \\
\text { und hielt sich fest, und wir sprachen kein Wort } \\
\text { mehr miteinander, bis Petra angezogen } \\
\text { herunterkam, mit Mantel und Kopftuch. }\end{array}$ \\
\hline [tag="\\
$."] & Satzende (beliebiges Zeichen am Satzende) & . , ] " : \\
\hline [tag="\\
$."\&word="\."] & Satzende: Punkt am Ende & . \\
\hline [word="\."] & Satzzeichen: Punkt & . \\
\hline [word="\»"] & Satzzeichen: Anführungszeichen "Guillemets" & $"$ \\
\hline
\end{tabular}




\begin{tabular}{|c|c|c|}
\hline Abfrage & Bedeutung & $\begin{array}{l}\text { Ergebnis in Fettschrift, Reihenfolge nach } \\
\text { Häufigkeit (wenn sinnvoll) }\end{array}$ \\
\hline [word="\"'] & $\begin{array}{l}\text { Satzzeichen: Anführungszeichen } \\
\text { "Gänsefüßchen" }\end{array}$ & $"$ \\
\hline [word="\?"] & Satzzeichen: Fragezeichen & $?$ \\
\hline [word="\!"] & Satzzeichen: Rufzeichen & $!$ \\
\hline [word="\!"] [word="\?"] & Satzzeichen: Rufzeichen und Fragezeichen & "Dann gibst du mir also recht!?" \\
\hline$<s />$ containing [tag="VVFIN"] containing [tag="PTKVZ"] & $\begin{array}{l}\text { Satz mit einem Vollverb in finiter Form, in } \\
\text { demselben Satz auch ein Verbzusatz (Als KWIC } \\
\text { erscheint der ganze Satz.) }\end{array}$ & $\begin{array}{l}\text { Der Vorsitzende nimmt an der Abstimmung } \\
\text { nicht teil. Es ist eines der drei Stücke, die Mama } \\
\text { auf dem Klavier spielen kann, geschrieben von } \\
\text { dem Komponisten Polívka, ich höre zu, und } \\
\text { mein Zorn vergeht. }\end{array}$ \\
\hline [tag="APPR"] [tag="ADJA"\&lemma=".*end"] & Partizipphrase (mit Partizip I) nach einem Artikel & $\begin{array}{l}\text { Sie holt die Chefin, Charlotte, die stürzt in } \\
\text { fliegender Eile hinauf. }\end{array}$ \\
\hline [tag="APPR"] [tag="ADJA"\&lemma=".*(en|t)"] & $\begin{array}{l}\text { Partizipphrase (mit Partizip II) nach einem } \\
\text { Artikel }\end{array}$ & $\begin{array}{l}\text { Erzeugnisse, die in unverändertem Zustand } \\
\text { ausgeführt werden }\end{array}$ \\
\hline [tag="ART"] [tag="ADJA"] [tag="NE"] & Eigenname mit Artikel und Attribut & $\begin{array}{l}\text { die falsche Kleopatra thronte immer noch auf } \\
\text { ihrem dunkelroten Kißchen; Aber die blonde } \\
\text { Käthe stolperte; Voraussetzung für die Schaffung } \\
\text { eines integrierten Europa }\end{array}$ \\
\hline [word="des"] [tag="NE"] & Eigenname nach des (Maskulin im Genitiv) & $\begin{array}{l}\text { der Sprachen des Balkans; die Anerkennung des } \\
\text { Kosovo; Die Wirklichkeit überholte auch die } \\
\text { tschechische Utopie des Karel Čapek; unter der } \\
\text { Statue des Jan Nepomuk, }\end{array}$ \\
\hline [word="des"] [tag="ADJA"] [tag="NE"\&!word=".*s"] & $\begin{array}{l}\text { Eigenname (im Genitiv) ohne }-s \text { mit bestimmten } \\
\text { Artikel des und Attribut }\end{array}$ & $\begin{array}{l}\text { Feudalist und Verehrer des alten Österreich; } \\
\text { Blicke waren nach oben auf das Reiterstandbild } \\
\text { des heiligen Wenzel gerichtet }\end{array}$ \\
\hline
\end{tabular}




\section{CQL-Abfragen (InterCorp): Alphabetische Reihung nach Bedeutung der Abfrage}

\begin{tabular}{|c|c|c|}
\hline Bedeutung & Abfrage & $\begin{array}{l}\text { Ergebnis in Fettschrift, Reihenfolge nach Häufigkeit (wenn } \\
\text { sinnvoll) }\end{array}$ \\
\hline $\begin{array}{l}\text { Abstand: Lemma essen und Wort } \\
\text { gern in einem Satz }\end{array}$ & [lemma="essen"][]*[word="gern"] within $<s />$ & Essen Sie gern Gorgonzola? \\
\hline $\begin{array}{l}\text { Abstand: Lemma halten im } \\
\text { beliebigem Abstand vom Wort } \\
\text { fest am Satzende }\end{array}$ & [lemma="halten"] []* [word="fest"] [tag="\\
$."] & hält die Kommission fest. \\
\hline $\begin{array}{l}\text { Abstand: Lemma Kopf und } \\
\text { Lemma Hand im Abstand von } \\
\text { maximal } 5 \text { Positionen }\end{array}$ & [lemma="Kopf"][]\{0,5\}[lemma="Hand"] & $\begin{array}{l}\text { Kopf in die Hände; Kopf in beide Hände; Kopf mit beiden } \\
\text { Händen; Kopf und die Hände }\end{array}$ \\
\hline $\begin{array}{l}\text { Abstand: Lemma Kuss oder Kuß } \\
\text { und Lemma Liebe in einem Absatz }\end{array}$ & [lemma="Ku(ss $\mid ß) "][] *[$ lemma="Liebe"] within $<\mathrm{p} />$ & $\begin{array}{l}\text { stoßweise gehender Atem und seine wilden Küsse ? Was } \\
\text { war das alles, wenn es keine Liebe war? }\end{array}$ \\
\hline Alternative: Schluß oder Schluss & [word="Schlu(ß|ss)"] & Schluss, Schluß \\
\hline Alternative ( $a$ oder $\ddot{u}$ ignorieren) & [wort="m[^a|ü]de"] & Mode \\
\hline Alternative (ü ignorieren) & [word="gr[^ü]ße"] & große, Größe \\
\hline $\begin{array}{l}\text { Eigenname (im Genitiv) ohne }-s \\
\text { mit bestimmten Artikel des und } \\
\text { Attribut }\end{array}$ & [word="des"] [tag="ADJA"] [tag="NE"\&!word=".*s"] & $\begin{array}{l}\text { Feudalist und Verehrer des alten Österreich; Blicke waren } \\
\text { nach oben auf das Reiterstandbild des heiligen Wenzel } \\
\text { gerichtet }\end{array}$ \\
\hline $\begin{array}{l}\text { Eigenname mit Artikel und } \\
\text { Attribut }\end{array}$ & [tag="ART"] [tag="ADJA"] [tag="NE"] & $\begin{array}{l}\text { die falsche Kleopatra thronte immer noch auf ihrem } \\
\text { dunkelroten Kißchen; Aber die blonde Käthe stolperte; } \\
\text { Voraussetzung für die Schaffung eines integrierten Europa }\end{array}$ \\
\hline $\begin{array}{l}\text { Eigenname nach des (Maskulina } \\
\text { im Genitiv) }\end{array}$ & [word="des"] [tag="NE"] & $\begin{array}{l}\text { der Sprachen des Balkans; die Anerkennung des Kosovo; } \\
\text { Die Wirklichkeit überholte auch die tschechische Utopie des } \\
\text { Karel Čapek; unter der Statue des Jan Nepomuk, }\end{array}$ \\
\hline Grundform/ Lemma & [lemma="Grund"] & Gründe, Grund, Grunde, Grundes \\
\hline
\end{tabular}




\begin{tabular}{|c|c|c|}
\hline Bedeutung & Abfrage & $\begin{array}{l}\text { Ergebnis in Fettschrift, Reihenfolge nach Häufigkeit (wenn } \\
\text { sinnvoll) }\end{array}$ \\
\hline $\begin{array}{l}\text { Grundform/ Lemma aller Wörter } \\
\text { auf -park in Werken von } \\
\text { Bachmann, Ingeborg }\end{array}$ & [lemma=".*park"]within <div·author="Bachmann, -Ingeborg"/> & Stadtpark, Stadtparks, Resselpark \\
\hline $\begin{array}{l}\text { Grundform/ Lemma beginnend } \\
\text { mit lieb- im Werk Scherz (von } \\
\text { Milan Kundera) }\end{array}$ & [lemma="lieb.*"]within <div·title="Scherz"/> & liebte, geliebt, lieben, lieber, liebste, Liebe \\
\hline $\begin{array}{l}\text { Grundform/ Lemma beginnend } \\
\text { mit Wien- oder wien- im Werk } \\
\text { Malina (von Ingeborg Bachmann) }\end{array}$ & [lemma="[Ww]ien. $\left.{ }^{*} "\right]$ within $<\operatorname{div}$ title="Malina" $/>$ & Wien; Wiener; wienerisches; Wienerwald; Wienerinnen \\
\hline $\begin{array}{l}\text { Negation: alle Wörter auf -lein, } \\
\text { jedoch nicht Klein/klein und } \\
\text { Allein/allein }\end{array}$ & [word=".*lein"\&!word="[k|K]lein |[a|A]llein"] & $\begin{array}{l}\text { Fräulein, Häuflein, Büchlein, Männlein, Henlein, Äuglein, } \\
\text { Bäuchlein, Bächlein... }\end{array}$ \\
\hline $\begin{array}{l}\text { Partizipphrase (mit Partizip I) } \\
\text { nach einem Artikel }\end{array}$ & [tag="APPR"] [tag="ADJA"\&lemma=".*end"] & $\begin{array}{l}\text { Sie holt die Chefin, Charlotte, die stürzt in fliegender Eile } \\
\text { hinauf. }\end{array}$ \\
\hline $\begin{array}{l}\text { Partizipphrase (mit Partizip II) } \\
\text { nach einem Artikel }\end{array}$ & [tag="APPR"] [tag="ADJA"\&lemma=".*(en|t)"] & $\begin{array}{l}\text { Erzeugnisse, die in unverändertem Zustand ausgeführt } \\
\text { werden }\end{array}$ \\
\hline $\begin{array}{l}\text { Phrase: jmdn. verlangt es nach } \\
\text { etw. (Reihenfolge im Satz: es - } \\
\text { verlangen - nach) }\end{array}$ & [word="es"] []*[lemma="verlangen"] []\{0,3\} [word="nach"] within $<$ s/> & $\begin{array}{l}\text { es hat mich so verlangt nach; es satt, und ihr Geist } \\
\text { verlangte nach }\end{array}$ \\
\hline $\begin{array}{l}\text { Phrase: jmdn. verlangt es nach } \\
\text { etw. (Reihenfolge im Satz: } \\
\text { verlangen - es - nach) }\end{array}$ & [lemma="verlangen"][]*[word="es"] []\{0,3\} [word="nach"] within $<s />$ & $\begin{array}{l}\text { Die Männer verlangte es nach weiblicher Gesellschaft; } \\
\text { verlangt es den Menschen mitunter nach dem monotonen } \\
\text { Rhythmus }\end{array}$ \\
\hline Präposition auf & [tag="APPR"\&word="auf"] & auf dem Bauch; auf dem Boden; auf den Boden \\
\hline Satzanfang: Dich am Satzanfang & $<s>[$ word="Dich"] & $\begin{array}{l}\text { Dich aber beneide ich; Dich kann nichts rühren; Dich so zu } \\
\text { erschrecken; Dich zu lieben bedeutet }\end{array}$ \\
\hline $\begin{array}{l}\text { Satzanfang: Modalverb mögen } \\
\text { am Satzanfang }\end{array}$ & $<\mathrm{s}>$ [tag="VMFIN"\&lemma="mögen"] & Mag, Möge, Mögen, Möchten... \\
\hline $\begin{array}{l}\text { Satzanfang: Modalverb, finite } \\
\text { Form am Satzanfang }\end{array}$ & $<s>[$ tag="VMFIN"] & $\begin{array}{l}\text { Kann ich Ihnen helfen ?; Sollte er das erzählen ?; Können Sie } \\
\text { bitte erklären, }\end{array}$ \\
\hline
\end{tabular}




\begin{tabular}{|c|c|c|}
\hline Bedeutung & Abfrage & $\begin{array}{l}\text { Ergebnis in Fettschrift, Reihenfolge nach Häufigkeit (wenn } \\
\text { sinnvoll) }\end{array}$ \\
\hline $\begin{array}{l}\text { Satz mit den Lemmata fest und } \\
\text { halten (Als KWIC erscheint der } \\
\text { ganze Satz.) }\end{array}$ & $<\mathrm{s} />$ containing [lemma="fest"] containing [lemma="halten"] & $\begin{array}{l}\text { Sie hielten mich erst in Bagram fest, dann in Kandahar und } \\
\text { schließlich in Guantánamo Bay. Er hob die Hände, ertastete } \\
\text { sich eine Stuhllehne und hielt sich fest, und wir sprachen kein } \\
\text { Wort mehr miteinander, bis Petra angezogen herunterkam, } \\
\text { mit Mantel und Kopftuch. }\end{array}$ \\
\hline $\begin{array}{l}\text { Satz mit einem Vollverb in finiter } \\
\text { Form und mit einem Verbzusatz } \\
\text { (Als KWIC erscheint der ganze } \\
\text { Satz.) }\end{array}$ & <s/> containing [tag="VVFIN"] containing [tag="PTKVZ"] & $\begin{array}{l}\text { Der Vorsitzende nimmt an der Abstimmung nicht teil. Es ist } \\
\text { eines der drei Stücke, die Mama auf dem Klavier spielen kann, } \\
\text { geschrieben von dem Komponisten Polívka, ich höre zu, und } \\
\text { mein Zorn vergeht. }\end{array}$ \\
\hline $\begin{array}{l}\text { Satzelemente: inverse } \\
\text { Wortstellung (frage ... ich) }\end{array}$ & [word="frage"] []* [word="ich"] within $<$ s/> & frage ich; frage mich, ob ich; frage ich mich, ob ich \\
\hline $\begin{array}{l}\text { Satzelemente: normale } \\
\text { Wortstellung (ich ... frage; } \\
\text { dazwischen } 1 \text { Wort) }\end{array}$ & [word="ich"][][word="frage"] & ich mich/ Sie/ ihn frage; ich freundlich frage \\
\hline $\begin{array}{l}\text { Satzende (beliebiges Zeichen am } \\
\text { Satzende) }\end{array}$ & [tag="\\
$."] & . , ] " : \\
\hline Satzende: Punkt am Ende & [tag="\\
$."\&word="\."] & . \\
\hline $\begin{array}{l}\text { Satzende: Wort allein am } \\
\text { Satzende }\end{array}$ & [word="allein"][word="\."] & allein . \\
\hline $\begin{array}{l}\text { Satzzeichen: Anführungszeichen } \\
\text { "Gänsefüßchen" }\end{array}$ & [word="\"'] & " \\
\hline $\begin{array}{l}\text { Satzzeichen: Anführungszeichen } \\
\text { "Guillemets" }\end{array}$ & [word="\»"] & " \\
\hline Satzzeichen: Fragezeichen & [word="\?"] & $?$ \\
\hline Satzzeichen: Punkt & [word="\."] & . \\
\hline Satzzeichen: Rufzeichen & [word="\!"] & ! \\
\hline $\begin{array}{l}\text { Satzzeichen: Rufzeichen und } \\
\text { Fragezeichen }\end{array}$ & [word="\!"] [word="\?"] & „Dann gibst du mir also recht ! ?“ \\
\hline
\end{tabular}




\begin{tabular}{|c|c|c|c|}
\hline Bedeutung & \multicolumn{2}{|l|}{ Abfrage } & $\begin{array}{l}\text { Ergebnis in Fettschrift, Reihenfolge nach Häufigkeit (wenn } \\
\text { sinnvoll) }\end{array}$ \\
\hline Substantiv auf -el & \multicolumn{2}{|l|}{ [tag="N.*"\&lemma=".*el"] } & Artikel, Mittel, Ziel, Beispiel... \\
\hline Substantiv auf -el, außer Artikel & \multicolumn{2}{|l|}{ [tag="N.*"\&lemma=".*el"\&!lemma="Artikel"] } & $\begin{array}{l}\text { Mittel, Ziel, Beispiel, Handel, Kapitel ... aber auch } \\
\text { Desubstantive auf -el: Geflügel, Flügel, Gürtel, Rätsel, Bündel ... }\end{array}$ \\
\hline Verb mit abgetrenntem Zusatz & \multicolumn{2}{|l|}{ [lemma="fordern"][]*[word="auf"] within <s /> } & $\begin{array}{l}\text { fordert die Kommission/ den Rat (nachdrücklich) auf; fordere } \\
\text { Sie auf, forderte ihn auf, fordert dazu auf }\end{array}$ \\
\hline $\begin{array}{l}\text { Verb mit abgetrenntem Zusatz } \\
\text { am Satzende }\end{array}$ & \multicolumn{2}{|c|}{ [lemma="halten"] []* [word="fest"] [tag="\\
$."] within <s ida=".*"/> } & $\begin{array}{l}\text { aber kaum hielt er sich derart fest, Für einen kurzen } \\
\text { Augenblick hielt ihr Blick den seinen fest. }\end{array}$ \\
\hline Verb mit Zusatz & \multicolumn{2}{|l|}{ [lemma="auffordern"] } & $\begin{array}{l}\text { auffordern, aufzufordern, auffordert, aufforderte, } \\
\text { aufforderten, auffordere }\end{array}$ \\
\hline $\begin{array}{l}\text { Verbalkomplex } \\
\text { („österreichische“ Reihenfolge) }\end{array}$ & \multicolumn{2}{|l|}{ [tag="VVINF.*"] [word="hätte"] [tag="VMINF.*"] } & $\begin{array}{l}\text { wie ich sie auffassen hätte können; Mehrwert, den er } \\
\text { erbringen hätte können; etwas stärker hervorheben hätte } \\
\text { müssen; einer wirklichen Selbstkritik unterziehen hätte sollen }\end{array}$ \\
\hline $\begin{array}{l}\text { Verbalkomplex („deutsche“ } \\
\text { Reihenfolge) }\end{array}$ & \multicolumn{2}{|l|}{ [word="hätte"] [tag="VVINF.*"] [tag="VMINF.*"] } & $\begin{array}{l}\text {..., daß sie seine Mutter hätte sein können.; , der genau hätte } \\
\text { sagen können,; ... die Preise , die ich ihnen hätte zahlen müssen }\end{array}$ \\
\hline $\begin{array}{l}\text { Verbzusatz am Ende eines } \\
\text { Fragesatzes }\end{array}$ & \multicolumn{2}{|l|}{ [tag="PTKVZ"\&word="auf"] [word="\?"] } & Nehmen Sie das auf ?; Warum regst du dich denn so auf ? \\
\hline Wortanfang & \multicolumn{2}{|l|}{ [word="rund.*"] } & rund, runde, rundherum, Runde, rundlich \\
\hline $\begin{array}{l}\text { Wiederholung von } 3 \text { bis } 5 \\
\text { Adjektiven }\end{array}$ & [tag="ADJA "]\{3,5\} & \multicolumn{2}{|c|}{$\begin{array}{l}\text { um die neuen globalen politischen Realitäten anzuerkennen; In dem ausgezeichneten } \\
\text { neuen britischen parlamentarischen Wahlkreis Daventry steht das Präzisionswerk...; } \\
\text { die für die koordinierte Einführung eines europaweiten öffentlichen zellularen } \\
\text { digitalen terrestrischen Mobilfunkdienstes in der Gemeinschaft bereitzustellen sind. }\end{array}$} \\
\hline Wortende & \multicolumn{2}{|l|}{ [word=".*rund"] } & aufgrund, Grund, rund, Hintergrund \\
\hline $\begin{array}{l}\text { Wort auf -schaft, jedoch NICHT } \\
\text { Gemeinschaft }\end{array}$ & \multicolumn{2}{|l|}{ [lemma=".*schaft"\&!lemma="Gemeinschaft"] } & Wirtschaft, Gesellschaft, Landwirtschaft \\
\hline Wortform & \multicolumn{2}{|l|}{ [word="rund"] } & rund \\
\hline
\end{tabular}




\subsection{Internetadressen}

\section{Adressen von Korpora}

\begin{tabular}{|c|c|c|c|}
\hline Sprache & Kürzel & Name & Adresse \\
\hline Deutsch & AAC & Austrian Academy Corpus & http://www.aac.ac.at/ \\
\hline Deutsch & BAS & Bayerisches Archiv für Sprachsignale & http://www.phonetik.uni-muenchen.de/forschung/bay arch sprsig/ \\
\hline Deutsch & ČNPK & $\begin{array}{l}\text { Česko-německý paralení korpus/ Das tschechisch-deutsche } \\
\text { Parallelkorpus }\end{array}$ & https://ske.fi.muni.cz/auth/corpora/ \\
\hline Deutsch & DDD & Deutsch Diachron Digital & http://www.deutschdiachrondigital.de/ \\
\hline Deutsch & DeReKo & Das Deutsche Referenzkorpus & http://www1.ids-mannheim.de/kl/projekte/korpora/ \\
\hline Deutsch & deTenTen & German TenTen corpus & https://ske.fi.muni.cz/ \\
\hline Deutsch & DeuCze & Das deutsch-tschechische Parallelkorpus & http://www.deucze.germanistik.uni-wuerzburg.de/ \\
\hline Deutsch & deWac & Deutsches Webkorpus & http://wacky.sslmit.unibo.it/doku.php auch unter \\
\hline Deutsch & DGD & Datenbank für gesprochenes Deutsch & http://dgd.ids-mannheim.de/ \\
\hline Deutsch & DWDS & Das Digitale Wörterbuch der deutschen Sprache & http://dwds.de/ \\
\hline Deutsch & Falko & $\begin{array}{l}\text { Ein fehlerannotiertes Lernerkorpus des Deutschen als } \\
\text { Fremdsprache }\end{array}$ & $\begin{array}{l}\text { https://www.linguistik.hu- } \\
\underline{\text { berlin.de/institut/professuren/korpuslinguistik/forschung/falko }}\end{array}$ \\
\hline Deutsch & FnhdC & Das Bonner Frühneuhochdeutschkorpus & http://www.korpora.org/Fnhd/ \\
\hline Deutsch & GeWiss & Korpus gesprochener Wissenschaftssprache & https://gewiss.uni-leipzig.de/ \\
\hline Deutsch & HGC & Huge German Corpus & http://www.ims.uni-stuttgart.de/forschung/ressourcen/korpora/hgc.html \\
\hline Deutsch & CHTK & Schweizer Text Korpus & http://chtk.unibas.ch/ \\
\hline Deutsch & InterCorp & InterCorp & http://www.korpus.cz/intercorp/ \\
\hline Deutsch & KGSR & Bochumer Korpus der gesprochenen Sprache im Ruhrgebiet & http://www.ruhr-uni-bochum.de/kgsr/ \\
\hline Deutsch & Korpus C4 & Korpus C4 & http://www.korpus-c4.org/ \\
\hline Deutsch & MHDBDB & Mittelhochdeutsche Begriffsdatenbank & http://mhdbdb.sbg.ac.at/ \\
\hline Deutsch & MULTEXT-East & $\begin{array}{l}\text { Multilingual Text Tools and Corpora for Central and Eastern } \\
\text { European Languages }\end{array}$ & http://nl.ijs.si/ME/ \\
\hline Deutsch & OPUS & The Open Parallel Corpus & http://opus.lingfil.uu.se/ \\
\hline Deutsch & Wortschatz-Portal & Korpusbasiertes Wortschatz-Portal der Universität Leipzig & http://wortschatz.uni-leipzig.de/ \\
\hline
\end{tabular}




\begin{tabular}{|c|c|c|c|}
\hline Sprache & Kürzel & Name & Adresse \\
\hline Englisch & BNC & British National Corpus & http://www.natcorp.ox.ac.uk/ \\
\hline Englisch & GloWbE & Global Web-Based English & http://corpus2.byu.edu/glowbe/ \\
\hline Englisch & ukWac & $\begin{array}{l}\text { Web corpus of British English } \\
\text { (Web-Korpus des britischen Englisch) }\end{array}$ & http://wacky.sslmit.unibo.it/ auch über: http://korpus.cz/ \\
\hline Französisch & CRFP & Le Corpus de Référence du Français Parlé & http://sites.univ-provence.fr/delic/corpus/index.html \\
\hline Französisch & frWac & $\begin{array}{l}\text { Corpus électronique de français constitué à partir du web } \\
\text { (französisches Web-Korpus) }\end{array}$ & http://wacky.sslmit.unibo.it/ auch über: http://korpus.cz/ \\
\hline Griechisch & EThEC/ HNC & 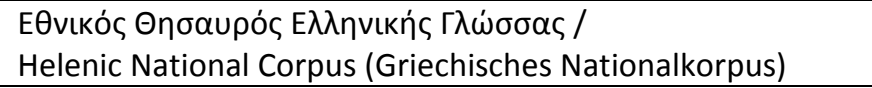 & http://hnc.ilsp.gr/en/ \\
\hline Italienisch & CORIS & Corpus di italiano scritto / Corpus of written Italian & $\underline{\text { http://corpora.dslo.unibo.it }}$ \\
\hline Italienisch & itWac & Web corpus di italiano (italiensches Web-Korpus) & http://wacky.sslmit.unibo.it/ auch über: http://korpus.cz/ \\
\hline Kroatisch & HNK & Hrvatski nacionalni korpus (Kroatisches Nationalkorpus) & http://www.hnk.ffzg.hr/ \\
\hline Polnisch & NKJP & $\begin{array}{l}\text { Narodowy Korpus Języka Polskiego (Nationalkorpus der } \\
\text { Polnischen Sprache) }\end{array}$ & http://nkjp.pl \\
\hline Russisch & NKRJ & $\begin{array}{l}\text { Национальный корпус русского языка (Nationales Korpus } \\
\text { der russischen Sprache) }\end{array}$ & http://www.ruscorpora.ru/ \\
\hline Slowakisch & SNK & Slovenský národný korpus (Slowakisches Nationalkorpus) & http://korpus.juls.savba.sk. \\
\hline Slowenisch & FIDAPLUS & $\begin{array}{l}\text { Korpus slovenskega jezika (Korpus der slowenischen } \\
\text { Sprache }\end{array}$ & http://www.fidaplus.net/ \\
\hline Spanisch & Corpus del Español & CORPUS DEL ESPAÑOL (Korpus des Spanischen) & http://www.corpusdelespanol.org/ \\
\hline Tschechisch & ČNK & Český národní korpus (Tschechisches Nationalkorpus) & http://korpus.cz/ \\
\hline Türkisch & TUD/ TNC & $\begin{array}{l}\text { Türkçe Ulusal Derlemi / Turkish National Corpus (Türkisches } \\
\text { Nationalkorpus) }\end{array}$ & http://www.tnc.org.tr \\
\hline Ungarisch & MNSz/ HuNC & $\begin{array}{l}\text { Magyar Nemzeti Szövegtár/ Hungarian National Corpus } \\
\text { (Ungarisches Nationalkorpus) }\end{array}$ & http://corpus.nytud.hu/mnsz/ \\
\hline
\end{tabular}




\section{Adressen von anderen (korpusähnlichen) Instrumenten}

\begin{tabular}{|l|l|l|}
\hline Kürzel & Name & Adresse \\
\hline ADABA & Österreichisches Aussprachewörterbuch/Österreichische Aussprachedatenbank & $\underline{\text { http://adaba.at/ }}$ \\
\hline FORWO & FORWO, das Aussprachewörterbuch & $\underline{\text { http://de.forvo.com/ }}$ \\
\hline Linguee & Linguee & $\underline{\text { http://www.linguee.de/ }}$ \\
\hline ParZU & ParZu - The Zurich Dependency Parser for German & $\underline{\text { http://kitt.cl.uzh.ch/kitt/parzu/ }}$ \\
\hline TextSTAT & TextSTAT 2.9. (Mathias Hüning) & $\underline{\text { http://neon.niederlandistik.fu-berlin.de/textstat/ }}$ \\
\hline
\end{tabular}




\section{Schlusswort}

Was können nun Sprachkorpora den Deutschlernenden bringen? Diese Frage stand als Impulsgedanke für diese Publikation und wurde hoffentlich zumindest teilweise beantwortet. Die Beschreibungen im Abschnitt IV und V können aufgrund der stetigen technischen und graphischen Entwicklung des jeweiligen Instruments bereits zum Zeitpunkt des Erscheinens dieses Buches veraltet sein. Vieles musste hier natürlich nur in Andeutungen bleiben, die entworfenen Forschungsfragen müssten in eigenständigen Studien tiefgreifender angegangen und detaillierter behandelt werden. Dies war jedoch nicht das Ziel dieser Arbeit. Vielmehr sollte die Breite der Nutzungsmöglichkeiten gezeigt werden, obwohl auch diese für DaF und DaZ viel breiter sind als der Platz in diesem Buch.

Die Nutzung von Korpora allgemein (nicht nur im $\mathrm{DaF} / \mathrm{DaZ}$ ) bedeutet für jede/-n selbstständige Arbeit mit der Sprache, Entdeckung neuer Erkenntnisse über die Sprache, über die Kultur dieser Sprache. Darüber hinaus bieten Korpora auch Einblicke in Ereignisse, die in der Gesellschaft passiert sind. Auch bekommt man nämlich oft Lust den ganzen Text zu lesen, aus dem der Beleg oder die Belege stammen.

Trotz dieser und anderer Argumente, die für die Nutzung von Korpora sprechen, ist unbestritten, dass die Arbeit mit Korpora (vor allem in der Anfangsphase) auch viel unnötig verlorene Zeit bedeuten kann. Es ist nur zu hoffen, dass mit diesem Buch der Zeit- und Arbeitsaufwand, der auf technische Probleme zurückzuführen ist (wie etwa das Suchen des richtigen Korpus, seiner Eigenschaften, der Formulierung der Abfrage, der Bedeutung eines Kürzels), verringert werden kann.

Auch muss man bei der Arbeit mit Korpora bedenken, dass jedes Korpus (auch das DeReKo mit seinen Milliarden von Textwörtern) lediglich einen ,winzigen“ Ausschnitt aus allen Texten darstellt, die in der jeweiligen Sprache getätigt werden. Wenn ein Wort oder ein Phrasem in einem Korpus nicht zu finden ist, bedeutet dies nicht, dass es in der Sprache nicht existieren. Aber: die Statistik lügt nicht. Wenn in einem großen Korpus (mit über einer Mrd. Worte) ein Wort nicht vorkommt, heißt es, dass dieses Wort allgemein gesehen und im Vergleich zu anderen Wörtern viel seltener gebraucht wird. Dieses Wort ist dann einerseits für die allgemeine Kommunikation eher unwichtig, muss daher nicht im allgemeinen Sprachunterricht vermittelt werden. Andererseits kann es aber sein, dass dieses Wort in einem spezifischen Sprachgebrauch häufig vorkommt.

Die Differenzen im Sprachgebrauch sind am besten zu sehen, wenn man Daten aus zwei unterschiedlich aufgebauten Korpora nimmt: In den Tabellen im Kap. 7 sind „Durchschnittswerte“ aus mehreren Korpora angeführt um die Objektivität der Angaben zu erhöhen. Vergleicht man die ersten hundert häufigsten Substantive eines ausgewogenen Korpus (z.B. DeReWo 2007) mit denselben eines nicht ausgewogenen Korpus (z.B. InterCorp_de), stellt man viele Überschneidungen, aber auch Unterschiede fest. Noch deutlicher ist es zu sehen, wenn man die zwanzig häufigsten Substantive (ihre Lemmata) in zwei unterschiedlichen Texten vergleicht.

Im Roman Malina von Ingeborg Bachmann sind es (nach Häufigkeit): Vater, Zeit, Leben, Herr, Tag, Frau, Hand, Haus, Mann, Nacht, Welt, Augen, Wasser, Gesicht, Menschen, Tür, Kopf, Namen, Wort, Mutter, in Pippi Langstrumpf von Astrid Lindgren hingegen: Mädchen, Kind, Pferd, Herr, Schule, Papa, Villa, Mama, Lehrerin, Damen, Hand, Tag, Hause, Weile, Leute, Seil, Kunterbunt, Kopf, Frau und Tisch. Daneben gibt es, obwohl die beiden Texte nur 
wenig gemeinsame Züge aufweisen, auch volle Übereinstimmungen in der Lexik: Frau, Hand, Haus, Herr, Kopf, Tag. Einige weitere Lexeme unterscheiden sich nur stilistisch: Vater/ Papa, Mutter/ Mama. Diese Lexeme findet man auch im Kap. 7.2 auf S. 188. Sie gehören unumstritten zum ,harten“ Kern des deutschen Wortschatzes.

Alle synsemantischen Wörter, Pronomina und sogar viele Verben sind fast ident.

Dieses Phänomen lässt sich mit der Tatsache vergleichen, dass jeder Mensch neben den allgemeinen sprachlichen Mitteln auch seine „eigene Sprache“ verwendet. Es betrifft selbstverständlich nicht nur den individuellen Wortschatz, sondern auch sprachliche Strukturen und kommunikative Strategien, die bei jedem Sprecher und jeder Sprecherin unterschiedlich beliebt sind und deswegen auch unterschiedlich häufig realisiert werden. Aus den Korpusdaten sind jedoch diejenigen sprachlichen Elemente zu erkennen, die (fast) jeder verwendet und versteht. Deswegen sollten eben diese den Sprachlernenden vermittelt werden.

Die Statistik lügt zwar nicht, sie darf aber auch nicht überschätzt werden. Die Daten der Korpora entsprechen nur der Realität der Texte, die im Korpus gespeichert sind - nicht der allgemeinen Realität der Sprache, die sowieso wegen ihrer Komplexität nicht fassbar ist. Darüber hinaus weisen alle Korpora noch viele technische, aber auch „faktische“ Mängel auf, die nicht in absehbarer Zeit behoben werden oder kaum jemals behoben werden können. Die Gründe sind zahlreich und spiegeln sich in den häufigsten Problemen wider, auf die man während der Korpusarbeit stößt. Sie lassen sich grundsätzlich in zwei markante Problemfelder zusammenfassen: Korpusmängel und Datenfehler. Deshalb muss jegliche Korpusarbeit mit dem Wissen ablaufen, dass Korpora mangelhaft im Aufbau und weitaus nicht frei von Fehlern jeglicher Art sind.

Ein großes Manko der Korpuslandschaft ist das Fehlen von wirklich repräsentativen Korpora der gesprochenen Sprache (dazu auch Heine 2008: 6). Wenn es diese in der (hoffentlich nahen) Zukunft auch geben sollte, wären sie zur Zeit ihrer Veröffentlichung bereits veraltet, denn die gesprochene Sprache ändert sich viel schneller als die geschriebene Sprache. Dazu kommt das bekannte Phänomen, dass man beim Schreiben automatisch eher auf die Norm achtet als beim Sprechen. Für die Objektivierung der Daten, aus denen normative Werke frei von (traditionell) puristischen Einflüssen und Eingriffen entstehen könnten, wären jedoch große Korpora der gesprochenen Sprache eine enorme Hilfe.

Parallele Korpora müssen (vermutlich langfristig) auf gesprochene Sprache völlig verzichten und müssen sich damit begnügen, was der aktuellen gesprochenen Sprache am nächsten kommt, nämlich gegenwärtige belletristische Werke in professioneller Übersetzung.

Der korpuslinguistische Grundsatz ,je mehr Daten, desto schärfer das Bild über das untersuchte Phänomen" kann abschreckende Wirkung haben. $\mathrm{Zu}$ viele Daten sind nicht bewältigbar, kein Mensch kann hunderttausende Konkordanzen in einer akzeptablen Zeitspanne sortieren. Deswegen ermöglichen gute Korpora eine Zufallsauswahl der Belege. Problematisch sind jedoch homonyme Formen, die von automatischen Textanalysatoren (Tagger, Parser) falsch erkannt wurden. Diese Fehler sind sehr häufig. Am besten ist dies an solchen Wörtern zu erkennen, die an ähnlichen Stellen im Satz (satztopographisch gemeint) unterschiedliche Funktionen einnehmen. Diese sind für die automatischen Analysatoren besonders schwierig richtig $\mathrm{zu}$ erkennen, daher ist die Fehlerquote extrem hoch. Beispielsweise kann die Wortform schon als Temporaladverb, Abtönungspartikel, Antwortpartikel oder (umgangssprachlich) als Imperativ des Verbs schonen vorkommen:

"Danke, und du schon dich auch!"

Wenn du schon mal wegen irgendwas an der Grenze erwischt wurdest

Wohl habe ich gestern schon dich gesehen und begrüßt. "

Schon ganz schön teuer! 
Diese Formen wurden im Korpus InterCorp_de und im DeReKo teilweise falsch erkannt. Hier muss man wieder der Statistik vertrauen und eine Zufallsauswahl durchführen. Aus den Konkordanzen der Zufallsauswahl muss man die prozentuelle Vertretung (hier der einzelnen Funktionen des Wortes schon ${ }^{58}$ ) berechnen und auf die Gesamtmenge der Konkordanzen übertragen. Wie viele Belege in die Zufallsauswahl einbezogen werden sollen, hängt sehr stark vom Ziel der Arbeit ab: Für Arbeiten, deren Aufgabe die erste Orientierung in der Problematik ist (Seminararbeiten, Forschungsprojekte), reichen oft bis zu hundert Belege. Kleinere wissenschaftliche Arbeiten sollten mit 500 bis 1.000 Belegen arbeiten, größere Arbeiten können erst mit mehreren (zehn)tausenden Belegen das gewählte Problem seriös beleuchten.

Die Fehlerquote der Annotation variiert sehr stark. In professionell erstellten Korpora werden der überwiegenden Mehrheit der Wörter sowohl ihre richtigen Grundformen als auch die richtigen morphosyntaktischen (oder syntaktisch-semantischen) Angaben zugewiesen. Die Fehlerquote liegt bei automatisch annotierten Korpora im Schnitt um 7 bis 10\%. Daher ist es notwendig, die Belege zumindest stichpunktartig durchzusehen und die Richtigkeit zu überprüfen. Die Annotationsinstrumente werden stets verbessert, daher sinkt auch die Fehlerquote. Das Aussortieren von falschen Belegen ist sicherlich eine der Arbeiten, die ohne Korpusdaten nicht existieren würden. Für gewisse qualitative Untersuchungen bzw. auch für die Vermittlung einiger Elemente braucht man nicht viele Belege, daher auch keine Korpora. Hier schließt sich bereits der Kreis zu den Gedanken im Kap. 1.

Ein häufiger Störfaktor sind auch Tippfehler. Viele Texte werden immer noch eingescannt, die Texterkennungsprogramme (OCR) entziffern nicht immer alle Buchstaben einwandfrei, darüber hinaus werden Texte unter dem heutigen hohen Zeitdruck mit vielen Fehlern herausgegeben.

Grundsätzlich sind aber Korpora Instrumente, die die Sprache (oder Sprachen im Vergleich) realitätsnah und objektiv erfassen können. Elemente oder Strukturen, über die früher hinweg geschaut wurde, können endlich objektiv überprüft werden. Beispiele, dass normative Werke diesbezüglich Lücken aufweisen, wurden im Kap. 6 gebracht. So gesehen sind Korpora Instrumente, mit denen ,im DaF-Unterricht ein gesundes Gleichgewicht zwischen der Präskription und Deskription zu erreichen [ist]“ (Stuyckens/ Brône 2009: 8). In Anbetracht der Tatsache, dass man sich von präskriptiven Werken emanzipieren kann, dass plurizentrische Sprachen differenziert untersucht und vermittelt werden können (vgl. auch Sorger 2013: 44), sind Korpora auch Instrumente für die Demokratisierung der Sprachbetrachtung und Sprachvermittlung.

Längst haben auch viele Wirtschaftsbereiche erkannt, dass Korpora bzw. einige Korpusinstrumente (v.a. die Kookkurrenz/Kollokationsanalyse) ein hilfreiches Mittel zur Aufdeckung komplexerer Zusammenhänge bieten. Es werden Analysen von Krankenberichten zur Erstellung neuer Diagnosen durchgeführt, Fehlerkomplexe in der Technik und Kommunikation erforscht oder kommerzielle Aufträge über verschriebene Medikamente vergeben.

Auch im neuesten Fach der angewandten Linguistik, in der forensischen Linguistik, spielen Korpusinstrumente zum Nachweis sprachlicher Besonderheiten der Täter eine wichtige Rolle.

58 Die Abfrage nach der Wortform schon liefert über 45.000 Belege, im DeReKo, Archiv TAGGED-T über eine Mio. Treffer. 
Diejenigen, die mit DaF und DaZ zu tun haben, wissen, wie unterschiedlich Lernende und ihre Bedürfnisse sind, wie schwierig es ist, ein passendes Lehrbuch für eine ganze Lernergruppe $\mathrm{zu}$ finden, noch dazu ein solches, das dem aktuellen Stand der Sprache, womöglich auch noch in der Region entsprechen würde. Korpora können Lehrbücher nicht ersetzen, sie können sie aber hervorragend ergänzen. Deshalb soll die Nutzung von Sprachkorpora für jede/-n Sprachlehrer/-in ein selbstverständlicher Teil der Arbeit werden. Noch mehr: die Grundkenntnisse der Korpusarbeit sollten auch an die Lerner/-innen weitergegeben werden.

Für die Erforschung der Sprachen, für den Vergleich von Sprachen und für eine effektive Vermittlung jeder Sprache sind Korpora ein Instrument, um das man nicht herumkommt. Ein Lehrer oder eine Sprachwissenschaftlerin ohne ein Sprachkorpus ist schlichtweg wie ein Pathologe ohne Leiche/ eine Bank ohne Tresor/ eine Chemielehrerin ohne Labor oder ein Gärtner ohne Dünger. 


\section{Literaturverzeichnis}

AAC (2014): Austrian Academy Corpus. Wien: Österreichische Akademie der

Wissenschaften. http://www.aac.ac.at/ [20.7.2014]

Abel, Andrea/ Zanin, Renata (Hg.) (2011): Korpora in Lehre und Forschung. Bozen: BU, Bozen Univ. Press.

ADABA (2005-2014): Österreichisches Aussprachewörterbuch/ Österreichische Aussprachedatenbank. Graz: Forschungsstelle österreichisches Deutsch. http://adaba.at/ [27.7.2014]

Aijmer, Karin/ Altenberg, Bengt (Hg.) (1991): English corpus linguistics: studies in honour of Jan Svartvik. London: Longman.

Baker, Paul/ Hardie, Andrew/ McEnery, Tony (2006): A glossary of corpus linguistics. Edinburgh: Edinburgh Univ. Press.

Barlow, Michael (2009): ParaConc. http://www.paraconc.com/ [17.9.2013]

Barnickel, Klaus-Dieter (1992): Falsche Freunde: ein vergleichendes Wörterbuch Deutsch Englisch. Heidelberg: Groos.

Baroni, Marco/ Bernardini, Silvia/ Ferraresi, Adriano/ Zanchetta, Eros (2009): The WaCky Wide Web: A Collection of Very Large Linguistically Processed Web-Crawled Corpora. In: Language Resources and Evaluation 43 (3). Springer Science + Business Media. S. 209-226.

BAS (1995-2014): Bayerisches Archiv für Sprachsignale. München: Institut für Phonetik und Sprachverarbeitung (IPS), Ludwig Maximilians Universität München.

http://www.phonetik.uni-muenchen.de/forschung/bay_arch_sprsig/ [12.5.2014]

Belica, Cyril (1995): Statistische Kollokationsanalyse und -clustering. Korpuslinguistische Analysemethode. Mannheim: Institut für Deutsche Sprache. http://corpora.idsmannheim.de/ [22.5.2012]

Berman, Stephen (2014): Sitemap zur Website des Proseminars Korpuslinguistik, SS 2014. Bochum: Ruhr-Universität. http://homepage.rub.de/Stephen.Berman/Korpuslinguistik/sitemap.html [28.7.2014]

Bibel Online (2014): CID - christliche internet dienst GmbH. Berlin. http://www.bibelonline.net/ [2.3.2014]

Biber, Douglas/ Conrad, Susan/ Reppen, Randi (1998): Corpus linguistics: investigating language structure and use. Cambridge: University Press.

BNC (2010): British National Corpus. Oxford: University of Oxford. http://www.natcorp.ox.ac.uk/ [25.7.2014]

Bopp, Sebastian (2010): Einführung in die Korpuslinguistik mit DeReKo und COSMAS II. Augsburg: Universität Augsburg, Germanistik. https://www.philhist.uniaugsburg.de/lehrstuehle/germanistik/sprachwissenschaft/mitarbeiter/stelspass/materiali en_lehrveranstaltungen/korpuslinguistik_dereko_cosmas2_bopp.pdf [20.7.2014]

Braun, Sabine (2005): Corpora4Learning.net. [Englische Korpora im Fremdsprachenunterricht]. Guildford, Surrey: University of Surrey. http://www.corpora4learning.net/ [17.7.2014] 
Breyer, Yvonne Alexandra (2011): Corpora in Language Teaching and Learning. Potential, Evaluation, Challenges. Frankfurt (M) u.a.: Peter Lang.

Bubenhofer, Noah (2006-2013): Einführung in die Korpuslinguistik: Praktische Grundlagen und Werkzeuge. http://www.bubenhofer.com/korpuslinguistik/ [7.4.2014]

Bubenhofer, Noah/ Konopka, Marek/ Schneider, Roman (2014): Präliminarien einer Korpusgrammatik. Tübingen: Narr.

Budin, Gerhard (2011): Die Sprache im Wandel der Zeit. In: Thema. Das Forschungsmagazin der ÖAW, 10/2011. Wien: Österreichische Akademie der Wissenschaften. S. 16-17.

Carstensen, Kai-Uwe/ Ebert, Christian/ Ebert, Cornelia/ Jekat, Susanne/ Klabunde, Ralf/ Langer, Hagen (Hrgs.) (2010): Computerlinguistik und Sprachtechnologie: eine Einführung. Heidelberg: Spektrum Akad. Verl.

Čermák, František (2005): Jak využívat Český národní korpus. Praha: Nakladatelství Lidové noviny.

Čermák, František/ Klégr, Aleš (2004): Modality in Czech and English. In: International Journal of Corpus Linguistics 9:1. S. 83-95.

Chiarcos, Christian/ Erjavec, Tomaz (2011): OWL/DL formalization of the MULTEXT-East morphosyntactic specifications. In: Proceedings of the 5th Linguistic Annotation Workshop (LAW-V). Portland (Oregon). S. 11-20.

Chomsky, Noam (1986): Knowledge of language: its nature, origin, and use. New York: Praeger.

CHTK (2008-2012): Schweizer Text Korpus. Zürich: Schweizer Textkoprus/ Schweizerisches Idiotikon. http://www.schweizer-textkorpus.ch/index.php/de/ [25.7.2014]

CORIS (2011) Corpus di italiano scritto/ Corpus of written Italian. Bologna: Università di Bologna. http://corpora.dslo.unibo.it [27.6.2014]

Corpus del Español (2002-2014): Davies, Mark: Corpus del Español: 100 million words, 1200s-1900s. http://www.corpusdelespanol.org [21.3.2014]

Cosmas II (2012): Corpus Search, Management and Analysis System. Mannheim: Institut für Deutsche Sprache. http://www.ids-mannheim.de/cosmas2/ [15.7.2014]

ČNK (2014): Czech Nation Corpus (and other available corpora). Praha: Univerzita Karlova, Ústav Českého národního korpusu. http://korpus.cz/ [26.7.2014]

ČNK-Tagset (2014): Hajič, Jan/ Cvrček, Václav: Internetová př́ručka ČNK. Morfologické značky (tagy). http://wiki.korpus.cz/doku.php/seznamy:tagy [27.7.2014]

ČNPK (2000-2005): Česko-německý paralení korpus/ Das tschechisch-deutsche Parallelkorpus. https://ske.fi.muni.cz/auth/corpora/ [15.7.2014]

CRFP (2014): Le Corpus de Référence du Français Parlé. http://sites.univprovence.fr/delic/corpus/index.html [20.6.2014]

Davies, Mark (2014): corpus.byu.edu. [Korpora an der Brigham Young University]. Provo, Utah: Birgham Young University. http://corpus.byu.edu/corpora.asp [12.7.2014]

DDD (2011): Deutsch Diachron Digital. Jena: Friedrich-Schiller-Universität u.a. http://www.deutschdiachrondigital.de/ [5.6.2014]

DeReKo (2014): Deutsches Referenzkorpus. Mannheim: Institut für deutsche Sprache. http://www1.ids-mannheim.de/kl.html [20.7.2014] 
DeReWo (2009): Korpusbasierte Wortlisten DeReWo. Mannheim: Institut für Deutsche Sprache. http://www.ids-mannheim.de/kl/derewo/ [15.4.2014]

deTenTen (2014): German TenTen corpus. Brno: Masarykova univerzita. https://ske.fi.muni.cz/ [15.7.2014]

deWac (2013): Deutsches Webkorpus. http://wacky.sslmit.unibo.it/doku.php [20.7.2014]

DGD (2014): Datenbank für gesprochenes Deutsch. Mannheim: Institut für Deutsche Sprache. http://dgd.ids-mannheim.de/ [15.7.2014]

Dovalil, Vít/ Káňa, Tomáš/ Peloušková, Hana/ Zbytovský, Štěpán/ Vavřín, Martin (2013): Korpus intercorp_de Version 6 vom 8. 4. 2013. Praha: Ústav Českého národního korpusu. http://www.korpus.cz [29.7.2014]

Drosdowski, Günther (1985): Die Dudenredaktion. In: Wimmer, Rainer (Hg.): Sprachkultur. Sprache der Gegenwart: Schriften des Instituts für Deutsche Sprache in Mannheim (63). Düsseldorf: Schwann.

Duden - Grammatik (2005): Kunkel-Razum, Kathrin/ Münzberg, Franciska (Redaktion): Duden: die Grammatik. Unentbehrlich für richtiges Deutsch. Mannheim: Dudenverlag.

Duden - Universalwörterbuch (2006): Wermke, Matthias/ Kunkel-Razum, Kathrin/ ScholzeStubenrecht, Werner (Hg.): Duden - Deutsches Universalwörterbuch, 6. Aufl. Mannheim: Bibliographisches Institut \& F. A. Brockhaus AG. [CD-ROM]

Ďurčo, Peter (1994): Probleme der allgemeinen und kontrastiven Phraseologie (am Beispiel Deutsch und Slowakisch). Heidelberg: Groos.

Ďurčo, Peter/ Banášová, Monika/ Hanzlíčková, Astrid (2010): Feste Wortverbindungen im Kontrast. Trnava: Univerzita sv. Cyrila a Metoda.

Ďurčo, Peter/ Kathrin Steyer (2012): Ein korpusbasiertes Beschreibungsmodell für die elektronische Sprichwortlexikografie. SprichWort-Plattform. http://sprichwortplattform.org/attach/Ergebnisse/SW_Modell_steyer_durco.pdf [22.7.2014]

Dürscheid, Christa/ Elspaß, Stephan/ Ziegler, Arne (2014): Variantengrammatik des Standarddeutschen. [Ein internationales Projekt der Universitäten Graz, Salzburg, Zürich.] http://www.variantengrammatik.net/ [20.7.2014]

DWB (1854-1961): Grimm, Jacob/ Grimm, Wilhelm: Deutsches Wörterbuch. http://woerterbuchnetz.de/DWB/ [15.5.2014]

DWDS (2014): Das Digitale Wörterbuch der deutschen Sprache. Berlin: BerlinBrandenburgische Akademie der Wissenschaften. http://dwds.de/ [15.7.2014]

Engel, Ulrich (1988): Deutsche Grammatik. Heidelberg: Groos.

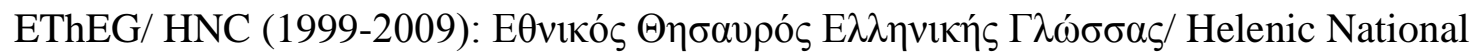
Corpus. Athena: [Institut für Sprache und Sprachverarbeitung] http://hnc.ilsp.gr/en/ [17.7.2014]

Facchinetti, Roberta (Hg.) (2007): Corpus Linguistics 25 Years on. Amsterdam u.a.: Rodopi.

Fachlexikon DaF/DaZ (2010): Barkowski, Hans/ Krumm, Hans-Jürgen (Hg.): Fachlexikon Deutsch als Fremd- und Zweitsprache. Tübingen/ Basel: Francke. 
Falko (2014): Ein fehlerannotiertes Lernerkorpus des Deutschen als Fremdsprache. Berlin: Humboldt-Universität. https://www.linguistik.huberlin.de/institut/professuren/korpuslinguistik/forschung/falko [20.7.2014]

FIDAPLUS (2014): Korpus slovenskega jezika. Ljubljana: Univerza v Ljubljani. http://www.fidaplus.net/ [27.7.2014]

Fleischer, Wolfgang (1969): Wortbildung der deutschen Gegenwartssprache. Leipzig: VEB Bibliographisches Institut.

FnhdC (2007): Das Bonner Frühneuhochdeutschkorpus. http://www.korpora.org/Fnhd/ [12.6.2014]

Forum Deutsch als Fremdsprache (1996-2012): Internetservice für den Unterricht Deutsch als Fremdsprache. Düsseldorf: Institut für Internationale Kommunikation e.V. http://www.deutsch-als-fremdsprache.de/ [14.3.2014]

FORWO (2008-2014): FORWO, das Aussprachewörterbuch. http://de.forvo.com/ [27.7.2014]

Francis, Nelson W. (1992): Language Corpora B.C. In: Svartvik, Jan (Hg.): Directions in Corpus Linguistics. Berlin: de Gruyter. S. 17-32.

frWac (2013): Corpus électronique de français constitué à partir du web. http://wacky.sslmit.unibo.it/ [27.7.2014]

GeWiss (2014): Gesprochene Wissenschaftssprache. Leipzig: Universität Leipzig. https://gewiss.uni-leipzig.de/ [20.7.2014]

GloWbE (2013): Davies, Mark: Corpus of Global Web-Based English: 1.9 billion words from speakers in 20 countries. http://corpus2.byu.edu/glowbe/ [17.4.2014]

Granger, Sylviane/ Lerot, Jacques/ Petch-Tyson, Stephanie (2003): Corpus-based Approaches to Contrastive Linguistics and Translation Studies. Amsterdam u.a.: Rodopi.

Harries, Tony/ Moreno Jaén, María (Hg.) (2010): Corpus linguistics in language teaching. Bern/ Wien u.a.: Peter Lang.

Hausmann, Franz Josef (2003): Was sind eigentlich Kollokationen? In: Steyer, Kathrin (Hg.): Wortverbindungen - mehr oder weniger fest. Berlin u.a.: de Gruyter. S. 309-334.

Heine, Antje (2008): Zur Nutzbarkeit der gegenwärtig verfügbaren deutschen Korpora für die Lerenerlexikografie Deutsch als Fremdsprache. Anspruch und Wirklichkeit. In:

Deutsch als Fremdsprache 45/1. Leipzig: Universität Leipzig.

Heringer, Hans Jürgen (2012): Chunking: Synonymik des Deutschen korpusbasiert. Tübingen: Narr.

HGC (2013): Huge German Corpus. Stuttgart: Universität Stuttgart. http://www.ims.unistuttgart.de/forschung/ressourcen/korpora/hgc.html [2.3.2014]

Hirschová, Milada (2013): Pragmatika v češtině. Praha: Univerzita Karlova, Karolinum.

HNK (2005-2013): Hrvatski nacionalni korpus. Zagreb: Sveučilište u Zagrebu. http://www.hnk.ffzg.hr/ [17.7.2014]

Hüning, Matthias (2013): TextSTAT - Simples Text Analyse Tool. Berlin: Freie Universität. http://neon.niederlandistik.fu-berlin.de/textstat/ [22.11.2013]

InterCorp (2011, 2014). Korpus InterCorp. Praha: Univerzita Karlova. http://ucnk.ff.cuni.cz/intercorp/?req=page:info [25.7.2014]

itWac (2013): Web corpus di italiano. http://wacky.sslmit.unibo.it/ [17.7.2014] 
Jelínek, Tomáš/ Petkevič, Vladimír/ Rosen, Alexandr/ Skoumalová, Hana (2012): Czech Treebanking Unlimited. Praha: Univerzita Karlova. http://utkl.ff.cuni.cz/synttb/Team_Czech_Treebanking_Unlimited.pdf [16.7.2014]

Káňa, Tomáš (2006): Korpuslinguistik - eine übersehene Herausforderung für den Deutschunterricht? In: Krumm, Hans-Jürgen (Hg.): Theorie und Praxis. Österreichische Beiträge zu Deutsch als Fremdsprache 9, 2005: Schwerpunkt: Innovationen - Neue Wege im Deutschunterricht. Innsbruck u.a.: StudienVerlag. S. 99-115.

Káňa, Tomáš (2006): Zur Problematik einiger tschechischer Ortsnamen in deutschen Texten. In: Lingua viva 2,1. České Budějovice: Jihočeská univerzita. S. 7-19.

Káňa, Tomáš (2007): Illokutive Kraft der Quellenangaben in Rundfunknachrichten. In: Balaskó, Maria/ Szatmári, Petra (Hg.): Sprach- und literaturwissenschaftliche Brückenschläge. München: Lincom.

Káňa, Tomáš (2008): Elektronische Sprachkorpora in Wissenschaft und Unterricht DaF/DaZ einige Vorschläge für die Nutzung der elektronischen Instrumente. In: Krumm, HansJürgen/ Portmann-Tselikas, Paul R. (Hg.): Theorie und Praxis: Österreichische Beiträge zu Deutsch als Fremdsprache. Schwerpunkt: Wortschatz (Serie A 11/2007). Innsbruck u.a.: StudienVerlag. S. 123-136.

Káňa, Tomáš (2010): Einige tschechische Flussnamen in elektronischen Korpora. In: Bock, Bettina (Hg.): Aspekte der Sprachwissenschaft. Hamburg: Verlag Dr. Kovač. S. 437445 .

Káňa, Tomáš (2012): Wortbildung: Umriss der Theorie mit Aufgaben und Übungen. Brno: Masarykova univerzita. https://is.muni.cz/elportal/?id=1071872 [15.6.2014]

Káňa, Tomáš / Hana Peloušková (2009): Deutsch und Tschechisch im Vergleich. Korpusbasierte linguistische Studien. Brno: Masarykova univerzita.

Káňa, Tomáš / Hana Peloušková (2011): Deutsch und Tschechisch im Vergleich: Korpusbasierte linguistische Studien II. Brno: Masarykova univerzita.

Káňa, Tomáš / Peloušková, Hana (2006): Elektronische Korpora in Tschechien und das tschechisch-deutsche Parallelkorpus. In: Kettemann, Bernhard/ Marko, Georg (Hg.) Planing, Gluing and Painting Corpora. Frankfurt (M): Lang. S. 27-46.

Káňa, Tomáš/ Peloušková, Hana (2010): Česko-německý paralelní korpus. Brno: Masarykova univerzita. http://www.ped.muni.cz/katedry-a-instituty/nemecky-jazykliteratura/aktivity/cesko-nemecky-paralelni-korpus/ [20.7.2014]

Káňa, Tomáš/ Vavř́n, Martin (2011): Das Korpus InterCorp (Deutsche Fassung). http://ucnk.ff.cuni.cz/intercorp/?lang=de [27.7.2014]

Káňa, Tomáš: (2010): Synthetische substantivische Diminutive im Tschechischen und ihre strukturellen Entsprechungen im Deutschen. In: Kratochvílová, Iva/ Wolf, Norbert Richard (Hg.): Kompendium Korpuslinguistik: Eine Bestandsaufnahme aus deutschtschechischer Perspektive. Heidelberg: Universitätsverlag Winter. S. 235-242.

KGSR (2014): Bochumer Korpus der gesprochenen Sprache im Ruhrgebiet. Bochum: RuhrUniversität. http://www.ruhr-uni-bochum.de/kgsr/ [17.7.2014]

Kilgarriff, Adam/ Husák, Miloš /McAdam, Katy/ Rundell, Michael/ Rychlý, Pavel (2008): GDEX: Automatically finding good dictionary examples in a corpus. In Proceedings 
of the XIII EURALEX International Congress. Barcelona: Institut Universitari de Lingüística Aplicada. S. 425-432.

Kilgarriff, Adam/ Baisa, Vít/, Bušta, Jan / Jakubíček, Miloš/ Kovář, Vojtěch/ Michelfeit, Jan/ Rychlý, Pavel/ Suchomel, Vít (2014): The Sketch Engine: ten years on. In: Lexicography ASIALEX (2014) 1. Berlin/ Heidelberg: Springer. S 7-36.

Kluge, Friedrich (2002): Etymologisches Wörterbuch. Berlin u.a.: de Gruyter.

Knittlová, Dagmar/ Grygová, Bronislava/ Zehnalová, Jitka (2010): Překlad a překládání. Olomouc: Univerzita Palackého v Olomouci.

Kocek, Jan/ Kopřivová, Marie/ Kučera (Hg.) (2000): Český národní korpus: Úvod a př́ručka uživatele. Praha: Univerzita Karlova, Ústav Českého národního korpusu.

Konopka, Marek/ Kubczak, Jacqueline/ Mair, Christian/ Štícha, František/ Waßner, Ulrich Hermann (Hg.) (2011): Grammatik und Korpora 2009. Dritte Internationale Konferenz. Mannheim, 22.-24.9.2009. Tübingen: Narr.

Korpus-C4 (2014): DWDS - AAC - Korpus Südtirol - Schweizer Textkorpus. http://www.korpus-c4.org/ [12.7.2014]

Kotulková, Veronika/ Kratochvílová, Iva/ Rykalová, Gabriela/ Wolf, Norbert Richard (20052010): DeuCze: Das deutsch-tschechische Parallelkorpus. Würzburg/ Opava: http://www.deucze.germanistik.uni-wuerzburg.de/ [16.6.2014]

Krajka, Jarosław (2007): Corpora and Language Teachers: From Ready-Made to TeacherMade Collections. CORELL: Computer Resources for Language Learning 1. S 36-55. http://www.ucam.edu/sites/default/files/corell/JKrajka.pdf [15.7.2014]

Kundera, Milan (1970): Scherz. München: Deutscher Taschenbuch Verlag.

Kurier (2014): Infokampagne zur MaHü: Experte glaubt nicht an Korruption. http://kurier.at/chronik/wien/infokampagne-zur-mariahilfer-strasse-experte-sickingerglaubt-nicht-an-korruption/44.661.945 [07.01.2014]

Lawler, John M./ Aristar-Dry, Helen (Hg.) (1998): Using computers in linguistics: a practical guide. London u.a.: Routledge.

Leech, Geoffrey (1991): The State of the Art in Corpus Linguistics. In: Aijmer, Karin/ Altenberg, Bengt (Hg.): English Corpus Linguistics. London: Longman. S. 8-29.

Leech, Geoffrey (1997): Teaching and Language Corpora: a Convergence. In: Wichmann, Anne/ Fligelstone, Steven/ McEnery, Tony/ Knowles, Gerry (Hg.): Teaching and language Corpora. London u.a.: Longman.

Lemnitzer, Lothar/ Zinsmeister, Heike (2010): Korpuslinguistik: eine Einführung. Tübingen: Narr.

Lewandowski, Theodor (1994): Linguistisches Wörterbuch. Heidelberg u.a.: Quelle \& Meyer.

Lewis, Michael (1996): The lexical approach: the state of ELT and a way forward. Hove: Language Teaching Publ.

Linguee (2013): Linguee. Köln: Linguee GmbH. http://www.linguee.de/ [17.7.2014]

Lišková, Danuša (2010): Peniaze - banky - burzy. In: Medvecká, L'ubica/ Šoltys, Jaroslav (Hg.): Odborný preklad 5: terminológia bankovníctva a finančníctva v súvislosti $\mathrm{s}$ prechodom na euro. Studia Translatologica Bratislavensia 20. Bratislava: AnaPress. S. 22-33. 
Lüdeling, Anke/ Kytö, Merja (Hg.) (2008): Corpus Linguistics. An International Handbook, vol. 1. Berlin u.a.: de Gruyter.

Lüdeling, Anke/ Walter, Maik (2010): Korpuslinguistik. In: Fandrych, Christian/ Hufeisen, Britta/ Krumm, Hans-Jürgen/ Riemer, Claudia (Hg.): Deutsch als Fremdsprache und Zweitsprache. Ein internationales Handbuch. Berlin u.a.: de Gruyter. S. 315-322.

Mair, Rebecca (2013): buk vs. backte; buken vs. backten; gebackt vs. gebacken [unveröffentlichte Seminararbeit]. Wien: Universität Wien.

McEnery, Tony/ Wilson, Andrew (2001): Corpus linguistics: an introduction. Edinburgh: Univ. Press.

Merkel, Silke/ Schmidt, Thomas (2009): Korpora gesprochener Sprache im Netz - eine Umschau. In: Gesprächsforschung - Online-Zeitschrift zur verbalen Interaktion, 10 (2009). http://www.gespraechsforschung-ozs.de/heft2009/heft2009.html [12.2.2013]

Metzler (2000): Glück, Helmut: Metzler Lexikon Sprache. Stuttgart u.a.: Metzler.

MHDBDB (2012): Mittelhochdeutsche Begriffsdatenbank. Salzburg: Universität Salzburg. http://mhdbdb.sbg.ac.at/ [27.7.2014]

MNSz (1998-2006): Magyar Nemzeti Szövegtár/ Hungarian National Corpus. Budapest: Magyar tudományos akadémia. http://corpus.nytud.hu/mnsz/ [27.7.2014]

Moudraia, Olga (2001): Lexical Approach to Second Language Teaching. Washington: Center for Applied Linguistics. (ERIC Digest, EDO-FL-01-02).

MULTEXT-East (2013): Multilingual Text Tools and Corpora for Central and Eastern European Languages. http://nl.ijs.si/ME/ [12.7.2014]

NKJP (2008-2012): Narodowy Korpus Języka Polskiego. http://nkjp.pl [27.7.2014]

NKRJa (2003-2014): Национальный корпус русского языка http://www.ruscorpora.ru/ [14.6.2014]

OPUS (2014): The Open Parallel Corpus. Uppsala: Uppsala Universitet. http://opus.lingfil.uu.se/ [12.6.2014]

Österreichisches Wörterbuch (2001): Back, Otto/ Benedikt, Erich/ Blümel, Karl/ Ebner, Jakob/ Hornung, Maria/ Möcker, Hermann/ Pohl, Heinz-Dieter/ Tatzreiter, Herbert: Österreichisches Wörterbuch. Wien: ÖBV \& HPT VerlagsGmbH.

ParZu (2014): ParZu - The Zurich Dependency Parser for German. Zürich: Universität Zürich. http://kitt.cl.uzh.ch/kitt/parzu/ [20.7.2014]

Peloušková, Hana (2013): Das Projekt InterCorp und seine Rolle in der Deutschlehrerausbildung und Forschung. In: Slowakische Zeitschrift für Germanistik. Banská Bystrica: Verband der Deutschlehrer und Germanisten der Slowakei.

Perkuhn, Rainer/ Belica, Cyril (2004): Eine kurze Einführung in die Kookkurrenzanalyse und syntagmatische Muster. Mannheim: Institut für Deutsche Sprache. http://www1.idsmannheim.de/kl/misc/tutorial.html [22.6.2014]

PONS Deutsch - Englisch (1998): Breitsprecher, Roland/ Terrell, Peter/ Schnorr, Veronika/ Morris, Wendy V.A: PONS Globalwörterbuch Deutsch - Englisch. Wien: ÖBV.

profil (2009): Interview "Hausbesetzer sind konservativ": Blixa Bargeld im Interview mit profil. http://www.profil.at/articles/0910/560/235752/hausbesetzer-blixa-bargeldinterview [14.6.2014] 
Reznicek, Marc/ Lüdeling, Anke/ Krummes, Cedric/ Schwantuschke, Franziska/ Walter, Maik/ Schmidt, Karin/ Hirschmann, Hagen/ Andreas, Torsten (2012): Das FalkoHandbuch. Korpusaufbau und Annotationen Version 2.01. Berlin: HumboldtUniversität zu Berlin. http://www.linguistik.huberlin.de/institut/professuren/korpuslinguistik/forschung/falko/FalkoHandbuch_Korpusaufbau\%20und\%20Annotationen_v2.01. [2.5.2014]

RFTagger - German. Brighton: Lexical Computing Ltd. http://www.sketchengine.co.uk/documentation/wiki/tagsets/german_rftagger [22.7.2014]

Römer, Ute/ Schulze, Rainer (Hg.) (2009): Exploring the lexis-grammar interface. Amsterdam u.a.: Benjamins.

Rosen, Alexandr (2012): Grammar-based treebank a happy marriage of empiricism and theory? Praha: Univerzita Karlova. http://utkl.ff.cuni.cz/synttb/Rosen_HappyMarriage.pdf [27.7.2014]

Rösch, Heidi (2011): Deutsch als Zweit- und Fremdsprache. Berlin: Akad.-Verl.

Rychlý, Pavel (2008): A Lexicographer-Friendly Association Score. In RASLAN 2008. Brno: Masarykova Univerzita. S. 6-9.

Sennrich, Rico/ Schneider, Gerold/ Volk, Martin/ Warin, Martin (2009): A New Hybrid Dependency Parser for German. In: Chiarcos, Christian/ de Castilho, Richard Eckart/ Stede, Manfred: Von der Form zur Bedeutung: Texte automatisch verarbeiten/ From Form to Meaning: Processing Texts Automatically. Proceedings of the Biennial GSCL Conference 2009. Tübingen. S. 115-124.

Schiller, Anne/ Teufel, Simone/ Stöckert, Christine (1995): Vorläufige Guidelines für das Tagging deutscher Textcorpora mit STTS. Stuttgart: Universität Stuttgart. http://www.cis.uni-muenchen.de/ schmid/tools/TreeTagger/data/stts_guide.pdf [18.5.2014]

Schmid, Helmut (1995): Improvements in Part-of-Speech Tagging with an Application to German. Proceedings of the ACL SIGDAT-Workshop. Dublin. ftp://ftp.ims.unistuttgart.de/pub/corpora/tree-tagger2.pdf [20.11.2013]

Schmid, Helmut/ Laws, Florian (2008): Estimation of Conditional Probabilities with Decision Trees and an Application to Fine-Grained POS Tagging. Manchester: COLING 2008. http://www.cis.uni-muenchen.de/ schmid/papers/Schmid-Laws.pdf [23.3.2014]

Sinclair, John McHardy (Hg.) (2004): How to use corpora in language teaching. Amsterdam u.a.: Benjamins.

Skalička, Vladimír (1957): Vztah morfologie a syntaxe. In: Slovo a slovesnost, Jg. 18 /1957, Nr. 2. S. 65-71. http://sas.ujc.cas.cz/archiv.php?art=885 [12.4.2014]

Sketch Engine (2014): Sketch Engine. Text corpora and corpus tools for all. Brighton: Lexical Computing Ltd. http://www.sketchengine.co.uk/ [22.7.2014]

SNK (2013): Slovenský národný korpus. Bratislava: Jazykovedný ústav L. Štúra SAV. http://korpus.juls.savba.sk [27.7.2014]

Sorger, Brigitte (2012): Der Internationale Deutschlehrerverband und seine Sprachenpolitik Ein Beitrag zur Fachgeschichte von Deutsch als Fremdsprache. Innsbruck:

Studienverlag. 
Sorger, Brigitte (2013): Institutions- und sprachenpolitische Aspekte des DACH-Konzepts. In: Demmig, Silvia/ Hägi, Sara/ Schweiger, Hannes (Hg.): DACH-Landeskunde. Theorie - Geschichte - Praxis. München: Iudicium. S. 32-48.

Sorger, Brigitte/ Káňa, Tomáš / Janíková, Věra/ Reitbrecht, Sandra/Brychová, Alice (2013): Schreiben in mehreren Sprachen. Deutsch nach Englisch: Mehrsprachigkeit und ihr Einfluss auf die Textkompetenz. Brno: Tribun EU.

Sparling, Don (1989): English or Czenglish? Praha: Státní pedagogické nakladatelství.

Steinbügl, Birgit (2005): Deutsch-englische Kollokationen: Erfassung in zweisprachigen Wörterbüchern und Grenzen der korpusbasierten Analyse. Tübingen: Max Niemeyer.

Storjohann, Petra (2005). Corpus-driven vs. corpus-based approach to the study of relational patterns. Proceedings of the Corpus Linguistics conference 2005 in Birmingham. http://www.corpus.bham.ac.uk/conference2005/index.htm [4.3.2014]

STTS Tag Table (1995/1999): Stuttgart - Tübinger Tag Set. Stuttgart: Universität Stuttgart. http://www.ims.uni-stuttgart.de/forschung/ressourcen/lexika/TagSets/stts-table.html [20.7.2014]

Stuyckens, Geert/ Brône, Geert (2009): Brauchbarkeit von Korpora des geschriebenen Deutsch für DaF-Lehrende. Eine Fallstudie. In: Deutsch als Fremdsprache. Jg. 46, 2009/1. Leipzig: Universität Leipzig.

Swan, Michael (1995): Practical English usage. Oxford: University Press.

Swan, Michael (2006): Chunks in the Classroom: Let's Not Go Overboard. The Teacher Trainer 20/3, 2006. http://www.mikeswan.co.uk/elt-applied-linguistics/chunks-in-theclassroom.htm [20.5.2014]

Tadić, Marko (2009): New version of the Croatian National Corpus. In: Hlaváčková, Dana/ Horák, Aleš/ Osolsobě, Klára/ Rychlý, Pavel (Hg.): After Half a Century of Slavonic Natural Language Processing. Brno: Masaryk University. S. 199-205.

TenTen corpora (2004-2014): Kilgarriff, Adam/ Rychlý, Pavel/ Smrž, Pavel/ Tugwell, David: The Sketch Engine. Proc EURALEX 2004. France: Lorient. S. 105-116. http://www.sketchengine.co.uk. [27.7.2014]

TextSTAT (2014): Hüning, Mathias: TextSTAT 2.9. http://neon.niederlandistik.fuberlin.de/textstat/ [12.6.2014]

Tiedemann, Jörg (2007): Building a Multilingual Parallel Subtitle Corpus. In Proceedings of CLIN 17. Belgium: Leuven, Alfa Informatica, University of Groningen. http://stp.lingfil.uu.se/ joerg/paper/clin17.pdf [27.7.2014]

Tiedemann, Jörg (2012): Parallel Data, Tools and Interfaces in OPUS. In: Proceedings of the 8th International Conference on Language Resources and Evaluation (LREC 2012). S. 2214-2218

Tiedemann, Jörg (2009): News from OPUS - A Collection of Multilingual Parallel Corpora with Tools and Interfaces. In: Nicolov, Nicolas/ Bontcheva, Kalina/ Angelova, Galina/ Mitkov, Ruslan (Hg.): Recent Advances in Natural Language Processing (vol. V). Amsterdam u.a.: Benjamins. S. 237-248.

TNC (2008-2012): Türkçe Ulusal Derlemi/ Turkish National Corpus. Mersin: Mersin Üniversitesi. http://www.tnc.org.tr/ [5.5.2014] 
Tschirner, Erwin (2005): Korpora, Häufigkeitslisten, Wortschatzerwerb. In: Heine, Antje/ Henning, Mathilde/ Tschirner, Erwin: Deutsch als Fremdsprache - Konturen und Perspektiven eines Faches. Festschrift für Barbara Wotjak zum 65. Geburtstag. München: Iudicum.

ukWac (2013): Web corpus of British English. http://wacky.sslmit.unibo.it/doku.php [27.7.2014]

Uri, Helene (2008): Nur die Stärksten überleben. München: Piper.

Variantenwörterbuch (2004): Ulrich Ammon/ Bickel, Hans/ Ebner, Jakob:

Variantenwörterbuch des Deutschen: die Standardsprache in Österreich, der Schweiz und Deutschland sowie in Liechtenstein, Luxemburg, Ostbelgien und Südtirol. Berlin u.a.: de Gruyter.

Westhof, Gerard J. (1987): Didaktik des Leseverstehens. Strategien des voraussagenden Lesens mit Übungsprogrammen. Ismaning: Hueber.

Westhof, Gerard J. (1991): Leseverstehen: Lesen, Lernen, Lehren. In: Andenmatten, Sigrid/ Bruder, Otto/ Faucherre, Alain/ Langer, Michael/ Schwarz, Alexander (Hg.): Verstehen im Deutschunterricht: Didaktik des Lese- und Hörverstehens im Fach Deutsch als Fremdsprache. Bulletin CILA (Schweizerische Hochschulkomission für angewandte Sprachwissenschaft), 1991/53. Neuchâtel: Université de Neuchâtel.

Wichmann, Anne/ Fligelstone, Steven/ McEnery, Tony/ Knowles, Gerry (Hg.) (1997): Teaching and language corpora. London u.a.: Longman.

Wikipedia: Übersetzungen (2014): Koordination von Übersetzungen aus anderen Sprachversionen der Wikipedia in die deutschsprachige. Absatz: Kopieren der Versionsgeschichte.

http://de.wikipedia.org/wiki/Wikipedia:\%C3\%9Cbersetzungen\#Lizenzfragen:_Urhebe rrecht_und_Originaltext. [13.1.14]

Wilson, Andrew/ Archer, Dawn/ Rayson, Paul (Hg.) (2006): Corpus linguistics around the world. Amsterdam u.a.: Rodopi.

Wortschatz-Portal (1998-2014): Deutscher Wortschatz. Leipzig: Universität Leipzig. http://wortschatz.uni-leipzig.de/ [15.7.2014]

Wray, Alison (2002): Formulaic Language and the Lexicon. Cambridge: University Press.

Zapletal, Štěpán/ Jungwirth, Karel/ Kouřimská, Milada (1980): Praktická mluvnice němčiny. Praha: Státní pedagogické nakladatelství.

Zifonun, Gisela/ Hoffmann, Ludger/ Strecker, Bruno/ Ballweg, Joachim/ Brauße, Ursula/ Breindl, Eva/ Engel, Ulrich/ Frosch, Helmut/ Hoberg, Ursula/ Vorderwülbecke, Klaus (1997): Grammatik der deutschen Sprache. Berlin u.a.: de Gruyter. 


\section{Index}

AAC

$28,48,54$

Austrian Academy Corpus

Abfrage $\quad \mathbf{1 5}$

Form oder Formel, die ins Suchfeld eingegeben wird (z.B. müd.* oder [tag="ITJ"]), inklusive Einstellung des Korpus

Abfragen COSMAS II

60ff

Abfragen DGD

Abfragen DWDS

Abfragen InterCorp

84ff

Abfragen Korpus-C4 $54 f$

Abfragen Wortschatz-Portal

ADABA

26, 70, 110

Österreichisches Aussprachewörterbuch, österreichische

Aussprachedatenbank; ein korpusähnliches Instrument

Affix

$58,61,114,203$

gebundenes Wortelement (existiert in der Sprache nicht frei);

Oberbegriff für Präfix, Suffix, (Zirkumfix, Infix)

Akkusativ

130, 133, 162, 186

Akkusativverbindung

Alignment

(in Parallelkorpora:) Anpassung des Textes zu einer anderen

Sprachparallele; (in multimedialen Korpora:) Synchronisierung des

Textes mit einer anderen medialen Form (z.B. Tonspur)

Allonym

unterschiedliche Namensformen

Anglizismus

sprachliche Entlehnung (Wort oder Wortverbindung) aus dem

Englischen

\section{Annotation}

zusätzliche Informationen zu den Korpustexten $(\rightarrow$ siehe

Metainformation) oder zu einzelnen Wörtern im Korpus $(\rightarrow$ siehe

Tag)

Appellativum $\rightarrow$ siehe Gattungsname

$65,90,127$

Attribut (allgemein)

$162,179,210$

Attribute

$83,88,97,102$

(in der Abfrage InterCorp) Charakteristik des Tokens: Wortform,

Lemma, Tag

Auslaut

19, 29, 80, 204

Laut(e) am Ende eines Wortes

117,121 
Auxiliar

136ff, 205

Hilfsverb

BAS

Bayerisches Archiv für Sprachsignale

Beleg

Endergebnis einer Abfrage (Konkordanz, Kollokationspartner, Tag, Textsequenz)

\section{Betonung}

Biegungsformen/ Flexionsformen

88, 135

BNC

British National Corpus (Britisches Nationalkorpus)

\section{CHTK}

Schweizer Text Korpus

\section{Chunk}

auch formulaic language; Kombination von mehreren sprachlichen Elementen, die sich in der Sprache häufig wiederholt (Phrasen, aber auch ,ritualisierte“ lockere Wortkombinationen)

\section{ČNK}

Český národni korpus (Tschechisches Nationalkorpus)

\section{ČNPK}

Česko-německý paralelní korpus (Das tschechisch-deutsche Parallelkorpus): ausgewogenes Parallelkorpus mit deutschen und tschechischen Originaltexten

\section{Computerlinguistik}

Wissenschaft zur Erforschung von Sprache(n) mithilfe der EDV

\section{CORIS}

Corpus di italiano scritto (Korpus des geschriebenen Italienischen)

\section{Corpus del Español}

Korpus des Spanischen

\section{COSMAS II}

Korpusmanager des DeReKo

CQL

Corpus Query Language: „Sprache“ des Korpusmanagers

\section{CRFP}

Le Corpus de Référence du Français Parlé (Referenzkorpus des gesprochenen Französischen)

\section{DaF/ DaM/ DaZ}

Deutsch als Fremdsprache/Muttersprache/Zweitsprache

\section{Dativ}

130, 165, 186 
Deutsch Diachron Digital (historische deutsche Korpora)

\section{Deklination}

Biegung/ Flexion der Substantive, Adjektive, Pronomina

\section{DeReKo}

25f, $56 f f$

Deutsches Referenzkorpus (bislang größtes) Korpus der deutschen Sprache

\section{deTenTen}

German TenTen corpus: milliardengroßes Korpus der deutschen Sprache (nur Internettexte)

deWac

Deutsches Webkorpus: Korpus mit deutschen Internettexten

DGD

Datenbank für gesprochenes Deutsch: Korpus der gesprochenen deutschen Sprache

\section{diachrones Korpus}

Korpus mit Texten aus mehreren Epochen

\section{DWDS}

Das Digitale Wörterbuch der deutschen Sprache

\section{Eingabe/ Suchfeldeingabe}

Form oder Formel, die ins Suchfeld eingegeben wird (z.B. müd.* oder [tag="ITJ"])

\section{EThEG}

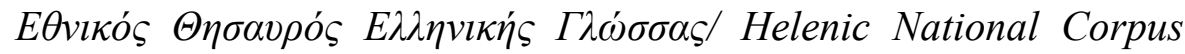
(Griechisches Nationalkorpus)

\section{Falko}

Fehlerannotiertes Lernerkorpus des Deutschen als Fremdsprache

\section{FIDAPLUS}

Korpus der slowenischen Sprache

Filter

Sortierungsmechanismus der Konkordanzen

Flexionsformen/ Biegungsformen

FnhdC

Das Bonner Frühneuhochdeutschkorpus

\section{FORWO}

(Internet-Selbsthilfe-)Aussprachewörterbuch

\section{FRANTEXT}

Base textuelle FRANTEXT (Korpus des geschrieben Französischen)

\section{Frequenz}

Anzahl (absolute F.) oder Häufigkeit (relative F.) des Vorkommens im Korpus 
Corpus électronique de français constitué à partir du web: französisches Web-Korpus

Gattungsname, Appellativum

$65,90,127$

allgemeine Bezeichnung für Gegenstände, Ereignisse etc. (kein Eigenname)

Genitiv

101, 186, 214

GeWiss

25,70

Korpus gesprochener Wissenschaftssprache (spezifisches Vergleichskorpus Deutsch, Englisch und Polnisch von L1- und L2Sprechern/-innen)

\section{GloWbE}

Corpus of Global Web-Based English: Korpusprojekt für englische Varietäten (nur Internettexte)

\section{Grammatik}

128,135

\section{graphische Eingabe}

Suchfeldeingabe mittels Buttons

\section{Häufigkeitsklasse}

Angabe über die relative Frequenz eines Wortes im Korpus/ in der Sprache bezogen auf das häufigste Wort

HGC

Huge German Corpus: Großes deutsches Korpus mit Zeitungsartikeln und Rechtstexten

\section{historisches Korpus}

Korpus mit historischen Texten

HNK

Hrvatski nacionalni korpus (Kroatisches Nationalkorpus)

Illokutionsverb

Verb, aus dem die Intention des Sprechers ersichtlich ist.

innere Annotation $\rightarrow$ siehe Tag

\section{InterCorp}

Parallelkorpora mit vielen Sprachen (manuell alignierte Texte unterschiedlicher Gattungen)

itWac

Web corpus di italiano: italienisches Web-Korpus

\section{KGSR}

Korpus der gesprochenen Sprache im Ruhrgebiet

\section{Kollokation}

$17,99 f f$

(allgemein) semantische Verbindung; hier: gemeinsames

Vorkommen der Elemente im Korpus (auch Kookkurrenz)

\section{Konjugation}

Biegung/ Flexion der Verben 
das gesuchte sprachliche Element mit Kontext

Konkordanzprogramm

$16,105 f f$

einfaches elektronisches Instrument für die Suche nach Wortformen in beliebigen elektronischen Texten

Kookkurrenz $\rightarrow$ siehe Kollokation

Korpus

(allgemein) Körper, Belegsammlung, Untersuchungsobjekt; hier: Sprachkorpus

\section{Korpus C4}

Korpusprojekt für deutsche Varietäten

\section{korpusähnliches Instrument}

ein Software- oder Webinstrument mit einigen Korpuseigenschaften

\section{Korpuslinguistik}

Wissenschaft zur Erforschung der Sprache(n) mithilfe von Sprachkorpora

\section{Korpusmanager}

Suchmaschine für das Suchen in Korpustexten

KWIC

Key Word in Context: auch Node; das gesuchte sprachliche Element

\section{Länderansicht}

Ergebnispräsentation sortiert nach Ursprungsland der Belege (im DeReKo: D-A-CH)

lc

lower case: Wortformen ohne Unterscheidung der Groß/Kleinschreibung

\section{Lemma}

Grundform eines Wortes

lemma_lc

lemma - lower case: Lemma ohne Unterscheidung der Groß/Kleinschreibung

Lemmatisierung

Zuweisung der flektierten Formen zu einer Grundform

\section{Linguee}

korpusähnliches Instrument (halbautomatisches

Übersetzungswörterbuch)

Metainformation/ Metadaten

Informationen über den Aufbau und Inhalt des Korpus

MHDBDB

Mittelhochdeutsche Begriffsdatenbank

MNSz

Magyar Nemzeti Szövegtár (Ungarisches Nationalkorpus) 
MULTEXT-East

Multilingual Text Tools and Corpora for Central and Eastern European Languages

NKJP

Narodowy Korpus Języka Polskiego (Nationalkorpus der polnischen Sprache)

NKRJ

Nationalkorpus der russischen Sprache

Node $\rightarrow$ siehe KWIC

16,98

OPUS

The Open Parallelkorpus: Parallelkorpora vieler Sprachen, automatisch alignierte Internettexte

Orthographie

orthographische Suche

Abfrage über das „normale“ (standardisierte) Schriftbild

Parallele

Korpustexte in einer Sprache, zu denen es in demselben Parallelkorpus Pendants noch in einer anderen Sprache gibt

\section{Parallelkorpus}

Korpus mit gleichen (übersetzten) Texten in mehreren Sprachen

Parser

Programm für syntaktisch-semantische Analysen von Korpustexten

ParZu

Online Parser

phonetische Suche

Abfrage über die phonetische Transkription

\section{Platzhalter/ Platzhalterzeichen}

Sonderzeichen für die Abfrage nach einer offenen Zeichenkette

\section{Präfix}

gebundenes Wortelement (existiert in der Sprache nicht frei)

positioniert vor der Wortbasis

\section{Position}

Stellung eines Tokens/ Elements im laufenden Text (oft Abstand zum KWIC)

\section{Regulärer Ausdruck}

Sonderzeichen oder eine Kette von (Sonder)Zeichen zur Erstellung von komplexeren Korpusabfragen

\section{RFTagger}

Korpustool für eine feine Annotation einzelner Wörter mit morphosyntaktischen Informationen (inkl. Zuweisung der Grundformen). 
sprachliche Angabe der Richtung, realisiert durch Lokalpräpositionen

\section{Segment}

(allgemein) ein Teil eines Textes (Absatz, Satz...); (im Parallelkorpus) minimale Einheit, die aligniert $(\rightarrow$ siehe Alignment $)$ wird.

SNK

Slovenský národný korpus (Slowakisches Nationalkorpus)

\section{Sprachkorpus}

elektronische Textdatenbank mit Suchmöglichkeiten nach sprachlichen Elementen

\section{STTS}

Stuttgart - Tübingen Tagset: Verzeichnis der morphosyntaktischen Zeichen (u.a. für Deutsch) in annotierten Korpora

Suchfeldeingabe $\rightarrow$ siehe Eingabe

Suffix

gebundenes Wortelement (existiert in der Sprache nicht frei) positioniert nach der Wortbasis

\section{synchrones Korpus}

Korpus mit gegenwärtigen Texten

syntagmatische Muster

69,166

Ergebnis einer Kookkurrenzanalyse (DeReKo) in Form konkreter Verbindungen (Chunks)

\section{Tag}

morphosyntaktisches Zeichen/ Angabe über morphosyntaktische Funktion des Tokens

\section{Tagger}

Programm für die morphosyntaktische Annotation von Korpustexten

\section{Tagset}

Liste der morphosyntaktischen Symbole und Zeichen

88, 204ff

\section{TextSTAT}

ein frei zugängliches Konkordanzprogramm

Token

formal selbstständige Einheit (Zeichenkette oder ein Zeichen) im Korpustext

\section{Tokenisierung}

Segmentierung der Texte in kleinere Einheiten: Absätze, Sätze, Wörter und Satzzeichen (in allen gängigen Korpora); in geparsten Korpora: Segmentierung in Phrasen und Satzkonstituente

\section{Treebank}

Korpora mit geparsten $(\rightarrow$ siehe Parser) Texten

\section{TreeTagger}

88, $206 f f$

Korpustool für Annotationen einzelner Wörter mit morphosyntaktischen Informationen inkl. Zuweisung der Grundformen 
Ergebnis einer Abfrage/ Konkordanzen

TUD

Türkçe Ulusal Derlemi (Türkisches Nationalkorpus)

ukWac

Web corpus of British English

Verbalkomplex

$155 f f$

Prädikat mit mehreren Verben

Verbzusatz

abtrennbarer Teil eines Verbkompositums

62ff, 146ff, 193

Vergleichskorpus

Korpus mit Texten in mehreren Sprachen zu vergleichbaren Themen (keine Übersetzungen)

\section{Word Sketch}

automatisch berechnete Verbindungen und grammatikalische Eigenschaften eines Wortes

Wortfamilie

Wörter mit gleichem Wortstamm

Wortform

(in der Korpuslinguistik) ununterbrochene Zeichen- oder Graphemkette (ohne Leerzeichen)

\section{Wortschatz-Portal}

25f, 49ff

korpusbasiertes Portal zum Wortschatz deutscher Internettexte (inkludiert auch andere Sprachen)

Wortteil

$12,14,51,71$

auch Intervall; Buchstabenkombination mit offenem Anfang oder Ende oder mit offenem Anfang und Ende

Wortwurzel

Basis eines komplexen Wortes

zeilenorientierte Eingabe

Sucheingabe direkt ins Abfragefenster 


\section{Sprachkorpora in Unterricht und Forschung DaF/DaZ}

Tomáš Káňa, Ph.D., Mgr.

Herausgegeben von der Masaryk-Universität 2014

Auflagenhöhe: 100 Exemplare

1. Auflage, 2014

MSD, spol. s r.o., Lidická 23, 60200 Brno, www.msdbrno.cz

ISBN 978-80-210-6994-7 